\title{
Development of a Public Transit Information System Using GIS and ITS Technologies
}

\author{
Submitted by Sarah J. Riley, B.Eng. \\ Carleton University \\ Ottawa, Ontario, 2002
}

\begin{abstract}
A thesis submitted to the Faculty of Graduate Studies and Research in partial
\end{abstract} fulfillment of the requirements for the degree of Master of Applied Science

\author{
Department of Civil and Environmental Engineering \\ Carleton University \\ Ottawa, Ontario
}

(C) Copyright 2002

Sarah J. Riley

The Master of Applied Science in Civil and Environmental Engineering Program is a joint program with the University of Ottawa, administered by the Ottawa-Carleton Institute for Civil Engineering. 
National Library

of Canada

Acquisitions and

Bibliographic Services

395 Wellington Street

Otawa ON K1A ONA

Canada
Bibliothèque nationale du Canada

Acquisitions et services bibliographiques

395, rue Wellington

Ottawa ON KIA ONA

Canada
The author has granted a nonexclusive licence allowing the National Library of Canada to reproduce, loan, distribute or sell copies of this thesis in microform, paper or electronic formats.

The author retains ownership of the copyright in this thesis. Neither the thesis nor substantial extracts from it may be printed or otherwise reproduced without the author's permission.
L'auteur a accordé une licence non exclusive permettant à la Bibliothèque nationale du Canada de reproduire, prêter, distribuer ou vendre des copies de cette thèse sous la forme de microfiche/film, de reproduction sur papier ou sur format électronique.

L'auteur conserve la propriété du droit d'auteur qui protège cette thèse. $\mathrm{Ni}$ la thèse ni des extraits substantiels de celle-ci ne doivent être imprimés ou autrement reproduits sans son autorisation.

\section{Canadàa}




\title{
Acceptance Form
}

The undersigned hereby recommended to the Faculty of Graduate Studies and Research acceptance of the thesis:

\section{Development of a Public Transit Information System Using GIS and ITS Technologies}

\author{
Submitted by: \\ Sarah J. Riley, B.Eng.
}

in partial fulfillment of the requirements for the degree of Master of Applied Science

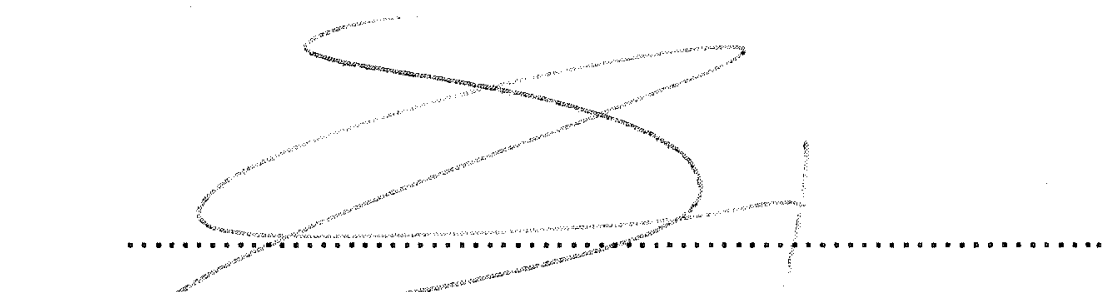

Acting Chair, Department of Civil and Environmental Engineering

A.O. Abd El Halim

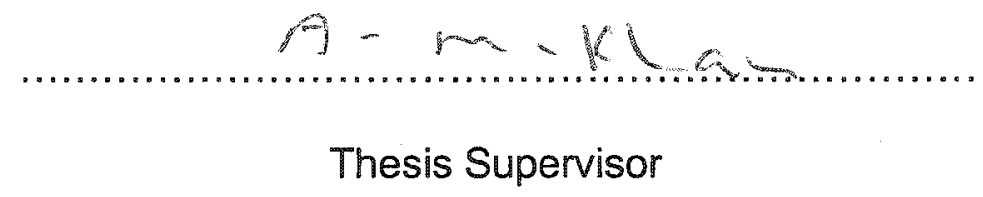

A.M. Khan

Carleton University

July 2002 


\section{Abstract}

In order to attract users to public transit, assist them in planning, and provide other useful information, most transit agencies use a telephone system and/or website that lists bus routes and schedules. But in order to determine an optimum route, an email or a phone call is required. This process is slow and tedious; therefore, an improved method is required to determine the optimal route for an individual, based on an origin and destination of travel.

To achieve this, data from OC Transpo and the City of Ottawa were used to develop a transit information system, which enables the identification of an optimum route for users. In solving these route problems, standard algorithms were modified in order to reflect the constraints of transit vehicles to adhere to fixed routes and schedules. Additionally, new methodology is intended to address the uncertainties in estimating travel time.

To facilitate this process, a Geographic Information System (GIS) software package called ArcView was used. This software utilizes the location of bus stops, scheduling, and route layouts in order to optimize a choice route. Bayesian statistical decision theory was also incorporated into the system to represent the possibilities of deficiencies in schedule adherence. In running the transit information system, the user enters start and end locations, the day, and time of travel. The output is given in the form of a map and trip itinerary.

The developed transit information system entails the latest advances in GIS technologies available, the Bayesian statistical decision theory addressing the uncertainty in schedule adherence and travel time, and accounting for the complexities of transit operation by the system. 


\section{Acknowledgements}

I would like to express my sincere gratitude to my supervisor, Dr. A. M. Khan, Carleton University, for his advice and support.

The City of Ottawa and OC Transpo provided transportation data and transit information. For this, I would like to thank two individuals, who for their invaluable time enabled me to attain the necessary schedule and statistical information. In particular, I would like to thank Jim Summers and Brian Barclay for their technical knowledge and feedback.

I would also like to thank my family and friends for their continued support and encouragement in completing this thesis. Finally, I would like to offer a sincere appreciation to my husband, Kevin, whose support and understanding has helped me pursue my goals. 


\section{Table of Contents}

\section{Pages}

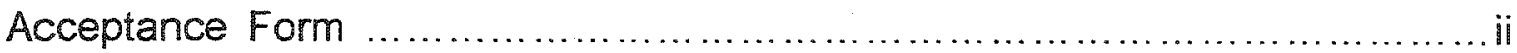

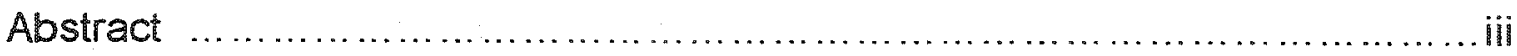

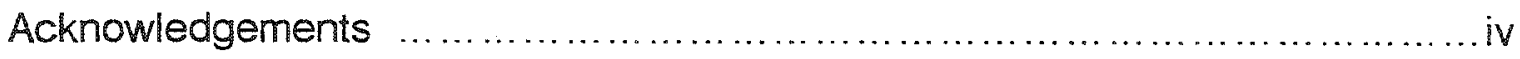

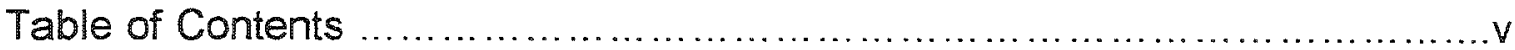

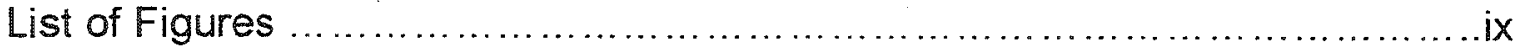

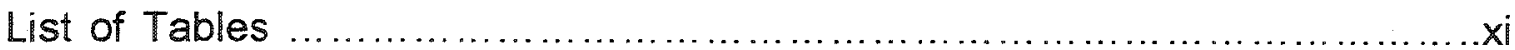

List of Appendices ............................................................ xii

List of Symbols and Acronyms ..................................................iii

\section{CHAPTER 1: PROBLEM IDENTIFICATION AND} METHODOLOGY FRAMEWORK .......................................................1

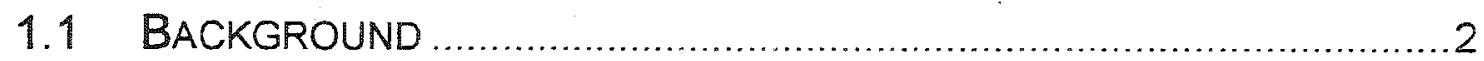

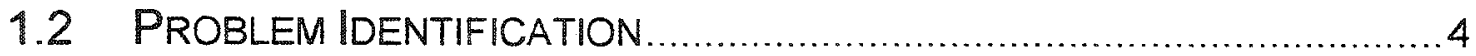

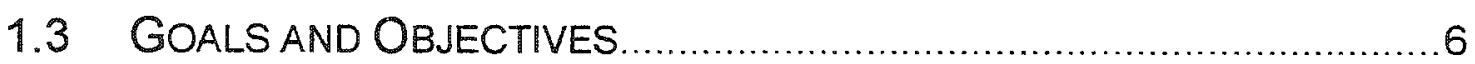

1.4 STUDY METHODOLOGY .........................................................

1.5 METHODOLOGICAL FRAMEWORK …............................................. 10

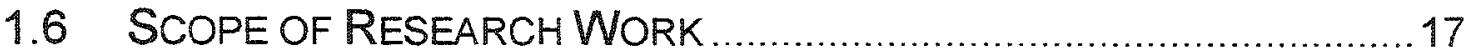

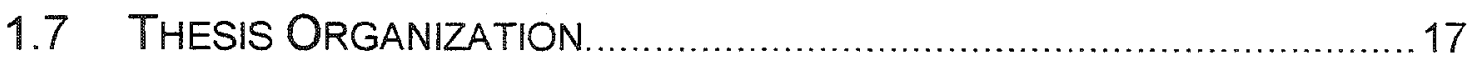

\section{CHAPTER 2: LITERATURE REVIEW.................................20}

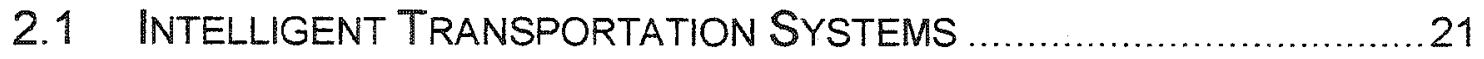

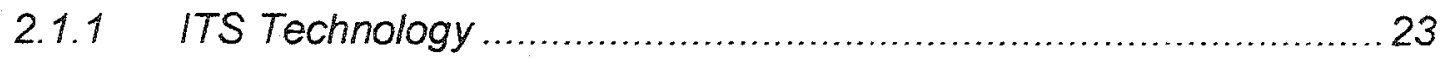

2.1.2 Advanced Public Transportation Systems.............................25

2.1.3 Automatic Vehicle Location (AVL) Devices and Operations .........26

2.1.3.1 AVL System Cost and Benefits..........................................31

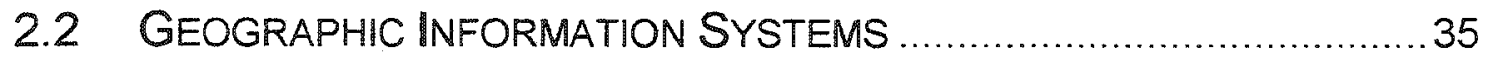

2.2.1 Applications to Public Transportation ....................................40

2.2.2 Fixed-Route Service Planning and Scheduling .......................47

2.2.3 Potential Obstacles to Transit GIS........................................ 49

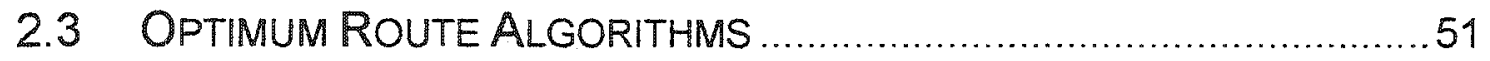

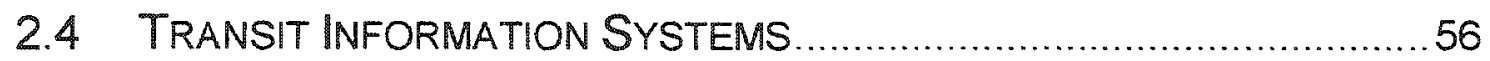




\section{CHAPTER 3: BUILDING BLOCKS OF TRANSIT INFORMATION SYSTEMS}

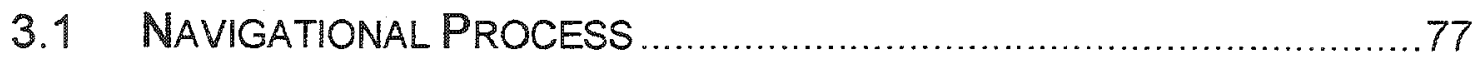

3.1.1 Path Finding Methods............................................................. 77

3.1.2 Requirements to Navigate Unfamiliar Journeys by Transit............78

3.2 GENERAL PRINCIPLES OF TRANSIT INFORMATION DESIGN ............80

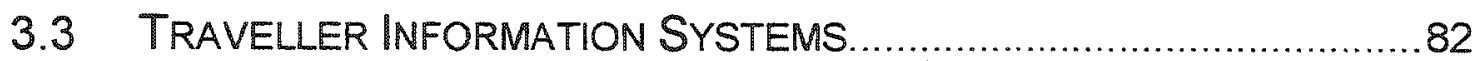

3.3.1 Traveller Information System Functions .................................. 85

3.3.2 Pre-Trip Information........................................................... 85

3.3.3 Effective Traveller Information Systems and Benefits ................88

3.4 TRANSIT SERVICE IN OTTAWA: INFORMATION SYSTEM AND OTHER

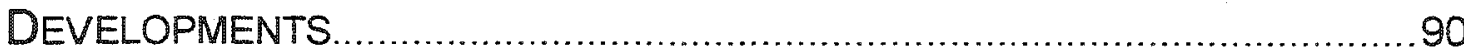

3.4.1 Long-Term Strategy............................................................ 92

3.4.1.1 Market driven and customer oriented services.......................93

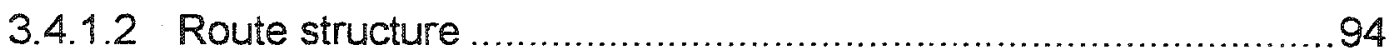

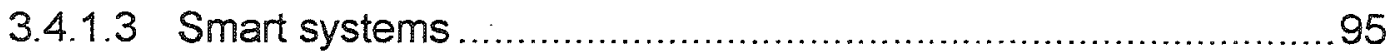

3.4.2 Automatic Passenger Counting (APC) at OCTranspo .................97

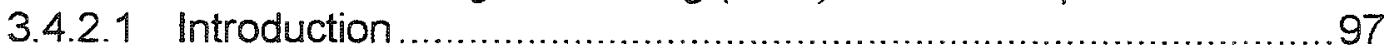

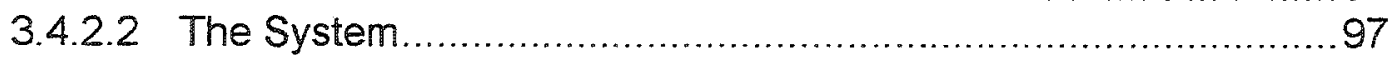

\section{CHAPTER 4: ARCVIEW GIS AND NETWORK}

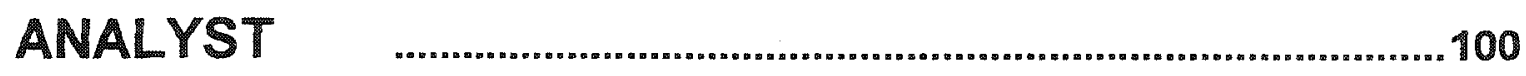

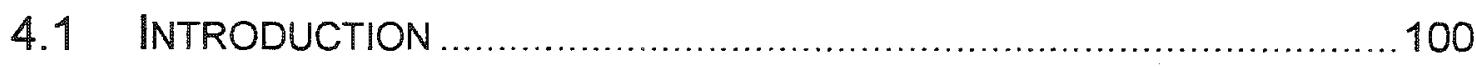

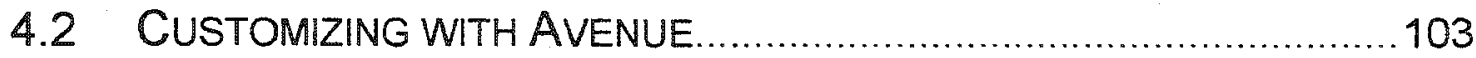

4.2.1 Network Analyst........................................................ 105

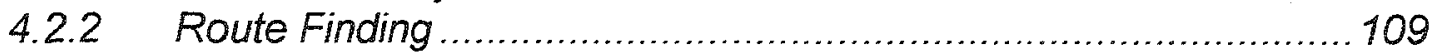

4.2.3 Dijkstra's Algorithm .......................................................... 110

\section{CHAPTER 5: APPLICATION PROCESS AND} ALGORITHM DEVELOPMENT

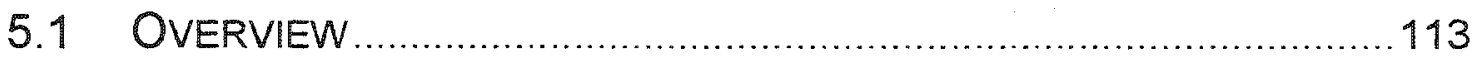

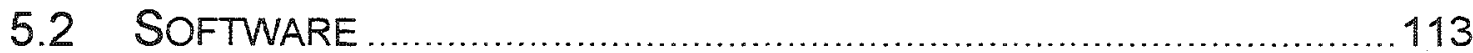

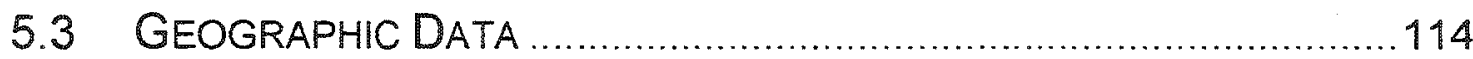

5.3.1 Data Source ............................................................... 114

5.3.2 Setting the Rules of the Network ..........................................116

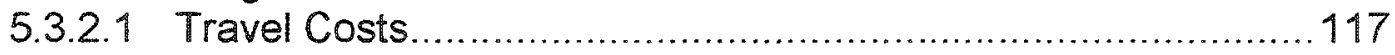




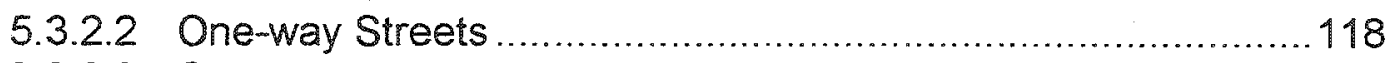

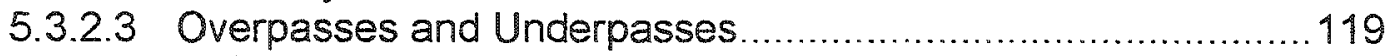

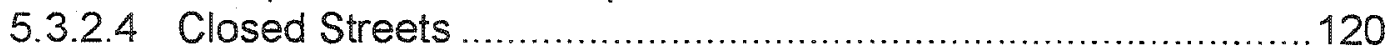

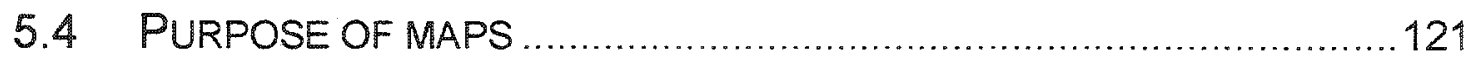

5.4.1 Base Street Network .................................................. 121

5.4.2 Individual Routes and Route Network ...................................122

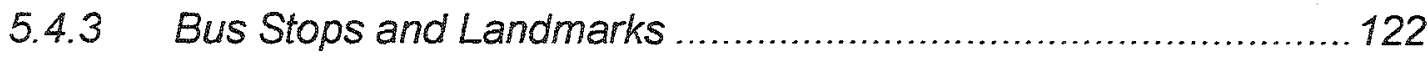

5.5 DATA PREPARATION PROBLEMS AND SOLUTIONS …....................123

5.5.1 Base Street Network.......................................................123

5.5.2 Individual Bus Routes and Route Network ............................ 124

5.5.3 Bus Stops and Landmarks ........................................... 125

5.5.4 Schedule Database ...................................................... 126

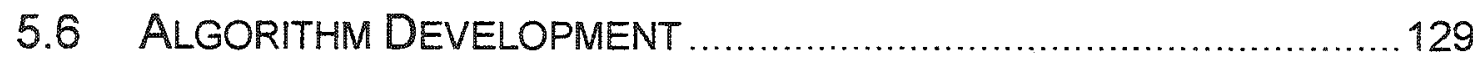

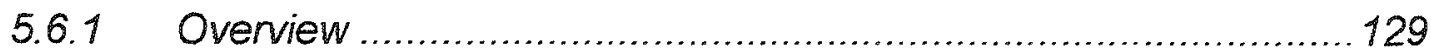

5.6.2 Development within ArcView ............................................. 130

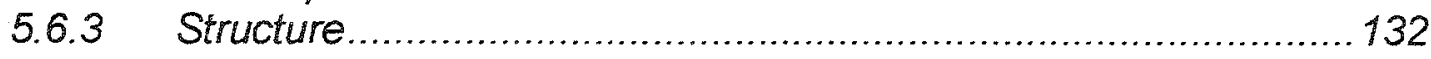

CHAPTER 6: STATISTICAL ANALYSIS ............................. 136

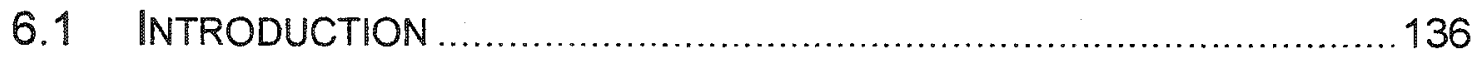

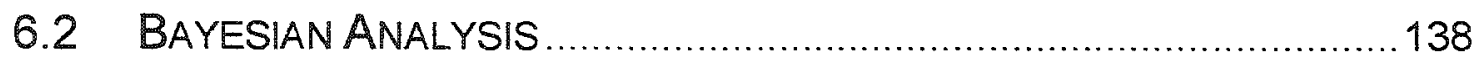

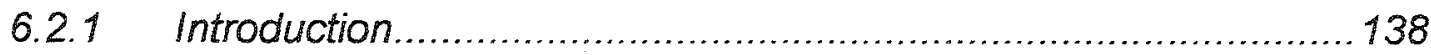

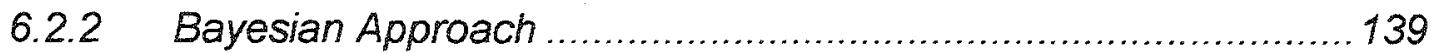

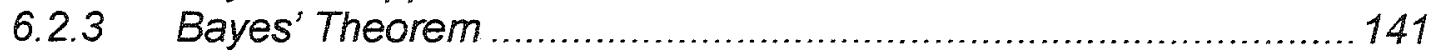

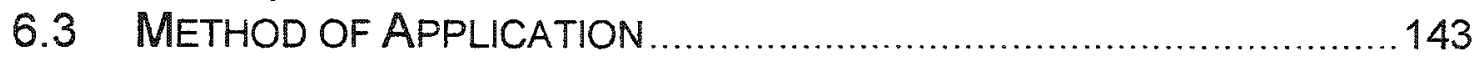

6.3.1 Information Updates ............................................................ 145

6.3.2 Conditional Probabilities ................................................... 145

6.3.3 Posterior Probabilities.............................................................. 149

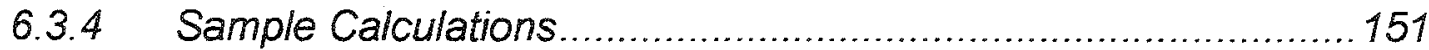

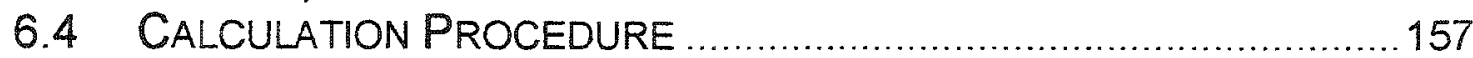

CHAPTER 7: SYNTHESIS AND APPLICATION OF THE TRANSIT INFORMATION SYSTEM .......................................159

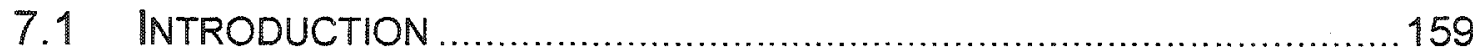

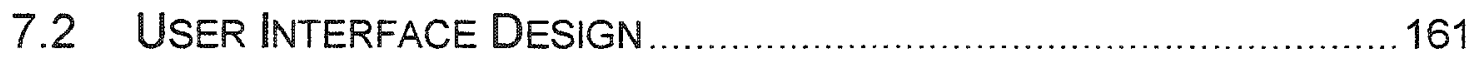




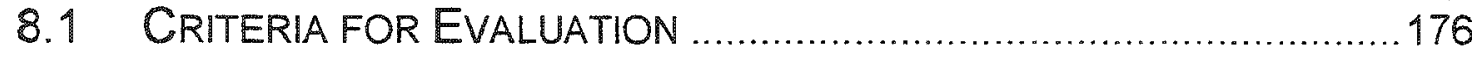
8.1.1 Is the Trip Planner Functioning Properly and Returning Accurate Information?

8.1.2 Is the System Attractive and Easy to Use?..........................176

8.1.3 Further Criteria ............................................................. 177

8.2 PERFORMANCE CRITERIA RESULTS ............................................177

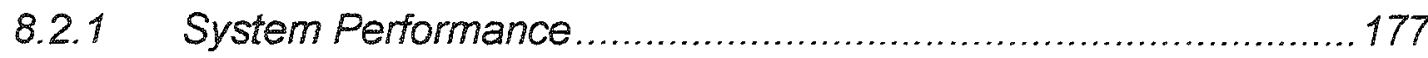

8.2.2 Attractiveness and Ease of Use......................................... 194

CHAPTER 9: SUMMARY, CONCLUSIONS, AND RECOMMENDATIONS ............................................................... 196

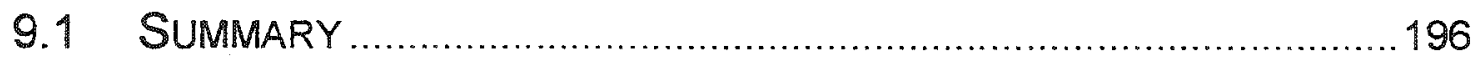

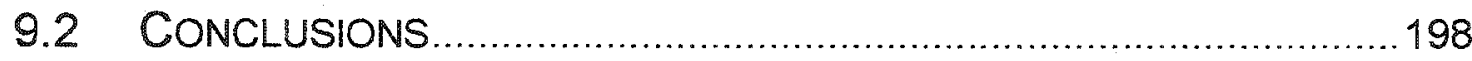

9.3 RECOMMENDATIONS FOR FUTURE RESEARCH...........................201

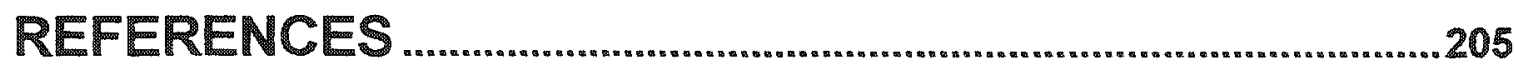




\section{List of Figures}

Pages

Figure 1.1: Study Methodology ...............................................

Figure 1.2: System Design Methodological Framework ........................11

Figure 1.3: Urban Transit Area of the City of Ottawa ...........................13

Figure 2.1: Concept of Overlay .............................................. 38

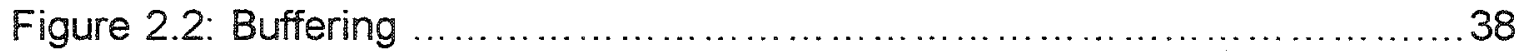

Figure 4.1: ArcView3.2a GIS Interface ..................................... 101

Figure 4.2: Shortest Path Routing ........................................ 108

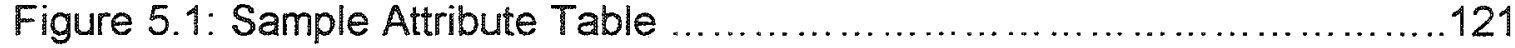

Figure 5.2: Relationships between Tables in the Schedule Database ..........128

Figure 5.3: Syntax of Closest Facility ...................................... 132

Figure 5.4: Algorithm Used Within Transit Information System ................134

Figure 6.1: The Bayesian Statistical Approach ................................140

Figure 6.2: Venn Diagram of Event A and Event B .........................141

Figure 6.3: Venn Diagram of Total Probability ............................... 142

Figure 6.4: Conditional Probabilities ..................................... 148

Figure 6.5: Early Arrival Case ............................................153

Figure 6.6: On Time Arrival Case .......................................... 154

Figure 6.7: Late Arrival Case ................................................. 155

Figure 6.8: Bayesian Statistical Analysis of Time ............................158

Figure 7.1: Overall Structure of Transit Information System ....................160

Figure 7.2: Initial View ...................................................... 162

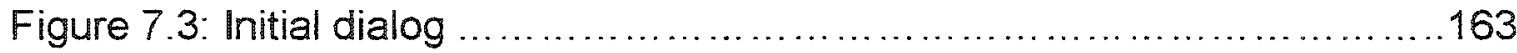

Figure 7.4: Verification Message Box ......................................... 163

Figure 7.5: Dialog Box for Schedule Information ............................. 164

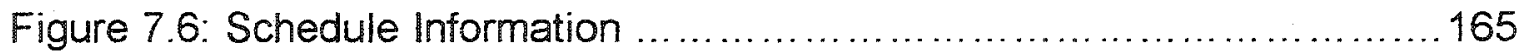

Figure 7.7: Dialog to Select Trip Information ...............................167

Figure 7.8a: Dialog to Enter the Origin Location ............................. 168

Figure 7.8b: Dialog to Enter the Destination Location ........................168 
Figure 7.9a: Origin Landmark Selection ....................................169

Figure 7.9b: Destination Landmark Selection ................................. 170

Figure 7.10: Message Box for Solving the Optimum Route .................171

Figure 7.11: Message Box for Selecting the Origin/Destination ............... 171

Figure 7.12: Message Box for Solving the Optimum Route using Point and

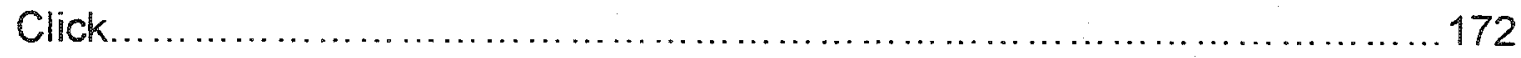

Figure 7.13: Buttons in Application ....................................... 173

Figure 7.14: Cost Selection ............................................... 174

Figure 7.15: Report Box Reporting Results ................................. 174

Figure 7.16: Results Image ................................................... 175

Figure 8.1: OC Transpo Route Network ......................................189

Figure 8.2: Path Change Due to Detour ..................................... 192

Figure 8.3: Route Number Change Due to Detour .............................193 


\section{List of Tables}

Page

Table 3.1: Types of Information Aids ........................................ 81

Table 5.1: Shape Types of Maps Used in the Transit Information System .....115

Table 6.1: Discrete Probabilities based on Defined Travel Periods by OC

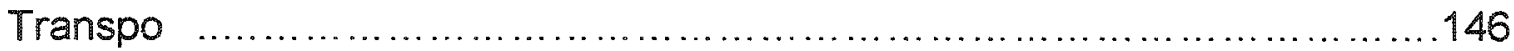

Table 8.1: Weekday Comparison Between Schedule and Algorithm ...........179

Table 8.2: Application of Probabilities to Weekday Trips .......................183

Table 8.3: Application of Probabilities to Saturday Trips ........................ 184

Table 8.4: Application of Probabilities to Sunday Trips .........................185 


\section{List of Appendices}

Page

Appendix A: Variables of Transit Operation ................................211

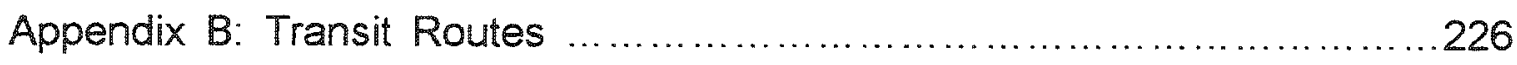

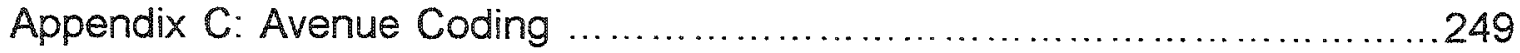

Appendix D: Attribute Tables ................................................412

Appendix E: Calalp.ave ................................................. 443

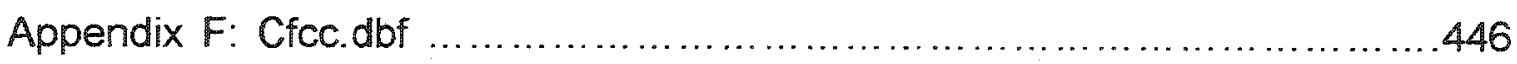

Appendix G: OC Transpo Operating Statistics ..............................450

Appendix H: Schedule Tables ...............................................452 


\section{List of Symbols and Acronyms}

AMS: Automated Mapping Systems

APC: Automatic Passenger Counter

APTS: Advanced Public Transportation Systems

ARTS: Automatic Radio Transmission Systems

ATIS: Advanced Traveller Information Systems

ATP: Automatic Trip Planning

AVL: Automatic Vehicle Location

AVLC: Automatic Vehicle Location and Control system

AVM/C: Advanced Vehicle Monitoring \& Communication System

BDS: Bus Dispatch System

BIS: Bus Information System

CADD: Computer-Aided Drafting and Design Systems

CBD: Central Business District

CUMTD: Champaign-Urbana Mass Transit District

DGPS: Digital Global Positioning System

ESRI: Environmental Systems Research Institute, Inc.

FHWA: Federal Highway Administration

FTA: Federal Transit Administration

GPS: Global Positioning System

GUI: Graphical User Interface

HCM: Highway Capacity Manual

ITS: Intelligent Transportation Systems 
IVHS: Intelligent Vehicle Highway Systems

IVR: Interactive Voice Response telephone systems

LACMTA: Los Angeles County Metropolitan Transportation Authority

MDI: Model Deployment Initiative

MDT: Mobile Data Terminals

O-D: Origin-Destination

O-OP: Object-Oriented Programming

OP: Official Plan

$P(R 1 \mid T 1):$ An example of Conditional Probability for T1

P1, P2, P3...Pn: Different probability conditions

PARIS: Passenger Routing and Information System

PCDs: Personal Communications Devices

PDA: Personal Data Assistant

R1: updated indication of early arrival; corresponds to T1

R2: updated indication of on-time arrival; corresponds to T2

R3: updated indication of late arrival; corresponds to T3

RCTIS: Regional Customer Telephone Information System

R-GRTA: Rochester-Genesee Regional Transportation Authority

SMS: Short Message Service

T1: early arrival at the destination

T2: on-time arrival at the destination

T3: late arrival at the destination

TATIS: Transit Advanced Traveller Information System 
TMP: Transportation Master Plan

TRB: Transportation Research Board

Tri-Met: Tri-County Metropolitan Transportation District of Oregon

USDOT: United States Department of Transportation

UTA: Urban Transit Area

VMS: Vehicle Management System

WAP: Wireless Application Protocol

WSTA: Winston-Salem Transit Authority

WWW: World Wide Web 


\section{Chapter 1: Problem Identification and Methodology Framework}

As a part of the development program for public transportation, and in order to improve the service and achieve customer satisfaction, new technologies are being introduced around the world. These technologies are helping with the increasing demand placed on the transportation networks. The ability to quickly respond to changing situations is essential to the success of transportation agencies. Questions such as where locations are, how to move around, how to quickly get to or get out of an area are faced by people of all walks of life on a daily basis.

The development of intelligent transportation systems (ITS) is bringing about a great change in the public transportation system. Traditional functions of transit services are undergoing important changes. Many transit agencies are considering, and some are implementing, new technologies to enhance their traditional services.

During the 1990's, Geographic Information Systems (GIS) has become one of the fastest growing software to be used within the field of transportation. One reason is that GIS is the ideal information management and analysis tool for many aspects of the transportation industry including both the public and the private sectors. Diverse areas of transportation, including highway, railway, 
airport, bus and rail service planning, transportation modeling, and others, are applying GIS to their work. GIS is emerging as an important planning and management tool for transportation professionals.

\subsection{Background}

One of the important functions of public transit services is to provide customer services, including transit information (Syed and Khan, 2000). Traditional customer information systems provide users with information about routing and schedules by offering route maps, printed transit schedules, and telephone assistance with itinerary trip planning. Generally, printed brochures depict routes in colourful maps and present schedules in a tabular fashion. Smaller systems often include all route schedules in one brochure, while larger systems print multiple brochures that cover the regional subsets of their system.

The telephone system allows a customer to dial a number corresponding to a transit stop. A computer-based system tells the user when a particular bus will arrive at the transit stop. Through a customer service representative, a user can either phone or email the transit agency and provide the origin and destination locations, as well as time requirements. The customer service agent plans a trip from a user's origin to destination based on the printed route map, bus schedules, and notices reflecting updates not yet made on the printed schedules. Some telephone services include software support to assist the transit 
representative in searching for an itinerary, while others simply require the representative to search standard printed route and schedule information. In all cases, the use of a customer service representative supplements standard route and schedule brochures (Smith, 2000). However, neither customer service agents nor users know whether the planned trip is the optimal one. Manual trip planning is a tedious, time-consuming, and often error-prone process, and information is sometimes inconsistent from one operator to another (Peng, 1997).

These traditional approaches have served transit customers well due to a number of strengths. First, printed brochures are portable, providing users with complete information in making decisions at their convenience. Also, they require little to no support from transit agencies' staff. On the other hand, the strength of the telephone service is that it provides immediate, interactive assistance to transit customers. Customers who may have difficulty deciphering the brochure can interact with a transit representative to identify a suitable trip itinerary.

Unfortunately, each approach also suffers from significant weaknesses. Printed brochures are static information distribution tools. They require that the transit user manually complete the complex analysis necessary to extract an itinerary that meets one's needs. In addition, if a change is made to a route or schedule, the brochures must be modified and reproduced. Not only is this costly, but it also results in the risk that a large portion of the transit users will continue to rely 
on outdated brochures to identify trips in a system with a new route/schedule structure (Smith, 2000).

Getting information from a customer representative has weaknesses as well. First, a user can access the service only when a transit representative is working. By expanding the availability of the service temporally, the transit agency is faced with higher operating costs. Finally, given that the service relies purely on verbal communications, it provides no visual information to the customer (Smith, 2000). If the customer is unfamiliar with the region, it may be difficult for a transit representative to describe verbally where a particular stop is located.

Clearly, there is room for improvement in the provision of route and schedule information. In particular, a service that maintains the strengths of brochures and telephone service, while eliminating their weaknesses is most desirable.

\subsection{Problem Identification}

Public transit systems are rarely thought of as a competitive business. In reality, the transit riders are captive consumers of transportation, the transit agency is a producer of transportation, and transportation itself is a competitive marketplace with many different options. For this reason, transit systems are always looking for ways to increase visibility, enhance rider services, and convey traveller information quickly, accurately and effectively. Currently, transit users are 
generally provided with only printed brochures, so there is a need to provide users with more effective tools to assist in selecting trip itineraries. Furthermore, existing methods are not capable of dealing with the uncertainties in estimating travel times from an origin of a trip to the destination.

The Ottawa-Carleton Transit Commission offers its users route and schedule information through its schedule booklets, a web site, and a telephone service. These methods for conveying information have been suitable for current transit users. However, new users sometimes have problems finding the appropriate information.

One problem is the difficulty in finding desired locations (origin or destination) on the available route maps. The bus route maps usually include only the portion of the region's road network that pertains to the particular route. However, accurately pinpointing one's location in relation to the closest bus stop may require a complete road map of the region along with the route map. Another problem is the general difficulty of determining a suitable route using the information available.

The subject of this research covers the methodology for the development of an optimum route identifier potentially applicable to OC Transpo and/or other transit agencies. This development will complement and enhance the efficiency of the current setup of the phone system and web information delivery. 


\subsection{Goals and Objectives}

The main purpose of this thesis is to improve on the current transit services available to users and potential users. The goal is to increase the efficiency of the trip planning process for users and transit customer service personnel. Therefore, a user-friendly, computer-based transit information system was developed. The transit information system determines an optimal trip itinerary based on the traveller's origin and destination at a specific time and day. Hence the system has the following five major attributes:

- List of trip locations and the capacity to enter addresses

- Location of the transit stops

- Schedule Information

- Improvement in estimating travel time

- Route Optimization

In developing this transit information system, the specific objectives were:

- To investigate the current state of transit information services and technology available throughout North America and major cities in Europe, Asia and Australia

- To assess and compare different path finding algorithms

- To develop a user-friendly interface and algorithm using Geographic Information Systems 
- To incorporate a method for dealing with the uncertainties in travel time estimates

- To enable the system to be applicable to any transit agency

- To carry out sample tests to postulate the effectiveness of the system

\subsection{Study Methodology}

A system development study of this nature has to deal with numerous issues and integrate many knowledge items. Figure 1.1 illustrates the general methodology followed for the conduct of this thesis research

Major elements of the study methodology include:

- Problem identification and definition of the various facets of the problem to be solved.

- Development of the methodological framework and identification of the constituent elements

- Literature review, covering ITS and GIS as these relate to the development of the transit information system.

- Definition of the variables of transit operations. 
- Identification of the building blocks of the transit information system, including navigational process, general principles of transit information design, the traveller information component, and an introduction to Ottawa's public transit system.

- Selection and characterization of the ArcView GIS software and Network Analysis.

- GIS-based application process and algorithm development.

- Development of the statistical analysis method.

- Synthesis of the transit information system and application.

- Validation of the transit information system.

- Compilation of summary, conclusions, and recommendations. 


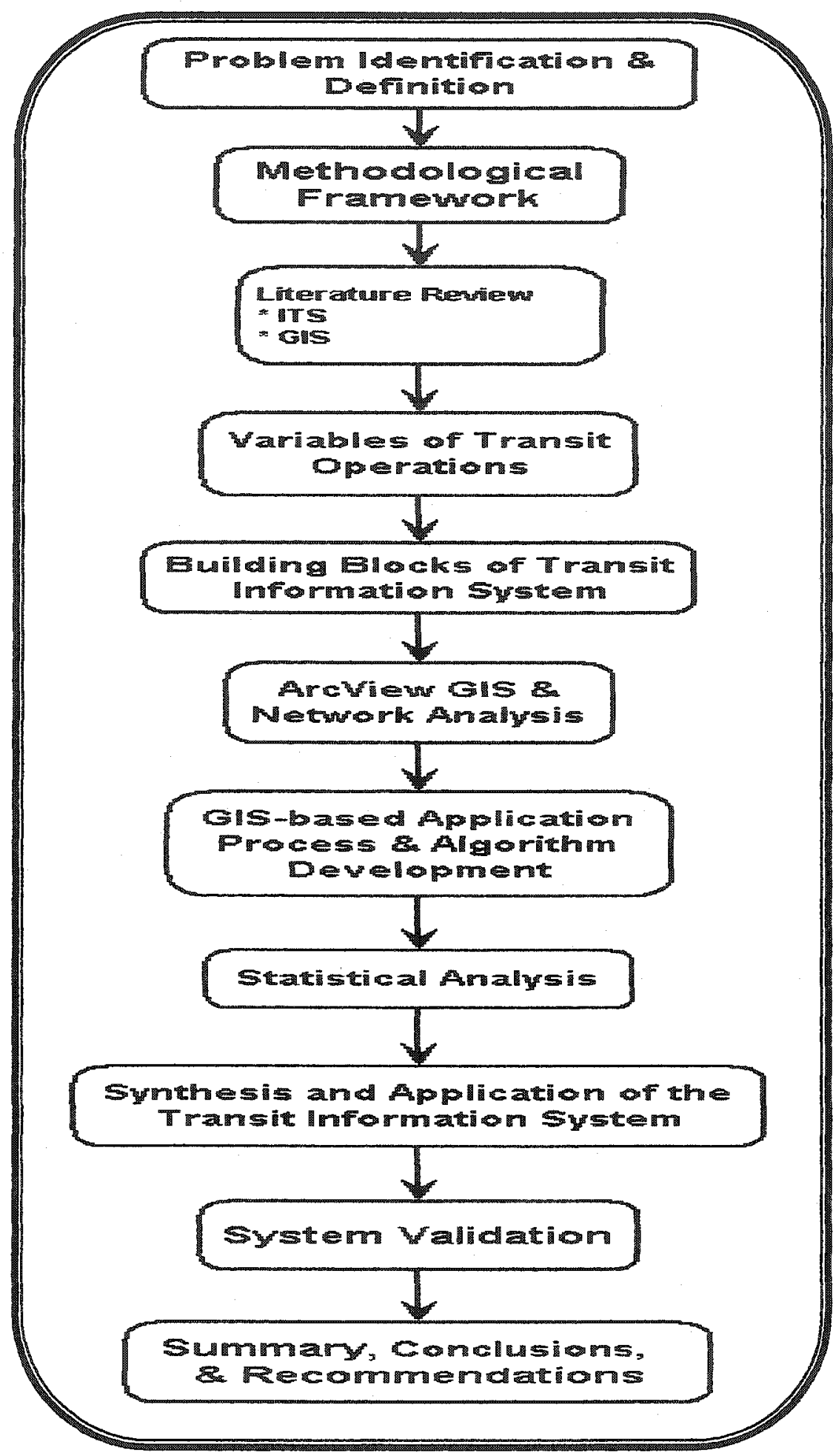

Figure 1.1: Study Methodology 


\subsection{Methodological Framework}

Major elements of the transit information system development process are shown in Figure 1.2. These are noted below:

- Identification of user requirements

- Definition of system elements

- Abstraction of transit system within a GIS software (ArcView)

- Provision for a link with transit company's on-line information on the status of buses (e.g. an automatic vehicle location and control system supplemented with prediction software for bus arrival)

- Algorithm development for the identification of the optimum route

- Incorporation of Bayesian Statistical Analysis into the overall algorithm for the identification of revised optimum route and time solutions

- Provision of outputs to the user 


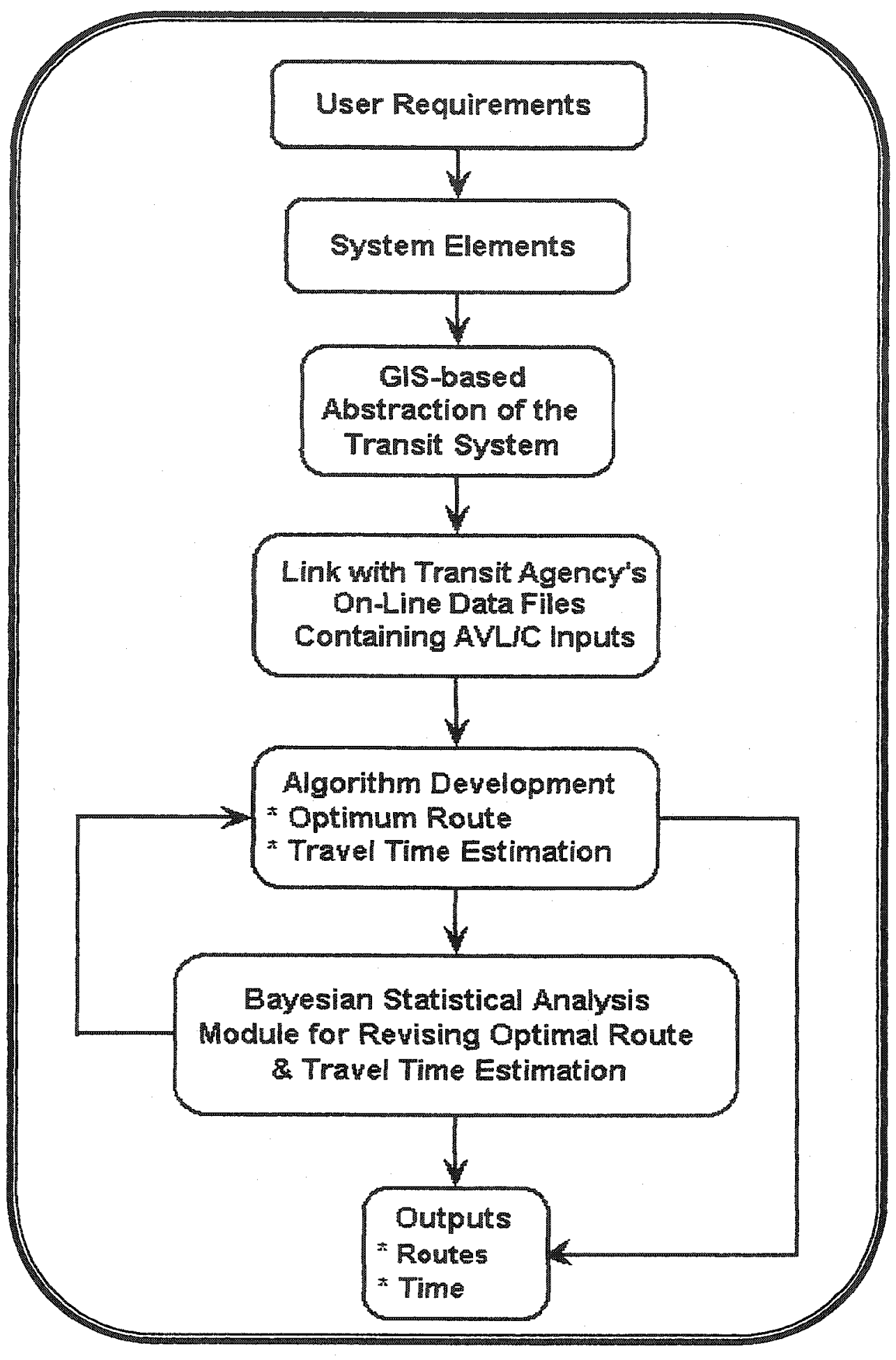

Figure 1.2: System Design Methodological Framework 
A number of explanatory notes would be helpful in describing the selected elements of the methodological framework.

1) Although the generic aspects of the system design form an important part of the methodology, much effort was devoted to an operation system based on OC Transpo data. A selection of routes provided by OC Transpo along with their corresponding bus stop locations was used. This small sample of routes was chosen based on the complexity of the system. In total, 23 routes (Appendix B) were used along with almost 2000 bus stops. These routes were selected from each service time period, namely regular, rush hour, express, and early morning. The school and shuttle services were excluded. These routes were selected in order to obtain a good representation of the Urban Transit Area (UTA) in Ottawa (Figure 1.3). As well, scheduling and service availability was also considered to account for service in both peak and off-peak periods. 


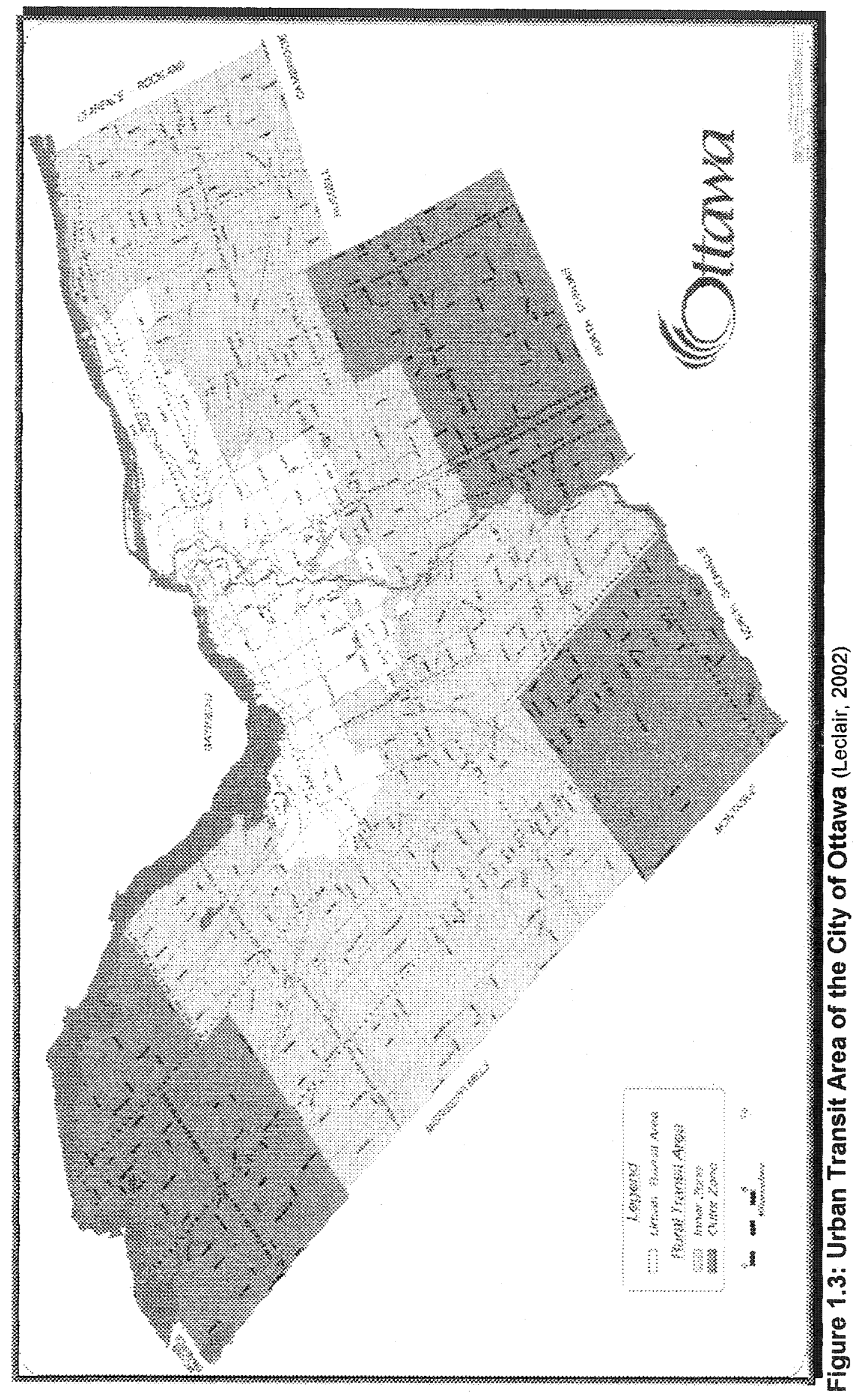


2) It was found that the path finding analysis capability was available in many GIS software programs. Based on a review of systems being used by transportation agencies, it became clear that ArcView and Arc/Info were predominant. Arc/Info is an excellent tool for storing, editing, and querying data as well as digitizing from images, but it requires a great deal of programming in order to perform tasks. ArcView can perform the above mentioned as well as provide an easy to use graphic user interface with menus, dialog boxes, and button bars for working. The system also enables simple programming capabilities as well as attainable system scripts for application. Therefore, ArcView3.2a was selected for use.

3) The algorithm developed will enable the user to request information based on origin and destination points as well as the time and day of departure. In solving the optimum path problem, many standard algorithms do not apply. Time travel on transit networks is constrained by a schedule as well as arrival times may be uncertain due to congestion, weather, or mechanical problems. As is well known, the transit network and service have many unique characteristics that differ from the street and highway network. This can become complex without real-time technology connections using AVL. Even if AVL is used, a prediction of bus arrival is necessary. Therefore it is assumed that users will time their arrivals with reference to the schedule and 
that by applying Bayesian statistics, decisions can be made under uncertainty.

4) In looking at solving an optimum path for transit, there are a few things to consider before applying a standard algorithm. Firstly, transit service is time dependent since it must adhere to a schedule. The availability and the level of transit service vary by time of day and day of week because many transit routes have express and limited services at different times. To plan a trip at a specific time, the trip planning system needs service information for specific times and locations. A trip planning system that is based only on transit route configuration and assumes ever-present availability of service on every route and at all stops will generate erroneous trip plans. Therefore, the most important database requirement of the transit information system design is an incorporation of transit routes and the relevant transit services.

5) As well, in order to assign transit riders to specific routes and stops from the rider's trip origin, a trip planning system needs information on the relationship among transit routes, bus stops, and street network. This requires a relational database to link bus stops, transit routes, and street network.

6) Not every bus stop has a scheduled arrival and departure time. The bus operator is only required to adhere to the schedule for those stops that are on 
the timetable. Therefore, for the purpose of trip planning the location of buses at specific times has to be estimated through linear interpolation.

7) Because of the unique features of a transit network noted above, a system has to be designed to address the time-dependent nature of transit services. It must be user-friendly and enable visualization of schedule information. In performing this task, network and geographic information is required along with a GIS software program.

8) The transit information system design process involves answering four major questions:

- Where is the nearest bus stop? (Location)

- When does the next bus leave? (Scheduling)

- How do I get from where I am to where I want to go? (Route Optimization)

- When will I arrive at my destination? (Travel Time)

In order to formulate these questions, the system will enable geocoding (method of deriving spatial coordinates for tabular data stored in an addressable form) of trip origin and destination points and link them to bus stops and routes. The respective bus routes will be selected and an optimum route will be found. The results will illustrate the preferred route and trip itinerary. 


\subsection{Scope of Research Work}

This thesis research involved the development of a state-of-the-art transit information system by incorporating the latest ITS and GIS technologies and statistical analysis methodology for incorporating new travel time information as it becomes available.

The information system developed is operational. OC Transpo can use it with minor further development work. The system can potentially be accessed wherever web capability is available (e.g. desktop computers, kiosks, and handheld devices such as a cell phone).

The generic nature of the system design enables its application by public transit agencies other than OC Transpo.

\subsection{Thesis Organization}

This thesis is organized into 9 chapters. The first chapter introduces the reader to some background information regarding transit services provided to the customer. Research objectives, study methodology, methodological framework and scope of research are described. Chapter 2 is devoted to literature review covering ITS, GIS, and their application to transit. In Chapter 3 , building blocks of transit information system are described. The navigational process is defined; the path-finding methods and the requirements for someone to navigate unfamiliar journeys by transit are explained. The general principles used in 
transit information design are illustrated that include the types of aids utilized and current suggestions recommended by the Transportation Research Board. The traveller information system and its functions are described. Pre-trip traveller information systems are explained along with some potential obstacles. An overview of transit service in Ottawa is given along with some pre-defined longterm strategies that OC Transpo intends to implement. A review of the SmartSystems and the current Automatic Passenger Counting System used by OC Transpo is described. In Chapter 4, ArcView3.2a is introduced along with its ability of customization. The programming language used in this application is explained as well as its relevance with the necessary extensions used during the development process. In addition, the route finding ability of ArcView is also explained. Chapter 5 begins the application process. In this chapter an overview of the steps taken in the design process are defined. The process used to input data, any modifications, and the applicable costs for the road network are explained. Any data preparation problems and solutions are also mentioned. The algorithm development used within the graphic user interface is explained. The statistical analysis used within the algorithm is introduced in Chapter 6. Here Bayesian statistical analysis is defined and how it is applied to the optimum route path. In Chapter 7 , the application development is explained. Within this chapter the user interface design is explained along with the overall structure in order to describe the process taken by a traveller when using this system. Chapter 8 demonstrates some sample runs of the program in order to compare schedule information to that obtained by the transit information system. A 
summary, conclusions, and recommendations along with some possible future work, are presented in Chapter 9.

This thesis also contains 8 Appendices. In Appendix A, the variables of transit operation are presented. It illustrates modal characteristics and trends along with present transit operations. The role of transit in urban centers is also illustrated as well as some parameters for bus facilities. Appendix B illustrates the transit routes used within the development of the transit information system. These are images of the entire route and the route classification (i.e. early morning, peak, regular). The following appendix is the coding developed for the user interface using the Avenue programming language. It includes the coding for user interface, the Bayesian analysis and the algorithm to determine the optimum route. Appendix $D$ lists the attribute tables used within the user interface. These include all of the locations listed for the user and the bus routes and stops. In Appendix $\mathbb{E}$ the avenue coding used to solve for segment lengths is given. This code must be run before the network analysis can proceed. Another necessity for the network analysis is given in Appendix F. This is a table that enables the coding of speed limits in order to solve for travel time along line segments. The following appendix lists some of OC Transpo's operating statistics. These include the service area and facts. The final appendix lists the schedule tables used within the transit information system. These include weekday, Saturday, and Sunday timetables. 


\section{Chapter 2: Literature Review}

in conducting this literature review, there were four main areas of concentration. Since the main objective of this thesis was to develop a transit information system, research was conducted in this area. This included uncovering past and present transit developments in providing users with customer services. The final section of this review gives an overview of predominantly American and European advances. It was found that very little has been done in the area of transit information systems in Canada.

Since technology plays an important role in the development of a transit information system, exploring technological and software advances in transportation was researched. It was discovered that the application of Intelligent Transportation Systems (ITS) is slowly being integrated to transit services. The most widely used subsections of ITS are the incorporation of Automatic Vehicle Location (AVL) devices and Geographic Information Systems (GIS). Hence, the literature begins with an overview of ITS technology and AVL system operations; the benefits and applications to transit services.

The following section discusses GIS, its components and areas of application. Since this transit information system was developed using a GIS software package, it was important to include an overview. One of the main areas that 
GIS is increasingly being integrated is public transportation; therefore it was essential to find areas where GIS has been applied in the past and illustrate how GIS has helped to resolve these constraints.

As the transit information system is being used to solve for an optimum route, it was crucial to find information pertaining to optimum path algorithms. A review of past developments in optimum route algorithms pertaining to transit is discussed in the third section. Those discussed include generic data modeling and system operation modeling.

This literature review identifies past research and it provides an intellectual context for this thesis enabling a comparison to other work in this area.

\subsection{Intelligent Transportation Systems}

Currently, in the field of transportation, there is a push towards implementing Intelligent Transportation Systems (ITS) technologies. ITS is a broad range of diverse technologies applied to transportation to make systems safer, more efficient, more reliable and more environmentally friendly, without necessarily having to physically alter existing infrastructure. The range of technologies involved includes sensor and control technologies, communications, and computer informatics and cuts across disciplines such as transportation, engineering, telecommunications, computer science, finance, electronic commerce and automobile manufacturing (Transport Canada, 2000). ITS is an 
emerging technology area and is benefiting public and private sectors alike. For example, ITS makes it possible to implement transportation safety compliance and road/bridge toll collection more economically, and to improve corporate productivity through time savings, reduced operating costs and energy consumption, and enhanced reliability and safety (Transport Canada, 2000).

The emphasis in much of ITS has been on road vehicular movement. Nonetheless, there is now a strong transit planning and management component to ITS efforts. Much of this takes the form of integrated information systems (kiosks, computer displays, Internet, postings, phone), common fare cards and SmartCards, articulated schedules (bus-rail), electronic payment, and variable fares. Also, ITS technology is being used to locate buses and improve schedule adherence, and research is continuing to improve ridership and travel time (Roess et al, 1998). Over the years, many other ITS applications have been implemented and planned both for roads and transit by a multiplicity of public and private sector organizations.

One of the preferred platforms for ITS technologies is a Geographic Information System. It is an area that has tremendous potential for better asset utilization and improved safety. Not all ITS applications involve GIS, but a growing number of implementations demonstrate that GIS adds a special new dimension to ITS, especially for incident management and traveller information. Most promising is the ability to use GIS to advise individual and fleet drivers of changing traffic 
conditions en-route using wireless technologies and spatial databases. Like traditional planning and analysis GIS applications, spatially enabled ITS can also be used as a decision support system by incident management staff. Timely, easy access to accurate data helps people make better decisions on both an individual and community wide basis.

The future of ITS is promising. Already, real systems, products and services are at work throughout the world. Still, the wide-scale development and deployment of these technologies represents a true revolution in the way people think about transportation (USDOT, 1998).

\subsubsection{ITS Technology}

In many ways the goal of the ITS movement is to apply modern computer and communications technologies in our transportation systems, resulting in improved mobility, safety, air quality, and productivity. ITS technologies comprise numerous products and services that can influence the public, including (USDOT, 1998):

- Intermodal transportation systems that will make life easier for travellers switching from one mode (e.g., automobile travel) to another (e.g., air or rail travel). 
- Intelligent traffic control systems that automatically adjust to the flow of traffic and reduce the time drivers stop at red lights for no apparent reason.

- In-vehicle technologies, such as traveller information and route guidance systems, and safety enhancement systems.

- Safety enhancement technologies, such as "smart" cruise control systems that take into account the location of the leading vehicle.

- Traveller advisory systems, including changeable message signs and advisory radio.

ITS technologies have the potential to improve the service and productivity of personalized public transit systems. These technologies are useful for both strictly curb-to-curb systems and hybrid systems. Various ITS technologies currently being used by the transit agencies include (Dessouky et al, 2001):

- Scheduling and dispatching software

- Automatic vehicle location devices (AVL)

- Advanced wireless communication

- Mobile data terminals (MDT) 
- Computerized vehicle navigation

- Geographic database

The following emerging technologies are under development (Dessouky et al, 2001):

- Internet dispatching

- Wireless internet dispatching

- Superphones

- Personal data assistants (PDA)

- Smartmaps

- Smart bus technologies

\subsubsection{Advanced Public Transportation Systems}

Advanced Public Transportation Systems (APTS) are advanced navigation and communication technologies that are used in all aspects of public transportation. These include the application of advanced electronic technologies to the deployment and operation of high occupancy vehicles, shared-ride vehicles, conventional buses, rail vehicles and the entire range of paratransit vehicles. They encompass pre-trip travel information, en-route transit information, ride matching and reservation, electronic payment services, public transportation management, personalized public transit, and public travel security (Bang, 1998). These systems 
enable transit agencies to make timely and needed transit information available to passengers, an element that is important to improving the convenience, reliability, and safety of public transportation.

APTS helps transit agencies manage a safe and efficient fleet and plan services to satisfy a broad range of consumer needs. When incorporated with a regional transportation system, APTS can also enable a system to manage its roadways with special accommodations for high occupancy vehicles. APTS applies advanced surveillance and communication technologies to rural area transportation systems with aim to improve safety, increase the efficiency of small community services and provide recreational travellers with location/navigation technologies (Bang, 1998).

\subsubsection{Automatic Vehicle Location (AVL) Devices and Operations} In regards to transit, AVL devices are instrumental in ITS development. They are an electronic communication system for tracking and reporting the location of the vehicles to a central dispatching center. By knowing the location of the vehicle at the time of scheduling (assigning a specific trip request to a specific vehicle), it may be possible to improve the productivity of the system by better matching the passenger's request location with 
the vehicle location. Actual position determination and relay techniques vary, depending on the needs of the transit system and the technology chosen (TRB, 1997). Typically, vehicle position information is stored on the vehicle for a time, which can be as short as a few seconds or as long as several minutes. Position information can be relayed to the control center in raw form or processed on-board the vehicle before its transmission. AVL systems also have the potential to reduce fleet sizes significantly (Dessouky et al, 2001, TRB, 1997).

Transit agencies have implemented AVL systems to assist them in a number of ways. These include the following (TRB, 1997):

\section{Operations}

- Improve schedule adherence.

- Improve service efficiency.

- Achieve better command and control of system.

- Improve bus schedules.

- Pre-process data for dispatcher.

- Facilitate systems integration.

- Improve information accuracy and availability.

- Provide better operations support.

- Reduce the number of street supervisors. 
- Simplify operation of vehicle for the operator.

- Provide customers with real-time service information.

\section{Safety}

- Improve safety on buses.

- Improve response times to incidents and emergencies.

The dominant technology deployed today for locating the vehicle for AVL is the use of the Global Positioning System (GPS). GPS technology uses signals transmitted from a network of 24 satellites in orbit around the earth and received by a GPS antenna placed on the roof of each bus. A GPS receiver calculates its position based on signals received from at least three satellites.

GPS works anywhere the satellite signals reach. However, satellite signals do not reach underground and can be interrupted by the presence of tall buildings or dense foliage. In areas where this is a problem, GPS is often supplemented by another method of position determination, for extrapolation of location from the last GPS reading until the next GPS measurement (TRB, 1997).

Until recently, the accuracy of GPS data was about 100 meters. Through processing, most AVL systems achieve better accuracy 
than standard GPS (USDOT, 2000). However, to further improve the position location accuracy, many transit agencies installed "differential GPS" (DGPS) when techniques such as signal priority were being employed or contemplated. In May 2000, the GPS accuracy was dramatically improved when the US military removed the intentional degradation to the signal that had been in place since GPS began operation. The accuracy of GPS today is between 10 and 20 meters (USDOT, 2000).

Prior to the availability of GPS, the most common form of AVL chosen by transit agencies was the signpost system in which a series of radio beacons is placed along the bus routes. An identification signal is transmitted by the signpost and is received by a short-range communication device on the bus. Since the location of each signpost is known, the location of the bus at the time of passing the signpost is determined. The distance traveled after passing the last signpost is measured by the bus odometer and is then used to estimate the bus position along its route (USDOT, 2000). However, this method can be limiting because signposts are placed at fixed locations. If a bus were to detour part of its route, it could not be tracked and if a transit route were altered additional signposts would be required. 
Transit agencies often incorporate other advanced public transportation system features in conjunction with AVL system implementations. These include the following (USDOT, 2000, Casey et al, 2000):

Normally Integrated with AVL Systems

- Computer-aided dispatch software

- Mobile data terminals

- Emergency alarm

- Digital communications.

Sometimes Integrated with AVL Systems

- Real-time passenger information

- Automatic passenger counters

- Automated fare payment systems

- Automatic stop annunciation

- Automated destination signs

- Vehicle component monitoring

- Traffic signal preferential treatment. 


\subsubsection{AVL System Cost and Benefits}

The capital cost of an integrated installation of AVL and other advanced public transportation system components is dependent on the size of the system, its level of sophistication, and the components to be included. Systems usually include a large amount of equipment (cameras, radio and microwave towers mobile data terminals) and new software. There is a significant cost for the equipment and software that reside at the operations/dispatch center. Often, the installation of AVL coincides with a new or major upgrade to the communications system (USDOT, 2000).

The benefit and cost factors of AVL are numerous and varied. Basically, the AVL technology provides up to the minute information on exactly where the bus is located. This information can be broadcast to an automated dispatch system. This will present bus operational information in real-time to travellers and perhaps affect passenger demand patterns. The improved information enhances passengers' acceptance of the system and improves the perceived reliability of the system (Bang, 1998).

For the transit agency, the real-time information allows operators to monitor the vehicle condition and respond quickly to problems such 
as falling behind schedule or traffic incidents. Enhanced control of vehicle operations and management improves service at lower cost (Bang, 1998). Since AVL technology is a major element of ITS, it provides a linkage by which transit agencies can participate in other fields of ITS applications. For example, a simple extension of the AVL can be applied to on-board passenger information, Advanced Vehicle Monitoring \& Communication System (AVM/C), Vehicle Management System (VMS), pre-trip scheduling system, and demand responsive dispatching (USDOT, 2000).

Many public transit systems where AVL technology has been implemented experience more efficient and on-time operations as their schedules are improved, they are better able to respond to disruptions, (e.g., a disabled vehicle), and bus operators are aware of their schedule adherence (Casey et al, 2000). Safety and security typically increase, since the dispatcher knows immediately where to send help. AVL information also provides very useful inputs to passenger information and traffic signal preferential treatment systems. In the long term, the compiled historic bus position data can be used for planning route, schedules, fleet and personnel deployment (Bang, 1998). 
From studies conducted by U.S. Department of Transportation, the following benefits from integrated implementation of AVL and other advanced public transportation system components were found (USDOT, 2000):

\section{Operations}

- Improved schedule adherence

- Improved transfer coordination

- Improved ability of dispatchers to control bus operations

- Facilitated on-street service adjustments

- Increased accuracy in schedule adherence monitoring and reporting

- Assisted operations during snowstorms and detours caused by accidents or roadway closings

- Effectively tracked off-route buses

- Effectively tracked paratransit vehicles and drivers

- Eliminated need for additional road supervisors

- Reduced manual data entry

- Monitored driver performance

- Received fewer complaints from operators. 


\section{Passenger Information}

- Provided capability to inform passengers of predicted bus arrival times

- Helped meet Americans with Disability Act requirements by using AVL data to provide stop annunciation

- Increased number of customer information calls answered

- Eliminated need to add customer information operators.

\section{Customer Relations}

- Received fewer customer complaints

- Used playback function in investigating customer complaints

- Used AVL data to substantiate agency's liability position

- Improved image of agency.

\section{Scheduling and Planning}

- Provided more complete and more accurate data for scheduling and planning

- Expected to ultimately reduce schedule preparation time and staff

- Aided in effective bus stop placement

- Generated more accurate ridership counts with automatic passenger counters

- Expected to improve bus productivity. 


\section{Safety and Security}

- Used AVL-recorded events to solve fare evasion and security problems

- Reduced the number of on-bus incidents by use of surveillance cameras

- Provided more accurate location information for faster response

- Foiled several criminal acts on buses with quick response

- Enhanced drivers' sense of safety.

\subsection{Geographic Information Systems}

As GIS was first introduced, most people used its software as methods of mapmaking, since manual drafting and map production was slow and tedious. This new method was more efficient and updates of maps were fast and more accurate in terms of data content (Clarke, 1997).

But to appreciate the aims of GIS, it is useful to understand the main components of its title. 'Geographic' is related to data and attributes that have some sort of spatial identity. These might be point features relating to specific sites such as schools, hospitals, or shopping centers; line features such as roads or rivers; or area features such as census wards, parliamentary constituencies, or postal districts/sectors. 'Information' is usually that, which defines something in decision making or planning. This may be present in data sets, data linkages or data 
models. Hence, an information system is a set of organized procedures which, when executed, provides information to support decision-making. Therefore, a geographic information system possesses these properties in relation to geographical data and information (Birkin et al, 1996).

GIS has not only made the production and analysis of geographic information more efficient, it is changing the way geographic information is perceived and used. It is technology that makes geographic data more manageable, more easily shaped by the user into the form best suited to the application at hand. It makes geographic information more easily customized. In a GIS, the storage of data is independent of the mode of presentation. The physical map becomes a relatively inexpensive output product that can be generated quickly and customized for a single application. In addition, the geographic data used to produce the map can be continuously updated. As a result, the physical map becomes a customized "snapshot" of a continuously changing geographic database (Birkin et al, 1996, Clarke, 1997).

The processing power of the GIS has also enabled geographic information to be used in a quantitatively different way. Complex analyses can be iteratively refined towards an optimum solution, an approach that would be prohibitively expensive using manual methods. 
A GIS has four necessary components: computer hardware (a personal computer or a workstation), a software package (for analysis of relationships and interfaces with databases), data (from various government, commercial and internal sources), and people to design it and use it.

Functionally, GIS provides tools for acquisition, editing, managing, analysis, modeling, display, output and dissemination of data. It is a computer-based tool that can perform the tasks of input, storage, retrieval, manipulation, analysis, and output for both spatial and non-spatial data (Clarke, 1997).

Data linkage is one of the most fundamental methods of adding value to data. In most geographic information systems, the concept of overlay is central to the process of linkage; Figure 2.1 illustrates an example of this. Data are stored in layers that can be retrieved from a system and then overlaid one on another to answer questions such as optimal site location. The overlay procedure is often undertaken in conjunction with spatial buffering (Aronoff, 1995). Buffering enables the user to determine an area at a chosen distance from a point or a line feature as shown in Figure 2.2. 


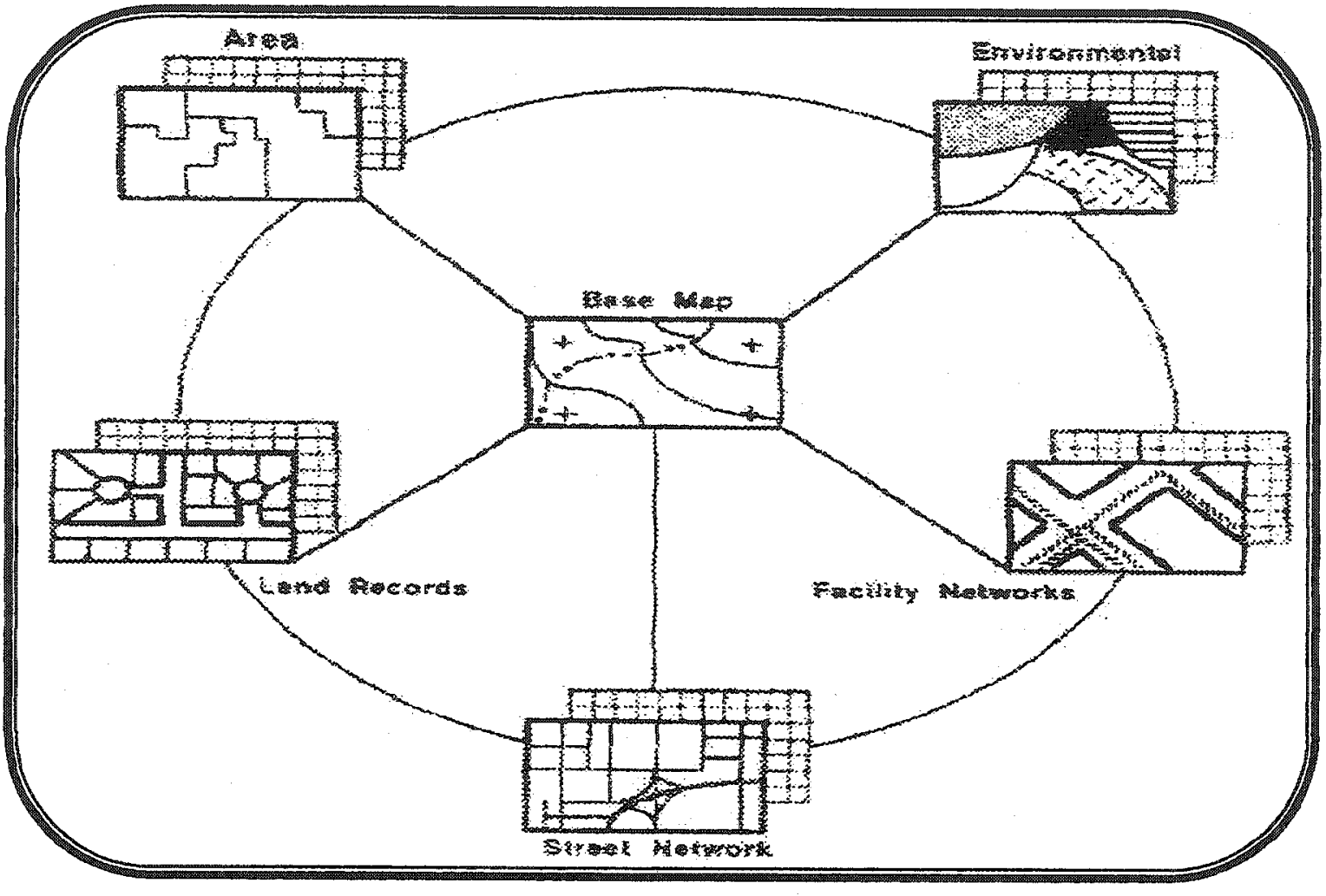

Figure 2.1: Concept of Overlay (Aronoff, 1995)

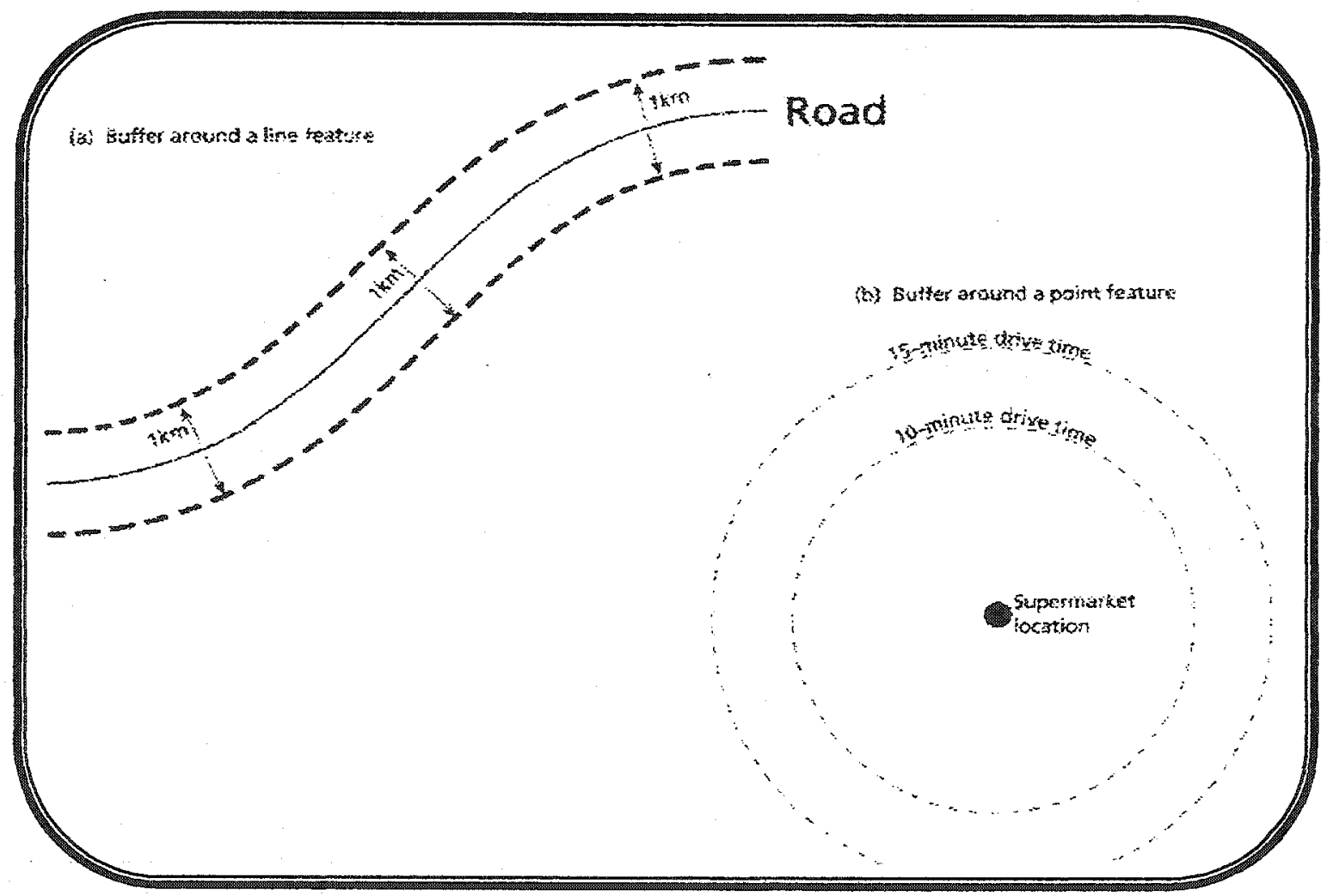

Figure 2.2: Buffering (Birkin, 1996) 
Once data have been given a spatial reference (geocoded) then the system can perform a number of spatial queries. For example, once a specific map location has been highlighted, the GIS will search through all of its information banks to retrieve all recorded information associated with that geographical feature.

It is important however, to realize that geographic information systems provide data systems rather than information for policy making. Typically, existing systems can synthesize and integrate spatial data effectively, but do not have the capacity to forecast or plan ahead, or to provide the basis for impact analysis. An ideal GIS incorporates statistical methods as well as forecasting models (Aronoff, 1995).

Using GIS in the field of transportation opens up a wide variety of possible applications. GIS provides applications for ensuring smooth flow of the distribution of goods and people through aiding in design, routing, traffic control, and real-time navigation. In essence, a GIS application in transportation is a merger of GIS with Intelligent Transportation Systems, where GIS no longer exists as a stand-alone product (Husdal, 2000). 


\subsubsection{Applications to Public Transportation}

Improving the mobility of today's citizens is a great challenge for public transit operators. Urban areas and employment centers are dispersing while automobiles have reduced the demand for public transportation. Therefore an economic and efficient system is required to enable transit agencies to provide sufficient services.

GIS has been in existence for many years, but only recently has its availability and application by transit been growing dramatically. GIS enables public transit to bring the power to communicate information through graphics to various aspects of transit planning, marketing, and decision-making. In an era of broad-based participation and complex assessments of information, GIS enables transit to leverage the evolution of computer technology and software development as a useful tool for improving the quality of transit services (Smith, 2000).

Transit planning applications in GIS are spreading throughout the world as new personal computer systems are being acquired and as several regional planning or city government GIS applications become familiar to transit planners. These applications range from simple uses of GIS for routine data analysis tasks to more sophisticated modeling tasks that usually involve programming 
interfaces and data exchanges with other planning tools (Attanucci and Halvorsen, 1993).

As stated earlier, GIS is defined as a tool that supports the processing of spatial data into information. By having this ability to support spatial analysis, while also simultaneously allowing for the management and analysis of large quantities of attribute data describing the spatial entities, makes GIS well suited to support the identification of transit trip itineraries, in this case, the information in the definition. Furthermore, GIS is currently used extensively by public transportation agencies, primarily to support planning functions.

When transit agencies implement the GIS software and tools; tracking, routing, and scheduling, provide the ability to answer the where and how to travel questions. GIS helps agencies to reach new levels of success, customer service, efficiency and technological excellence (mww.esri.com).

The beauty of GIS is in its wide scope and applicability to virtually any type of industry and activity. Thus GIS is not restricted to the conventional view of movement and transportation e.g. roads and railroads but extends to any from of motion and activity. GIS can 
provide the tools one needs to gain the insight (www.corpweb.semcor.com).

Drivers, dispatchers, maintenance workers, route planners, management personnel, and even riders can make better decisions about their jobs and transportation options when they can make safe, reliable choices based on real information and not guesses. Information on bus routes, current location and schedule adherence, transit stop location, emergency situations and locations, road condition, demographic changes, employment centers, rideshare programs, and neighborhood characteristics are all the factors that can be used to improve transit performance. It enables transit operators to meet this challenge by putting information in the hands of people who can use it (www.esri.com).

There are a number of different uses for GIS in transit. Among its many applications, a GIS can be used for the display and/or analysis of the following (Casey et al, 2000):

- Bus routes, streets, parking lots, facilities, shelter locations, ridership loadings, running times, scheduling, bus assignments, dead-head routings, accidents, and customer complaints - for service and facilities planning; 
- Street and route maps, service performance monitoring, vandalism location and history, and emergency call location identification - for operations purposes;

- Land uses, employer sites, demographic data, and travel patterns - for market development;

- Bus route maps, trip planning route choices, on-time performance data, multi-media displays, pass sales outlet planning, and customer complaint data - for customer information/service purposes;

- Customer address location, service qualification determination, and service performance statistics - for Americans with Disabilities Act service operations; and origin and destination of ridesharing applicants, custom bus service requests, and high occupancy vehicle (HOV) lane violations - for other transportation service analyses.

This extensive use of GIS is particularly advantageous in that most transit agencies will likely possess the data and software required to serve as the foundation for a transit trip itinerary planner. GIS 
has the potential to significantly increase the quality of urban transportation planning data while reducing the cost of data collection and preparation by enabling transit and other local agencies to share and disseminate data (Schweiger, 1992). To further explore the applicability of GIS to this problem, consider some of the fundamental spatial analyses required by a transit trip planner. As stated earlier, proximity analysis (buffering) is important. It is key to determine if a candidate bus stop serves as a viable origin or destination point. The basic GIS tools of "overlays" and "buffers" provide the needed functionality to support this requirement. For example, once a customer's point of origin is located within the network, a GIS software can be used to see if that point falls within a user-defined distance from a bus stop. Furthermore, the use of a map-based GIS interface will allow a customer to determine if certain objects comprise unacceptable barriers to reaching a potential transit stop.

Nearly all commercially available GIS are built upon a relational database management system (RDBMS). The RDBMS serves as the engine that supports the management of attribute data describing the spatial entities (DeMers, 1999). This capability provides the functionality needed to store and analyze schedule information. The database, therefore, plays a key role in the trip 
itinerary planner system. It can organize data describing the transit system, and provides a mechanism to store and manage the preferences of the user as they interact with the system.

The capabilities that GIS can bring to transit agencies help with the growing needs of the industry. For example, the ability of GIS to communicate detailed data in a format more easily understood by decision makers and citizens makes it extremely valuable. Transit agencies can also use GIS for planning and analysis and to deliver more cost-effective services. GIS can be used to analyze the service area of a transit system. The analysis is also useful in addressing a variety of policy and service planning issues (Smith, 2000).

Since GIS can associate large data files with particular geographic points, routes, or small street segments, it is also an ideal tool for organizing large amounts of data associated with public transit routes, schedules, and facilities. It provides a transit planner with the following general capabilities (Attanucci and Halvorsen, 1993):

- Database Management: A GIS will allow transit managers to store data such as route descriptions, bus stop files, ridership 
information, population and employment demographic data, street and neighborhood characteristics.

- Geographic and Thematic Mapping: A GIS allows a planner to display and plot each database contained within it on a geographically accurate electronic map.

- Planning Analyses and Evaluation: Most GIS include a variety of preprogrammed data analysis routines that provide the transit planner with automated tools to aggregate and disaggregate various datasets according to predetermined criteria.

- Presentation Tool: The unique combination of accurate mapping and graphic representation of demographic and other statistical data provides transit planners with a new tool to present information to colleagues or to the public.

The capabilities that GIS brings to the professional planner and transit properties complement the evolving needs of the industry. For instance, the ability of GIS to communicate detailed data in a format more easily understood by decision makers and citizens makes it extremely valuable. Transit properties can also use GIS 
for planning and analysis and for addressing issues such as those related to the equitable allocation of resources (Crowson et al, 1997).

GIS can provide a great deal of constructive and cost-effective assistance to transit agencies as they strive to deliver efficient and effective customer-focused transportation services.

The evolution of user-friendly GIS software, efficient desktop personal computers, and refined geographic and demographic databases has resulted in moving GIS from a highly specialized capability requiring extensive training and support to one in which anyone can develop the necessary skills to provide a costeffective GIS capability for transit properties (Ball, 1996).

\subsubsection{Fixed-Route Service Planning and Scheduling}

Transit service planning and scheduling encompass service demand and supply, as well as the spatial equity and efficient service allocations. This analysis of the transit demand and supply is accomplished by identifying spatial distribution of transit service, ridership, and their interaction with demographic and land use characteristics (Peng et al, 1998).

The developed GIS database provides transit planners a tool for planning analysis and performance evaluation. For example, service planners can use the database to identify and display major 
sources of transit ridership by time of day at the stop level, route segment level or route level, so that they can determine the level of service on the route level and evaluate the route-on-route segment performance based on the obsened ridership (Smith et al, 1998).

The challenge for route planning is to cover as many transit origins and destinations as possible while providing users with direct routings. The GIS database allows planners to identify potential transit stops to be served and to generate transit routes automatically. Transit planners can use the GIS data to identify the demographic and land use characteristics within walking distance of a proposed transit route and can then estimate potential transit ridership at the route-level (Peng et al. 1998). Every time planners design a new route or intend to make a route configuration change, they can know the demographic and land use characteristics of the route coverage area, and the potential ridership changes.

The relational structure in the GIS database that links temporal scheduling and spatial network is very valuable in service schedule planning. The GIS database allows the user to identify the topological and temporal relationship of different transit routes and stops. This is particularly helpful to facilitate the integration of fixed- 
route bus and light rail dispatch to identify key system transfer points between bus and rail service (Smith et al, 1998).

\subsubsection{Potential Obstacles to Transit GIS}

The obvious question that may be asked at this point is if GIS is so great, why doesn't every transit agency use it? This question may be very difficult to answer if the same situation exists a few years from now, but for now some obstacles still exist to establishing an effective transit GIS. These include at least the following real or perceived problems.

Firstly, there are some difficulties in learning and using GIS software. Earlier versions of commercial GIS software required hours of training and repetitive use of different options to master the programs. While points and small segments of routes are generally handled well by most GIS, full or partial transit routes using multiple street segments are still difficult to manipulate using some commercial products. It should also be noted that the routing and scheduling features vary widely and only a few GIS packages can effectively address certain transit applications such as paratransit, trip itinerary planning, and other network modeling tasks. In general, the ease-of-use of the better GIS products has improved (Attanucci and Halvorsen, 1993). 
Secondly, starting with no existing data can be a difficult task. Fortunately, GIS vendors now often can provide preloaded U.S., Canadian, or world data and other customized database development services through ESRI. In addition, many transit agencies can now look to other regional agencies to get at least a start on their databases

Thirdly, with as many as 100 different commercial GIS packages available, incompatibility of data files is likely in some cases when different agencies use different GIS tools. Even when the software outputs are compatible, inter-agency cooperation in sharing recent data can be lacking. Presently, users are investigating how a particular GIS handles other competitive systems' data before deciding on a standard for any specific application (Attanucci and Halvorsen, 1993).

Subsequently, and perhaps the largest obstacle to the most effective use of GIS in transit agencies is a lack of consideration about its broader use within the agency. In many cases, a particular department starts using the GIS application without determining how other databases might be interfaced or be inputted into their application. As a result, duplicate data entry is often 
required with other text-based applications such as bus stop inventory programs or ridership count analysis software (Attanucci and Halvorsen, 1993).

Finally, until recently, initial GIS software and hardware purchases required substantial resources, with new mainframe and minisystems running into hundreds of thousands of dollars and even up into the millions. While software and hardware prices have dropped, full-featured GIS packages are still not in the same league as typical office automation commercial software. The costs of specific application development within a GIS environment can vary widely depending on the product and the feature required (Attanucci and Halvorsen, 1993). GIS applications development will likely continue and resources need to be regularly budgeted to take advantage of a fast-developing new technology.

\subsection{Optimum Route Algorithms}

The transit network is much more complex than the road network because it is strongly time-dependent and highly dynamic. Transit-oriented network data models and routing algorithms are considerably underdeveloped compared to those of highway networks. With the growing demand for online transit information services and trip itinerary planning, there is an increasing need for efficient transit network data model and trip planning aigorithms. 
The analysis of transportation networks is one of many application areas in which the computation of shortest paths is one of the most fundamental problems. Several algorithms and data structures for algorithms have been put forward since the classic shortest path algorithm by Dijkstra (1959). In its modified version, this algorithm computes a one-to-all path in all directions from the origin node and terminates when the destination has been reached. Most computer applications, including ArcView, use this method to calculate an optimum route path to travel.

When it comes to developing an algorithm for transit, compared to its highway counterpart, transit networks receive much less effort in terms of data modeling. Transit is usually mentioned in a few words in the development of the general transportation data model.

Nevertheless, this all changed in the 1990's when data modeling for public transportation emerged. Work in the field of generic data model for Linear Referencing Systems brought together ideas from Koncz, Greenfeld, and Mouskos (1996), Deuker and Butler (1998), Peng and Huang (2000). These researchers are GIS-T professionals and practitioners and most of the research is focused on Linear Reference Systems for highways or on generic linear data models. 
Koncz et al (1996) designed an approach to develop an algorithm and strategy for transit providers to find best route alternatives for the user, and to demonstrate how a geographic information system can be used in the development of transit advanced traveller information system (TATIS) to meet these needs. The major difference between this algorithm and others previously developed are the capabilities of handling multiple modes of transit, providing paths that include walking distances and provisions of multiple optimal paths to allow the user flexibility in choosing a path.

Dueker and Butler (1998) developed a generic GIS-T enterprise data model that is independent of map scale, specific entity attributes, mode of travel, and location measurements methods. With this data mode, criteria for path finding are attached or linked to traversal segment or nodes. Single or combined variable optimization based path finding algorithms, such as shortest path algorithm, can be employed to search the network. When this model is implemented in transit, a bus route with a direction can be expressed, as a traversal, and bus stops and transfer nodes are point events. This data model and others like it, have been implemented in many headway-based shortest path algorithms.

Another group of researchers in the transportation area are more focused on transit operations. Instead of working on GIS data modeling, they are more concerned with system operation, optimization, statistics and planning. Within 
this group, transit is studied in terms of route assignment, trip planning, scheduling and providing service (Tong and Richardson (1984), Wong and Tong (1998), Peng and Huang (2000)).

Path finding and route assignment algorithms developed by this group fall into two categories: headway based and schedule based. Headway based algorithms assign passengers to the first arriving vehicle based on the combined bus frequencies on the same street. Several probabilistic or stochastic models can be linked to this category. The headway-based approaches are often applied to congested transit systems and work well with frequent service environment with headway of 5 minutes or less.

The optimal path search by schedule-based algorithms is strictly dependent on the schedule. Schedule-based algorithms are sometimes referred to as heuristic or deterministic approaches. In the model developed by Wong and Tong (1998), the estimation of time-dependent origin-destination (O-D) matrices for transit network based on observed passenger counts is given. The dynamic assignment framework uses a schedule-based transit network model to help determine the time-dependent least cost paths between all O-D pairs. An entropy-based approach is then employed to estimate the time-dependent O-D matrices based on the observed passenger counts at those observed links in the network. It was found that there was a good agreement between predicted and observed matrices. 
Tong and Richardson (1984) developed a "branch-and-bound" algorithm approach to solving schedule-based optimum route finding. This time-dependent algorithm finds the minimum path between two stations in a multi-route, multi-mode transit system. Selection of the minimum path can be based on either journey time or on weighted time. This model can be used for generating route schedule information to guide transit users, for assisting in route schedule coordination, and for analyzing transit system accessibility.

Based on the data model, Peng and Huang (2000) developed two basic schedulebased path-finding algorithms and one basic non-schedule based minimal transfer path algorithm. Taking a spatio-temporal perspective, they developed an objectoriented data model in which the network itself is modeled as an object. The network object is composed of a set of subclass objects including a time map mechanism that monitors temporal behaviour and builds dynamic network topology. In addition, the Object-Oriented model substantially reduced network redundancy and enhances path search efficiency. Based on the data model, the authors develop two basic schedule-based path-finding algorithms and one basic non-schedule based minimal transfer path algorithm. By using various combinations of the basic algorithms an array of path options are generated to mimic human cognition in path finding. 
As an interesting final note, three researchers in India developed a model for public transportation routing and scheduling using genetic algorithms. Rao, Muralidhar, and Dhingra (2000) presented the applicability of genetic algorithms as a possible technique instead of the current heuristic procedures used.

The design process was done in two phases based on two main objectives. These are to minimize the in-vehicle travel time and the transfer time for the whole network and the minimization of an overall cost generally a combination of user cost (sum of in-vehicle travel time, waiting time and transfer time) and the operator's cost.

This was applied to a small network where most of the node pairs had non-zero demands whereas in real networks transit demand matrices tend to be sparse, with fewer non-zero entities. The results were favorable but they found that work was needed to investigate in detail the effect of various parameters on larger networks.

\subsection{Transit Information Systems}

Within the last few years there has been an increase in the development and enhancement of aids to helping transit passengers travel more efficiently. Most of the early research and development has been done in Europe. The developed concepts and ideas have performed well and have recently spread to parts of 
North America and other major cities around the world. Most have implemented new telephone operated systems, kiosk monitors and real-time Internet based systems. These variations of systems, all build on existing infrastructure and technology. Currently, most transit agencies rely on schedule information found in procures and on the Internet.

The most widely used and well-known system in the United States is NextBus. This company combines existing technology to provide real-time information for commuters in California (San Francisco, Oakland, Emeryville, Glendale, Santa Barbara), Boston and Lowell, Massachusetts, Vail, Colorado and Fairfax, Virginia. NextBus was the first company in the United States to serve passenger-focused information to the Internet. (Casey et al, 2000)

NextBus is not a static schedule listing; it is actual arrival information, updated at regular intervals. NextBus uses satellite technology and advanced computer modeling to track vehicles on their routes. GPS receivers are installed on transit vehicles, and this tracking information is used to calculate when a vehicle will arrive at a particular stop (www. NextBus.com).

Each bus receives frequent signals from satellites that are used to give an accurate location of the bus. This information is then passed by radio to a central computer and to displays at bus stops. Each display then determines the waiting time to bus arrival which is shown with updates every 30 seconds. Taking into 
account the actual position of the buses, their intended stops, and the typical traffic patterns, NextBus can estimate vehicle arrivals with a high degree of accuracy (www.NextBus.com).

The accuracy of the predictions varies depending upon changes in traffic patterns, accidents, or the amount of Internet activity (number of users at one time). Therefore, NextBus cannot specify a precise overall accuracy. In general though, the margin of error is less than one minute for predictions of five-minutes or less. For ten-minute predictions, the margin of error is less than two minutes. These predictions are made available on the World Wide Web and to wireless devices including signs at bus stops and business, Internet capable cell phones, Palm Pilots, and other Personal Digital Assistants (PDAs). (mww. NextBus.com)

Although NextBus is the most widely used system in the United States, there are other companies that have developed Automated Trip Planning Systems. These include Tidewater Consultants Inc., Megadyne Information Systems, and TeleRide Sage.

The system developed by Tidewater Consultants Inc. is designed to run in a Windows-based environment on a standard PC. The system relates stops, routes, and schedules to a GIS based on a Rapid Routing Module, an algorithm that computes a trip plan for a customer who has called. Travellers call the transit agency's telephone information center; give their origin and destination, 
and the system computes a trip plan, generally in under 10 seconds. Looking at a pull-down menu, the telephone center agent then can describe to the caller the proposed itinerary, or can fax it or send it in the mail. These printouts can be multi-lingual, in Braille and/or in large type (Casey et al, 2000).

Megadyne markets several unique software products, as well as related marketable technology. One of Megadyne's products is directed toward public transit/transportation. Among the many Megadyne Information Systems developed most activities range from system development/integration, to professional services, to software products, and to process management. The systems developed by Megadyne include:

- PARIS: Passenger routing and information system

- PARISpeak: Interactive voice response system

- V-TRAX: Automated vehicle monitoring system

- SPARTAN: Schedule planning and real-time assessment

- ATHENS: Automated vehicle dispatch system

- Dial-A-Ride: Automated ride pooling system

- Rideshare: Carpooling and vanpooling system

First among these products are PARIS and PARISpeak. PARIS provides fast, accurate, and detailed information on transit services; generates optimum trip itineraries; and provides schedules, fares, stops, routes, travel times, multitransfer itineraries, service mode, dispersed sales outlets, emergency updates, 
handicapped access, and related management, trend, and cost information. PARISpeak is an automated voice response software that works either as a stand-alone product or in conjunction with PARIS. The greatest utility is achieved when the two products work together (muw. Megadyneinfo.com).

Teleride Inc., a TTi Systems Company, is a global organization providing a unique spectrum of products and solutions to the transit industry. The products developed enhance the performance of transit agencies through scheduling and run cutting, itinerary planning, passenger information, driver management, demand-responsive scheduling and dispatch. All products are engineered on the Windows NT platform, using state-of-the-art Rapid Application Development tools and Object-oriented programming methodologies (uww. Teleride.com).

The TeleRider Interactive Voice Response (IVR) System defines the state of the art for transit passenger information systems. It diverts calls from telephone information center operators, reducing abandoned calls, busy signals and call waiting times. It is available to passengers 24 hours a day, 7 days a week and can obtain schedule information within 20 to 30 seconds. It tells passengers if their bus is on time, delayed, detoured or canceled ( $\mathrm{mww}$. Teleride.com).

TeleRider Plus provides route recommendations and route and schedule information on the basis of current transit network data. When a starting point and destination or a specified stop, address or point recognized by the 
Geographic Information System and a date or day of the week and time of day of travel are entered, TeleRider Plus calculates the most effective connections. It is a client-server solution offering users access over a standard network. All transit information is calculated online by the TeleRider Plus server and then made available to users for viewing, further calculation, printing and/or fax distribution (wnw. Teleride.com).

TeleRider Plus is also Internet-enabled, allowing travellers with Internet access the convenience of planning their upcoming trips. It utilizes a highly developed data format to provide and calculate transit information efficiently on-line. A specially developed program is used to convert transit schedule and route information from the data management module into the format utilized by the TeleRider Plus server. TeleRider Plus offers the traveller accurate, detailed recommendations based on the actual date of requested travel (unw. Teleride.com).

Individual cities and counties have also implemented programs themselves through their department of transportation. In San Diego County, the system implemented is called InfoExpress. It is an interactive voice response system available 24 hours a day, seven days a week. Callers can get basic route, schedule, and general information in Spanish or English for seven transit agencies in greater San Diego. This enables information agents to help with more complicated questions like trip planning (Casey et al, 2000). 
The Winston-Salem Transit Authority, through the Mobility Manager model project being developed under the FTA's APTS program, is providing users with a menu of transportation services by telephone. By calling a single number, a prospective passenger will immediately be able to schedule a trip, ask about the status of a trip, make arrangements to transfer more readily from one mode of travel to another, or receive a schedule of transportation service available in the regional area. In the future, the Mobility Manager project will be expanded to include electronic variable message signs to provide passengers with real-time information about a vehicle en route, such as updates on delays or revised schedules (Casey et al, 2000).

In Los Angeles, California, CalTrans is directing the Smart Traveller program, which is a free automated information service to provide commuters with up-tothe-minute freeway conditions and traffic speeds, customized transit route planning, and real-time carpool matching. It has a telephone information service operating 24 hours a day, which provides Los Angeles County Metropolitan Transportation Authority information. As well, there are kiosks available. They utilize a personal computer based system, which is connected via dedicated digital telephone lines to a telecommunications system. This system accesses the rideshare, transit, and freeway conditions information residing in databases maintained by Commuter Transportation services, Inc., Los Angeles County 
Metropolitan Transportation Authority, and CalTrans Transportation Management Center (Casey et al, 2000).

The kiosks provide personalized itineraries including routes, fares, schedules, and origin-to-destination travel times; carpooling possibilities; real-time freeway conditions; and videos on various transportation topics. The user interface is a touch-screen monitor. Freeway flow status is displayed on a map. All other information is displayed in textual form (Casey et al, 2000).

In Seattle, Washington, the King County Department of Metropolitan Services is employing several forms of electronic communication technology to access vital transportation information relating to the greater Seattle/Puget Sound region. Riderlink is an on-line information resource available on the Internet via the World Wide Web. It gives instant access to Seattle Metro bus routes, timetables, and maps, as well as information about vanpool and ridematch services, bicycle transportation, freeway congestion, the state's commute trip reduction law, and a variety of other transportation topics (Casey et al, 2000).

Seattle Metro's automated Bus-Time system makes schedule information available to anyone with a touch-tone phone; people can access information about their route and bus stop. As callers respond to a series of questions to identify their route and bus stop, a digitized script is packaged and passed on to 
where schedule, route, and bus stop information, and a digitized vocabulary are stored (Casey et al, 2000).

Along with the current telephone system in Seattle, Washington and Portland, Oregon, MyBus was created. The goal of MyBus is to present to riders, in realtime, the predicted departure times of buses at specific locations throughout a transit region.

MyBus is designed as a distributed application. Schedule data and real-time AVL data streams flow between components. These components include an AVL source, a prediction generator, a set of filters for bad or erroneous AVL data, and a web server for final text formatting and delivery over the web (Dailey, 2001).

The buses are equipped with GPS units that daily capture observations. Onboard computers and Automatic Vehicle Location equipment are linked with the Bus Dispatch Center to improve communication with bus operators, locate buses in real time and enhance data collection. A stop record is created every time a bus passes a stop or a door is opened. Automatic Passenger Counters record the number of passengers boarding and leaving. In addition, an event record is created to record other activities, such as a bus travelling on or off schedule. Bus drivers also send and receive digital messages, and key in more 
than 30 pre-programmed codes to capture field information. All records include the time and coordinate position (Kalmer and Beckel, 1999).

The prediction component of MyBus uses three inputs: a schedule data set, a set of historical trip realizations, and a real-time AVL stream. The schedule data provides the expected time for each event. Historical data provides ensembleaveraged statistics to the algorithm that predicts vehicle departure. The AVL stream supplies instantaneous bus location information approximately every 1 to 3 minutes per vehicle (Dailey, 2001).

The predictor algorithm uses an optimum filtering technique based on Kalman filter technique. An underlying assumption is that any real-time arrival prediction algorithm depends upon reliable real-time transit vehicle location information. A separate prediction process is started for each goal to minimize statistical errors. The prediction algorithm produces the optimal estimate of the departure time given the information provided to the filter. To compare the predictions with the vehicle behaviour, the actual departure time needs to be estimated. Since the vehicle is tracked irregularly, there is no guarantee that the location will be reported just as the vehicle departs. To get an estimate of the real departure, the location report just before arrival and just after the departure can be recorded and linearly interpolated to the actual departure time. The filter is continuously predicting the departure as a function of both time and space. The statistics of the deviation of the predictions from the actual can be expressed as a probability 
surface in space and time. Moreover, predictions are accurate both far in advance of the scheduled departure time, as well as near departure time (Dailey, 2001).

The second major component of MyBus is the web server program. This component is responsible for receiving input from the predictor component and for storing and formatting this prediction data in a manner suitable for output over the Internet. Currently, the Seattle predictor and the combined Seattle/Portland web site run as two Java Virtual Machines on the same Windows NT host (www.MyBus.org).

Touch screen kiosks, featuring full-motion color video, stereo sound, on-screen maps, personalized public transit itineraries, and carpool matches for commuters, have been installed in the Coachella Valley area of Riverside County, California. The pilot project, called TransAction Network, has four kiosks at shopping centers with high pedestrian traffic. The TransAction Network is being introduced by Commuter Transportation Services Inc. and SunLine Transit Agency. SunBus, the personalized public transit option enables the user to obtain transit information. By entering a destination, arrival, or departure time and a transportation option, a user can receive a free printout of a complete SunBus itinerary including route, bus stop, fare, and schedule, as well as a carpool match list (Casey et al, 2000). 
The Mass Transit Administration of Maryland's transit information center has been updated through the upgrading of the computer system to contain all of the latest schedule information. When a customer calls the information center, the operator will call up the schedule on a computer screen, instead of looking through books. Moreover, the system will keep a running memory of the customer's call based on the number where the call came from. The caller's last question asked and home address can be brought up on screen by the operator, which saves time in processing requested the information. There are also five kiosks installed in greater Baltimore to provide the same information as the operators. The full intent is to have all information be real-time, after the AVL system is fully installed and refined (Casey et al, 2000).

The Rochester-Genesee Regional Transportation Authority (R-GRTA) has implemented an automated telephone system. When a call comes into the system, Direct Talk sends the request for route, time of day, direction, and location to the mainframe, and then translates the data into a verbal response to the caller. The typical time to complete this transaction is two minutes. The request for information begins with the caller giving the route number. If the route number is not known, a listing is provided, and the caller can choose from the list to complete the request. If further information is needed, the caller can cut back into the system to speak with a customer service representative without having to hang up and redial to obtain the necessary information (Casey et al, 2000). 
The Metropolitan Atlanta Rapid Transit Authority has implemented a trip planning system that supports MARTA's customer information center and kiosks that were available for the Olympics. The Passenger Routing and Information System (PARIS) (provided by Megadyne) requires an origin and destination to produce a nearest bus stop-to-nearest bus stop itinerary. The itinerary does not provide instructions to reach a bus stop, but it does take into account walking time and impossible walks (e.g., crossing a river without a bridge). PARIS ranks all of the options for a trip and the highest ranked option is provided. In addition, a limited amount of highway-related information can be obtained from PARIS (mww.Megadyneinfo.com).

The AVL system, which was implemented by Transportation Management Solutions for the Olympics, provides real-time vehicle status information to the itinerary planning system. This capability enables the trip planning system to provide itineraries based on actual vehicle arrivals and departures as well as providing information on service disruptions (Casey et al, 2000).

In the Minneapolis/St. Paul, Minnesota area, transportation officials are looking to advanced technology to make transit operations more efficient and attractive to commuters. Called Travlink, this demonstration project is part of Minnesota Guidestar, Minnesota DOT's program for ITS, which is actively testing and deploying new technologies that improve the movement of people, goods and 
services. Travlink represents the integration of a computer-aided dispatch and AVL system based on the global positioning system, an advanced traveller information system (ATIS) and an automatic vehicle identification system in the I394 corridor in the Minneapolis/St. Paul metropolitan area. Travlink is using a variety of devices and systems to distribute both real-time and static transit and traffic information to travellers. Travlink is designed to encourage commuters to consider alternatives to single-occupant travel, especially public transit (Casey et al, 2000).

In the state of Florida, the Department of Transportation has developed a software program that allows one to quantify and visualize the mobility provided by a transit system at different times of the day and week at any location within the system's service area. Other helpful tools provided by the TLOS software include the ability to measure transit level of service, the ability to create mailing lists tailored to potential customers served by particular transit routes, and the ability to estimate the percentage of persons using transit, in those locations and during those hours when transit is available as an option to them. The results of a TLOS analysis can be used in a variety of transportation planning applications, including transportation planning models and geographic information systems (mww11.myflorida.com).

The newest version of the TLOS software adds route-finding ability, allowing a user to find the minimum and average travel times for a trip, and the transit 
route(s) one would take to make the trip. The software can also determine the number of people and jobs located within a user-defined transit travel distance of a location, calculate transit travel times between all combinations of transit services within an area, and calculate the auto-transit travel time level of service measurement. It is expected that these new features will have a variety of applications, from transit planning, to welfare-to-work, to customer service (muw11.myflorida.com).

The Champaign-Urbana Mass Transit District (CUMTD) currently, in addition to schedule booklets, provides bus schedules through the World Wide Web (WWW). Recently a Bus Information System for CUMTD, which is an interactive and easy-to-use WMW-based application, was introduced. Dynamap 2000 by ESRI provided the street information and addressing and MapObject, NetEngine, and Arc/lnfo were used to develop the system. This system provides users with the shortest travel path and transfer information once the users enter an origin and a destination. The common algorithm provided by NetEngine 1.1, was used along with some modifications that combined the schedule data with the network data from the entire bus route network (Lee, Baumgartner, Kim, 1999).

BusView was successfully designed and demonstrated using real-time transit coach locations on a digital map to the University of Washington campus community. The project was further enhanced to encompass developing a graphical transit information system using data from King County Metro's existing 
automatic vehicle location (AVL) system and the Puget Sound's regional intelligent transportation systems (ITS). It was created and launched on the World Wide Web providing real-time bus location information (Dailey, MacLean, Pao, 2000).

During the Seattle Smart Trek Model Deployment Initiative (MDI), the ideas developed in the campus-based version of BusView were used to create a version that could be widely supported on the Internet. Two new applications were created to provide real-time transit information. The first project, BusView Plus, is a desktop display of the real-time location of all the transit vehicles operated by the regional transit carrier. The second project is Transit Watch, which is a real-time arrival prediction system suitable for deployment in transit centers. Both of these applications are designed to operate over the Internet as Java Applets and are designed to be sufficiently general so that they can easily be ported to other cities (Dailey, 2001).

The first project, BusView Plus, implements a Java applet to display real-time transit vehicle locations on a variety of computing and operating system platforms. BusView is platform-independent with the goal of making transit vehicle location information accessible to anyone on the Internet. An additional goal is to develop an interactive interface that promotes modal change and encourages the use of transit (Dailey, 2001). 
The second project called Transit Watch deployed an APTSIATIS that provides a prediction of the arrival status of scheduled transit coaches. This prediction is categorized into four stages: on-time, delayed $\mathbf{N}$ minutes, departed, and no information. The database engine is central to the application. The data storage, relational queries, and serialization capabilities are used to synchronize the Predictor and Display Server components. The database stores information from the AVL stream necessary for the Predictor to make arrival time predictions as well as storing the resulting predictions for use by the Display Server (Dailey, 2001). Once reliable predictions are available in the database, the second specialized component of the Transit Watch architecture is used to distribute the information either to applet clients at transit centers or on the Internet (Dailey, 2001).

An interactive trip planner for users of PENTRAN, public transit for the Peninsula region of Virginia has been created. The interactive routing feature, or trip planner, allows a user that is unfamiliar with the PENTRAN routes and/or service area to plan a trip from their origin to a specific destination. The user will enter their origin and the routing program will calculate possible trips between the origin and destination by examining possible bus stops within a user-selected radius of the origin and destination. These trips will then be presented to the user to select and view a particular trip. Once a trip is selected, a trip itinerary will be presented to the user with all of the necessary travel information. Also included are a trip map that shows the desired bus stops for pickup and delivery 
and their relation to the origin and destination through the use of a street network (Smith et al, 1998).

In Helsinki, Finland, the metropolitan area public transportation services has become more popular with the implementation of a system called mTravel to inquire about the routes and timetables. The system enables the user to search a route by entering two addresses. This facility tells the user immediately which public-service vehicle to get on to reach the desired destination. The user can also choose a certain point of time, on the basis of which the route service provides the next departure time of the public-service vehicle. The time can be the departure time of the vehicle or the time by which the user of the service wants to reach the intended destination (www. Novogroup.com).

The journey-planning algorithm compiles the best route at any given time by combining public transport connections and walking links, based on digital road networks. mTravel can also accommodate real-time positioning feeds from vehicles. This information can be used to correctly estimate arrival and departure times (www. Novogroup.com).

In 1996 the Copenhagen Transportation Company implemented a project called Priobus involving the real-time tracking of buses throughout the metropolitan area. The system, which was developed and implemented by the Dutch company Peek Traffic B.V., helps dispatchers prioritize buses moving about the 
city and track arrival information via dynamic displays to passengers waiting at bus stops (mww.esri.com).

Implementing the Priobus project included continuous accurate real-time localization calculated with on-board computers in the vehicles using a Digital Global Positioning System (DGPS). The information is communicated using radio transmission to the central system. All configuration and real-time information is stored in an Oracle database. The database is used to import new schedules that are automatically downloaded to the vehicles and to create reports with statistical information. In order to provide intuitive control over the system, a geographical interface was implemented using MapObjects (www.esri.com).

In South East Queensland, Australia, a journey planner has been implemented called Transinfo. It provides integrated public transport information on buses, ferries and trains. The service provides callers with detailed information on timetables, routes, stop locations, fares, connections, special events and other service provider information. The TransInfo journey planner helps on to organize travel times and explore alternate trip options by providing users with travel fare information on public transport for a selected journey. (mww.esriau.com)

This area is also provided with a trip finder that is available through the Internet, kiosks, and mobile phones (using Wireless Application Protocol (WAP) and Short 
Message Service (SMS) technology). The Tripfinder provides route planning and timetable information direct to public transport travellers. The user must choose an origin and a destination location and select a departure day and time from lists provided. The route planning and timetable algorithms have been developed based on predetermined routes to the list of origins and destinations (mww.SydneyBuses.nsw.gov.au).

From each of these transit information systems mentioned, a strong conclusion can be made in that the uncertainty in the estimation of travel time is never a factor in the development process. While the simplest systems use a telephone system to let passengers know how the bus is running, the more complex systems rely heavily on the technology of AVL or GPS. This technology is put in place to allow for real-time placement of transit vehicles. A user can track the bus, but not be aware of travel time conditions. In other cases, a prediction method or a filtering system is used to estimate when a bus will arrive at the next transit stop. The prediction may be accurate but what happens if this bus never makes it to the next transmission point. By not considering the uncertainty in the travel time a bus can still be predicted incorrectly.

The system developed here is also building upon those systems that do not rely heavily on technology. Some agencies do not have the budget to acquire the latest technology, so they must design and adapt accordingly. By considering the advantages and disadvantages of each system described, an improved 
methodology can be used to develop a well thought out system that can be applied to cities without the latest real-time technology. More options are also made available to the users within the user interface and the process of solving the optimum route considers uncertainty. As technology changes and becomes more affordable, this system can be updated.

Careful consideration is also required in making sure the system is generic in nature. While NextBus is widely used, many of the other sytems described were designed to serve a specific purpose of a particular city.

Overall the developed transit information system entails the latest advances in GIS technologies available. By applying the Bayesian statistical decision theory, the uncertainty in schedule adherence and travel time is considered. Finally, the system accounts for complexities of transit operation by the system. 


\section{Chapter 3: Building Blocks of Transit Information Systems}

\subsection{Navigational Process}

\subsubsection{Path Finding Methods}

Whether walking through a building, driving in a city, or selecting routes on public transit, a traveller develops a mental image of the surrounding environment, of his or her own location within the environment, and of his or her progression through the environment to the desired destination. Such knowledge is acquired in three basic stages: (TRB, 1999)

In the first stage of path finding, a person identifies landmarks and begins to orient himself/herself using these landmarks as references. As landmark knowledge develops into route or procedural knowledge, a person starts to build travel directions and decisions around the framework of landmarks and can visualize travel plans as a series of actions that will take him or her from an origin to a destination.

Finally, with enough navigational experience in a particular environment, a person will develop a mental picture of that environment, including knowledge of the landmarks, the routes 
from any one place to another, and approximate or relative distances between them. This survey knowledge allows travellers to describe routes they may never have traveled, by defining them in terms of this picture.

Although survey knowledge can be developed eventually from route knowledge and real-world navigation through the environment, it can often be acquired more quickly from map study. A printed map of landmarks and the spatial relation-ships between them helps to form the picture in a person's mind (TRB, 1999).

3.1.2 Requirements to Navigate Unfamiliar Journeys by Transit Like any path finding experience, navigation through a transit system involves the three stages: orientation via landmarks, development of route knowledge to travel between those landmarks, and, finally, survey knowledge of the transit system. Transit information aids must translate the many elements of a transit system (the geography, connections, operations, and rules) into a base of knowledge that will allow a rider to identify and make decisions about the routes, transfers, and boarding and disembarking locations that will deliver them to the correct destination (TRB, 1999). User-friendly transit information aids provide this information in a way that allows the rider to travel confidently and easily through the various segments of a trip. 
Ideally, passenger information should be available at every stage of the rider's transit trip. Pre-trip information helps the rider to plan routes and connections. In-transit information assists the rider at each decision point during the trip. Supportive/confirming information repeats and reinforces data and decisions and helps the rider to feel more confident that he or she is progressing toward the desired destination (TRB, 1999).

As defined by the Transportation Research Board, the pre-trip information requires the following:

- Location of the nearest bus stop,

- Routes that travel to the desired destination and transfer locations,

- Fare,

- Time of departure and approximate duration of the trip.

While in-transit information needs consist of the following:

- At the departure point: identification of the correct bus to board,

- On the bus: identification of bus stops for transfers or disembarking,

- At transfer points: how to transfer to another route; cost, time limits, and restrictions; and identification of the correct bus to board, 
- At the destination: area geography and return trip information.

\subsection{General Principles of Transit Information Design}

Transit information aids are designed wherein users can learn transit routes, itinerary, and other relevant information like ticket costs very quickly. Users can educate themselves on transit information through the repetitive motion of viewing transit maps, speaking to others or through the media. This helps reduce the vagueness of how a transit system works. Transit information should be simple and easy to understand. Common names, terms, and references to known locations make it easier for users to navigate themselves through the transit network. When designing multiple aids, consistency should be maintained so the user is not confused. The rider should be able to build on initial information with data that confirm or reiterates the trip information (TRB, 1999).

Transit information can be presented in various ways like oral instruction, printed maps, and signage at bus stops or on buses. Each type of aid has benefits and drawbacks (see Table 3.1). Although no single information aid can meet all of the information needs of transit passengers, a combination of information types will accommodate different learning styles, different levels of transit experience, and different stages of a rider's transit trip (TRB, 1999).

Table 3.1: Types of Information Aids (TRB, 1999) 


\begin{tabular}{|c|c|c|}
\hline Information Aids & What They Provide & What They Don't Provide \\
\hline $\begin{array}{l}\text { Oral Instructions } \\
\text { (Telephone } \\
\text { information, bus } \\
\text { operator, other } \\
\text { passengers) }\end{array}$ & $\begin{array}{l}\text { Straightforward and } \\
\text { personalized information. } \\
\text { - Simplicity for new riders } \\
\text { and for those who have } \\
\text { difficulty reading maps. } \\
\text { - Instant accessibility. }\end{array}$ & $\begin{array}{l}\text { An overall picture of the } \\
\text { transit system. } \\
\text { - Reference material for } \\
\text { future or continued travel. } \\
\text { Flexibility or easy error } \\
\text { correction; if a rider misses } \\
\text { a step in the process, } \\
\text { his/her frame of reference } \\
\text { is lost unless he/she can } \\
\text { converse further with the } \\
\text { information source. }\end{array}$ \\
\hline Maps & $\begin{array}{l}\text { "Bird's-eye" view of the } \\
\text { transit system; spatial } \\
\text { relationships of landmarks, } \\
\text { routes, and connections. } \\
\text { - Flexibility for changing trip } \\
\text { plans. } \\
\text { - Supportive information } \\
\text { during a trip. } \\
\text { "Portable" information, } \\
\text { useful for pre-trip and in } \\
\text { transit. }\end{array}$ & $\begin{array}{l}\text { Instant accessibility. Not } \\
\text { only is the map a physical } \\
\text { object that a potential rider } \\
\text { must obtain before trip } \\
\text { planning can begin, but } \\
\text { map reading presents } \\
\text { difficulties for many people. }\end{array}$ \\
\hline Signs & $\begin{array}{l}\text { Information at "decision } \\
\text { points": bus stops, transfer } \\
\text { points, terminals. } \\
\text { Supportive information. }\end{array}$ & $\begin{array}{l}\text { Detailed information and } \\
\text { explanations. } \\
\text { Portable information; no } \\
\text { help during pre-trip } \\
\text { planning or on-board. }\end{array}$ \\
\hline Timetables & $\begin{array}{l}\text { Portable information. } \\
\text { - Detailed route information. }\end{array}$ & $\begin{array}{l}\text { Instant accessibility. Many } \\
\text { riders have trouble reading } \\
\text { and using timetables. }\end{array}$ \\
\hline
\end{tabular}

Although some transit riders use maps and timetables readily and regularly, many find difficulties in reading or understanding pieces of the information. The 
information provided by the transit system's information operators or by automated recordings updates users on information pertaining to static maps and schedules. For many riders, the telephone will remain their primary choice for obtaining information about new trips. Even with the best system of signs and rider information, some still find the principal source of information about the transit system remains the bus operator. People with visual disabilities, cognitive problems, or functional illiteracy rely on the operator for route guidance, transfer information, and times (TRB, 1999).

\subsection{Traveller Information Systems}

Traveller Information Systems apply many technologies to allow customers to receive roadway, transit network, and other information important to their trip. This information assists the customers in selecting their mode of travel (car, train, bus), route and departure time. Transit schedule and status information may be obtained from Transit Management Systems. Most of the roadway-based information is collected by surveillance equipment (vehicle detectors, cameras, automated vehicle location systems) and is processed by computers in transportation management centers for further distribution to traveller information systems. Other information used in a traveller information system are map databases, emergency services information, and information on motorist services and tourist attractions and services (USDOT and FTA, 1998). 
In the beginning of public transit, trip planning involved a person and a bus timetable. This timetable was divided into different stops and all of the different times of day a bus would be at that stop. Transit schedules fluctuated based on the current day of the week, so usually more than one timetable was needed. In the case of a large transit system, it was impossible to include every stop on the schedule, so occasionally only high volume stops would be given. A user had to know the physical location of a particular stop, relative to those listed on the schedule if it was not a high volume stop. In addition, the user would have to approximate the time, since the stop was not listed on the schedule. Of course, this assumed the user knew which stops to use and any route transfers that might be needed. Thus, route maps became important so a user could locate an origin and destination, and determine the needed routes for the trip. In the case of large transit systems, the map could be cluttered and confusing, and thus would have to be split into sections. What was needed was a single map, where a user could zoom in and out of view and not lose sight of the big picture. With the growth of the personal computer, this suddenly became possible. In a GIS environment, a user can search a route map of all available stops, with tools such as zooming and panning included. As transit systems have developed and become more complicated, the need has increased for a computer program to analyze the information quickly and accurately (Smith et al, 1998, Culp, 1994).

GIS can serve a variety of purposes for a transit provider in addition to trip planning. For example, the analysis of census data can benefit the overall transit 
planning of the agency, including the determination of optimal locations for bus stop shelters. Executing an interactive trip planner is just one of the many applications of GIS, making it a worthwhile tool for any transit system (Attanucci and Halvorsen, 1993).

Traveller Information Systems provide travellers with information on one or more modes of transportation to facilitate decision-making before their trip and during the trip (en-route). Information can be provided to trip makers at home, work, transportation centers, wayside stops, and on-board vehicles. With links to automatic vehicle location, traveller information systems specifically for transit are beginning to provide ready real-time information, such as arrival times, departure times, and delays (Marks, 2001). The performance of such systems can be improved if models of bus delay are used to supplement AVL/C data (Abdelfattah and Khan, 1998). Travellers can access this information through a variety of media, including telephones, monitors, cable television, variable message signs, kiosks, and personal computers (Casey et al, 2000, Marks, 2001). 


\subsubsection{Traveller Information System Functions}

Trip planning information and assistance may be provided pre-trip or en-route. Pre-trip planning assistance provides travellers with roadway information, including road condition, traffic information and travel times, and transit information which can be used to select route, mode, and departure time. This support may be requested from home, the workplace, park and ride facilities, transit stations, and other locations. En-route traveller assistance provides the traveller with roadway and transit information while travelling, including traffic information, roadway conditions, transit information, route guidance information, and other information such as adverse travel conditions, special events, and parking (USDOT and FTA, 1998).

\subsubsection{Pre-Trip Information}

Traffic volumes in cities across the world are consistently increasing every year, while transit ridership remains relatively static or is decreasing. Getting travellers to give up the convenience of driving their own cars is a difficult task. In doing so, it is essential to focus on a change in travel behavior, which entails providing accurate and timely information to travellers before their trip. This information will enable the traveller to make an informed decision about mode(s), as well as route(s) and departure times. 
Pre-trip traveller information systems help travellers make decisions about the choice of transportation mode, route, and departure time before they begin their trip (Casey et al, 2000).

Pre-trip planning systems provide travellers transit information that can be used to plan a trip, including which route to take and what time to depart. Whether provided to travellers at home, the workplace, park and ride facilities, or transit stations, pre-trip traveller information can help relieve congestion by allowing travellers to make informed decisions. Pre-trip traveller information also supports itinerary planning, which can provide information on the entire trip from one point to another. In providing pre-trip traveller information, the focus is on travel behavior and decision support. This requires providing accurate and timely information to travellers before their trip. Touch-tone telephones, personal computers, personal digital assistants (PDAs), kiosks, and automated data retrieval systems that augment existing humanoperator interfaces have the potential to substantially improve the accessibility of traveller information, thus influencing traveller behavior (USDOT and FTA, 1998, Marks, 2001).

Automated data retrieval systems to expand existing humanoperator interfaces can provide information to the caller in a timely 
manner. These media allow transit users to have good connection with transit information center. The Internet may be the most promising one because of its growing popularity and low cost. But many of transit users do not have internet access. So the accessibility is an issue for Internet for the time being. The kiosk is good to deliver pre-trip information as well but with a higher cost. The combination of Internet with kiosks would increase the accessibility of Internet and enhance the information contents of kiosks. Telephones have good interactive function through the oral communication between passengers and telephone operators. It is a convenient tool especially for visual impaired people, but there is a high cost associated with a customer service agent. An automatic message center with real time connection with AVL information is a lower cost alternative (Peng, Jan, 1999).

Pre-trip information systems in the past were primarily directed towards riders who knew the transit system already, and only wanted updates on schedules and transfers. New systems provide relatively obtainable information to the beginner as well as the experienced transit passenger. Typically, these systems can be accessed by a touch-tone telephone. Newer systems include map displays of the service area based on geographic information systems (mostly for the operator providing information to the 
customer who has requested information) and schedule information that is provided on the Internet through the World Wide Web. These newer systems, whether accessed directly or through a customer service operator can locate the closest transit stop to the caller's origin, provide directions to this stop, identify the closest stop to the caller's destination, provide detailed directions on transit between the origin and destination stops, and provide directions to the final destination from the last stop (Casey et al, 2000, Marks, 2001).

With the expanding implementation of Automatic Vehicle Location systems, the potential for providing real-time information on bus arrival and departure times is slowly becoming reality. Real-time schedule information is beginning to be provided by several transit agencies through a variety of media, including electronic signs and kiosks.

\subsubsection{Effective Traveller Information Systems and Benefits}

Effective traveller information systems should provide information that is timely, accurate, reliable, and relevant to making travel decisions. For travel information to be useful, it needs to be current and received in time to allow a user to act on it (e.g., route, time). Travellers also want and need to know that the travel information is 
correct, whether it concerns the arrival time of the next bus or the location. As well, potential and current users want information to be available when needed, and are consistent in quality. The information provided should reflect the entire service area and it must contain sufficient details about locations, times, and possible alternates to be useful in planning travel. The system should be easily integrated with other ITS systems like emergency management, freeway management, and traffic signal control to obtain adequate traveller information. In designing a system, the developer must consider the user friendliness and access capabilities. Traveller information should be accessible in a variety of forms and locations such as personal devices, kiosks, and home computer and be easy to use, since users perceive access time as an additional cost associated with using traveller information. Finally, it must be easy to maintain and not require excessive costs and time to operate (USDOT, 1999).

In general, each traveller information system must be designed to meet the objectives of users within an urban area. Overall, traveller information systems attempt to reduce travel times and delays to users. In doing so, traveller stress for trips to unfamiliar destinations can be reduced. In many cases traveller information systems have demonstrated benefits in several areas including 
travel time, consumer satisfaction, on-time performance, and environmental impacts (USDOT, 1999). Although users are receiving the necessary information in order to plan a trip within an urban area, improvements would be desirable.

\subsection{Transit Service in Ottawa: Information System and Other Developments}

OC Transpo has long been recognized as a leader in the public transit field. Its ridership levels are the highest among mid-sized North American cities, and its innovative Transitways have allowed the cost-effective introduction of rapid transit services throughout the region (OCTranspo, 1999)

OC Transpo provides transit service to the communities within the City of Ottawa, which include Nepean, Vanier, Rockcliffe, Gloucester, Kanata, and Cumberland. Some OC Transpo routes also operate to downtown Hull, Quebec. OC Transpo serves a population of 625,000 and on a typical weekday, ridership averages about 302,000 person trips. During a peak hour one-way trip, OC Transpo can carry approximately 10,000 passengers at the busiest location on approximately 190 buses per hour. Most of these operations occur on exclusive, gradeseparated $80 \mathrm{~km} / \mathrm{h}$ busways (OCTranspo, 1999).

Its operations include 878 buses, distributed over 204 routes, and provide 5,500 bus stops. OC Transpo's Transitway system is by far the most comprehensive 
bus based Rapid Transit System in North America. It provides a 31-kilometre Transitway that runs across the city with 28 bus stations and 5 light rail stations. It also has $3 \mathrm{~km}$ of mixed-use traffic in the downtown area, $21 \mathrm{~km}$ of freeway shoulder lanes, and an additional 10km of exclusive bus lanes and 32 stations ( 6 in CBD). Currently OC-Transpo is expanding the length of the Transitway to include areas outside the greenbelt. Along the Transitway there are 5 park-andride lots: Baseline, Greenboro, Place d'Orleans, Eagleson Rd/Kanata, and Fallowfield Rd/Barrhaven (www.OCTRANSPO.com). For more information on OC Transpo's operating statistics, please refer to Appendix G.

With an aging population and the changing nature of work, OC Transpo has struggled to keep its ridership. There has been a reduced significance of employment in the downtown core and the growth of suburban business parks that has led to OC Transpo's expansion in order to serve this population. The number of buses is up more than $6 \%$ in the last 5 years along with an increase of $35 \%$ in the number of routes. This value is mostly due to the introduction of school-service routes to decrease the congestion on regular routes. The number of bus stops has increased $10 \%$ due to the development of new suburban areas in the east and west of Ottawa. OC Transpo has been committed to the goals of providing economic, dependable, customer-friendly service from all levels and components of the organization and hence has seen an increase in annual ridership of $7 \%$ in the last 5 years (OCTranspo, 1999). 
Currently, OC Transpo has setup a daily timetable for each bus route within its service area. Lists of buses, schedules and maps are presently available in paper format and on the website. These times are to include an estimated arrival time at each bus stop location. Also there is a telephone information system (the 560 system) for public use in order to update their schedule. In this system, a recorded message informs the users and announces the estimated arrival time for each bus stop location. The current interactive information system, run by OC Transpo, is a telephone and email service. Users are able to gain desired information regarding bus routes and schedules by speaking directly with telephone operators or sending them requests through emails. First, the users provide their starting point (origin), where they want to go (destination), and what time they want to travel. The operators, using information available to them and personal knowledge, find the most suitable route and travel time for the caller.

\subsubsection{Long-Term Strategy}

The long-term objectives for OC Transpo support the policies of the City's Official Plan (OP) and Transportation Master Plan (TMP) to reduce Ottawa-Carleton's dependency on private automobile use and promote more environmentally friendly modes of transportation. To meet these objectives, the City has adopted regional modal share targets that would significantly increase the proportion of trips made by more sustainable transportation modes and reduce dependence on automobile travel (OCTranspo, 1999). 


\subsubsection{Market driven and customer oriented services}

Transit cannot be all things to all people therefore OC Transpo, like any other agency needs to give priority to key markets and provide effective services to them. Emphasis should be placed on providing a dependable basic system, supplemented with specific services emphasizing speed and convenience in markets where transit can compete with the private automobile. OC Transpo has laid out a long-term strategy that involves changes to the route structure; these are listed below:

- The development of an effective base network providing priority service on major arteries to address suburban trip patterns and to serve cross-town trips not necessarily oriented to the Central areas.

- More community oriented services that provide feeder service toffrom the base network and Transitway stations.

- More express and limited stop services to major activity and employment centers located in suburban locations.

A second feature of the service strategy is to accommodate the travel needs of an aging population and an increasing number of 
people with disabilities with an integrated fixed route/paratransit system. Low floor, accessible buses, accessible light rail vehicles, accessible Transitway stations and paratransit services designed to feed into the conventional system should help promote the integration and efficient use of the fixed route and paratransit services (OCTRANSPO, 1999).

A third feature of the service strategy is the transit priority network for all of the bus routes comprising the base network. This faster and more reliable service can be achieved through traffic signal pre-emption, responsive signal phasing, queue jump lanes, reserved bus lanes, automatic vehicle location and control and advanced passenger information (OCTRANSPO, 1999).

Enhanced passenger information systems will provide pre-trip information to plan a trip, bus arrival time at stops, next stop information, and other data. This will complement the "560" telephone system and traditional transit shelter maps, printed schedules, and ride guides (OCTRANSPO, 1999).

\subsubsection{Route structure}

The transit system currently consists of a hierarchy of routes that brings most urban residents within 400 meters of a transit route 
(www.OCTranspo.com). The strength of the system is the route structure that provides basic region-wide mobility for residents, local services for communities, and express services for commuters. The Transitway and base routes in the main travel corridors, the community services supporting these routes, and the extensive express bus network throughout the region are the main reasons for the transit system's high ridership when compared to similar sized cities in North America (OCTranspo, 1999).

OC Transpo has expanded its route network in the suburbs to keep pace with the decentralization of population and employment, but has had to offset the increased service hours and costs by stretching its services in the older more established parts of the service area where ridership is highest. As a result, the transit routes have become more oblique, the transit services have become less frequent, and running times have become tighter and more difficult to control over the years (OCTranspo, 1999)

\subsubsection{Smart systems}

Recent advances in the effective use of information technology for transit referred to as 'smart systems' have provided a relatively low cost means for increasing and sustaining ridership. OC Transpo has already begun to implement smart system enhancements. An 
action plan to cover the next five years has been developed and addresses the following:

- Electronic fare collection: A single "smart" card could replace paper tickets and passes, as well as reduce the volume of cash and paper transfers used.

- Fleet operations management: A vehicle location monitoring system would be more cost-effective to operate than $\mathrm{OC}$ Transpo's current system, it would support the ParaTranspo fleet, and it would provide the basis for improvements in on-time performance, through real-time fleet location information.

- Traffic signal priority: Along certain routes, buses tend to be delayed due to unpredictable traffic problems. Adjustments to the traffic signals, to provide a longer green phase can be essential in restoring on-time performance.

- Passenger information: Enhancements to OC Transpo's current techniques for communicating passenger information, including the "560" telephone information system, the website and the station displays will be made. In general, additional information will be provided to passengers before and during their trips at an 
expanded range of locations. The station displays will provide ongoing updates to passengers about expected arrival times while they are waiting at major stations and terminals.

3.4.2 Automatic Passenger Counting (APC) at OCTranspo

\subsubsection{Introduction}

While many transit companies have found the idea of Automatic Passenger Counting tempting few have pursued this technical challenge and even fewer have been successful. As stated by Brian Barclay at OC Transpo, APC has been in full operation since 1986, collecting data on passenger movement and running times, and storing the data in a flexible database for reporting. Planners, Schedulers and Managers have a wide variety of APC reports at their disposal.

\subsubsection{The System}

The system uses a fleet of approximately 80 specially equipped buses and more recently Ottawa's own light rail service has been added. The APC fleet represents about ten percent of the total OCTranspo fleet, with each type of vehicle represented to ensure APC operation is unrestricted. Using a sample based approach, adequate data are gathered from these vehicles during one of four service periods that divide the year. Samples rates are often over 
ninety percent. Many of the trips are captured multiple times, and with close to 7,500 scheduled one-way trips each day, this translates into over 21,000 trips in the database. Assignments for APC buses are prepared the day before using an automatic assignment system that weighs user needs and requests against the vehicles available for the next day.

Each data bus (or train) has been equipped with infrared sensors that monitor doors for passenger movement. Passengers are counted as they enter and exit at each stop; time spent in this exchange is also recorded. Vehicle location is tracked by tapping into the vehicle odometer so that relative distances can be recorded with each activity. Later during data processing passenger activities stored in memory are associated to actual bus stops by using distance and interpreted bus movement combined with a strict set of parameters. This "to the bus stop level" reporting offers data users the greatest possible detail for their work. To assist with vehicle location accuracy, several on street microwave transmitters are used to add location benchmarks to the data as the bus travels its route. Coded signals are received by the buses roof top receiver. GPS is being phased in to replace these transmitters. This change in technology will add even greater accuracy to location work. 
Each night data are uploaded from all APC vehicles using a Automatic Radio Transmission Systems (ARTS). Data are also processed overnight. Processing puts captured data through very tight quality checks to maintain a high level of reliability. Reports can be run by the APC system staff each morning although, most requests use a completed service period to give a larger sample.

Passenger counting is only part of the work done by APC. Equally as important is APC's ability to report on running times collected by the system. The running time between any two bus stops on a route or collection of routes can be checked for any time period. Time utilization between stops can also be analyzed showing traffic congestion and time wasted; this is done using specialized logs (data records).

Schedule adherence reporting is yet another of the performance monitoring roles APC performs. APC also reports on revenue/cost ratio statistics. By adding the cost of service to the ridership and fare information, planners are quickly supplied with data that highlights those trips that are not operating efficiently. APC has gained acceptance through its demonstrated ability to provide timely input for solutions to practical problems. 


\section{Chapter 4: ArcView GIS and Network Analyst}

\subsection{Introduction}

Transportation data providers, including government agencies and private organizations, are discovering the convenience of publishing and disseminating transportation information with the use of a geographic information system, like ArcView GIS.

As defined by the developer, Environmental Systems Research Institute, Inc. (ESRI), ArcView3.2a is a desktop geographic information system (GIS). With ArcView3.2a one can create intelligent, dynamic maps using data from virtually any source and across most popular computing platforms. ArcView3.2a provides the tools to let one work with maps, database tables, charts, and graphics all at once. It enables one to link information to location (it connects the what to the where), allowing one to see and analyze data in new and useful ways.

Below is a typical ArcView3.2a project, which was developed by the author. A map, chart, and table have been used to depict route characteristics and to illustrate general locations throughout Ottawa. 


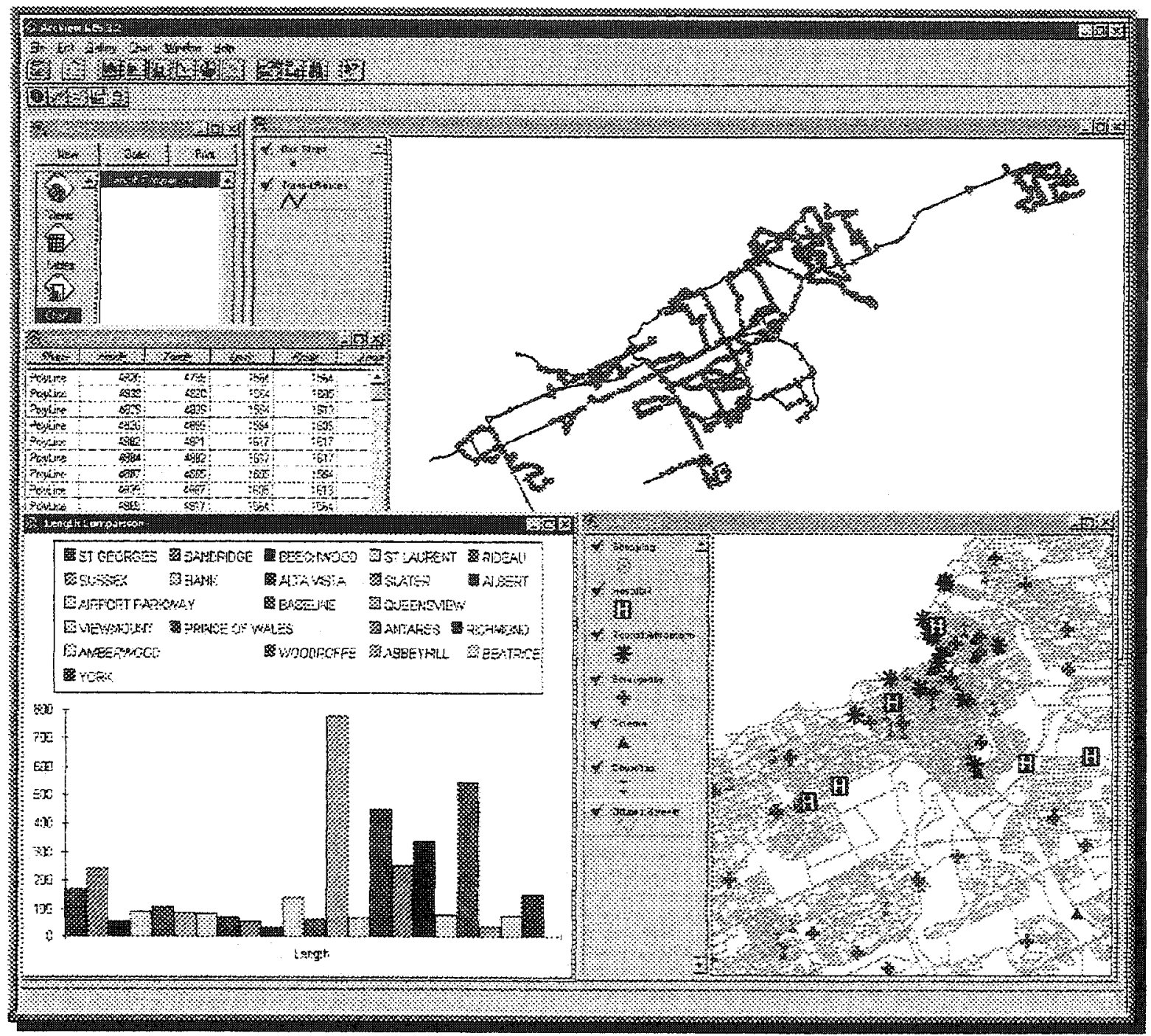

Figure 4.1: ArcView3.2a GIS Interface

The ArcView3.2a interface consists of windows that present information in different ways. Rows of menus, buttons, and tools at the top of the main application window allow you to view and perform analytical operations on the data in the database. 
ArcView3.2a has a number of features that make it an excellent tool for learning and using GIS. Unlike most GIS software of the past, ArcView3.2a provides a graphical user interface. This provides some benefits as defined by ESRI:

- No need to remember thousands of commands and command arguments.

- Most commands and operations are available by navigating the ArcView3.2a menu and dialog structure.

Only recently have computers become cheap and powerful enough to supply the resources needed for a fully functional GIS on a typical office or school desktop. At the same time, much effort has been undertaken by software manufacturers to increase the functionality and ease of use of their products. In the recent past, GIS software could be used only on high-end UNIX or VMS workstations. Today, PCs are powerful enough to handle GIS applications, and the software is easy enough to get started. It is possible that a user can become productive within the first few hours of using ArcView3.2a (www.esri.com).

ArcView3.2a has a strong programming language called Avenue. These scripts (algorithms) can be used to automate complex or repetitive tasks, or to create entire applications

Several extensions are shipped with ArcView3.2a, and others are available for free or at additional cost. These extensions increase the functionality of 
ArcView3.2a by adding features commonly found in other GIS software such as the Network Analyst. Some of the built-in optional extensions are used for direct use of CAD data, digitizer support, projections, and modeling. It is also possible to create personal extensions to run custom tasks or applications.

\subsection{Customizing with Avenue}

ArcView3.2a can meet a wide variety of GIS needs as stated earlier but it can also be customized. This means that the user interface can be modified for personal or public use. Examples are noted below (ESRI, 1996):

- Buttons not required can be removed,

- ArcView scripts can be accessed (created either by the programmer or obtained from other application developers) along with other applications such as Netscape and Windows based programs,

- New elements that are either specialized for a particular application or that give access to additional code that is not accessible within ArcView can be added.

The customization process is relatively simple. Changes made to the ArcView3.2a interface can be applied to a specific project, a user's default project, or to the system default ArcView project. This provides a great deal of 
freedom to ArcView3.2a programmers in developing applications that fit the needs of system users (ESRI, 1997).

When customizing in ArcView3.2a, Avenue is used to accomplish different tasks. One can use Avenue software to customize the standard graphical user interface (GUI) that comes with ArcView3.2a. For example, one can reorganize the controls (menus, buttons, tools, and popups), change text or icons, and add or remove choices. With Avenue, new functions for a specific application can also be created. One can combine a series of steps frequently used into a single click of a button. Avenue can also be used to develop a complete application that has its own GUI.

Avenue gives an easy-to-use framework for customizing controls and creating new functions. Dialog boxes can be created to modify controls and the Script Editor to write Avenue programs, called scripts. The scripts contain code that executes a new function. By using controls and scripts together, a new ArcView3.2a GUll is built (ESRI, 1997).

Avenue is able to perform all of these tasks because it was created as an objectoriented programming language. An object is an element, such as a view, theme, button, or a symbol, that one works with in ArcView3.2a. Objects with common characteristics belong to the same class. Each object is associated with a set of actions or requests. For example, requests for a layout object 
include opening, closing, and printing. A marker symbol object has a different set of requests, such as setting marker size and colour. If one can identify objects and their associated requests, one can write Avenue scripts (ESRI, 1996).

Object oriented programming addresses many problems inherent in the procedural approach to programming. The principal advantage of the objectoriented language is its ability to handle complexity in a clear manner. Using this programming, functionalities are placed inside the objects and out of one's sight. Consequently, to execute a specific functionality, one simply makes a request to the object (ESRI, 1997). This type of programming eliminates redundant code. Also, this saves time by building a program with objects, and that the quality of the programs improves because object functions are protected from the code that is written.

\subsubsection{Network Analyst}

Within ArcView3.2a, extensions can be installed for greater analysis than a basic query. One of these extensions is Network Analyst. This extension enables the user to find the best route, closest facility or service area. In ArcView3.2a, any system of interconnecting line features is a network. The user chooses the points of interest and manually steps through choosing specific constraints and options. ArcView3.2a uses the information and 
creates a Network theme be it a line or a polygon, to be used for interpolation.

When the Network Analyst is installed there are several new Avenue classes and requests added for solving network problems. These classes and requests can be used to automate tasks, add new capabilities, and build applications. Each of these can be changed or manipulated through the user interface in order to solve different and complex network problems (ESRI, 1997).

The Network class is the primary class used in solving network problems. It represents the network problem to be solved. Each of the problem solving requests operates on the Network class, which include finding routes, closest facilities, and service areas. Besides representing the network problem, the Network class can have several properties associated with it, such as cost fields or a customized search tolerance. There are several requests that operate on the Network class to set and retrieve these additional properties (Meyer and Miller, 2001).

The Network Analyst can solve network problems on any theme containing lines that connect. This extension is designed to help 
use networks more efficiently. The theme can be a shapefile, an ARC/INFO coverage, or a CAD drawing (Meyer and Miller, 2001).

Network analysis is one of the most frequently used components of ArcView3.2a. Once a road system has been digitized, a GIS can quickly be used to answer questions such as "what is the quickest way from $A$ to $B$ ?". It can be used to model the real world conditions and hence provide the shortest or quickest route as long as the network, calling points (stops) and the cost of traversing the individual links are identified. Two parameters normally define the cost of traversing a link, the length of the link and the time taken to traverse the link. The response algorithm can be made more complicated by adding impedances or "costs" onto major roadways. These may include speed restrictions, one-way systems, or temporary blockages caused by accidents or road repairs (Aronoff, 1995). Figure 4.2 summarizes the simple network operations of routing a vehicle or person through a street network. Based on predefined costs assigned to route segments, the illustration indicates the optimum path to travel. 


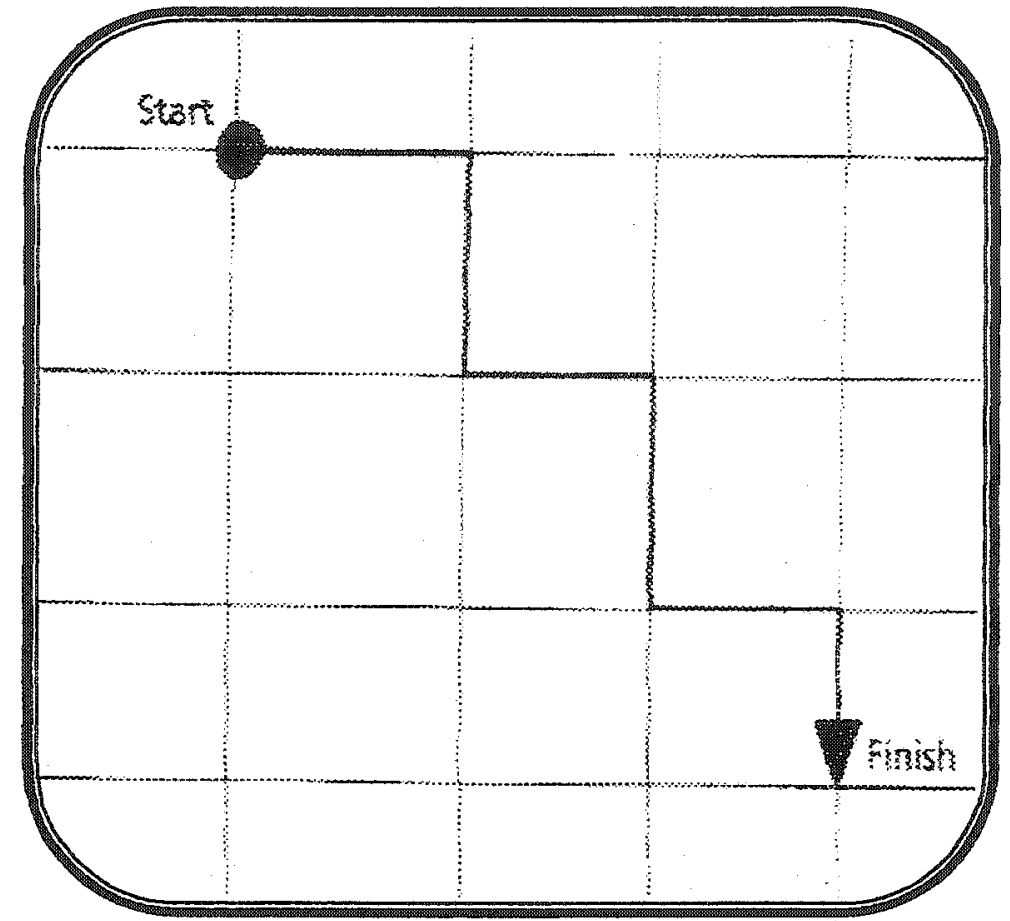

Figure 4.2: Shortest Path Routing (Aronoff, 1995)

Network Analyst supports different objectives, such as travelling quickly, travelling by the shortest route, travelling by the most interesting route or any criteria the user specifies. Network Analyst only needs a specified cost field in the line theme's attribute table when solving the problem. The cost fields that are used can be in any units of distance or travel time, such as minutes for finding a route that minimizes travel time, or kilometers for finding a route that minimizes travel distance.

An example of an application of a geographic information system's network analysis is a car navigation system based on GPS, designed to give up-to-date information as to how to avoid the 
latest construction or accident hold-ups. As well, it could keep precise knowledge on the locations of fire engines and ambulances so that the nearest vehicle can always be dispatched and response times improved. Coupled with navigation systems, these allow the drivers to find the quickest and safest routes to accidents or emergencies given the latest local traffic conditions.

\subsubsection{Route Finding}

The obvious approach to finding the shortest path to travel is to examine all the possible paths. A large network contains so many possibilities that it becomes impractical. Several algorithms have been developed to find the shortest (or least cost) path through the network. Network Analyst uses the most well known of these, developed by Dijkstra. In solving for the optimum route, ArcView scans the network for connectivity and travel cost relationships among the nodes. Once it has this information it derives the optimum path.

Route planning is a process that helps a person plan a route prior to or during a journey. It is widely recognized as a fundamental issue in the field of vehicle navigation. A variety of route optimization criteria may be used in route planning. The quality of a route depends on the distance, the travel speed, prohibited turns, 
one-way streets and the number of stops. All of these factors are referred to as travel costs. If the existing network has these rules of the road, they should be represented in the roads' line theme through cost factors before the Network Analyst is used. This will enable realistic solutions.

In the computer literature, people often refer to finding a route from point $A$ to point $B$ as the shortest path problem. By using Dijkstra's algorithm, ArcView can determine this least cost path between stops.

\subsubsection{Dijkstra's Algorithm}

Dijkstra's algorithm computes the shortest path from a given node, called the root node, to every other node in the network. A node exists wherever line segments connect. The set of paths found forms a sub-graph of the original network. This sub-graph turns out to be a tree (a tree is a graph having no loops in it). Hence, Dijkstra's algorithm finds the shortest path tree. (www.princeton.edu, 1959)

Consider a network, $R=\{N, A\}$ where $N$ is the set of nodes and $A$ is the set of arcs. Let $a_{i j}$ denote the length of $\operatorname{arc}(l, j) \in A$. 
Dijkstra's algorithm is an iterative procedure where at each iteration, one new node is added to the shortest path tree. The process starts with no edges in the shortest path tree and with the root node as the only node in the tree.

At each iteration, an array is built that contains the shortest distance from each node to the root node that is subject to the constraint that all nodes in the connecting path belong to the pathtree constructed. Initially, the array is equal to zero at the route node, then it is set to the distance from the root node to every adjacent node within the route system, and all other node paths are set to infinity. (www. princeton.edu, 1959)

Also, there is another array that indicates the node in the shortestpath-tree to which each node $j$ not yet in the tree has to be connected in order to obtain a path from the root node to this node. Initially, all adjacent nodes are equal to the route node, and the other $j^{\text {th }}$ nodes are set to some prohibited value (used as a flag). (www.princeton.edu, 1959) 
Until all of the nodes have been included in the shortest path tree, the following steps are performed:

- Loop through the nodes not in the shortest path tree to find the node with the smallest value of path length connecting it to the path tree. This node is now the new node. If its path length is infinite, then the network must be disconnected.

- The new node is added to the shortest-path-tree and an arc is added to the shortest-path-tree linking the new node back to the origin node.

- The path length and origin node are updated for every adjacent node that still needs to be added to the tree. Each new length is scanned for the lowest value. Once found, this adjacent node now becomes the new node, an arc is added, and the system advances until all nodes have been accounted for.

During the process, the program is determining the connectivity and travel cost relationships among nodes. Once it has the information, it derives the shortest path. This process can be done for any relevant cost field. (Ormsby and Alvi, 1999) 


\section{Chapter 5: Application Process and Algorithm Development}

\subsection{Overview}

The overall goal of this project was to design a user interface that would enable a person to find the optimum transit route to travel from an origin point to a destination point. It would enable visualization of schedule information and link to OC Transpo's website. As well, it would account for uncertainty in schedule adherence. In performing this task, network and geographic information is required along with a GIS software program.

\subsection{Software}

The software utilized is listed below.

ArcView3.2a/Avenue: ArcView3.2a desktop GIS software provides a userfriendly Graphic User Interface (GUI). Avenue, as an object-oriented programming language, provides an application development environment. Developing a Transit Information System in ArcView3.2a utilizing Avenue can make full use of the existing capabilities of ArcView3.2a.

Dialog Designer Extension: ArcView3.2a Dialog Designer provides flexibility to design and build custom user interfaces for different data entry and for organizing functions of the transit information system. 
Network Analyst Extension: Enables analysis of networks based on cost restrictions. It can solve common network problems on any theme containing lines that connect.

\subsection{Geographic Data}

\subsubsection{Data Source}

Before using the data each geographic file and database had to be created and/or prepared. Data were collected from the City of Ottawa and OC Transpo. This included full street network files both database and shape information from a compact disc provided by the city. These files were digitized by the City and contain service and characteristic information about the street networks. Bus stop locations, route layouts, schedules and timetables were collected from OC Transpo. Latitude and Longitude information pertaining to each bus stop was used to input the data into ArcView3.2a. The schedule information was entered into database tables. A small sample of landmark addresses was gathered from the phone book. All of these point locations were individually digitized into ArcView3.2a.

The following table provides the shape type for each map. The graphic and data information can be found in Appendix $D$. 
Table 5.1: Shape Types of Maps Used in the Transit Information System

\begin{tabular}{|l|c|}
\hline Majs & Shape Tloe \\
\hline Ottawa Street Network & Line \\
\hline Transit Route Network & Line \\
\hline Individual Bus Routes & Line \\
\hline Bus Stops & Point \\
\hline Landmarks & Point \\
\hline
\end{tabular}

Once the data have been added to the view, the map units are set so that the interface will yield meaningful distance units. These have been set to 'meters'. In the project, there are seventeen themes, the street network, the transit route network, geocoded locations (cinemas, churches, emergency, hospitals, hotels-motels, libraries, museums, tourist attractions, schools, shopping malis, grocery stores, recreation centers), bus stops, an empty theme for input information, and a hotlink.

The geocoded addresses were created from a list of digitized points. This is a function under the Theme / Properties menu in ArcView3.2a. For further information consult the ArcView3.2a help menu. The empty theme is used when the user enters a location by an address and to join multiple locations through landmark selection. This theme is then geocoded for use in finding the 
optimum route. The hotlink theme is used to link the user to $O C$ Transpo's website.

5.3.2 Setting the Rules of the Network

Road networks have rules about how vehicles progress through them. For a given street network, roads may be closed, or one-way streets and speed limits in place. These could determine if a street is traveled or not. Therefore, if the Network Analyst is to solve a problem based on the existing street network, care is required in representing correct traffic control to enable a realistic solution. The cost fields are important because ArcView3.2a uses these to determine either the quickest or shortest route to travel. The cost field is assigned the units of distance (kilometers, meters, miles) or travel time (minutes, hours).

In the Network Analyst, the rules of the road are as follows:

- One-way streets

- Prohibited turns

- Overpasses and underpasses

- Closed streets or authorized vehicles only

The following sections define these rules and how they were entered. 


\subsubsection{Travel Costs}

The units specified in the projection are used to specify length in the transit routes' and street's attribute tables. The script 'calalp.ave' (Appendix E) is used to input the length field into the tables. When the script is run, it calculates the length of each line segment and inputs a field named 'length' into the attribute tables. This script is an ArcView3.2a system script available for anyone's use.

A speed limit for each road was entered into the same attribute tables manually using the field calculator. This field is called 'speed_lim' and the units are specified in kilometers per hour. As well, a new field must be added called 'cfcc'. These are codes based on the type of road (freeway, two-way with median, residential) the speed limit corresponds to. All of the coding is available from a systems table (refer to Appendix F). A sample extension called Speed Limit Calculator applies speed limit information to a cost distance field (e.g. minutes, meters). By selecting the option of Speed Limit Calculator from the Network Analyst menu, this new field is created. The 'minutes' field represents travel according to drive time. 
In addition to the above speed limit costs, the dwell time costs of opening and closing doors, boarding and deboarding passengers, and merging back into traffic was added on to the sections of the network that contained bus stops. As defined earlier in chapter 2, dwell time can be assumed 2 to $5 \mathrm{~s}$ for door opening and closing plus an additional time to be 60 s for $\mathrm{CBD}$, transit stations, or major on-time transfer points; 30 s for major outlying stops; and 15 s for typical outlying stops. These values were added on in accordance to the location of the stop along the route and the location within the city.

\subsubsection{One-way Streets}

The city of Ottawa has many one-way streets, especially in the downtown. Therefore this cost cannot be assumed insignificant. A field was created to represent streets that can only be traveled in one direction. This is a string field that is added to the roads theme. Its name must be ONEWAY in order for the Network Analyst to recognize it.

When setting one-way streets, the direction of the digitizing must be known. The direction of the digitization can be found by having the roads indicated by arrows in the view. Once this is known, the oneway field can be populated with the correct string information. If the 
one-way street runs in the same direction as the digitization, then the field should have the value of 'FT'. If it runs in the opposite direction it has a value of 'TF'. ' $\mathrm{F}$ ' corresponds to 'from' and ' $T$ ' corresponds to 'to'. If there is no entry permitted, it can be represented by using ' $N$ '. Any other value or a blank cell represents travel in both directions (Ormsby, 1999).

\subsubsection{Overpasses and Underpasses}

Street themes contain many locations where one line feature crosses another. Some of these locations designate an intersection where it is possible to make a left or a right turn from one street onto another. Other locations designate an overpass or underpass, where in reality there is no physical connection between the two streets. Modeling overpasses and underpasses allows a definition of which streets are physically connected (Ormsby, 1999).

The overpasses in this network were not specified as a field in a table. This is because the map was digitized with nonplanar streets for overpasses and underpasses. This means that the streets do not have connections or nodes. This method involves two unbroken lines, one passes completely over the other to represent an overpass. Therefore it is not necessary to explicitly set them 
because Network Analyst considers them not to be connected and does not stop at them to consider a route left or right.

\subsubsection{Closed Streets}

Streets currently closed to traffic or certain types of streets to avoid should be used so that the Network Analyst knows not to select them for travel. For this project, the closed streets cost was performed at the same time as the one-way streets' field was created. As mentioned under one-way streets, an ' $\mathrm{N}$ ' was used to indicate a road is closed to traffic. This cost field can also be indicated as a separate field. The other methods used can be found by consulting the ArcView3.2a help menu.

Figure 5.1 shows a sample of the transit route attribute table showing the added fields. As seen in this figure, the fields created are: speed_lim, length, and minutes. 


\begin{tabular}{|c|c|c|c|c|c|c|c|}
\hline \multicolumn{6}{|c|}{ Stransit routes dibl } & \multicolumn{2}{|c|}{ - $3 x$} \\
\hline 20 & $3 x+510$ & $4 x$ & hos & 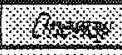 & $12 \times$ & 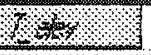 & \\
\hline 463 & 25 & 41.514 & 0.628 & $F$ & D: & 0 & 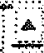 \\
\hline$A 63$ & 25 & 38.361 & 0.624 & $F T$ & 0 & 0 & \\
\hline 225 & 55 & 115.173 & 0.645 & & 0 & 0 & \\
\hline$A 63$ & 25 & 46233 & 0.636 & $F$ & 0 & 0 & \\
\hline 863 & 25 & 47.187 & 0.637 & $F$ & 0 & 0 & \\
\hline$\$ 25$ & 55 & 237.769 & 0.728 & & 0 & 0 & \\
\hline $\mathrm{A41}$ & 25 & 90.069 & 0.450 & TF & 0 & 0 & \\
\hline A41 & 25 & 76.949 & 0.15 & TF & 0 & 0 & \\
\hline A25 & 55 & 115.303 & 0.078 & & 0 & 0 & \\
\hline A41 & 25 & 91.243 & 0.438 & TF & 0 & 0 & \\
\hline$A 41$ & 25 & 105.045 & 0.474 & TF & 0 & 0 & \\
\hline 863 & 25 & 229.026 & 0.909 & $F T$ & 1) & 0 & \\
\hline$\Delta 41$ & 25 & 68.446 & 0.102 & TF & 0 & 0 & \\
\hline A25 & 55 & 144.935 & 0.098 & & 0 & 0 & \\
\hline Ad1 & 25 & 51.601 & 0.403 & TF & 0 & 0 & \\
\hline$A 41$ & 25 & 1142255 & 0487 & IF & 0 & 0 & \\
\hline A41 & 25 & 101.515 & 0.468 & TF & 0 & 0 & \\
\hline$A 41$ & 25 & 102346 & 0.470 & TF & 0 & 0 & \\
\hline 801 & 30 & 278.07 & 0.913 & TF & D & 0 & \\
\hline 84 & 25 & 83844 & 0.125 & TF & 0 & 0 & \\
\hline$B 41$ & 25 & 149.979 & 0.791 & IF & 0 & 0 & \\
\hline 8.41 & 25 & 94726 & 0.141 & $\mathrm{TF}$ & 0 & 0 & \\
\hline 425 & 55 & 465589 & 0.803 & & 0 & 0 & 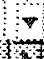 \\
\hline
\end{tabular}

Figure 5.1: Sample Attribute Table

\subsection{Purpose of maps}

\subsubsection{Base Street Network}

The base street network provides the core geographic data for the transit information system. It is used to create individual bus routes, display street map to users, and provide geocoding capabilities for data preparation and for operation. 


\subsubsection{Individual Routes and Route Network}

Like the base network, individual route information allows specific routes to be displayed as needed by analysis results. Individual routes are also used to create the entire route network and allow for easy maintenance of the network if one route from the network requires alterations.

Network Analyst was used to find the optimum travel path. Individual costs were inserted so that additional information would be included in the calculation so that the optimum travel path was implemented.

\subsubsection{Bus Stops and Landmarks}

By using the bus stop map, the location of the closest bus stop to the users' specified locations can be pinpointed. In addition, these points are used to display for users the places where they would start and end their trip in relation to their selected origin and destination. The maps of landmarks are not only utilized for displaying landmark locations, but also allow users to select their origin and destination location directly from the list of landmarks. 


\subsection{Data Preparation Problems and Solutions}

As the project progressed various obstacles and data problems presented themselves. The problems encountered and the degree to which a solution was necessary to continue with the research project are discussed below.

\subsubsection{Base Street Network}

Although the Ottawa area street network from the city of Ottawa was acquired in September 2001, some of the data it contained needed to be updated and addresses fixed. A limited number of routes were acquired; therefore if there were major problems with certain parts of the street network where roads were missing these routes were eliminated. This aided in the development process. For, if these routes were used, the street network would have required additional digitizing risking problems with compatibility with the original street network design.

In addition to missing streets, the geocoded information provided on the map also created problems. First, address ranges provided by the city were incomplete or incorrect. In some cases additional maps were required to fix the problems in order for the geocoding process to work correctly. Due to the scope of the project, corrections made to the acquired map were limited, as correcting all 
of the errors in the geocoding information of the base map was not possible. However, during manual digitization of missing streets, respective address ranges were entered as accurately as possible to minimize additional errors in address matching.

\subsubsection{Individual Bus Routes and Route Network}

Shape files (themes) of individual routes in addition to the entire route network were essential to the system. To create these shape files, each bus route was digitized from the base road network and saved independently. Merging the individual routes into one shape file using an ArcView3.2a tool created the route network.

Unlike the base network, the problems encountered when creating the individual bus routes were not as extensive. The primary concern regarded the street network. Again, it was not possible to make manual corrections in every case. Corrections were made in extreme cases that were recognized early enough to not create an exorbitant amount of backtracking in the project progression.

Cases also arose where bus routes travel through parking lots that are not represented on the base network map. In these cases, the road closest to the location was used as the approximate route or a segment was digitized to represent approximately where the route would travel. Information was also added to describe what the 
segment represented (e.g., Nepean SportsPlex, Carlingwood Mall). In the future, buildings/parking lots will be added and the corresponding costs to travel these areas will be incorporated. In some instances the wait time through these areas (i.e., General Hospital) will most likely be more significant than that presented in this thesis.

\subsubsection{Bus Stops and Landmarks}

Although the OC Transpo schedule book only lists times at specific points (time points) on the routes, all stops were required to be extracted from the entire route network. Therefore all of the stops were digitized based on latitude and longitude coordinates collected by OC Transpo to create the bus stop shape file. Although the stops were recognized, their descriptions needed to be entered. Users need an explanation or intersection name to locate a bus stop, because providing them with a number would just create confusion. Thus, intersection names (e.g., Merivale and Meadowlands, Montreal and St. Laurent) or location descriptions (e.g., on Baseline Station at stop 1A) were manually entered for respective bus stops to complete the bus stop map and database.

To create the Landmark geographic file, an Excel database of landmarks was created. Using the addresses in the Excel 
database, the landmark shape file was created through address matching (geocoding) with addition location corrections made manually in the shape file.

It is essential that the final location of each landmark in the landmark database is accurate; therefore an initial inspection of addresses was made. Each address was checked to make sure that it matched the base network. Problems encountered after inspection included landmarks not recognized by the system because the address on the streets was incorrect or out of date. Hence, the base network was recoded for address placement and the landmarks were re-matched.

\subsubsection{Schedule Database}

One of the important elements in the system is the OC Transpo schedule database, developed in Excel. The database contains information for the entire bus schedule, data of time-points, which are the bus stops provided in the schedule booklet, and descriptions of routes and time-points for weekend, Saturday and Sunday (Appendix H). The database is used for finding the shortest bus route and to provide schedule information to the users. 
The schedule databases are considered relational databases made up of multiple tables rather than a single table. These tables are related to each other through the information they contain. To establish the relationship, a common field between the tables is needed. This field enables the tables to be linked together and can be used to search for information throughout the tables.

Three tables are contained in the developed schedule database. They are the schedule table, the bus route table, and the bus stop table. The schedule table contains the entire schedule for each trip of each route. The bus route table includes information related to routes such as route names, descriptions, and their availability during the week. Lastly, the bus stop table provides data related to bus stops, such as the stop number, descriptions, and arrival times. Figure 5.2 provides a flow chart describing the relationship between these three tables and highlights the identifiers between each table that allow for the link to exist. 


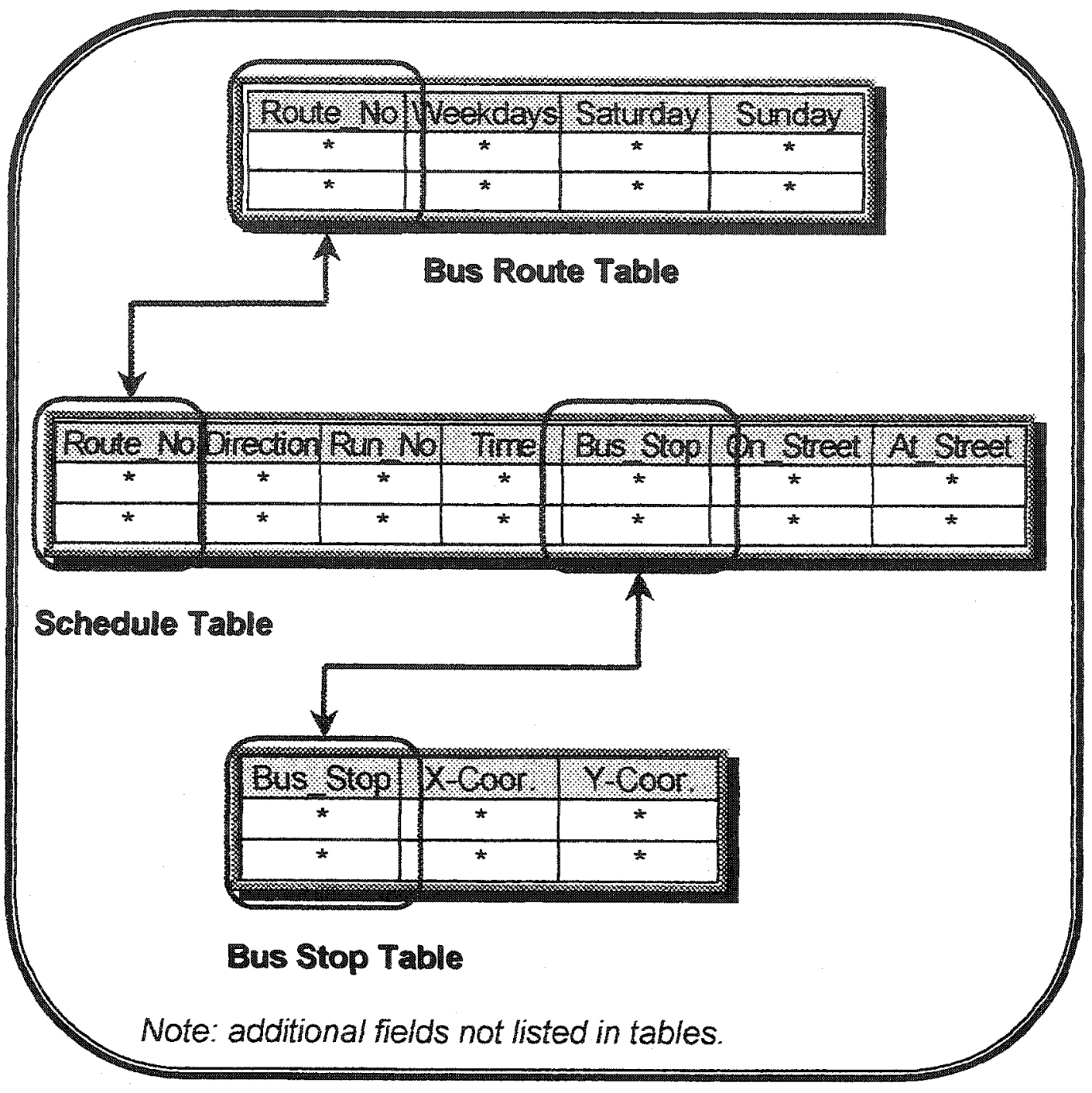

Figure 5.2: Relationships between Tables in the Schedule Database

As shown, there are two relationships among the tables: one between the schedule table and the bus route table, and the other between the schedule table and the bus stop table. The field establishing the relationship between the bus route table and the schedule table is given as "Route_No", which is the route number, 
while "Bus_Stop" is the field that establishes a link between the schedule table and the bus stop table.

Detailed route and stop information can be derived through the schedule table because of these links. Even if the schedule table does not physically contain detailed information concerning routes and stops, information in the bus stop table and the bus route table can be located using the links between the tables. Therefore, after the calculations in the schedule table, other detailed user information can readily be derived from the bus route and stop tables.

\subsection{Algorithm Development}

\subsubsection{Overview}

A crucial part of the development of the transit information system is the algorithm for finding the optimum path. In solving optimum path problems, static methods have been the foremost approach. However, on transit networks, there is a level of uncertainty pertaining to arrival times due to factors such as traffic congestion, demand on the route, and malfunctions. Therefore true representation of transit networks should be probabilistic. In addition to being probabilistic, travel time on transit networks is constrained by a schedule; transit schedules are time-dependent. 
The optimal path varies according to the time of entry into the system (Koncz, Greenfeld, Mouskos, 1996). If the system was not time dependent then a Dijkstra type algorithm can be used. However, optimum paths that are time-dependent can be found equally efficiently by using a Dijkstra algorithm with some modifications (Tong, Richardson, 1984). Therefore, it is assumed that transit travel will adhere to arrivals according to a schedule and that Bayesian statistics will be applied to compensate for the probabilistic nature of travel.

Since the transit system varies from that of a private automobile. The departure and arrival time from the origin to the destination are dictated by the bus schedule; they vary from daytime to evening, and from weekdays to weekends. Therefore, by combining the schedule data, extracted from the OC Transpo schedule database, with the network data from the entire bus route network, a new algorithm is developed.

\subsubsection{Development within ArcView}

In order to perform this task effectively, ArcView3.2a is used since it applies Dijkstra's algorithm (Chapter 4). ArcView3.2a contains four capabilities that are useful and necessary for building a transit information system. First is the capability of the input of standard street network files by any agency. Second is the ability to do 
address matching or geocoding. Third is the ability to do pathfinding, whereby an optimal path based on time or length is found from an origin to a destination through any desired stops. Last is the ability to answer spatial analysis questions or queries such as finding the nearest facility. These capabilities, in addition to the visualization tools provided in a GIS, allow for ease of implementation (Koncz and Greenfeld, 1995).

For further modification, instead of starting at time zero at the origin node, the actual clock time is used. The arrival time at various nodes in the network will also be recorded as the actual clock time. As well, the length of an arc is dependent on the arrival time at the beginning of the arc. Hence, in order to determine the shortest travel time from node $A$ to $B$ the procedure is to search through the network file and find all arcs which match, and then for each of these arcs in turn read their start time from node $\mathrm{A}$ and then read the arrival time at node $B$ and find the difference in time.

Before assigning a time to a bus stop, it is assumed that the bus gets to the time point on time. That is, the scheduled time at a time point is the real time a bus gets to that point. To assign a time to each stop, it is necessary to know the direction and distance of a stop to a time point where the scheduled time is known, as well as the speed of the bus between two time points. The distances of 
each time point and bus stop has been obtained through one of ArcView3.2a's system algorithm (calalp.ave). Therefore, the distance and the direction between a stop and a time point can be identified. Through linear interpolation, each bus stop will read an arrival time when the information system is run.

The nearest bus stops to the chosen origin and destination are found using another ArcView3.2a script. This script enables a bus stop to be chosen within a distance of the chosen origin and destination points. An example of this script is shown below.

\section{Syntax}

aNetwork.FindClosestFac (aOriginPointList, aFacilityPointList,

NumFacs, CutOff, ToFacilityBoolean)

\section{Example}

' Calculate the closest tow trucks to a list of cars.

NumTowTrucksToFind $=1$ ' find the closest tow truck

CuttOffDistance $=0$ ' search the entire network

ToFacilityBoolean $=$ False ' travel is from the tow trucks to the cars

aNumFoundList $=$ aNetwork.FindClosestFac(aPointListOfBrokenDownCars, aPointListOFTowTrucks, NumTowTrucksToFind, CuttOff, ToFacilityBoolean)

Figure 5.3: Syntax of Closest Facility (ArcView3.2a)

\subsubsection{Structure}

In developing the structure of the transit information system, an attempt was made to simulate the communication that takes place 
when the telephone service is utilized. Like telephone service interaction, the structure of the transit information system has three components: input of users' information, calculation of the shortest bus route, and display of the result. The following steps provide a description of the process used by the algorithm (Figure 5.4):

- Obtain information from user and step through user interface

- Geocode the origin and destination stops

- Search for bus arrival times at the origin point closest to the user's input

- Search all bus routes containing the origin or the destination point

- Obtain the closest bus stop to the origin and destination

- Obtain the nearest bus stop with a time point for linear interpolation

- Calculate the arrival time at the bus stop closest to the origin and destination

- Using the applied costs, calculate the travel time from the origin to destination

- Apply Bayesian statistics to the travel time

- Output the results graphically and with a written itinerary 


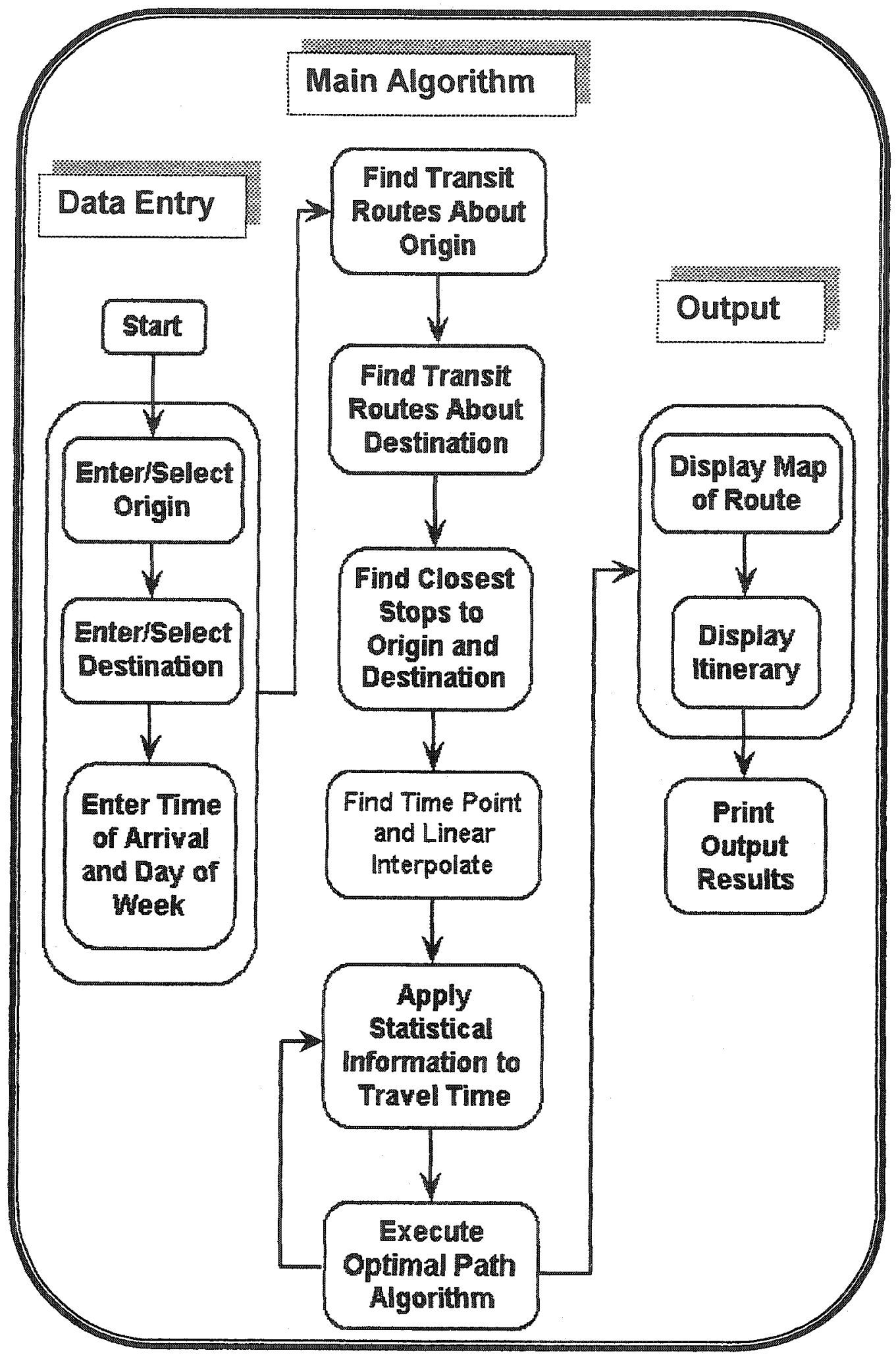

Figure 5.4: Algorithm Used Within Transit Information System 
Within the transit information system, the database plays an important role. It organizes data describing the transit system, and provides a mechanism to store and manage the preferences of the user as they interact with system. Considerable effort is required when designing the structure of the database tables. 


\section{Chapter 6: Statistical Analysis}

\subsection{Introduction}

Statistics are generally defined as the collection, classification, presentation, and analysis of numerical data (McGrew, Monroe, 1993). The use of statistics allows one to:

- Describe and summarize spatial data

- Make generalizations concerning complex spatial patterns

- Estimate the probability of outcomes for an event at a given location.

Engineers commonly use statistical analysis to solve problems and make decisions based on limited information about one or more of the parameters of the problem. The types of information available to them can be either objective or subjective. Objective information is based on experimental results or actual observations of a phenomenon. Subjective information is based on experience, intuition, other previous problems that are similar to the one under consideration, or the physics of the problem (Ayyub and McCuen, 1997).

Variables in the data sets used can be characterized as either discrete or continuous. A discrete variable has some restriction placed on the values that the variable can assume. A continuous variable has an infinitely large number of possible values along some interval of a real number line. In general, discrete 
data are the results of counting, and potential values are limited to whole integers. Continuous data are the result of measurement, and values can be expressed as decimals (McGrew and Monroe, 1993).

If objective information has been gathered, it can be dealt with using the theories of probability and statistics. In this type, probability is interpreted as the frequency of occurrence assuming sufficient repetitions of the problem, its outcomes, and parameters, as a basis of the information. Subjective information reflects the judgment of the decision maker, the engineer, or analyst studying the problem. Subjective probabilities are used for decision making under uncertainty. These reflect the state of knowledge of the engineer or the analyst (Ayyub and McCuen, 1997).

It is common in engineering to encounter problems that are solved with both objective and subjective types of information. In these cases, it is desirable to utilize both types of information to obtain solutions or make decisions. The subjective probabilities are assumed to constitute a prior knowledge about a parameter, with gained objective information (or probabilities). Combining the two types produces posterior knowledge. The combination is performed based on Bayes' theorem (Ayyub and McCuen, 1997). 
Bayes' theorem follows from the basic rules of probability. Its importance is that it enables analysts to update their knowledge, expressed in probabilistic terms, as new data are obtained.

\subsection{Bayesian Analysis}

Although Bayesian methods are attracting increasing attention there have been relatively few Bayesian applications in the transportation field. Researchers in other disciplines are adopting Bayesian techniques because they provide a principled approach for incorporating non-sample prior information, and they avoid erroneous approximations (Brownstone, 2000). For transit applications, consistent examinations of route conditions are required so that the public is informed and updated. By applying Bayesian techniques, scheduled travel information can be continually updated based on observations and expert knowledge. Therefore, the Bayesian technique was adopted for this research.

\subsubsection{Introduction}

This transit information system takes into account the initial estimate of travel time based on the latest available information (schedule information). The system then checks to see if $O C$ Transpo has given any revised information based on travel conditions. 
Within the database, travel time estimates are noted as R1, R2, R3, meaning early, on time, or late respectively. The actual travel times are treated as uncertain, and are revised based on gathered statistical information

Once the journey is complete, the actual time from origin to destination becomes known. The actual time can be different from the estimated time, given a large number of sources of uncertainty. To name a few, weather-related delays, lane blockage due to snow clearing activities, accidents or incidents that result in lane blockage, unforeseen traffic conditions on mixed traffic links, blockage of bus-only lanes on city streets, passenger loading/unloading and other processes on the Transitway that cause deviations from the schedule.

\subsubsection{Bayesian Approach}

The Bayesian statistical approach to model a phenomenon is to systematically combine prior knowledge and experience with new data to improve the predictive relationship. The Bayesian methodology enables decisions to be made in the short term while improvements to the data, judgment, and the model continue to be made. The Bayesian solution achieves a balance between two 
solutions based on initial observations and updated information (CSHRP, 2000).

In assembling information, the data collected are supplemented with prior knowledge. This approach is summarized Figure 6.1. When further information is obtained, the probability is updated using Bayes' theorem. The distribution before updating is called the prior distribution and the updated distribution is the posterior distribution (McGrew and Monroe, 1993).

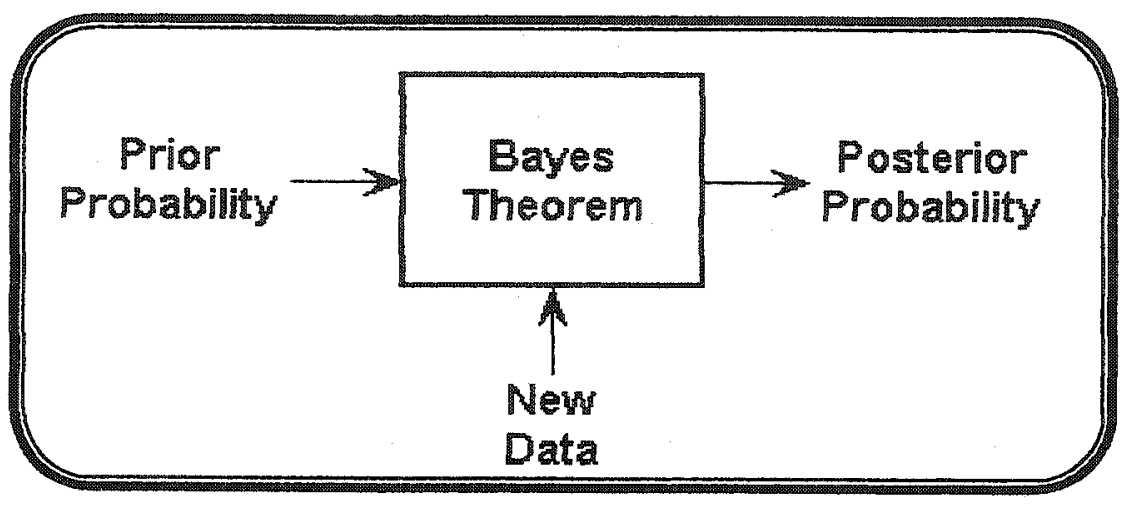

Figure 6.1: The Bayesian Statistical Approach (adapted from CSHRP, 2000)

The advantages of using Bayesian methods justify the increased effort in computation and prior determination. Bayesian methods provide one with the ability to formally incorporate prior information. The assumptions are conditional on the actual data and all of the analyses follow directly from the posterior distribution; no separate theories of estimation, testing, multiple comparisons are needed. 
Any question can be directly answered through Bayesian analysis (Carlin and Louis, 2000).

\subsubsection{Bayes' Theorem}

Bayes' Theorem allows one to assess the probability of A given that $B$ has already occurred. For this development, there are two simple formulas from probability theory. If $\mathrm{A}$ and $\mathrm{B}$ are two events, then:

$$
P(A \mid B)=P(A \text { and } B) \quad \text { if } P(B) \neq 0
$$

$P(A)=P(A$ and $B)+P[A$ and $($ not $B)]$

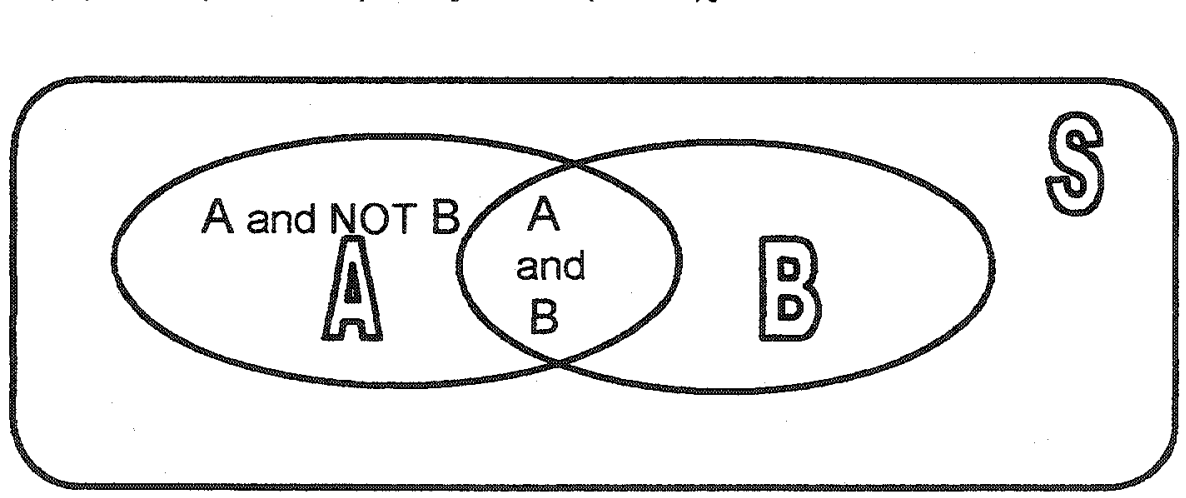

Figure 6.2: Venn Diagram of Event $A$ and Event $B$

Now if a set of mutually exclusive events $A_{1}, A_{2}, . . A_{N}$ (prior, subjective information) form a partition of a sample space where $A_{1}$ $+A_{2}+. .+A_{N}=S$ and $E$ (objective information) is an arbitrary event 
(Ayyub and McCuen, 1997) as shown in the figure below, then the theorem of total probability states that:

$P(E)=P\left(A_{1}\right) P\left(E \mid A_{1}\right)+P\left(A_{2}\right) P\left(E \mid A_{2}\right)+\ldots+P\left(A_{N}\right) P\left(E \mid A_{N}\right)$

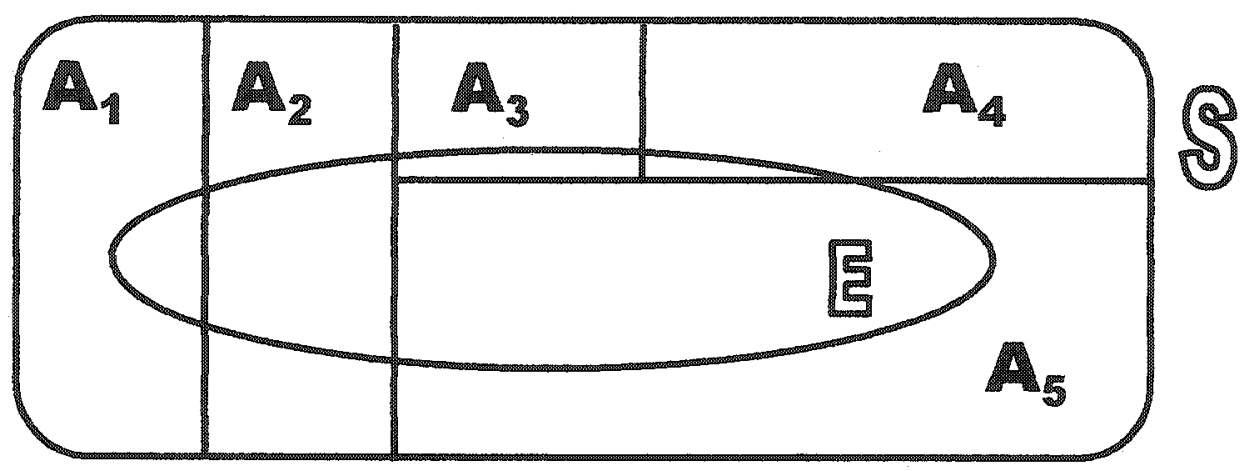

Figure 6.3: Venn Diagram of Total Probability

This theorem is very important in computing the probability of the event $E$, especially in practical cases where the probability cannot be computed directly, but the probabilities of the partitioning events and the conditional probabilities can be computed.

Bayes' theorem is very useful in computing the posterior (or reverse) probability of the type $P\left(A_{i} \mid E\right)$, for $i=1,2, \ldots, n$. (Raiffa, 1968, Ayyub and McCuen, 1997). The posterior probability can be computed as follows:

$P\left(A_{i} \mid E\right)=\frac{P\left(E \mid A_{i}\right) P\left(A_{i}\right)}{P\left(E \mid A_{1}\right) P\left(A_{1}\right)+P\left(E \mid A_{2}\right) P\left(A_{2}\right)+\cdots+P\left(E \mid A_{n}\right) P\left(A_{n}\right)}$ 
Or in more general terms as:

$$
P\left(A_{i} \mid E\right)=\frac{P\left(E \mid A_{i}\right) P\left(A_{i}\right)}{\left.\sum_{j=1}^{n} P\left(E \mid A_{j}\right) P\left(A_{j}\right)\right)}
$$

The denominator of this equation is $P(E)$, which is based on the theorem of total probability. According to the above, the prior knowledge, $P\left(A_{i}\right)$, is updated using the objective information, $P(E)$, to obtain the posterior knowledge, $P\left(A_{i} \mid E\right)$. In general as more and more data are added to the problem, the posterior will continue to become more and more definitive.

\subsection{Method of Application}

This approach treats origin-destination travel time as uncertain. To begin the analysis, the system was assigned three states-of-nature (T1, T2, T3), which are uncertain travel times. T1, T2, and T3 correspond to early, on time, and late arrival respectively. As defined by OC Transpo during data collection: "The allowance for 'early' is tight but the 'late' allows a few minutes". As explained further, a bus was categorized as early if it was one to two minutes earlier than scheduled. A bus was considered late if it arrived 3 to 4 minutes after the scheduled time. Therefore the probabilities obtained are based on these conditions. 
Since the Ts are discretely defined, meaning the travel time can either be one of three choices, the probabilities have been regarded as 'discrete' and assigned on the basis of such rationale as: low probability of early arrival at the destination, high probability of on-time arrival, moderate probability of late arrival. The sum of these equals one. The probabilities have then been estimated on the basis of frequency analysis of OC Transpo data.

When the data were compiled, there was a relatively lower number collected over the weekend. The lower number of observations is acceptable for Bayesian analysis because as more data are collected, the system can be updated and new posterior probabilities can be obtained.

For the purpose of calculating travel time, the three travel times were based on OC Transpo's information on what is categorized as early, on time, and late. Therefore T1, T2, and T3 were assigned travel times as follows:

T1: Travel time - 1 min or more

T2: Travel time (if arrival is less than 1 minute early or less than 3 minutes late) T3: Travel time +3 min or more

The prior probabilities of the Ts, $P^{\prime}(T)$, are found in Table 6.1. These have been defined based on OC Transpo's time periods. These are for off peak periods $(18: 31-6: 30 \& 9: 31-15: 30)$, peak periods $(6: 31-9: 30 \& 15: 31-18: 30)$, and peak hours $(7: 31-8: 30 \& 15: 31-16: 30)$. As an overall summary of all day performance, these were also compiled. To represent the sample routes within the application, probabilities were estimated for both Transitway $(95,97)$ and 
non-Transitway $(3,118)$ routes. The data values were obtained through OC Transpo's AVL/C system.

Therefore, the initial expected travel time based solely on prior probabilities is calculated as follows:

Expected Travel Time $=P(T 1)(T 1)+P(T 2)(T 2)+P(T 3)(T 3)$

\subsubsection{Information Updates}

By making frequent checks with road conditions and travel times, the system can obtain the latest estimates of time. Within the transit information system, the transit agency must specify their perception of how the buses are running. A code is entered into the transit route table corresponding to early, on time or late conditions; R1, R2, and R3 respectively.

\subsubsection{Conditional Probabilities}

The conditional probability, for example $P(R 1 \mid T 1)$, is the probability of getting the indication of 'early arrival', given that the actual state is T1 (early arrival). In other words, if it turns out that the traveller will arrive early, what is the probability that the information obtained implies the same. Likewise $P(R 2 \mid T 1)$ defines the probability of getting the on-time indication, given that the true state is early arrival. 


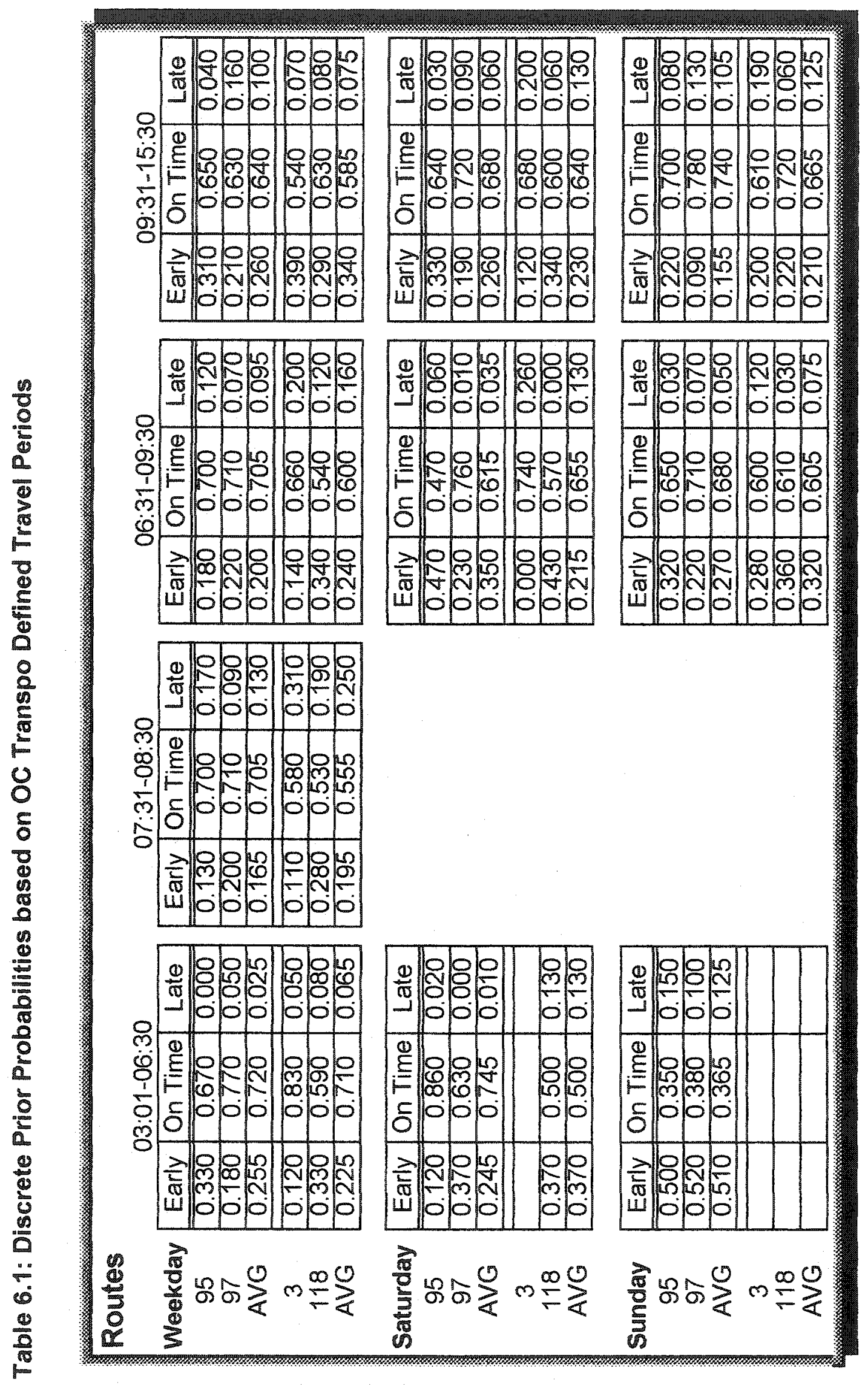




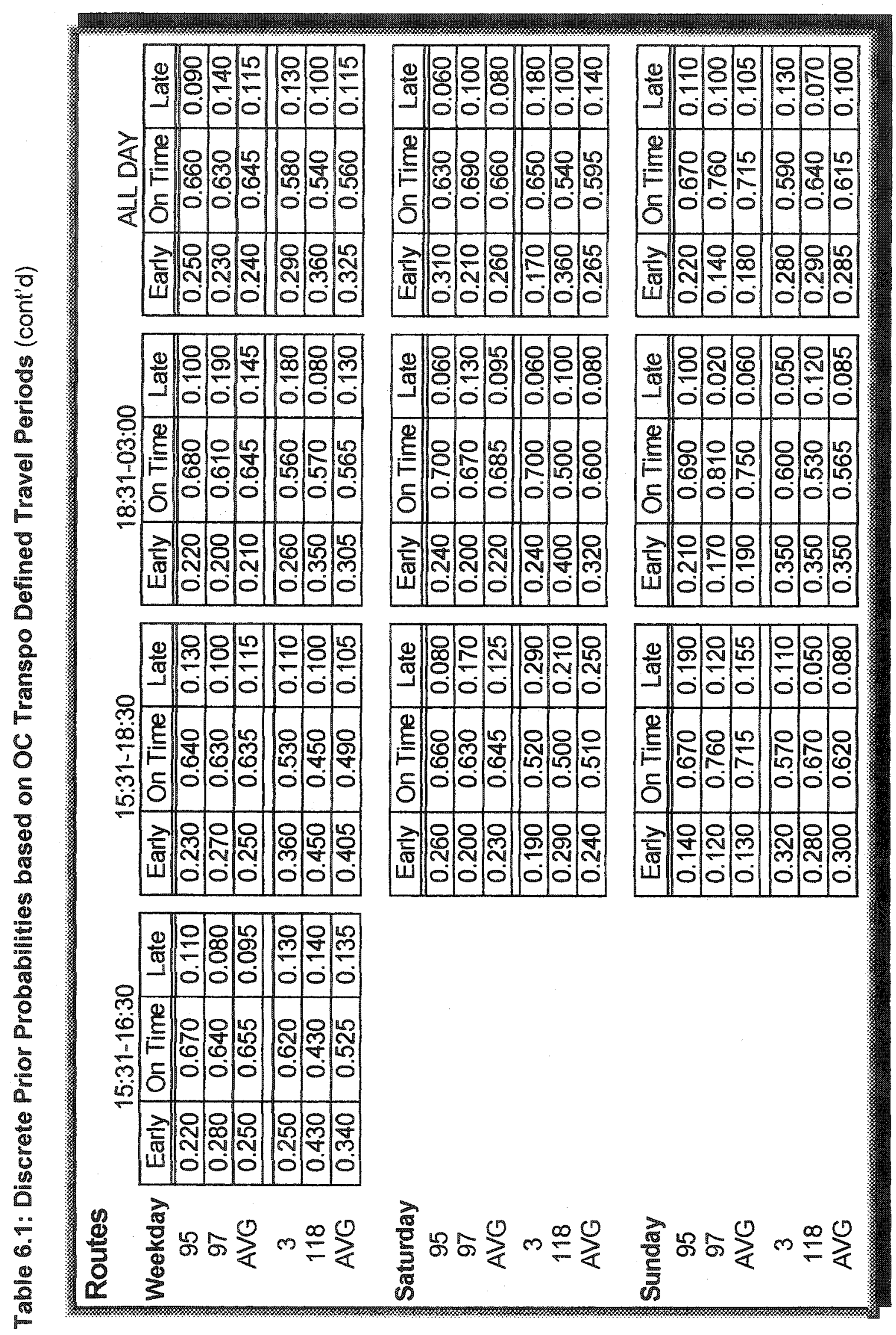


This conditional probability information was obtained through $O C$ Transpo's AVLC system. Based on previous experience, the conditional probabilities were estimated. These are shown in Figure 6.4. That is to say if the actual travel time turns out to be 'early', the probabilities of getting the advisory as early, on time or late are $70 \%, 25 \%$, and $5 \%$, respectively. The probabilities of receiving advisory are early, on time, or late given that the true travel time will be 'on time' are $15 \%, 70 \%$, and $15 \%$, respectively. And the probabilities of receiving advisory as early, on time, or late given that the true travel will be 'late' are $5 \%, 25 \%$, and $70 \%$.

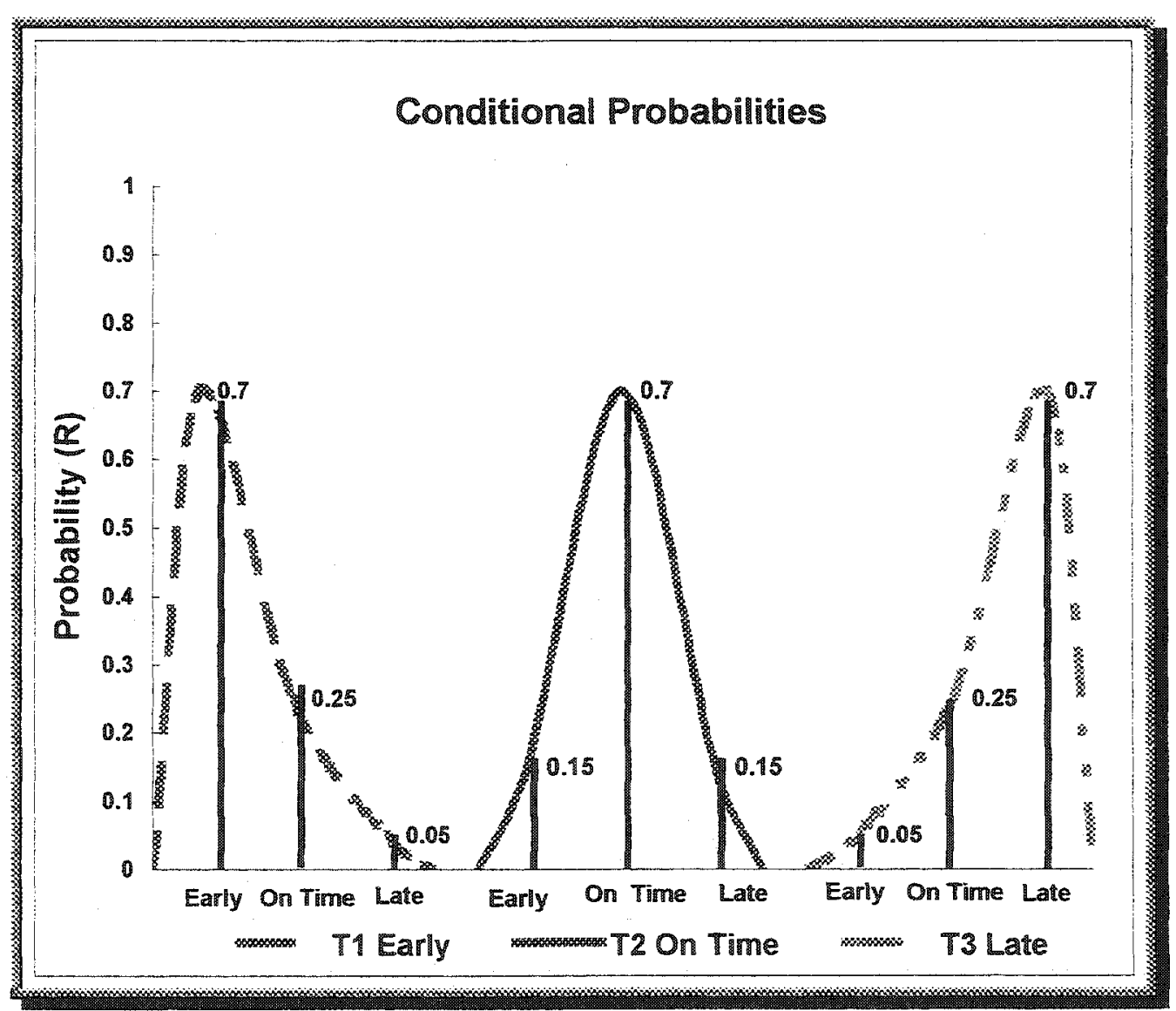

Figure 6.4: Conditional Probabilities 
These conditional probabilities can be regarded as the reliability of information. As more data are obtained on a seasonal or yearly basis, modifications can be made.

\subsubsection{Posterior Probabilities}

These are revised probabilities of states of nature, found by combining old information with new information. Bayes' Theorem is used to calculate posterior probabilities.

If $\mathrm{R} 1$ is received as the information:

$$
\begin{aligned}
& P^{\prime \prime}(T 1 \mid R 1)=\frac{[P(T 1) P(R 1 \mid T 1)]}{[P(T 1) P(R 1 \mid T 1)+P(T 2) P(R 1 \mid T 2)+P(T 3) P(R 1 \mid T 3)]} \\
& P^{\prime \prime}(T 2 \mid R 1)=\frac{[P(T 2) P(R 1 \mid T 2)]}{[P(T 1) P(R 1 \mid T 1)+P(T 2) P(R 1 \mid T 2)+P(T 3) P(R 1 \mid T 3)]} \\
& P^{\prime \prime}(T 3 \mid R 1)=\frac{[P(T 3) P(R 1 \mid T 3)]}{[P(T 1) P(R 1 \mid T 1)+P(T 2) P(R 1 \mid T 2)+P(T 3) P(R 1 \mid T 3)]}
\end{aligned}
$$

If $\mathrm{R} 2$ is received as the information:

$$
P^{\prime \prime}(T 1 \mid R 2)=\frac{[P(T 1) P(R 2 \mid T 1)]}{[P(T 1) P(R 2 \mid T 1)+P(T 2) P(R 2 \mid T 2)+P(T 3) P(R 2 \mid T 3)]}
$$




$$
\begin{aligned}
& P^{\prime \prime}(T 2 \mid R 2)=\frac{[P(T 2) P(R 2 \mid T 2)]}{[P(T 1) P(R 2 \mid T 1)+P(T 2) P(R 2 \mid T 2)+P(T 3) P(R 2 \mid T 3)]} \\
& P^{\prime \prime}(T 3 \mid R 2)=\frac{[P(T 3) P(R 2 \mid T 3)]}{[P(T 1) P(R 2 \mid T 1)+P(T 2) P(R 2 \mid T 2)+P(T 3) P(R 2 \mid T 3)]}
\end{aligned}
$$

If $R 3$ is received as the information:

$$
\begin{aligned}
& P^{\prime \prime}(T 1 \mid R 3)=\frac{[P(T 1) P(R 3 \mid T 1)]}{[P(T 1) P(R 3 \mid T 1)+P(T 2) P(R 3 \mid T 2)+P(T 3) P(R 3 \mid T 3)]} \\
& P^{\prime \prime}(T 2 \mid R 3)=\frac{[P(T 2) P(R 3 \mid T 2)]}{[P(T 1) P(R 3 \mid T 1)+P(T 2) P(R 3 \mid T 2)+P(T 3) P(R 3 \mid T 3)]} \\
& P^{\prime \prime}(T 3 \mid R 3)=\frac{[P(T 3) P(R 3 \mid T 3)]}{[P(T 1) P(R 3 \mid T 1)+P(T 2) P(R 3 \mid T 2)+P(T 3) P(R 3 \mid T 3)]}
\end{aligned}
$$

Once the posterior probabilities are found, the expected travel time can be calculated as follows:

For R1 case:

The Expected Travel Time =

$P^{\prime \prime}(T 1 \mid R 1)(T 1)+P^{\prime \prime}(T 2 \mid R 1)(T 2)+P^{\prime \prime}(T 3 \mid R 1)(T 3)$ 
For $\mathrm{R} 2$ case:

The Expected Travel Time $=$

$P^{\prime \prime}(T 1 \mid R 2)(T 1)+P^{\prime \prime}(T 2 \mid R 2)(T 2)+P^{\prime \prime}(T 3 \mid R 2)(T 3)$

For R3 case:

The Expected Travel Time $=$

$P^{\prime \prime}($ T1|R3)(T1) + P"(T2|R3)(T2) + P" (T3|R3)(T3)

The following section illustrates the above equations through an example.

\subsubsection{Sample Calculations}

With the above defined, sample calculations have been performed to observe how Bayesian analysis can handle the uncertainty with travel time.

\section{Carleton University to My House Joumey}

$\mathrm{T} 1=21$ minutes

$\mathrm{T} 2=22$ minutes

$\mathrm{T} 3=25$ minutes 
Priors: Using 'ALL DAY' WEEKDAY probabilities for Transitway routes.

$$
\begin{aligned}
& P^{\prime}(T 1)=0.24 \\
& P^{\prime}(T 2)=0.645 \\
& P^{\prime}(T 3)=0.115
\end{aligned}
$$

\section{Expected Time Based on Prior Probabilities}

Exp. Time $=(0.24)(21)+(.645)(22)+(.115)(25)=22.1$ minutes

\section{Conditional Probabilities}

$$
\begin{array}{lcc}
P(R 1 \mid T 1)=0.700 & P(R 1 \mid T 2)=0.150 & P(R 1 \mid T 3)=0.050 \\
P(R 2 \mid T 1)=0.250 & P(R 2 \mid T 2)=0.700 & P(R 2 \mid T 3)=0.250 \\
P(R 3 \mid T 1)=\frac{0.050}{1.000} & P(R 3 \mid T 2)=\frac{0.150}{1.000} & P(R 3 \mid T 3)=0.700 \\
& 1.000
\end{array}
$$

\section{Posterior Probabilities}

$$
\begin{array}{lcc}
P^{\prime \prime}(T 1 \mid R 1)=0.621 & P^{\prime \prime}(T 1 \mid R 2)=0.053 & P^{\prime \prime}(T 1 \mid R 3)=0.064 \\
P^{\prime \prime}(T 2 \mid R 1)=0.358 & P^{\prime \prime}(T 2 \mid R 2)=0.836 & P^{\prime \prime}(T 2 \mid R 3)=0.511 \\
P^{\prime \prime}(T 3 \mid R 1)=\frac{0.021}{1.000} & P^{\prime \prime}(T 3 \mid R 2)=\frac{0.111}{1.000} & P^{\prime \prime}(T 3 \mid R 3)=0.425 \\
\hline
\end{array}
$$

As shown in Figures 6.5 to 6.7 , there is a significant change in the probabilities due to a change in the travel advisory. The prior probabilities are $24,64.5$, and 11.5 percent for early, on time, and late travel respectively. 


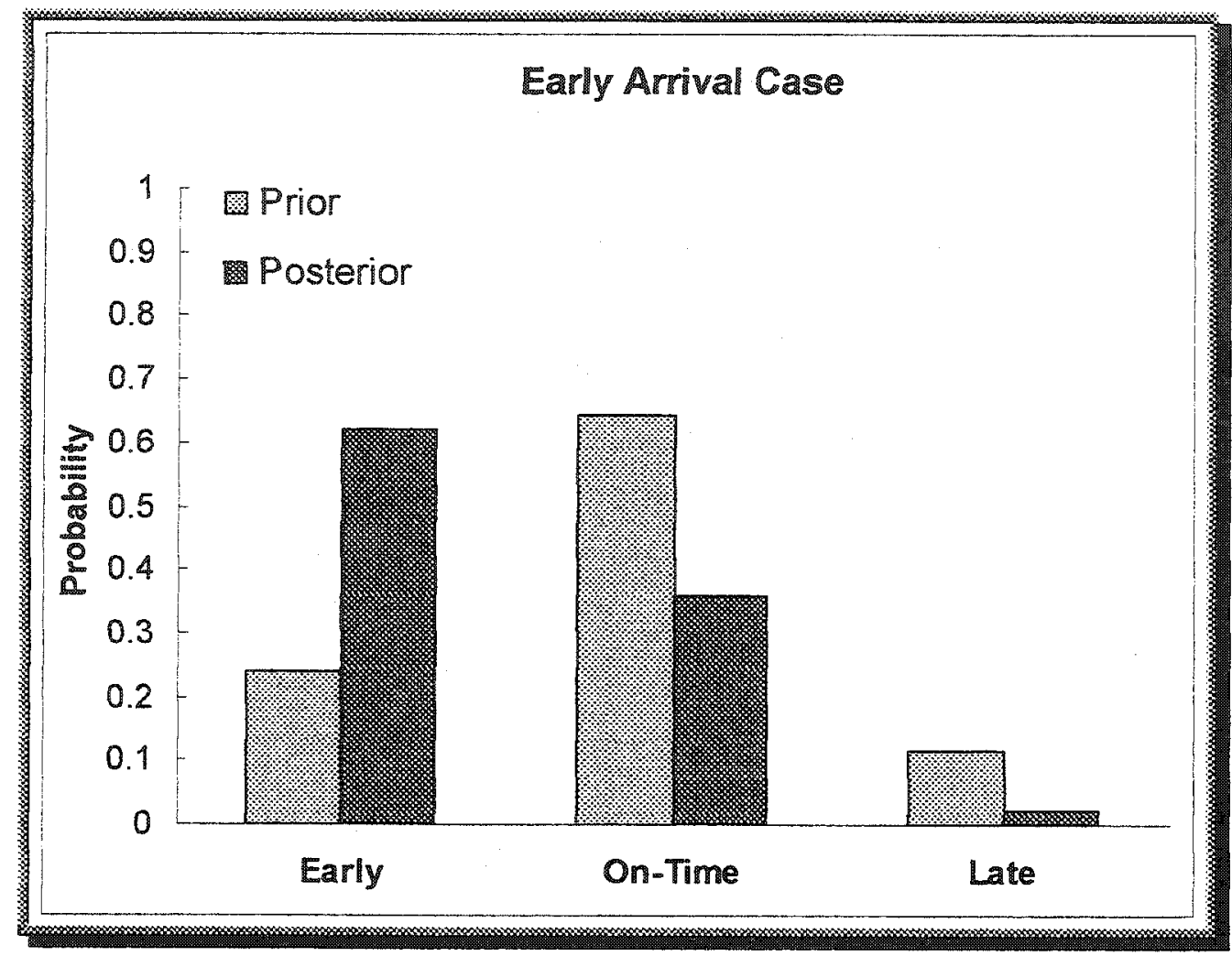

Figure 6.5: Early Arrival Case

Given that the arrival is early, Figure 6.5 illustrates the probabilities of a bus being early, on time, or late. As expected, the probability of early arrival is significantly increased. To compensate, there is a significant decrease in the on time probability and a small change is found in the late probability. If the bus is travelling early, there is a high probability of it arriving early, while it will seldom arrive late. Once these posterior probabilities are applied to update the travel time, the uncertainty in early travel can be noticeably reduced. 


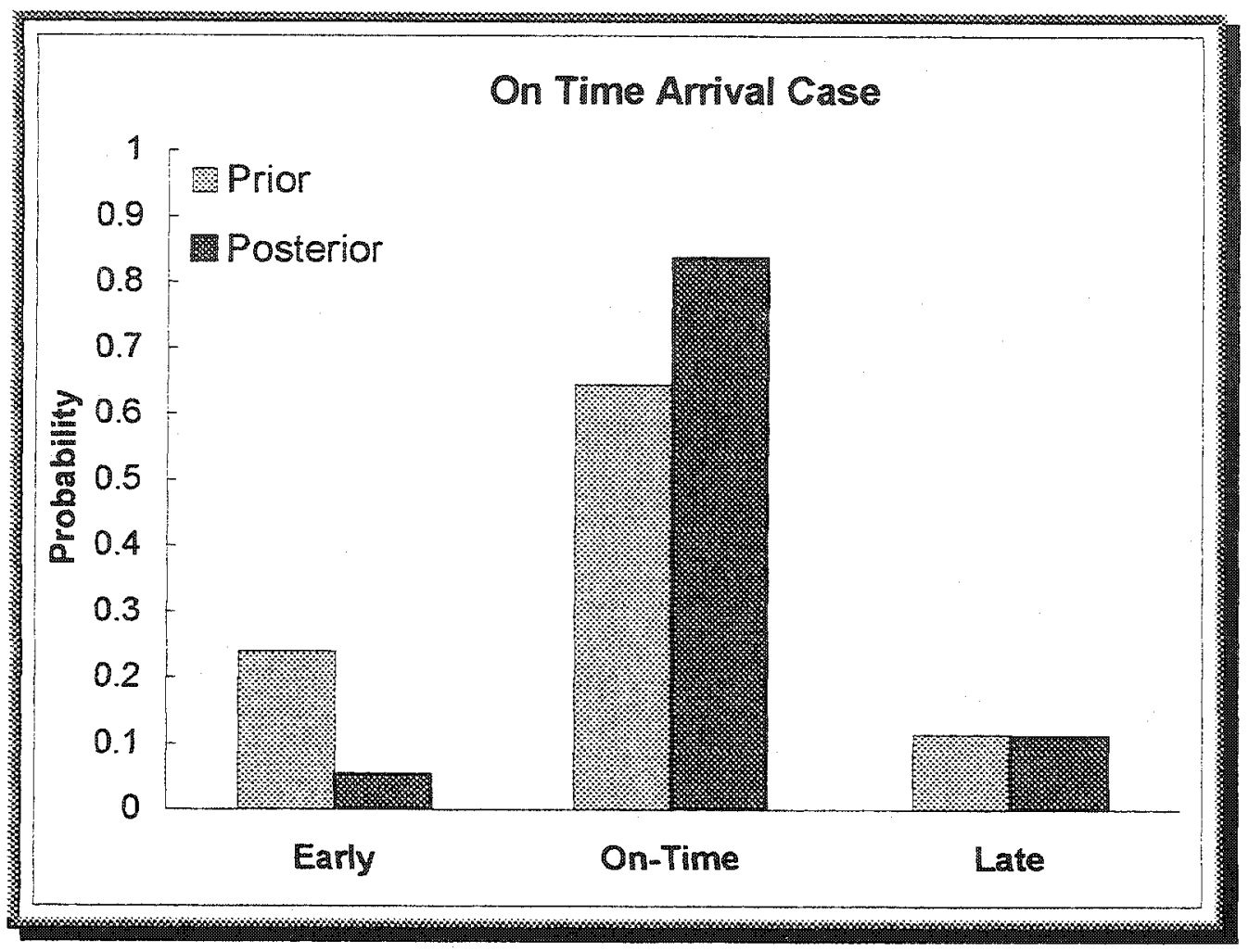

Figure 6.6: On Time Arrival Case

In Figure 6.6, the on time advisory is given. As expected in this case, the probability for being on time is increased. To compensate for this increase, the early and late probabilities have decreased. When applied to update the travel time, the user will notice that the travel time probability will shift from having a fairly good probability of being on time to a clear-cut on time probability. 


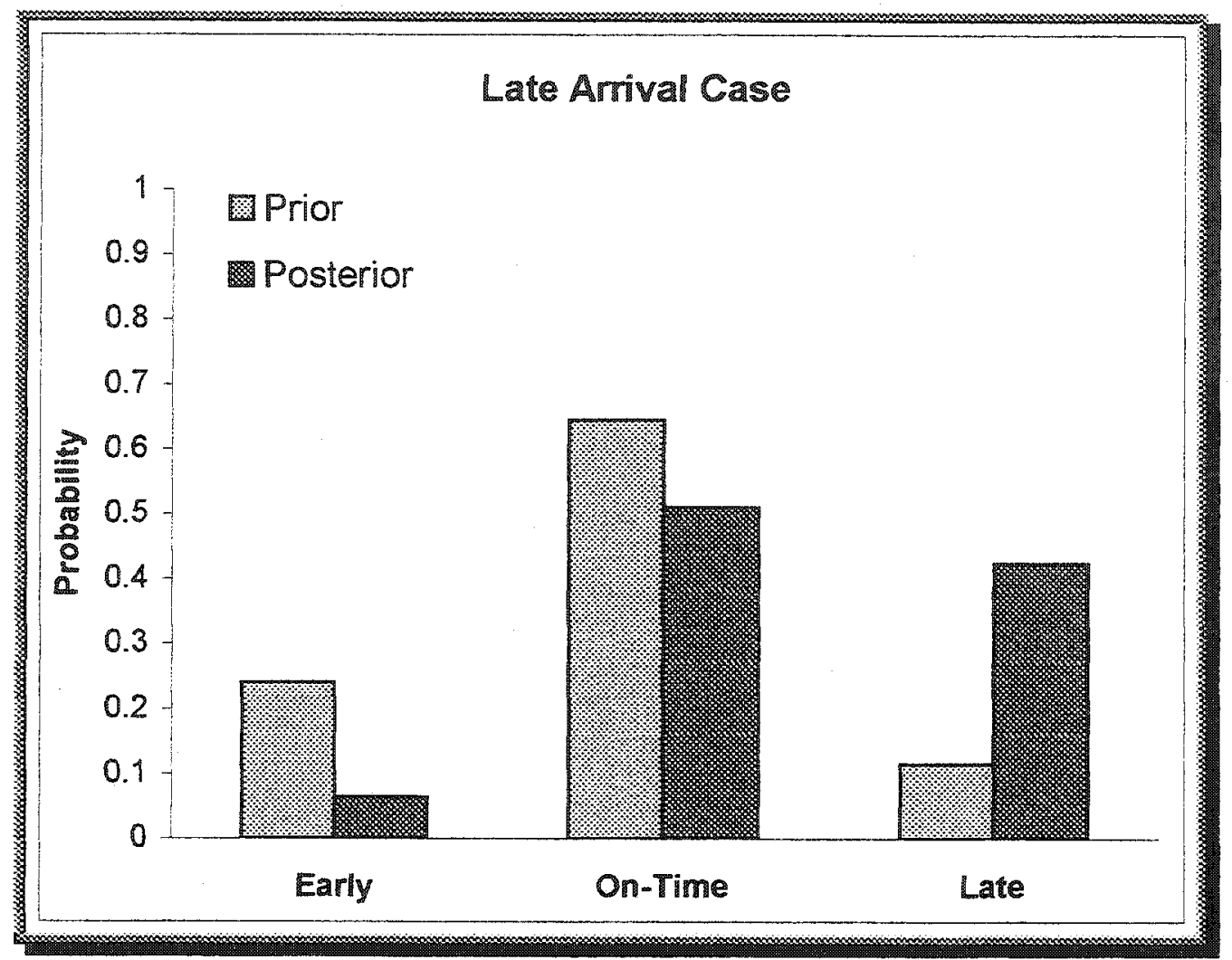

Figure 6.7: Late Arrival Case

Likewise for the late advisory shown in Figure 6.7, the late probability has significantly increased. As expected, when a bus is travelling late, the probability of it arriving late is high. The likelihood of a bus arriving early is small, where there is still a notable probability of it arriving on time. Since the prior probability of being late is quite small in comparison to the other conditions, the on time posterior probability is still greater than the late probability. But, with the large increase in the late probability, the uncertainty has been greatly minimized for the user. 


\section{Expected Time Based on Posterior Probabilities}

Expected Travel Time: if $\mathrm{R} 1$ is the information received:

Time $=(0.621)(21)+(0.358)(22)+(0.021)(25)=21.44$ minutes

Expected Travel Time: if $\mathrm{R} 2$ is the information received:

Time $=(0.053)(21)+(0.836)(22)+(0.111)(25)=22.28$ minutes

Expected Travel Time: if $\mathrm{R} 3$ is the information received:

Time $=(0.064)(21)+(0.511)(22)+(0.425)(25)=23.21$ minutes

From this example, it can be seen that the Bayesian analysis results in more realistic estimates of travel time as compared to a complete reliance on scheduled travel time. Likewise, a complete reliance is not placed on the bus status advisory, given that such information on a projected basis is not absolutely reliable. By applying Bayesian analysis, the uncertainty is considered and minimized to help users plan their arrivals at bus stops and their trips more efficiently. 


\subsection{Calculation Procedure}

Figure 6.8 shows the computational procedure used for producing inputs to the optimal route algorithm. Following the establishment of the states of nature T1, T2, and T3 and the assignment of prior probabilities, the next step is to check on the transit agency's on-line database for changes in bus arrival times (e.g. a bus running late).

If there is no change from the initial times, prior probabilities are used to find expected travel time. On the other hand, if there is a travel time change advisory, then the posterior probabilities are computed and used as a basis for estimating expected travel time.

The estimated travel time information is provided to the optimum route algorithm for establishing the best route and finding the estimated travel time. 


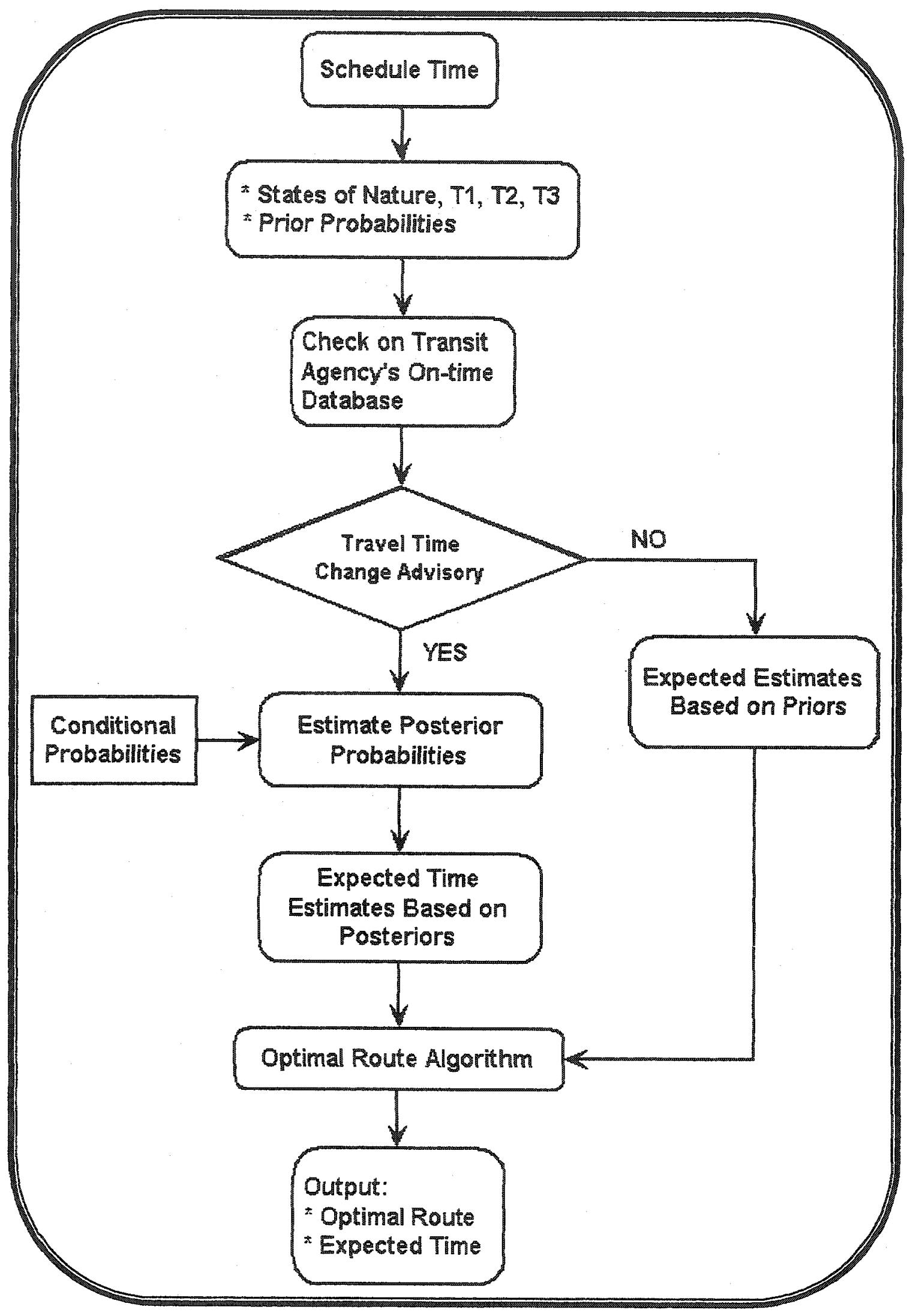

Figure 6.8: Bayesian Statistical Analysis of Travel Time 


\section{Chapter 7: Synthesis and Application of the Transit Information System}

\subsection{Introduction}

The Transit Information System design process using GIS is an application process requiring many steps. The design process starts with defining a GIS data structure to represent a logical relationship among individual elements of transit data. Next, the design enables the representation of the control relationships among functions. Further, the design focuses on the interface by showing how the application communicates within itself and with users. Finally, the transit information system was designed utilizing ArcView3.2a, Avenue, and Dialog Designer. The final product of the system consists of 75 scripts (algorithm), and 7 dialogs. The scripts can be found in Appendix C.

The transit information system was developed as a user-interface. In the userinterface, there are three parts: setting the user's preference, inputting the user's desired origin and destination or schedule information, and displaying the results. 


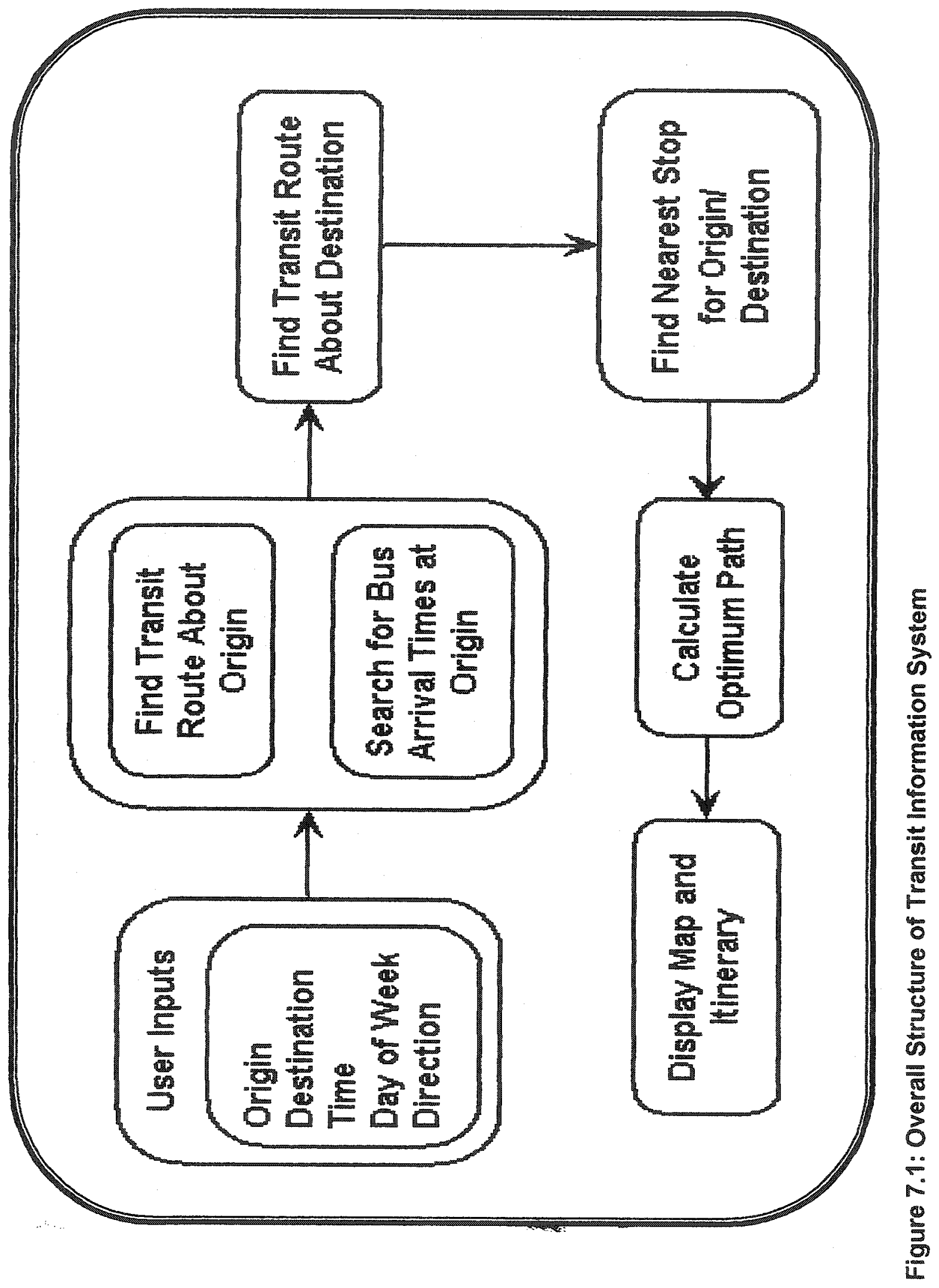


Figure 7.1, outlines the process. First, according to the set user preferences the application generates the subsequent pages, for selecting the origin and destination points. From these pages, the user's origin and destination data flow into the application for locating the points and their respective bus stops. Once it finds the origin and destination, and bus stops, the application displays these points and calculates the optimum path and the result is displayed to users to print.

\subsection{User Interface Design}

When the user enters the application, a screen showing the route network and bus location points is visible. If the user would like to view other categorical landmarks they can be chosen by selecting one or more on the left hand side of the screen. When implemented an instructions/help menu will also be available for further instructions and explanations for the user. Figure 7.2 illustrates this screen. To continue the user presses the button labeled 'RUN TIS' located on the main view. 


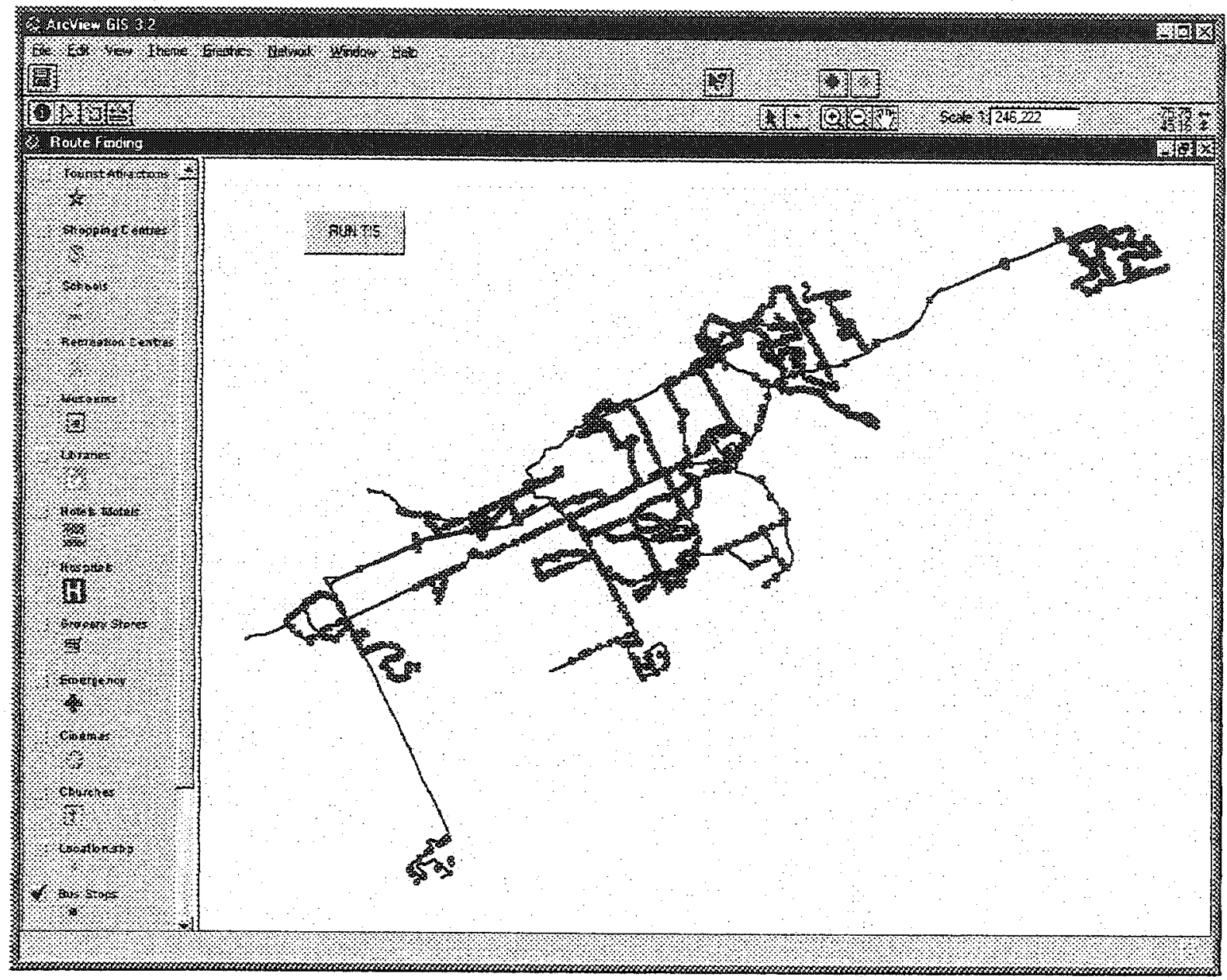

Figure 7.2: Initial View

When the user enters the transit information system, the window shown in Figure 7.3 appears. The user can either obtain schedule information pertaining to a specific route or continue to the user interface in order to find the optimum route. If exit is chosen, a message box will appear to verify this is what the user desires. This message box is shown in Figure 7.4. 


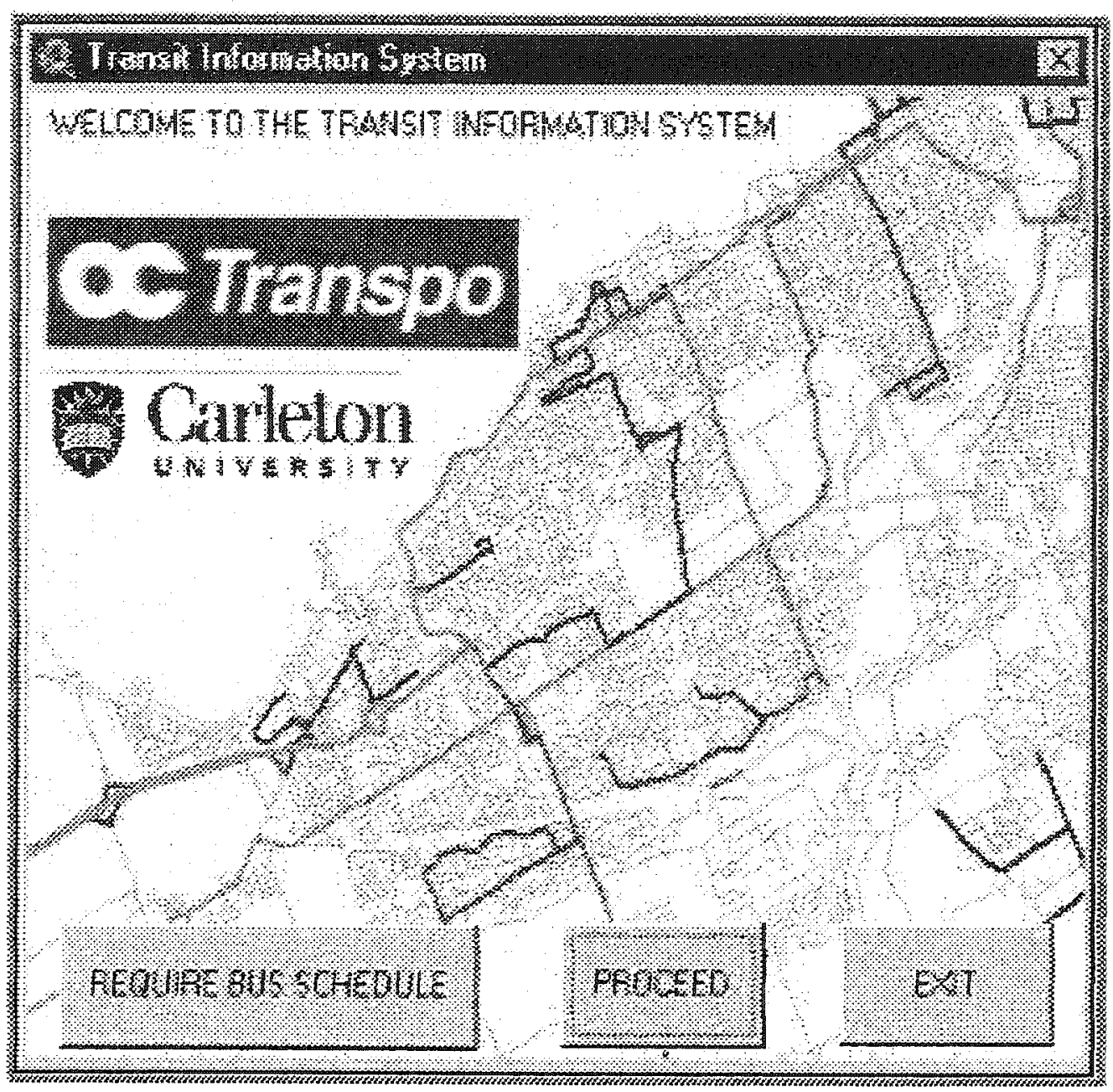

Figure 7.3: Initial dialog

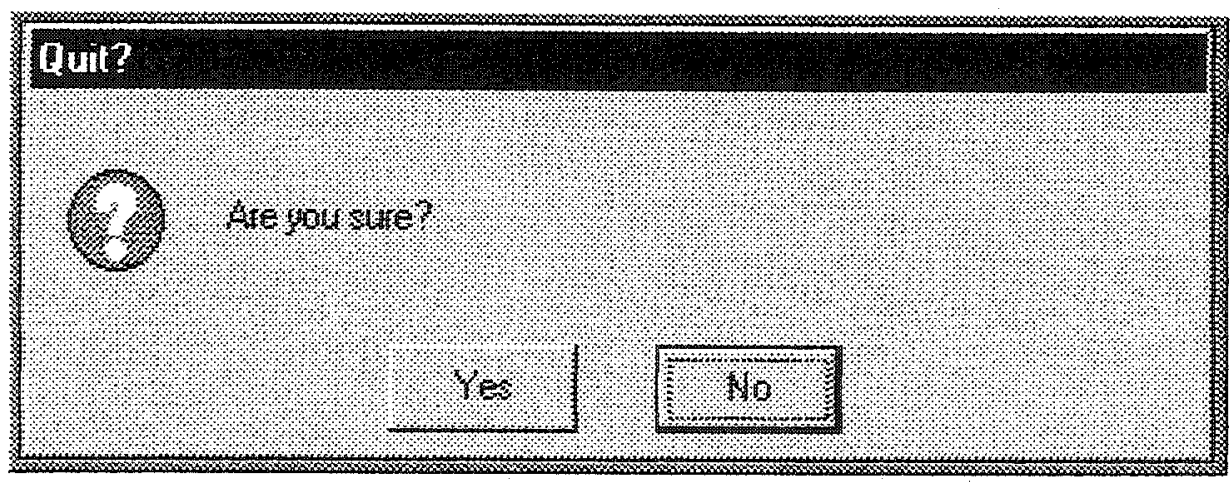

Figure 7.4: Verification Message Box 
If the user selects 'REQUIRE BUS SCHEDULE' the dialog box shown in Figure 7.5 will appear. In this step, the user is asked to provide the time (hour, minute, and AM or PM) his/her trip will begin and the day of the week (Weekday, Saturday, or Sunday) the trip will take place. Radio buttons prevent the user from selecting both AM and PM or every day of the week. Since this dialog will produce a schedule, the user must also select a route number from the drop down menu. When all of the information is entered, the user has the option of canceling and restarting or to get the schedule information.

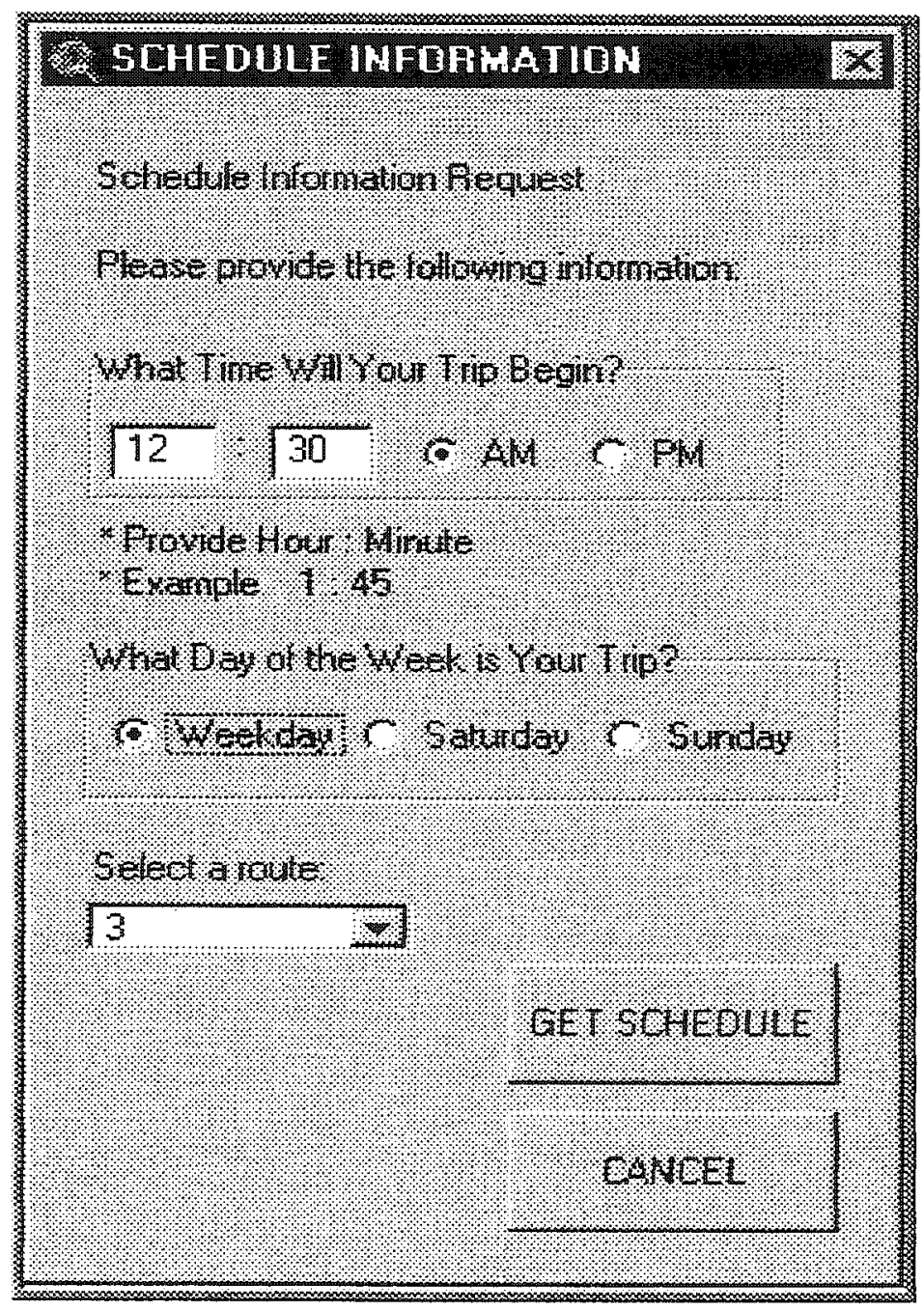

Figure 7.5: Dialog Box for Schedule Information 
When the 'GET SCHEDULE' button is selected, the algorithm produces a highlighted table with the bus route and corresponding times of arrivals at different bus stops. The figure below shows an example of this.

\begin{tabular}{|c|c|c|c|c|c|c|c|}
\hline \multicolumn{8}{|c|}{ x meekdaydbf } \\
\hline$x_{x} x, x_{2}$ & $10,8<1$ & $30, x$ & 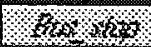 & $6 x_{n}$ & $40,2 x y$ & W & \\
\hline 19 & H & 0148 & 205 & $\mathrm{HH} / \mathrm{dp}$ & 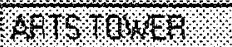 & $8 \%$ & \\
\hline 41 & 4 & 4189 & U6 5 & HSH & $4760 \times 4$ & 65 & \\
\hline 18 & Q & 1518 & $\left.(2)_{1}\right)$ & 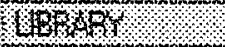 & 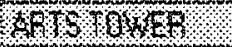 & 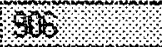 & \\
\hline 17 & $\mathrm{OA}$ & $83 \times 9$ & 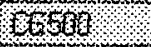 & HER 6 & \$1010m & 31 & \\
\hline 11 & 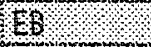 & 460 & 5101 & 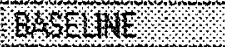 & & 02 & \\
\hline \%1 & E⿰ & $6+14$ & Ifo6 & 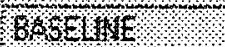 & & 84 & \\
\hline 11 & $\mathrm{~EB}$ & 140 & 81000 & Dass & & 99 & \\
\hline 11 & E & 4,60 & 5030 & MESOEWOKHOS & $\mathrm{XIXX \textrm {NAO }}$ & $31 \%$ & \\
\hline 4 & 80 & E 78 & 6080 & 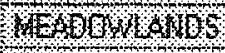 & MHW & $6 \%$ & \\
\hline 11 & $\mathrm{~GB}$ & 11619 & 5833 & MEOOON $4,1, O S$ & 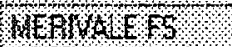 & $04 \%$ & \\
\hline 11 & 40 & 1160 & $5 \mathrm{~B}>0$ & 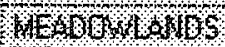 & 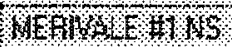 & 67 & \\
\hline 11 & 46 & 46 & $\$ 2321$ & Wf & 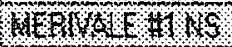 & 819 & \\
\hline $1 \%$ & 10 & 1168 & $30 \times 1$ & 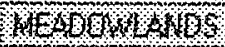 & 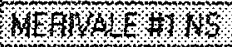 & 93 & \\
\hline 11 & 40 & 1598 & $5+493$ & W & & 918 & \\
\hline 11 & 10 & 10109 & 3804 & $6 \%$ \& B IU & मै। & 84 & \\
\hline X & 16 & 1612 & 18 80 & 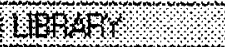 & $471 S 10 \times 4 \mathrm{P}$ & 998 & \\
\hline 111 & 18 & E6s) & Ousula & (D & 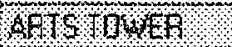 & 68 & \\
\hline 95 & $E B$ & 9543 & EE905 & BLAIR & 18 & 2318 & \\
\hline 86 & $\mathrm{~EB}$ & 8602 & $A L 020$ & ELMVALE SC & 11th STOP & 928 & \\
\hline 97 & $E B$ & 9702 & $N 1915$ & LINCOLN FIELOS & 2A:DOWNTOWN & 444 & \\
\hline 86 & $E B$ & 8603 & ALO20 & ELMVALE SC & $12 \mathrm{hSTOP}$ & 947 & \\
\hline 4 & $F$ & $80 \pi ?$ & CHAON. & 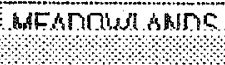 & MERIMIFMIS & 1253 & $\frac{1}{1}$ \\
\hline
\end{tabular}

Figure 7.6: Schedule Information

If the user selects 'PROCEED', step one of the transit information system is run. This window, shown in Figure 7.7 establishes how the system will proceed through the system. In this step, the user is asked to provide the time, as previously mentioned, when the trip will begin, what day of the week (Weekday, 
Saturday, or Sunday) the trip will take place and the direction. The travel information is established in this fashion due to the structure of OC Transpo's schedule system. In addition, the user establishes the selection method (by Address, Landmark, or Point_Click) that will be used to select his/her desired origin and destination. As per entering the time and day of travel, radio buttons also prevent the user from choosing more than one method of selecting an origin or destination. Under each method, a user may only select on of three choices. If the user selects Address, then the next dialog to appear will have the user enter a street address. It is not case sensitive, nor will the user need to enter a street type (Rd, St, Ave). The street name and number should be inputted correctly, although there is a tolerance for spelling errors.

If the user were to select Landmark, the next dialog to appear would have the user select a category (school, library, church), then the corresponding location from drop down menus. Finally, if Point_Click were chosen, the main view would re-appear, and the user would get instructions to select locations and solve the optimum route.

Depending on which selection the user has chosen for the destination, the transit information system will have the corresponding dialog appear for the user to enter his/her destination. Each dialog will appear in the same manner as described above. 


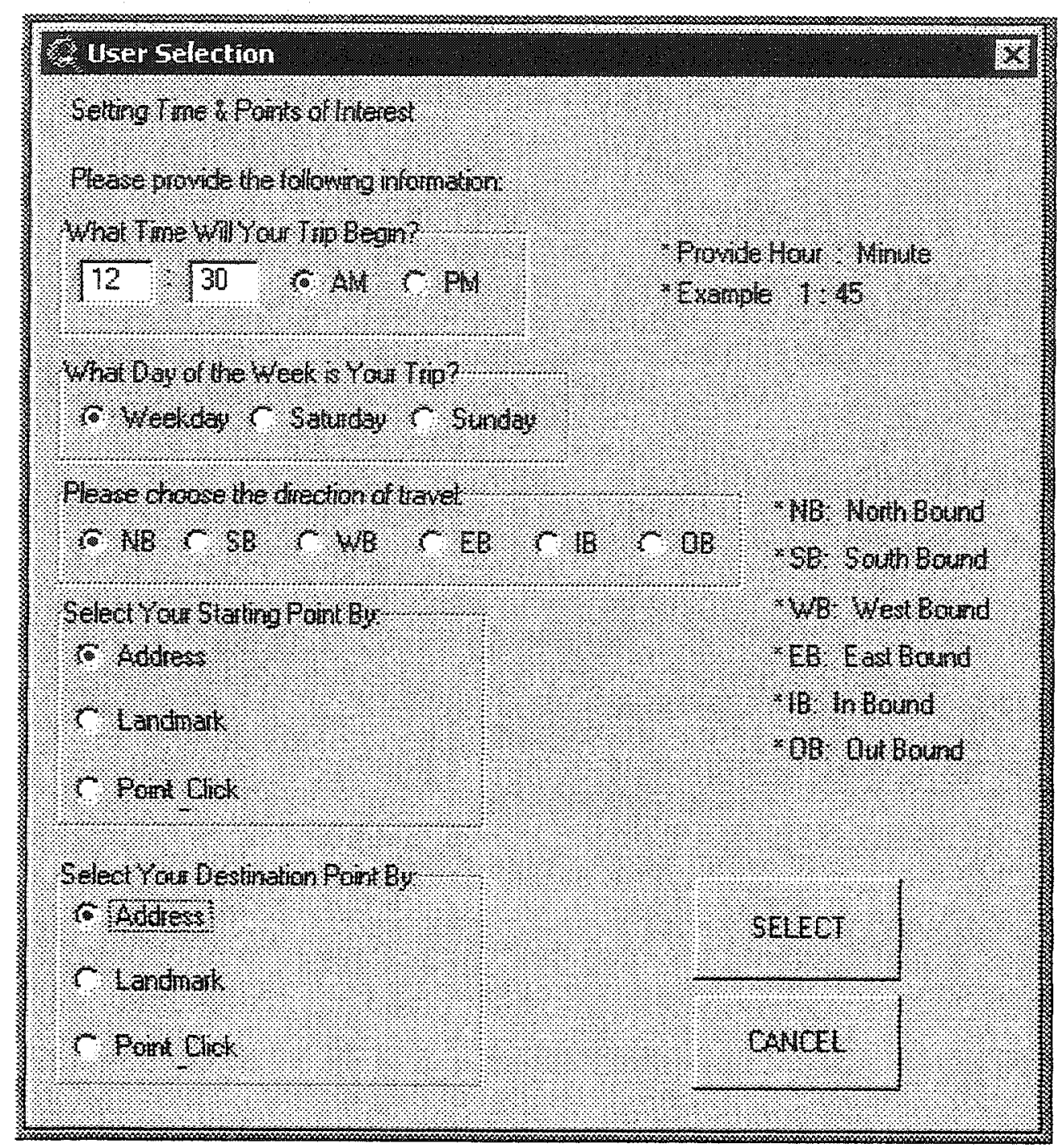

Figure 7.7: Dialog to Select Trip Information

When all information is provided, buttons allow the user to either continue to the next step or to cancel and restart the application.

Step two allows the user to select his/her desired starting point per the set selection methods requested in step one. Depending on the selection method set by the user, the page will vary to accommodate the information required. 
If the user selects Address for either the origin or destination locations, the windows in Figure $7.8 \mathrm{a} / \mathrm{b}$ will appear. The user enters the origin and/or destination location. When the user selects 'OK', this address is geocoded into the network through address matching with the street network. A point will appear in the view.

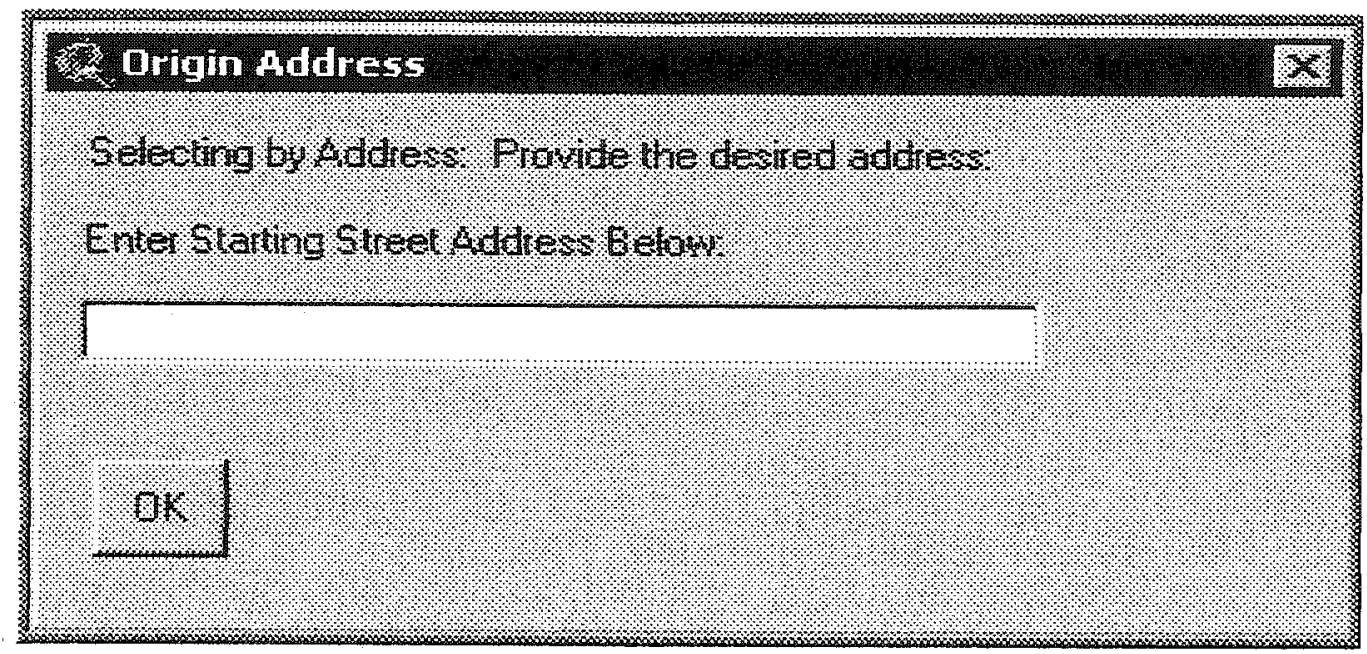

\section{Figure 7.8a: Dialog to Enter the Origin Location}

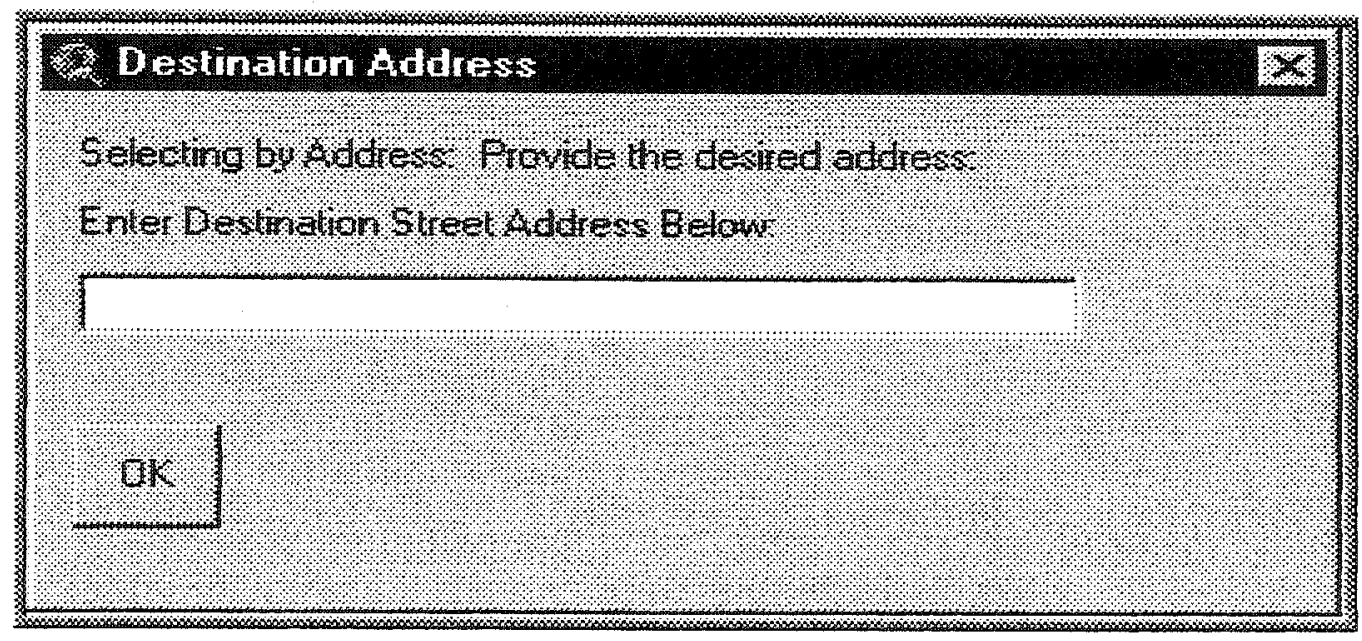

Figure 7.8b: Dialog to Enter the Destination Location 
If the users were to select Landmark Selection from the User Interface, then the dialog shown in Figure 7.9a/b will appear. The users have the option of selecting their origin and/or destination location via a list provided by the application. These are the point themes added through address matching. The user will select a category and the corresponding attributes (landmarks) from the drop down menus. These will be added into the 'Location' table for route analysis.

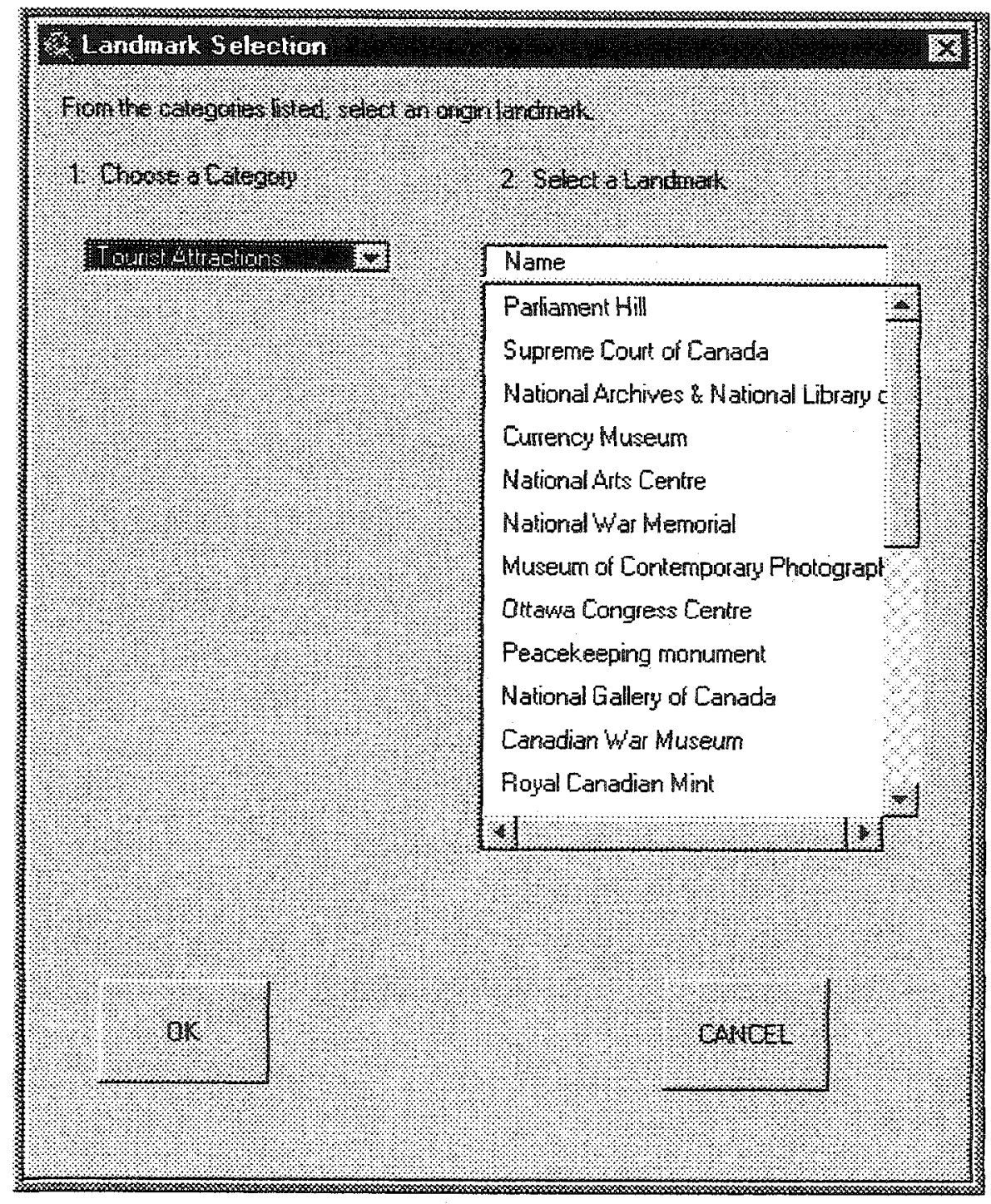

Figure 7.9a: Origin Landmark Selection 


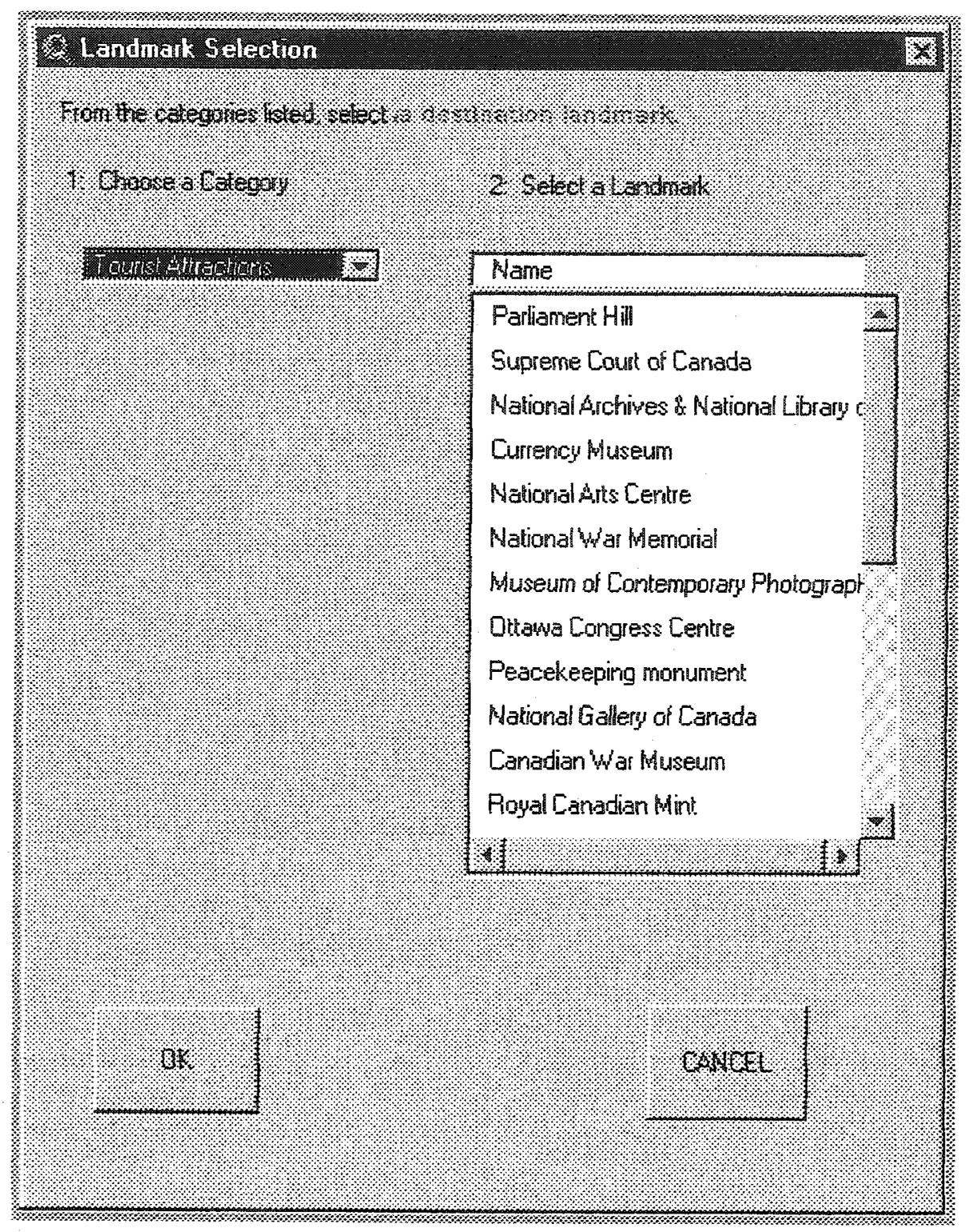

Figure 7.9b: Destination Landmark Selection

Once the user selects 'OK' for either the Address or Landmark Selection the message box in Figure 7.10 will appear. This directs the user to select the route button to solve the optimum route problem. 


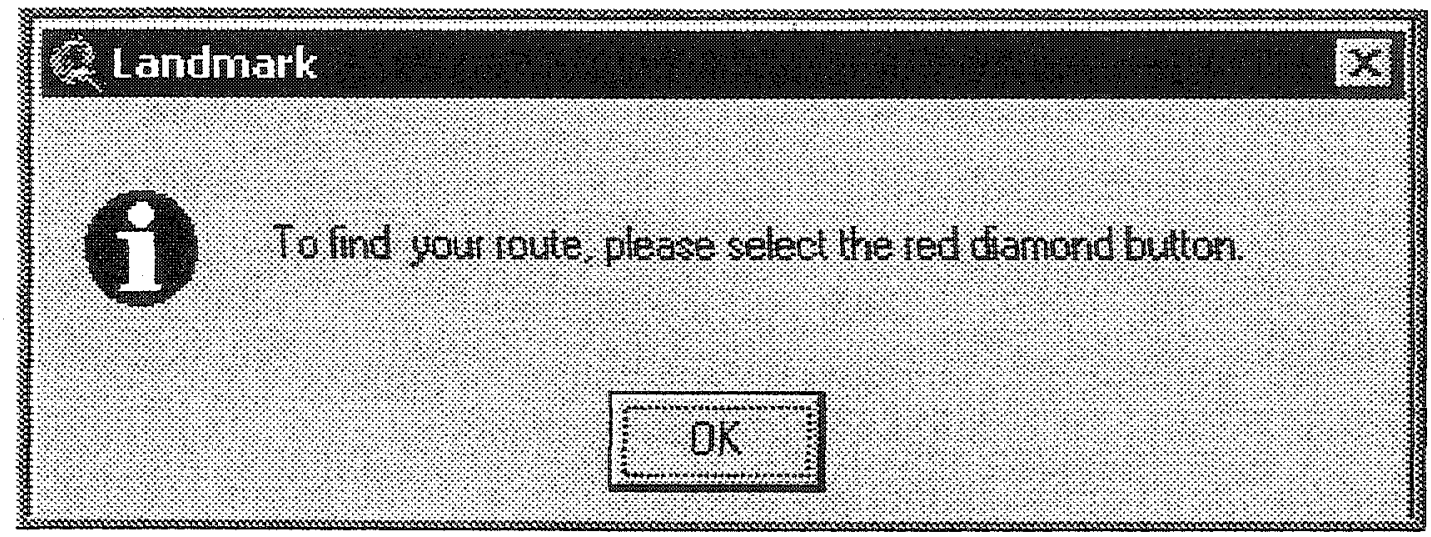

Figure 7.10: Message Box for Solving the Optimum Route

If the user is to select the origin and/ or destination by Point and Click then the dialog structure will close. Two message boxes will appear (Figures $7.11 \& 7.12$ ) indicating to the user, the method to select the origin and/or destination points to solve the route.

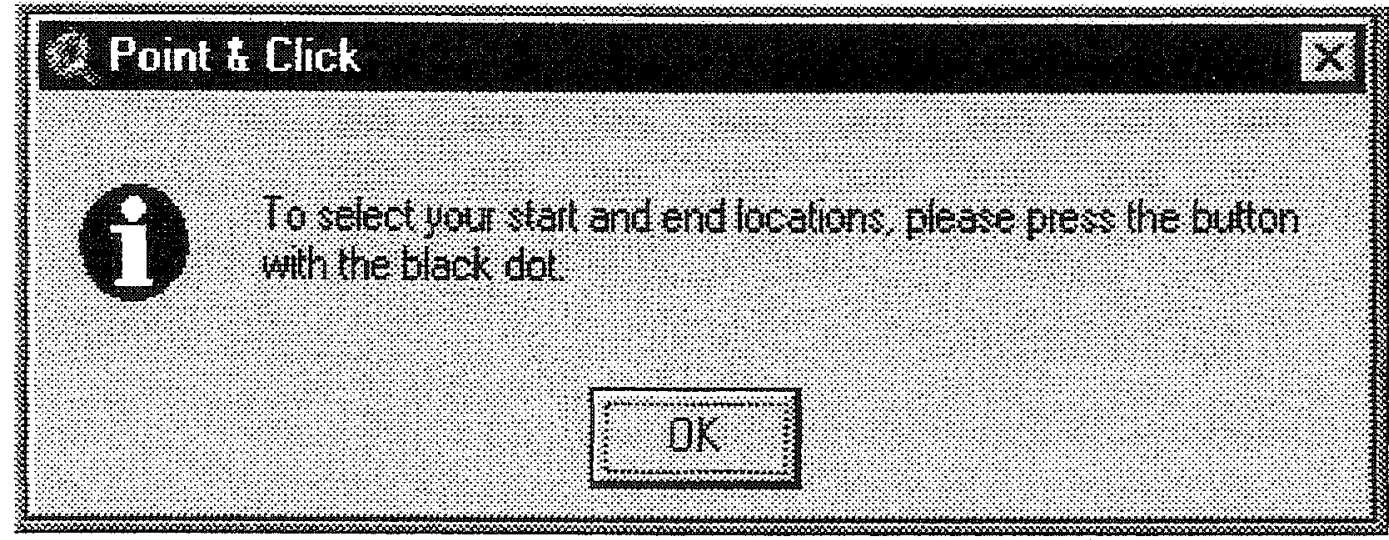

Figure 7.11: Message Box for Selecting the Origin/Destination 


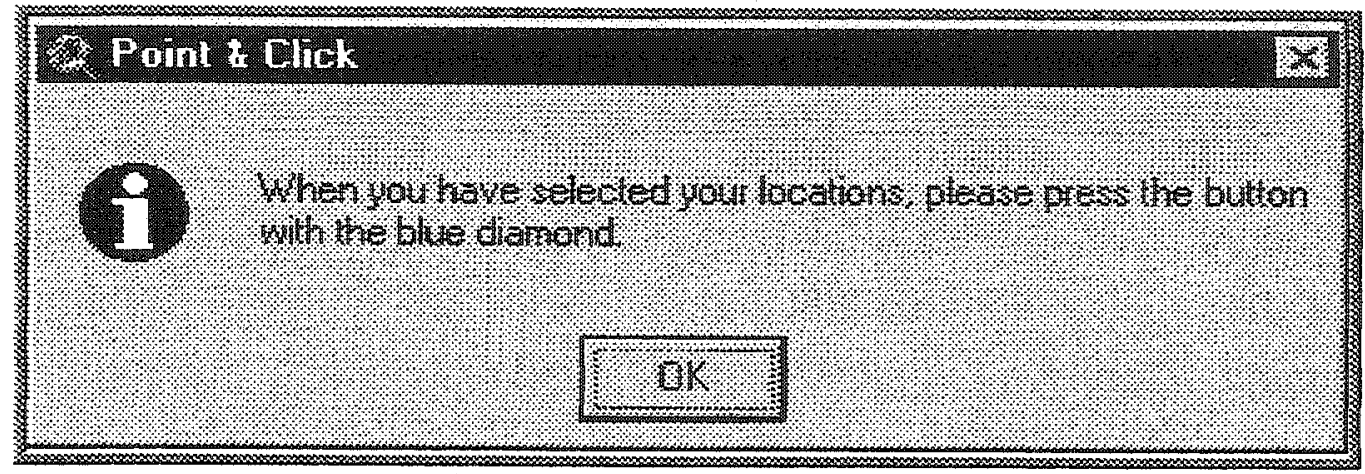

Figure 7.12: Message Box for Solving the Optimum Route using Point and Click

When designating the desired origin or destination point by using the Point_Click the user is provided with the transit routes network. This interface includes the map and functions to manipulate the map. These functions include zoom in, zoom out, pan, and select and are clearly located for easy access. Figure 7.13 illustrates the buttons and their location in the view. The Point_Click has a different route button since there is an additional step in solving the route problem. Given that the points entered by the user are graphics, they must be first converted to a theme in order for the application to solve the optimum route. This additional step is added into the route button along with the optimum route algorithm. 


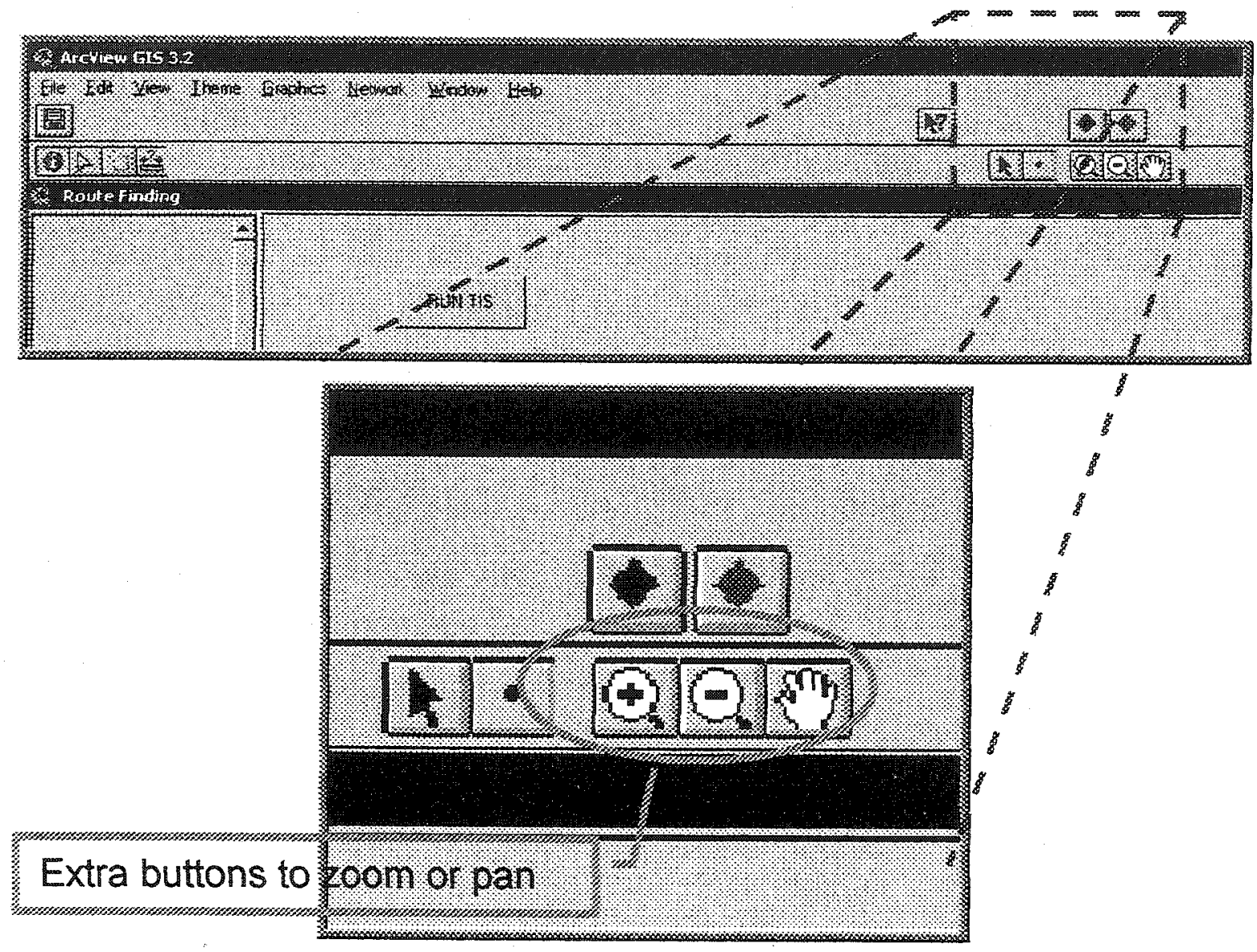

Figure 7.13: Buttons in Application

The final step is producing the results. To date the user must select the travel cost before the optimum route is found. This window produces only one cost (minutes) for the user to select. To eliminate this step the core system scripts for the Network Analyst need to be changed. This is a simple procedure, but due to the network violations it would create, this was not performed. If this application is to be implemented, the core system can be modified before use. Figure 7.14 illustrates this cost window. 


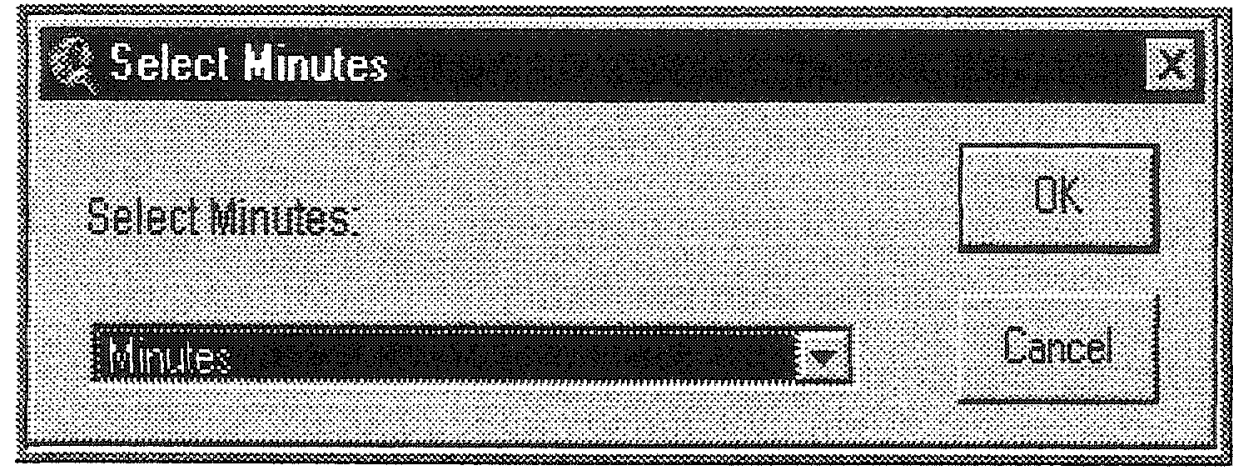

Figure 7.14: Cost Selection

The final step provides the user with the query results. This page consists of two parts. The first is an itinerary, which includes information on where the user must go to take the bus, which bus the user will board, when that bus will arrive, and approximately how long the trip will take. The second part of the results page is a map showing the origin and destination points and the optimum route. Sample itineraries and route illustrations are shown in Figures 7.15 and 7.16 respectively.

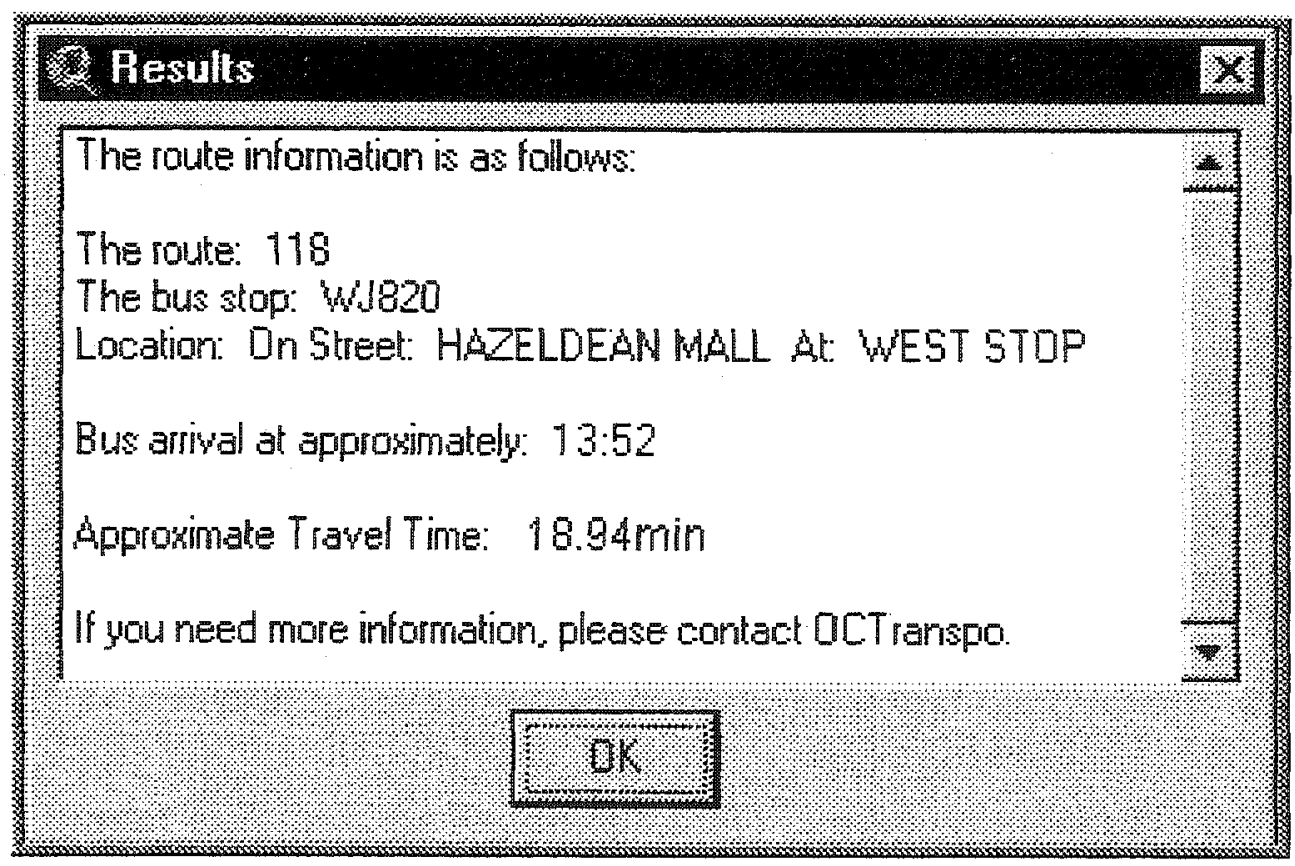

Figure 7.15: Report Box Reporting Results 


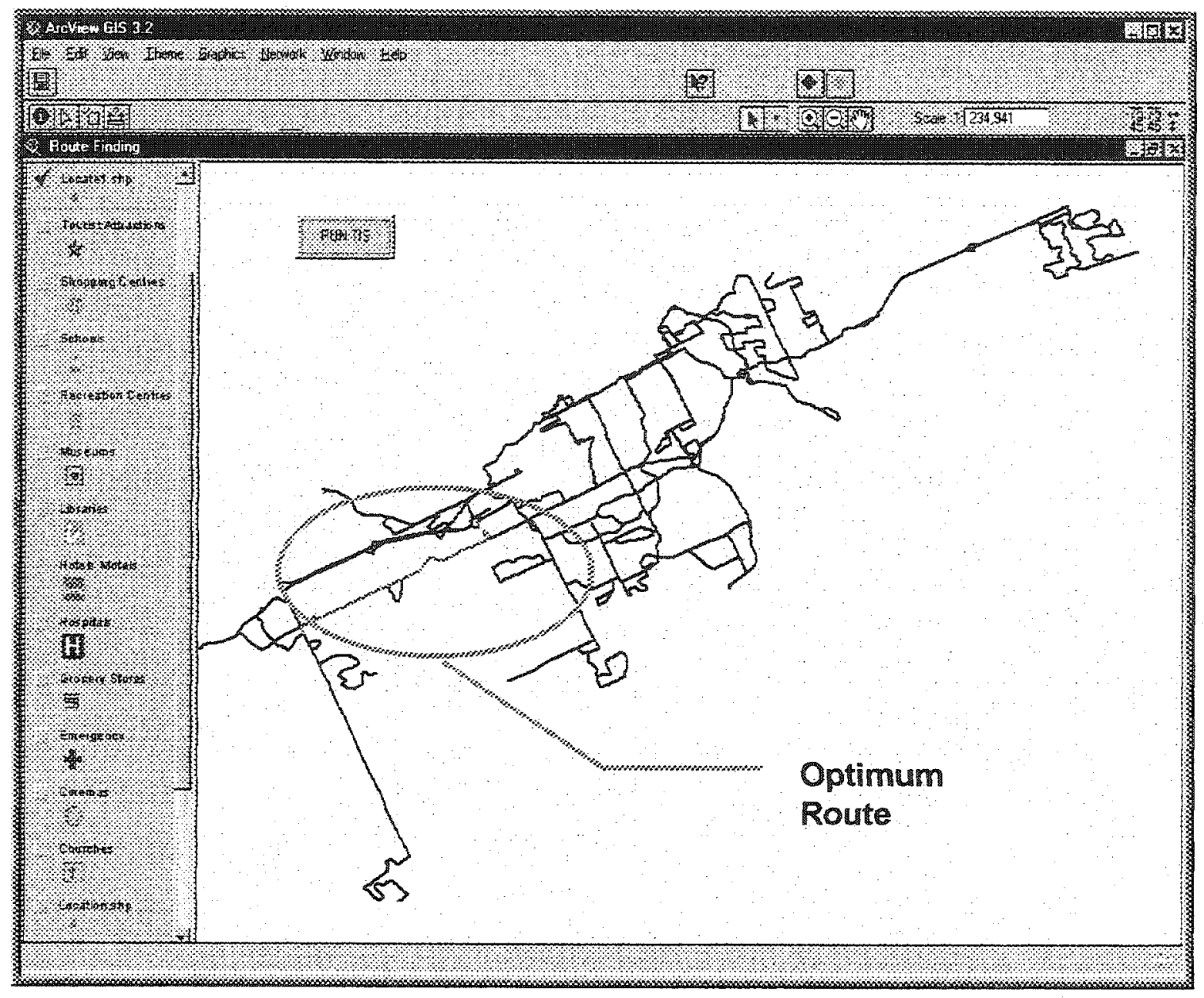

Figure 7.16: Results Image

The written results Itinerary will automatically print once the 'OK' button is selected and the user has the option to print the graphic route in the figure above. As well, the user can re-enter the transit information system to plan another trip or select landmarks from the left hand side of the view. 


\section{Chapter 8: System Validation}

Once an application like this has been created, it needs to be tested. For this step, the key is to find all possible errors in the algorithm that a user might encounter. For this step several trial runs of the systems were conducted in an attempt to simulate possible user inputs. To date this has been successful and any errors encountered have been eliminated. A final version of the transit information system will ultimately indicate if this system is completely operational.

\subsection{Criteria for Evaluation}

8.1.1 Is the Trip Planner Functioning Properly and Returning Accurate Information?

This criterion is the most important for a successful trip planner. It is a necessity that the address entered by the user be recognized and located accurately by the transit information system. The system must be able to calculate an accurate route from the inputted information.

8.1.2 Is the System Attractive and Easy to Use?

For some transit riders, this trip planner will be their first introduction to OC Transpo's services. For this reason it is a necessity to create a positive impression and a pleasant trip 
planning experience. The overall design and layout of the system should be colourful and the graphics should attract attention. If this service is successful, then the user will anticipate the same from the transit service. On the other hand, if the trip planner is not adequate, then OC Transpo will lose credibility with the users.

\subsubsection{Further Criteria}

Other criteria for evaluation could be developed when the system is implemented. These might be if the trip planner is used regularly. If it is then this will indicate that the public is aware of the new technology. If the public is not aware then further advertisement could be made. As well, criteria might be the satisfaction of the public with the trip planner. The main measure of the success of a topic like this is the response from the transit users themseives. If a person uses the trip planner to receive transit information and successfully completes the trip to his/her satisfaction then this system will be a success. This evaluation could be accomplished through user feedback through the Internet or informal surveys.

\subsection{Performance Criteria Results}

\subsubsection{System Performance}

Upon testing the system, sample runs were done in order to simulate a user trying to enter addresses, selected landmarks or 
points. To date the system has been able to recognize addresses with minimal spelling errors and has no difficulties moving between selections.

In addition, sample trips between stations were made to test the optimum route algorithm with Transitway and non-Transitway trips. For the purposes of evaluation, routes 3,7 , and 118 were chosen for non-Transitway routes and routes 95 and 97 for Transitway routes. These trips were performed for weekday and weekend trips. Since the schedule databases for both weekday and weekend trips produced the same headways between time points only weekday trips are presented here. In Table 8.1, a comparison of the scheduled travel times designated by OC Transpo and that calculated through the transit information system is illustrated. Since it is necessary to validate the accuracy of the route algorithm, Bayesian Analysis was not initially applied at this stage. The percent difference is used to demonstrate the precision between the travel times. 


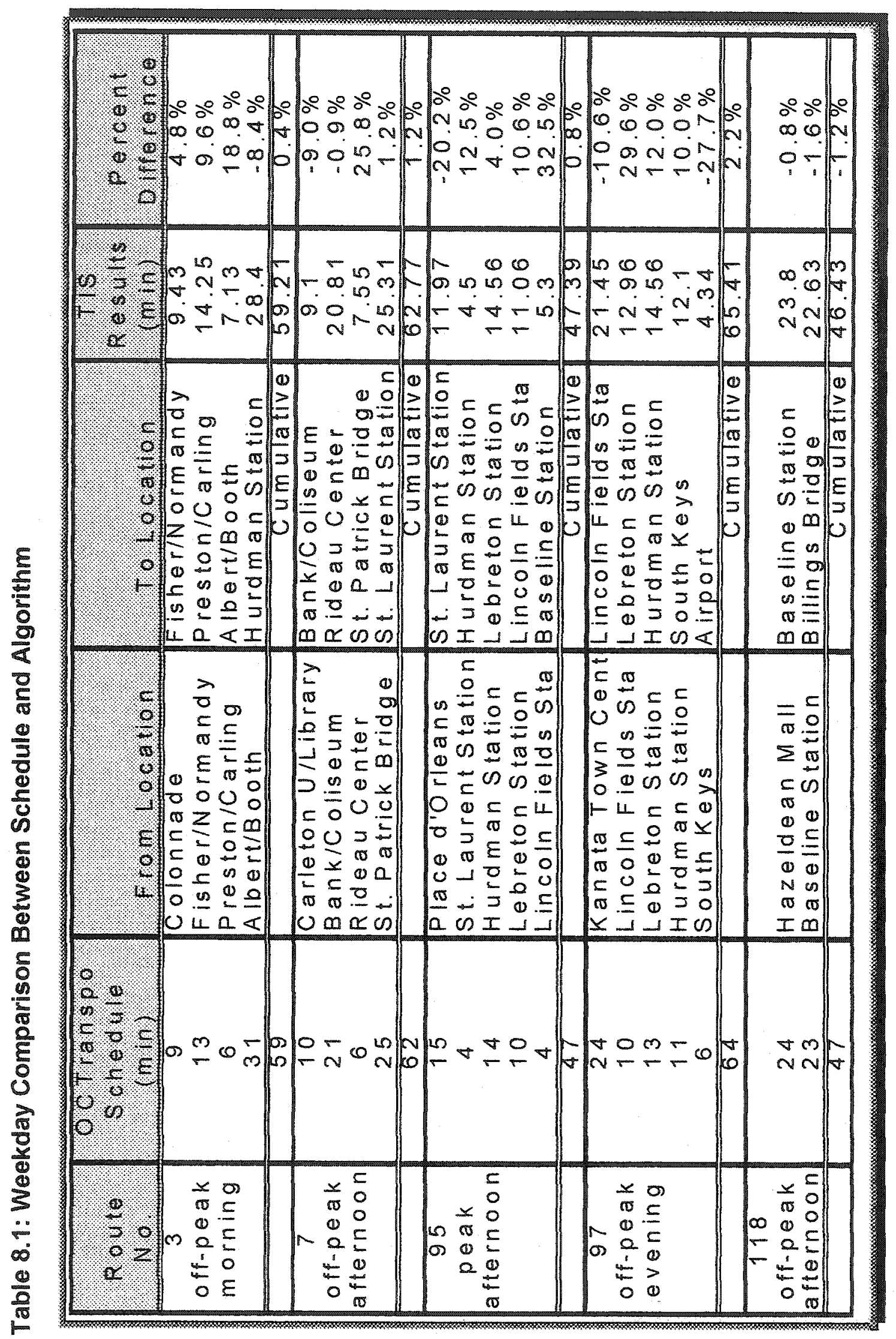


As seen, there are some discrepancies with some of the travel times. Many of these occur in short distances, where a difference of 30 seconds causes a significant percent difference. Route 95 is an example of this where half a minute to a minute causes a percent difference of up to $30 \%$.

When the delay costs were entered into the algorithm, it took into consideration the wait time at stations from travellers leaving and boarding the bus. Linear interpolation is also used to estimate the arrival of buses at stops where there is no scheduled time. This can also add to the error within the calculated travel time. Estimates of the arrival of buses at certain bus stops based on the travel distance carry error which could explain the additional time in comparison to the schedule. Additional work may entail obtaining trip frequency results from OC Transpo on the travel time between stations for comparison. These trips could then be statistically analyzed for error calculations and incorporated into the algorithm.

In the case of travel time being less than that of the schedule, these occur in large distances between stations where travel speeds are greater than $80 \mathrm{~km} / \mathrm{hr}$ (Queensway and/or Transitway). This can be seen from routes 95 and 97 . Both these routes travel 
predominantly on the Transitway as well as a portion on the Queensway. Route 95 travels from St. Laurent to Place d'Orleans on the Queensway while route 97 travels the Queensway from Kanata to Lincoln Fields. In both cases there are no or few stops between stations leaving these travel times to the uncertainty of the road conditions. Future work may entail comparing travel times on high speed zones in order to represent these sections of the route more accurately. Nevertheless, in most cases when a bus actually arrives early at a time point, it waits until the designated time to depart. This allows for later arriving travellers to obtain their transit ride.

As observed in the cumulative trip time, the percent differences have been minimized. It balances because depending on the location of where the particular graphic point was placed within the transit stations; it would include or not include the individual wait time. As a suggestion to avoid this in the future, stations should be coded differently from bus stops. Presently the transit stations include multiple stop locations and if the user was unsure where the bus arrives or departs, an inconsistency would ensue. If a user was to put the point at the beginning of a transit station or at the end then the travel time would vary because the transit station costs would either be included or excluded. Overall, from one end 
of the route to the other the costs have been accounted for accordingly and appropriate travel times have been recorded.

Based on the results of the algorithm, trial runs were executed given each condition of early, on time, or late. Bayesian analysis was applied for both weekday and weekend trips to visualize the different travel conditions instituted by the probabilities. These results are illustrated Tables 8.2 to 8.4 .

As indicated in chapter 6, the prior probabilities are applied where there is no travel time change advisory. In this case, the travel time is recalculated using the appropriate prior probabilities. Where there is a travel time change advisory, conditional probabilities are used to estimate posterior probabilities that are then applied to update the travel time. 


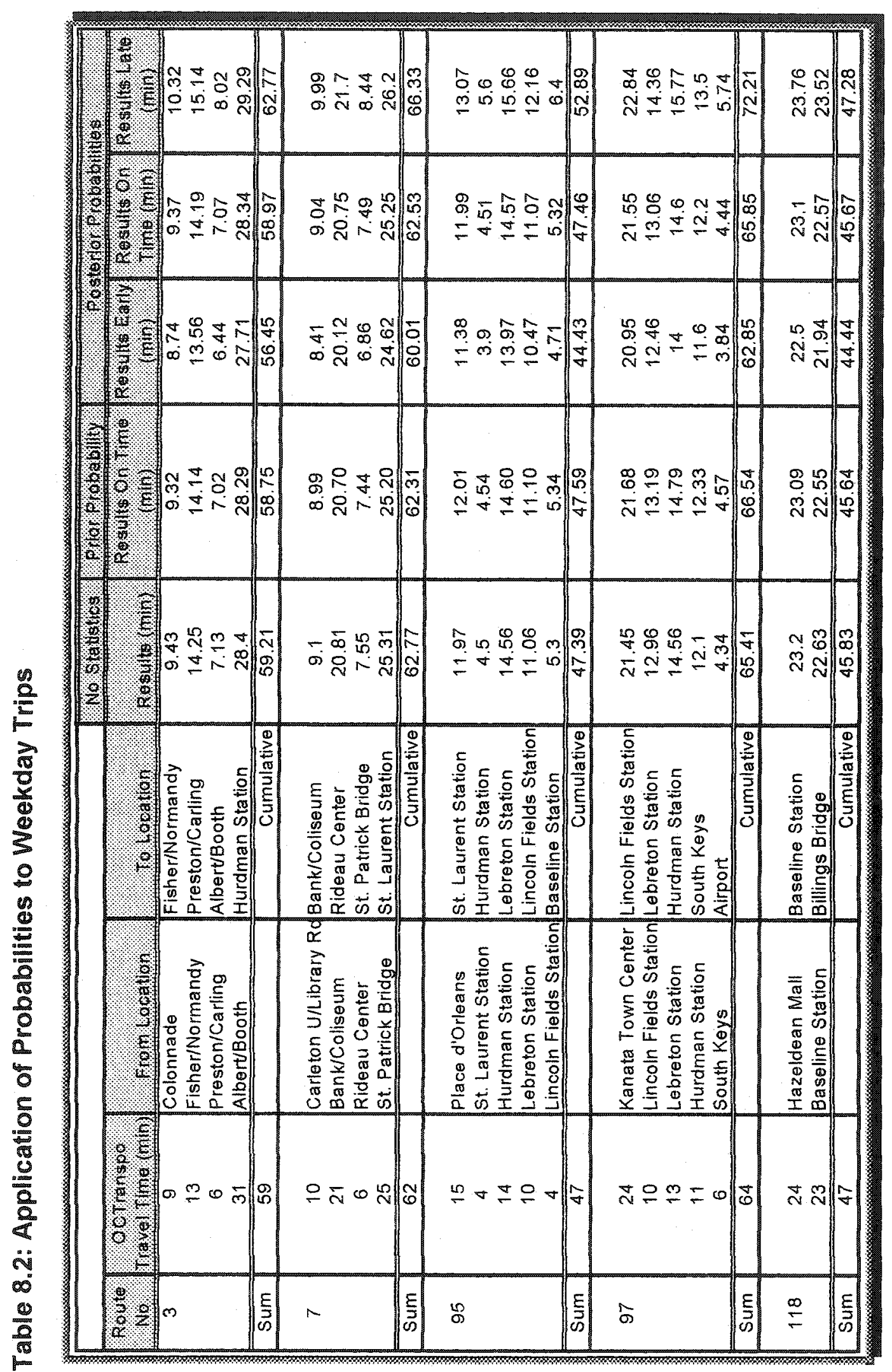




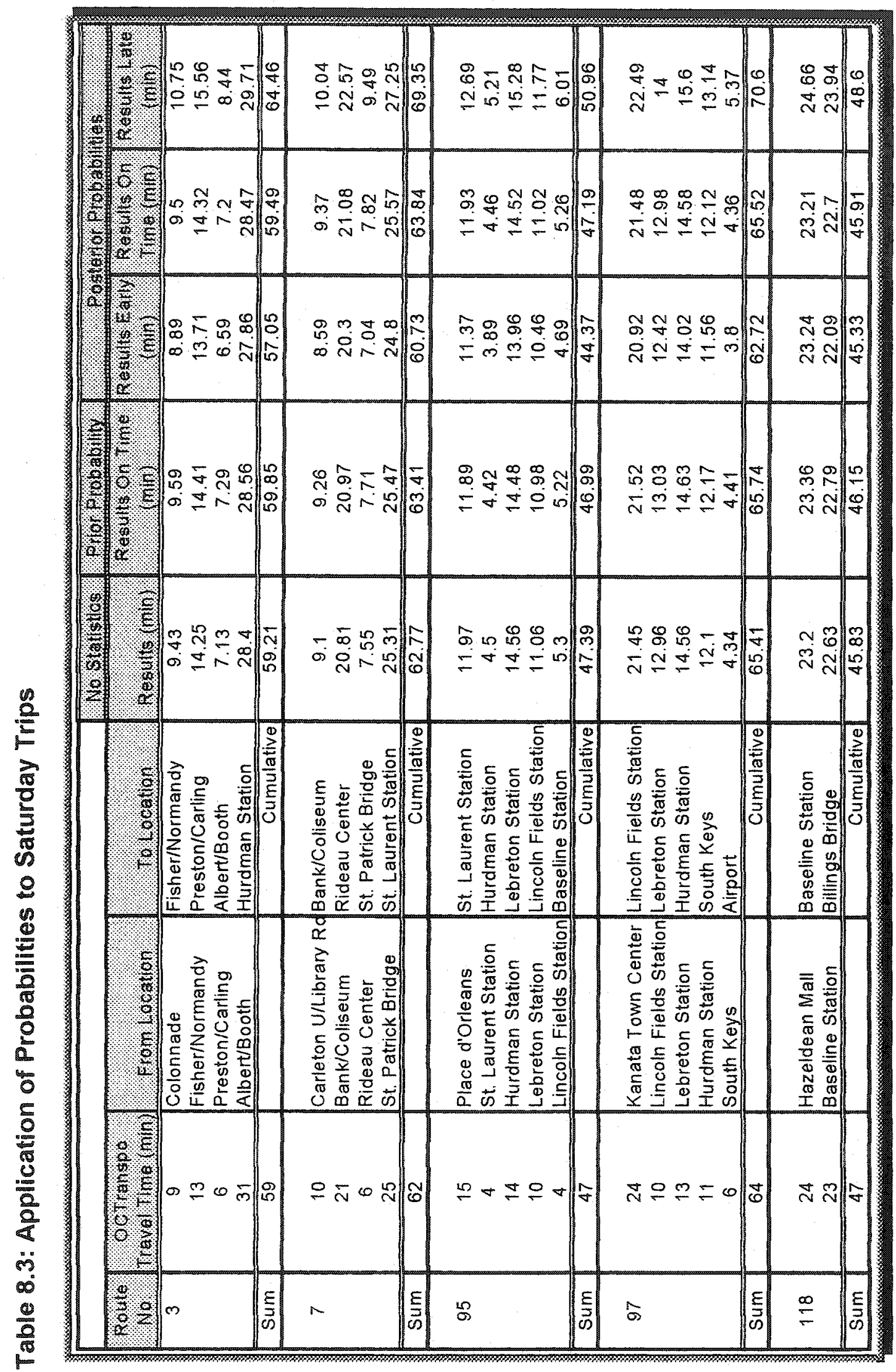




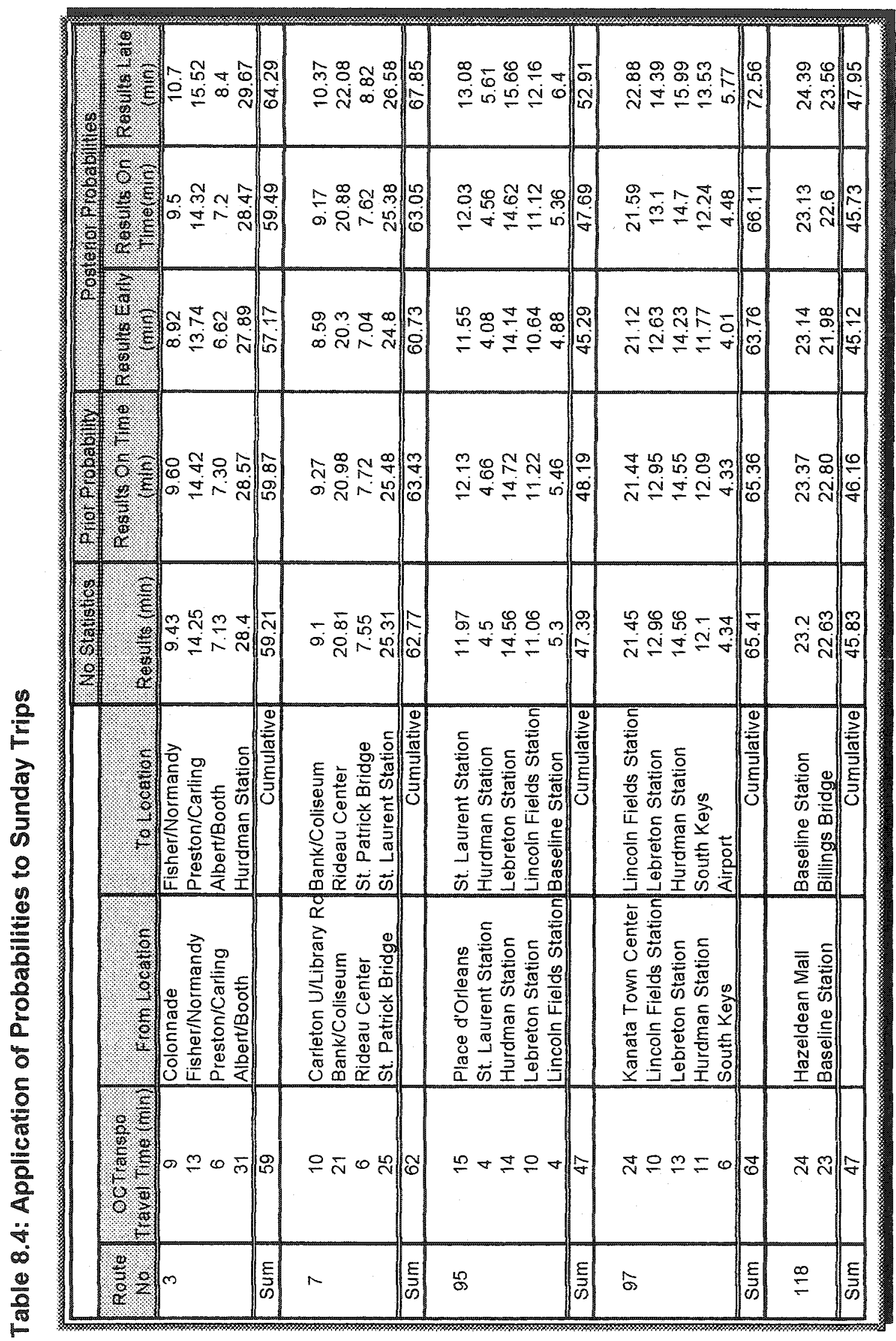


In the previous tables, the results obtained from the Bayesian Analysis clearly show how the uncertainty of transit travel can be minimized. There is an obvious difference in travel time given the criteria of being early, on time, or late.

Depending on the applied probabilities to the corresponding time period, the bus arrival can vary up to $10-15 \%$ representing early to late at a particular bus stop. This percentage demonstrates how a bus could arrive between 3 to 8 minutes early or late as compared to the actual scheduled time.

An excellent example of this variability is shown in routes 95 and 97 for weekday travel. The total travel time varies from 3 minutes early to roughly 5 to 7 minutes late. This difference can significantly impact when a person arrives at a transit stop and the entailed waiting time. By minimizing the uncertainty, the anxiety and frustration of waiting or missing a bus is reduced. People can be more aware and plan daily routines more accurately given the travel time with the inclusion of uncertainty through Bayesian Analysis. 
The probabilities also indicate how variability changes from weekday, Saturday, and Sunday travel. Given the departure time is within the same time period, the resulting travel times vary from day to day. Since travel time on weekends tend to vary differently due to reduced passenger loads and congestion changes, the probabilities are altered.

Depending on the route and which area of the city it serves, the probabilities will differ. If a route serves a large number of passengers and the impact by congestion from mixed traffic does not change significantly, the results obtained from weekday travel to weekend travel can be small.

An example of this is shown using Route 118. From the previous three tables, route 118 only has travel times varying from 1 to 3 minutes of being early or late arriving at a transit stop. This route travels directly between Kanata and Billings Bridge stations having two shopping centers at either end. The route uses two major roads where traffic conditions do not vary significantly between weekday and weekend travel. Therefore, its uncertainty between days varies less throughout the week. . 
As shown in chapter 5, a loop back in to the algorithm is performed to check for another route with less travel time based on the Bayesian application. An attempt was made to show a change in the optimality of a route due to the Bayesian Analysis. Unfortunately due to the limited number of routes used for the transit information system, no alternate routes were obtained for illustration purposes. Since only 23 routes were used instead of the entire network, many routes do not overlap or travel to identical origins and destinations. There are restrictions to where and when transit vehicles travel, therefore it is difficult to obtain another optimal route with a small number of routes. Given the opportunity of applying the entire network, as shown in Figure 8.1, the possibility of having the Bayesian Analysis altering an optimal bus route would be greatly enhanced. 


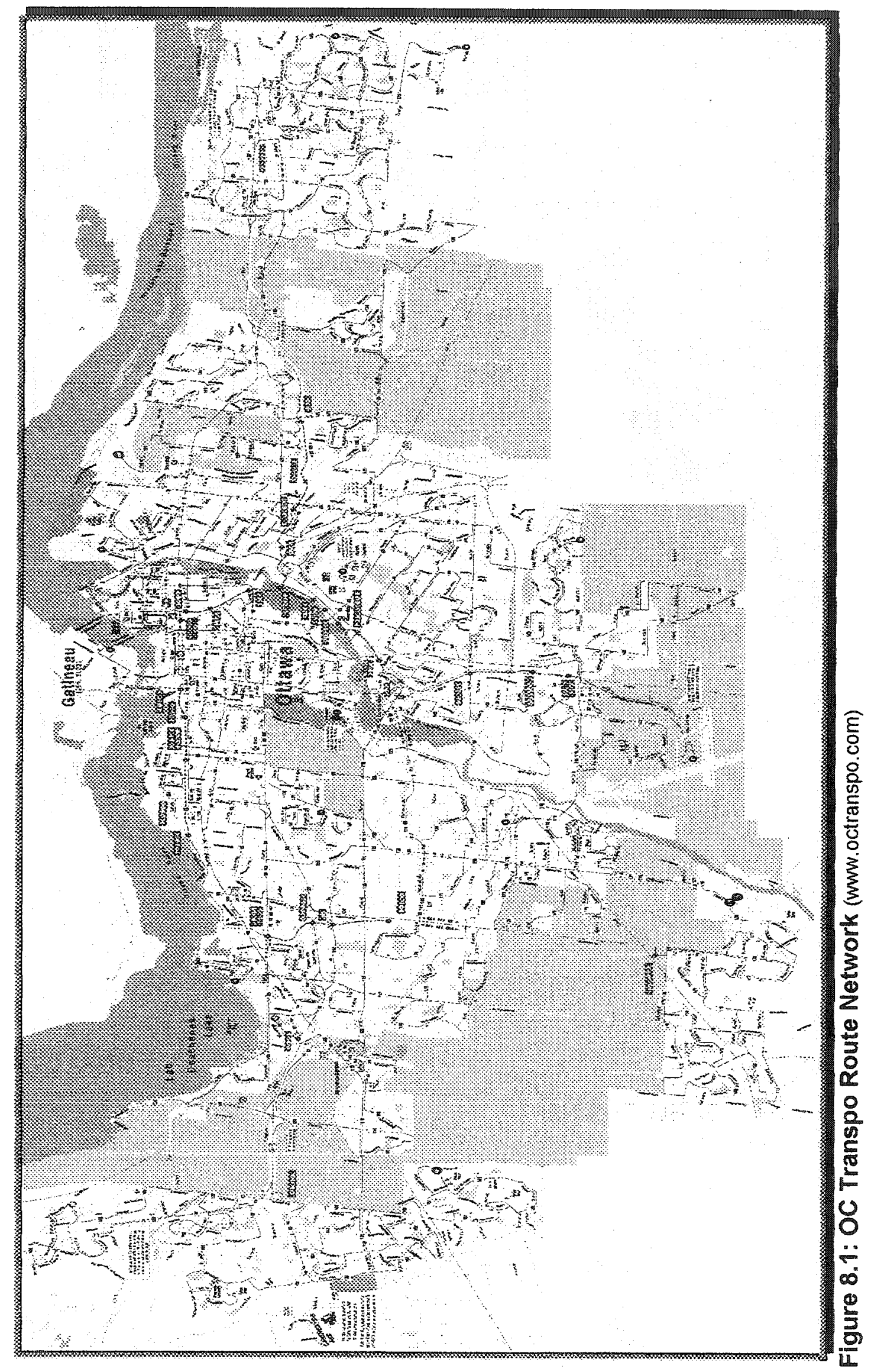


The Bayesian analysis is also useful and is applied in cases where a route could be detoured or diverted for a long or short period of time due to road construction, protests, Canada Day festivities, or a major fire. In cases where there is a major event being celebrated or construction occurring, the City of Ottawa and OC Transpo work together to let the public know of street closures and schedule changes ahead of time. OC Transpo and the emergency services in Ottawa presently work together to alert each other of problems along the roadways. So, if there is a major traffic accident along a particular route, drivers can let the dispatchers know and can then work together to help in establishing a detour in order to avoid the situation.

When route changes are required, $O C$ Transpo has the ability to recode the line segments pertaining to the specific route within the attribute table. As stated in Chapter 5, line segments can be coded to represent one-way streets and closed streets. Using the appropriate code, routes may be changed and an advisory given for a late arrival. The Bayesian analysis would be applied to the new travel time with respect to the revised route and the suitable conditional probabilities will be assigned based on the time period. 
A new travel time is calculated based on the pertaining costs for the new section of road. For the most part, the bus will not stop and pick-up or drop-off any passengers until the regular route is once again being traveled upon. Therefore the new costs only include the speed limit and other relevant road restrictions. For the bus stops not being used for this period of time, OC Transpo can code them as 'not in use' for the designated period of time.

An example is given below where Route 137 has been given a detour and a late advisory. In Figure 8.2, the prior route is shown in the solid line and the new route upon given the detour is shown in the dashed line. In this case, a section of Centrum Road is closed and the bus must now travel along St. Joseph Blvd. The travel time given by the schedule is 14 minutes and the prior expected travel time is 14.63 minutes. Once the late advisory is given, the new travel time is calculated to be 16.86 minutes based on the new travel route and the conditional probabilities. In this case the route travel time has increased since the detour is somewhat lengthier and the late advisory takes into consideration any uncertainty that may arise within the detour. 


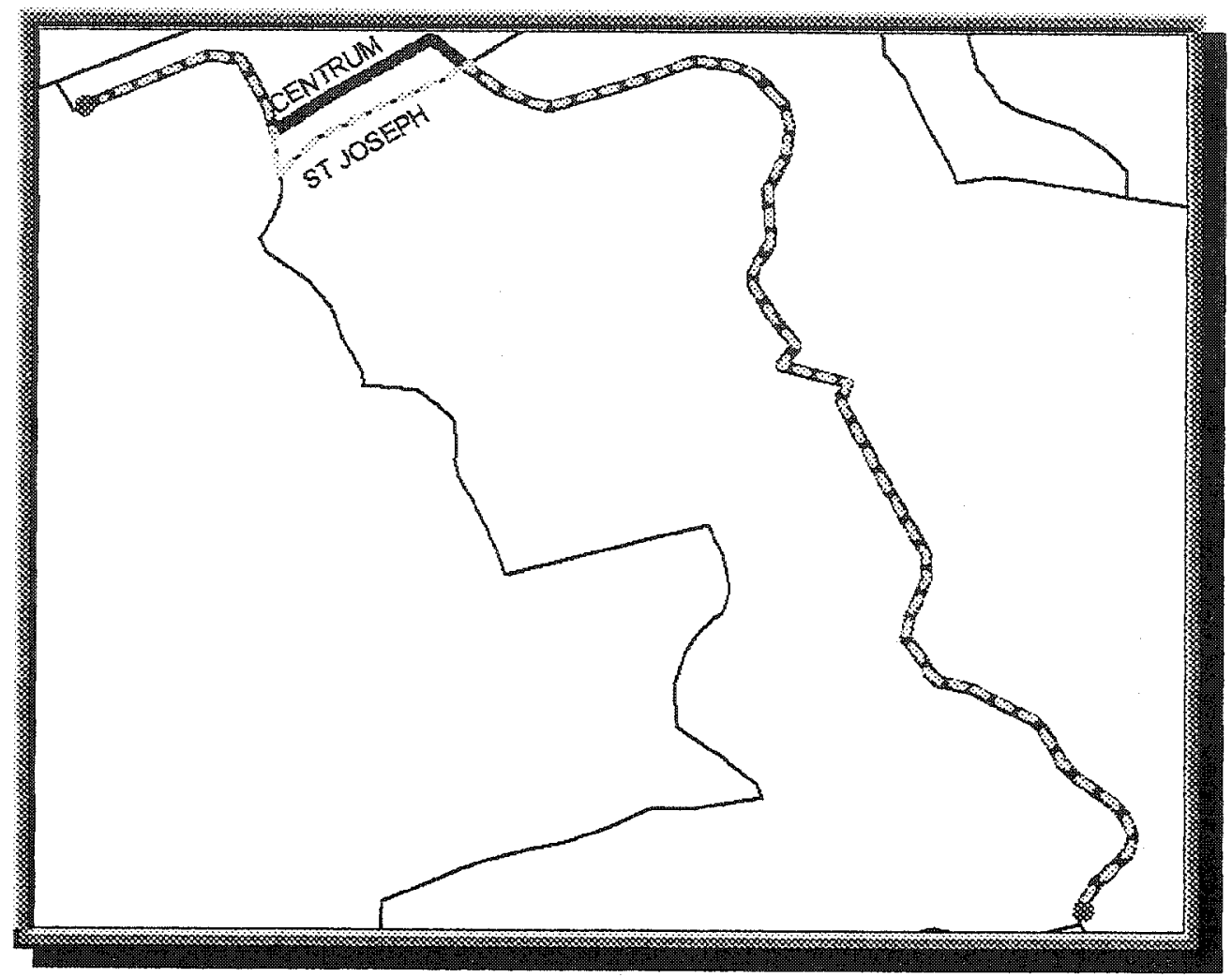

Figure 8.2: Path Change Due to Detour

In another example, the bus route number to travel may change. In this case the sub optimal, indirect route may be required. Two bus routes travel to the same location but one has been blocked off due to a residential fire. The indirect route is being used to detour traffic. As shown below, the prior path is route 116 (solid line) travelling from Meadowlands and Woodroffe to Merivale Mall. This expected travel time found through the travel information system is 8.92 minutes. 


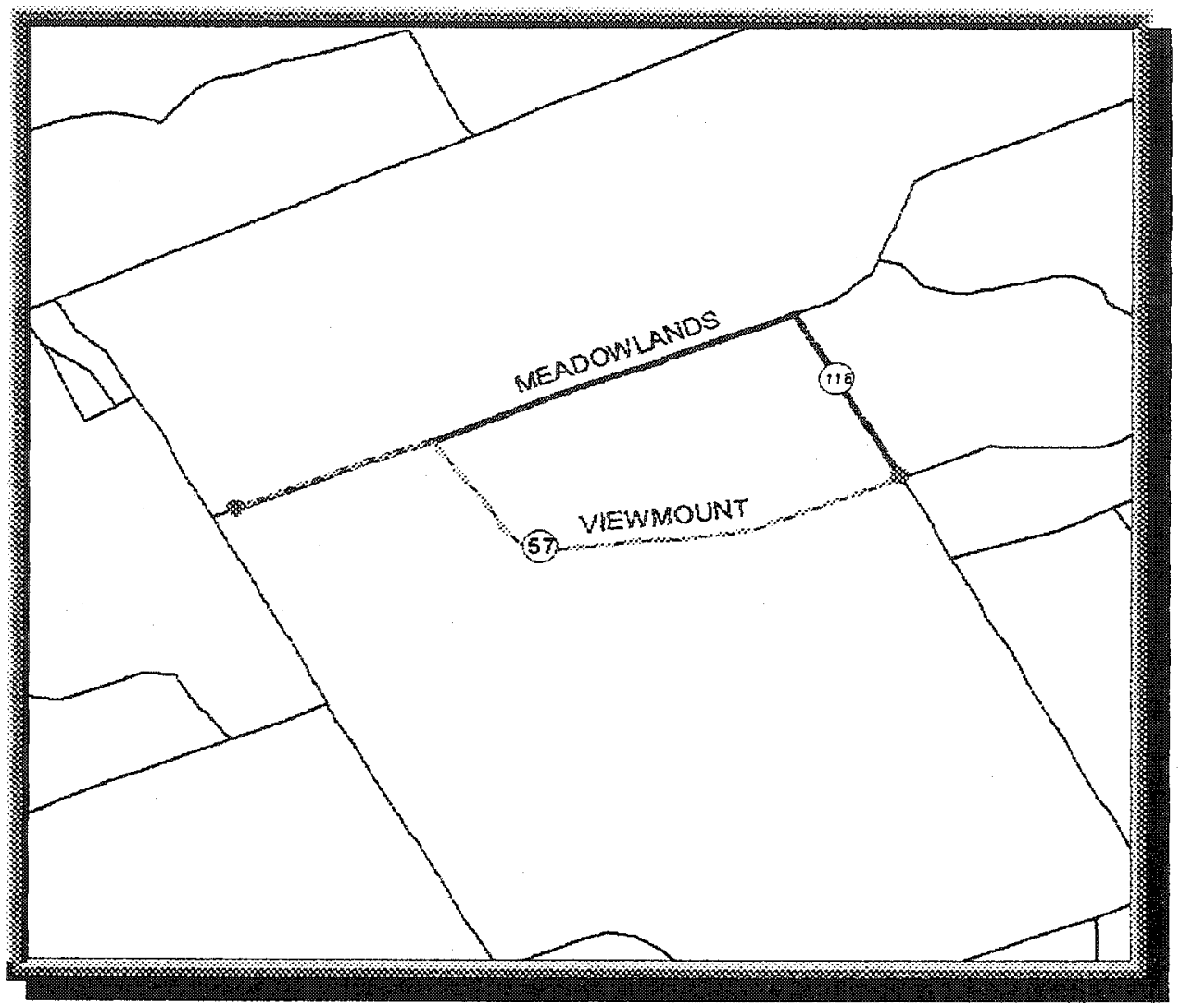

Figure 8.3: Route Number Change Due to Detour

Given that Meadowlands Drive from Viewmount Drive to Merivale Road is blocked off, vehicles must now travel via Viewmount Drive. The new route found from the transit information system is route 57 (dashed line) and its expected travel time is 9.91 minutes. As mentioned above, OC Transpo would give a late advisory and the corresponding conditional probabilities would be applied to give a new expected travel time of 11.2 minutes. This new time takes into consideration route 57's uncertainty due to passenger loading and congestion. The bus stops located along the closed section of 
Meadowlands Drive would be given a 'not in use' label and the closest bus stop to Viewmount Drive would be designated for the user.

Since a detour is a major deterrent, OC Transpo can also include a written advisory on its web site and within the transit information system to allow users to plan their trips wisely and allow extra time for travel.

Overall, by distinguishing between the travel time periods of peak and off-peak, evening to early morning, the travel time uncertainty is appropriately accounted for. Each time period and day of the week vary in accordance with the amount of congestion on the route be it passenger or traffic. By applying Bayesian analysis, the user can obtain reasonable information pertaining to route conditions and plan their arrival at transit stops more efficiently. They become more confident and less worrisome of travel time to a destination. Passengers appreciate knowing how travel will vary from day to day.

\subsubsection{Attractiveness and Ease of Use}

In regard to the attractiveness and ease of use of the system, this can only be truly evaluated once executed. As it stands, the author 
believes, the overall design of the system is structured for easy understanding, the layout is colourful, and the graphics draw attention. Since, this trip planner will be the first of this type introduced to OC Transpo's services, it is assumed that it will create a positive impression and a pleasant trip planning experience. The transit information system should be a successful and credible implementation. 


\section{Chapter 9: Summary, Conclusions, and Recommendations}

\subsection{Summary}

Like most businesses and government facilities, transit agencies are under increasing pressure to do more with less and to provide better customer services. The development of a transit information system provides part of the high-tech approach for transit agencies to seek more productive solutions. The system enables transit agencies to provide more efficient customer services to transit users.

The success of GIS implementation at transit agencies demands an integrated GIS database to meet the needs of a wide range of applications. The challenge of the transit GIS database design is to integrate spatial features in the transit network and the temporal service variations.

The spatial features in the transit network (route, segments, patterns, stops and time points) can be logically linked to linear and location referencing. It also registers the transit network to the street network and other geographic features like landmarks, which provides the basis for geocoding and trip planning. Considerable effort was required when designing the structure of the database tables. By considering the maintenance of the system throughout the design 
process, changes can be easily completed through the databases, without changing the core source code.

By developing this user-friendly transit information system, transit agencies can produce efficient customer service while using an integrated geographic information system. Within the application, an analysis of the Ottawa street network, transit routes and transit stops is performed. Schedule databases were created to link to transit attribute tables while addresses were geocoded, network costs entered, and speed limits allocated. It provides a quick method to find a route and obtain a printed copy of an itinerary. It has the capability of instantly planning an optimal transit trip based on the user's trip origin, destination and the time of travel.

With the lack of real-time information and the limitations of making prediction of arrival times, the application of Bayesian statistics helps to reduce the uncertainty found with transit travel by applying the appropriate probability based on the travel time period and the AVL/C advisory. With this technique, the user can obtain information pertaining to the route in order to travel efficiently. Be it weekday or weekend travel, the suitable probabilities will be applied to represent the conditions of the route.

While this application has been developed based on the information gathered from OC Transpo and the City of Ottawa, careful consideration was made during 
the design process to ensure that it is generic. This system can be integrated into any transit system by simply changing the graphical information (routes, bus stops, landmarks). It is designed so the agency can make changes easily and quickly to the spatial data in the GIS environment. The core algorithm merely requires the statistical information to be updated to enable accurate system performance. In accordance with the long-term strategies of OC Transpo, this implementation would enhance the current passenger information systems available and overall, this system can be widely used and become very useful to potential and current passengers.

\subsection{Conclusions}

An advanced transit information system is developed by incorporating the ITS and GIS technologies and statistical analysis methodology for updating travel time information on the basis of transit advisories regarding bus movements. Results obtained from the developed transit information system suggest that this system will be useful. Sample runs indicate the ease of use and identification of procedures in order to find the optimal route of travel and corresponding travel time. This kind of analysis enables visual orientation with written instructions. Overall, the following conclusions can be drawn that pertain to the development and applicability of the transit information system. 
- The research presented in this thesis illustrates the development of a transit information system using GIS and ITS technologies as well as Bayesian Statistical Analysis.

- This system is unique by its consideration of the uncertainty in travel time and schedule adherence as compared to other transit path finding methods.

- The Bayesian methodology is used to handle uncertainty in schedule adherence. This feature makes the best use of AVL/C information obtained from the transit agency.

- The object-oriented data model design simplifies data management and maintenance as well as increases the efficiency of network analysis, including spatial search, query, and shortest path finding.

- The user interface identifies user inputs and enables abstraction of the transit system in order to determine an optimum route.

- It considers the conditions of travel as assigned by the transit agency. If the travel time is consistent with the scheduled time, then the prior probabilities applied account for the possibility of lack of adherence due to the projected nature of a journey yet to be completed. If the advisory indicates that travel 
running off of the schedule, then this new information is used to estimate posterior probabilities to calculate travel time.

- The developed information system demonstrates that GIS is a powerful tool to develop flexible and versatile functions and to deliver large amount of information to the user.

- The use of ArcView GIS software was found to be very helpful because many functions are integrated into the software and require a minimal amount of new programming software code for any specific routine, with the exception of the transit route-planning and statistical analysis algorithm.

- The interactive map-based software allows the system to incorporate other information such as shopping centers, cinemas, and other local attractions.

- The optimum route finding algorithm combines schedule-based information and statistical analysis to fit the unique characteristics of transit networks.

- By linking to the transit agency, information on the status of buses is acquired for the algorithm procedures.

- This system has the capability of instantly planning an optimal transit trip based on the user's origin, destination, and the day and time of travel. 
- The system ignores the routes that are not in service at the time of travel.

- An optimal route is found based on the information given by the user and updated travel times are outputted.

\subsection{Recommendations for Future Research}

The transit information system has been developed mainly as a stand-alone system, with the exception of assistance from transit agency's database on the status of buses. However, by linking it with other ITS applications and the World Wide Web it can be further enhanced. The next step is to obtain Global Positioning points for the entire Ottawa area. These points can be used to adjust the base map in its entirety for positional accuracy. Additional information can be collected at each bus stop and it can then be used in the creation of a database that will be used to maintain information on the attributes of each stop. GPS would not only provide a tool for operations to improve service, it could also improve relations between the public and the transit system. A GPS-based AVL system would enrich the coverage and accuracy of real-time data on transit vehicles. In turn, the real-time location information would enhance the trip planning process. Users could then receive more accurate bus schedules and route information from the system. 
The transit information system can also be linked with real-time bus scheduling, dispatch, and control. That will take incidents such as accidents, traffic congestion, and special events into consideration to make trip planning more efficient and more effective. The system can be further linked with paratransit scheduling and the advanced highway traveller information system to become a multi-modal traveller information system.

The data for a project like this needs to be standardized. Since no geographic data for transit systems exist and street networks are generally designed for the automobile mode of transportation, not bus, new data needs to be generated from the existing street network. Although transit is affected by characteristics of the street network, a bus network has unique characteristics, such as designated bus stops and operation times. The data should be designed to be interoperable between GIS software programs and transit GIS usage. Any transportation agency should have the ability to use the data proficiently. The transit sytem data must be capable of being linked to the road network used by passenger vehicles.

Within the system, more travel options can be offered to the user so that travel preferences will better fit the individual. The current system provides the users with only the shortest route determined by the travel time. Possible options can be travelling on buses with bike-racks throughout the trip or riding low-floor buses. In addition, a possible travel criterion could be added to enable the user 
to select a desired arrival time. Currently, the system can only accept desired departure time. Additionally, by adding in walk times to and from the origin and destination and drive time to park and ride lots, this would enable the users to better plan their schedule. Again, by offering users more options for setting their travel preferences each query can be personalized to meet the priorities of the specific user.

In a completely accurate system, the topography would also be integrated. This would include, railway lines, lakes, rivers, and streams. The central business district could also be incorporated, by enabling the representation of buildings where no passage is allowed between streets. This could also include no trespass areas corresponding to private property.

A level of complexity transpires when more than one path exists from an origin to a destination. Often the most direct or simplest path is given to the user. This is often misleading in that there may be several routes that are in the vicinity of the passenger's origin, or a variety of routes with different travel speeds at a nearby transit stop. Additionally, better routes may be possible by making one or two transfers with or without walking links.

Although, the application is complete, there are some trivial requirements before implementation. Presently, the system only has a small sample of the existing routes and bus stop locations within the UTA. Therefore to complete the 
application, the remaining routes and stops need to be added. These include the school routes, shuttle buses and the O-Train.

In determining the transit information system is operational, it now requires that it be monitored upon implementation. The true test will be user response to assess the effectiveness of improved customer service. 


\section{References}

Abdelfattah, Ali and Ata M. Khan. Models for Predicting Bus Delays. Transportation Research Record 1623, 1998, pp. 8- 15.

Aronoff, Stan. Geographic Information Systems: A Management Perspective. Ottawa: WDL Publications, 1995.

Attanucci, John P., Rick Halvorsen. What GIS Can Do For Transit Planning. Papers for the Operational And Service Plannings Symposium, Dec. 1993. http://www.fta.dot.gov/library/technology/symops/attanucc.htm

Ayyub, Bilal M., Richard H. McCuen. Probability, Statistics, and Reliability for Engineers. New York: CRC Press, 1997.

Ball, William. The Use of GIS in Public Transportation. 1996. http://www.azfms.com/DocReviews/Oct96/art16.htm

Bang, Chulho. Integrated Model to Plan Advanced Public Transportation Systems. 1998. http://scholar.lib.vt.edu/theses/available/etd-122898-222857/

Birkin, Mark, Graham Clarke, Martin Clarke, Alan Wilson. Intelligent G/S Location Decisions and Strategic Planning. New York: Pearson Professional Ltd., 1996.

Brownstone, David. Discrete Choice Modeling for Transportation. IATBR Travel Behavior Conference, Australia, July 2000.

http://orion.oac. uci.edu/ dbrownst/discret.pdf

BusView http://mww.its. washington edu/busview

Canadian Strategic Highway Research Program (CSHRP) Bayesian Modeling: A User's Guide. 2000.

http://uww.cshrp.org/products/bavesian/guide/gchone.htm

Carlin, Bradley P., Thomas A. Louis. Bayes and Empirical Bayes Methods for Data Analysis, $2^{\text {nd }} \mathrm{Ed}$. New York: Chapman and Hall/CRC, 2000.

Casey, Robert F., Lawrence N. Labell, Leisa Moniz, Jackson W. Royal, Michael Sheehan, Terry Sheehan, Alex Brown, Malinda Foy, Margaret Zirker, Carol L. Schweiger, Buck Marks, Bruce Kaplan, and Doug Parker. Advanced Public Transportation Systems: The State of the Art Update 2000. US 
Department of Transportation, Federal Transit Administration, December 2000. http://www. itsdocs. fhwa.dot.gov//JPODOCS/REPTS TE//13583.pdf

Clarke, Keith C. Getting Started with Geographic Information Systems. New Jersey: Prentice Hall, 1997.

Conditions and Performance Report http://www.

Crowson, Jane L., David E. Leasure, Robert W. Smith, Frederick P. Worthen. A GIS for Public Transit. ESRI International User Conference 1997. http://www. esri.com/library/userconf/proc97/proc97/to400/pap359/p359.htm

Culp, Linda. Short Range Transit Planning and Marketing Using Desktop Geographic Information Systems. San Diego Association of Governments, December 1994. www.fta.dot.gov/library/planning/CULP/CULP.htm

Dailey, D. J. Smart Trek: A Model Deployment Initiative. Washington State Transportation Commission and US Department of Transportation Federal Highway Administration, May 2001.

http://www. its. washington. edu/pubs/smart trek report.pdf

Dailey, D. J., S. MacLean, I. Pao. BusView: An APTS Precursor and a Deployed Applet. Washington State Transportation Commission and US Department of Transportation Federal Highway Administration, June 2000. http://mww. its. washington.edu/pubs/busview_report.pdf

DeMers, Michael. Fundamentals of Geographic Information Systems. New York: J. Wiley \& Sons Inc., 1999.

Dessouky, Maged M., Randolph W. Hall, Rutvij Shah, Majid Aldaihani. Evaluating the Impact of ITS on Personalized Public Transit. March 2001. www. its. berkeley.edu/publications/UCB/ 2001/PWP/UCB-ITS-PWP-2001-12.pdf

Dijkstra's Algorithm, 1959.

http://www. princeton.edu/ alaink/lab8_html/lab8.html

Dueker and Butler. GIS-T Enterprise Data Model with Suggested Implementation Choices. The Center for Urban Studies, Portland State University, 1998.

Edwards, John D. Transportation Planning Handbook. New Jersey: Prentice Hall, 1992.

ESRI. Getting to Know ArcView GIS. Redlands, CA: ESRI Press, 1997.

ESRI. Avenue Customization and Application Development for ArcView. Redlands, CA: ESRI Press, 1996. 
http://corpweb.semcor.com/gis/solutions/type/routel

http://www esri.com

http://wmw.esriau.com.au/ozri/info.asp

htto://umw.sydneybuses.nsw.gov.au/sta/tp UI.html

http://muw11.myflorida.com/transit/Pages/

Husdal, J. Road Transportation Management - Vehicle Routing and Tracking. University of Leicester, UK. 2000.

http://www.husdal.com/mscgis/transport.htm

Kalmer, Bibiana, Beckel Marc. A Magic Bus, Portland's Mass Transit System Benefits from GIS/GPS Technology. GeoWorld, September, 1999.

Khisty, C. Jotin, B. Kent Lall. Transportation Engineering An Introduction, $2^{\text {nd }}$ Edition. New Jersey: Prentice Hall, 1998.

Khon, Harold M. Factors Affecting Urban Transit Ridership. Statistics Canada, June 2000. http://www. statcan ca/cgibin/downpub/listpub.cgi?catno=53F0003XIE

Koncz, Nicholas, Joshua Greenfeld, Kyriacos Mouskos. A Strategy for Solving Static Multiple-Optimal-Path Transit Network Problems. Journal of Transportation Engineering, American Society of Civil Engineers, Vol. 122, No. 3 , May/June, 1996, pp218-225.

Koncz, Nicholas, Joshua Greenfeld. GIS-Based Transit Information Bolsters Travel Options. GIS World, July 1995.

Leclair, R. T. Transit Service In Villages and Rural Areas Report to Transportation and Transit Committee and Council. City of Ottawa, March 2002. http://www. city.ottawa.on.ca/calendar/ottawa/citycouncil/ttc/2002/0320/ACS2002-TUP-TRN-0003.htm

Lee, Jong Sung, Jenny A. Baumgartner, and Tschangho John Kim. Champaign-Urbana Mass Transit Web-based Bus Information System. 1999 ESRI User Conference Proceedings http://www.esri.com/library/userconf/proc99/proceed/papers/pap494/p494.htm

Marks, James. How Transit Agencies are Leveraging the Web for Traveller Information, 2001. http://wmw. nawgits.com/icdn/transitweb.html 
McGrew, J. Chapman, Charles B. Monroe. An Introduction to Statistical Problem Solving in Geography. Dubuque, lowa: Wm. C. Brown Publishers Inc., 1993.

\section{MegaDyne http://www.megadyneinfo.com}

Meyer, Michael D., Eric J. Miller. Urban Transportation Planning $2^{\text {nd }}$ Edition. Toronto: McGraw-Hill, 2001.

Mouskos, Kyriacis C., Joshua Greenfeld, Louis J. Pignataro. Toward a MultiModal Advanced Traveller Information System. NJIT Research, Vol. 4, Spring 1996.

Mouskos, Kyriacos C., Joshua Greenfeld. A GIS-Based Multimodal Advanced Traveller Information System. Computer-Aided Civil and Infrastructure Engineering Vol. 14, 1999, pp267-279.

MyBus http://wnw.mybus.org

NextBus http://www.nextbus.com

NovoGroup. Increasing Popularity of Public Transport Route Search Facility. Press Release September 2001. http://umw. novogroup.com

OC Transpo Comprehensive Review The Way Ahead Becoming The Best of The Best. Ottawa: February, 1999. http://www.octranspo.com/admin/Comprehensive Review.htm

OC Transpo Statistics. http://umw.octranspo.com/admin/

Ormsby, Tim, Jonell Alvi. Extending ArcView GIS. ESRI Press, 1999.

Orski, C. Kenneth. The Traveller Information Industry Today, 2000. http://www.nawgits.com/ko_ti_today.html

Peng, Zhong-Ren, Jonathan N. Groff, Kenneth J. Dueker. An Enterprise GIS Database Design for Agency-Wide Transit Applications. URISA Journal, Vol. 10, No. 2, 1998.

Peng, Zhong-Ren, Oliver Jan. Assessing Means of Transit Infomration Delivery for Advanced Public Transportation Systems. Transportation Research Record 1666, 1999.

Peng, Zhong-Ren, Ruihong Huang. Design and development of interactive trip planning for web-based transit information systems. Transportation Research Part C, Vol. 8, pp409-425, 2000. 
Peng, Zhong-Ren. A Methodology for Design of a GIS-Based Automatic Transit Traveller Information System. Computer, Environment, and Urban Systems, Vol. 21, No. 5, pp359-372, 1997.

Peng, Zhong-Ren. GIS Data in the Internet: Issues and Solutions. http://mww.bts.gov/gis/reference/abstracts/abs23.html

Raiffa, Howard. Decision Analysis Introductory Lectures on Choices under Uncertainty. Addison-Wesley Publishing Company Inc., 1968.

Rao, K. V. Krishna, S. Muralidhar, S. L. Dhingra. Public Transport Routing and Scheduling Using Genetic Algorithms, 2000. http://www. winforms. phil.tubs.de/caspt/program.htm

Region of Ottawa Carleton. Transportation Master Plan. 1997.

Regional Municipality of Ottawa-Carleton. Transportation in Ottawa-Carleton. Status Report, December 1994

Roess, Roger P., William R. McShane, Elena S. Prassas. Traffic Engineering $2^{\text {nd }}$ Edition. New Jersey: Prentice Hall, 1998.

Schweiger, Carol L. Current Use of Geographic Information Systems in Transit Planning. Transportation Research Record 1349, 1992.

Shaw, Shih-Lung, Phillip Lall Dass. A GIS Analysis of Geographic Variations in Travel Characteristics. May 1996.

http://www. esri.com/library/userconf/proc96/TO300/PAP295/P295.HTM

Smith, Brian L. Using Geographic Information Systems and the World Wide Web for Interactive Transit Trip Itinerary Planning. Journal of Public Transportation, Vol. 3, No. 2, 2000.

Smith, Brian L., Priya K. Durvasula, Michael J. Demetsky, Stephen C. Brich. Decision Support Software for Real-Time Route Deviation Transit Service. 1998 User Conference Proceedings. http://www.esri.com/library/userconf/proc98/PROCEED/TO300/PAP272/P272.HT M

Syed, Sharfuddin and Ata M. Khan. Factor Analysis for the Study of Determinants of Public Transit Ridership. Journal of Public Transporation, Vol. 3, No. 3, 2000, pp.1-18.

Teleride http://mww.teleride.com

Tong, C. O., A. J. Richardson. A Computer Model for Finding the TimeDependent Minimum Path in a Transit System with Fixed Schedules. Journal of Advanced Transportation, Vol. 18, No. 2, pp145-161, 1984. 
Transport Canada. What is ITS? 2000. http://mww itssti.gc.calen/its what is its. htm

Transportation Research Board (TRB), National Research Council. AVL Systems for Bus Transit. National Academy Press, 1997.

http://ntl.bts.gov/display.cfm?sub=d2\&.cat $=4$

Transportation Research Board (TRB), National Research Council. TCRP Report 45 Passenger Information Services: A Guidebook for Transit Systems. Transit Cooperative Research Program, Sponsored by the Federal Transit Administration, 1999. www.nationalacademies.org/trb/publications/ tcrp/tcrp_rpt_45.pdf

Transportation Research Board (TRB). Highway Capacity Manual, 2000.

U. S. Department of Transportation (USDOT). Developing Traveller Information Systems Using the National ITS Architecture. Intelligent Transportation Systems Joint Program Office, August 1998. http://wnw. itsdocs. fhwa.dot.gov/jpodocs/repts te/37n01!.pdf

US Department of Transportation (USDOT) and Federal Transit. Administration (FTA). Traveller Information. http://www. fta.dot.gov/library/technology/APTS/update/CHAP3.HTM

US Department of Transportation (USDOT). Automatic Vehicle Location Successful Transit Applications A Cross Cutting Study Improving Service and Safety. August 2000. http://ntl.bts.gov/data/8v301.pdf

US Department of Transportation (USDOT). Conditions and Performance Report. Sponsored by Federal Highway Administration and Federal Transit Administration, 1999. http://mww. fhwa.dot.gov/policy/1999cpr/ch-2/cpm02_8.htm

Wong, S. C., C. O. Tong. Estimation of Time-Dependent Origin-Destination Matrices for Transit Networks. Transportation Research-B, Vol. 32, No. 1, pp 3548, 1998. 


\section{Appendix A \\ Variables of Transit Operation}




\section{Appendix A: Variables of Transit Operation}

\section{A.1 Modal Characteristics and Trends}

Throughout the world, the last few decades have seen an upgrading of bus systems and an expansion of rail transit systems. Urban expansion and growth have triggered this need for public transportation development. In the United States and Canada, transit investment reflects expansion of downtown office space; suburban and urban traffic congestion; the desire to provide a viable alternative to the car and freeway; and the realization by many local and federal governments that the automobile cannot exclusively provide commuter transportation (Edwards, 1992).

Bus transportation is the dominant form of public transport in most North American cities. Most bus service operates in mixed traffic over streets and highways. Bus priority lanes are provided on city streets and highways. Transitways have been introduced in some cities including Ottawa.

The speed, capacity, and reliability of buses can be enhanced by a variety of techniques, such as:

- Exclusive or preferential transit lanes on sections of streets and freeways.

- Exclusive transit turns at intersections. 
- Passive traffic signal priority measures, such as cycle length adjustments, split phases, and timing plans selectively favouring buses.

- Exclusive transit streets, malls, and ramps.

- Exclusive busways.

\section{A.2 Role of transit}

Within the urban environment, the automobile is by far urban transit's greatest competitor having the remainder walk or use bicycles. Around the world, those that have implemented policies that support urban transit have, in some cases, encouraged people to switch from the automobile to forms of public transportation such as buses, streetcars and subways. Prohibitive taxing and pricing policies have the potential to force drivers out of their cars into urban transit vehicles. Nevertheless, with the exception of a handful of major population centers, most communities in Canada are sparsely populated, which makes the provision of urban transit services relatively expensive and difficult to offer on a comprehensive basis (that is, providing all parts of the community with frequent service). Changes in work patterns such as work-at-home, shift work outside the traditional 9 to 5 time frame, the location of work sites away from downtown areas and other similar factors have given transit planners a multitude of challenges. 
However transit plays three major roles in North America. First, it accommodates choice riders; those who choose transit for their trip making even though they have other means of travel, such as a passenger vehicle. Many commuters choose transit because they are unwilling to deal with traffic congestion during peak periods for work trips. Choice riders dominate transit during the peak periods for work trips. In this way, transit increases the number of people who can be carried by urban transportation systems and constrains the growth of more passenger vehicles. Accommodating choice riders is especially critical in cities with high central business district densities and costly and limited parking (TRB, 2000).

Transit assists in congestion relief. If transit service consistently provides doorto-door travel times that are competitive with those of private automobile trips, then transit will provide a meaningful substitute for automobiles as the travel mode of choice. In doing so, transit can effectively reduce roadway congestion. This is especially important for commuting trips, which are often made during times of peak period congestion. With the busways and light rail systems riders are provided with a separate system that avoids all automobile traffic and congestion (USDOT, 1999).

The other major role of transit is providing basic mobility for segments of population that are too young, too old, or otherwise unable to drive due to physical, mental, or financial situations. These transit users have been called 
captive riders. These users benefit from a transit system that provides regular access to multiple destinations at a low cost (USDOT, 1999, TRB, 2000).

These three roles will obviously overlap with and support each other. For example, a transit vehicle may primarily serve as a congestion relief tool during peak travel periods while supporting basic mobility in off-peak hours. An individual may choose a housing location near a transit station in order to both avoid rush-hour congestion and to access shopping and entertainment activities in the evening.

The cost of providing comprehensive services, especially for communities that are characterized by urban sprawl, has meant a requirement for subsidization. In 1998, governments in Canada paid approximately $\$ 2.4$ billion in capital and operating subsidies to urban transit companies (Khon, 2000). Nevertheless, transit companies have sought out new sources of revenue such as fees from parking lots and advertising. Revenues from these sources grew from $\$ 82.4$ million in 1995 to $\$ 110.4$ million in 1998 (Khon, 2000).

Most would agree that there are many factors that affect both the demand for and supply of urban transit. These include (Khon, 2000): 
- Family size - Families with children may choose to use personal vehicles rather than urban transit because the monthly cost of transit passes may be more expensive;

- Economic changes such as employment opportunities, taxes, fuel costs, parking fees, automobile insurance costs, and vehicle operating costs;

- Demographic impacts such as population growth, immigration rates and fertility rates;

- Ridership loyalty;

- Parking rates and distance to work;

- Other factors such as convenience, a change in work schedules and the work-at-home phenomenon;

- Community size - Large cities with long commutes, expensive downtown parking and relatively greater distances to destination points may positively influence the use of public transit because of the level and frequency of service as well as time and cost savings. Travel times in smaller communities may not be as lengthy, although in smaller communities, the availability of urban transit may not be as comprehensive as in larger cities.

Factors that affect supply and demand are complex, constantly changing and difficult to identify and discern. Therefore it is safe to say that many factors individually or combined influence transit ridership. 


\section{A.2.1 Transit Characteristics}

Several characteristics differentiate transit from the automobile in terms of availability and capability. Although the automobile has widespread access to roadway facilities, transit service is available only in certain locations during certain times. Roadway capacity is available 24 hours a day seven days a week once constructed, but transit capacity is limited by the number of transit vehicles operating at a given time.

Transit passengers frequently rely on other modes to gain access to transit. Transit use is greatest where population densities are highest and pedestrian access is good. A typical transit user does not have transit service available at the door and must walk, bike, or drive to a transit stop and then must walk or bike from the transit discharge point to the destination. In contrast, suburban areas are mainly automobile-oriented, with employment and residents dispersed, often without sidewalks, and without direct access to many transit lines. If potential passengers cannot have access to transit from both their trip origin and destination, transit is not an option.

Finally, transit is about moving people rather than vehicles. Transit operations at their most efficient involve relatively few vehicles, 
each potentially carrying a relatively large number of passengers. In contrast, roadway analysis traditionally involves relatively large numbers of vehicles, each usually only one occupant. When evaluating transit priority measures for transit and automobile users, it is the number of people affected that should be compared, rather than the number of vehicles (TRB, 2000).

\section{A.2.2 Classification}

Transit users can be classified in two groups "captive" and "choice" riders. Captives are those who have no alternative transportation mode available; they have no access to an automobile either as driver or passengers, nor is walking or bicycling a viable alternative for those trips for which transit is used. The majority of this group depends on transit so frequently that they must choose their home, work location, shopping, and other facilities in relation to wellestablished transit lines. Choice riders are attracted to transit if they feel that the travel time and cash costs involved are competitive with those of the automobile alternative. This is most likely to occur for peak period trips with one end in the CBD because of the likelihood of congestion at the approaches to the downtown area and the high parking costs within it (Khisty and Lall, 1998). 
A.2.3 Types of service

Bus transit can be either fixed route or demand responsive. Fixedroute service is ideal for large, densely populated urban areas. In less dense areas, which cannot support fixed-route service, demand responsive transit can be an essential part of transportation of the non-driving population (TRB, 2000). In Ottawa, OC Transpo operates as a fixed route system.

Local transit networks operate in mixed traffic (shared right-of-way) on suitable streets and freeways. For environmental reasons, transit routes are generally confined to major arterials and collector streets. However to serve outlying neighbourhoods adequately, some residential streets may have to be used. Where applicable busways are built to accommodate bus traffic and more efficient service (khisty and Lall, 1998).

There is no precise definition of what differences in right-of-way, technology, or service constitute a specific transit type. Commonly, transit is classified into three generic classes, based mostly on right-of-way (Region of Ottawa-Carleton, 1997):

- Street Transit-(surface transit) designates transit operated on roadways with mixed traffic. Its reliability is often low because 
of various interferences, and its speed is lower than the speed of traffic due to the time spent at passenger stops. Buses, trolley buses and streetcars fall into this category.

- Semi-Rapid Transit uses mostly longitudinally separated right of way although other classes of right of way may be used. Some LRT and bus-based systems fall into this category.

- Rapid Transit operates exclusively on fully separated right-ofway. They generally feature high speed and capacity. Busways such as the OC Transpo's Transitway fall into the category of rapid transit. Busways are controlled access facilities dedicated for bus services separated from general traffic, often with grade separated right-of-way. Buses, therefore, operate under conditions achieving speeds equal to or in excess of light rail systems. The busway combines the flexibility of a bus, which can go anywhere there is an adequately paved street, with freedom from general traffic conditions (Region of OttawaCarleton, 1997).

A.2.4 Quality of service Whether or not transit is provided near a person's origin and destination is key in use of transit. Ideally, transit service is 
provided within a reasonable walking distance of the origin and destination, or demand-responsive service is available. The reasonableness of the walking distance varies from source to source and depends on the situation (TRB, 2000).

If transit service is not provided near the origin, other options include driving to a park-and-ride lot or riding a bike to transit. Both of these options require that the transit operator provide additional facilities, such as parking lots, bicycle storage and racks.

However, if transit service is not provided near the destination, the choices are more limited. A bicycle might be carried on a rack, but a customer must have some degree of confidence that space will be available in the bike rack when the bus arrives.

How often and when a transit service is provided are important factors in the decision to use transit. The more frequent the service, the shorter the wait when a bus or train is missed or when the exact schedule is not known, and the greater the flexibility customers will have in selecting travel times. The number of hours during the day when service is provided is also important. It does not matter whether a transit stop is located within walking distance 
if service is not provided at the desired time of travel; transit then cannot be an option (TRB, 2000).

\section{A.2.5 Reliability}

Reliability affects the amount of time passengers must wait at a transit stop, as well as the consistency of a passenger's arrival time at a destination from day to day. Reliability encompasses on-time performance as well as the regularity of headways between successive transit vehicles. Uneven headways result in uneven passenger loadings, so that a transit vehicle arriving late picks up not only its regular passengers but also others who have arrived early for the following vehicle. As a result, the vehicle falls further and further behind schedule. In contrast, the vehicles following will have lighter-than-normal passenger loads and will tend to run ahead of schedule. Reliability is influenced by transit conditions, staff availability and vehicle maintenance, and by how well vehicle operators adhere to schedules (TRB, 2000).

\section{A.2.6 Total Trip Time}

Total trip time includes the travel time from the origin to a transit stop, waiting time for a transit vehicle, travel time onboard a vehicle, travel time from transit to the destination, and any required transfers between routes during the trip. In general, both the 
absolute travel time and the travel time in relation to competing modes will factor in a traveller's decision about transit (TRB, 2000). However, the apparent inconvenience of a longer transit trip can be diminished if the passenger can use the time onboard productively (i.e. reading, preparing or reviewing work, or even catching up on sleep).

Travel speed is a useful route segment performance measure because it reflects how long a trip may take without depending on how long a route segment might be. Transit priority measures, improvements to fare collection procedures, the introduction of lowfloor buses and other similar actions implemented along a route segment will be reflected as improvements in travel speed (TRB, 2000)

Total trip time is influenced by several factors, including the route spacing, the service frequency, the frequency of stops, traffic congestion, signal timing, and the fare collection system (TRB, 2000). Regardless of the kind of bus facility being analyzed, there are some fundamental components common to each that are required to calculate the facility's vehicle and person capacity. Dwell time is the most important of these, but all have some effect on capacity. 
The average speed along a route is a function of stop spacing and either the top speed capacity of the vehicle or train or the speed limit imposed by law or physical conditions. Most choice riders seek the shortest total travel time door to door, and that maximizing the speed of transit routes at the cost of accessibility is not necessarily advantageous. However, if accessibility remains about the same, increases in speed are beneficial to users (Khisty and Lall, 1998).

\section{A.2.7 Capacity}

Transit capacity is different from highway capacity. It deals with the movement of both people and vehicles; it depends on the size of the transit vehicles and how often they operate; and it reflects the interaction of passenger traffic and vehicles flow. Transit capacity depends on the operating policy of the transit agency, which specifies service frequencies and allowable passenger loadings. Accordingly, the traditional concepts applied to highway capacity must be adapted and broadened (TRB, 2000).

\section{A.2.8 Dwell time}

Just as dwell times are key to determining capacity, passenger demand volumes and passenger service times are key to 
determining dwell times. Dwell times may be governed by boarding demand, alighting demand, or total interchanging passenger demand. In all cases, dwell time is proportionate to the boarding and alighting volumes times the service time per passenger. Dwell time also can influence a transit operator's service costs; if average vehicle speeds can increase by reducing dwell time, and it the cumulative change exceeds the route headway, then fewer vehicles may be required to provide the same service frequency (TRB, 2000).

As defined by the Highway Capacity Manual, there are six main influences on dwell time. Two relate to passenger demands; the others relate to passenger service time:

- Passenger demand and loading.

- Stop and station spacing.

- Fare payment procedures.

- Vehicles types.

- On-board circulation.

- Wheelchair and bicycle boarding.

Dwell time can be summed as the time required to serve passengers at the busiest door plus the time required to open and 
close the doors. A value of 2 to $5 \mathrm{~s}$ for door opening and closing is reasonable for normal operations (TRB, 2000).

Dwell time can be measured in the field. Field measurement of dwell time is best suited for determining the capacity and LOS of an existing transit line. In the absence of other information, dwell time can be assumed to be 60 s for CBD, or transit stations; 30 s for major outlying stops; and 15s for typical outlying stops (TRB, 2000).

\section{A.2.9 Clearance Time}

Clearance time includes two components, the time for a bus to start up and travel its own length while exiting a bus stop and the reentry delay associated with the wait time for a sufficient gap in traffic to allow a bus to pull back into the travel lane. Various studies have looked at these factors, either singly or together. Research has found that bus start-up times range from 2 to $5 \mathrm{~s}$. The time for a bus to travel its own length after stopping is approximately 5 to $10 \mathrm{~s}$, depending on acceleration and traffic conditions. Other research recommends a range of 10 to $15 \mathrm{~s}$ for clearance time (TRB, 2000). 
Appendix B

Transit Routes 


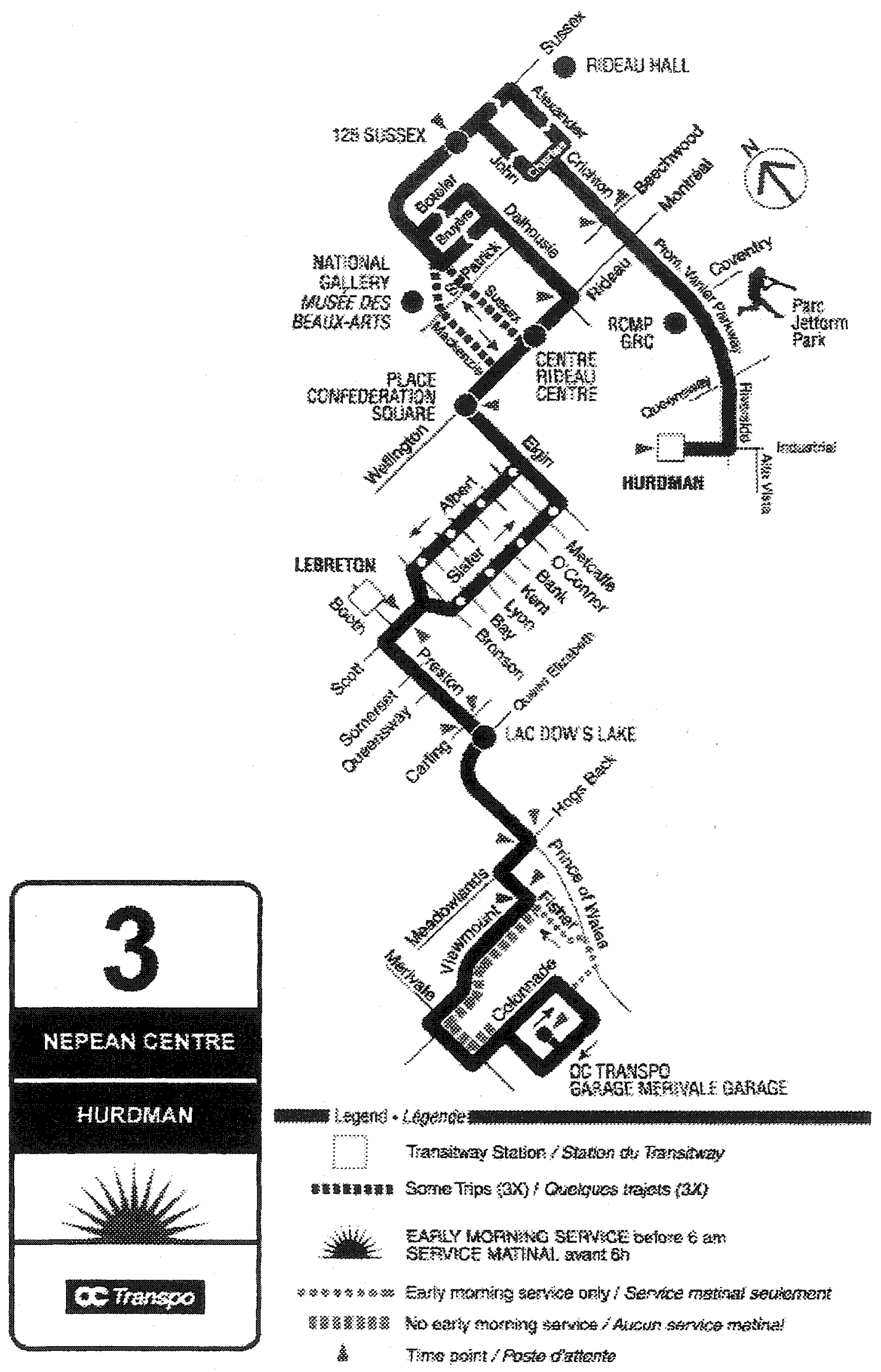



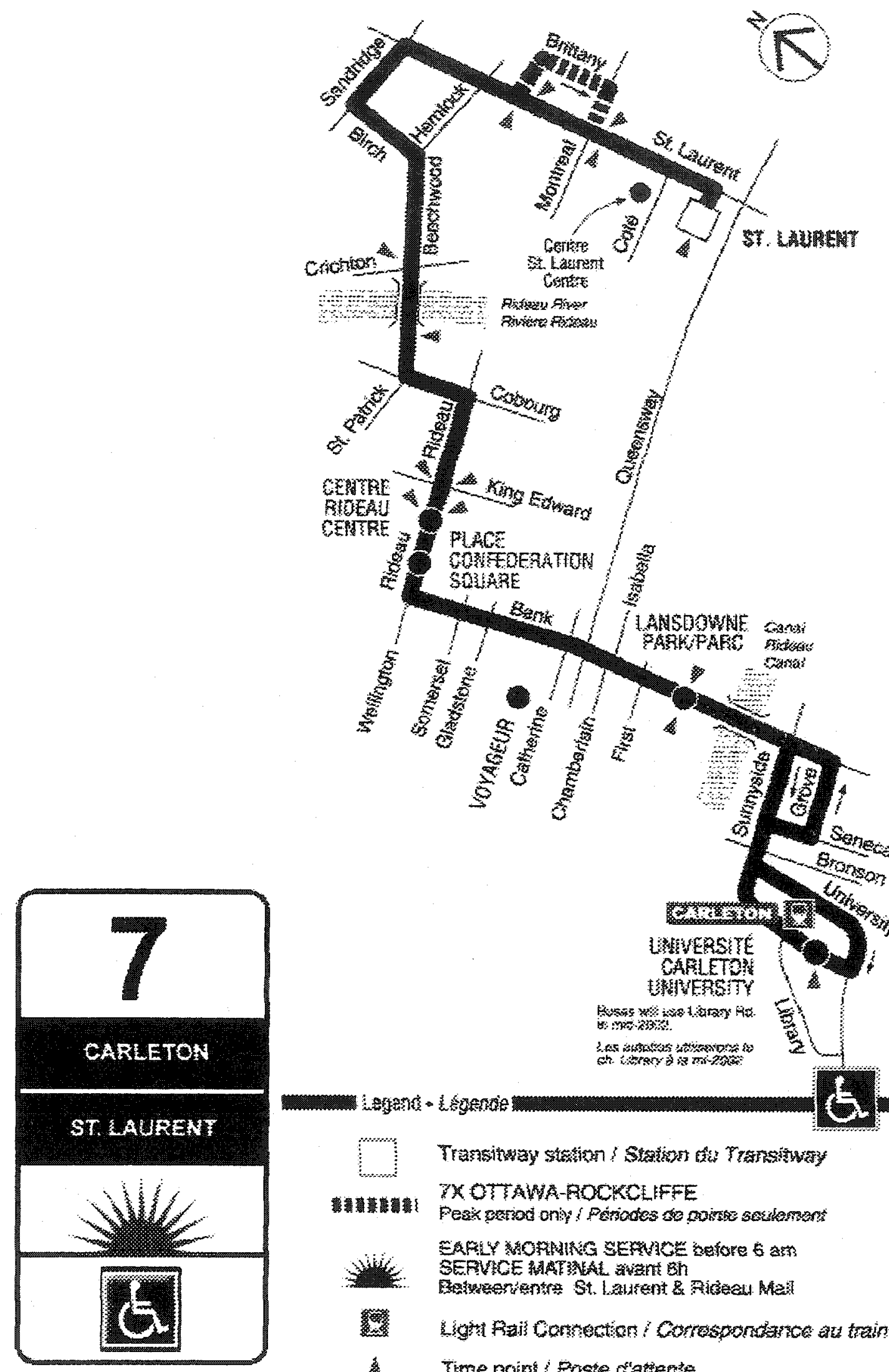

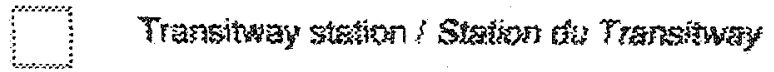

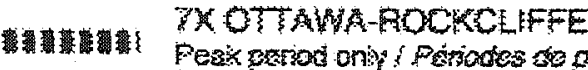

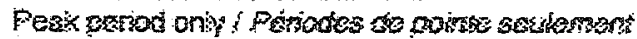

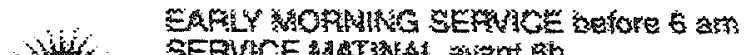

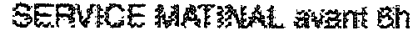

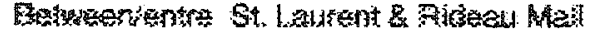

筑等

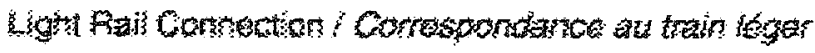

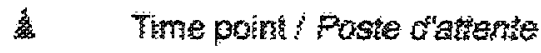




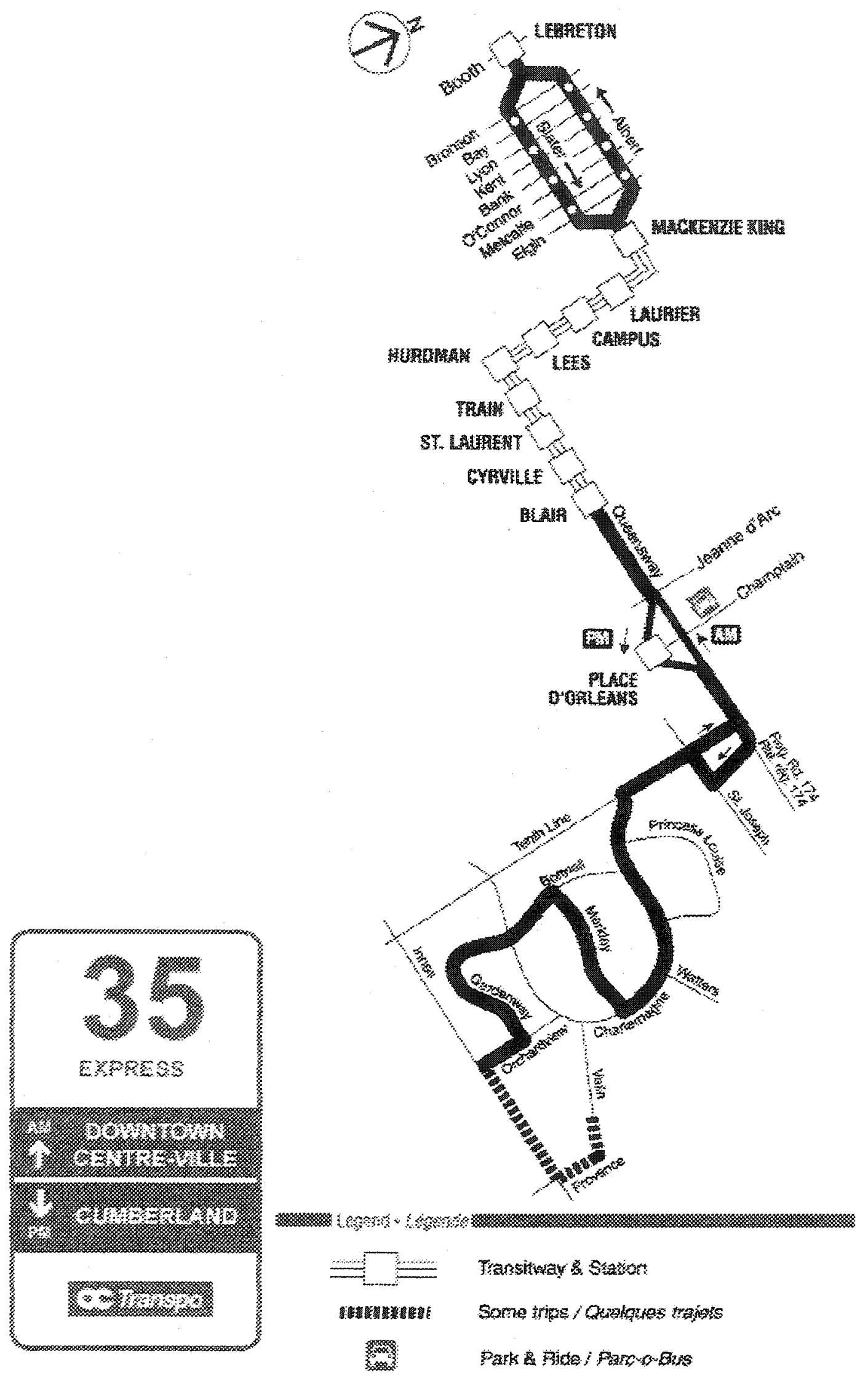



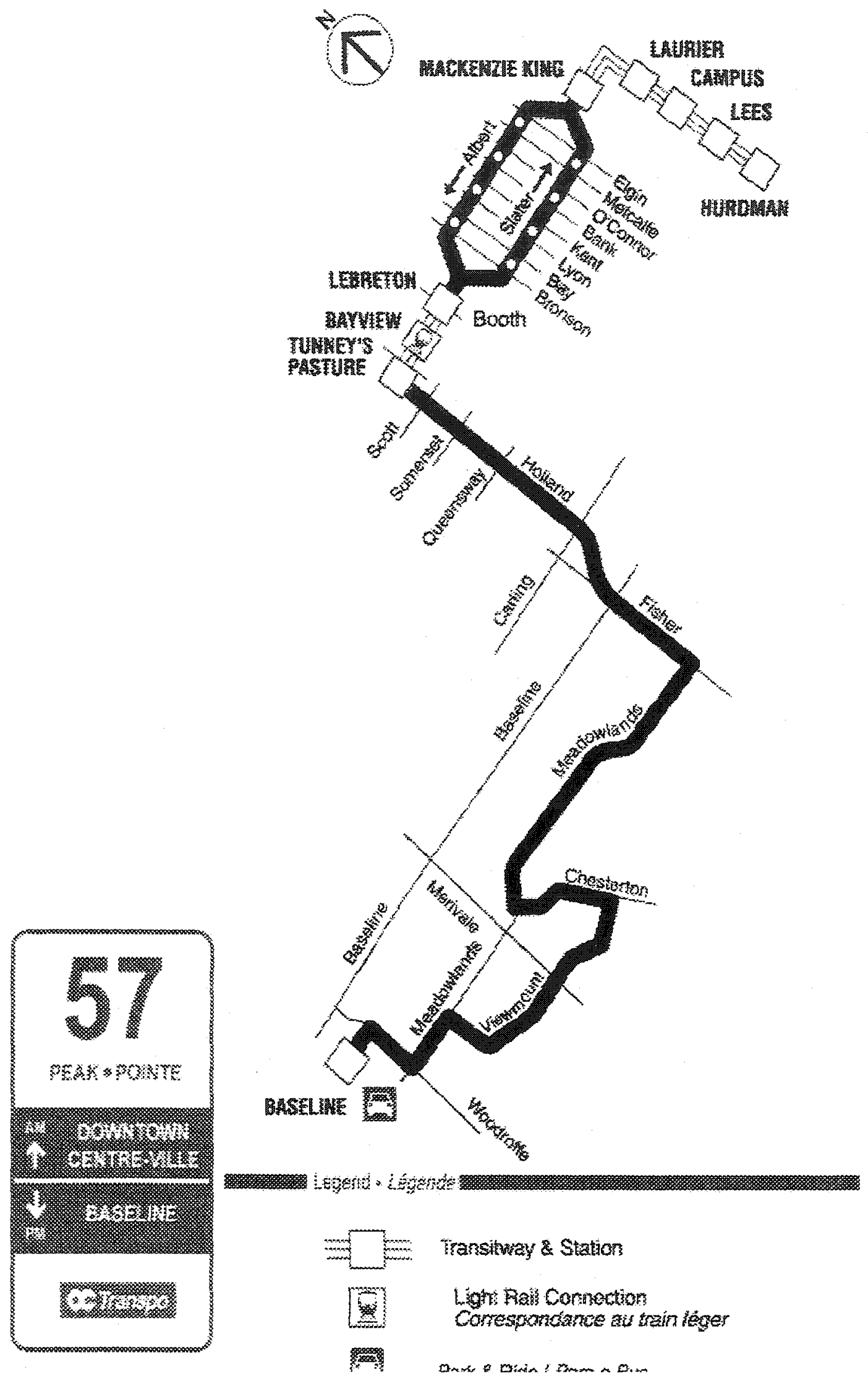


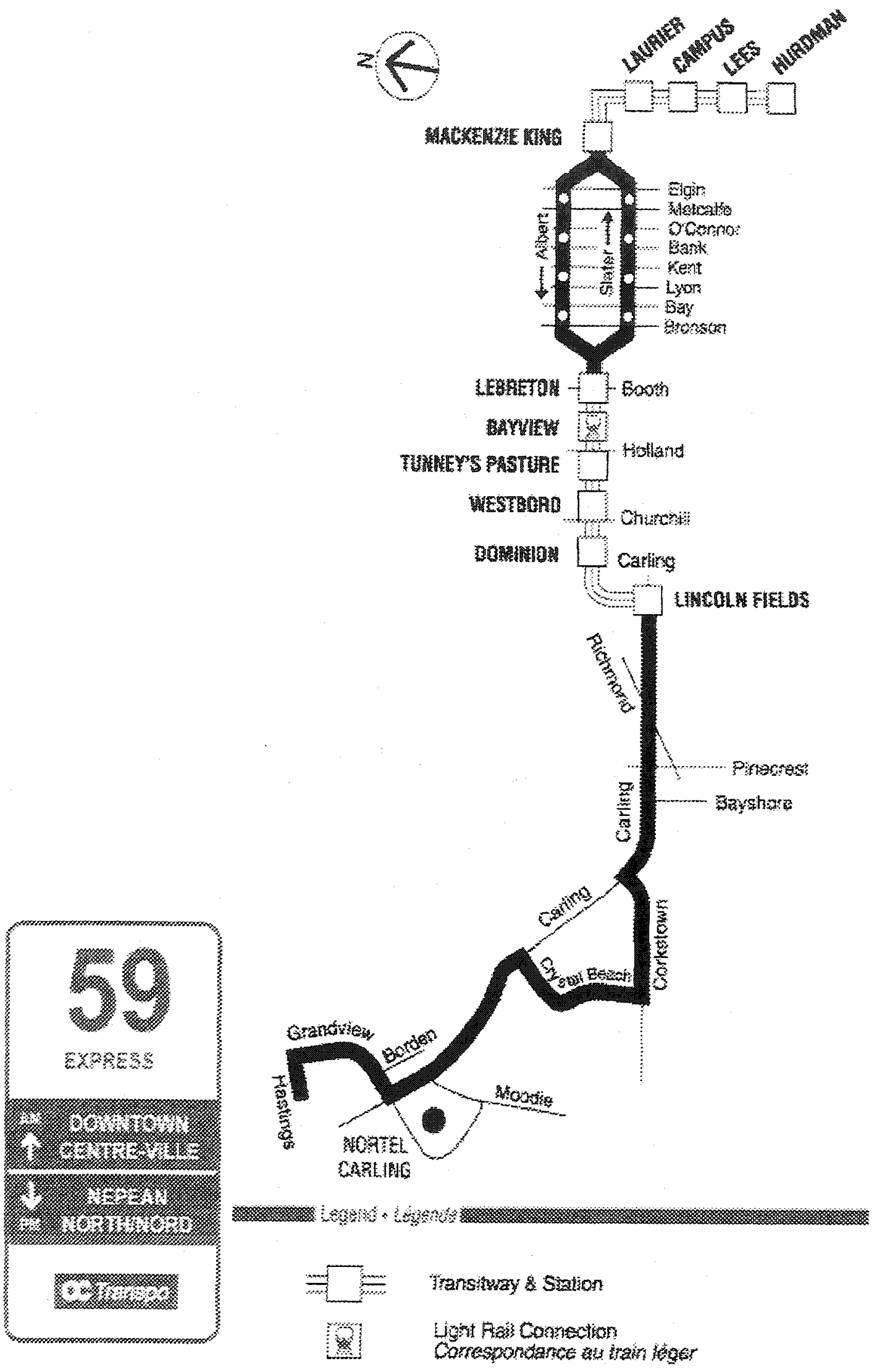



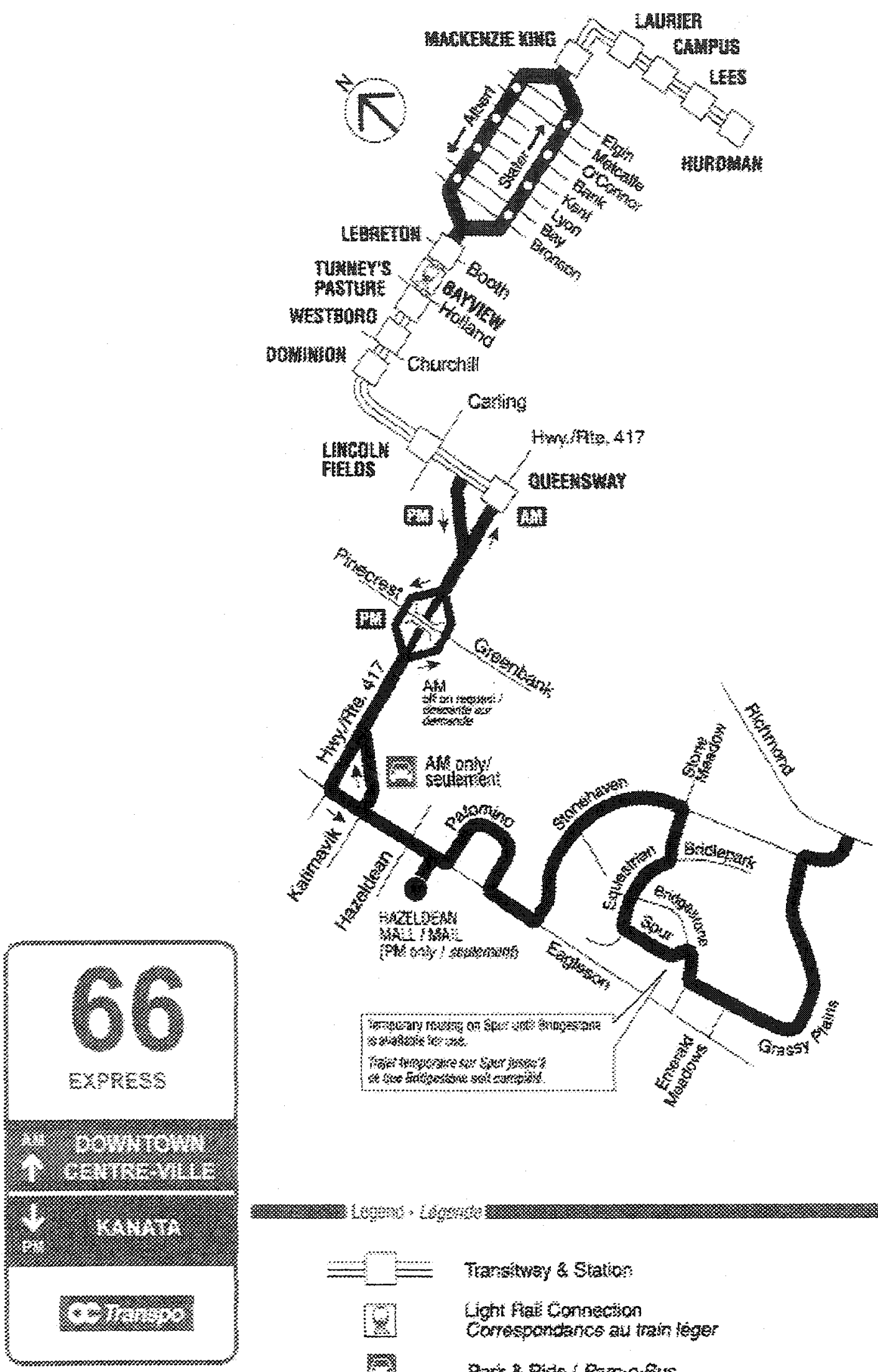

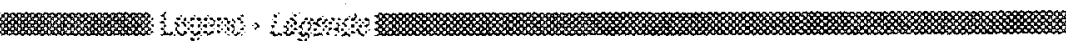

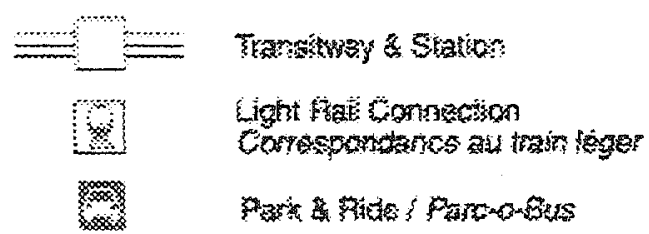



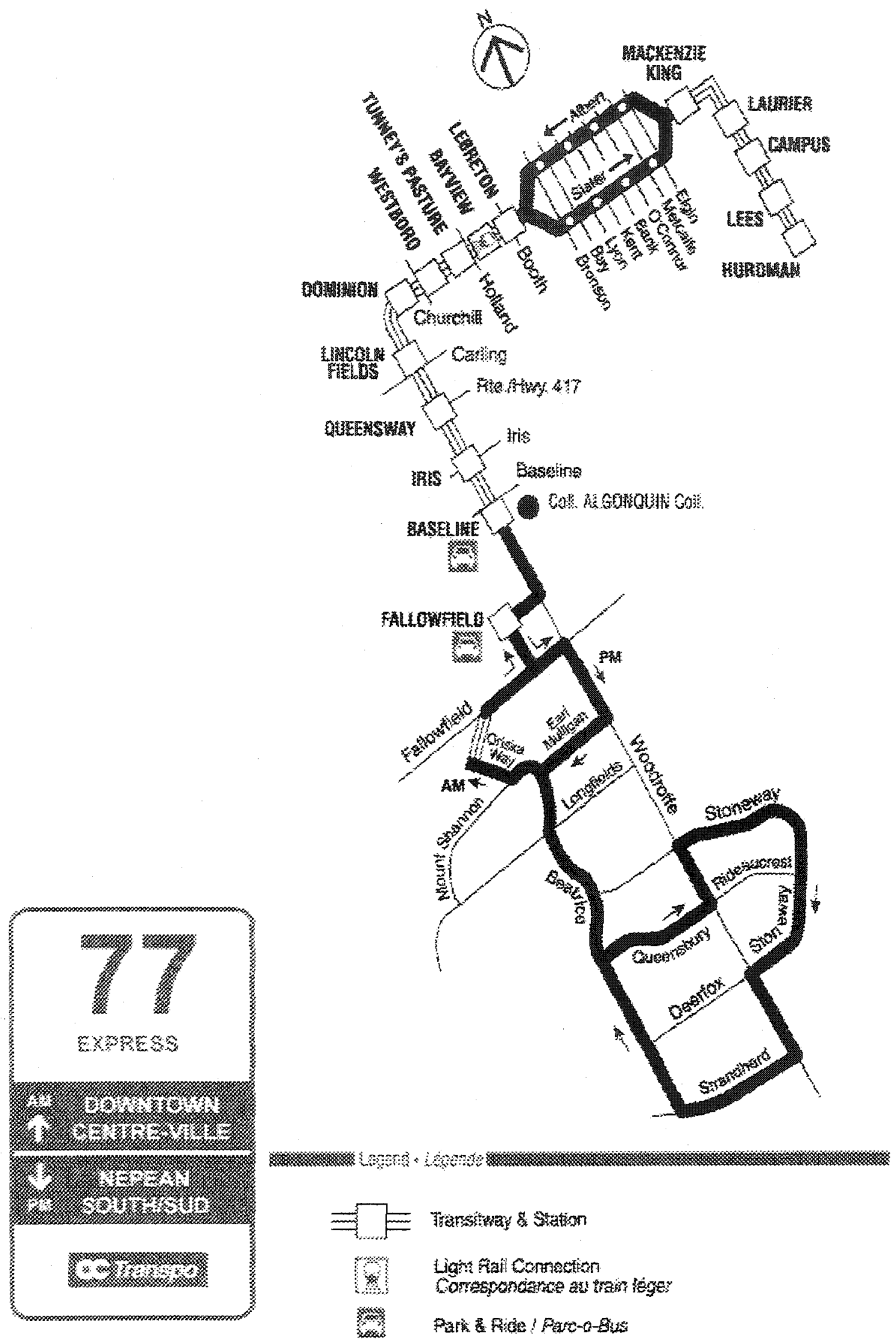


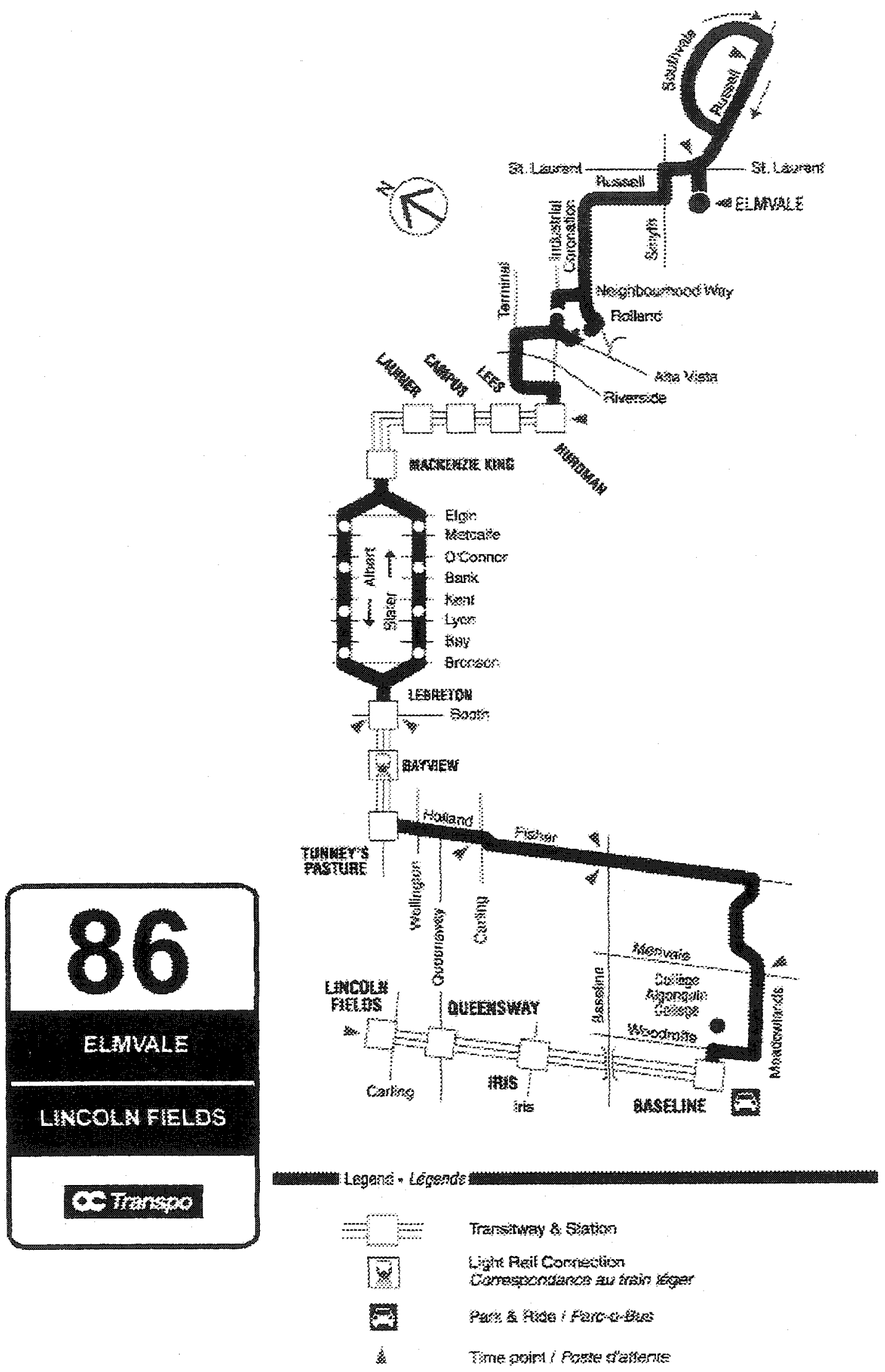




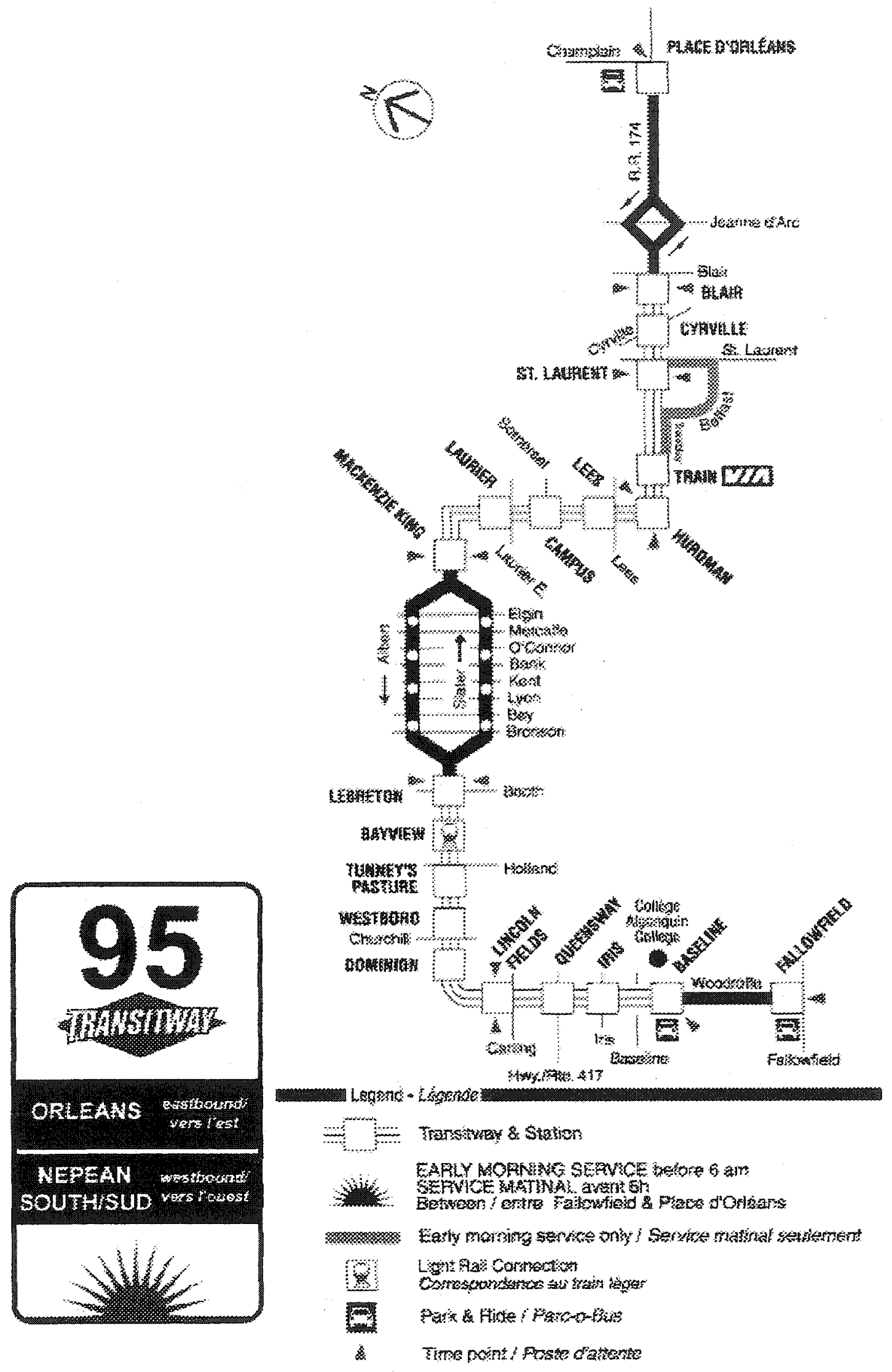



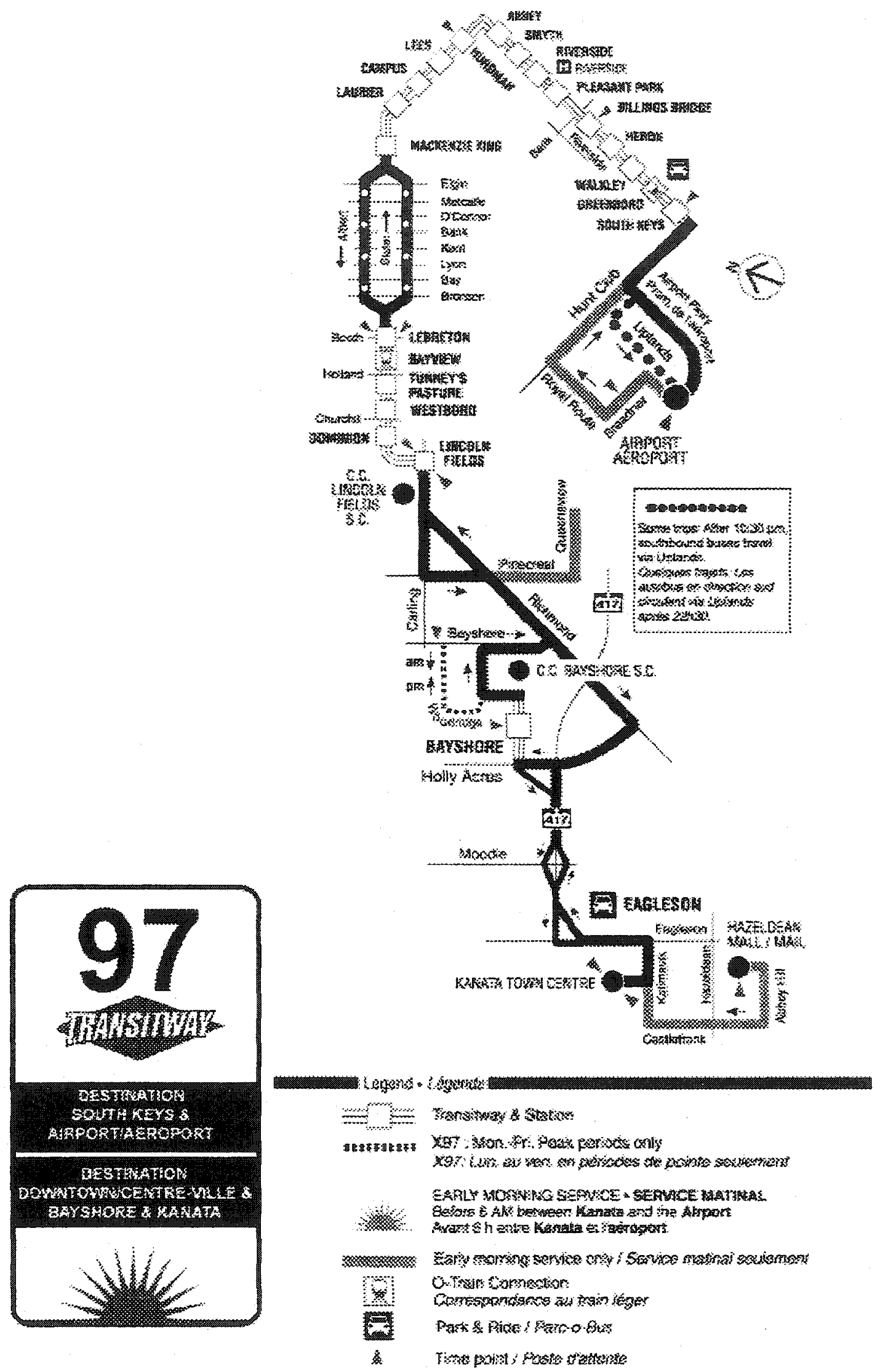


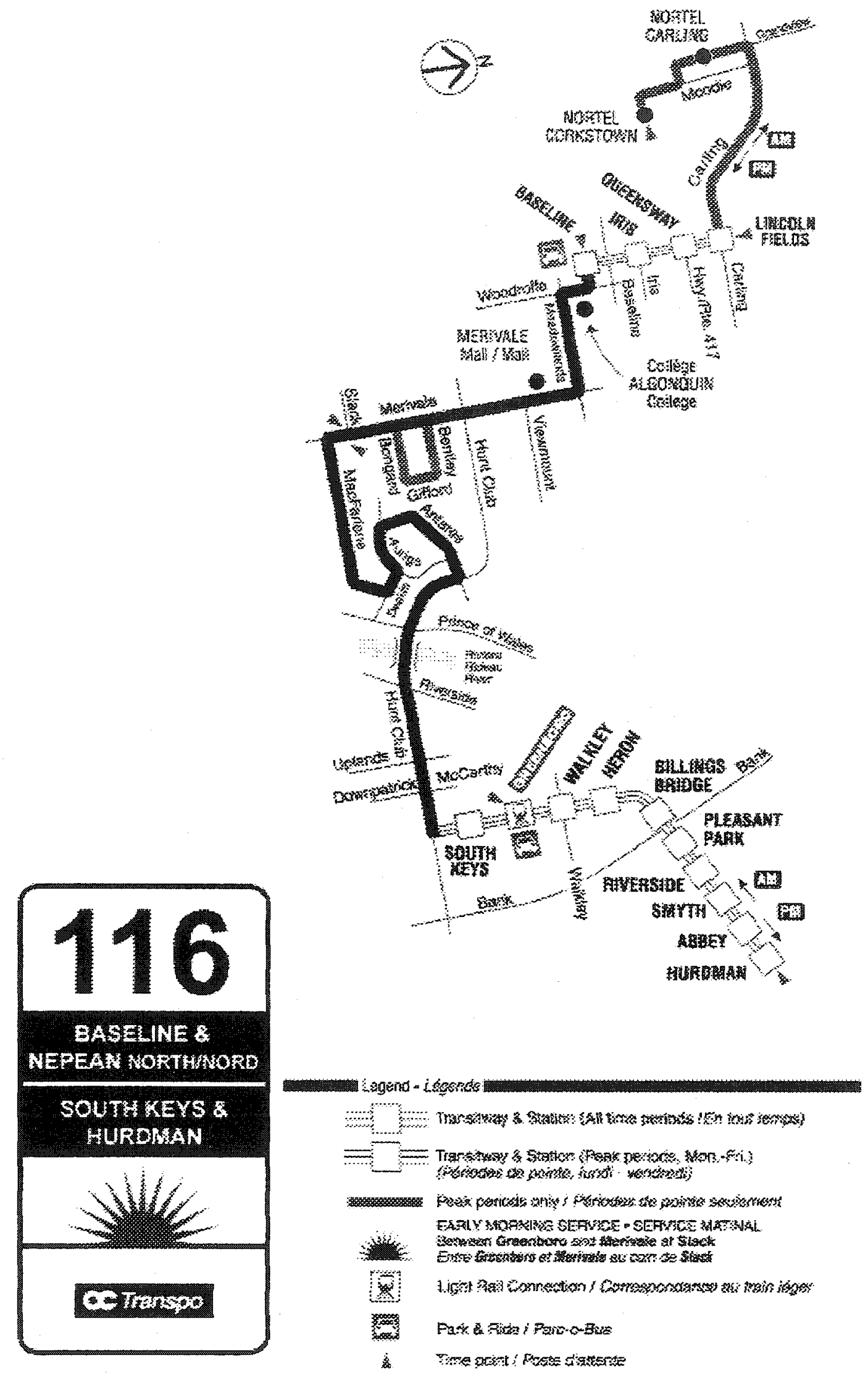



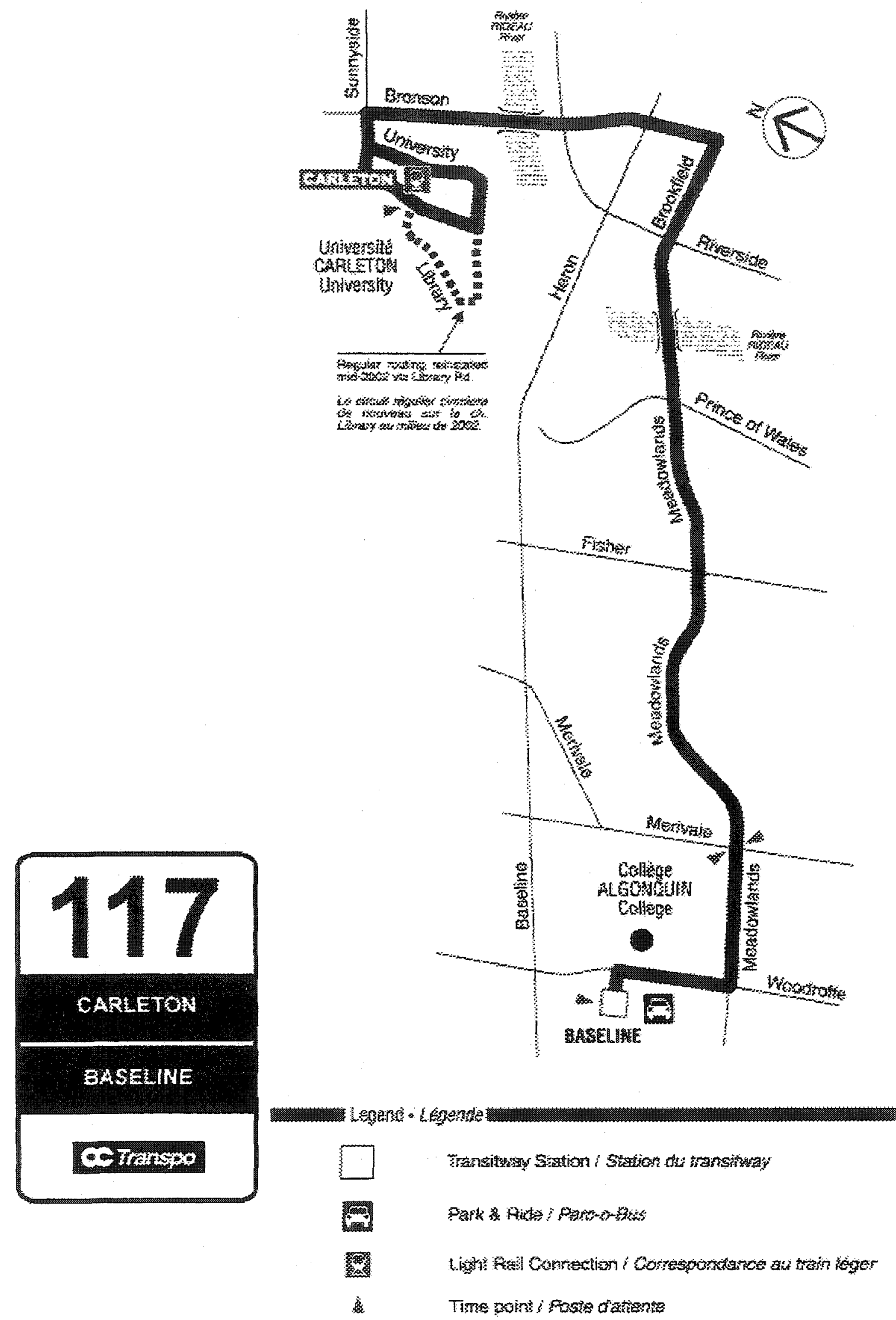


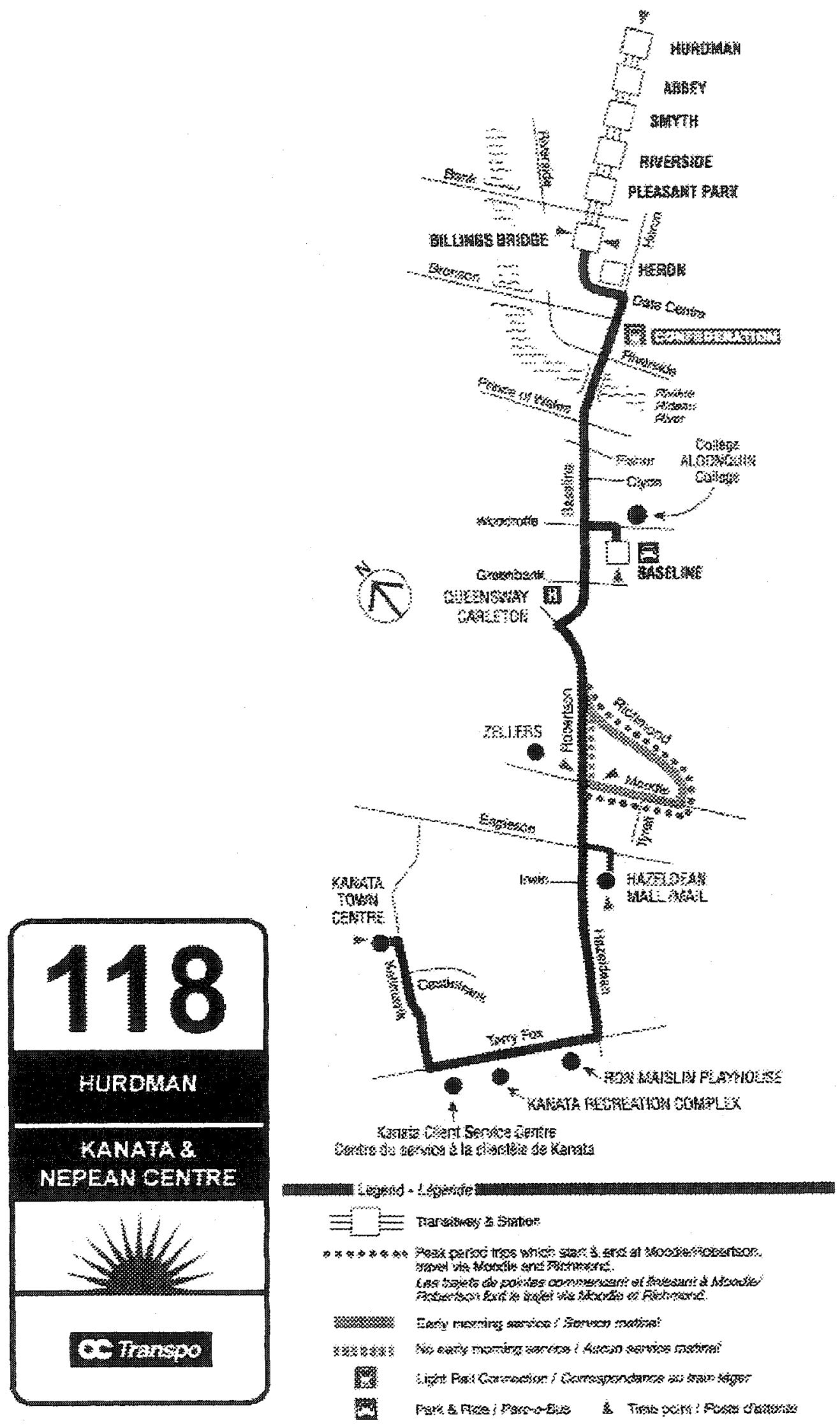




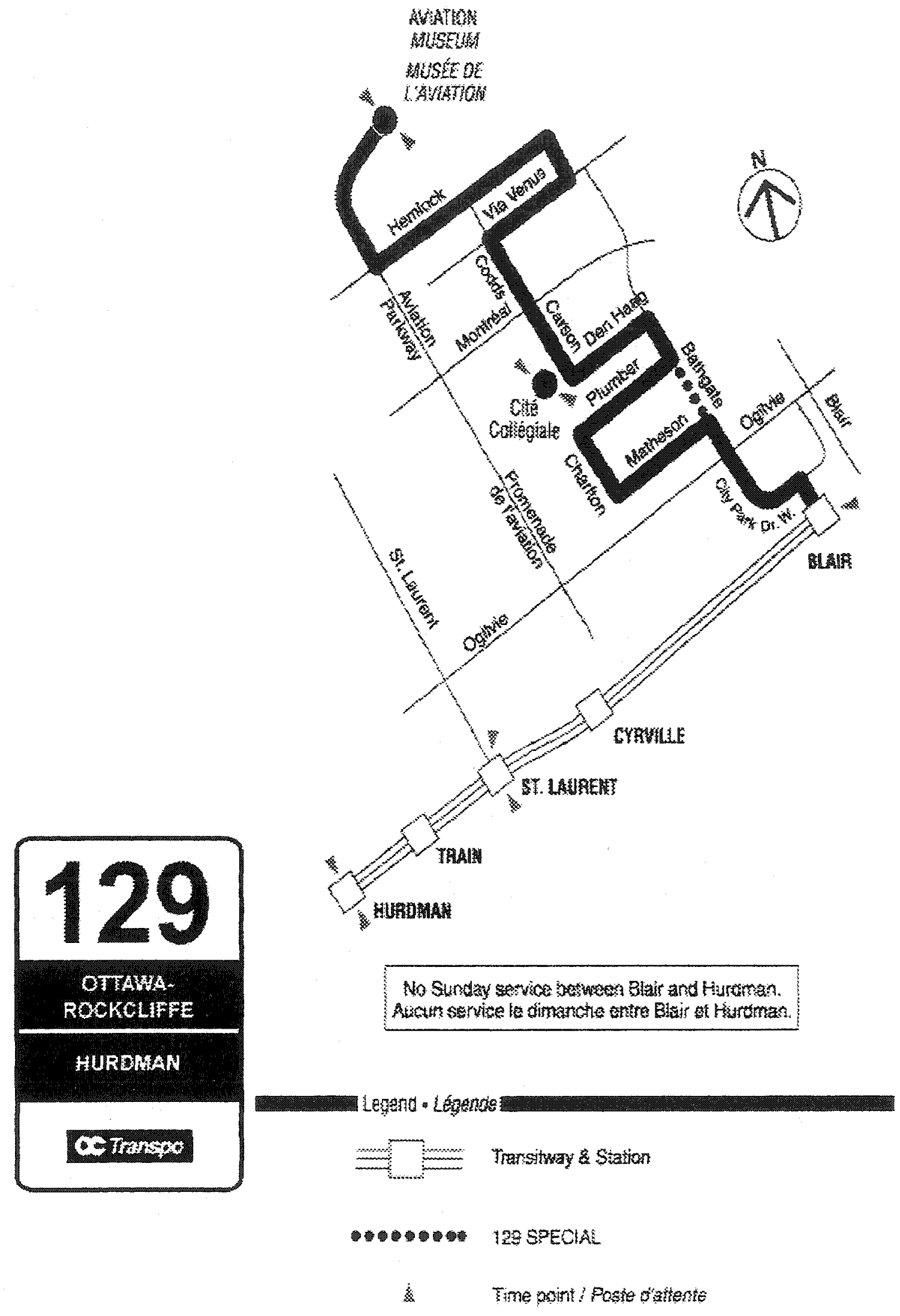




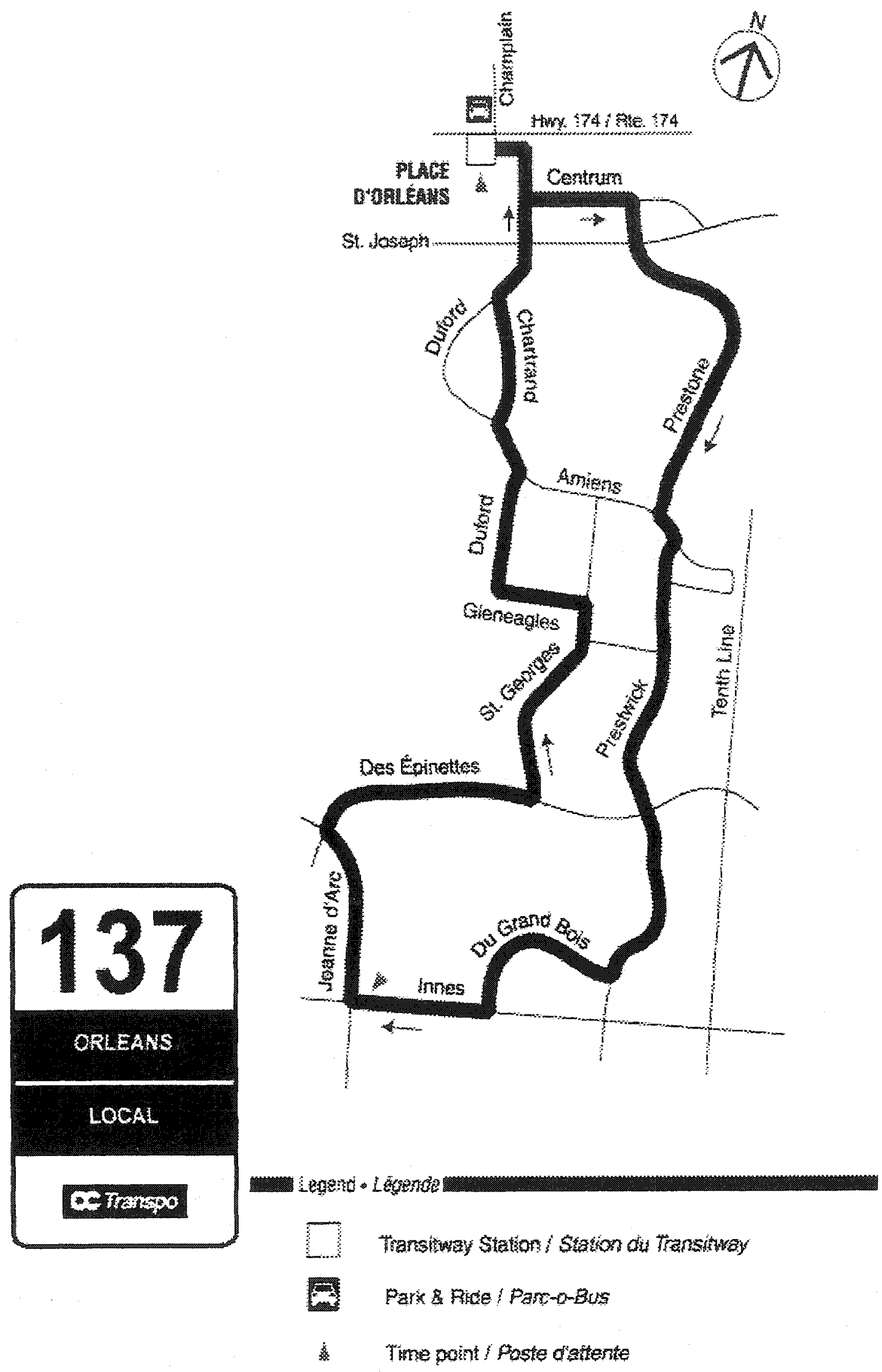




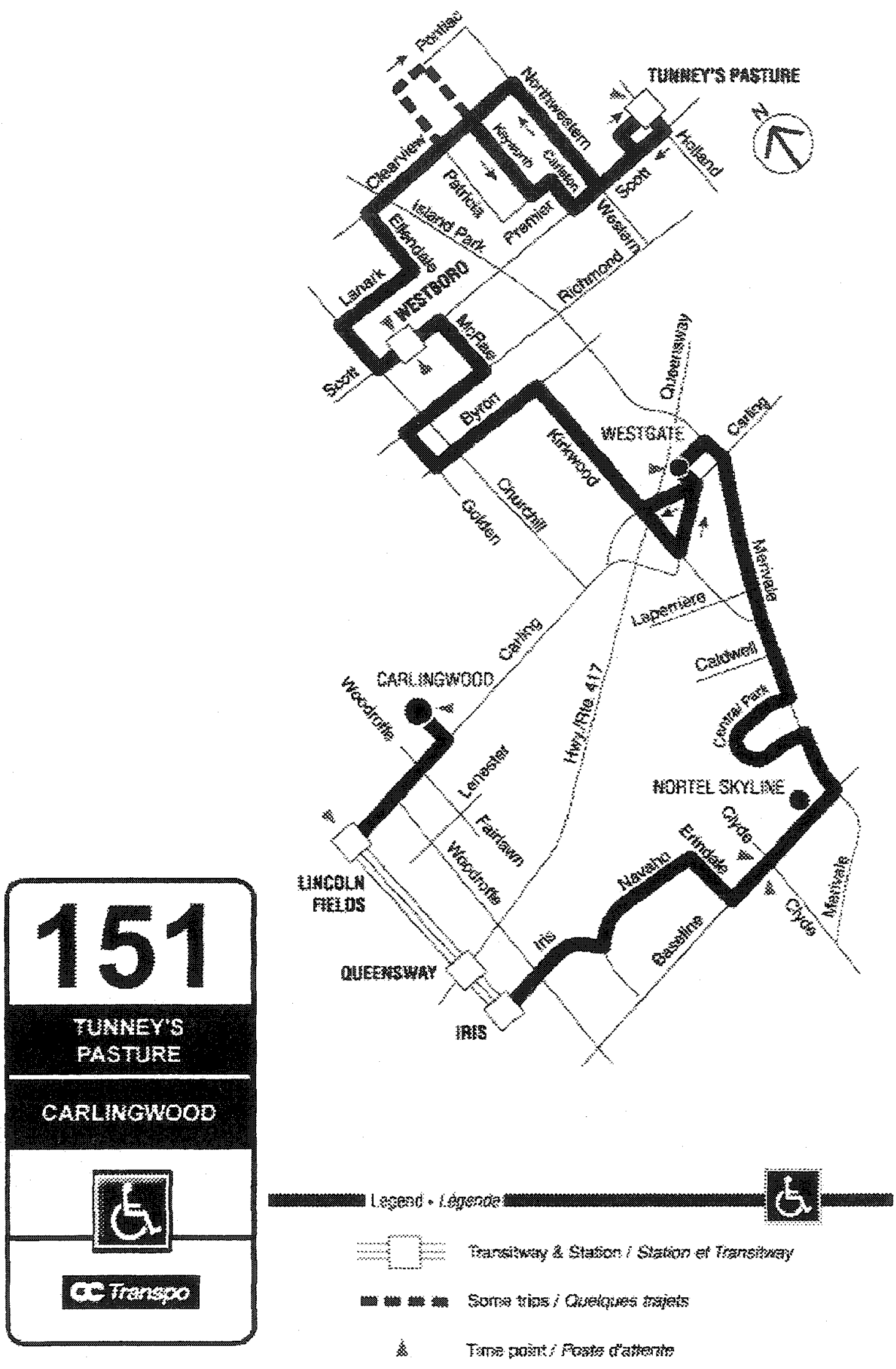




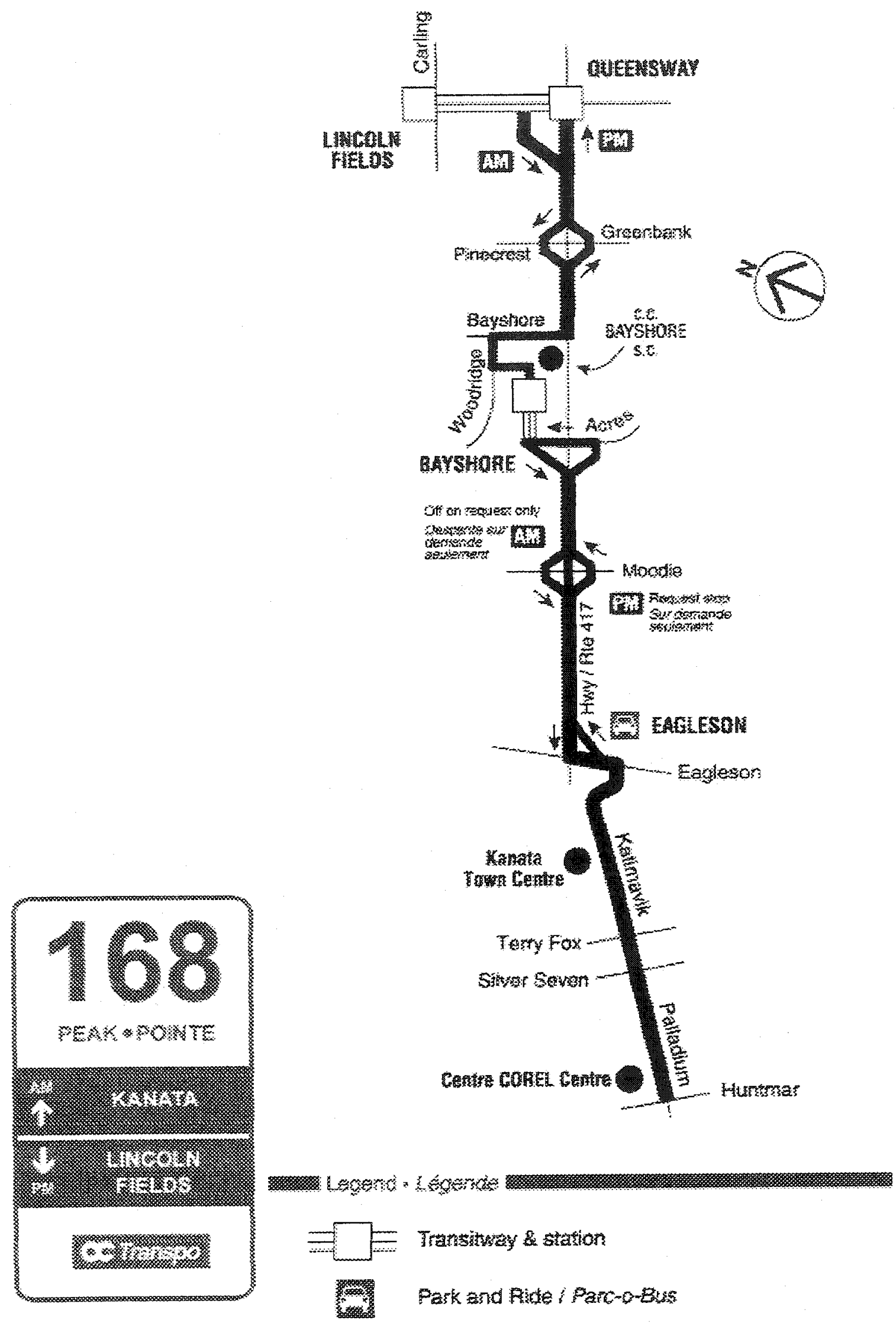




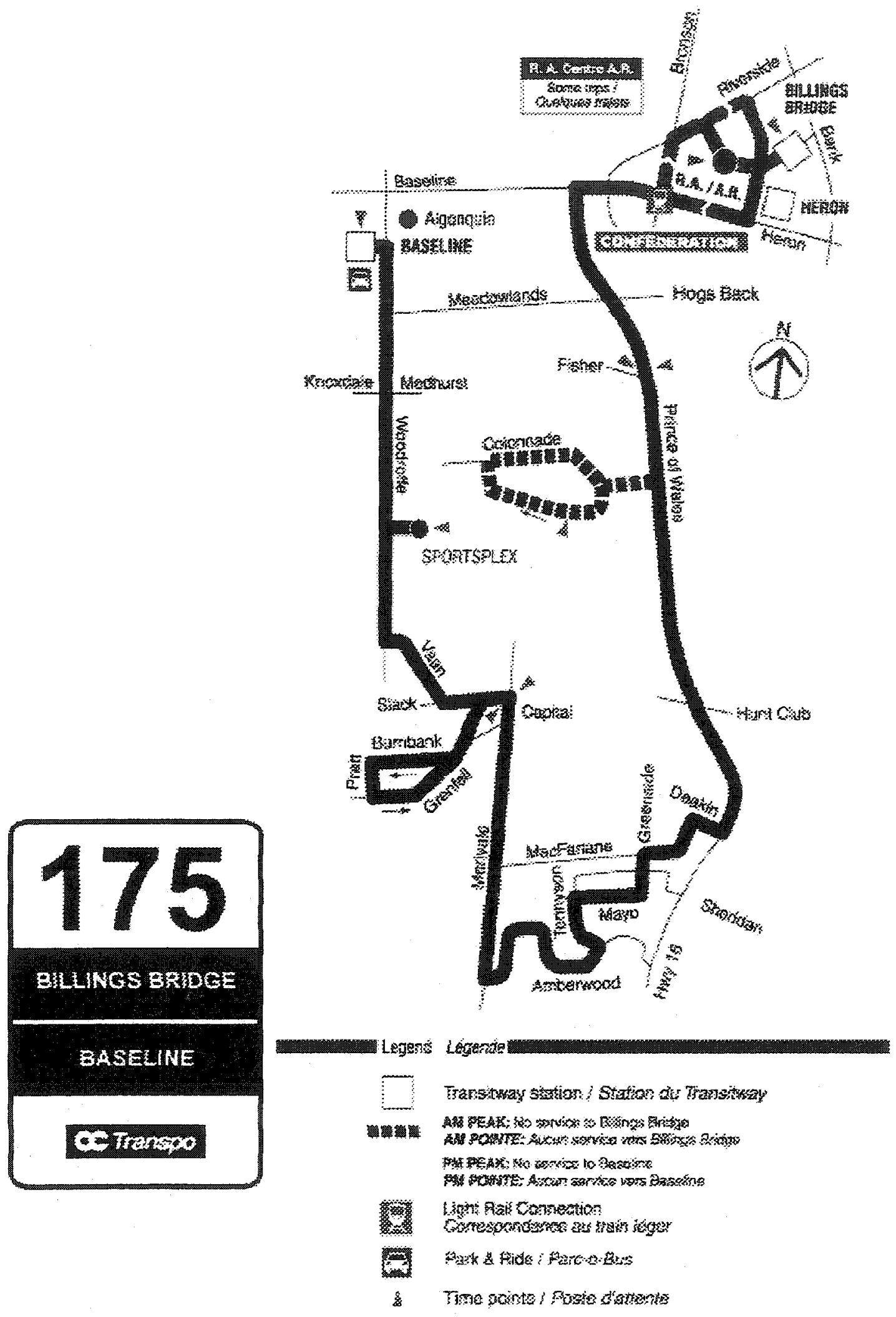




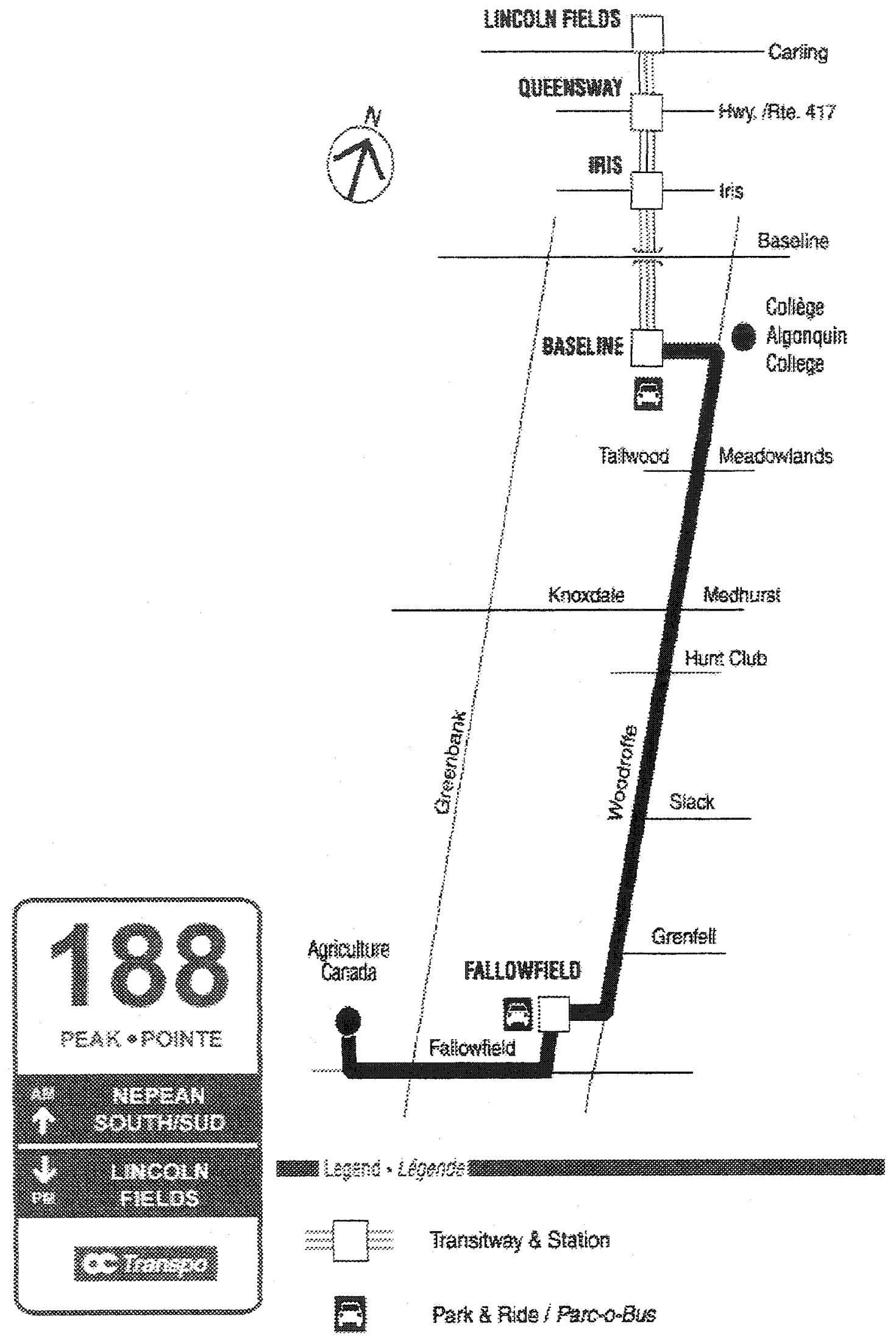




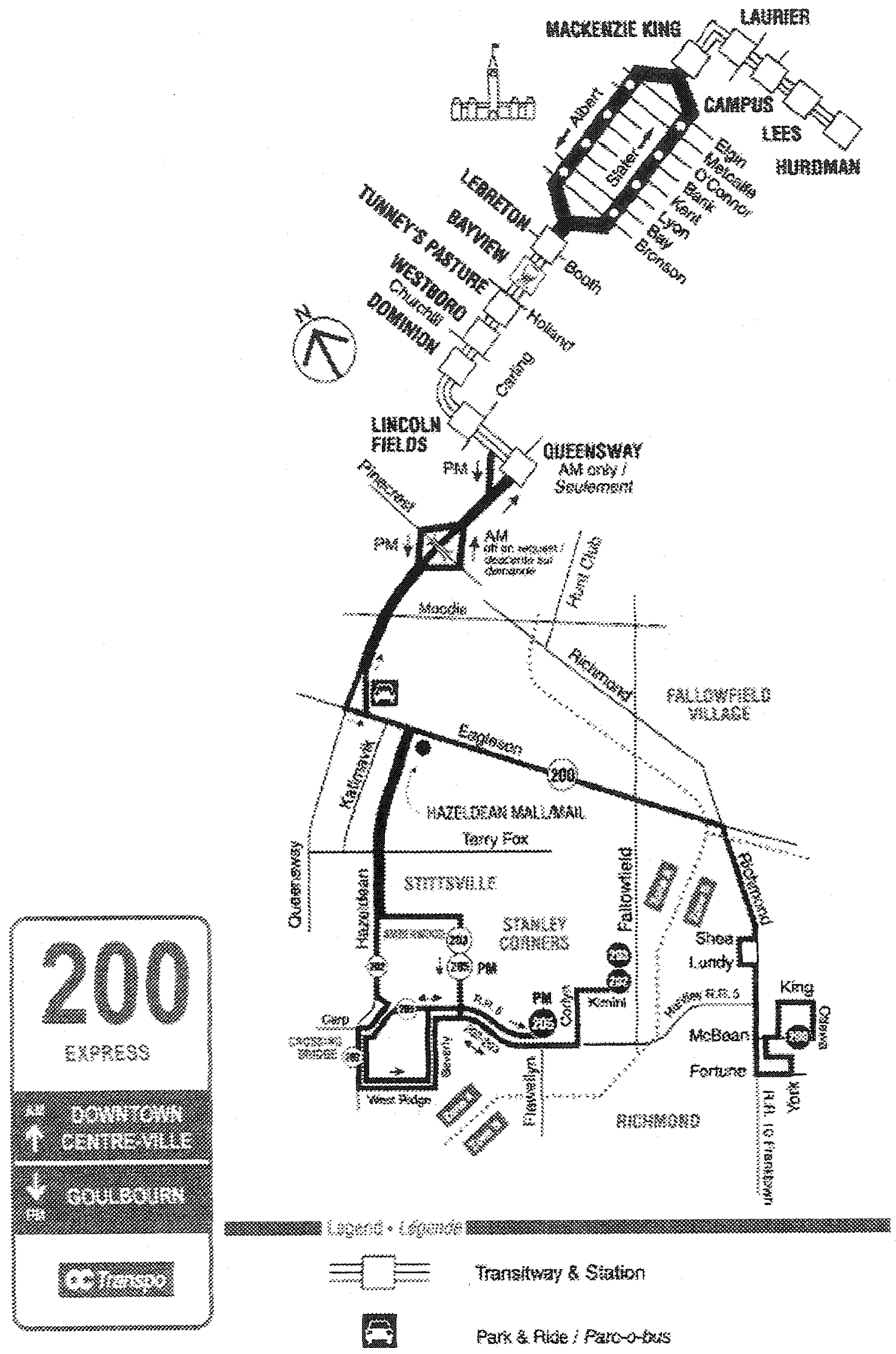



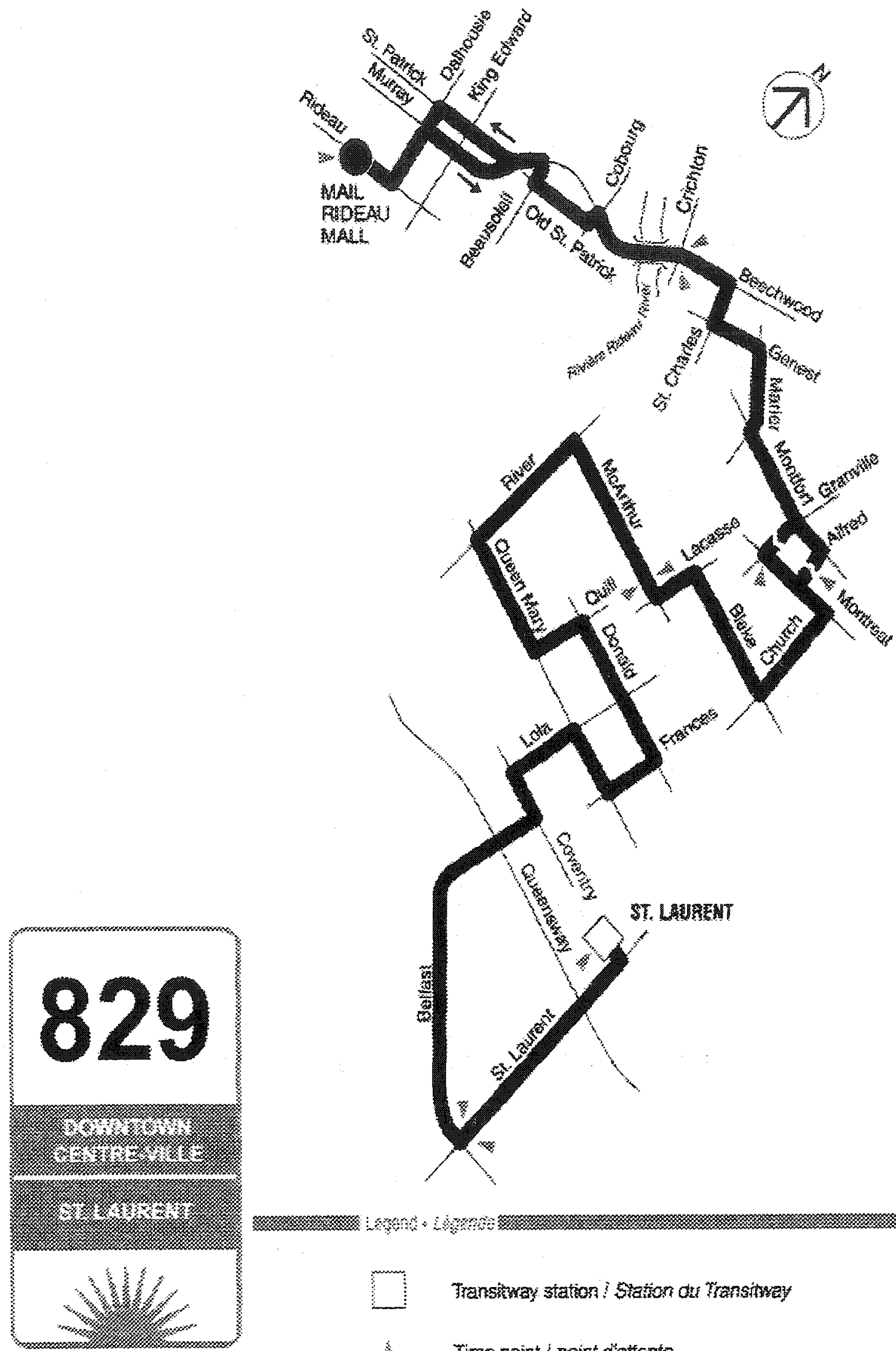

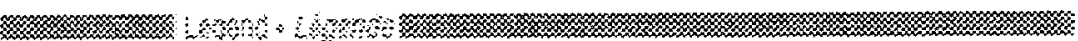

Transimay station $i$ Starion du Transiduay

2. Time point pount dattende 

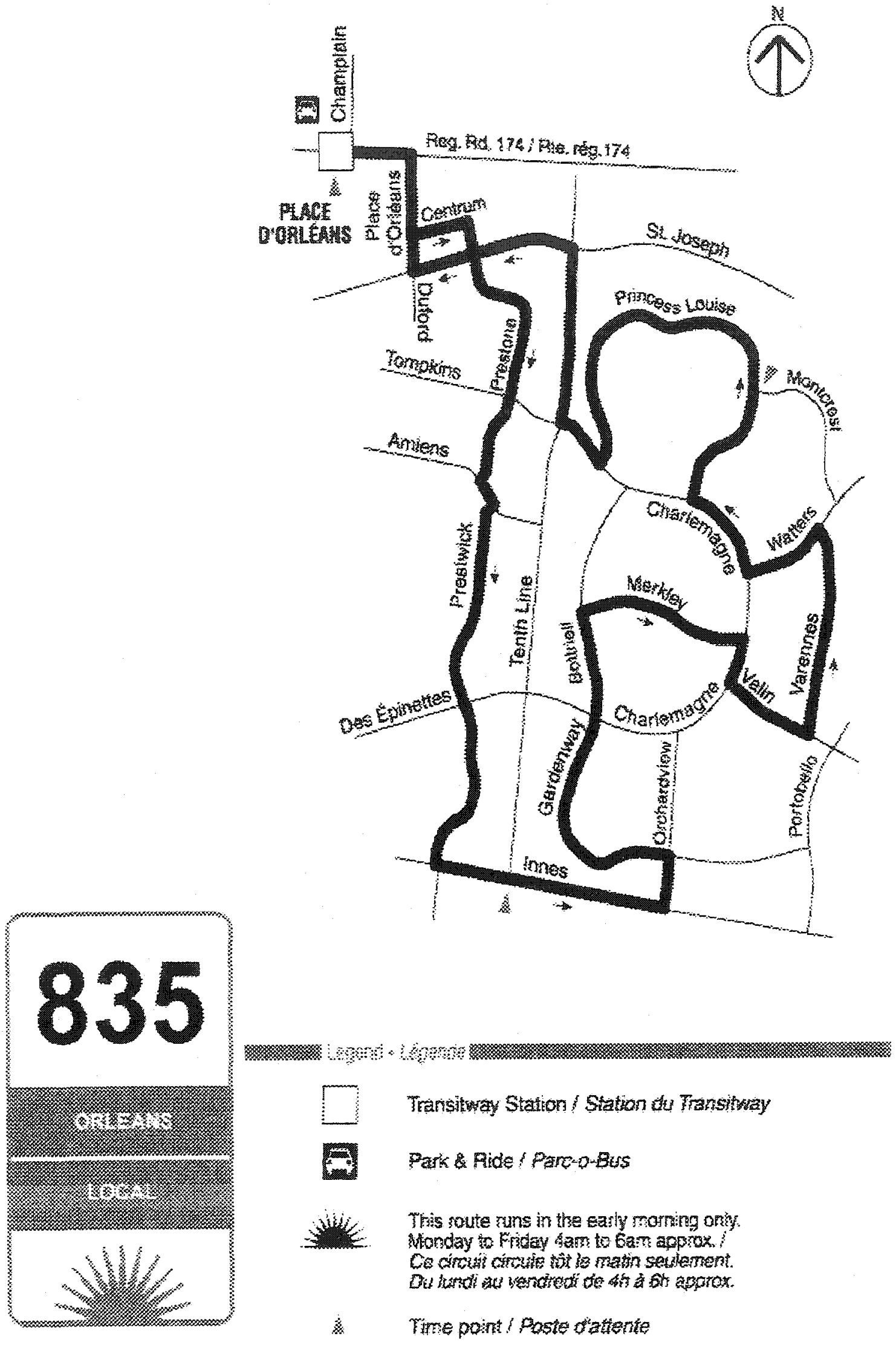

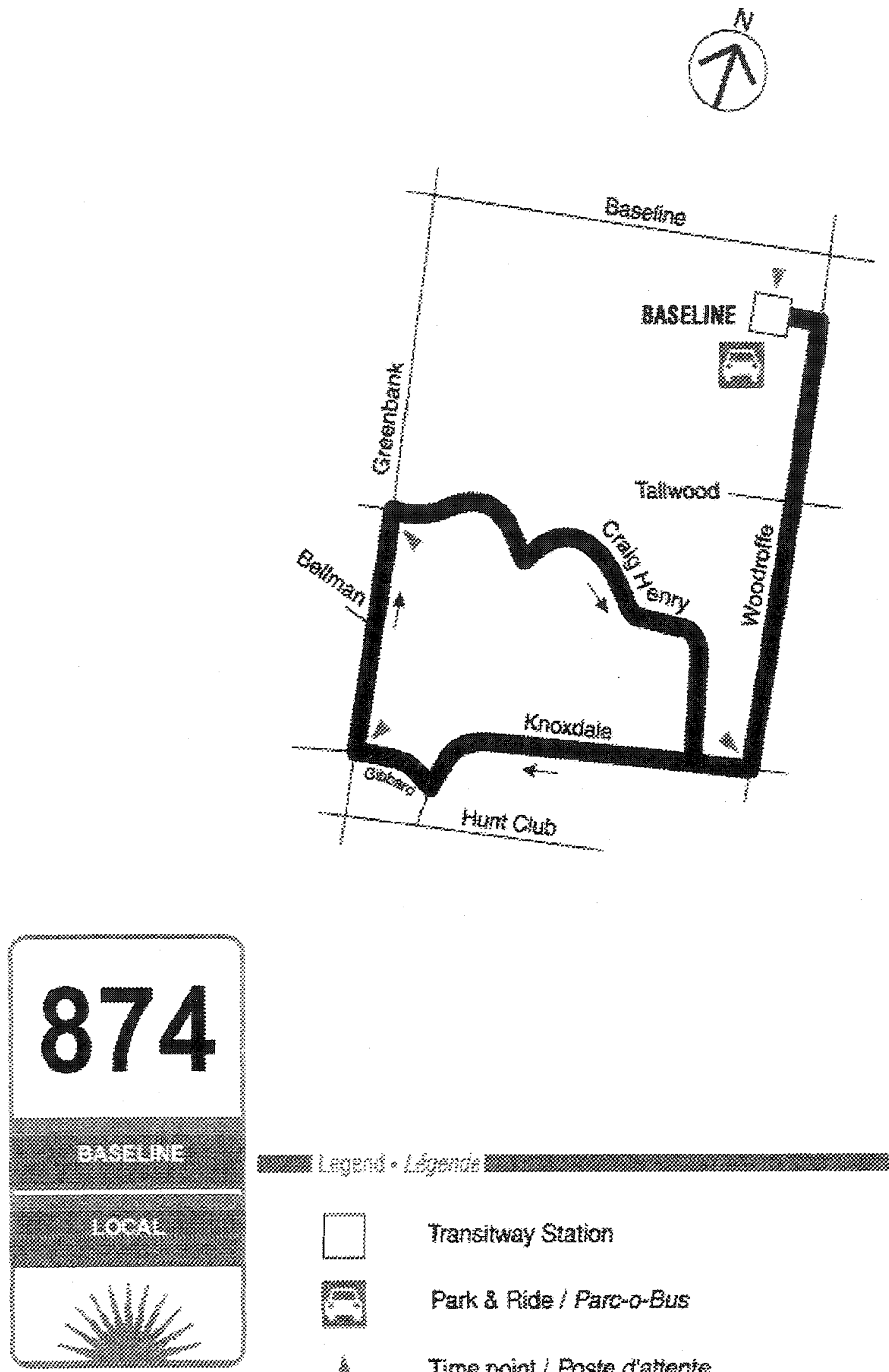

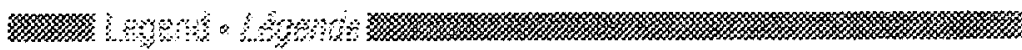

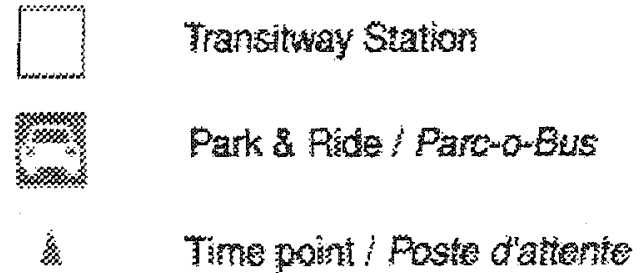


Appendix C

Avenue Coding 


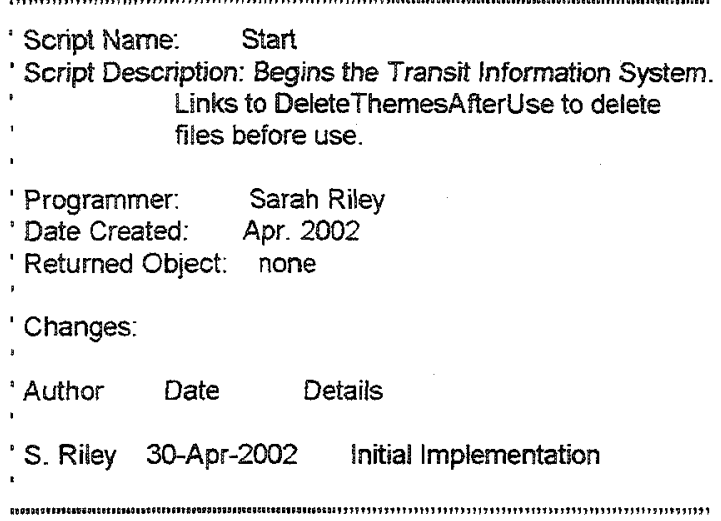

'delete themes, tables, graphics before use

av.run("DeleteThemesAfterUse", "n")

'starts the transit information system

av.FindDialog("Transitinfo").Open 


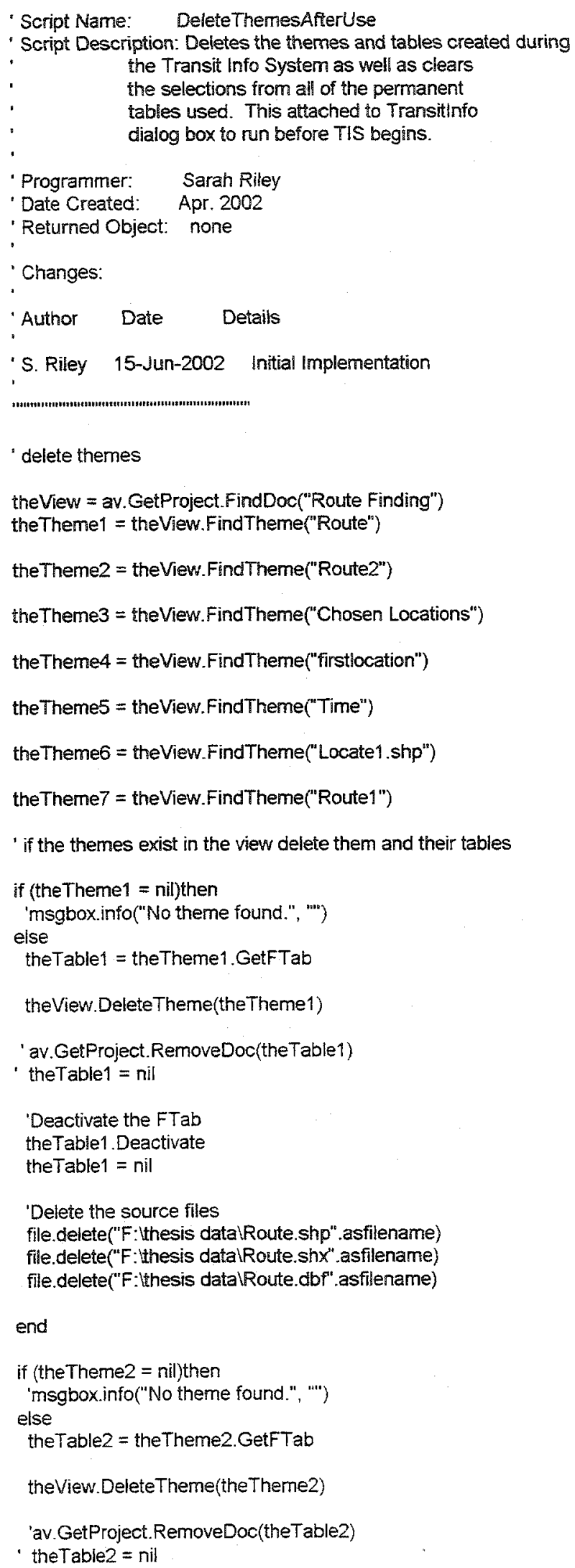




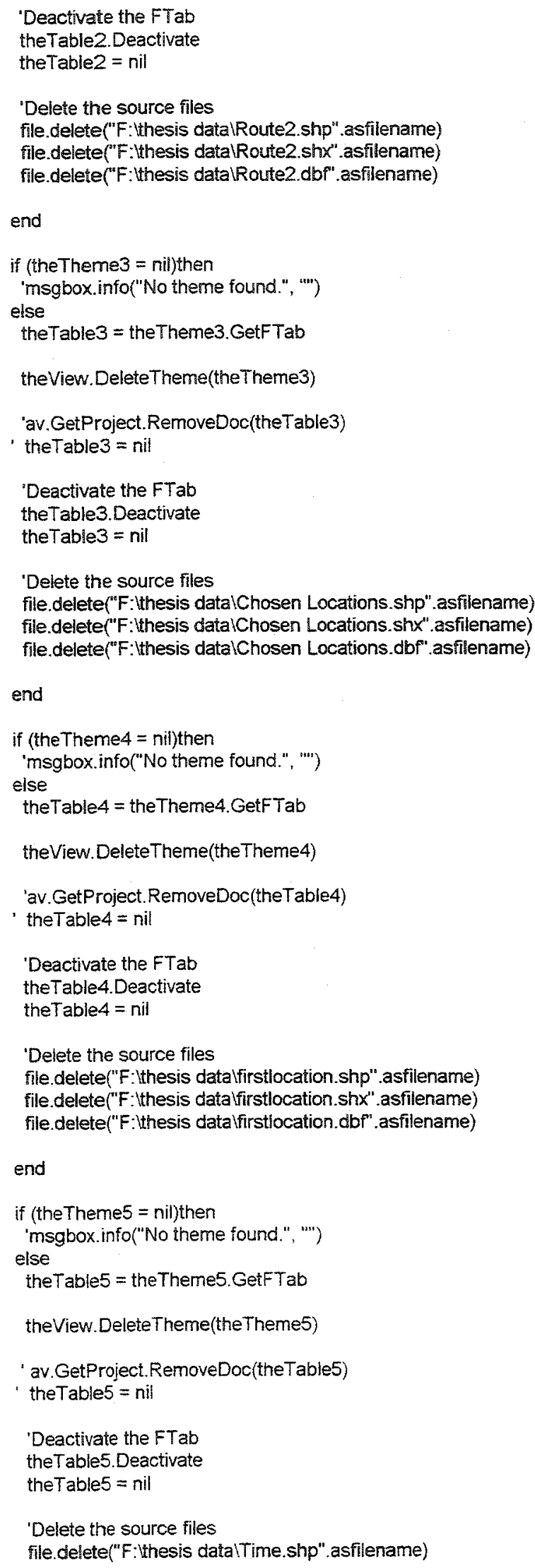


file.delele(" $F$ :thesis dataiTime.sh $x$ "asflename) file.delete(" $F$ : ithesis datalTime.dbf'.asflename)

end

if (theThemes $=$ nil)then

"msgbox.info("No theme found.", "'")

else

theTable 6 = the Theme6. GetFTab

theView.DeleteTheme(the Theme6)

'av. GetProject.RemoveDoc(the Table6)

- the Table6 $=$ nil

'Deactivate the FTab

the Table6. Deactivate

the Table6 $=\mathrm{nil}$

'Delete the source files

file.delete(" $F$ : thesis dataLocate1 sho".asfilename)

file.delete(" $F$ : thesis datalLocate 1. sh $x$ ". asfilename)

flle.delete("F: thesis datallocatel dbf".asfilename)

end

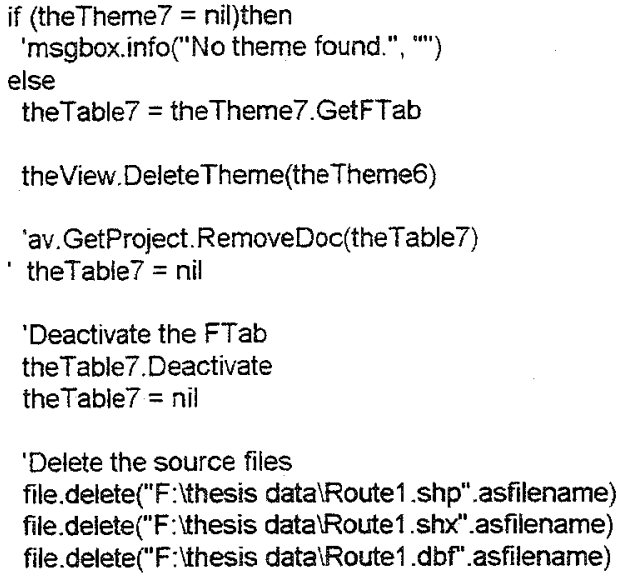

'After you delete the themes from the View, 'send the command:

theView.Invalidate

'This will update the view with no themes in it

'Also have to delete the tables that were created

'Remove the Table doc of the FTab if it exists

the Table8 $=$ av.FindDoc("near.dbf")

if (theTable8 = nil)then

'msgbox info("No table found.", "'")

eise

theVTab8 $=$ the Table8. GetVTab

av. GetProject.RemoveDoc(theTable8)

the Table $8=$ nil

'Deactivate the VTab 
theVTab8. Deactivate
theVTab8 = nil

'Delete the source files

file.delete(" $F$ : thesis datainear.dbf".asfilename)

end

theTable9 $=$ av.FindDoc("Results. dbf ")

if (theTable9 $=$ nil)then

"msgbox.info("No table found.", "')

else

theVTabs = the Table9.GetVTab

av.GetProject.RemoveDoc(theTable9)

the Tableg $=$ nil

'Deactivate the VTab

theVTab9. Deactivate

theVTab9 $=$ nil

'Delete the source files

file delete("F:thesis datalResutts.dbf".asfilename)

end

'Delete extra source files

file delete("F:thesis datalRoute1 shp".asfilename)

file.delete(" $F$ :thesis datalRoute1.shx".asfilename)

file.delete("F: thesis datalRoute1.dbf".asfilename)

file.delete("F: Ithesis datalRoute2.shp".asfilename)

file.delete("F:thesis datalRoute2.shx".asfilename)

file.delete("F:thesis datalRoute2.dbf".asfilename)

file.delete("F:ithesis datalRoute3.shp".asfilename)

file.delete(" $F$ :thesis datalRoute3.sh $x$ ".asfilename)

file.delete("F:thesis data\Route3.dbf".asfilename)

file.delete("F:Ithesis datalTime1.shp".asfilename)

file.delete("F:thesis dataiTime1.shx" asfilename)

file.delete(" $F$ : thesis datalTime1.dbf".asfilename)

file.delete("F:thesis datalTime2.shp".asfilename)

file.delete(" $F$ : thesis datalTime2.sh $x$ ". asfilename)

file.delete("F: thesis dataiTime2.dbf".asfilename)

'delete the temp script file created

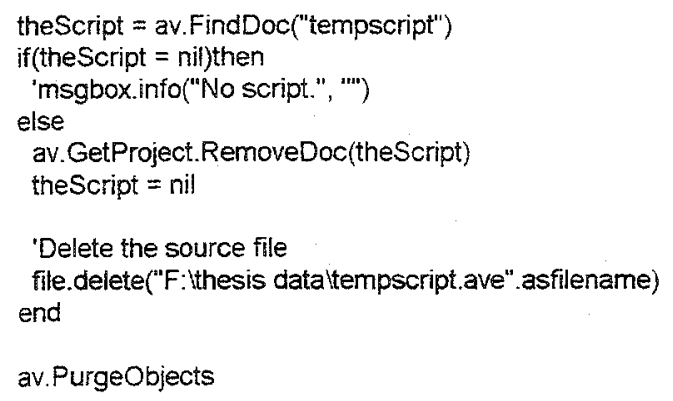

'Delete the source files

file.delete("F:thesis datal doff".asfilename)

theProject $=$ av. GetProject

the Docs $=$ theProject GetSelectedDocs 


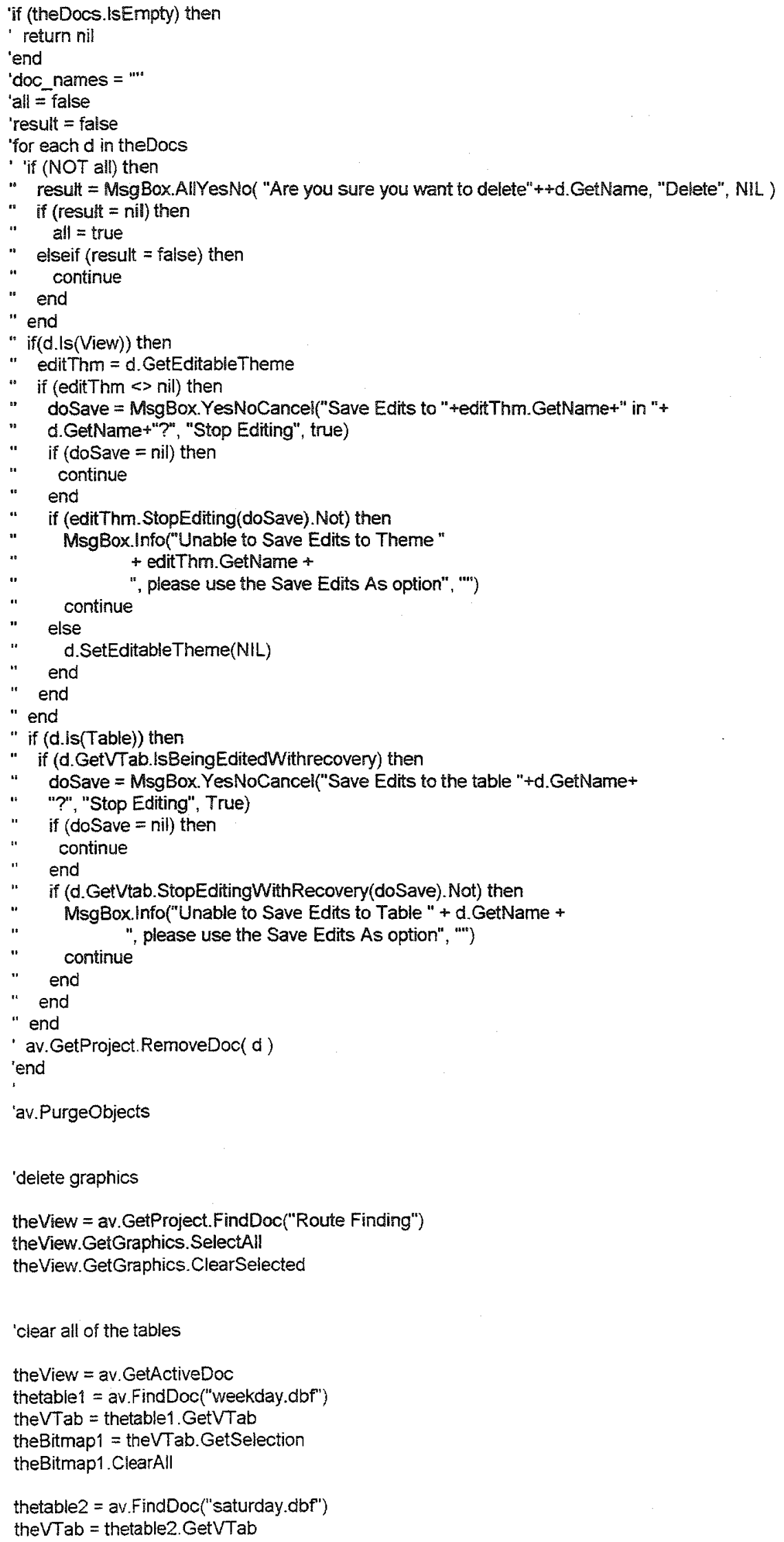


theBitmap2 = theVTab.GetSelection

theBitmap2.ClearAll

thetable $3=$ av.FindDoc("sunday.dbf")

theVTab = thetable3. GetVTab

theBitmap3 = theVTab.GetSelection

theBitmap3.ClearAll

theTherne1 = theView.FindTheme("Bus Stops")

theFTab = theTheme1. GetFTab

theBitmap4 = theFTab. GetSelection

theBitmap4.ClearAll 


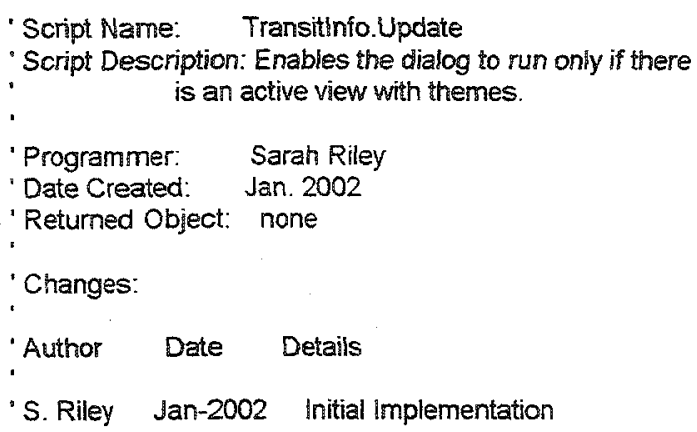


- Script Name: Transithfo.schedule lbt. Update ' Script Description: Enables the schedule button.

'Programmer: Sarah Riley

' Date Created: Apr. 2002

'Returned Object: none

'Changes:

'Author Date Details

'S. Riley 16-Apr-2002 initial implementation

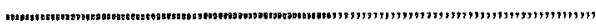

SELF.SetEnabled(true) 
- Script Name: Transitinío.schedule lbt.Click

'Script Description: Script runs when the schedule button is pressed. It calls to open the userinterface 2 dialog.

' Programmer: Sarah Riley

' Date Created: Apr. 2002

' Refurned Object: none

'Changes:

'Author Date Details

'S. Riley 16-Apr-2002 Initial Implementation

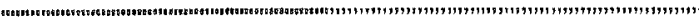

'SELF refers to the button

SELF.SetEnabled(true)

'closes the dialog

SELF.GetDialog.Close

'run the userinterface 2 dialog

av.FindDialog("userinterface2"). Open 
' Script Name: Transitinfo.proceed_Ibt.Update

'Script Description: Enables the proceed button in the dialog.

- Programmer: Sarah Riley

' Date Created: Jan. 2002

' Returned Object: none

' Changes:

'Author Date Details

'S. Riley Jan-2002 Initial Implementation

\section{SELF.SetEnabled(true)}


Script Name: Transitlnfo.proceed_ibt.Click

' Script Description: Script runs when the proceed button is pressed. It calls to open the userinterface dialog.

'Programmer: Sarah Riley

Date Created: Jan 2002

'Returned Object: none

'Changes:

'Author Date Details

'S. Riley Jan-2002 initial Implementation

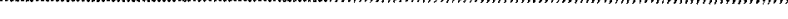

'SELF refers to the button

SELF.SetEnabled(true)

'closes the dialog

SELF.GetDialog. Close

'run the userinterface dialog

av. FindDialog("userinterface").Open 


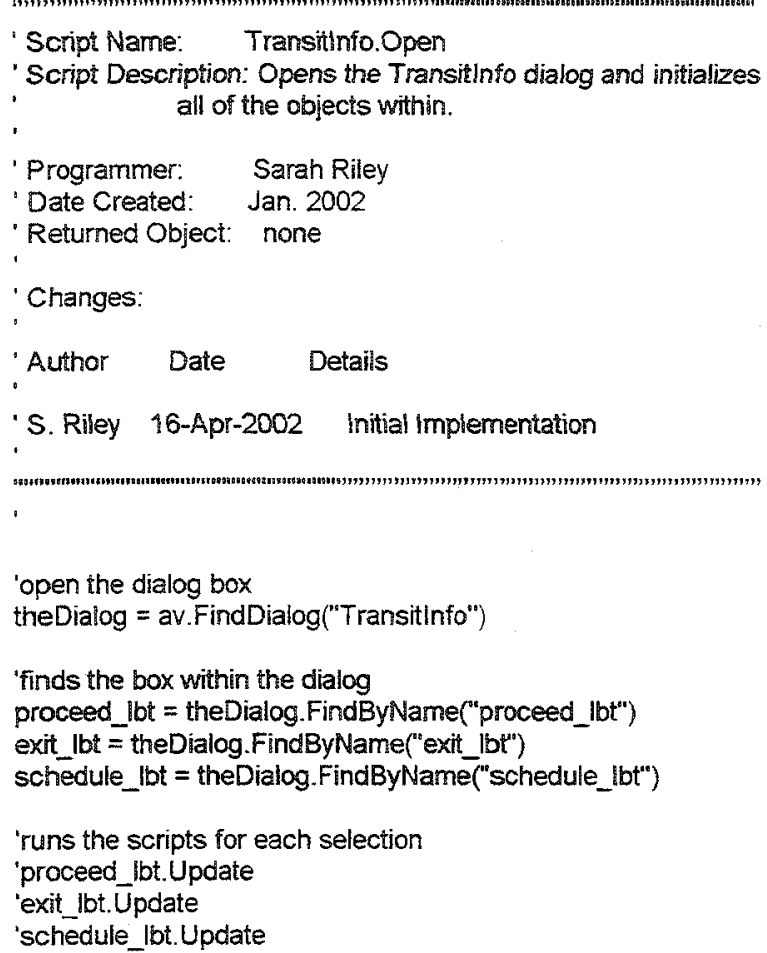




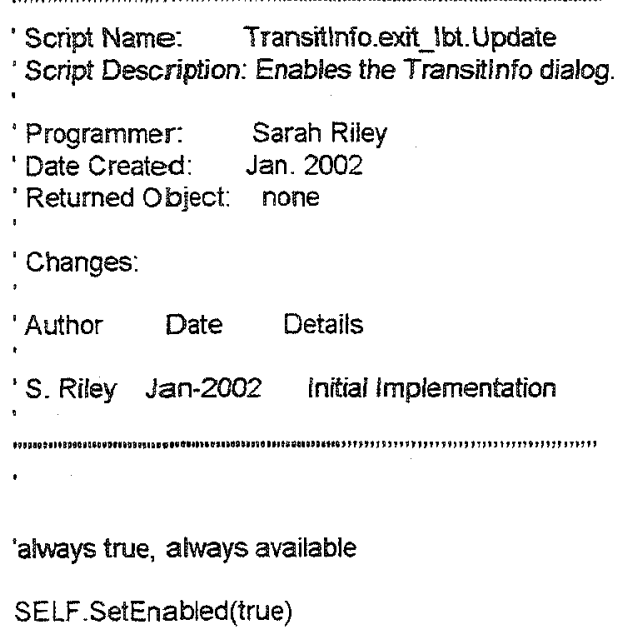


- Script Name: Transitinfo.exit_lbt.Click

- Script Description: Script runs when the exit button is pressed. It closes the dialog and makes sure the user really wants to.

'Programmer: Sarah Riley

- Date Created: Jan. 2002

' Returned Object: none

' Changes:

'Author Date Details

'S. Riley Jan-2002 Initial Implementation

'SELF refers to the button

'closes the dialog

if(msgbox.yesno("Are you sure?", "Quit?", false)) then

self.GetDialog.Close

quit $=$ true

end

'SELF.GetDialog.Close 


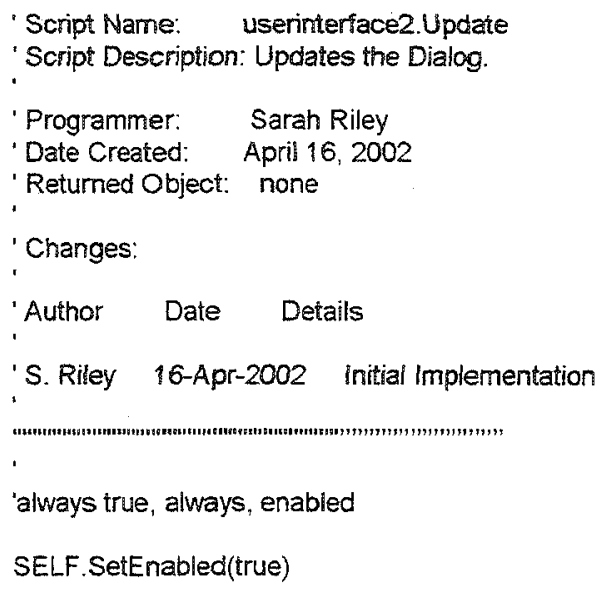


- Script Name: userinterface2.self.Update

' Script Description: Broadcast the update events.

- Programmer: Sarah Riley

' Date Created: 16-Apr. 2002

- Returned Object: none

' Changes:

*Author Date Details

S. Riley 16-Apr-2002 initial implementation

"

SELF.BroadcastUpdate 
- Script Name: userinteríace2 select ibtupdate

' Script Description: Makes sure the select button is always available for selection.

- Programmer: Sarah Riley

- Date Created: Apr. 16, 2002

'Returned Object: none

' Changes:

- Author Date Details

'S. Riley 16-Apr-2002 Initial Implementation

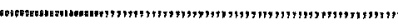

'ahways true, always avaiiable

SELF.SetEnabled(true) 


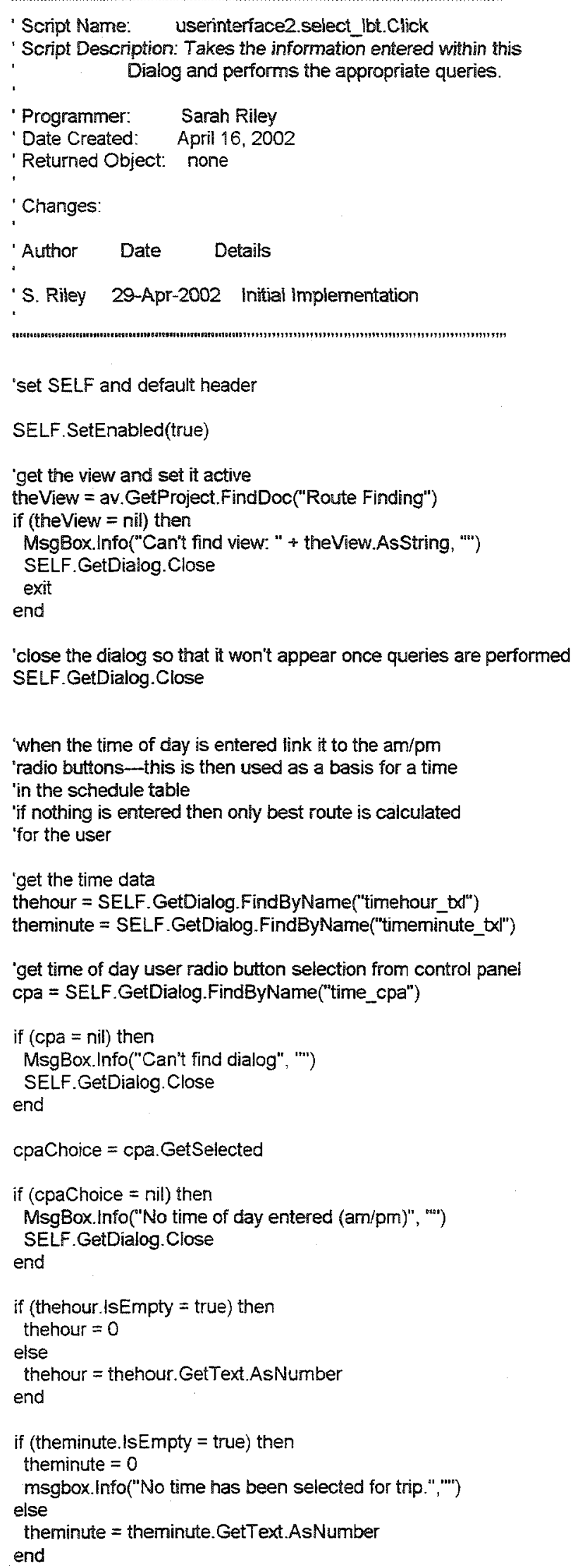




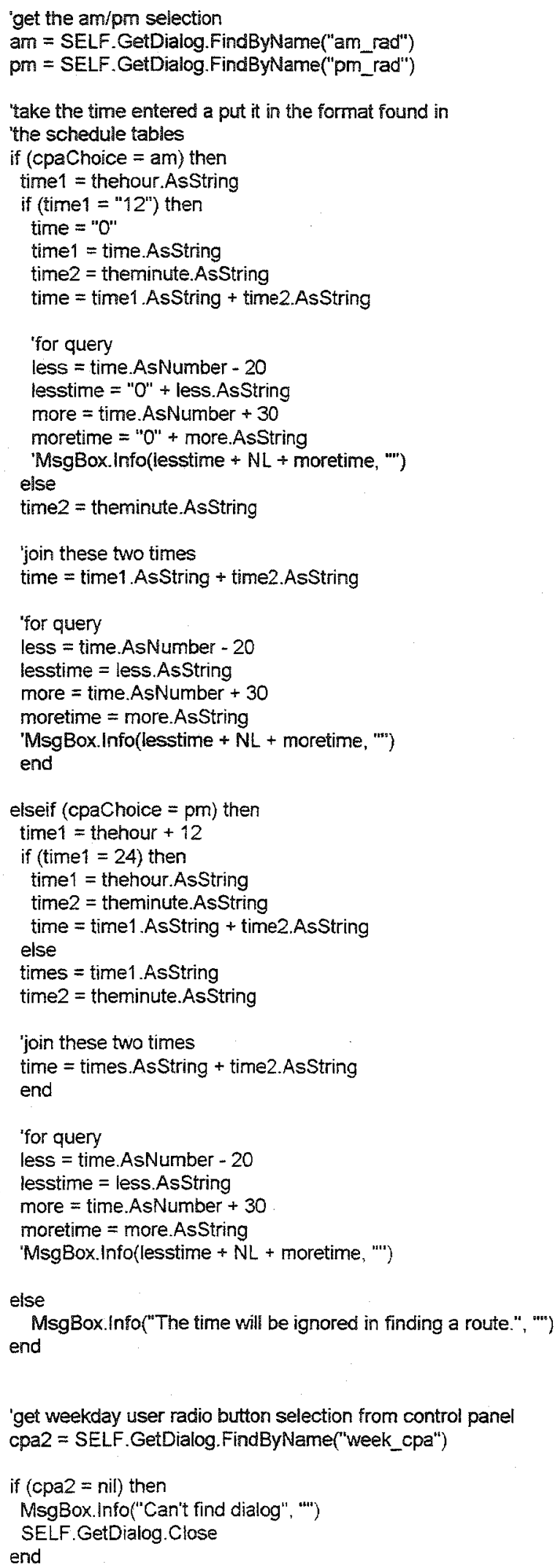




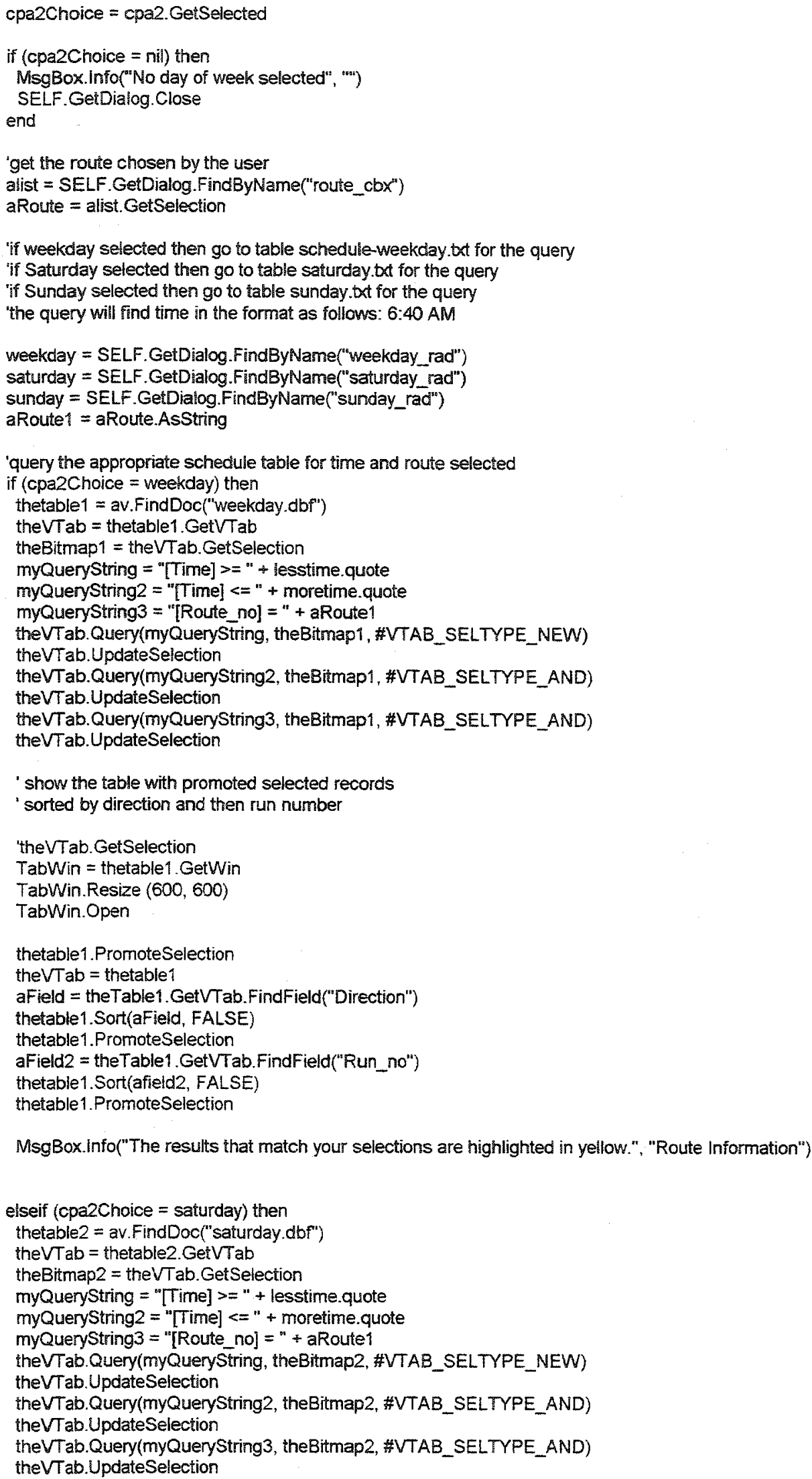




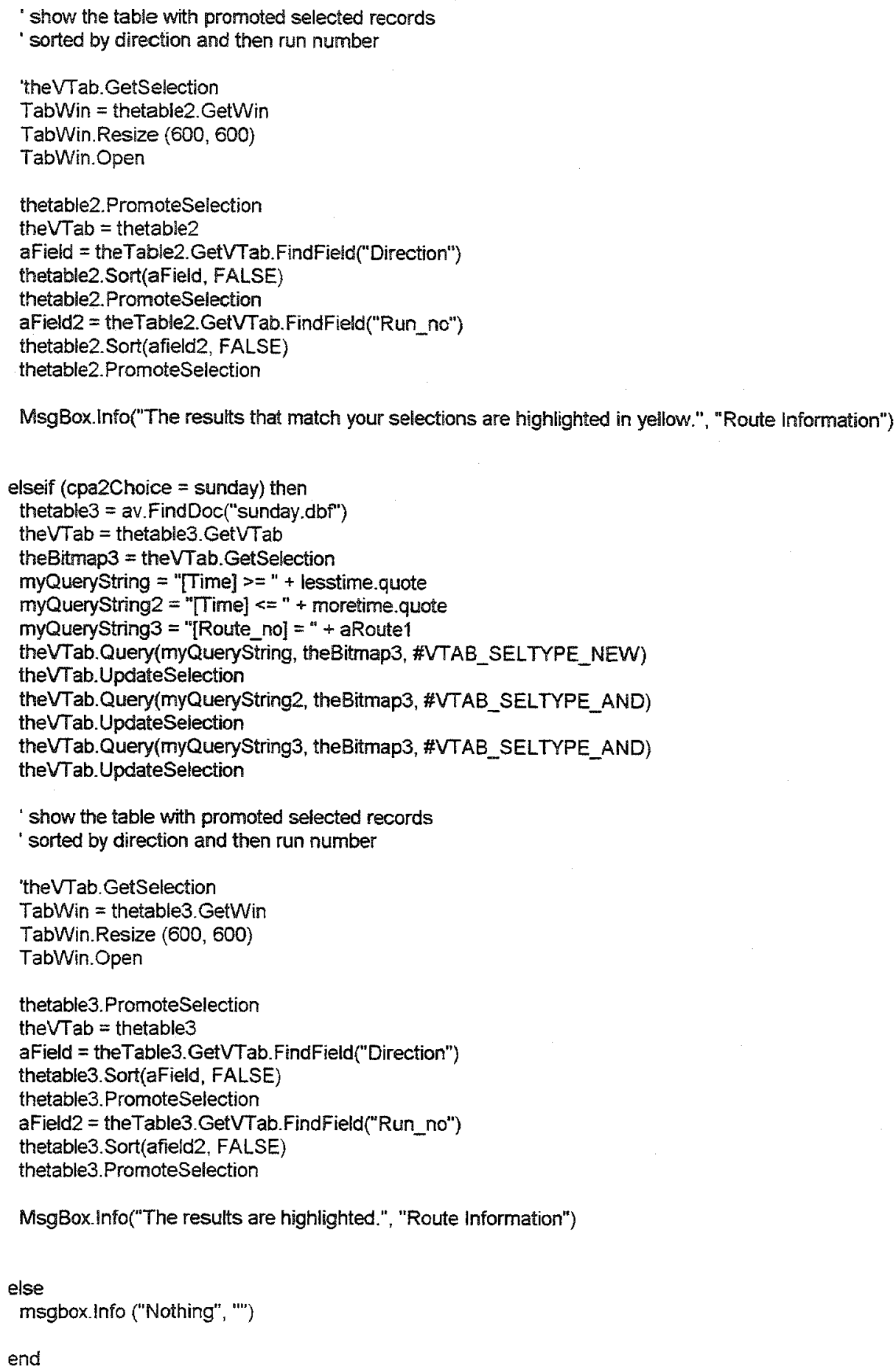




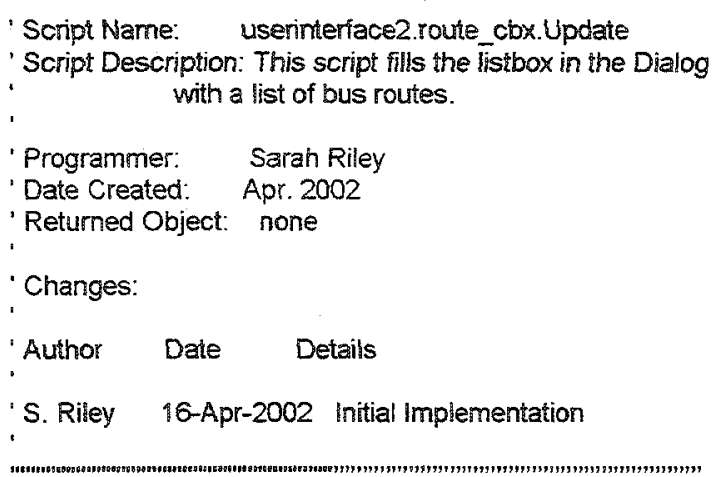

' Returned Object: none

- Changes:

- Author Dale Details

S. Riley 16-Apr-2002 Initial Implementation

".

'create a list of numbers

alist $=\{3,7,35,57,59,66,77,86,95,97,116,117,118,129,137,151,168,175,188,200,829,874\}$

Add the list contents to the listbox

self.DefineFromList(alist) 


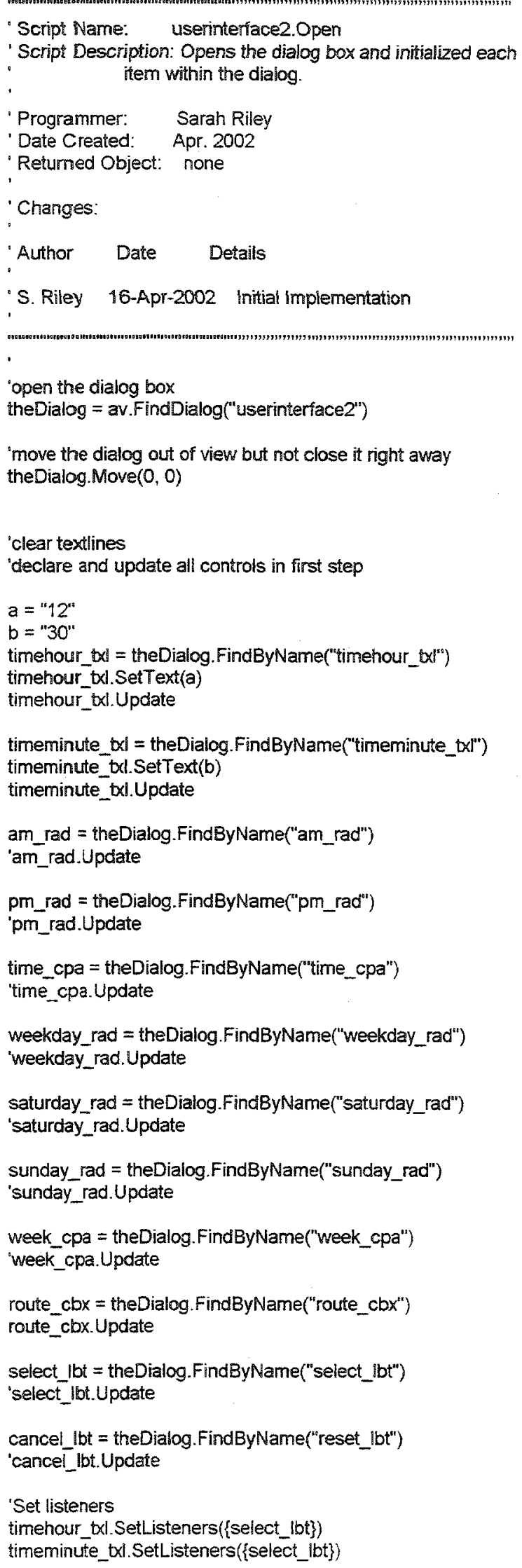


time_cpa.SetListeners(\{select_lbt\})

week_cpa.SetListeners (\{select_lbt\})

route_cbx.SetListeners(\{select_bt\})

'make one radio button active as a default

am_rad = theDialog.FindByName("am_rad")

am_rad.Select'(true)

weekday = theDialog.FindByName("weekday_rad")

weekday. Select'(true) 
Script Name: $\quad$ userinterface2.cancel lbt.Update

' Script Description: Allows the cancel button to always be available.

' Programmer: Sarah Riley

'Date Created: April 2002

' Returned Object: none

' Changes:

'Author Date Details

'S. Riley 16-Apr-2002 Initial Implementation

'always true, always available

SELF.SetEnabled(true) 
- Script Name: userinterface2.cancel ibt.Click

'Script Description: Closes the dialog when the cancel button is selected.

- Programmer: Sarah Riley

- Date Created: Apr. 2002

' Returned Object: none

' Changes:

- Author Date Details

'S. Riley 16-Apr-2002 Initial Implementation

'close the dialog box

SELF.GetDialog.Close 
'Script Name: . userinterface. Update

'Script Description:

'Programmer: Sarah Riley

' Date Created: Jan. 2002

'Returned Object: none

' Changes:

'Author Date Details

'S. Riley Jan-2002 Initial Implementation

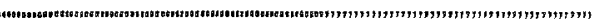

'always true, always, enabled

SELF.SetEnabled(true) 
- Scripl Name: userinterface.self.Update

- Script Description: Broadcasts the update events.

- Programmer: Sarah Riley

' Date Created: Jan. 2002

'Returned Object: none

'Changes:

'Author Date Details

'S. Riley Jan-2002 initial implementation

SELF.BroadcastUpdate 
' Script Name: userinterface.select ibt. Update

' Script Description: Enables the select button. Always active.

- Programmer: Sarah Riley

' Date Created: Jan. 2002

' Returned Object: none

' Changes:

' Author Date Details

'S. Riley Jan-2002 Initial Implementation

'always true, always available

SELF.SetEnabled(true) 


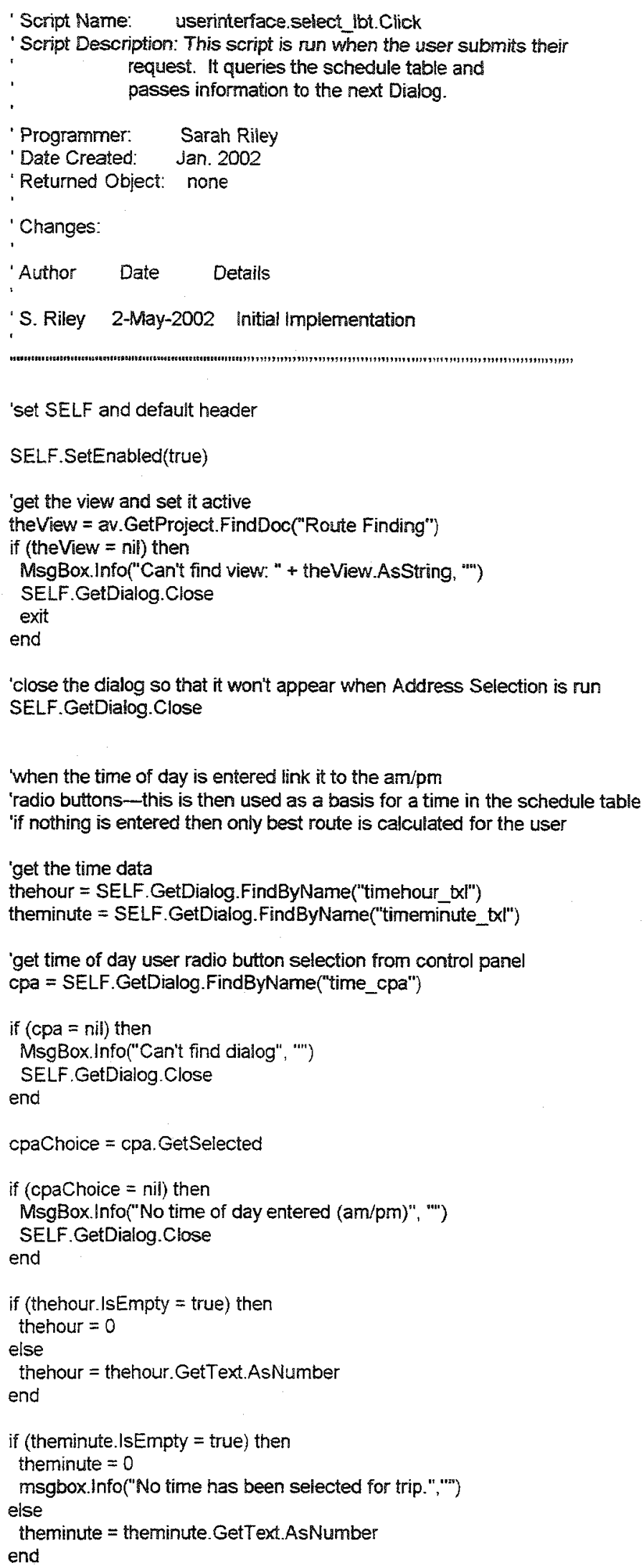




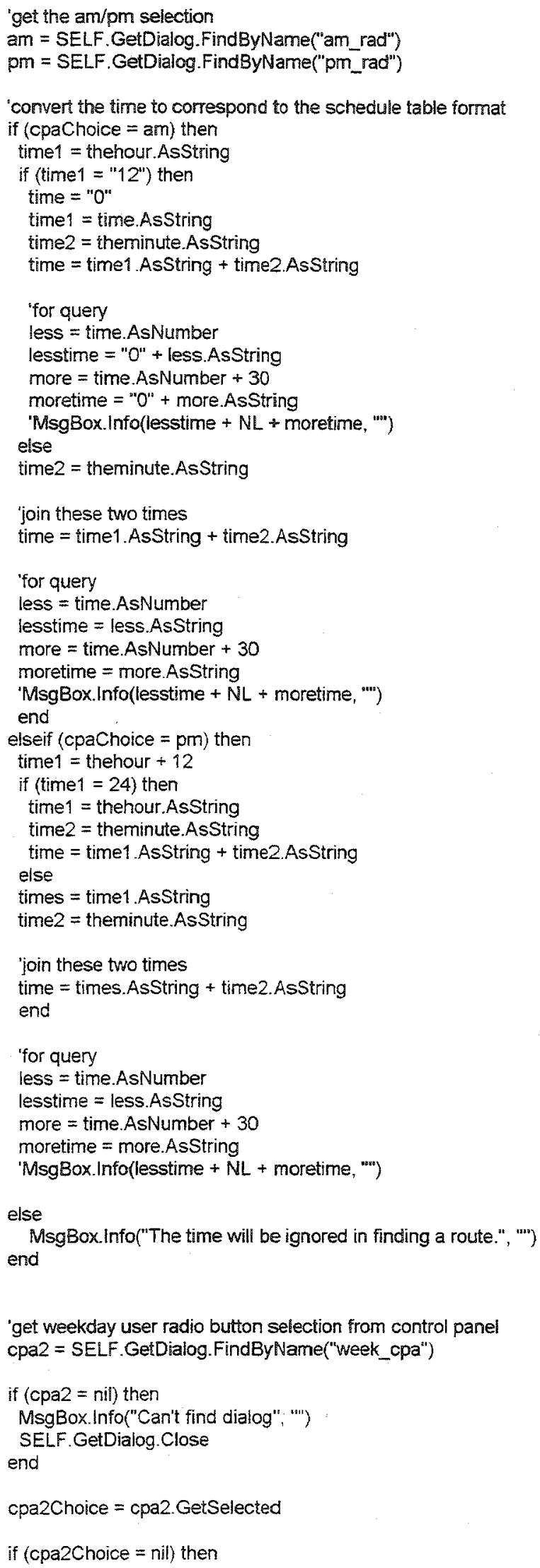




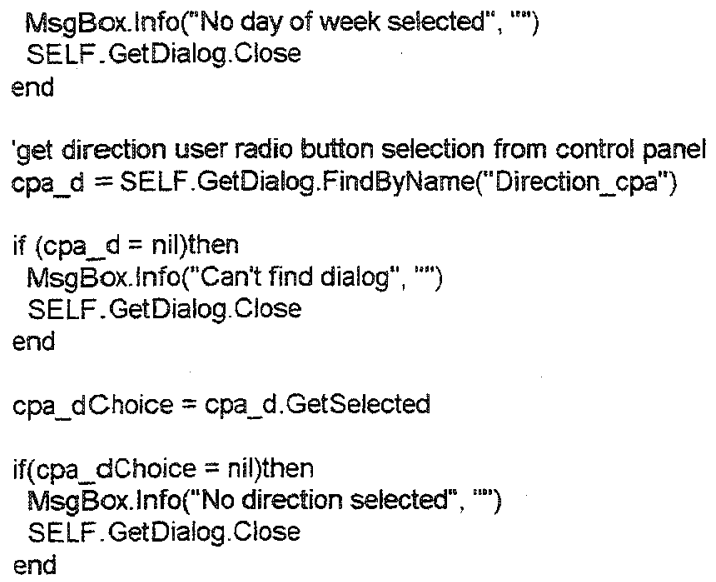

'if weekday selected then go to table weekday.dbf for the query 'if Saturday selected then go to table saturday.dbf for the query 'if Sunday selected then go to table sunday.dbf for the query 'the query will find time in the format as follows: 6:40 AM

'in this step, the direction of travel is also selected from the tables

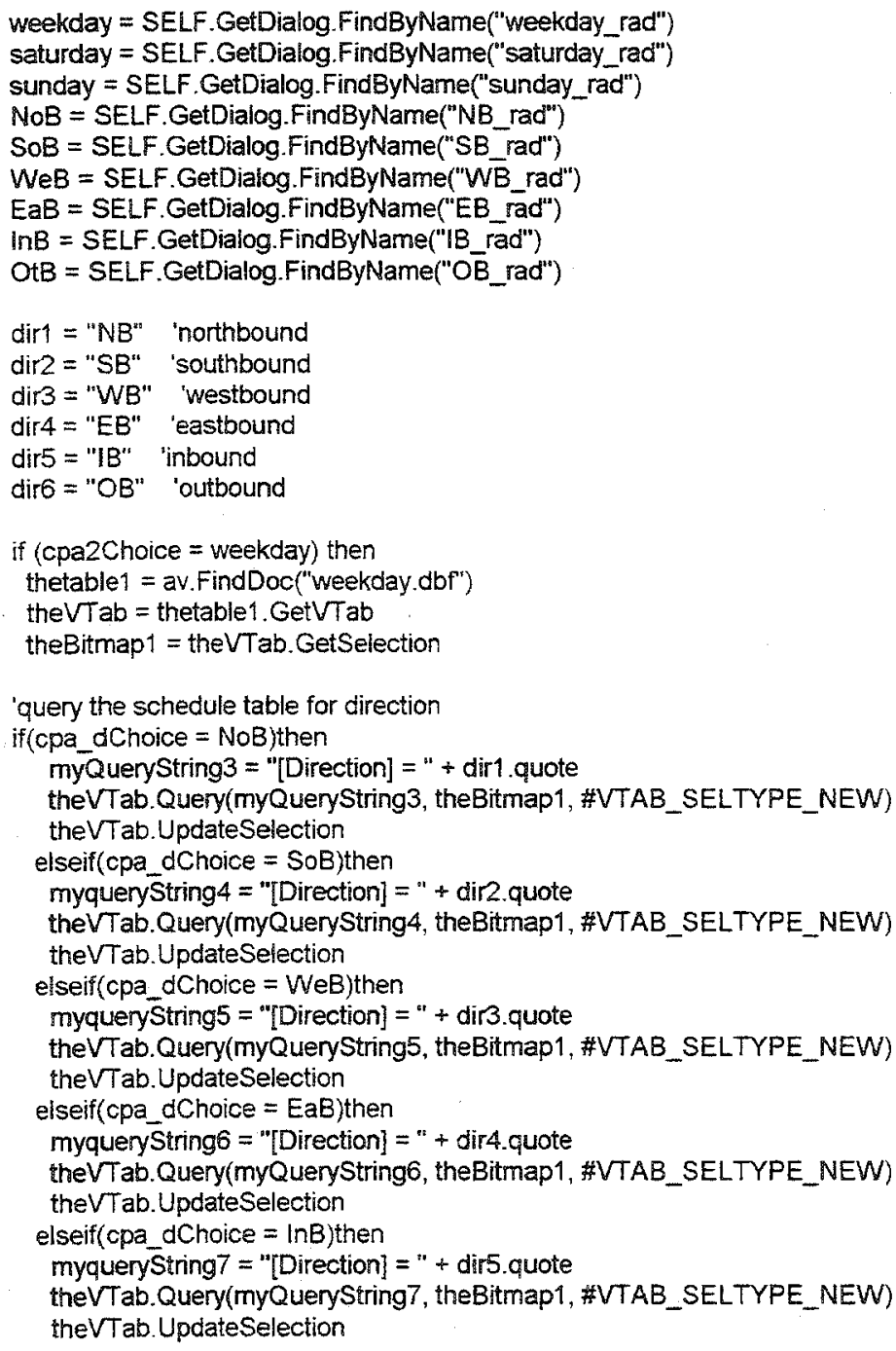


elseif $(c p a$ dChoice $=O t B)$ then

myquerystring $8=$ "[Direction] $="+$ dir6.quote

theVTab.Query(myQueryString8, theBitmap1, \#VTAB_SELTYPE_NEW)

theVTab. Updateselection

else

msgbox.Info ("Nothing", "'.")

end

'query the schedule table for the time

myQueryString $="[$ Time] $>="$ + lesstime.quote

myQuerystring2 $=$ "[Time $<="$ + moretime.quote

theVTab.Query(myQueryString, theBitmap1, \#VTAB_SELTYPE_NEW)

theVTab.UpdateSelection

theVTab.Query(myQueryString2, theBitmap1, \#VTAB_SELTYPE_AND)

theVTab.UpdateSelection

'the schedule tables are permanently linked by me before

the program starts, but this is the code that is used

"link to the bus stop theme to a schedule table

"theField = theVTab. FindField("Bus stop") 'from dbf

" theTheme = theView. FindTheme("Bus Stops") 'TTheme

- $a V T a b=$ theTheme. GetFTab

- aField = aVTab.FindField("Bus_stop"

theVTab.Link(theField, aVtab, aField)

elseif (cpa2Choice $=$ saturday) then

thetable2 $=$ av.FindDoc("saturday.dbr")

the $V T a b=$ thetable2. GetVTab

theBitmap2 = theVTab.GetSelection

'query the schedule table for direction

if $($ cpa_dChoice $=$ NoB $)$ then

myQueryString3 $=$ "[Direction $]=$ " + dir1.quote

theVTab.Query(myQueryString3, theBitmap1, VTAB_SELTYPE_NEW)

theVTab.UpdateSelection

elseif $(\mathrm{cpa}$ dChoice $=$ SoB $)$ then

myquerystring $4=$ "[Direction $]="+\operatorname{dir}$.quote

theVTab.Query(myQueryString4, theBitmap1, WTAB SELTYPE NEW)

theVTab. UpdateSelection

elseif $(\mathrm{cpa}$ dChoice $=$ WeB $)$ then

myquerystrings $=$ "[Direction] $="+\operatorname{dir} 3$.quote

theVTab. Query(myQueryString5, theBitmap1, \#VTAB SELTYPE NEW)

theVTab.UpdateSelection

elseif $(c p a$ dChoice $=E a B$ )then

myquerystring6 $=$ "[Direction $]="+\operatorname{dir} 4$.quote

theVTab.Query(myQueryString6, theBitmap1, \#VTAB_SELTYPE_NEW)

theVTab. UpdateSelection

elseif $($ cpa_dChoice $=\ln B)$ then

myquery $\overline{\text { tring } 7}=$ "[Direction $]="+\operatorname{dir} 5$.quote

theVTab.Query(myQueryString7, theBitmap1, VTAB_SELTYPE_NEW)

theVTab. UpdateSelection

elseif $(c p a$ dChoice $=O \mathrm{OtB})$ then

myquerystring $8=$ "[Direction] $="+$ dir6.quote

theVTab.Query (myQueryString8, theBitmap1, \#VTAB_SELTYPE_NEW)

theVTab.UpdateSelection

else

msgbox.Info ("Nothing", "'")

end

'query schedule table for the time

myQueryString = "[Time] $>="$ + lesstime.quote

myQueryString2 $=$ "[Time] $<="$ + moretime.quote

theVTab.Query(myQueryString, theBitmap2, \#VTAB SELTYPE NEW)

theVTab. UpdateSelection

theVTab.Query(myQueryString2, theBitmap2, \#VTAB_SELTYPE_AND)

theVTab.UpdateSelection

'the schedule tables are permanentiy linked by me before 
"link to the bus stop theme

- theField = theVTab.FindField("Bus_stop") "from dbf

- theTheme = theView.FindTheme("Bus Stops") "fTheme

- $a$ Tab = the Theme.GetFTab

- aField = aVTab.FindField("Bus stop")

' theVTab.Link(theField, aVtab, aField)

elseif (cpa2Choice = sunday) then

thetable $3=$ av. FindDoc("sunday. $d b f ")$

theVTab = thetable3.GetVTab

theBitmap 3 = theVTab. GetSelection

'query schedule table for direction

if $(\mathrm{cpa}$ _dChoice $=$ NoB $)$ then

myQuerystring $3=$ "[Direction] $=$ " + dir1.quote

theVTab.Query(myQueryString3, theBitmap1, WTAB SELTYPE NEW)

theVTab.Updateselection

elseif(cpa dChoice $=$ SoB $)$ then

myquerystring $4=$ "[Direction] $="+$ dir2.quote

theVTab.Query(myQueryString4, theBitmap1, HTAB_SELTYPE_NEW)

theVTab. UpdateSelection

elseif $(c p a$ dChoice $=$ WeB $)$ then

myqueryString5 $=$ "[Direction] $=$ " + dir3.quote

theVTab.Query (myQueryString5, theBitmap1, \#VAB_SELTVPE_NEW)

theVTab. Updateselection

elseif(cpa_dChoice $=$ EaB)then

myquerystring6 $=$ "[Direction $]=$ " dir4.quote

theVTab.Query(myQueryString6, theBitmap 1, \#VTAB_SELTYPE_NEW)

theVTab. UpdateSelection

elseif (cpa dChoice $=\ln B$ ) then

myquerystring $7=$ "[Direction] $=$ " + dir5.quote

theVTab.Query(myQueryString7, theBitmap1, \#VTAB_SELTYPE_NEW)

theVTab. UpdateSelection

elseif (cpa dChoice $=$ OtB)then

myquerystring8 $=$ "[Direction $]="+$ dir6.quote

theVTab.Query (myQueryString8, theBitmap1, WTAB_SELTYPE_NEW)

theVTab.UpdateSelection

else

msgbox.Info ("Nothing", "')

end

'query schedule table for the time

myQueryString $=$ "TTime] $>="$ + lesstime.quote

myQueryString2 = "[Time $<="$ + moretime.quote

theVTab.Query(myQueryString, theBitmap3, \#VTAB_SELTYPE_NEW)

theVTab. Updateselection

theVTab.Query(myQuerystring2, theBitmap3, \#VTAB_SELTYPE_AND)

theVTab. Updateselection

'the schedule tables are permanently linked by me before

the program starts, but this is the code that is used

"link to the bus stop theme

" theField = theVTab.FindField("Bus_stop") "from dbf

" theTheme = theView.FindTheme("Bus Stops") 'fTheme

- $\mathrm{VVT} \mathrm{ab}=$ the Theme. GetFTab

- aField = aVTab. FindField("Bus stop")

' theVTab.Link(theField, aVtab, afield)

else

msgbox.Info ("Nothing", "')

end

get origin user radio button selection from control panel 


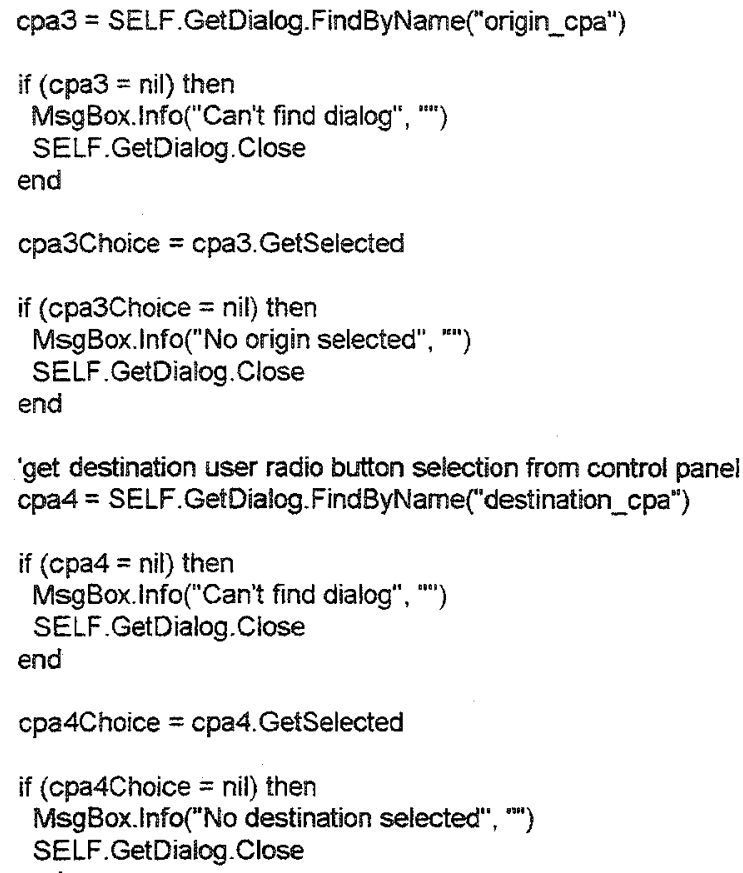




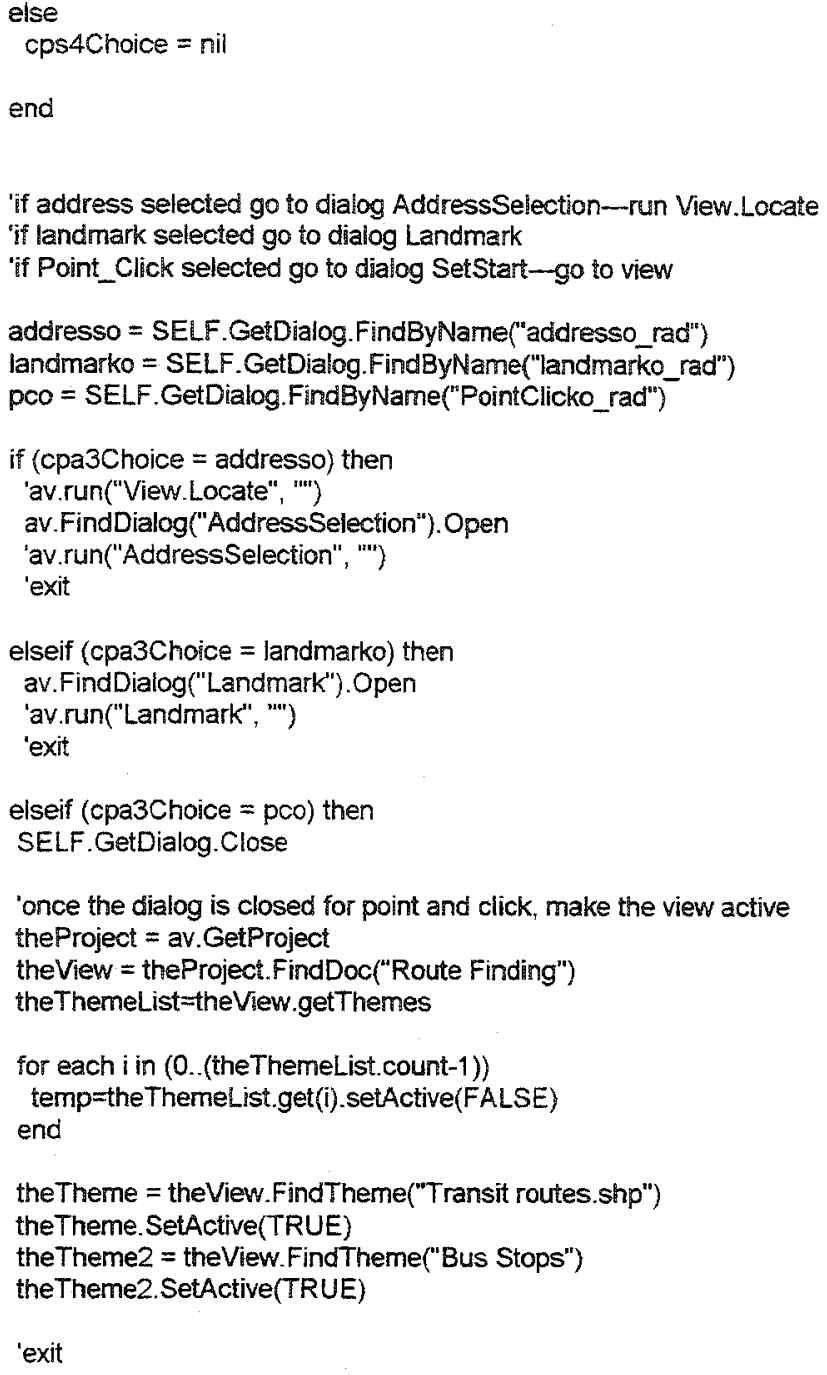




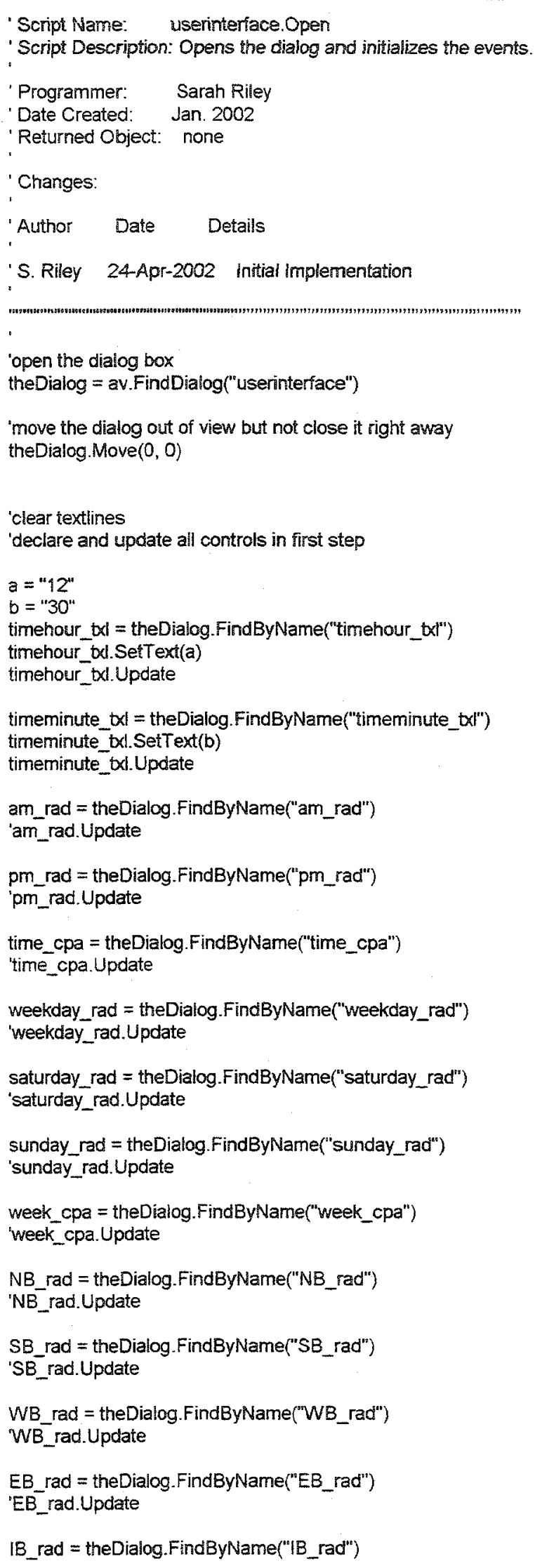




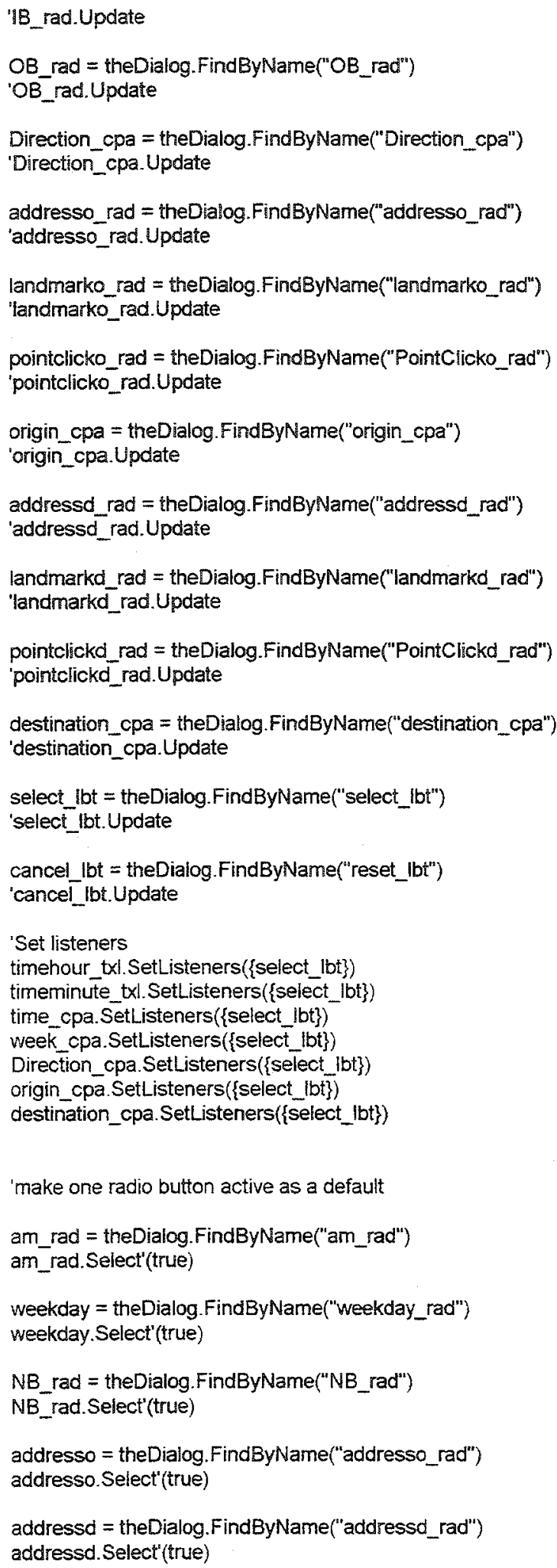


- Script Name: userinterface.cancel libt.Update

' Script Description: Enables the cancel button, sets it active.

- Programmer: Sarah Riley

' Date Created: Jan. 2002

'Returned Object: none

' Changes:

'Author Date Details

'S. Riley Jan-2002 initial Implementation

'always true, always available

SELF.SetEnabled(true) 
'Script Name: userinterface.cancel lbt.Click

' Script Description: Closes the dialog when the cancel button is selected.

- Programmer: Sarah Riley

Date Created: Jan. 2002

- Returned Object: none

- Changes:

- Author Date Details

'S. Riley Jan-2002 Initial Implementation

"

"close the dialog box

SELF.GetDialog. Close 


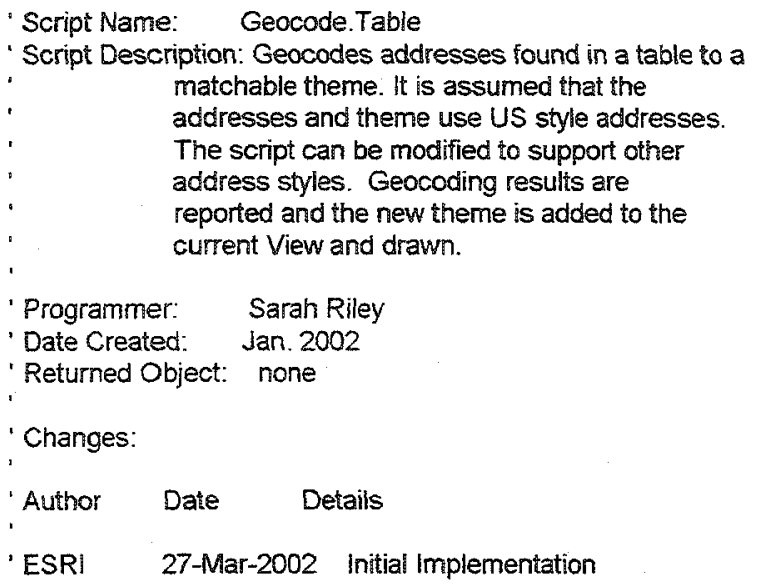

' Collect information used to create the the new geocoded point theme.

- The theme to match against, the table containing addresses, and the ' name of the field containing address information are passed from the ' calling script...

'get the match source

theView = av.GetProject.FindDoc("Route Finding") aMatchSource $=$ theView.FindTheme("Ottawa Street Network"). GetMatchSource

' Get the address event table.

addressTable $=$ av.GetProject.FindDoc( "Attributes of Location.shp" ).GetVTab addressField $=$ address Table.FindFieid("Address")

addressFTab $=$ address Table $:$ GetFTab

' Get the matchable feature source and double check that it is valid...

'if (aMatchSource $=\mathrm{Nil})$ then

" MsgBox.Error("Theme + match Theme.GetName+t"is not matchable.",

' exit

'end

' Open or create index to optimize performance...

aMatchSource.Openindex

' Specify the output point shapefile that will be created from the " matched addresses..

aGeoName = GeoName.Make $($ aMatchSource, addressFTab, addressfield, addressField )

fnOutfile $=$ "F:lhesis datalChosen Locations". AsFileName

'fnOutFile = FileDialog.Put(av.GetProject.MakeFileName("Chosen Locations", "shp"),

" "shp","Output Shapefile")

if (fnOutFile $=$ nil) then

exit

else

aGeoName.SetFileName(fnoutFile)

end 


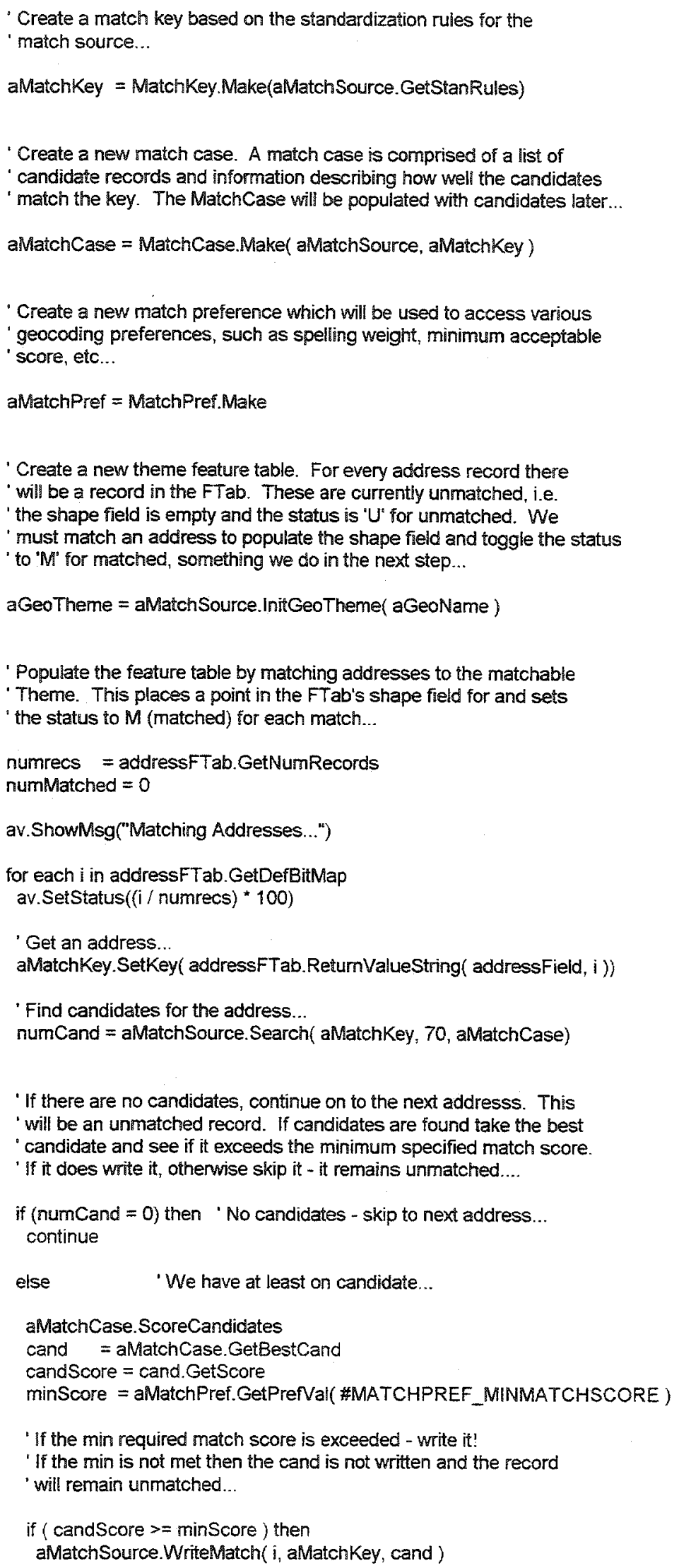

- Populate the feature table by matching addresses to the matchable - Theme. This places a point in the FTab's shape freld for and sets 


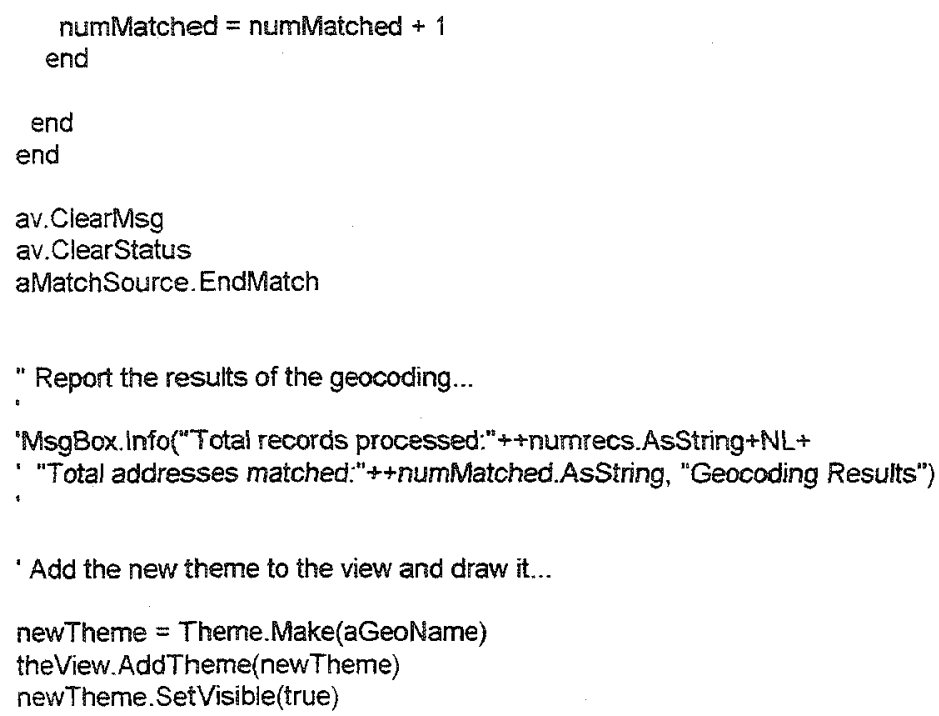

" Report the results of the geocoding...

"MsgBox.Info" Total records processed:"++numrecs.AsString+NL+

" "Total addresses matched."++numMatched.AsString, "Geocoding Results")

- Add the new theme to the view and draw it...

newTheme $=$ Theme. Make(aGeoName)

theView.AddTheme(newTheme)

newTheme.SetVisible(true) 


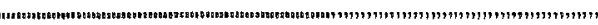

'Script Name: Geocode

' Script Description: Runs the Geocode. Table script.

'Programmer: Sarah Riley

Date Created: Apr. 2002

'Returned Object: none

' Changes:

'Author Date Details

'S. Riley 29-Apr-2002 intitial implementation

1

ay.run("Geocode.Table", 
'Script Description:

- Programmer: Sarah Riley

' Date Created: Jan. 2002

' Returned Object: none

' Changes:

'Author Date Details

'S. Riley Jan-2002 Initiaf implementation

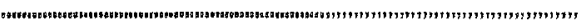

'always true, always enabled

SELF.SetEnabled(true) 


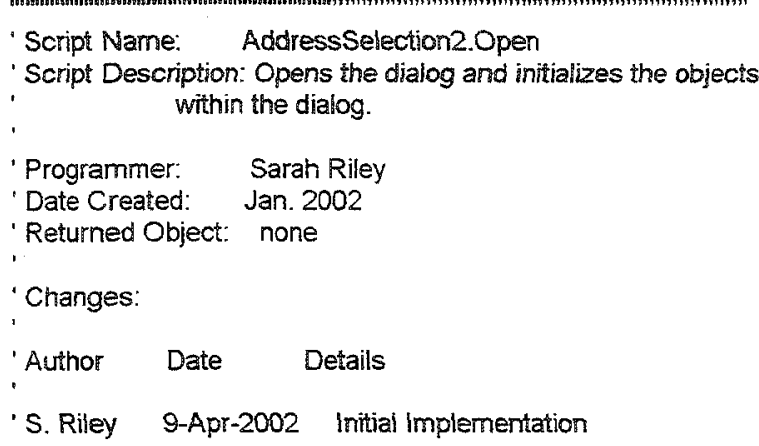

'This script will run when the dialog is opened 'SELF refers to the dialog "find the project and table Addresses and set editable 'for input from dialog

theView = av.GetProject. FindDoc("Route Finding") the Theme $=$ theView. FindTheme("Location.shp") 'the Theme. SetActive(true)

"theView.SetEditableTheme("Location.shp")

the Table $=$ the Theme. GetFTab

fldAddress = theTable.FindField("Address")

'theTable.SetActive(true)

the Table.SetEditable(true)

'the Theme.StopEditing(TRUE)

"delete contents first

"Table. SelectAll

"theSelection = the Table. GetSelection

the Table. GetFTab. Get Selection. SetAll

'the Table.GetFTab.UpdateSelection

theTable GetSelection

'av. GetProject.SetModified(true)

the Selection = the Table. GetSelection

theSelection.SetAll

$\mathrm{SC}=$ the Selection Count

if $(S C>1)$ then

'Table.DeleteRecords

'if there is more than 1 record then delete, if not

just make the table ready for adding records

'the Table = av. GetActiveDoc

the $V T a b=$ the Table '. GetFTab

the Vtab.BeginTransaction

theVTab.RemoveRecords(theVTab. GetSelection. Clone)

theVTab. EndTransaction 
"Table. DeleteRecordsUpdate

theVTab = av. GetActiveDoc.GetVTab

- SELF.SetEnabled(theVTab.CanRemoveRecords and (theVTab.GetSelection.Count > 0))

else

theTable = av. GetActiveDoc. Get.VTab

fldAddress = theTable.FindField("Address")

SELF.SetServer(theTable)

end

'clears out the textline

thetextline = theDialog.FindByName ("destinationaddress_bul")

thetextline. Empty 
- Script Name: AddressSelection2.ok_lbt.Update

' Script Description: Enables the OK button to aways be availabie.

'Programmer: Sarah Riley

- Date Created: Jan. 2002

- Returned Object: none

- Changes:

- Author Date Details

S. Riley Jan-2002 Initial implementation

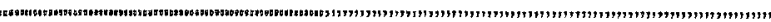

'ahways true, always enabled

SELF.SetEnabled(true) 
- Script Name: AddressSelection2.ok ibt.Click

'Script Description: Runs when the OK button is selected. Enters information into a table.

'Programmer: Sarah Riley

- Date Created. Jan. 2002

' Returned Object: none

'Changes:

- Author Date Details

'S. Riley 12-Apr-2002 Initial Implementation

'SELF refers to the button

SELF.SetEnabled(true)

'gets the address entered by user

address_bd = SELF.GetDialog.FindByName("destinationaddress_tal")

'reference the attributes of address.shp vtab--table has been made

theView = av.GetProject.FindDoc("Route Finding")

the Theme = theView.FindTheme("Location.shp")

"theView.SetEditableTheme("Location.shp")

the Table $=$ theTheme.GetFTab

'theTable = SELF. GetServer

fldAddress = the Table. FindField("Address")

add the new record

theTable.SetEditable(true)

recNewAddress = theTable.AddRecord

theTable.SetValue (fldAddress, recNewAddress, address_tol.GetText)

theTable.SetEditable(false)

theTable.Refresh

'close the dialog box

self.GetDialog. Close

'geocode points

av.run("Geocode", "'")

'have user select red button to find route

message $=$ "To find your root, please select the red diamond button."

MsgBox.Info(message, "Address Selection") 


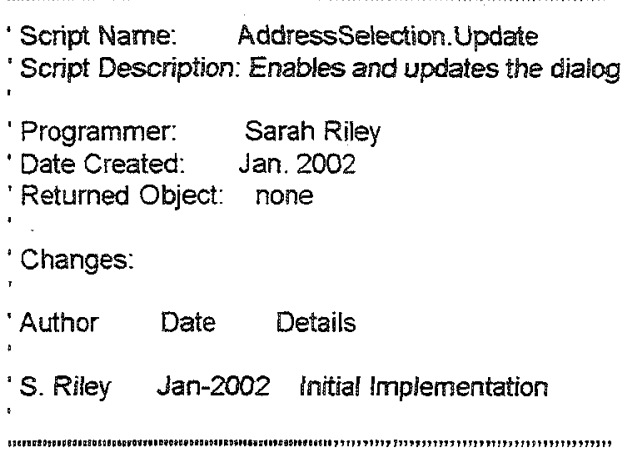

'always true, always enabled

SELF.SetEnabled(true) 


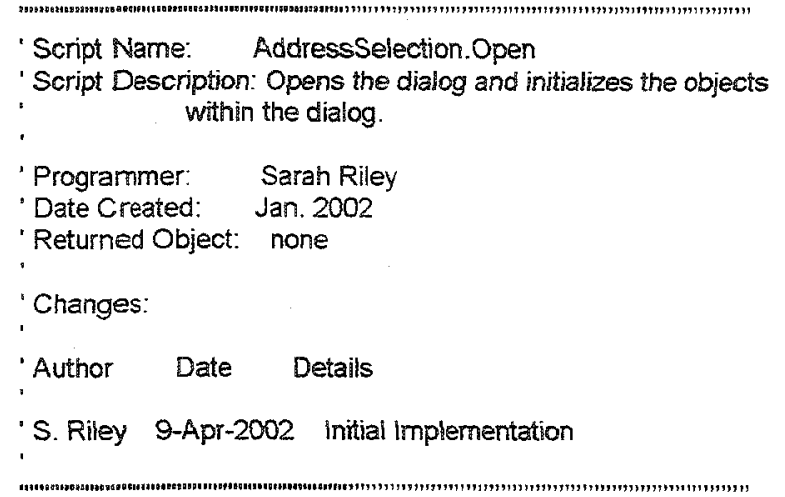

'open the dialog box

theDialog = av.FindDialog("AddressSelection")

'declare and update all controls in the dialog

address_t $|x|=$ theDialog.FindByName("address_t $x \mid ")$

address_txl.update

ok_lbt = theDialog.FindByName("ok_lbt")

'ok_lbt.Update

'This script will run when the dialog is opened

'SELF refers to the dialog

"find the project and table Addresses and set editable

'for input from dialog

theView = av.GetProject. FindDoc("Route Finding")

theTheme = theView.FindTheme("Location.shp")

"theTheme.SetActive(true)

"theView.SetEditableTheme("Location.shp")

theTable $=$ theTheme.GetFTab

fldAddress = the Table.FindField("Address")

theTable.SetActive(true)

theTable.SetEditable(true)

'theTheme.StopEditing(TRUE)

'delete contents first

'Table.SelectAll

'for every record i delete

'theSelection = the Table. GetSelection

'theTable.GetFTab GetSelection. SetAll

'theTable.GetFTab.UpdateSelection

theTable.GetSelection

'av.GetProject.SetModified(true)

theSelection $=$ the Table. GetSelection

theSelection.SetAll

$\mathrm{SC}=$ the Selection. Count

if $(S C>1)$ then

'Table.DeleteRecords

if there is more than 1 record then delete, if not

'just make the table ready for adding records

the Table $=$ av.GetActiveDoc

theVTab = the Table '.GetFTab

the Vtab.BeginTransaction 
theVTab.RemoveRecords(theVTab.GetSelection.Clone)

theVTab.EndTransaction

- 'Table.DeleteRecordsupdate

- theVTab = av.GetActiveDoc. GetVTab

- SELF.SetEnabled(theVTab.CanRemoveRecords and (theVTab.GetSelection.Count >0))

else

'the Table = av. GelActiveDoc.Get.VTab

fidAddress = theTable.FindField("Address")

SELF.SetServer(theTable)

end

'clears out the textline

thetextline = theDialog.FindByName("address_tx")

thetextline.Empty 
Script Name: AddressSelection.OK Ibt.Update

'Script Description: Enables the OK button to always be available.

' Programmer: Sarah Riley

'Date Created: Jan. 2002

' Returned Object: none

'Changes:

'Author Date Details

'S. Riley Jan-2002 Initial Implementation

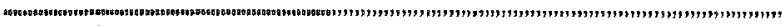

'always enabled, atways true

SELF.SetEnabled(true) 


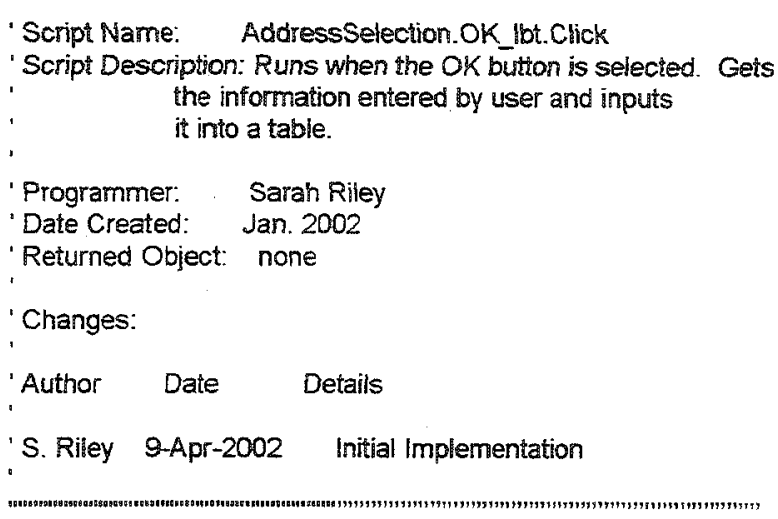




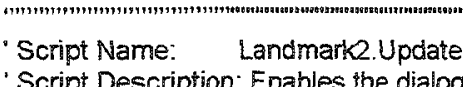

- Script Description: Enables the dialog.

'Programmer:

Sarah Riley

' Date Created: Jan. 2002

- Returned Object: none

' Changes:

'Author Date Details

'S. Riley 27-Mar-2002 Initial Implementation

"

'always enabled, always true

SELF.SetEnabled(true) 


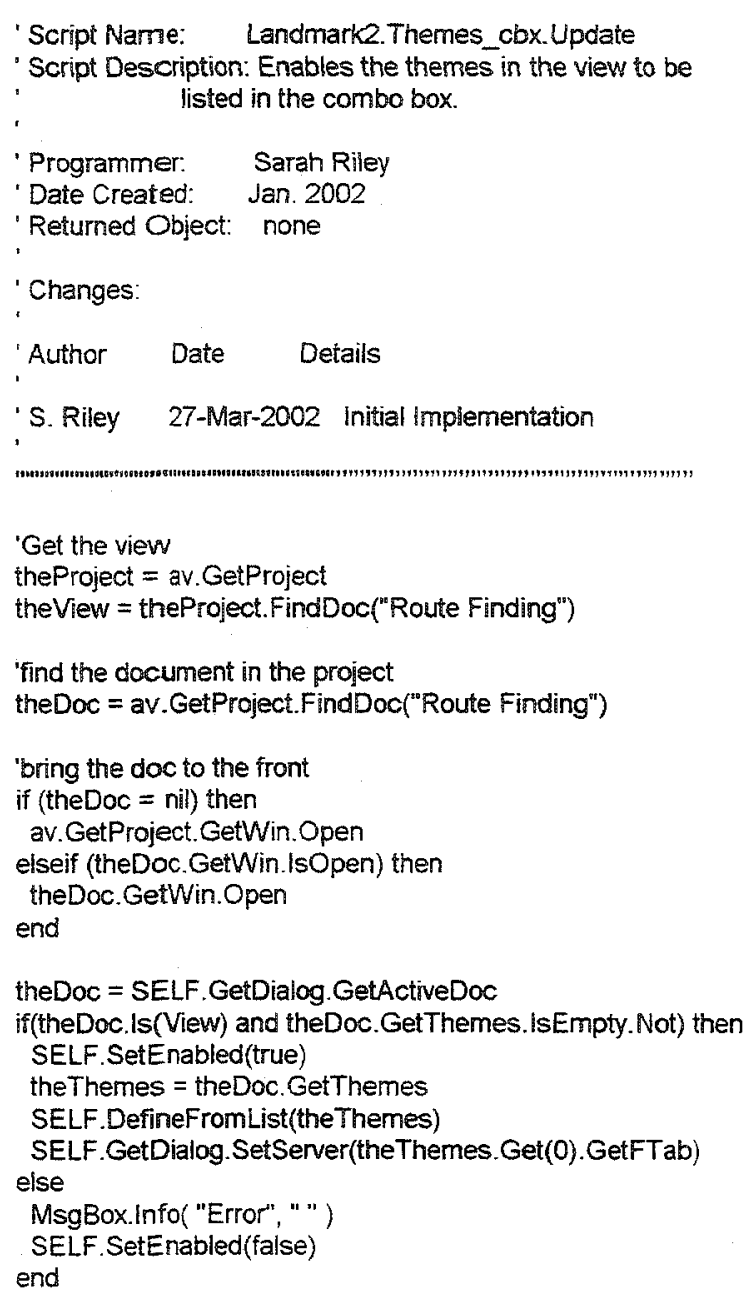




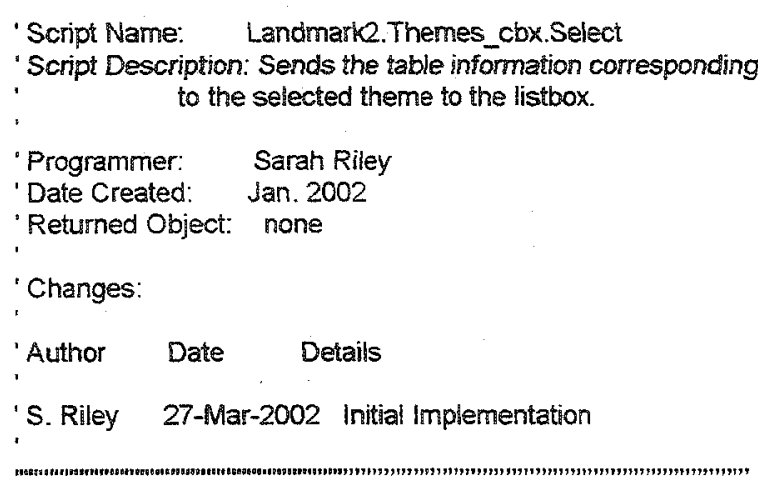

'display current themes in the view the Theme = SELF. GetCurrentValue

theFTab = theTheme. GetFTab

SELF.GetDialog.SetServer(theFTab)

SELF.BroadcastUpdate 


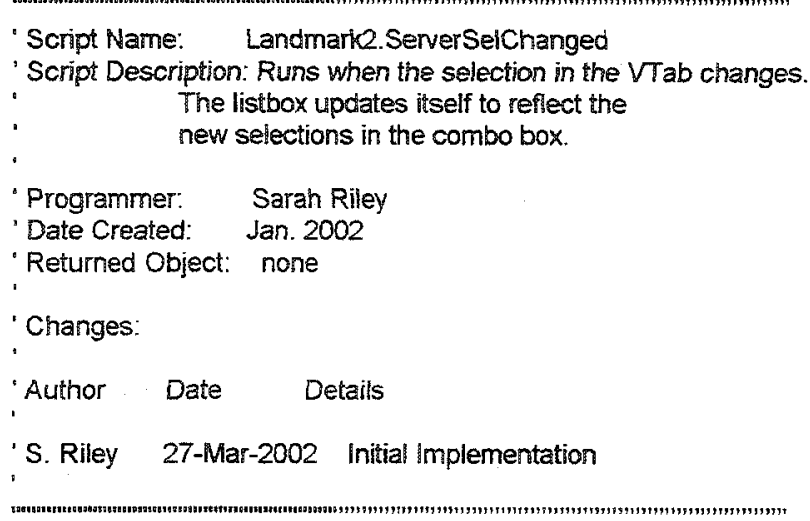

SELF.FindByName("Landmark_lbx").Update 
' Script Name: Landmark2.Open

' Script Description: Opens and initializes the items within the dialog.

' Programmer: Sarah Riley

- Date Created: Jan. 2002

' Returned Object: none

' Changes:

'Author Date Details

'S. Riley 27-Mar-2002 Initial implementation

'open the dialog box

theDialog = av.FindDialog("Landmark2")

'center the dialog

theDialog.Move $(0,0)$

'declare and update all controls

ok_lbt = theDialog.FindByName("OK_lbt")

ok_lbt.Update

cancel $\_b t=$ theDialog.FindByName("cancel_bt")

cancel_Ibt.Update

themes_cbx = theDialog. FindByName ("Themes_cbx")

themes_cbx.Update

landmark_lbx = theDialog.FindByName("Landmark_lbx")

landmark_lbx.Update

themes_cbx.SetListeners $(\{$ landmark_lbx\} $\})$ 
Script Name: Landmark. OK lbt. Update

'Script Description: Enables the OK button. Sets it active.

'Programmer: Sarah Riley

' Date Created: Jan. 2002

' Returned Object: none

' Changes:

'Author Date Details

' S. Riley 27-Mar-2002 initial Implementation

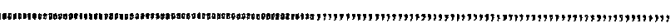

'always enabled, always true

SELF.SetEnabled(true) 


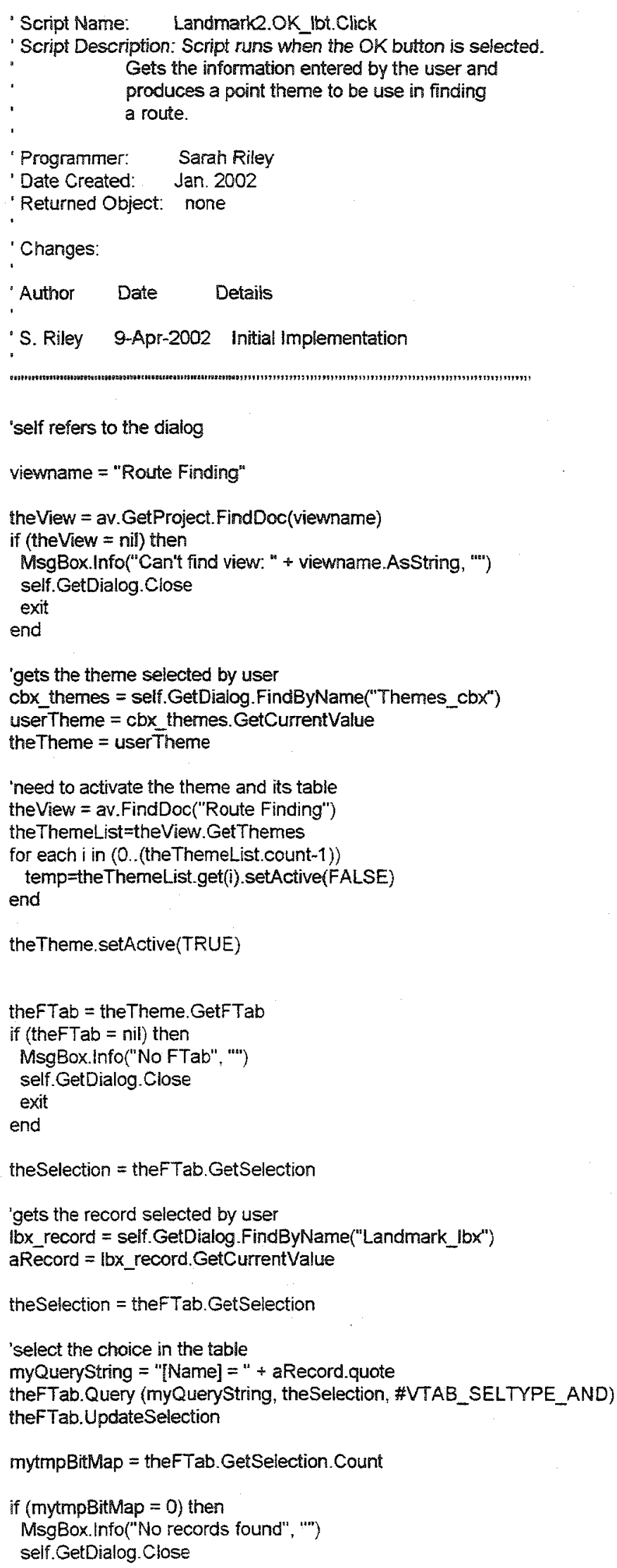


'The following makes the location list point theme by merging the start and the end locations.

'This theme is used in the network analyst routine.

theProject $=$ av. GetProject

theView = theProject. FindDoc("Route Finding")

themesToMerge = List. Make

themes ToMerge.add(theView.FindTheme("firstlocation"))

"themes ToMerge.add(theView.FindTheme("Location.shp"))

themes ToMerge.add(theView.FindTheme(theTheme.AsString)) 'theme corresponding to location selected by user "themes ToMerge.add(theView. FindTheme("firstlocation"))

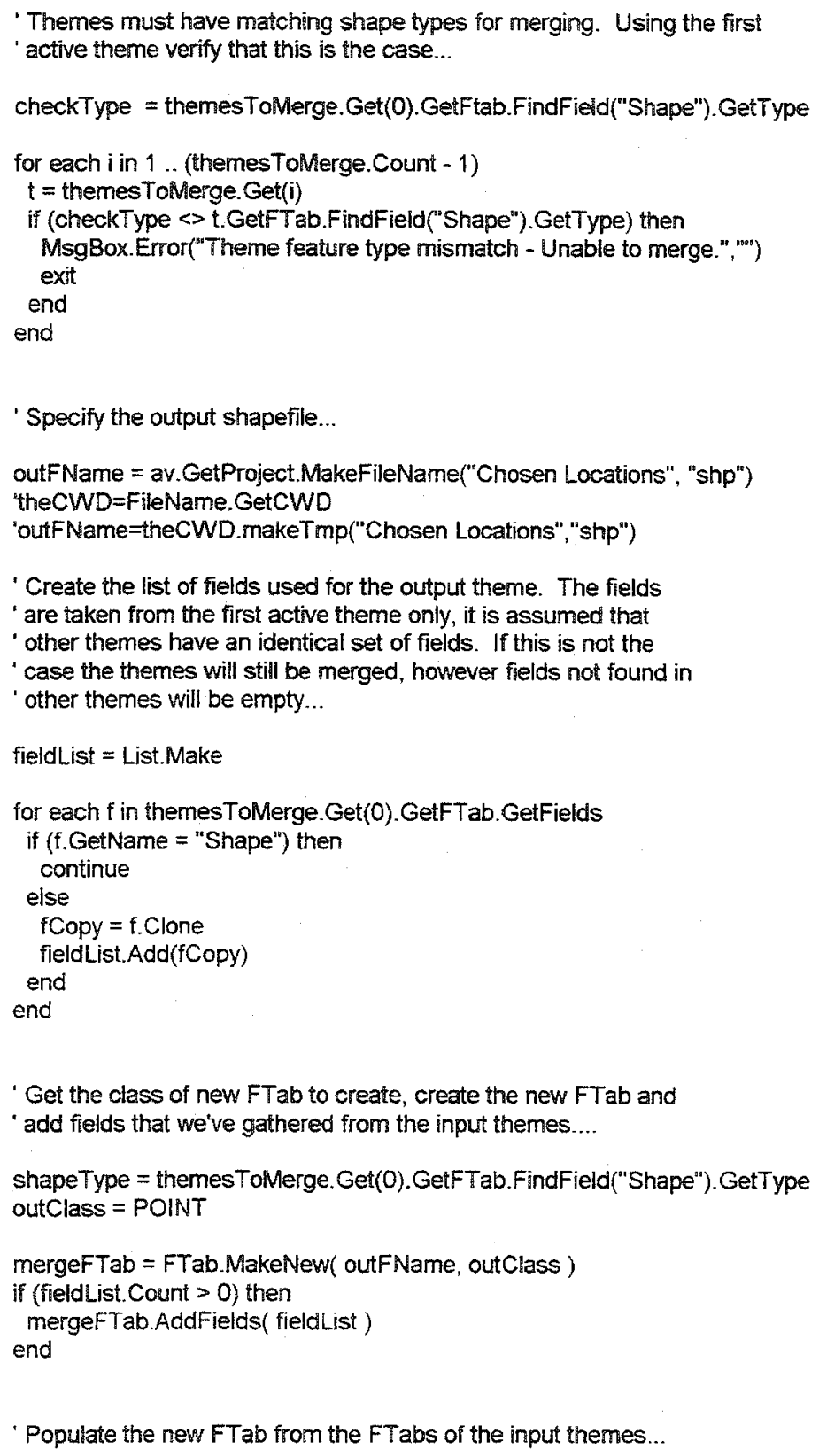




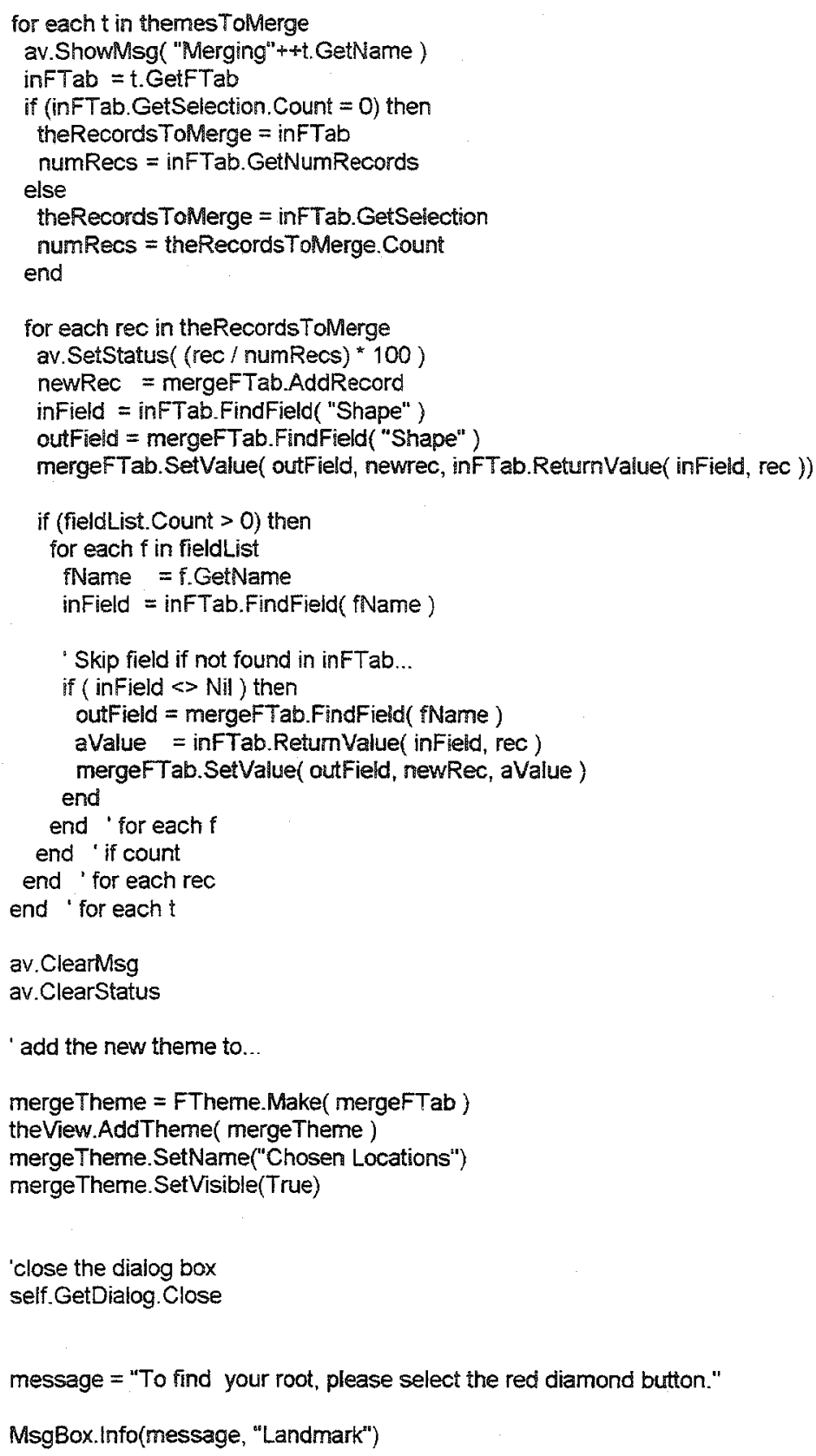




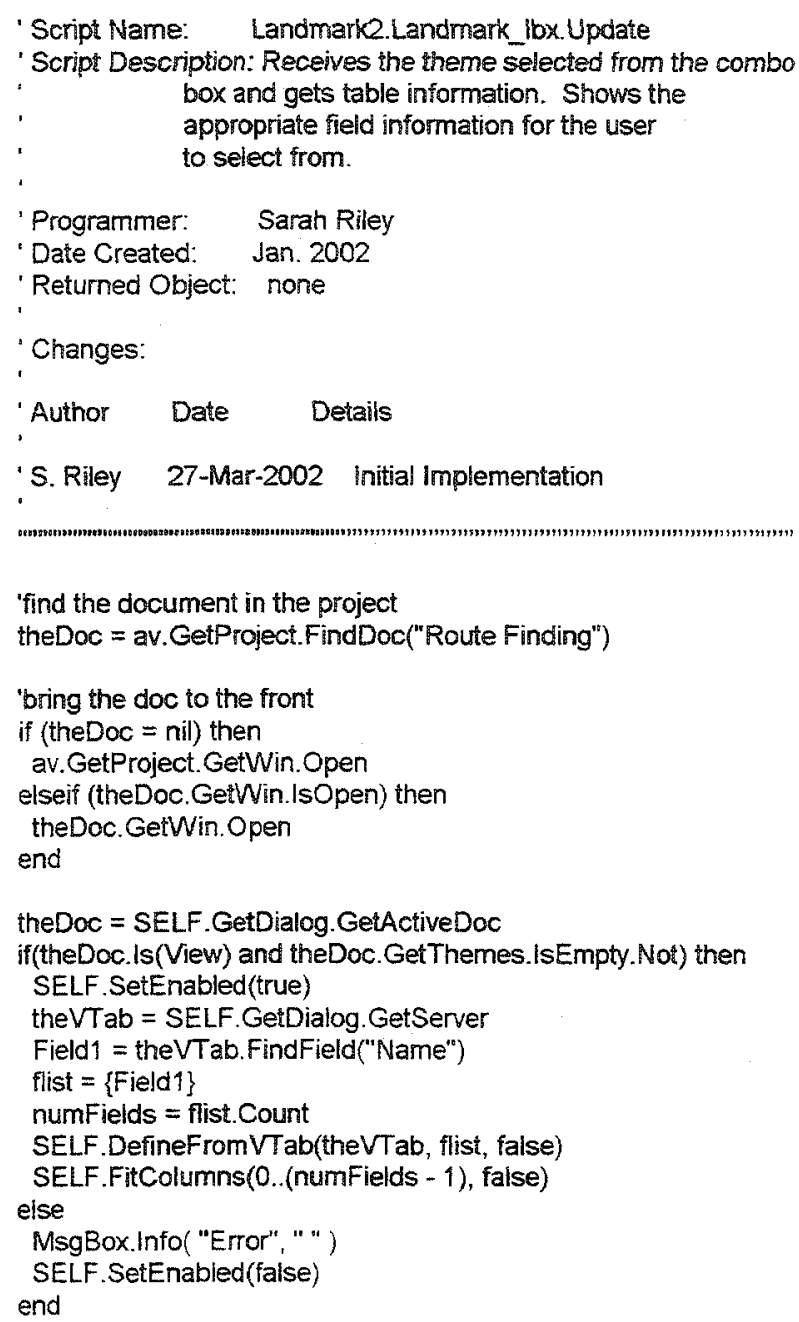




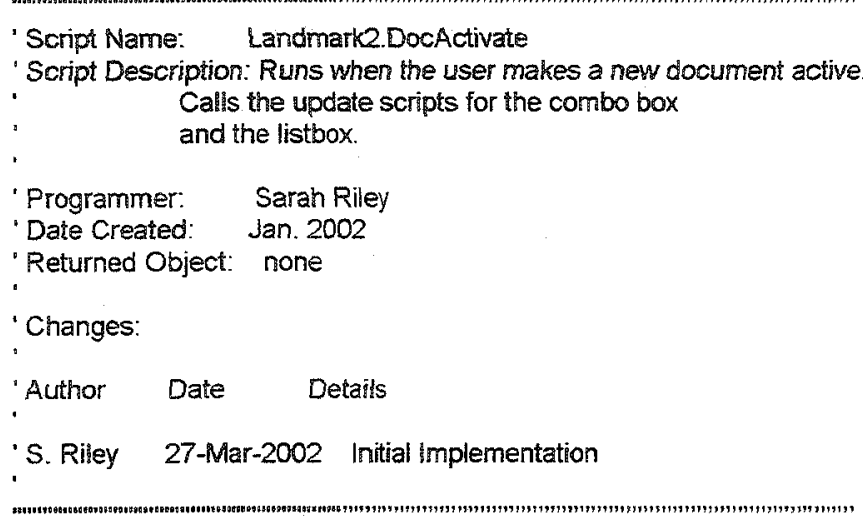

SELF.FindByName("Themes_cbx").Update SELF. FindByName("Landmark $\left.1 b x^{\prime \prime}\right)$.Update 


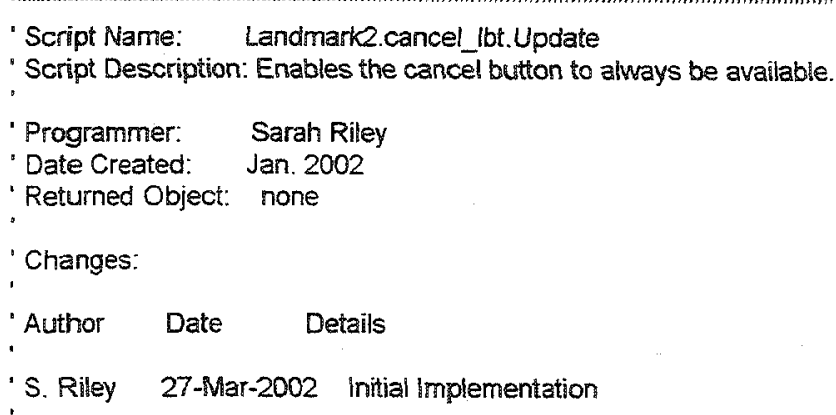

- Returned Object: none

' Changes:

- Author Date Details

- S. Riley 27-Mar-2002 Initial implementation

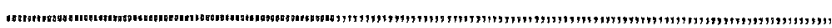

'always true, always available

SELF.SetEnabled(true) 
- Script Name: Landmark2 cancel lbt.Click

Script Description: Closes the dialog when the cancel button is selected

'Programmer: Sarah Riley

Date Created: Jan. 2002

'Returned Object: none

- Changes:

'Author Date Delails

'S. Riley 27-Mar-2002 Initial Implementation

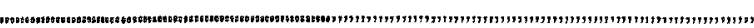

'close the dialog box

SELF.GetDialog.Close 


\begin{abstract}
'Script Name: Landmark. Update
' Script Description: Enables dialog.

' Programmer: Sarah Riley

' Date Created: Jan. 2002

' Returned Object: none

' Changes:

'Author Date Details

'S. Riley Jan-2002 Initial Implementation

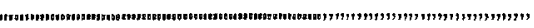

'always enabled, always true

SELF.SetEnabled(true) 


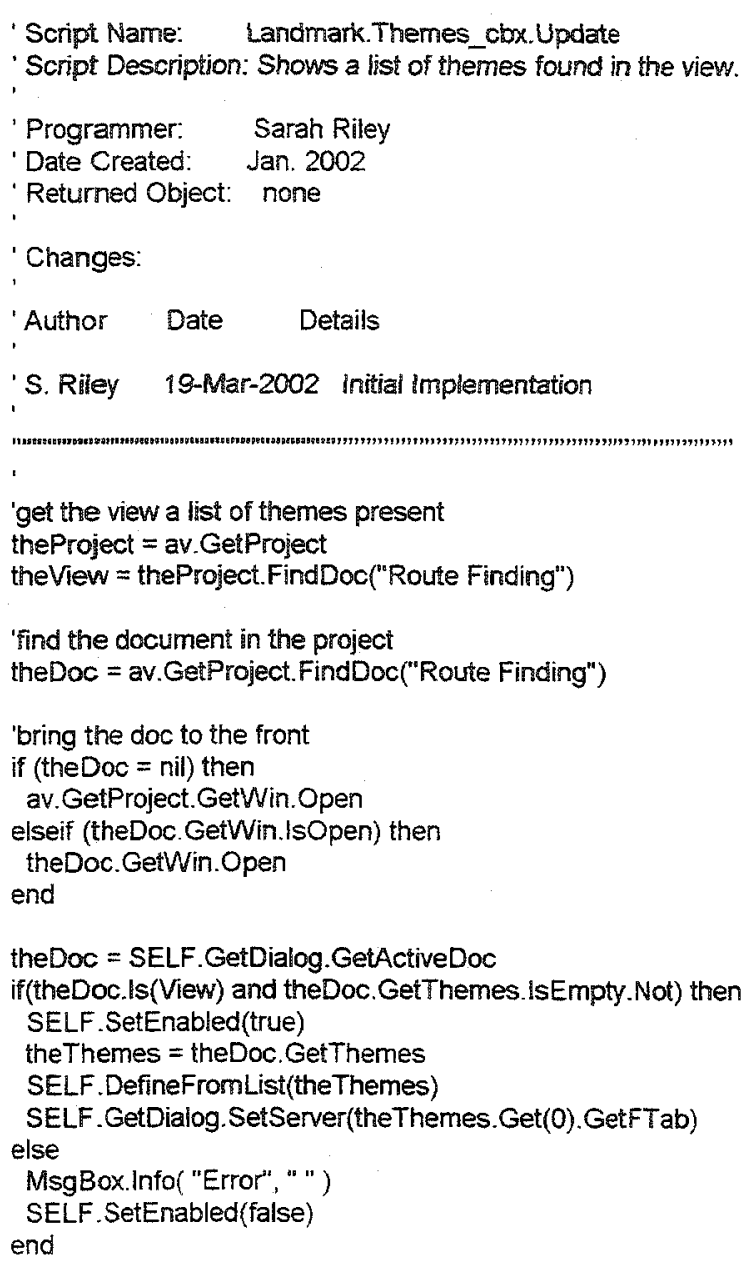


年,

- Script Name: Landmark.Themes_cbx.Select

' Script Description: Broadcasts the selected theme's table

- information to the listbox.

- Programmer: Sarah Riley

'Date Created: Jan. 2002

'Returned Object: none

' Changes:

- Author Date Details

'S. Riley 19-Mar-2002 Initial Implementation

,

'display current themes in the view

the Theme $=$ SELF . GetCurrentValue

theFTab = theTheme.GetFTab

SELF.GetDialog.SetServer(theFTab)

SELF.BroadcastUpdate 
'Script Name: Landmark.ServerSelChanged

'Script Description: Runs when the selection in the VTab changes.

The listbox updates itself to reflect the

new selections in the combo box.

' Programmer: Sarah Riley

' Date Created: Jan. 2002

'Returned Object: none

' Changes:

'Author Date Details

'S. Riley Jan-2002 Initial Implementation

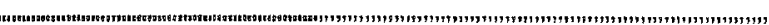

SELF.FindByName("Landmark_Ibx").Update 


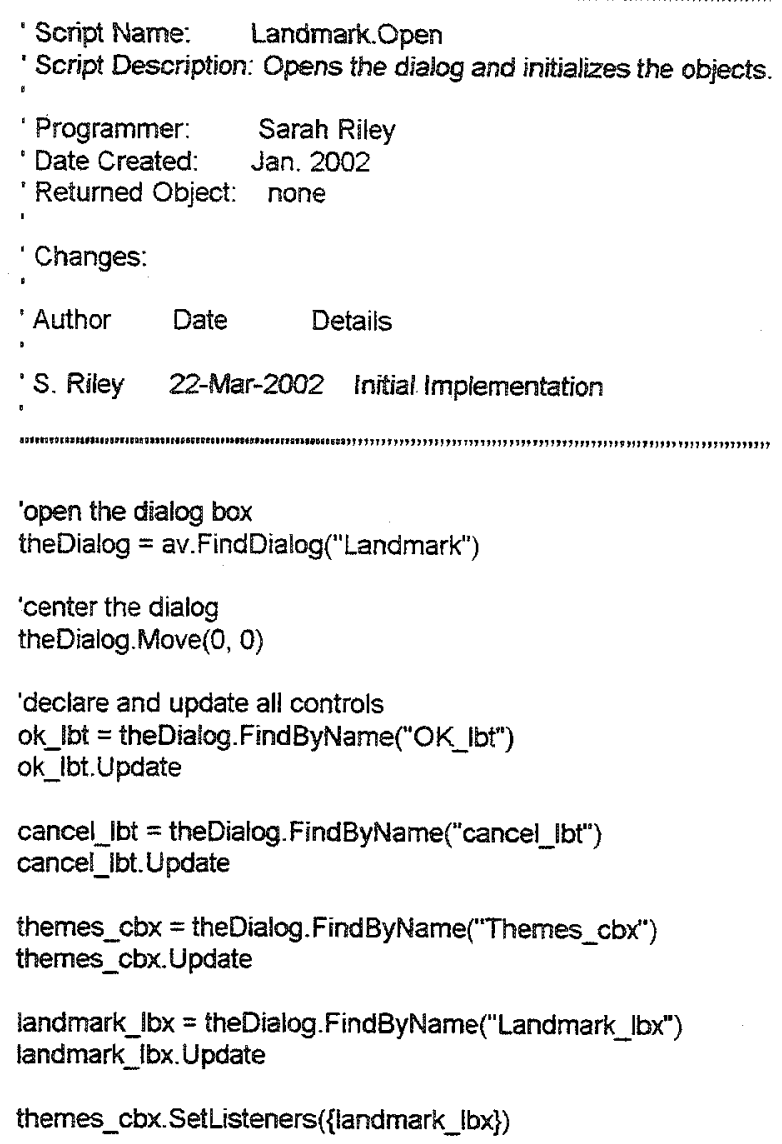


' Script Name: Landmark.OK Ibt.Update

'Script Description: Enables the OK button to always be available.

' Programmer: Sarah Riley

' Date Created: Jan. 2002

'Returned Object: none

' Changes:

'Author Date Details

'S. Riley Jan-2002 Initial Implementation

'always enabled, always true

SELF.SetEnabled(true) 


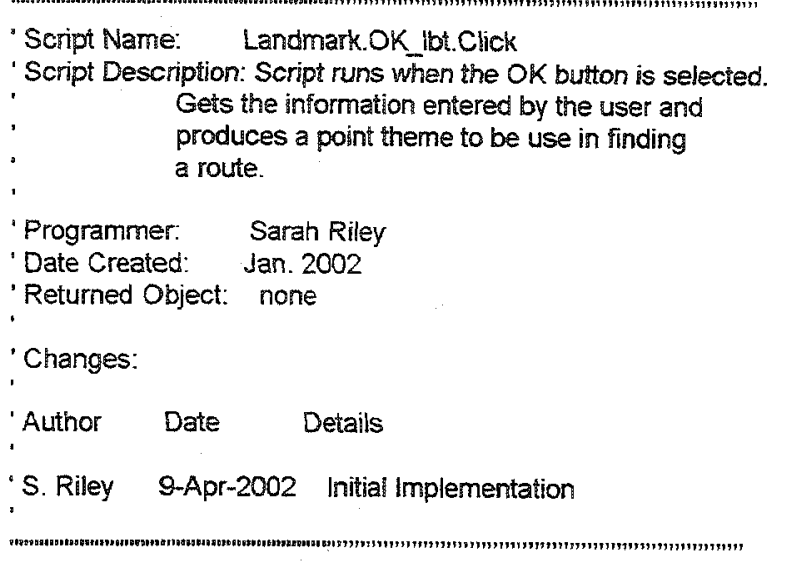

'self refers to the dialog

viewname $=$ "Route Finding"

theView = av.GetProject.FindDoc(viewname)

if (theView = nil) then

MsgBox.Info("Can't find view: " + viewname.AsString, "'")

self.GetDialog.Close

exit

end

'gets the theme selected by user

cbx_themes = self.GetDialog.FindByName("Themes_cbx")

userTheme $=c b x$ themes. GetCurrentValue

the Theme $=$ user Theme

'need to activate the theme and its table theView = av.FindDoc("Route Finding")

theThemeList=theView. GetThemes

for each $i$ in (0..(the ThemeList.count-1))

temp=theThemeList.get(i).setActive(FALSE) end

theTheme.setActive(TRUE)

theFTab = theTheme. GetFTab

if (theFTab = nil) then

MsgBox.Info("No FTab", "')

self.GetDialog.Close

exit

end

theSelection $=$ theFTab. GetSelection

'gets the record selected by user

lbx_record = self.GetDialog.FindByName("Landmark_lbx")

aRecord $=\mathrm{lbx}$ _record. GetCurrentValue

theSelection $=$ theFTab.GetSelection

'select the choice in the table

myQueryString = "[Name] $=$ " + aRecord.quote

theFTab.Query (myQueryString, theSelection, \#VTAB_SELTYPE_AND)

theFTab.UpdateSelection

mytmpBitMap $=$ theFTab.GetSelection. Count

if (mytmpBitMap $=0$ ) then

MsgBox.Info("No records found", "')

self.GetDialog. Close 
exit

end

'The following makes the location list point theme by merging the start and the end locations.

'This theme is used in the network analyst routine.

'get the view and the necessary themes

theProject $=$ av. GetProject

theView = theProject.FindDoo("Route Finding")

theFTheme = theView.FindTheme("Location.shp")

theTable $=$ theFTheme. GetFTab

fldAddress = the Table.FindField("Address")

'theTable.SetActive(true)

theTable.SetEditable(true)

theSelection = the Table. GetSelection

theSelection.SetAll

$\mathrm{SC}=$ theSelection. Count

if $(\mathrm{SC}>=2)$ then

'Table.DeleteRecords

'if there is more than 1 record then delete them

if not just make the table ready for adding records

'the Table $=$ av. GetActiveDoc

theVTab = theTable '.GetFTab

theVtab.BeginTransaction

theVTab.RemoveRecords(theVTab.GetSelection.Clone)

theVTab. EndTransaction

else

'theTable = av.GetActiveDoc. GetVTab

fldAddress = theTable.FindField("Address")

SELF.SetServer(theTable)

end

themesToMerge = List.Make

themes ToMerge.add(theView. FindTheme("Location.shp"))

themesToMerge.add(theView.FindTheme(theTheme.AsString)) 'theme corresponding to location selected by user

"themesToMerge.add(theView.FindTheme("Location.shp"))

'MsgBox.List(themes ToMerge, "'", "'n')

- Themes must have matching shape types for merging. Using the first

' active theme verify that this is the case..

checkType $=$ themesToMerge.Get(0).GetFtab.FindField("Shape").GetType

for each i in 1 .. (themesToNerge.Count - 1)

$t=$ themesToMerge. Get(i)

if (checkType < t.GetFTab.FindField("Shape").GetType) then

MisgBox.Error("Theme feature type mismatch - Unable to merge.", "'")

exit

end

end

' Specify the output shapefile

outFName = av.GetProject.MakeFileName("firstlocation", "shp")

theCWD=FileName. GetCWD

'outFName=theCWD.makeTmp("firstlocation" ,"shp")

- Create the list of fields used for the output theme. The fields

' are taken from the first active theme only, it is assumed that

' other themes have an identical set of fields. If this is not the

' case the themes will still be merged, however fields not found in

' other themes will be empty. 


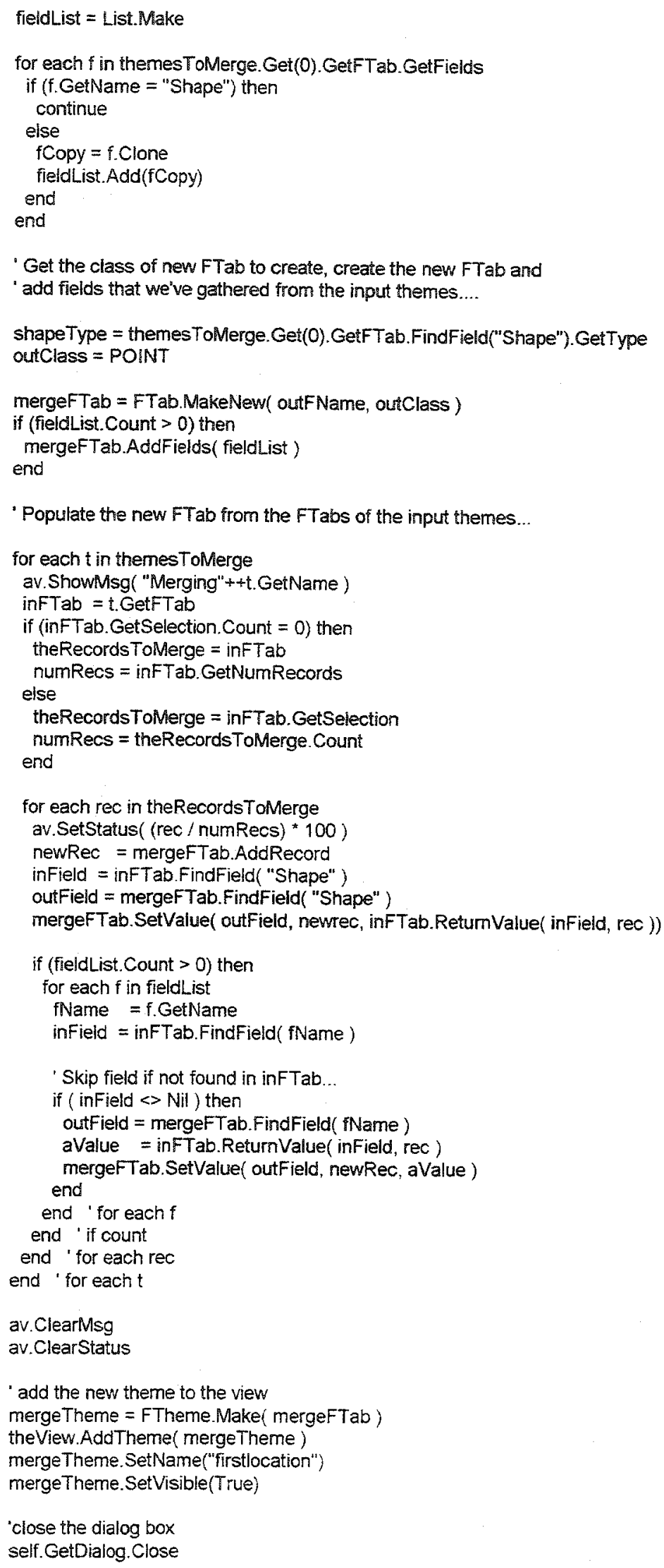

- Populate the new FTab from the FTabs of the input themes... 


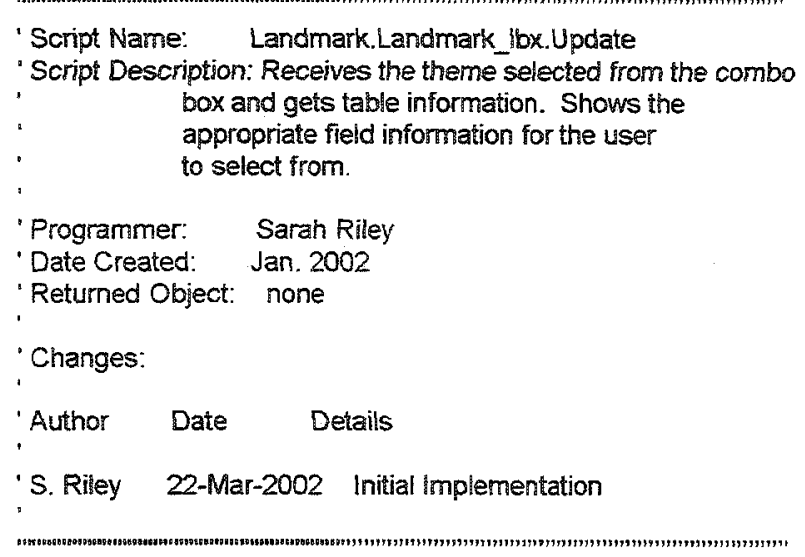

'find the document in the project

theDoc $=a v$. GetProject.FindDoc("Route Finding")

"bring the doc to the front

if (theDoc = nil) then

av.GetProject.GetWin. Open

elseif (theDoc.GetWin.IsOpen) then

theDoc.GetWin.Open

end

theDoc $=$ SELF.GetDialog. GetActiveDoc

if(theDoc.is(View) and theDoc.GetThemes.IsEmpty.Not) then

SELF.SetEnabled(true)

theVTab = SELF. GetDialog. GetServer

Field1 $=$ theVTab. FindField $\left(" N a m e^{n}\right)$

flist $=\{$ Field 1$\}$

numFields $=$ flist . Count

SELF.DefineFromVTab(theVTab, flist, false)

SELF. FitColumns ( 0 ..(numFields - 1 ), false)

else

MsgBox.Info( "Error", " ")

SELF.SetEnabled(false)

end 


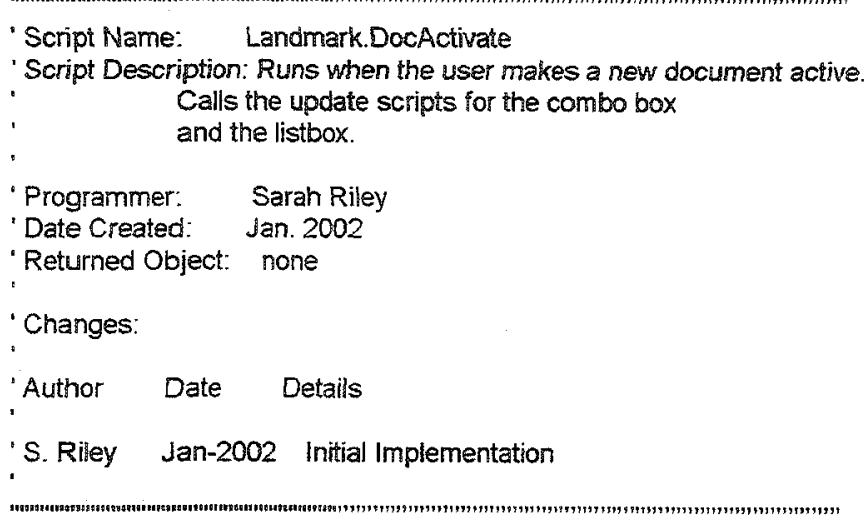


- Script Name: Landmark cancel lbt.Update

'Script Description: Enables the cancel button to always be available.

- Programmer: Sarah Riley

' Date Created: Jan. 2002

- Returned Object: none

' Changes:

-Author Date Details

'S. Riley Jan-2002 Initial Implementation

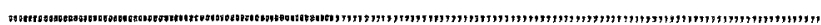

'always true, always available

SELF.SetEnabled(true) 


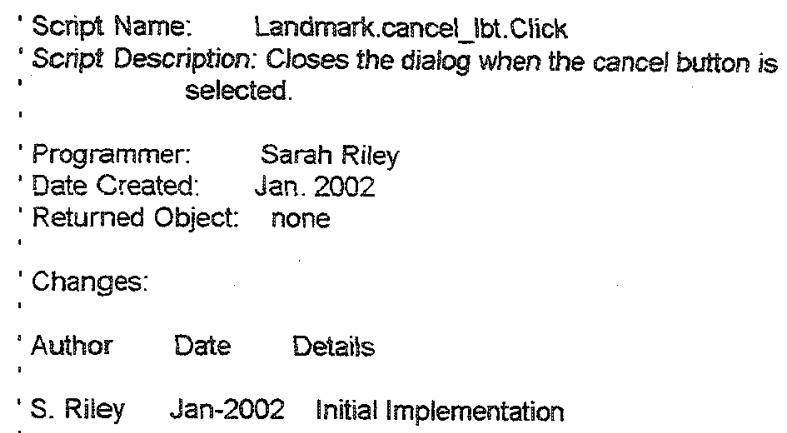

' Returned Object: none

' Changes:

'Author Date Details

'S. Riley Jan-2002 Initial Implementation

sce

'close the dialog box

SELF.GetDialog. Close 


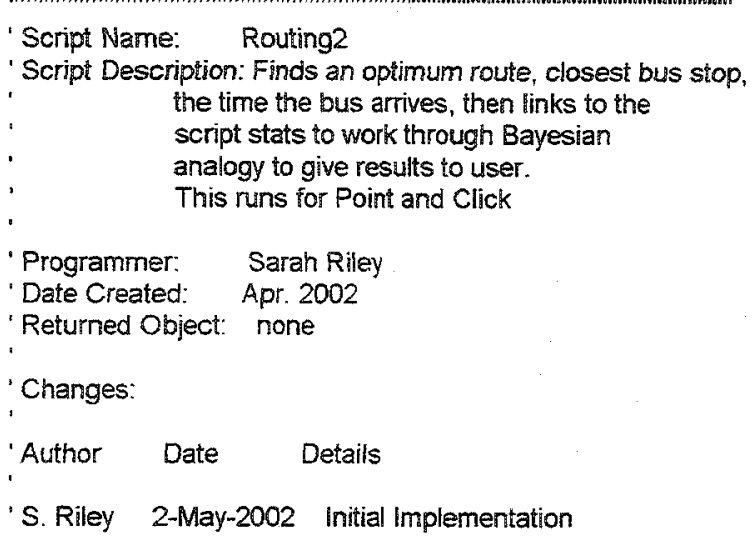




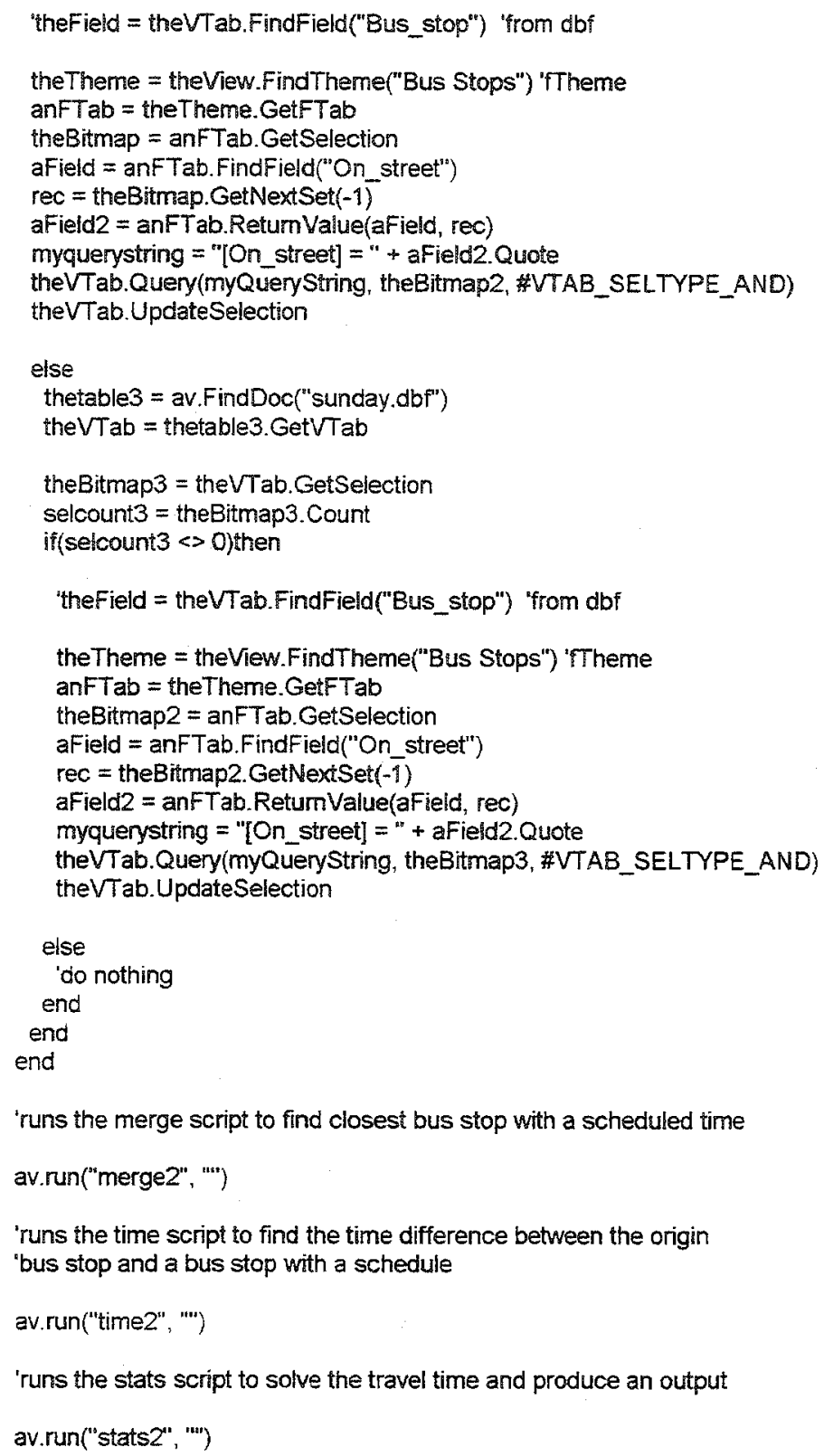

'runs the merge script to find closest bus stop with a scheduled time

av.run("merge2", "'s")

'runs the time script to find the time difference between the origin 'bus stop and a bus stop with a schedule

av.run("time2", "')

'runs the stats script to solve the travel time and produce an output

av.run("stats2", "') 


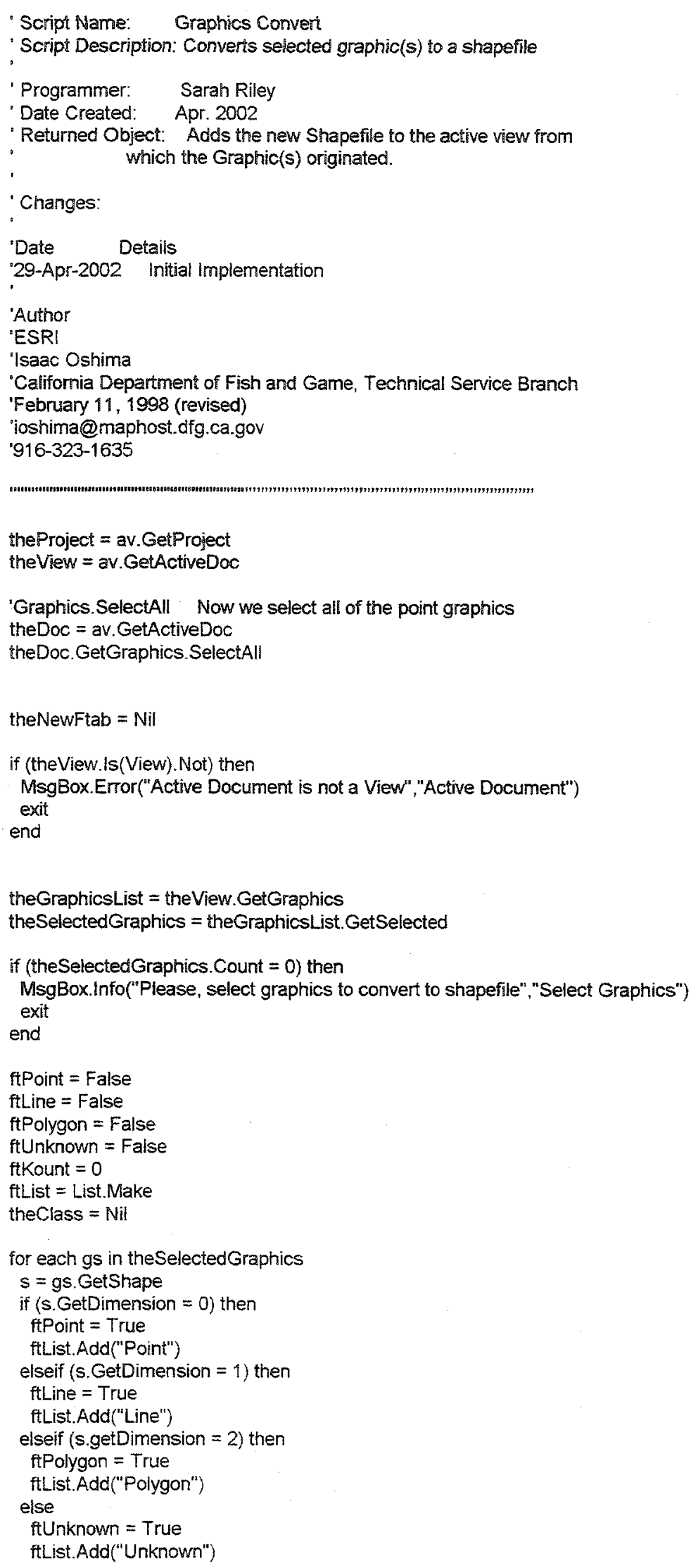




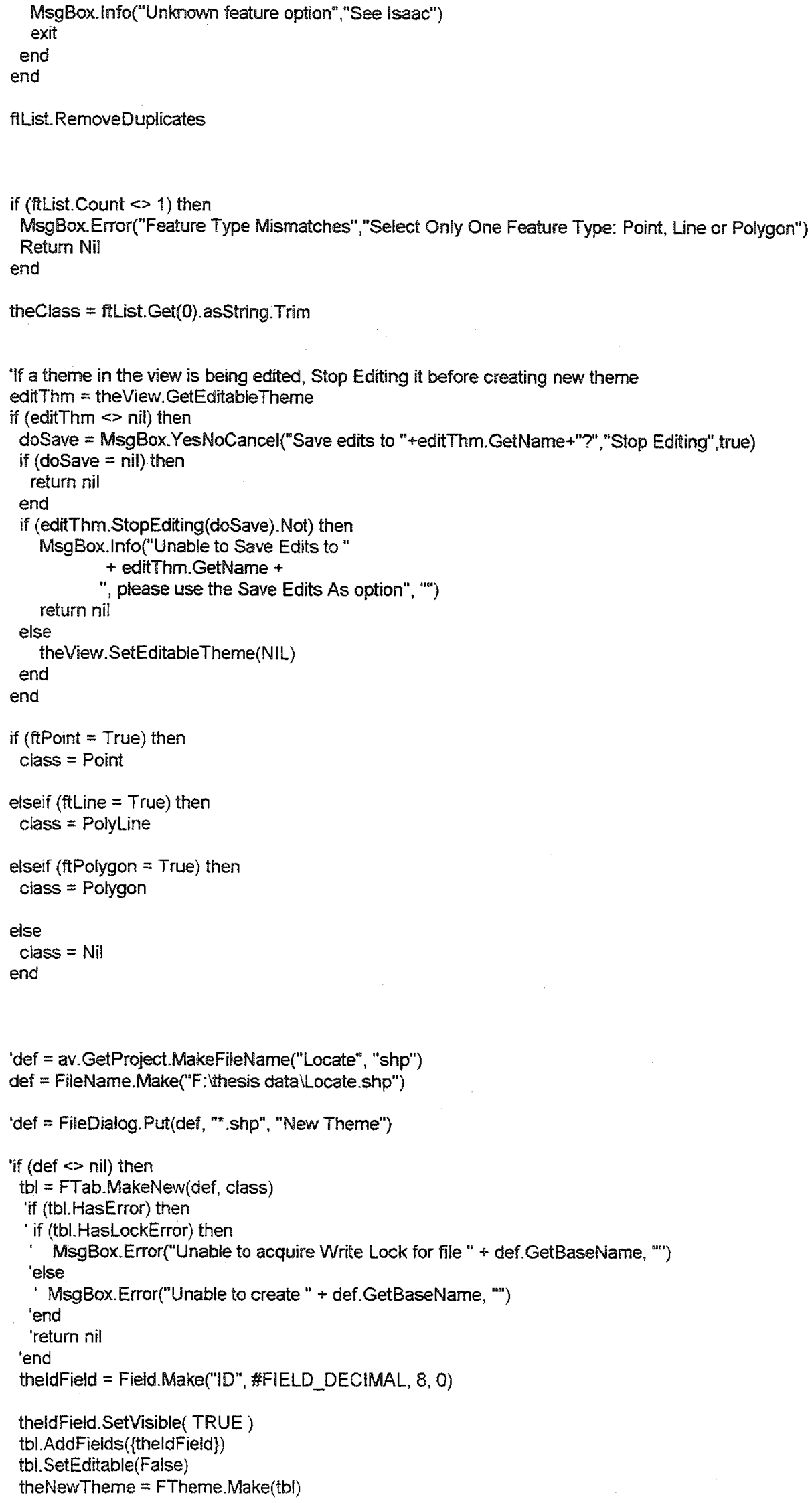




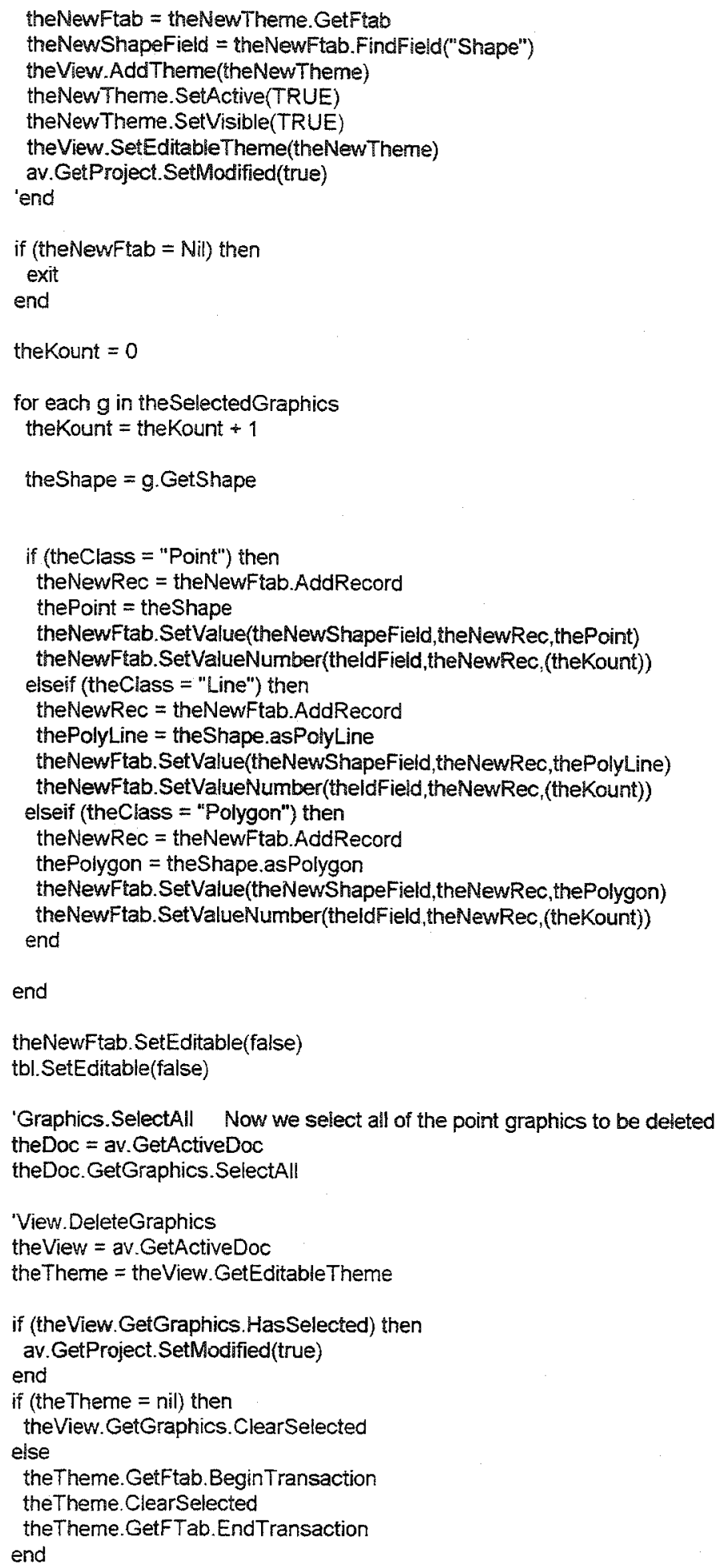




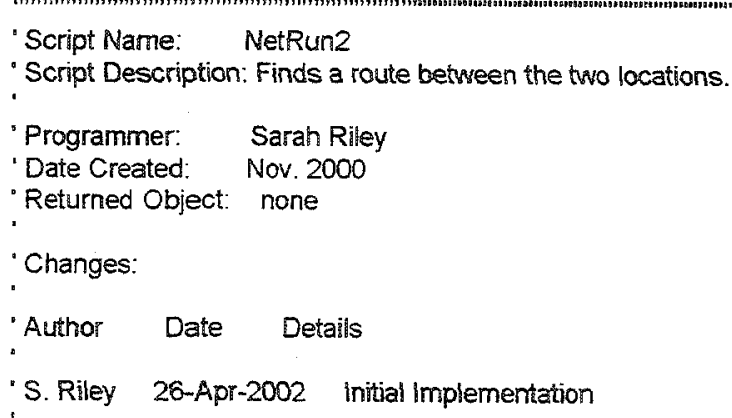

' Set the cost for solving a routing problem

' It then solves the problem and displays

' directions for the route.

' Get a list of available costs for this line theme. Choose a cost from the list.

aNetCostFieldList $=$ theNetDef.GetCostFieids

aNetCostField = MsgBox.Choice\{aNetCostFieldList, "Select Mintues", "Select Minutes")

' If a cost was chosen, set it.

if (aNetCostField < $>$ nil) then theNetwork.SetCostField(aNetCostField) end

'The above can be replaced by changing the hard code for Network Analyst wherein 'there will only be the chosen cost of MINUTES to select.

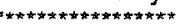

TravelDistance $=$ theNetwork.FindPath(thePointList, True, False) 'msgBox.Info( TravelDistance.AsString, "Total"++reportUnitStr)

'theView.GetGraphics.add(GraphicShape.Make(theNetwork.ReturnPathShape))

aSymbol = Symbol.Make(\#SYMBOL_PEN)

aSymbol.SetSize(2.0)

asymbol.SetColor(Color.GetMagenta)

theGL=theView. GetGraphics

theRoute $=$ GraphicShape. Make(theNetwork. ReturnPathShape)

theRoute.SetDisplay(av.GetActiveDoc.GetDisplay)

theroute. SetSymbol(aSymbol)

theGL.Add(theRoute)

'theroute.GetSymbol.SetColor(color.GetMagenta) SetSymbol(aSymbol)

theRoute.Draw

theroute. Invalidate 
'set view size for public viewing

theView = av.getproject.finddoc("Route Finding")

theWin = the View. GetWin

theWin.Resize(1025,600)

"theWin. Moveto $(0,0)$

theWin.Open

- Write the route result FTab, this is necessary to get directons.

tmpFileName $=$ FileName.Make ("F: thesis datalRoute.shp")

theNetwork. WritePath (tmpFileName)

resFTab = FTab.Make(SroName.Make(tmpFileName.AsString))

"Show the directions then remove the temporary flle.

"theDirections $=$ "Your route directions are as follows" $+\mathrm{NL}+\mathrm{NL}+$

" theNetwork. GetPathDirections(resFTab)

'msgBox.Report(theDirections, "Route Information")

'add theme to view

newTheme $=F$ Theme.Make $($ resFTab $)$

theView.AddTheme(newTheme)

newTheme.SetName("Route")

newTheme.SetVisible(True) 


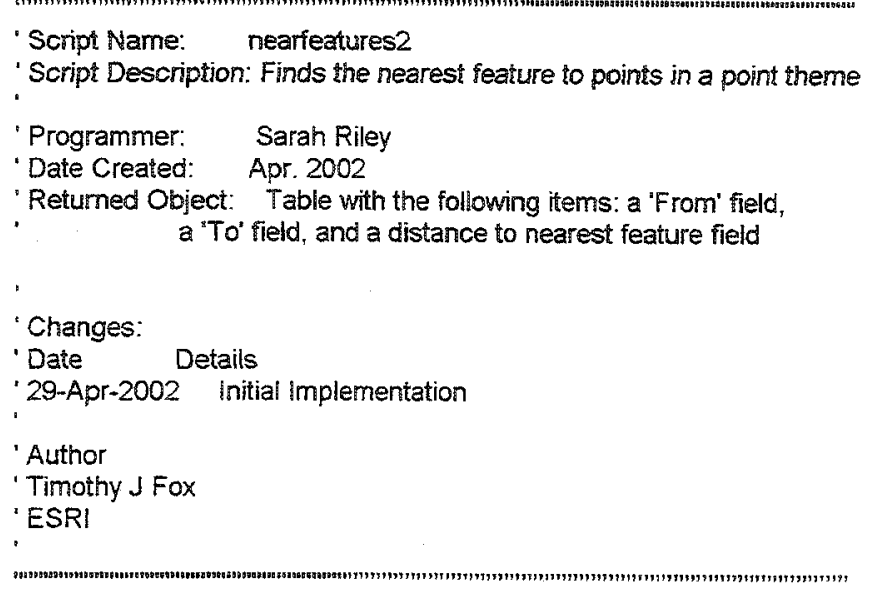

'Get 'From' theme and field - check for 'From' selection, and field

$f=$ v.FindTheme("Locate.shp")

"f = msgbox.list ( $t$, "Select the theme that contains" + NL + "the 'From' features." "From' theme")

if $(f=$ nil) then

- exit

'end

fromName $=$ f. GetName

fromShpFId = f.GetFTab.FindFieid("Shape")

-fFieldList $=$ f.GetFTab. GetFieids

'ff = msgbox.multilist (fFieldList, "Select "From' field to preserve" ,"From" field")

Field $1=$ f.GetFTab. FindField ("ID")

"Field2 = f.GetFTab.FindField("Address")

'fFieldList $=\{$ Field1, Field2 $\}$

'if (ff $=$ nil) then

" msgbox.warning("you must select a field" ,w)

- exit

'end

if (f. GetFTab. GetSelection. Count $=0$ ) then

fromFeatures $=$ f.GetFTab

from Total $=$ f. GetFTab. GetNumRecords

else

fromFeatures $=$ f.GetFTab. GetSelection

from Total $=$ fromFeatures . Count

end

'get ' $T o$ ' theme and field

$t=v$.FindTheme $(" B u s$ Stops")

't = msgbox.list ( $t$, "Select 'To' theme", "To' theme")

'if $(t=$ nil $)$ then

' exit

'end

toName $=$ t.GetName

toShpFid $=$ t.GetFTab.FindField("Shape")

'tFieldList $=$ t.GetFTab.GetFields

'ti = msgbox.multilist (tFieldList, "Select field to preserve", "To' field") 
'if $(t f=$ nil $)$ then

' msgbox.warning("you must select a field", w)

' exit

'end

Field3 = t.GetFTab.FindField("Bus_stop")

Field4 $=$ t.GetFTab.FindField ("On street")

Field5 = t.GetFTab.FindField("At_street")

'tFieldList $=\{$ Field 3, Field4, Field5 $\}$

\section{'get 'To' Theme's selection}

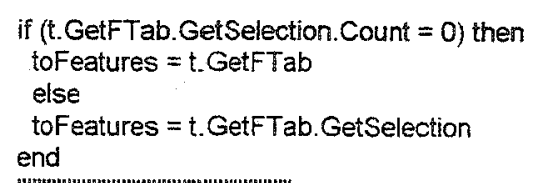

'create new table

tempFilename = FileName.Make("F.thesis datainear.dbf")

"theFilename = FileDialog.Put(tempFilename, "^.dbf", "Save Table where?")

'if (theFilename $=$ nil) then

' exit

'end

tempFilename.SetExtension("dbf")

'theFilename.SetExtension("dbr")

'newVTab = VTab.MakeNew (theFilename, dBASE

newVTab = VTab.MakeNew (tempFilename, dBASE)

'fID = ff.clone

'tID $=$ tf.clone

$\mathrm{f} 1=$ Field 1 . clone

' $\mathrm{f} 2$ = Field2.clone

$\mathrm{t} 1=$ Field 3 .clone

$12=$ Field 4 . clone

$\mathrm{t} 3=$ Field5 .clone

distField = Field.Make("NF Dist",\#FIELD_DECIMAL,8,2)

newfields $=\{11, t 1, t 2,13$, distField $\}$

newVTab.AddFields(newFields)

' Find the closest feature to the point...

av.ShowMsg("NF analysis: From"+ fromName + "To " + toName )

for each frec in fromFeatures

fPoint $=$ f.GetFTab. ReturnValue(fromShpFld,frec)

theCount $=$ theCount +1

for each trec in ToFeatures

tShape $=$ t.GetFTab.ReturnValue(toShpFid,trec)

dist $=$ tShape. Distance (fPoint)

if (dist < shortDist) then

shortDist $=$ dist

shorTRec $=$ trec. Clone

end

end

'Write to table: 'distance','From' and 'To' fields

'tempTid = t.GetFTab. ReturnValue(tf, shortTRec)

tempFid $1=$ f.GetFTab. ReturnValue(Field 1, frec)

'tempFid2 = f.GetFTab. ReturnValue(Field2, frec)

tempTid3 = t.GetFTab. ReturnValue(Field3, shortTRec)

tempTid $4=$ t.GetFTab.ReturnValue(Fieid4,shortTRec)

tempTid5 = t.GetFTab. ReturnValue(Field5, shortTRec)

newVTab. SetEditable (true)

newRec = newVTab.AddRecord

newVTab.SetValue (f1, newRec,tempFid1)

'newVTab.SetValue(f2, newRec,tempFid2) 
newVTab.SetValue(t1, newRec,tempTid3)

newVTab. SetValue(t2, newRec,tempTid4)

newVTab.SetValue(t3, newRec,tempTid5)

'newVTab.SetValue(ti, newRec, tempTid)

newVTab.SetValue(dist Field, newRec,shortDist)

shortDist $=100000000$

av.SetStatus ((theCount $/$ from Total) $* 100)$

end

av.ClearStatus

av.ClearMsg

newVTab.SetEditable (false)

' create table from VTab \& display table

newTab = table. Make (newVTab)

newTab.setName ("near.dbf")

newTabWin = newTab. GetWin

newTabWin.Resize $(350,250)$

newTabWin.Open 


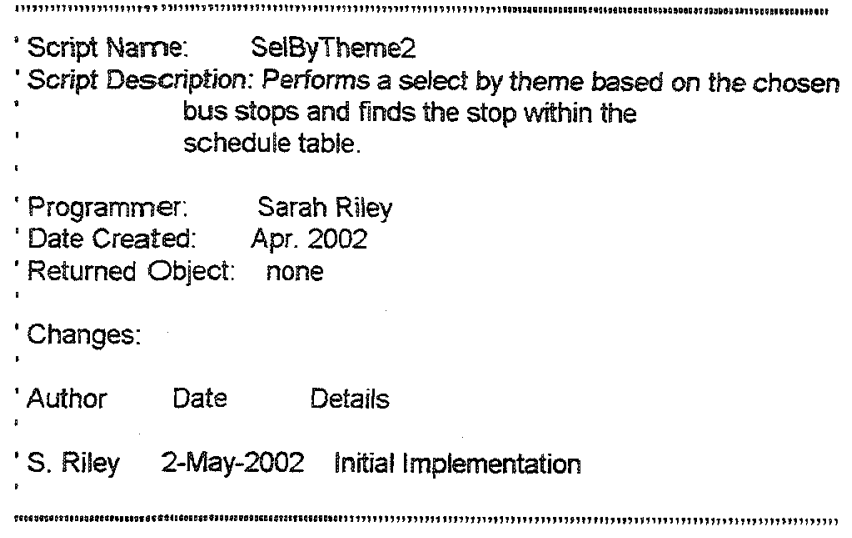

'Features will be selected from aTheme

'based on anotherTheme

aView = av.GetProject.FindDoc("Route Finding")

theWin $=$ a View. GetWin

theWin. Open

theThemeList=aView.get Themes

for each $i$ in (0..(the Themelist.count-1))

temp=the ThemeList.get(i). setActive(FALSE)

end

aTheme = aView. FindTheme("Bus Stops")

aTheme.SetActive(TRUE)

anotherTheme = aView. FindTheme("Locate.shp")

theFTab = anotherTheme.GetFTab

'get the first selected record for merging

if $($ theFTab $=$ nil)then

MsgBox.Info("No table...", "')

exit

end

theBitmap = Bitmap.Make(theFTab.GetNumRecords)

for each rec in (0..(theFTab.GetNumRecords-1)) by 2

theBitmap.Set(rec)

end

theFTab. SetSelection(theBitmap)

theFTab. UpdateSelection

'msgbox.info(av.GetActiveDoc.GetDisplay.GetUnits.AsString, "')

aDistance = Units.Convert(1.5, \#UNITS_LINEAR_KILOMETERS, av.GetActiveDoc.GetDisplay.GetUnits )

aReIType = \#FTAB_RELTYPE_ISWITHINDISTANCEOF

'aDistance $=2$ 'make sure this is $\mathrm{km}$ else it would be meters

'- this can change-maybe do a for statement until a bus stop is found

aSeIType = \#TAB_SELTYPE_AND

aTheme.SelectByTheme(anotherTheme, aReIType, aDistance, aSeIType) 


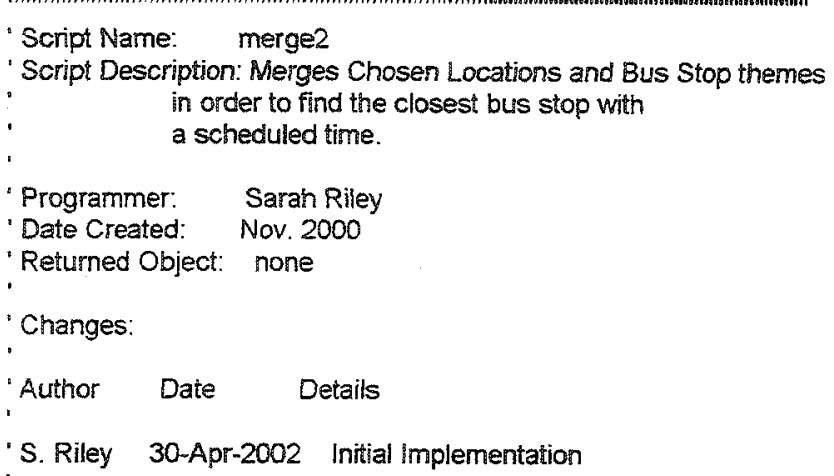

' Create the list af fields used for the output theme. The fields ' are taken from the first active theme only, it is assumed that ' other themes have an identical set of fields. If this is not the ' case the themes will still be merged, however fields not found in other themes will be empty..

fieldList $=$ List.Make

for each $f$ in themesToMerge.Get(0).GetFTab.GetFields 


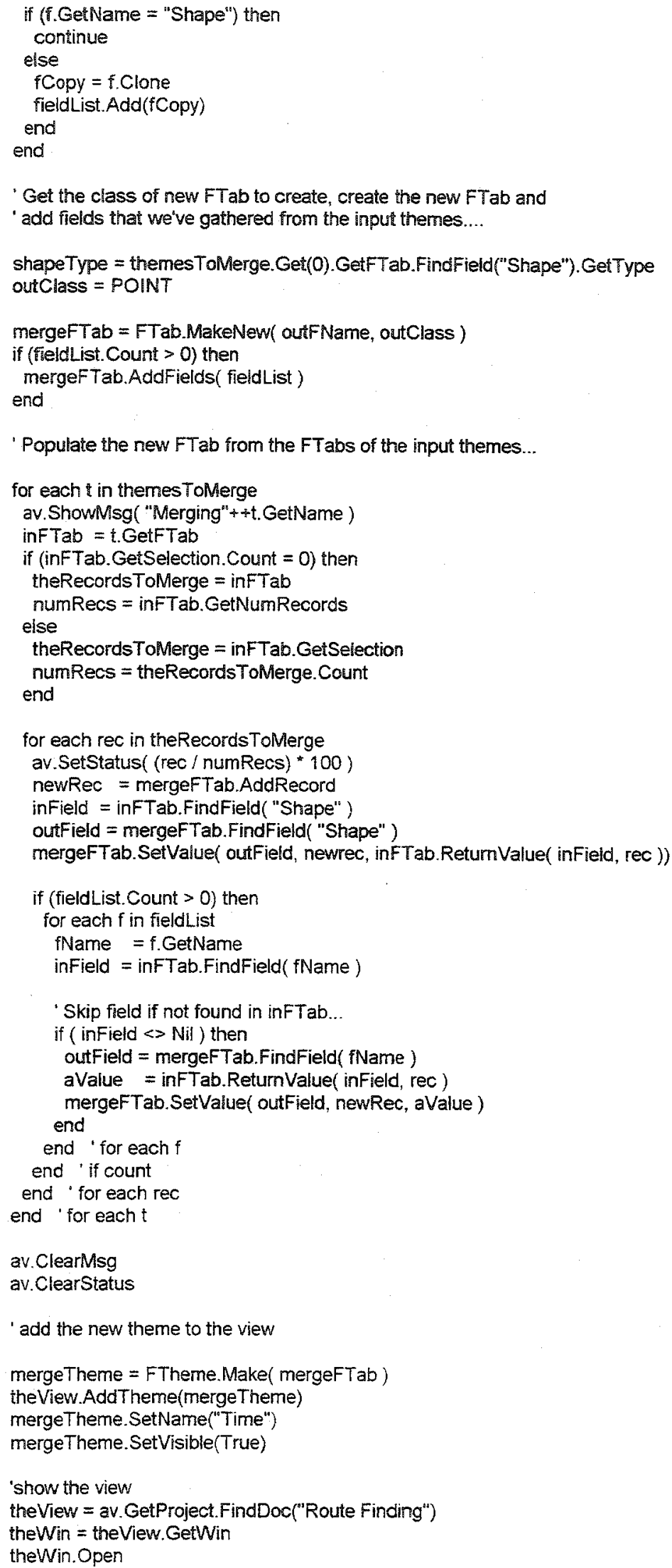




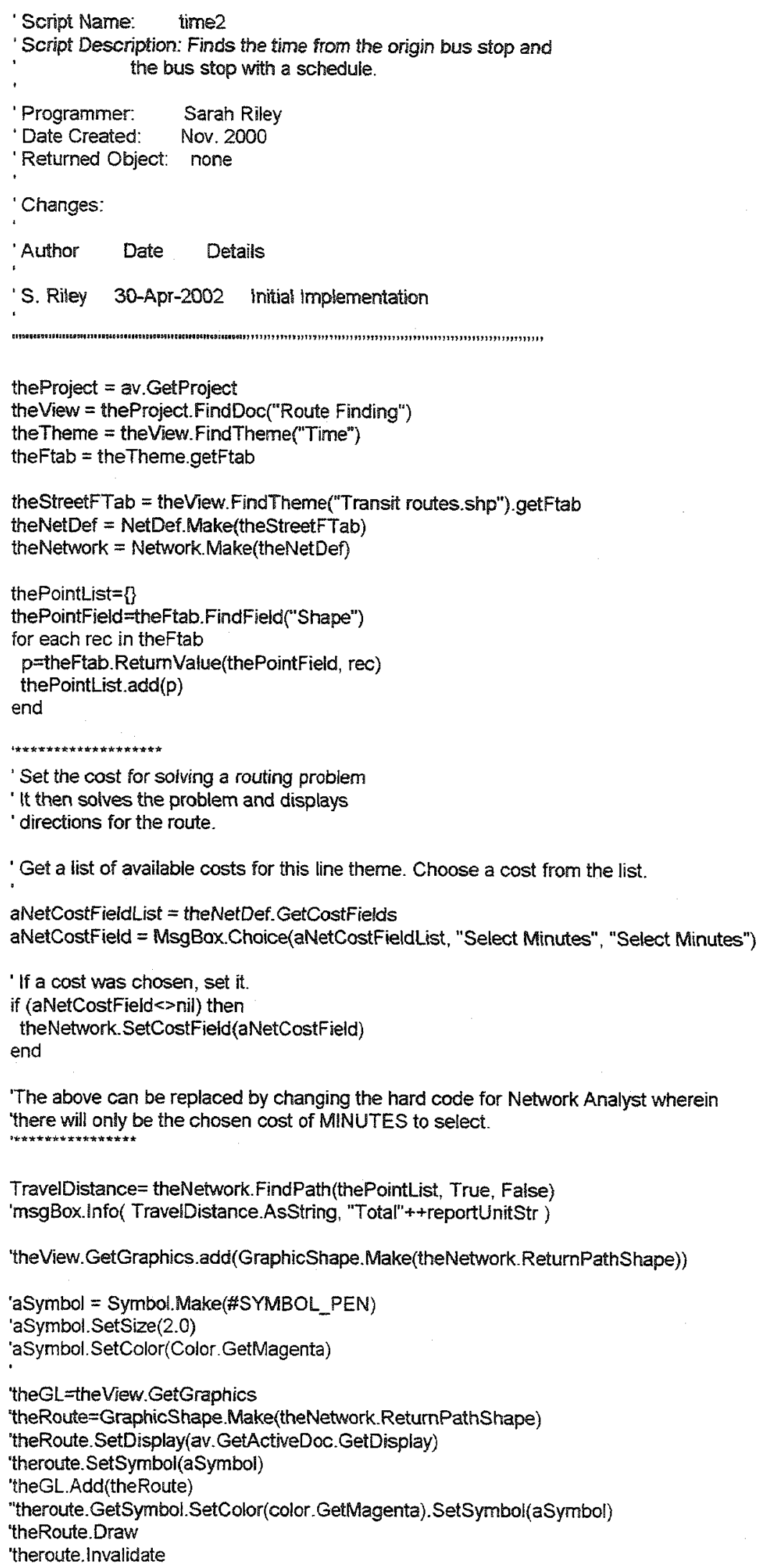


'set view size for public viewing

theView = av.getproject.FindDoc("Route Finding")

theWin = theVlew. GetWin

'theWin.Resize(1025,600)

theW in.Moveto $(0,0)$

theWin. Open

'Write the route result FTab, this is necessary to get directons.

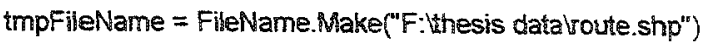

theNework. WritePathtmpFileName)

resFTab = FTab. Make(SrcName.Make(tmpFileName.AsString))

' Show the directions then remove the temporary file.

theDirections $=$ "Your route directions are as follows" $+N L+N L+$

- theNetwork. GetPathDirections(resFTab)

insg Box.Report(theDirections, "Route Information")

'gets the view, sets the time invisible

theView = av.GetProject.FindDoc("Route Finding")

theTheme = theView.Find Theme("Time")

the Theme. SetActive(FALSE)

theTheme.SetVisible(FALSE)

'add theme to view

new Theme $=$ FTheme.Make (resFTab)

theView.AddTheme(newTheme)

newTheme.SetName("Route2")

newTheme.SetVisible(True) 


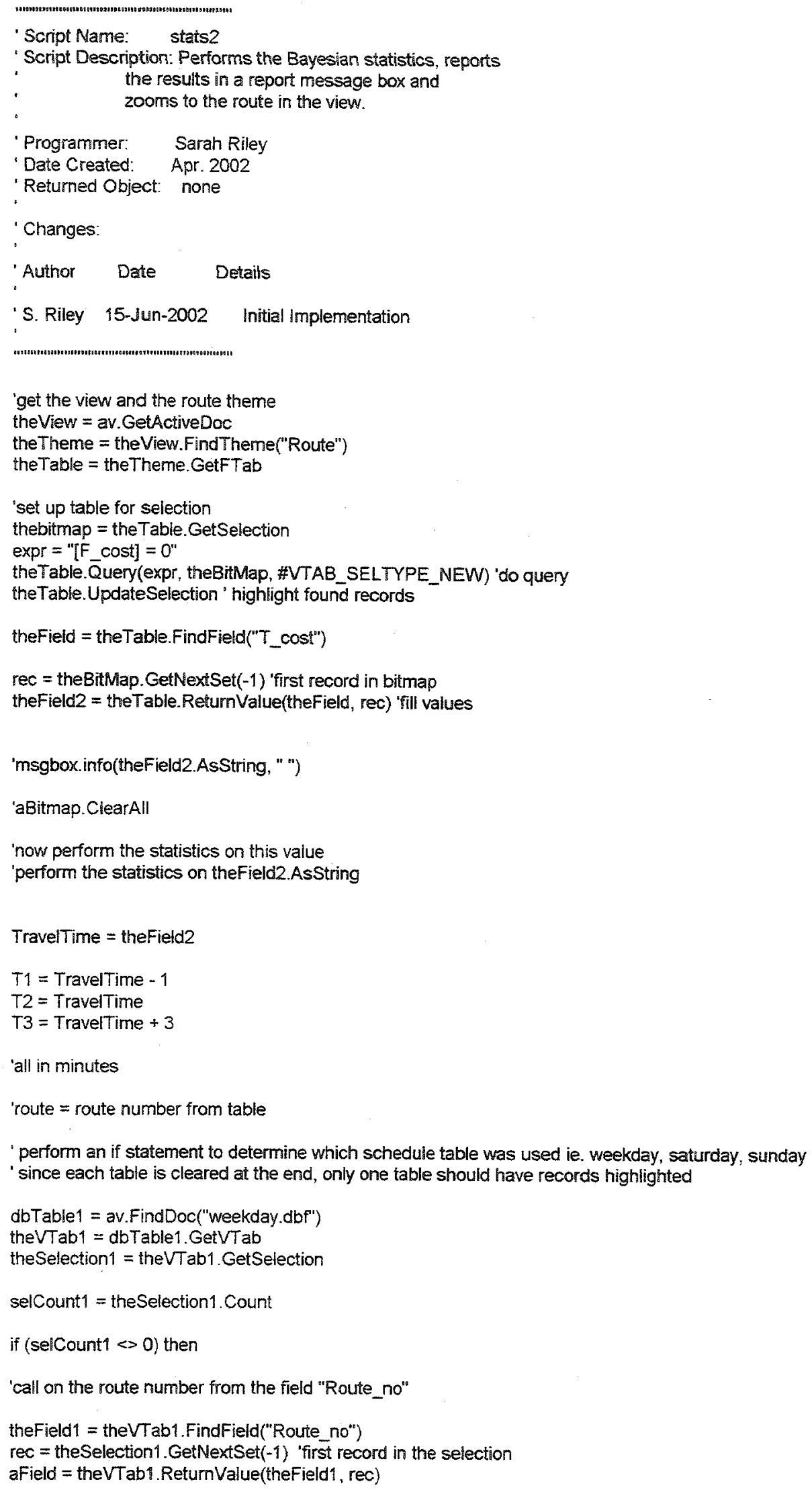




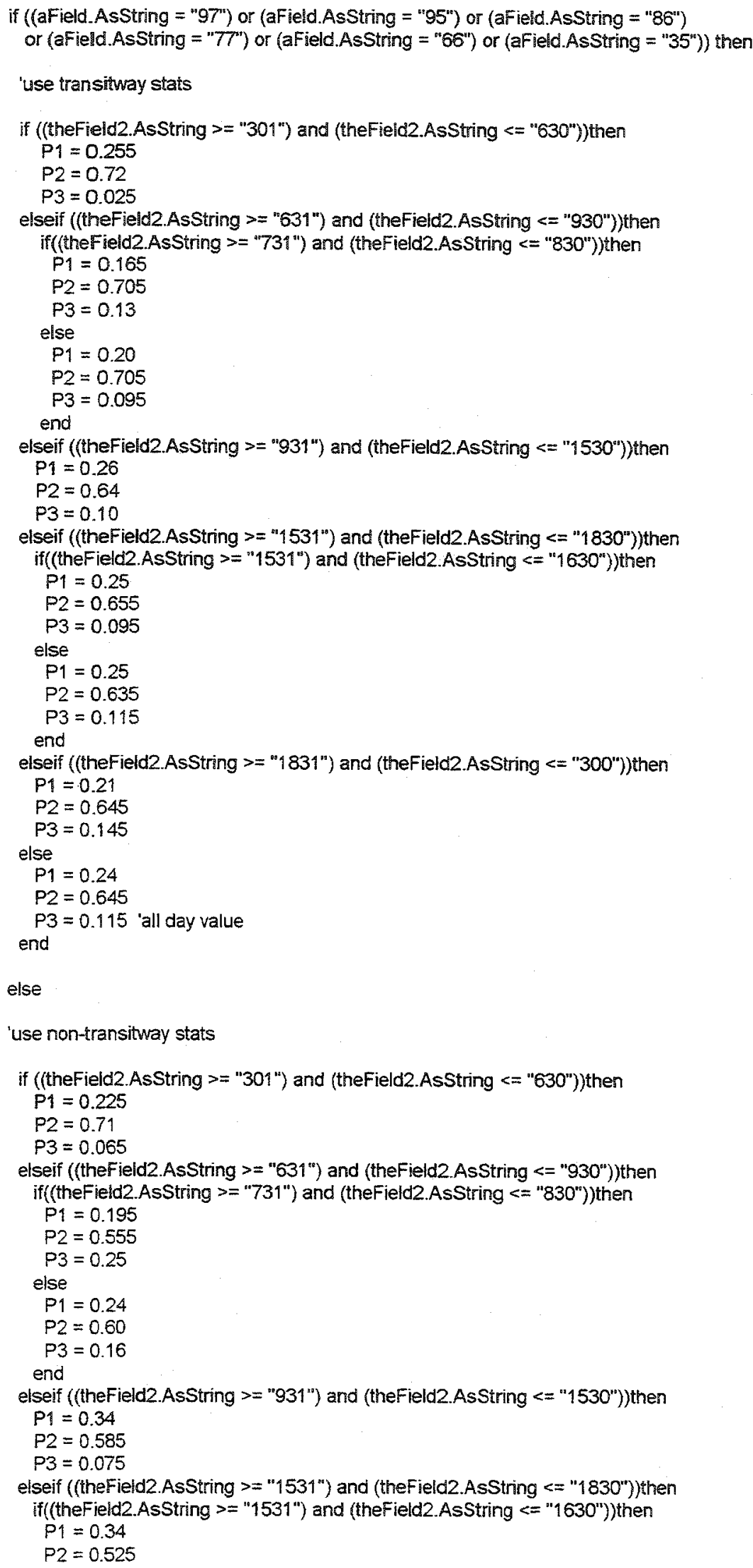




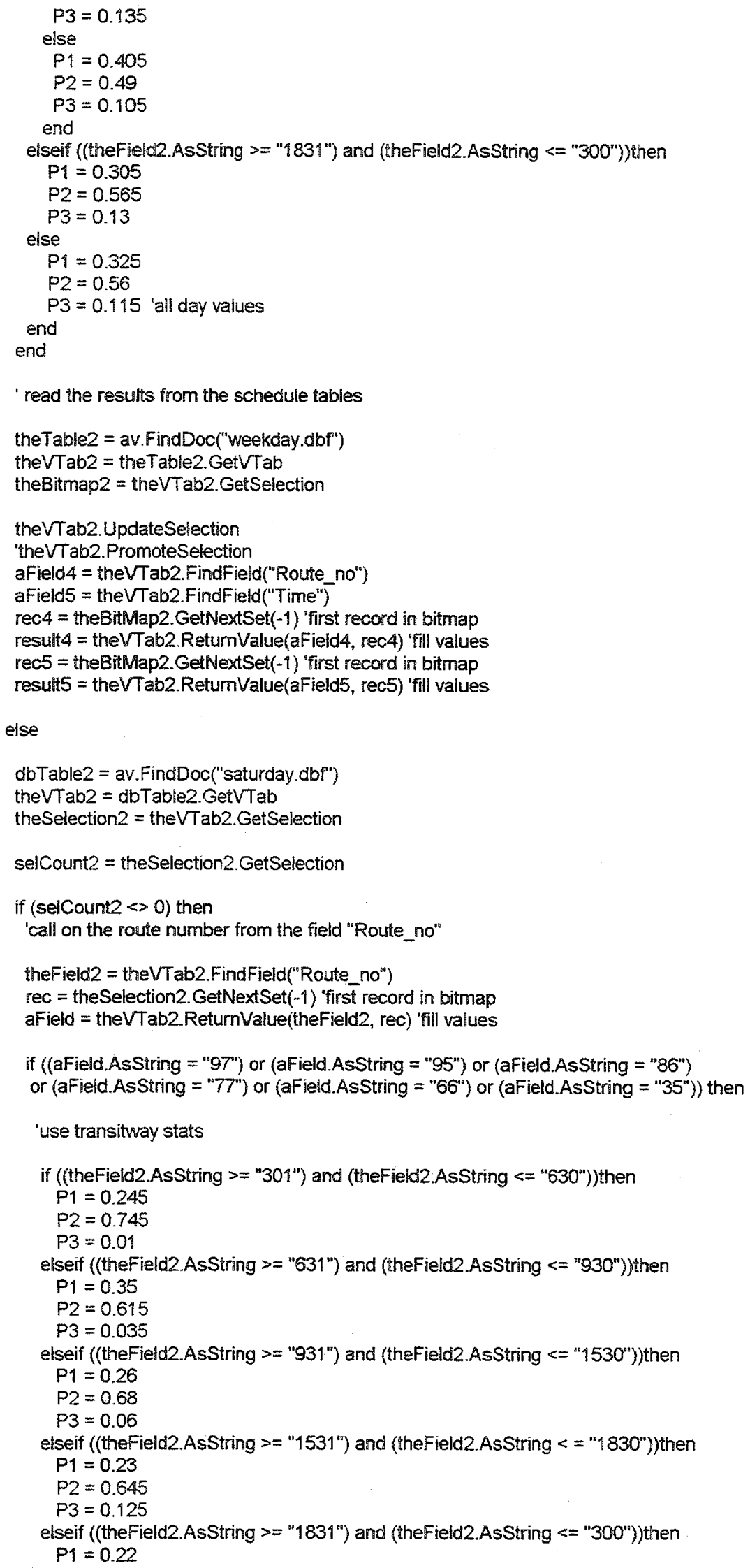




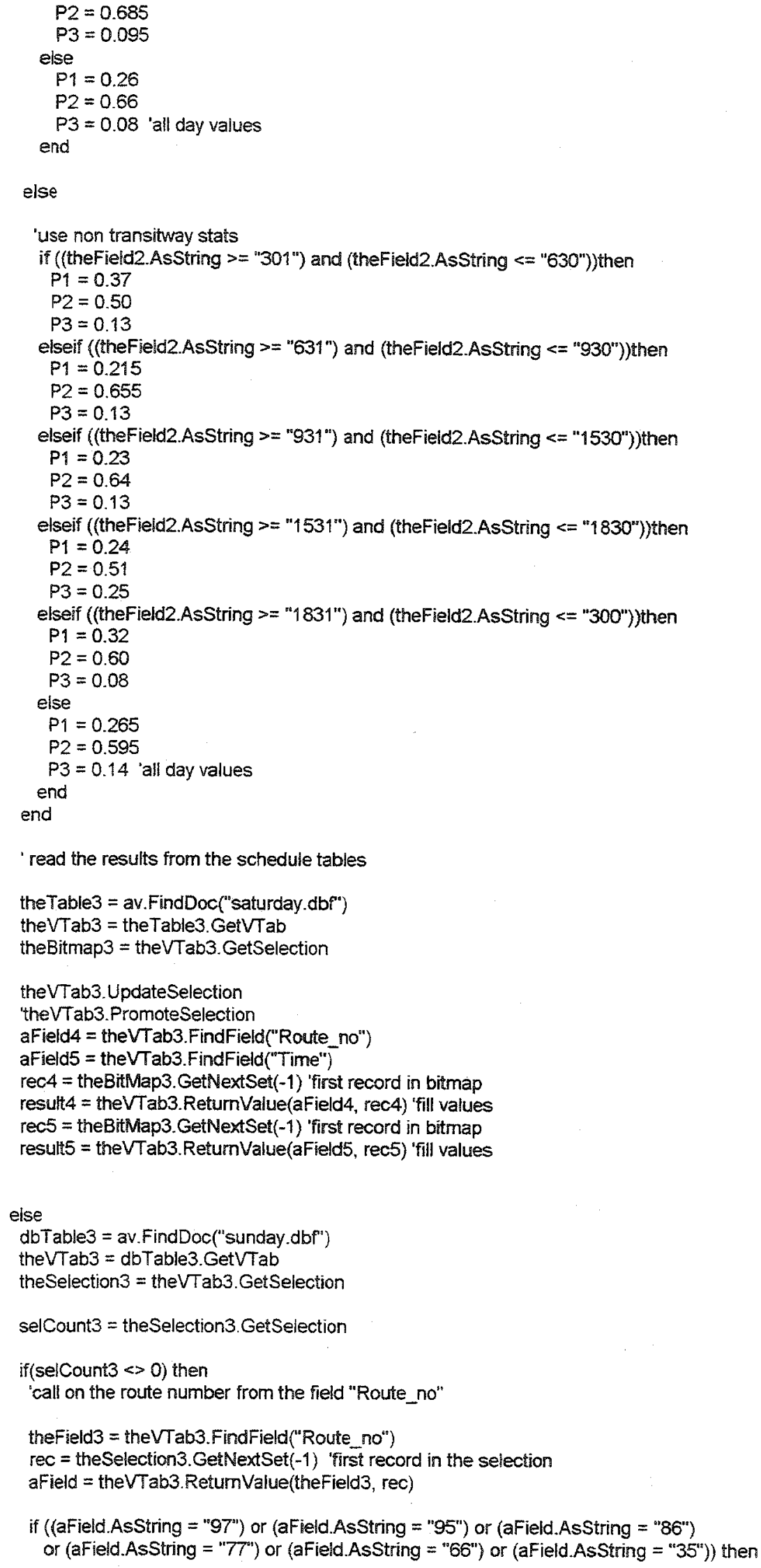




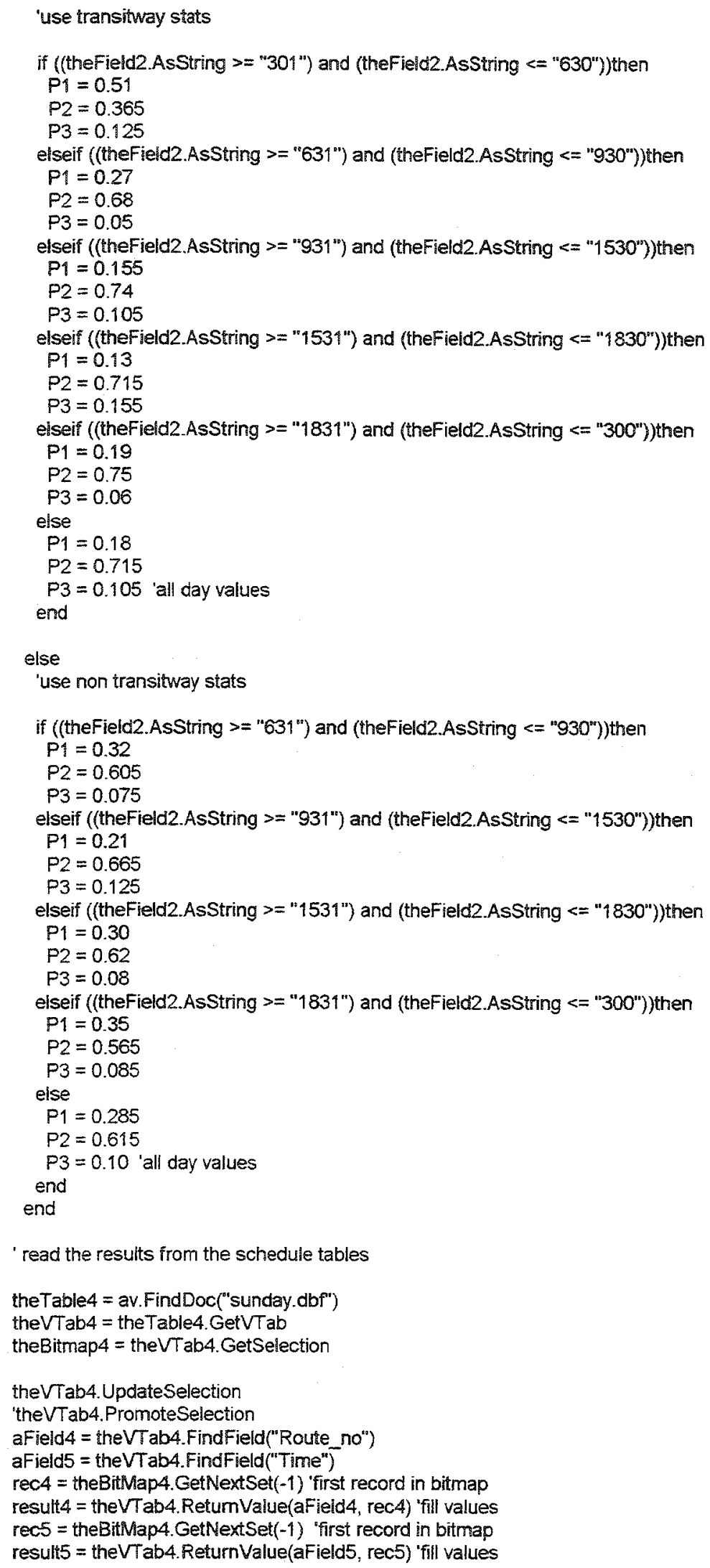




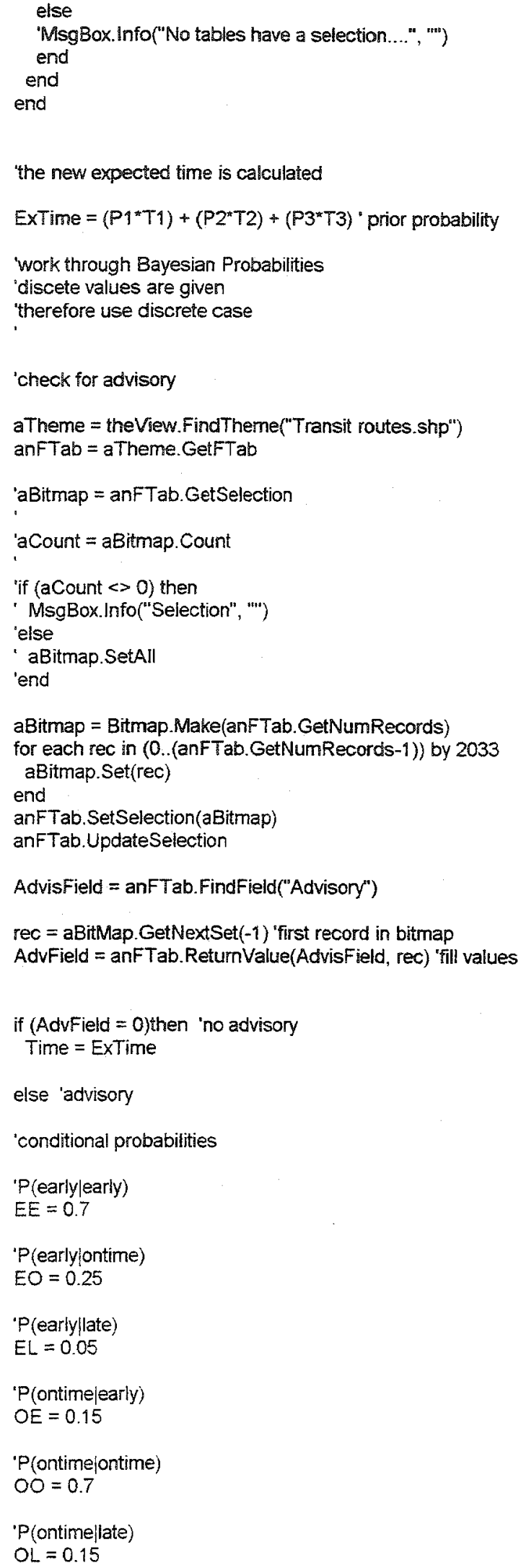




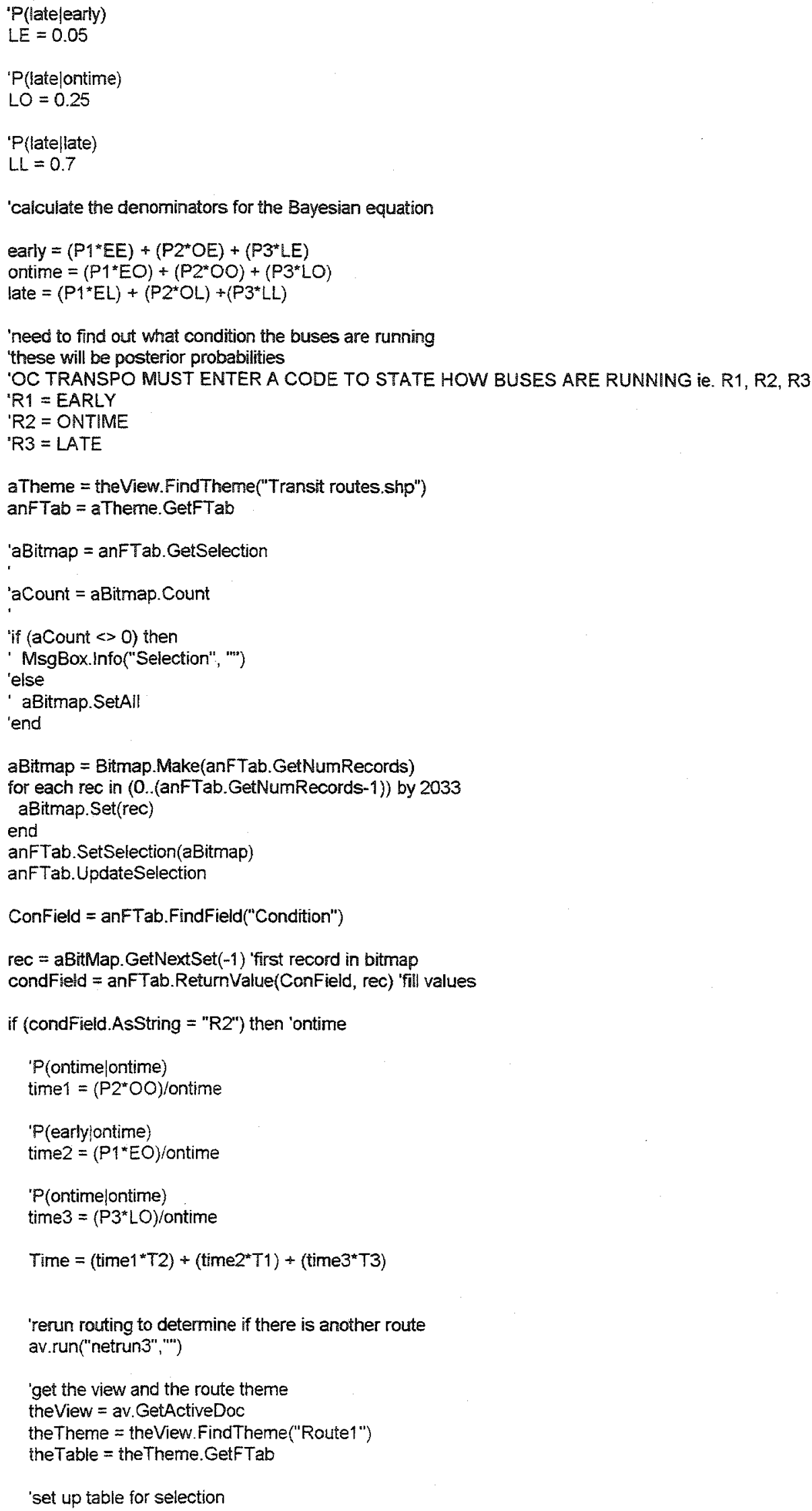




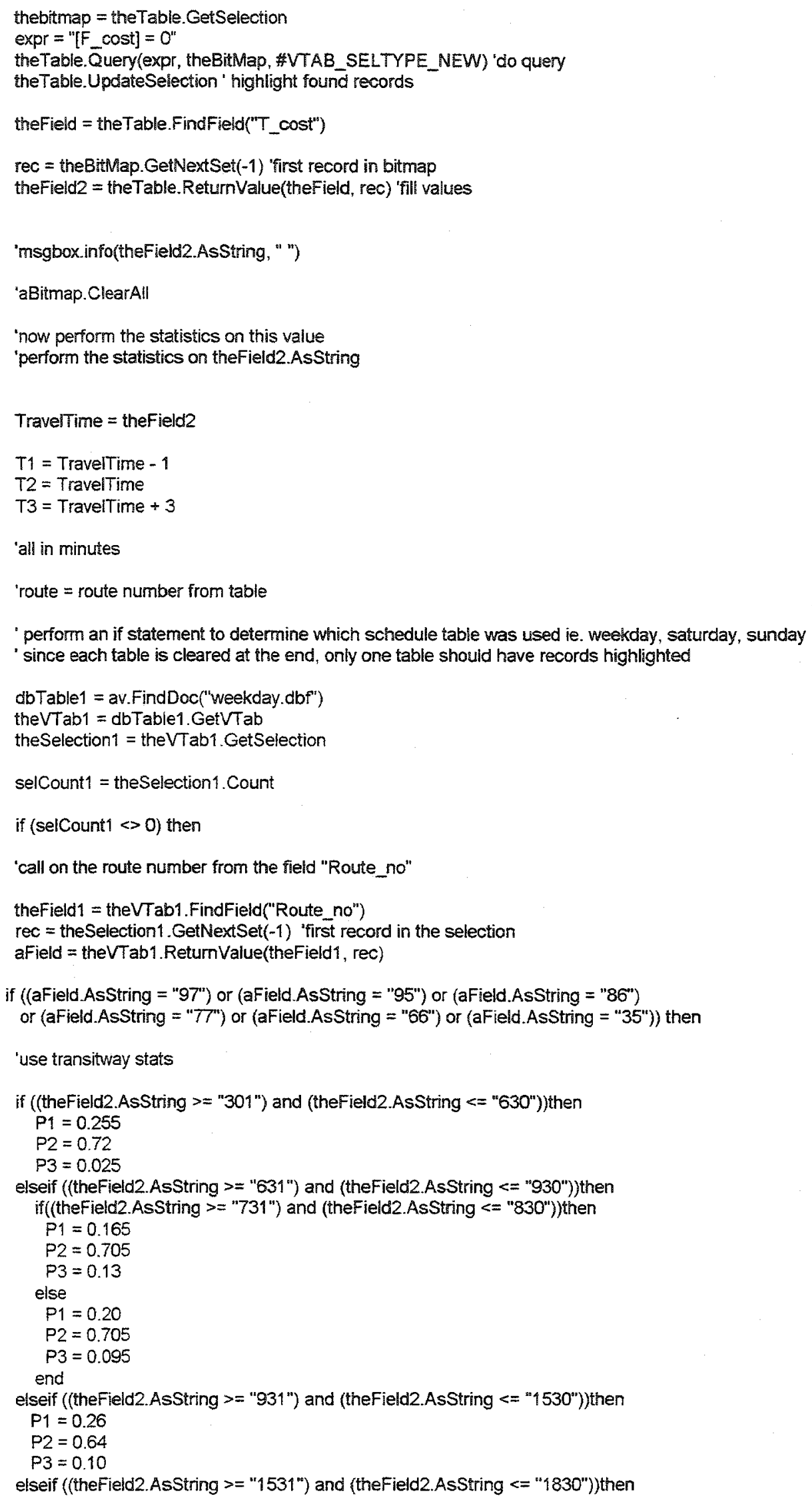




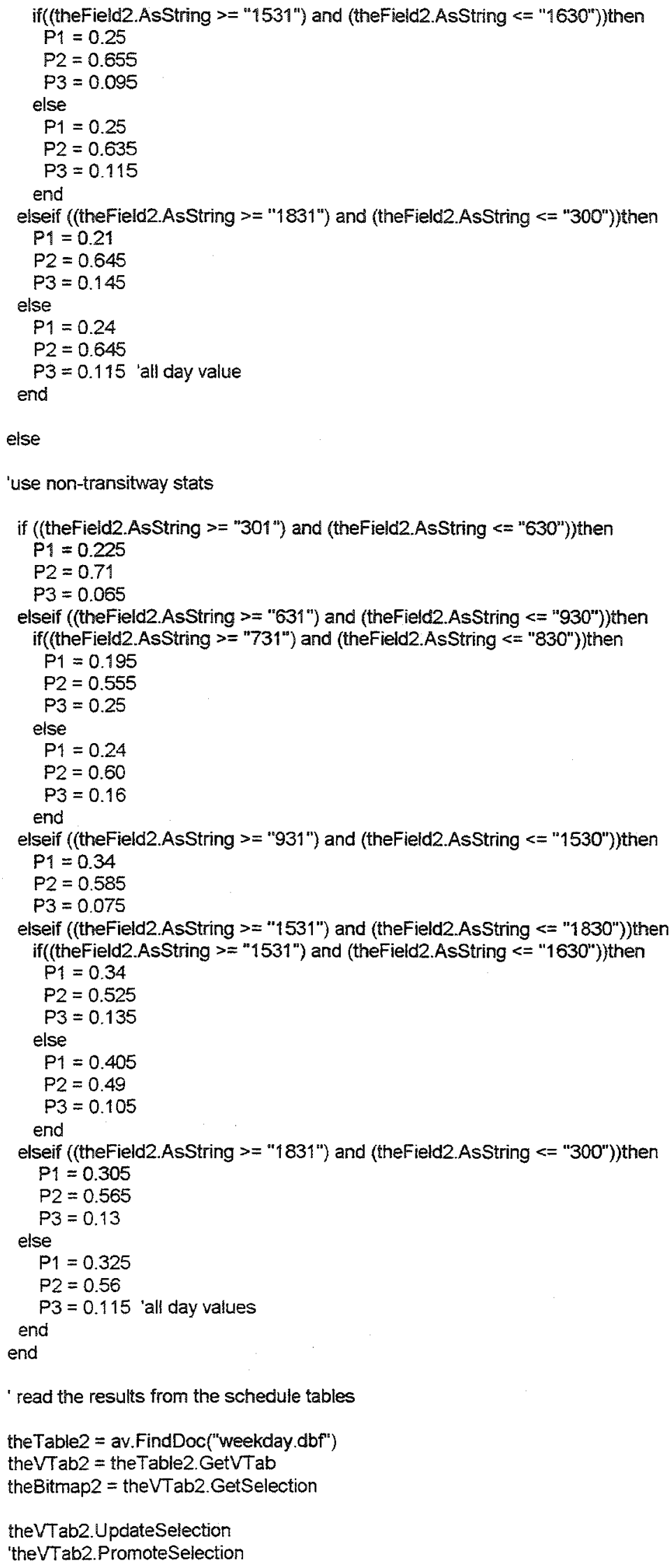




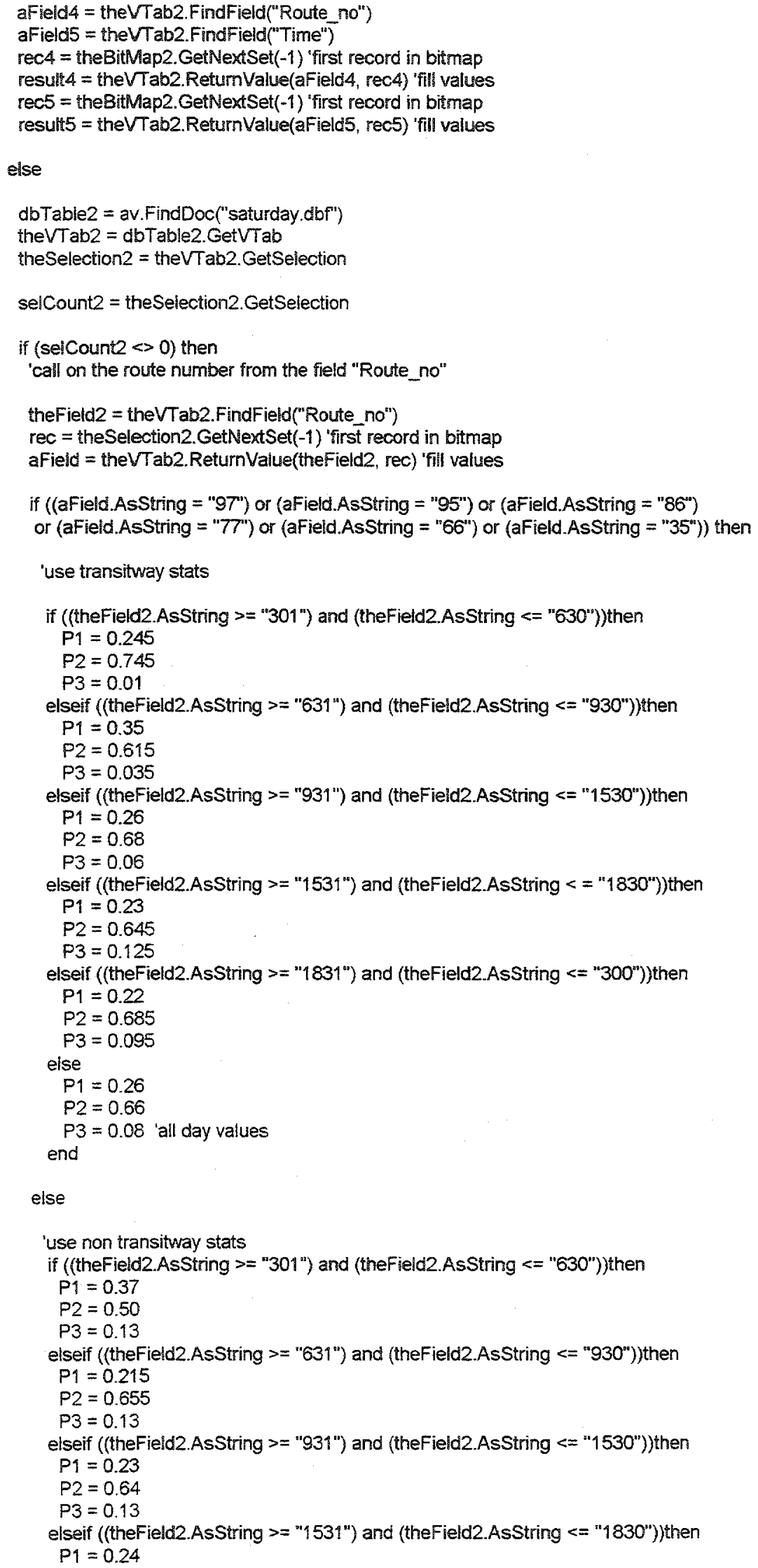




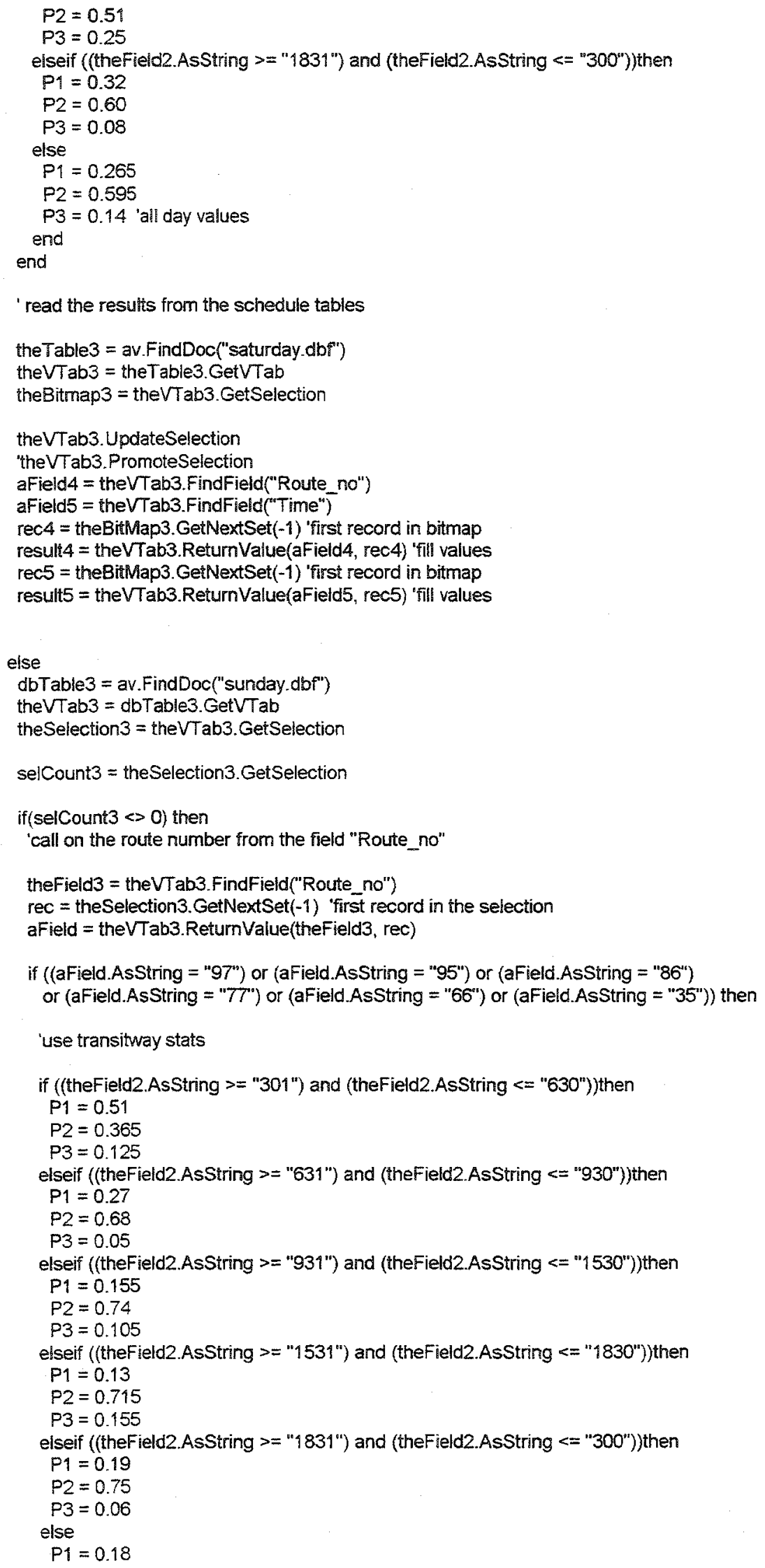




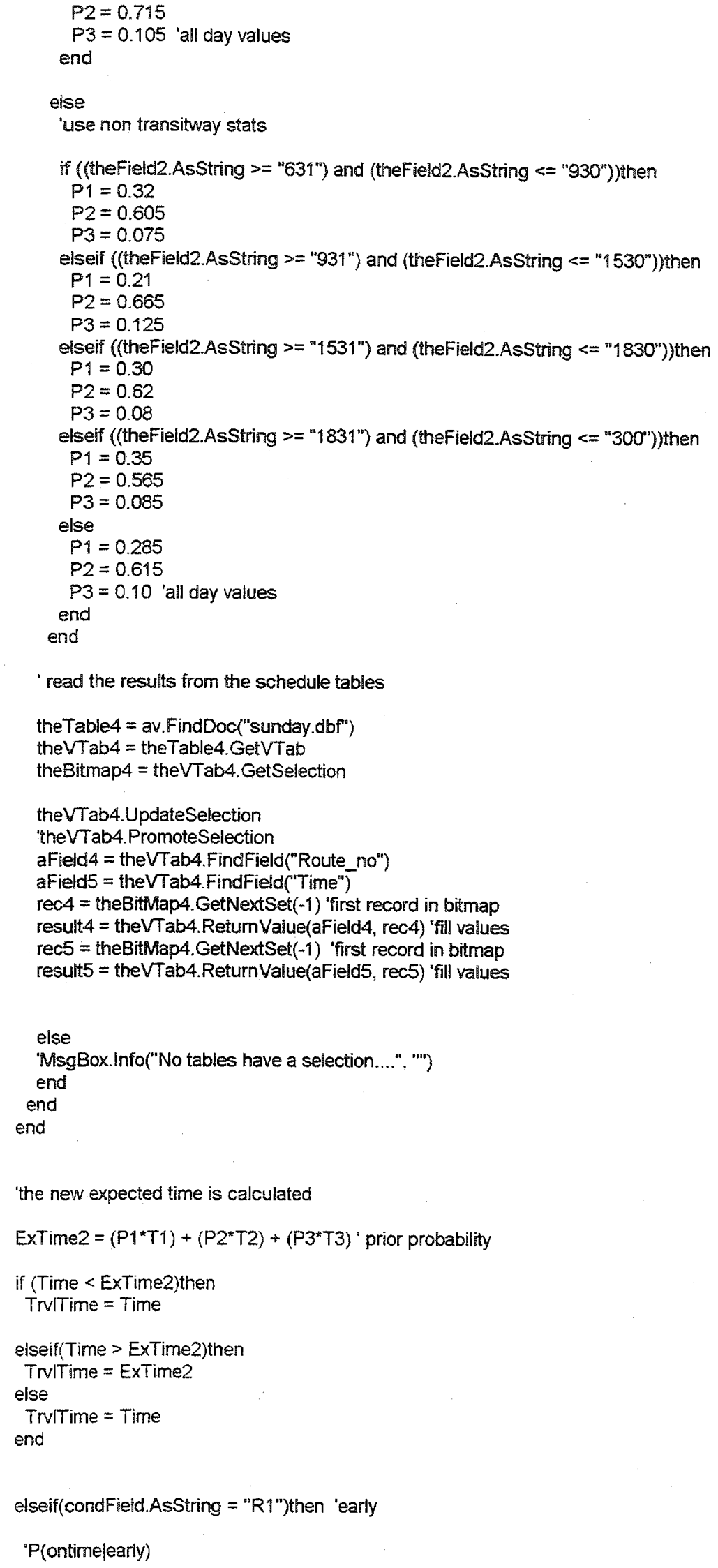




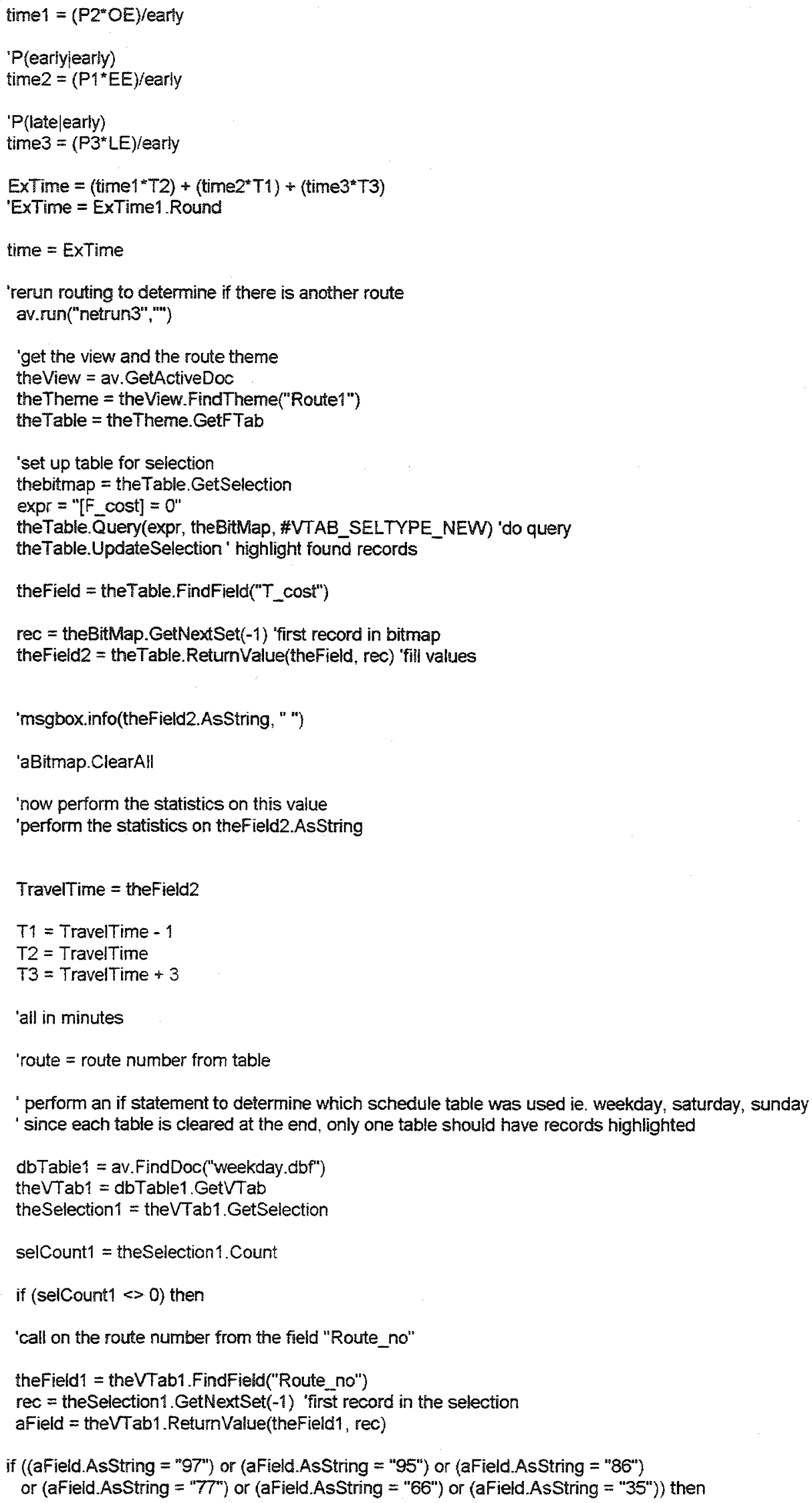




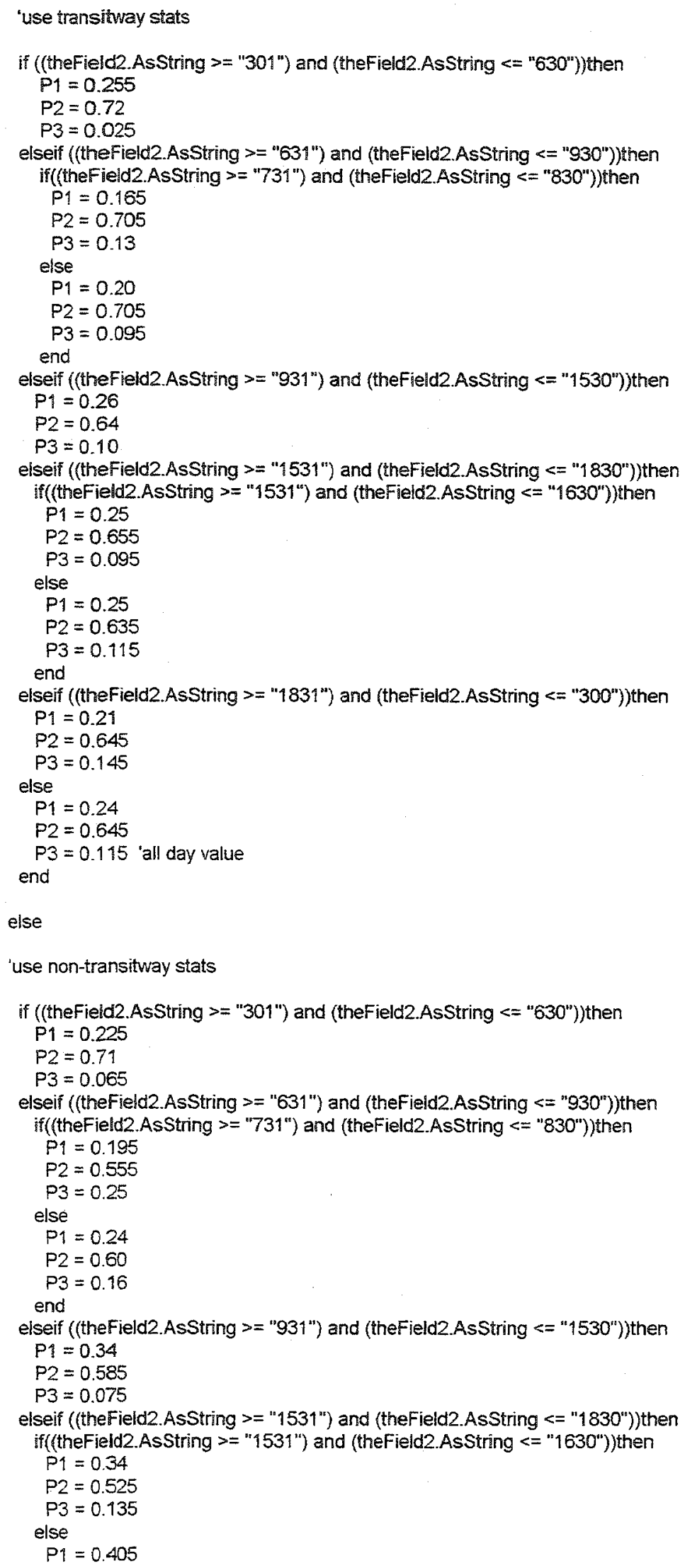




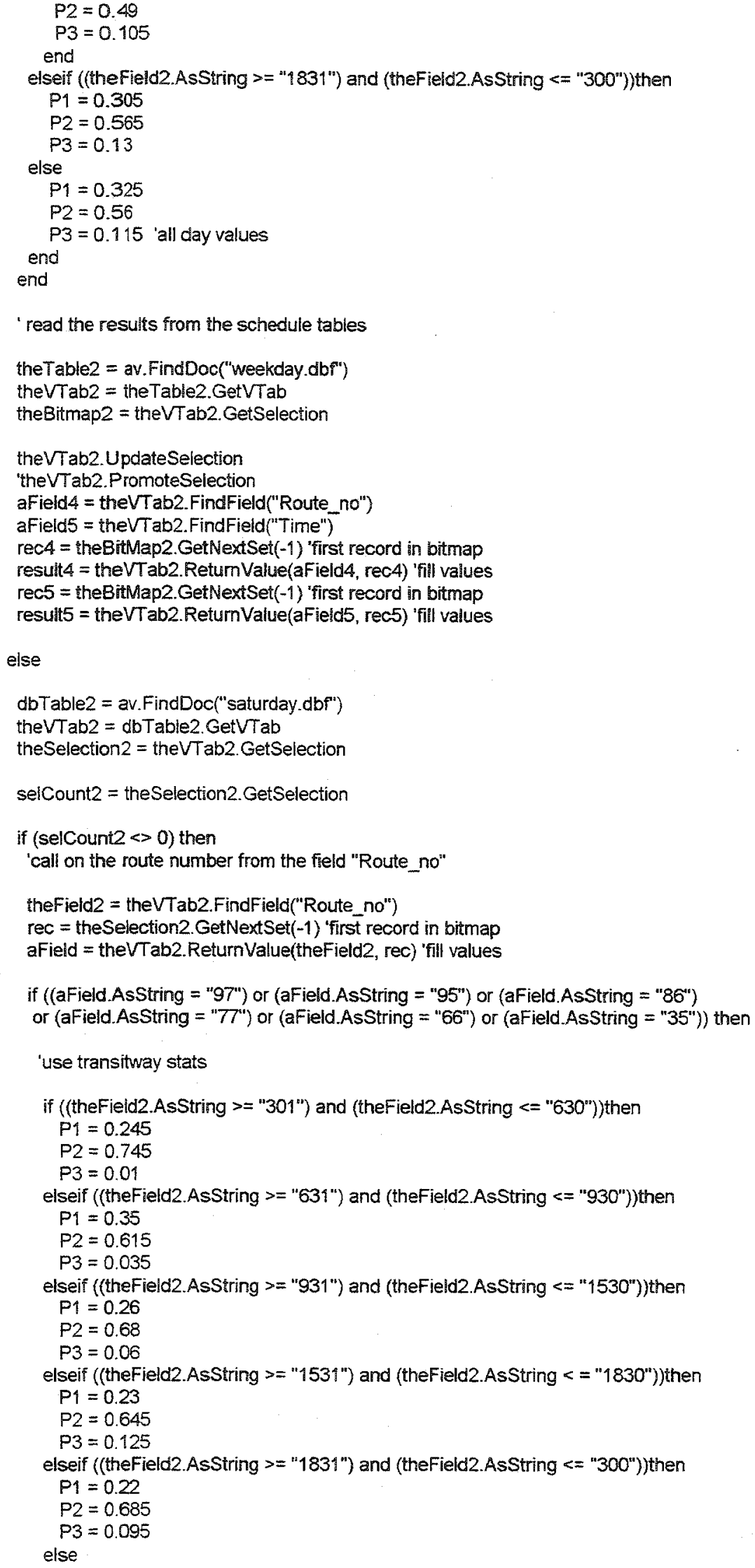




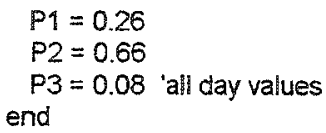




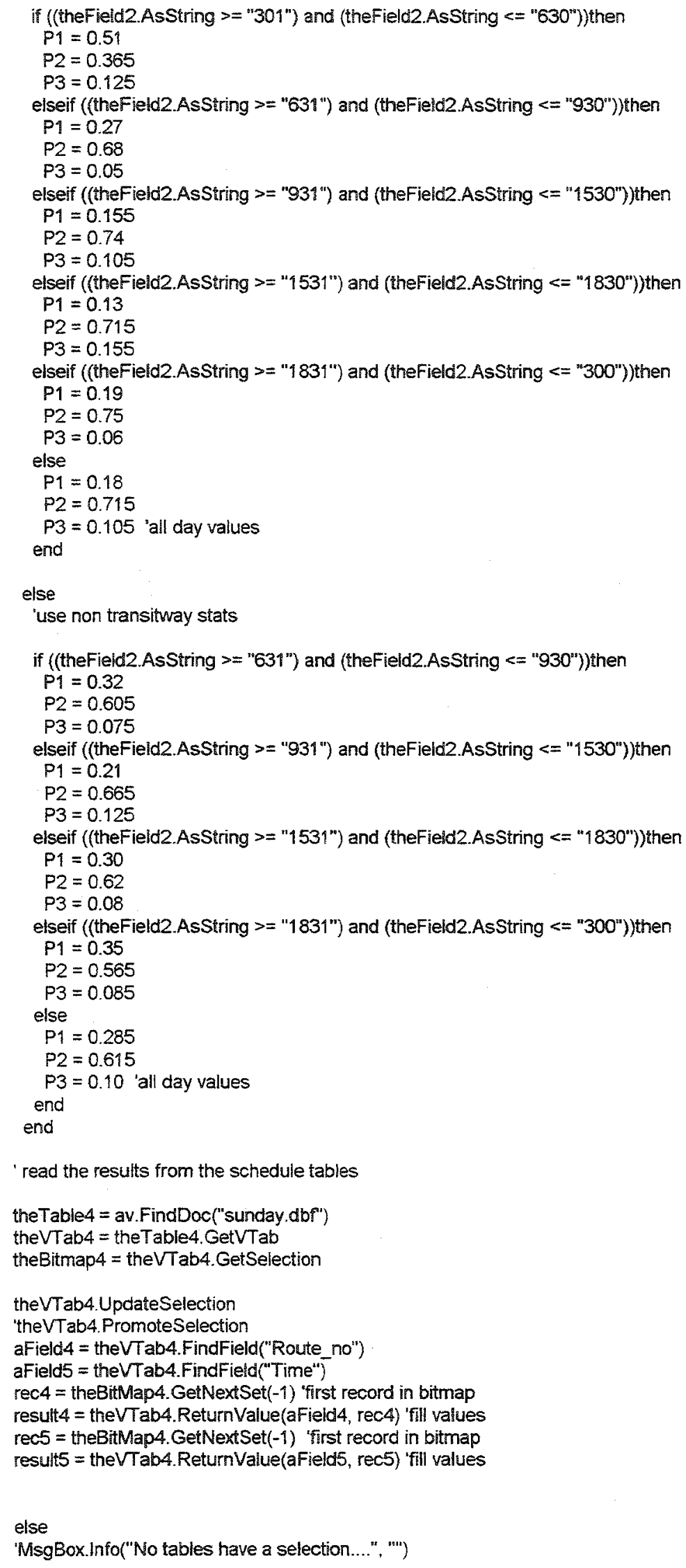




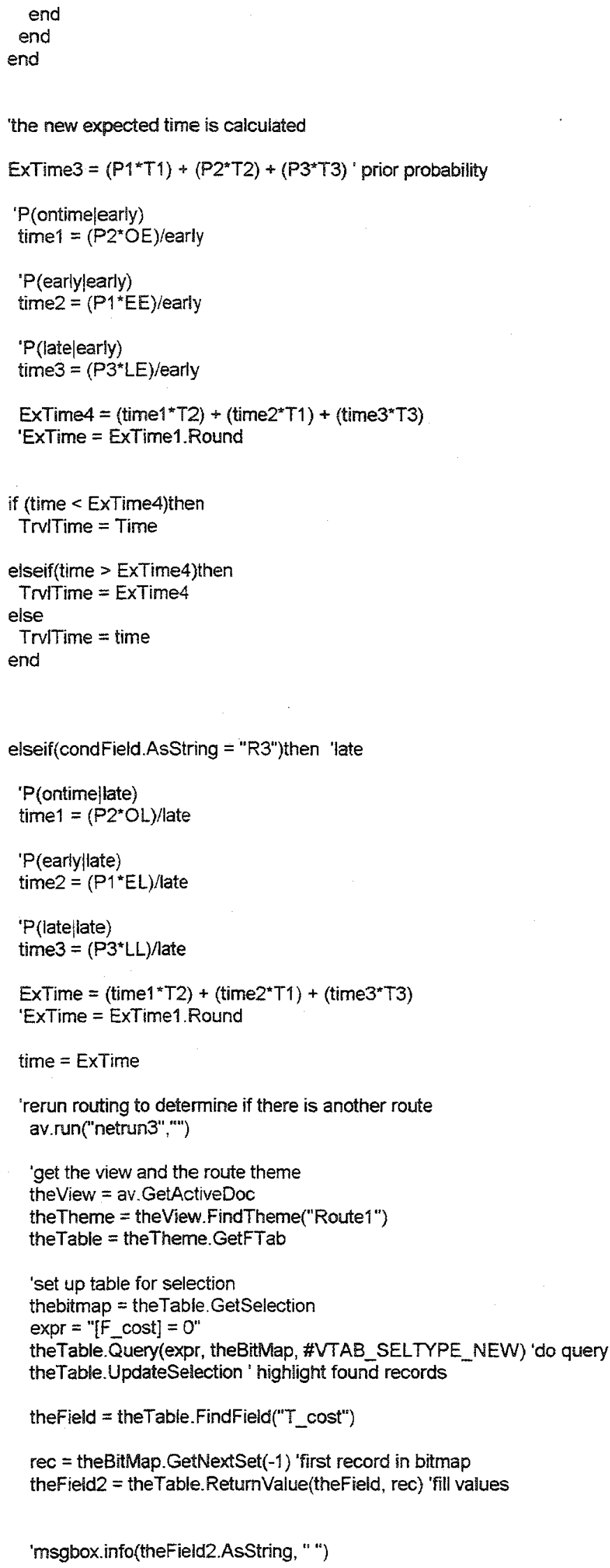




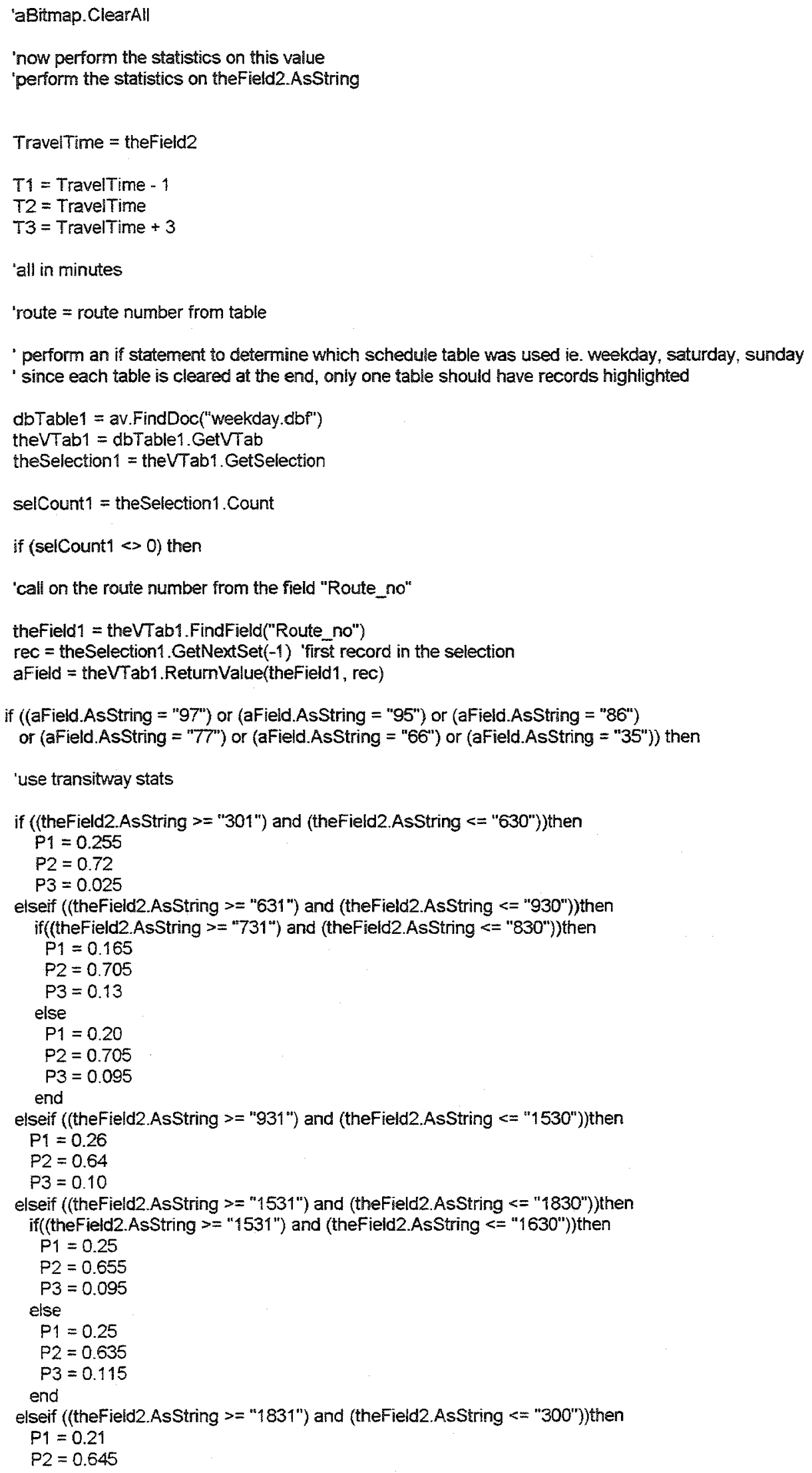




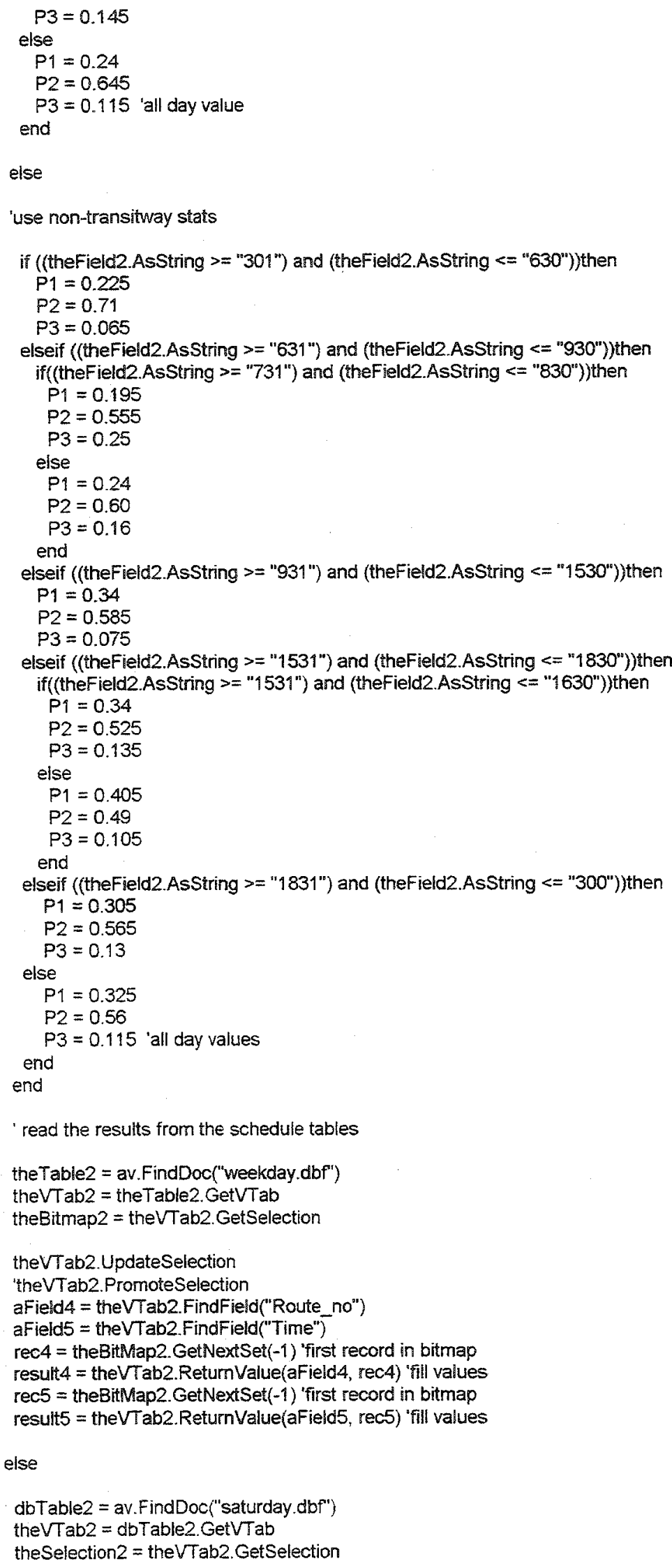




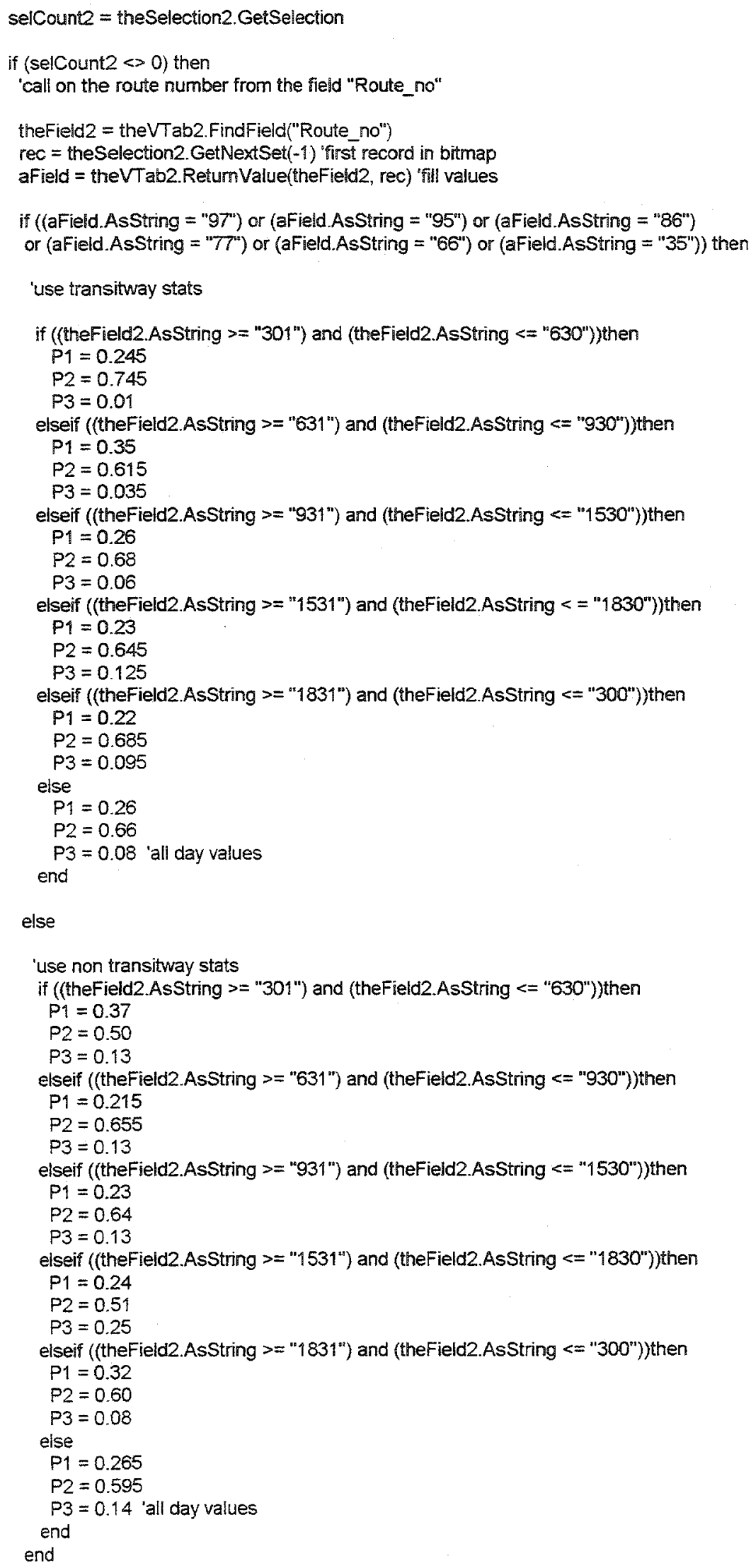




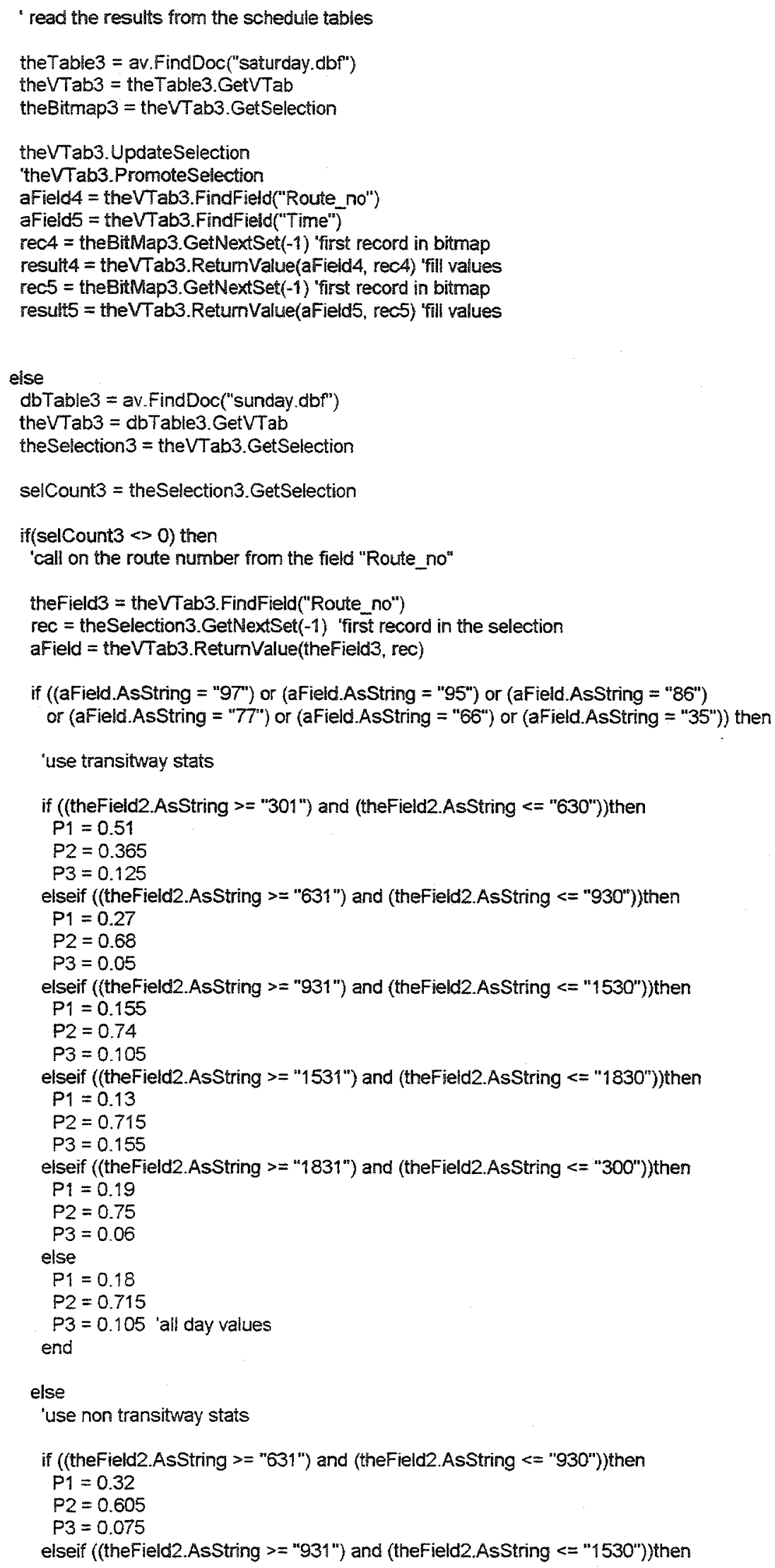




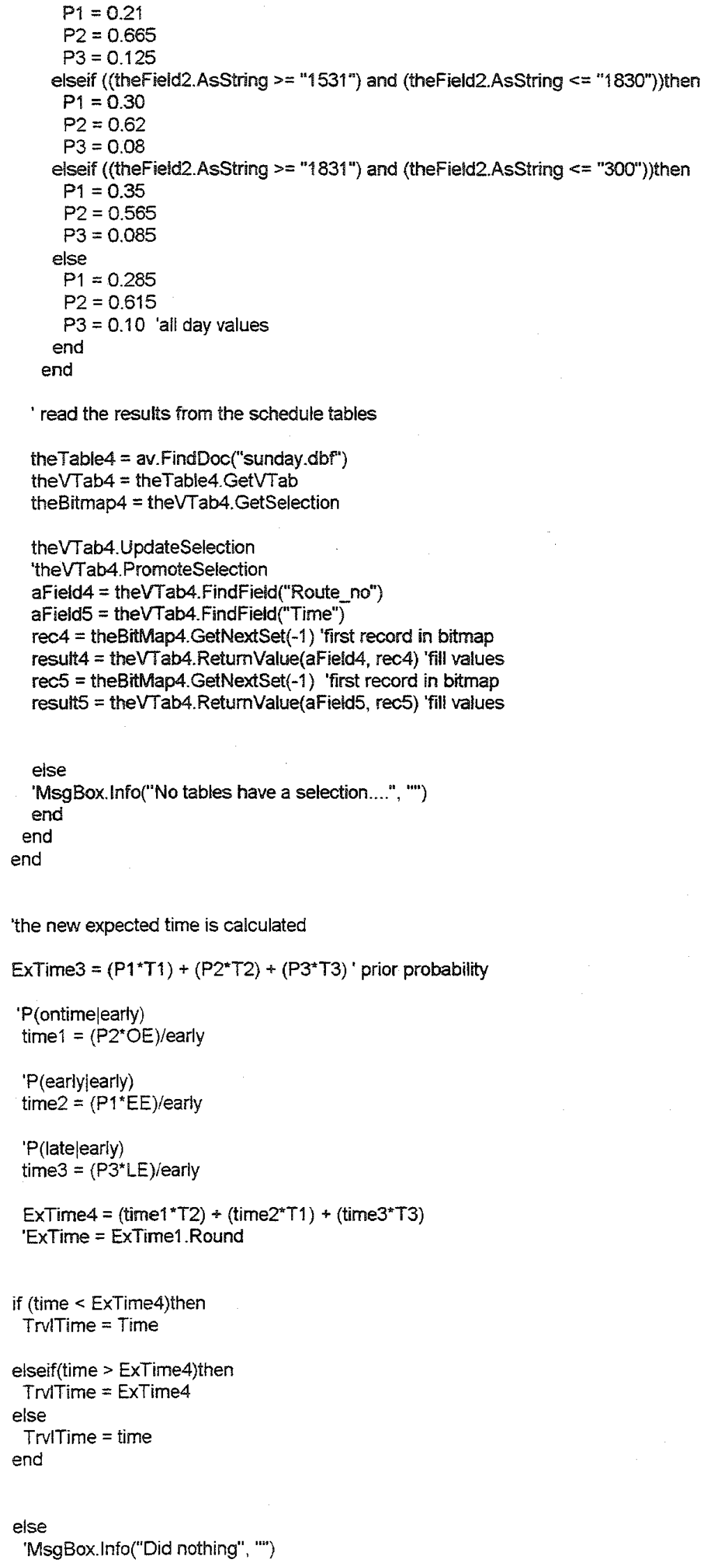


end

end

'read the results from the table created by Nearest Feature 'called near.dbf

theTable1 = av.FindDoc("near.dbf")
theVTab1 = theTable1.GetVTab
theBitmap1 = theVTab1.GetSelection
theBitmap1. SetAll
theVTab1.UpdateSelection
aField1 = theVTab1.FindField("Bus_stop")
aField2 = theVTab1. FindField("On_street")
aField3 = theVTab1. FindField("At_street")

rec1 = theBitMap1. GetNextSet(-1) 'first record in bitmap result1 = theVTab1. RetumValue(aField1, rec1) "fill values rec2 = theBitMap1. GetNextSet(-1) 'first record in bitmap result 2 = theVTab1. RetumValue(aField2, rec2) "fill values rec3 $=$ theBitMap1. GetNextSet(-1) 'first record in bitmap result $3=$ theVTab1.ReturnValue(aField3, rec3) "fill values

'resutt5 needs the T_cost added to it-time distance between the bus stop "with a time and the bus stop without a time-mif the distance is zero then "result5 = "Time"

theView = av.GetProject.FindDoc("Route Finding")

theTheme = theView.Find Theme("Route 2 ")

theTable = the Theme.GetFTab

theBitmap = the Table. GetSelection

Querystring $=$ " $\left[F_{\text {_ cost }}\right]=0^{\prime \prime}$

theT able.Query(QueryString, theBitmap, \#VAB_SELTYPE_NEW)

theTable.UpdateSelection

theField = theTable.FindField ("T_cost")

aRec $=$ theBitmap. GetNextSet(-1) 'first record in bitmap

Tvalue $=$ the Table. ReturnValue(theField, aRec) 'fill values

ArrivalTime $=$ result5. AsNumber + Tvalue. Round

'get view and zoom to route

theView $=$ av. GetActiveDoc

the Theme $=$ theView. FindTheme("Locate.shp")

the Theme.SetActive(TRUE)

'View.ZoomToTheme

av. GetProject.SetModified(true)

the View $=$ av. GetActiveDoc

theThemes $=$ theView.GetActiveThemes

$r=$ Rect.MakeEmpty

for each $t$ in the Themes

$r=r$.UnionWith(t. ReturnExtent)

end

if (r.IsEmpty) then

return nil

elseif ( r.ReturnSize $=(0 @ 0)$ ) then

theView.GetDisplay.PanTo(r.ReturnOrigin)

else

theView.GetDisplay.SetExtent(r.Scale(1.1))

end

theView = av.GetProject.FindDoc("Route Finding") the Theme = theView. Find Theme("Bus Stops")

the Theme.SetVisible(FALSE)

theTherne 2 = theView.FindTheme("Locate.shp")

theFTab $=$ theTheme2.GetFTab 


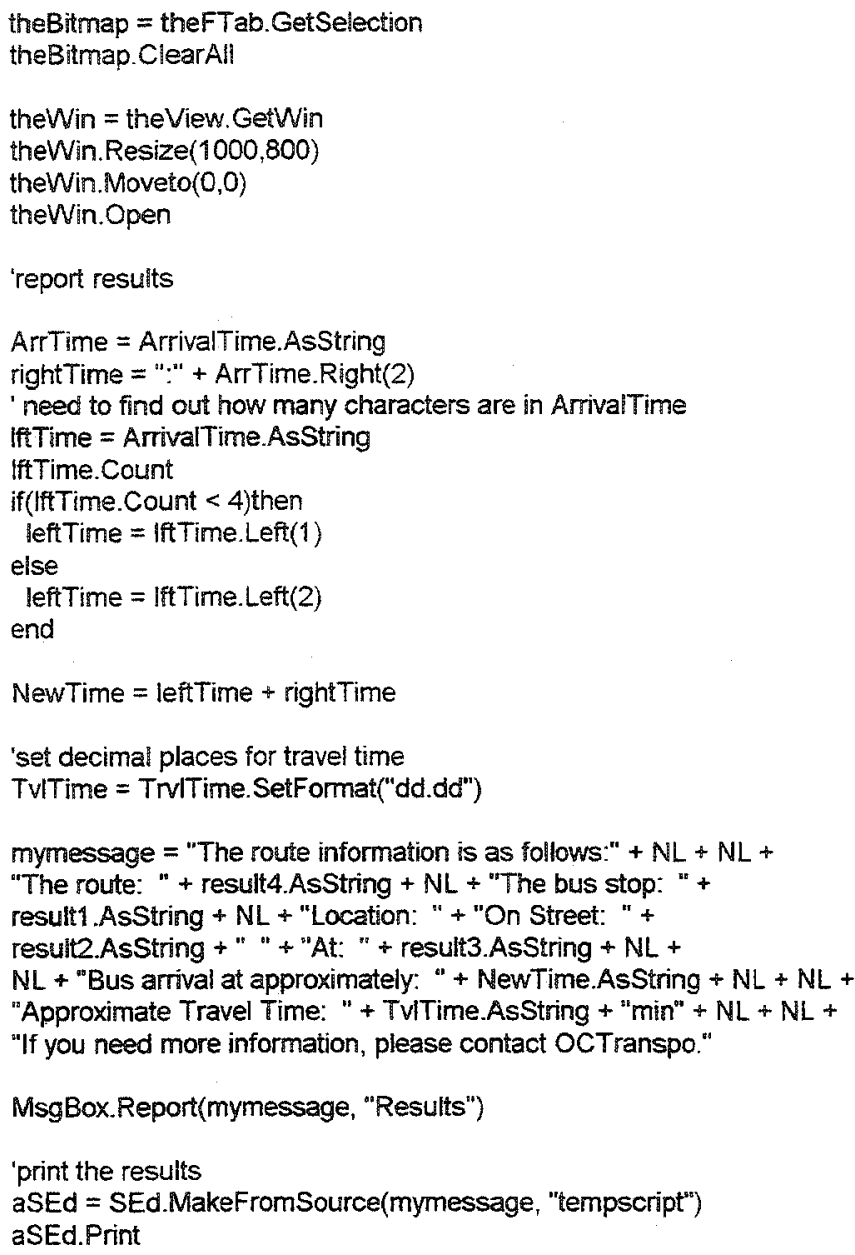




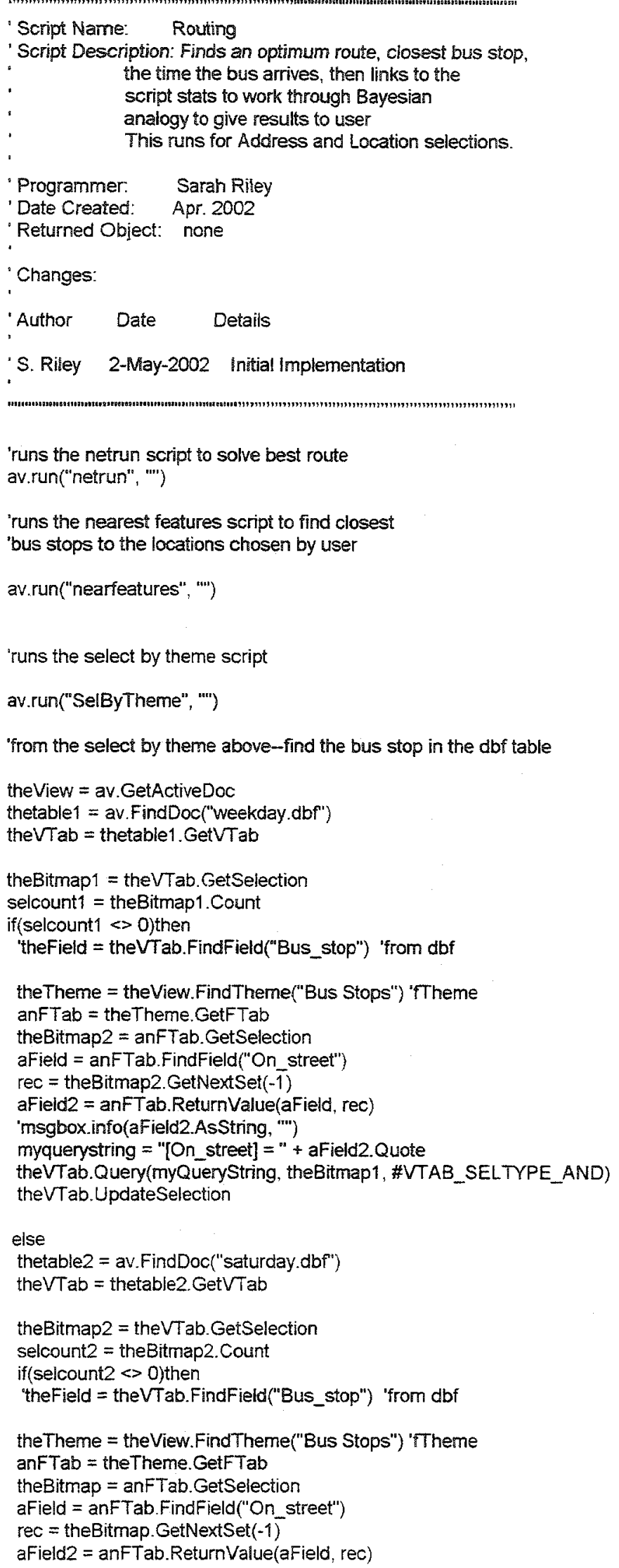




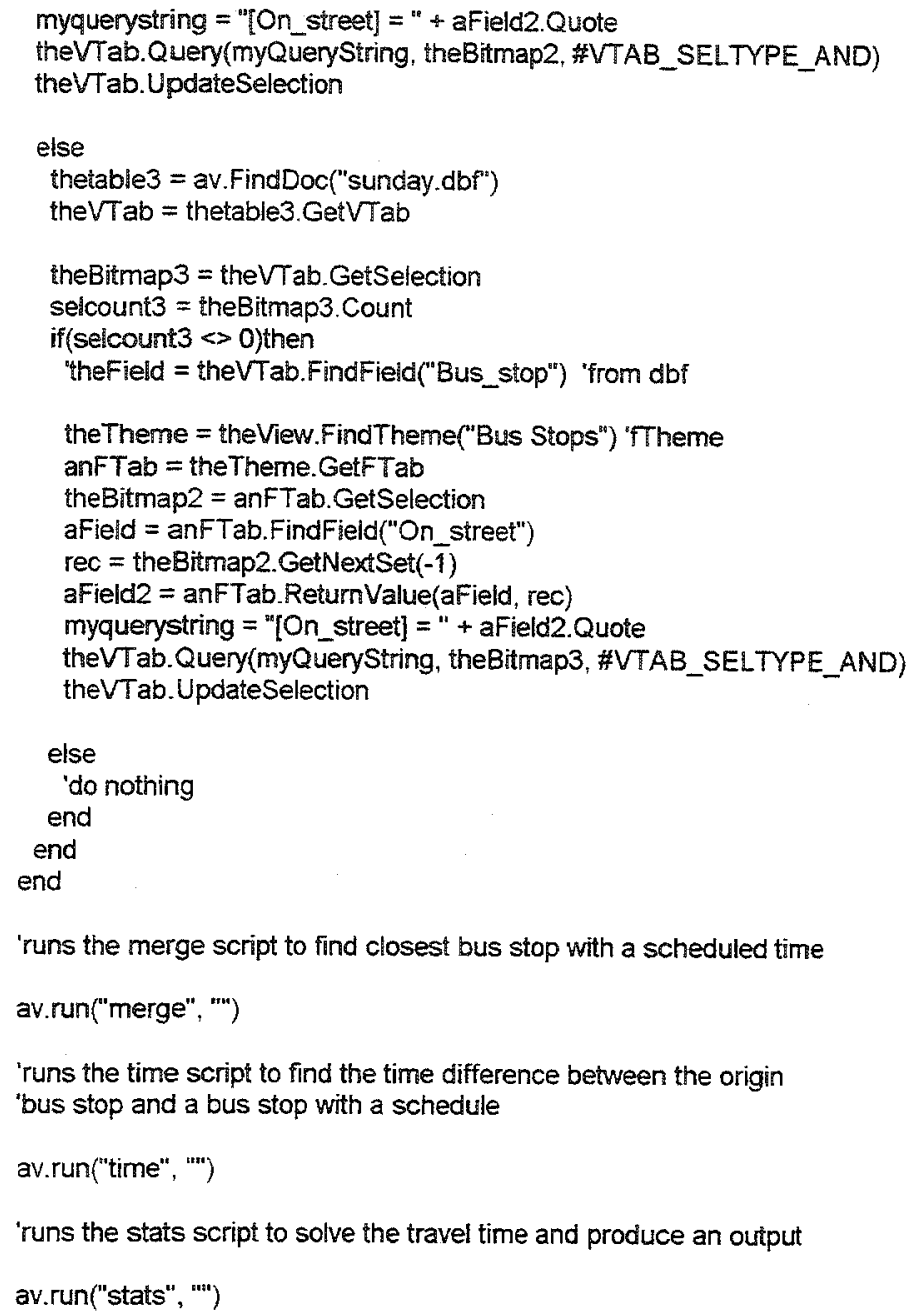




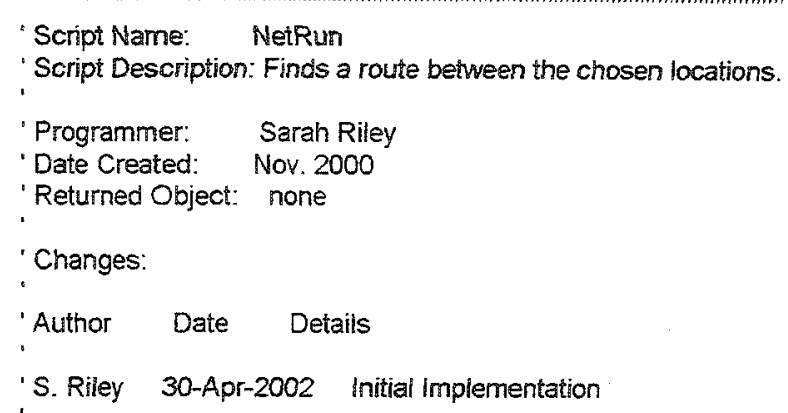

' Get a list of available costs for this line theme. Choose a cost from the list.

aNetCostFieldList $=$ theNetDef.GetCostFieids

aNetCostField = MsgBox.Choice(aNetCostFieldList, "Select Minutes", "Select Minutes")

- If a cost was chosen, set it.

if (aNetCostField <>nil) then

theNetwork.SetCostField(aNetCostField)

end

'The above can be replaced by changing the hard code for Network Analyst wherein 'there will only be the chosen cost of MINUTES to select.

*

TravelDistance $=$ theNetwork.FindPath(thePointList, True, False)

'msgBox.Info( TravelDistance.AsString, "Total" ++reportUnitStr)

'theView.GetGraphics.add(GraphicShape.Make(theNetwork.ReturnPathShape))

aSymbol = Symbol.Make (\#SYMBOL_PEN)

aSymbol.SetSize(2.0)

asymbol.SetColor(Color.GetMagenta)

the GL=theView. Get Graphics

the Route=GraphicShape. Make(theNetwork.ReturnPathShape)

theRoute.SetDisplay(av.GetActiveDoc.GetDisplay)

theroute.SetSymbol(aSymbol)

the GL.Add(the Route)

"theroute.GetSymbol.SetColor(color.GetMagenta).SetSymbol(aSymbol)

theRoute. Draw

theroute. Invalidate 
'set view size for public viewing

the View $=$ av.getproject.finddoc $($ "Route Finding"

theWin = the View. GetWin

'theWin.Resize (1025,600)

theWin.Moveto $(0,0)$

thewin.Open

- Write the route result FTab, this is necessary to get directons.

tmpFileName = FileName.Make("F:thesis dataiRoute.shp")

theNetwork.WritePath(tmpFileName)

resFTab = FTab.Make(SrcName.Make(impFileName.AsString))

' Show the directions then remove the temporary file.

theDirections $=$ "Your route directions are as follows" $+N L+N L+$

theNework.GetPathDirections(resFTab)

'msgBox.Report(theDirections, "Route Information")

'add theme to view

newTheme = FTheme. Make(resFTab)

theView.AddTheme(newTheme)

newTheme.SetName("Route")

newTheme.SetVisible(True) 


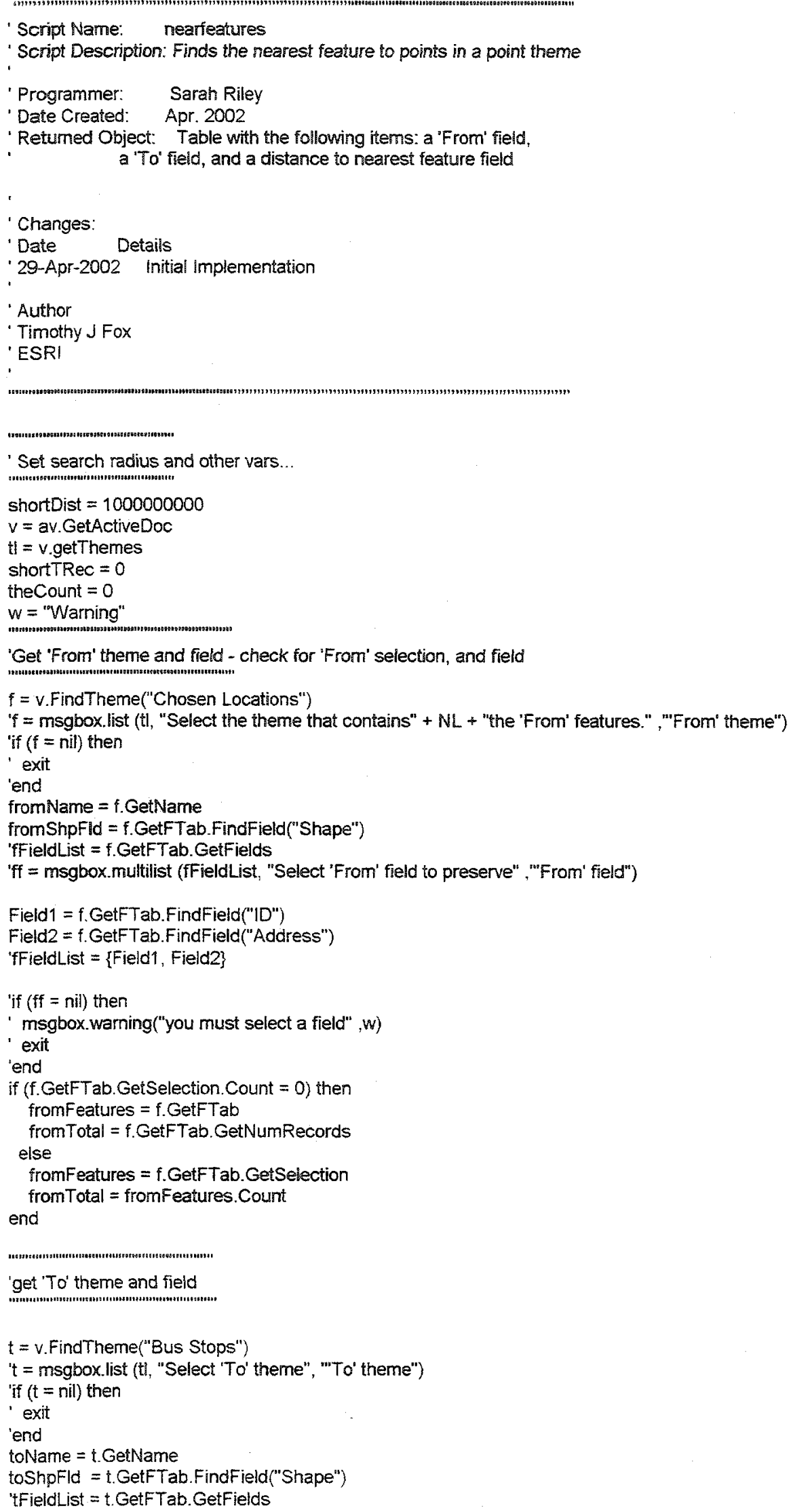




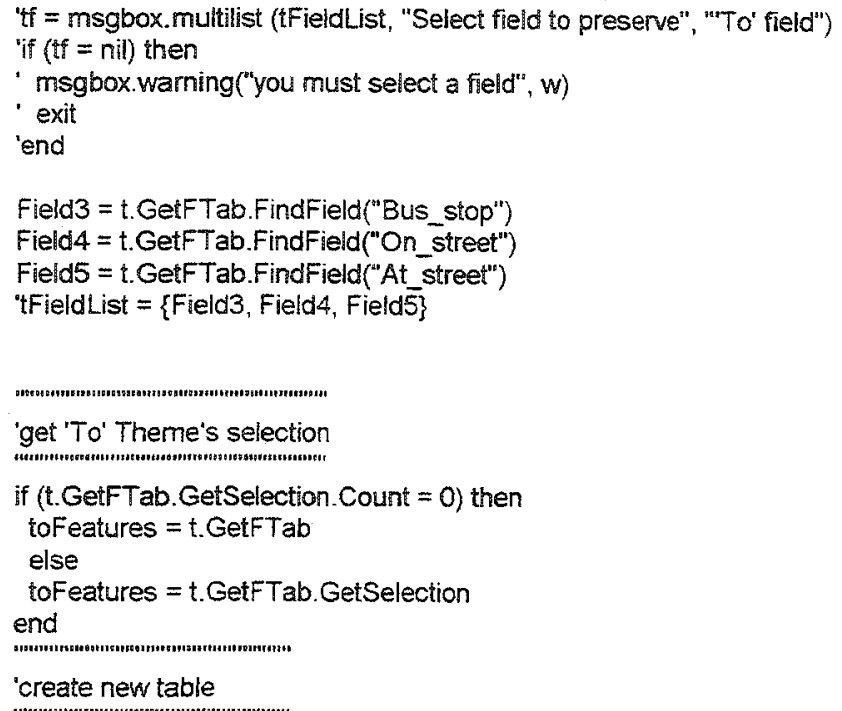




\author{
newVTab.SetValue(f2, newRec,tempFid2) \\ newVTab.SetValue(t1, newRec,tempTid3) \\ newVTab.SetValue(t2, newRec,tempTid4) \\ newVTab.SetValue(t3, newRec,tempTid5) \\ 'newVTab.SelValue(tf, newRec, tempTid) \\ newVTab.SetValue(distField, newRec, shortDist) \\ shortDist $=1000000000$ \\ av.SetStatus $(($ theCount / fromTotal $) * 100)$ \\ end \\ av.ClearStatus \\ av. ClearMsg \\ newVTab.SetEditable (false) \\ create table from VTab \& display table \\ newTab = table.Make (newVTab) \\ newTab.setName ("near.dbf") \\ newTabWin = newTab.GetWin \\ newTabWin.Resize $(350,250)$ \\ newTabWin Open
}




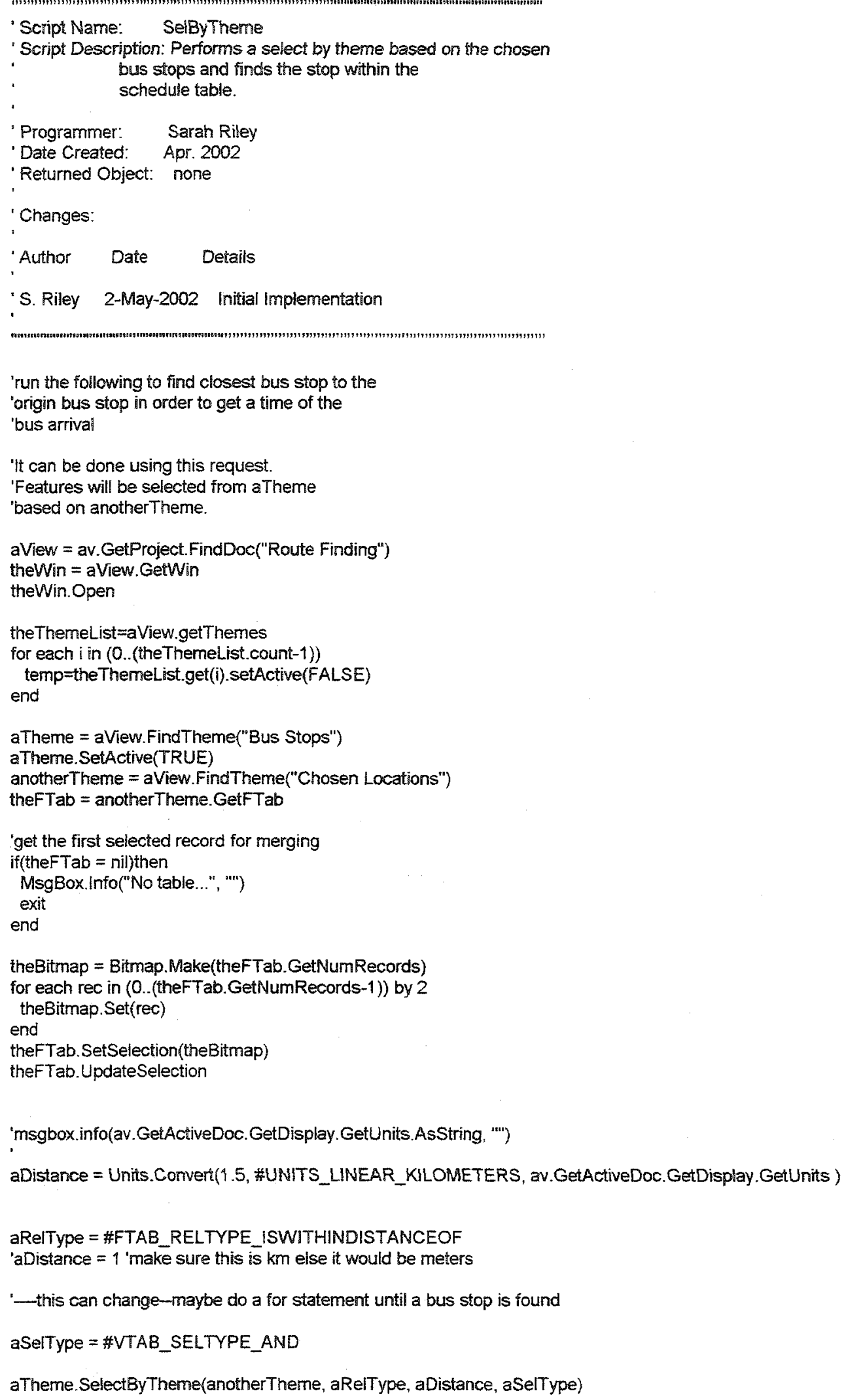




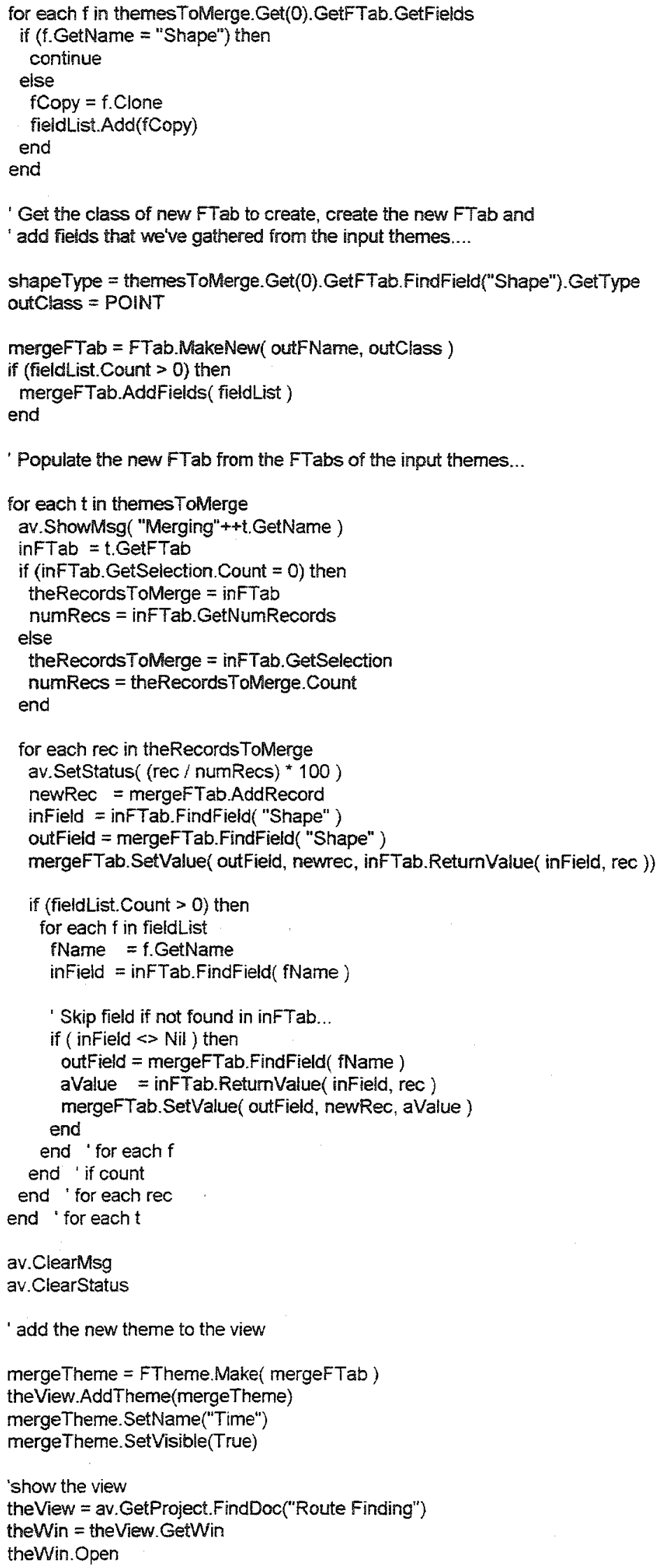




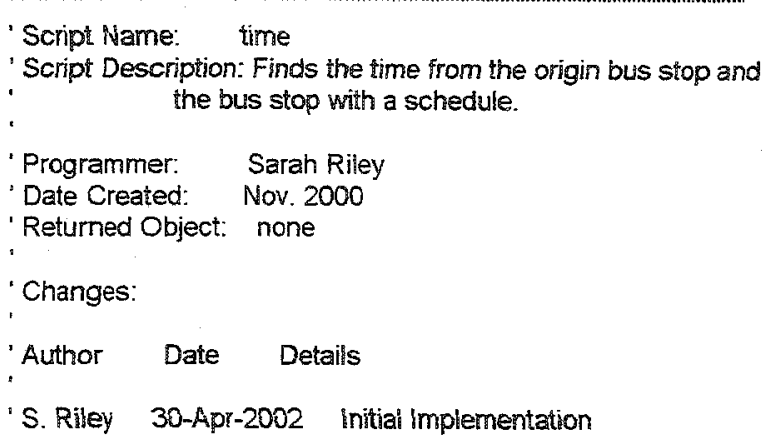

'The above can be replaced by changing the hard code for Network Analyst wherein 'there will only be the chosen cost of MINUTES to select.

TravelDistance $=$ theNetwork. FindPath(thePointlist, True, False)

'msgBox.Info( TravelDistance.AsString, "Total" ++reportUnitStr)

'theView.GetGraphics.add(GraphicShape.Make(theNetwork.ReturnPathShape))

'aSymbol = Symbol Make(\#SYMBOL PEN)

'aSymbol.SetSize(2.0)

'aSymbol.SetColor(Color. GetMagenta)

'the $G L=$ theView. GetGraphics

'theRoute $=$ GraphicShape. Make (theNetwork.ReturnPathShape)

theRoute.SetDisplay (av.GetActiveDoc.GetDisplay)

'theroute. SetSymbol(aSymbol)

theGLAdd(theRoute)

"theroute.GetSymbol.SetColor(color.GetMagenta).SetSymbol(aSymbol)

theRoute. Draw

'theroute. Invalidate 
"set view size for public viewing

theView = av.getproject.finddoc("Route Finding")

the Win = theView. GetWin

"theWin.Resize(1025,600)

'theWin.Moveto $(0,0)$

theWin.Open

-Write the route result FTab, this is necessary to get directons.

tmpFileName = FileName Make $($ "F $:$ thesis datatroute.shp")

theNetwork. WritePath(tmpFileName)

resFTab = FTab.Make(SrcName.Make(tmpFileName.AsString))

"Show the directions then remove the temporary file.

"theDirections="Your route directions are as follows" $+N L+N L+$

- theNetwork GetPathDirections(resFTab)

'msgBox.Report(theDirections, "Route information")

'make the Time theme invisible

theView = av. GetProject. FindDoc("Route Finding")

the Theme = theView. Find Theme("Time")

'theTheme. SetActive(FALSE)

the Theme. SetVisible(FALSE)

'add theme to view

newTheme $=$ FTheme.Make $($ resFTab)

theView.Add Theme(newTheme)

newTheme.SetName("Route2")

newTheme.SetVisible(True) 


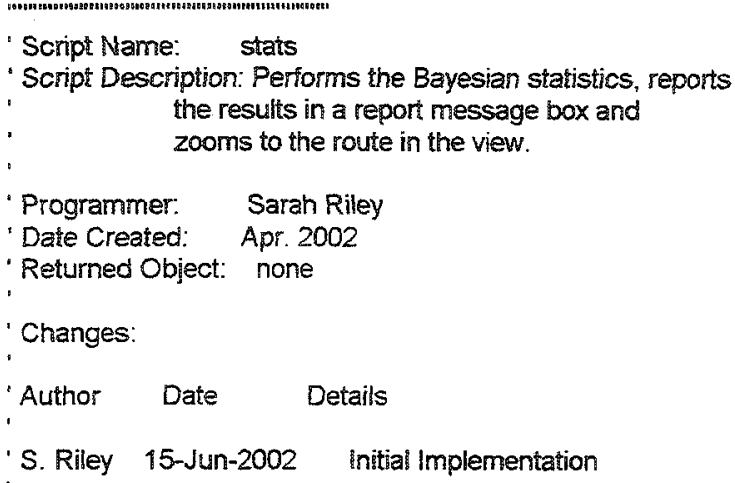




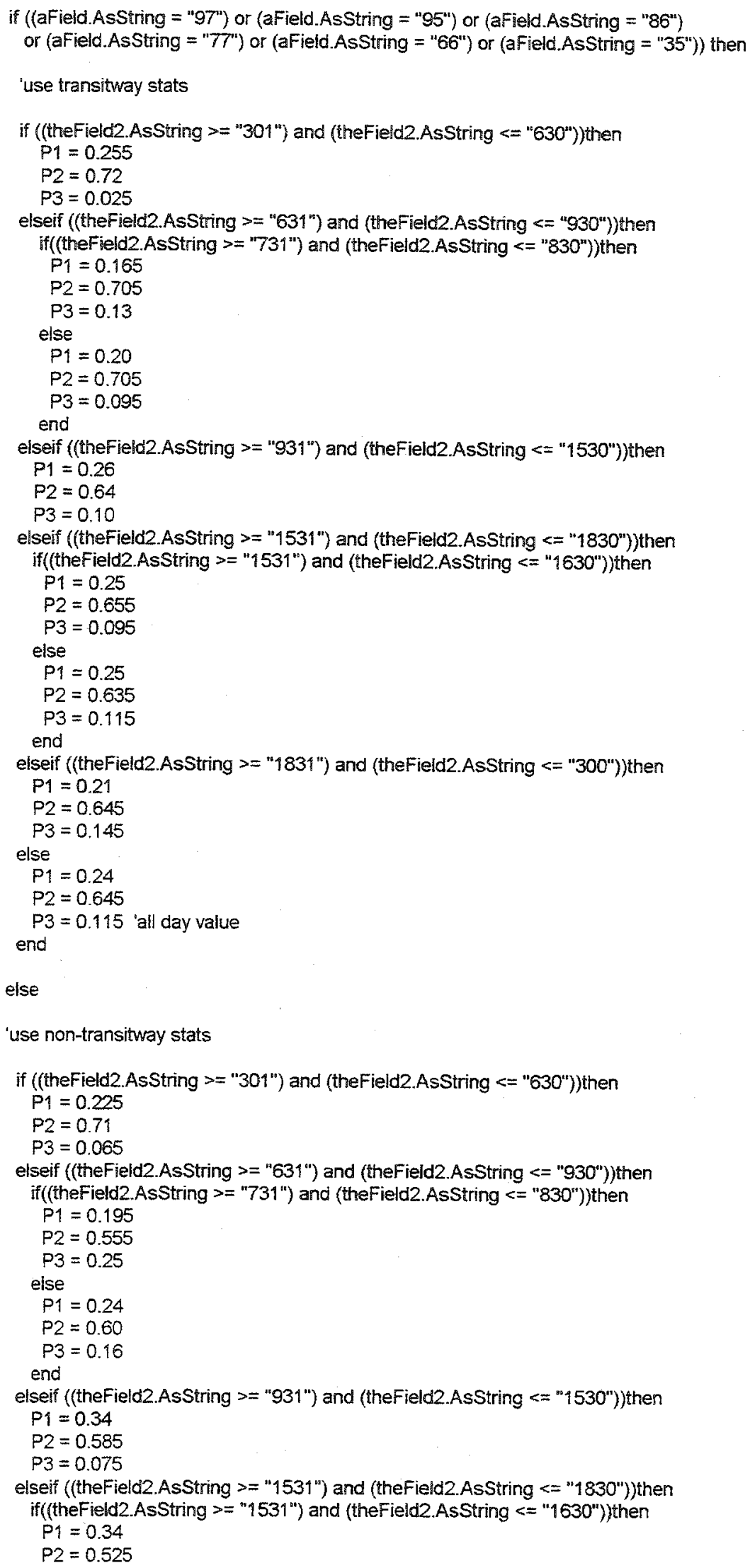




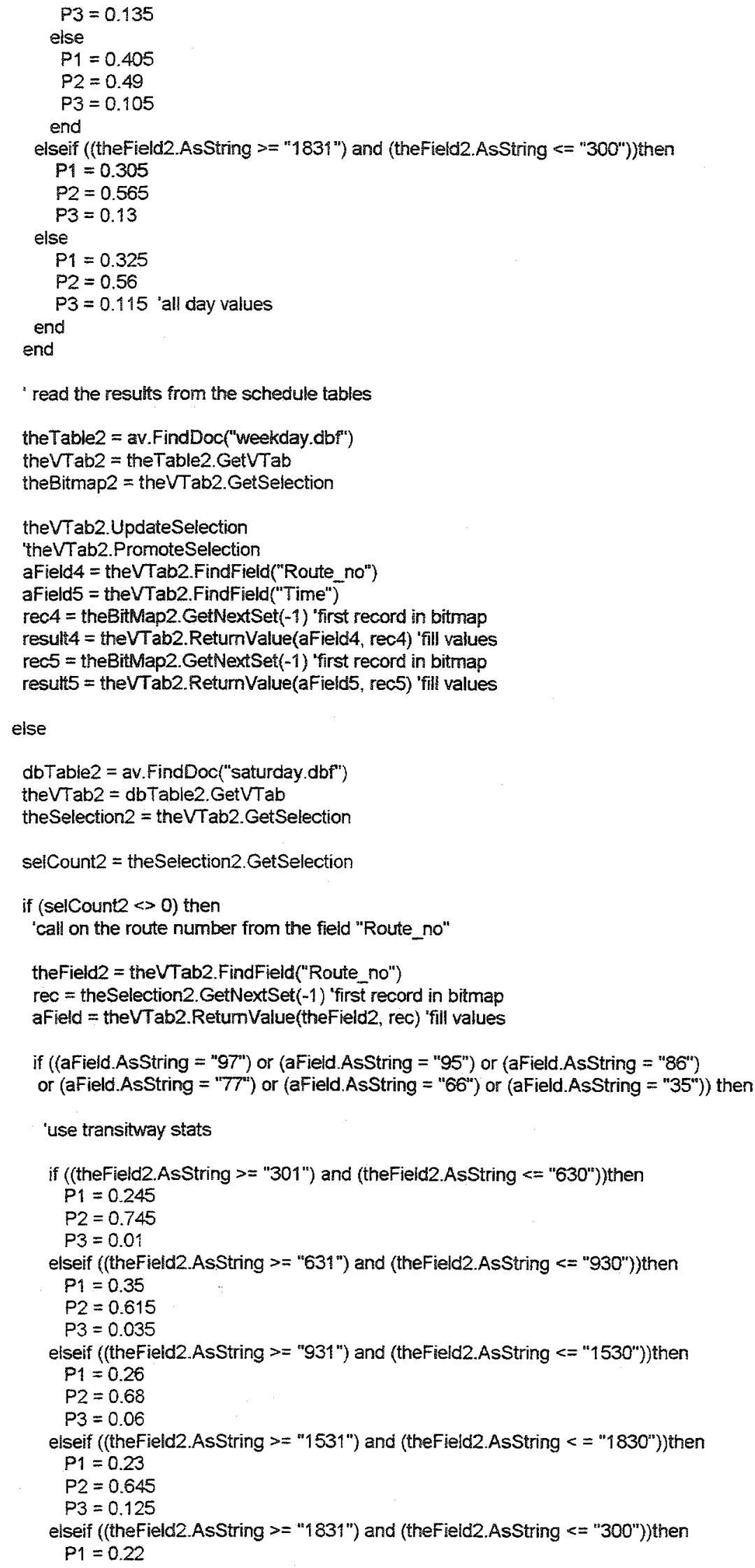




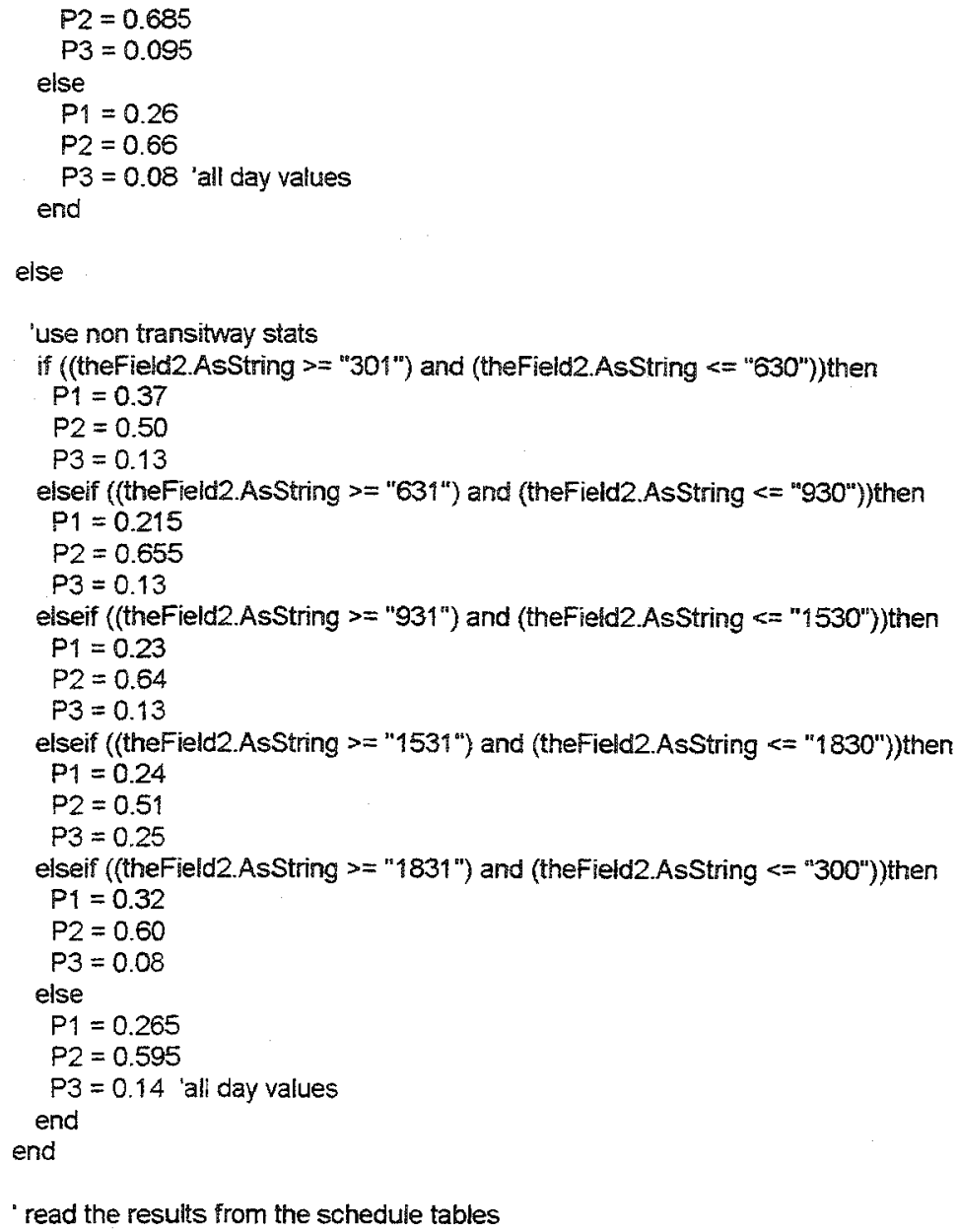




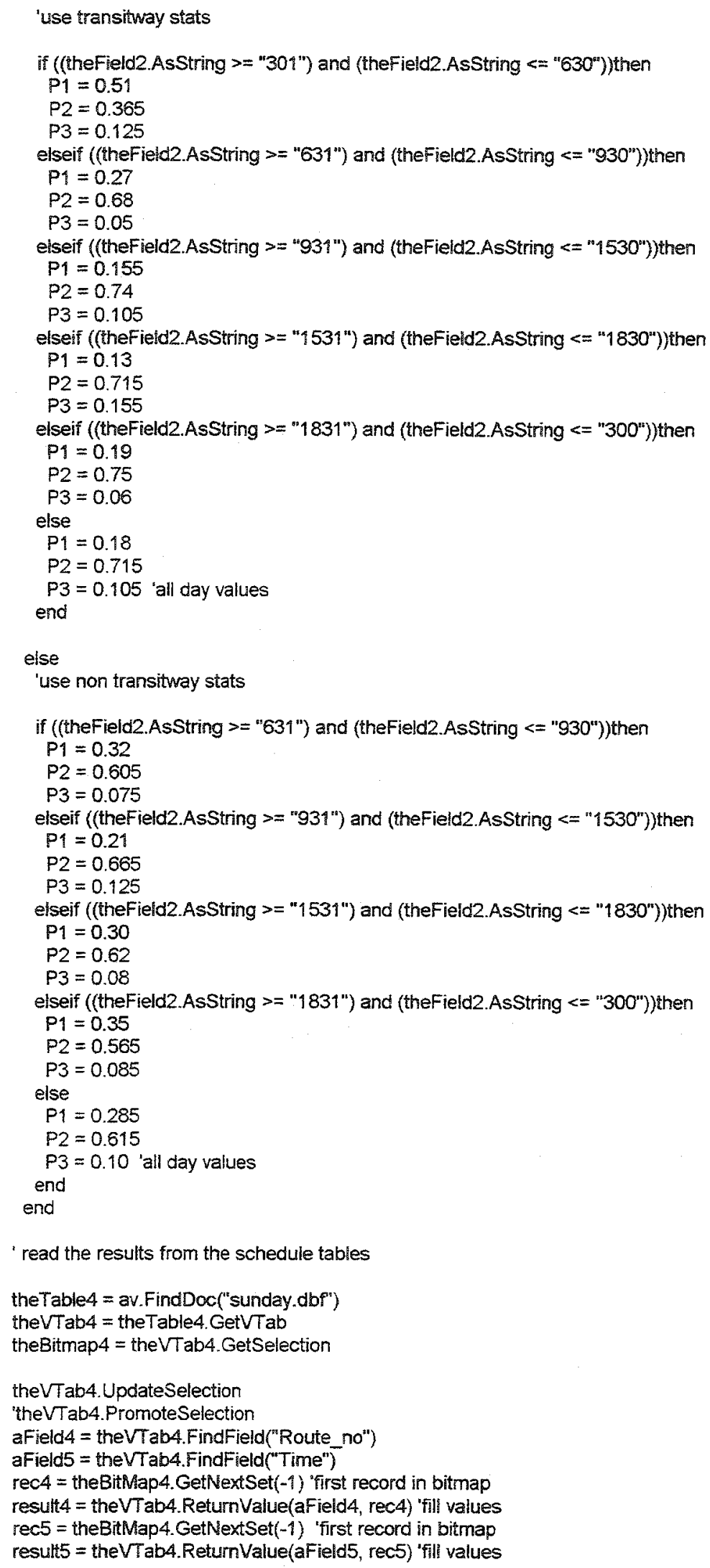




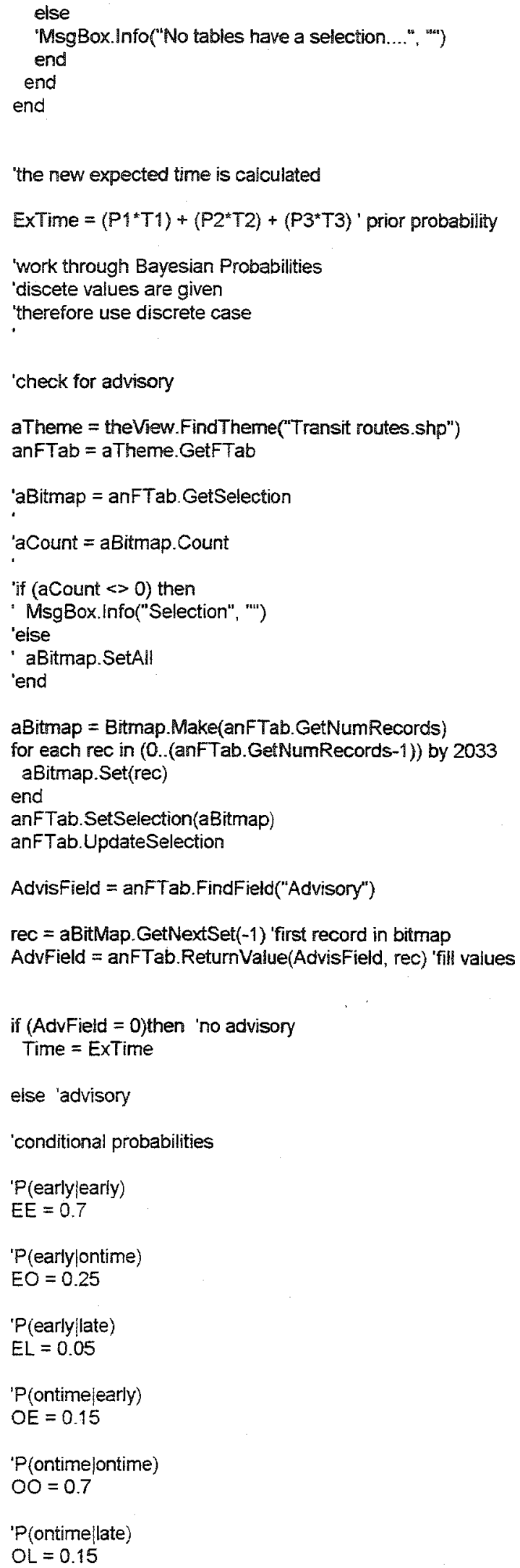




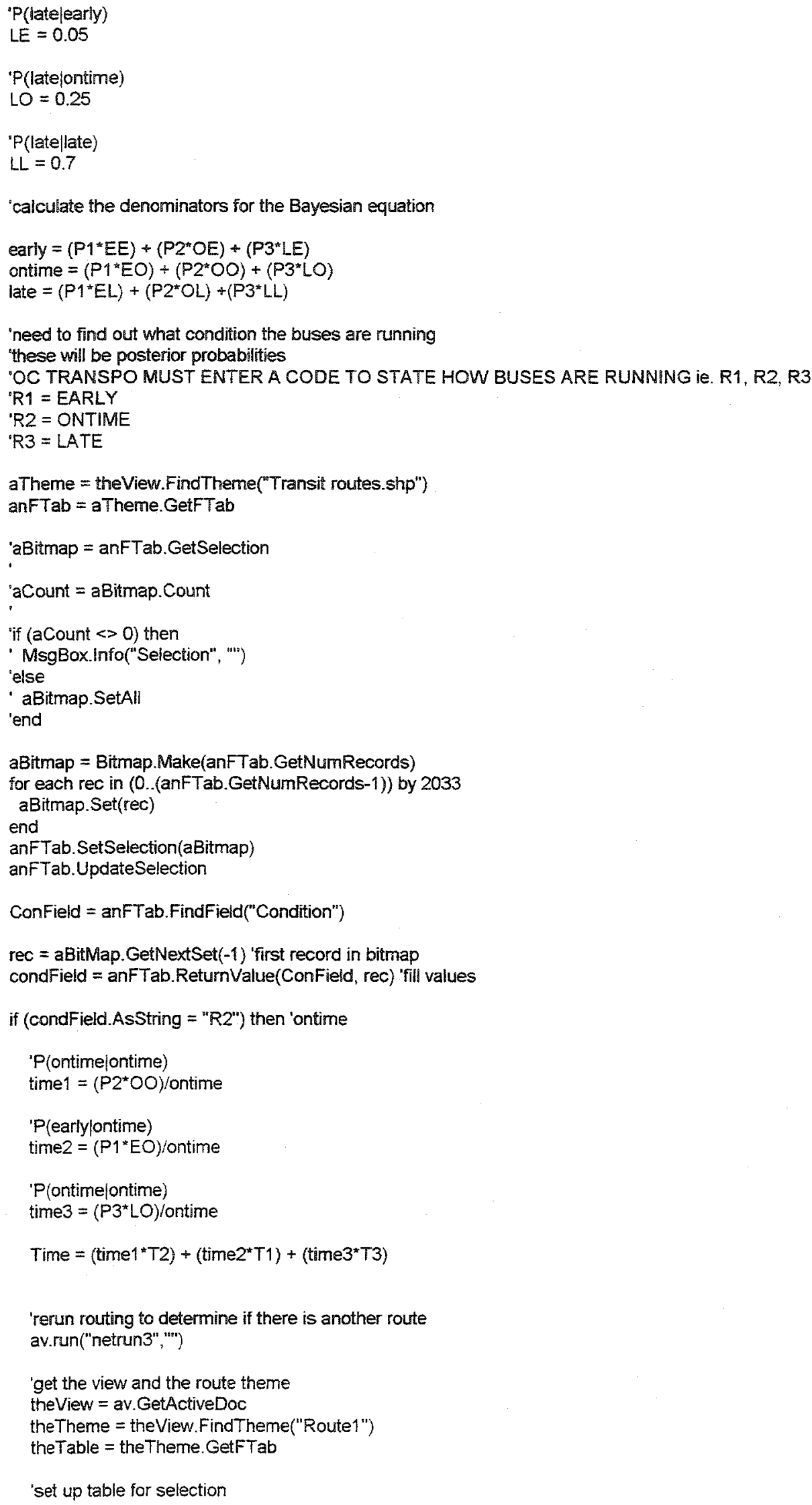




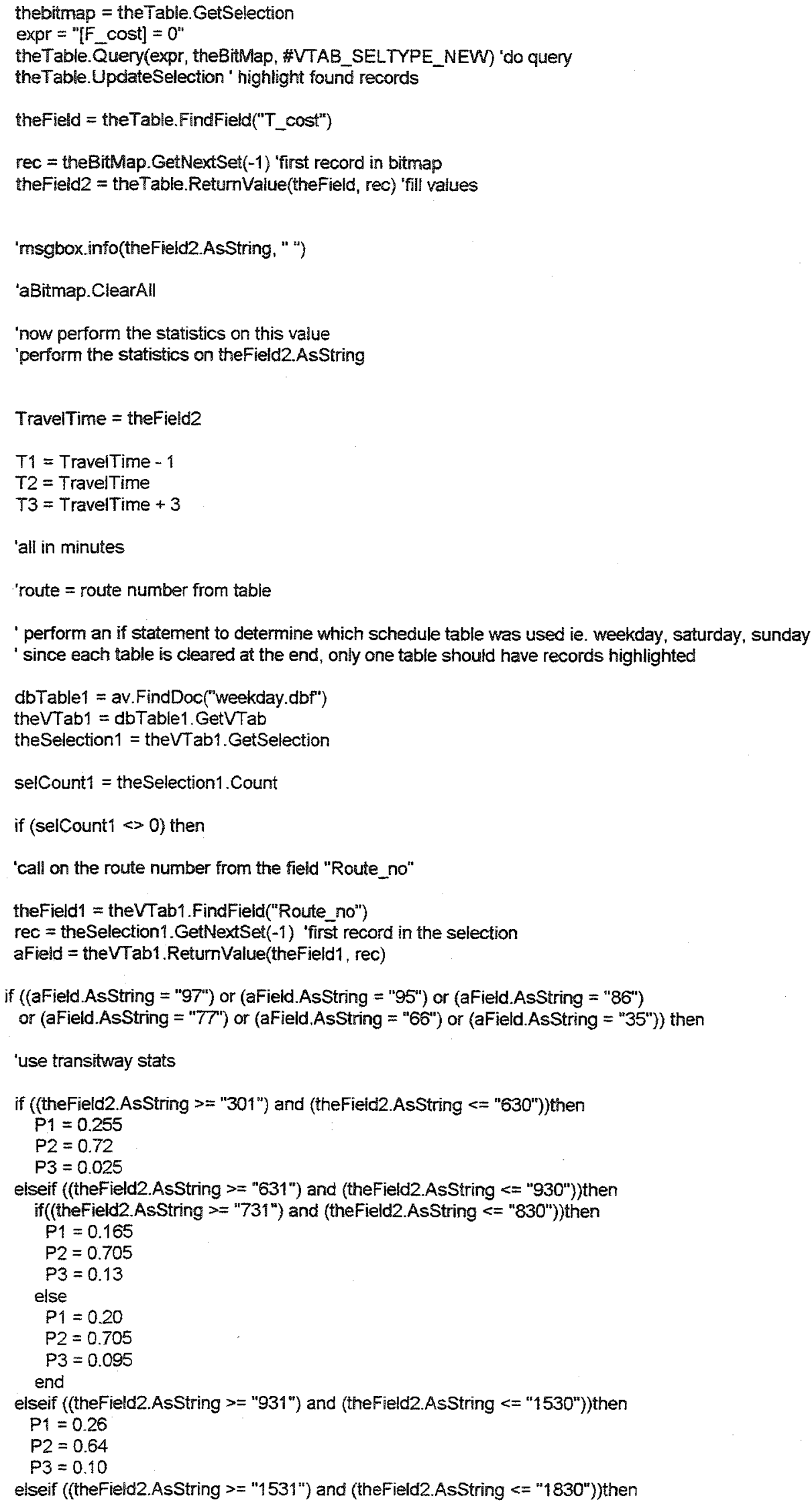




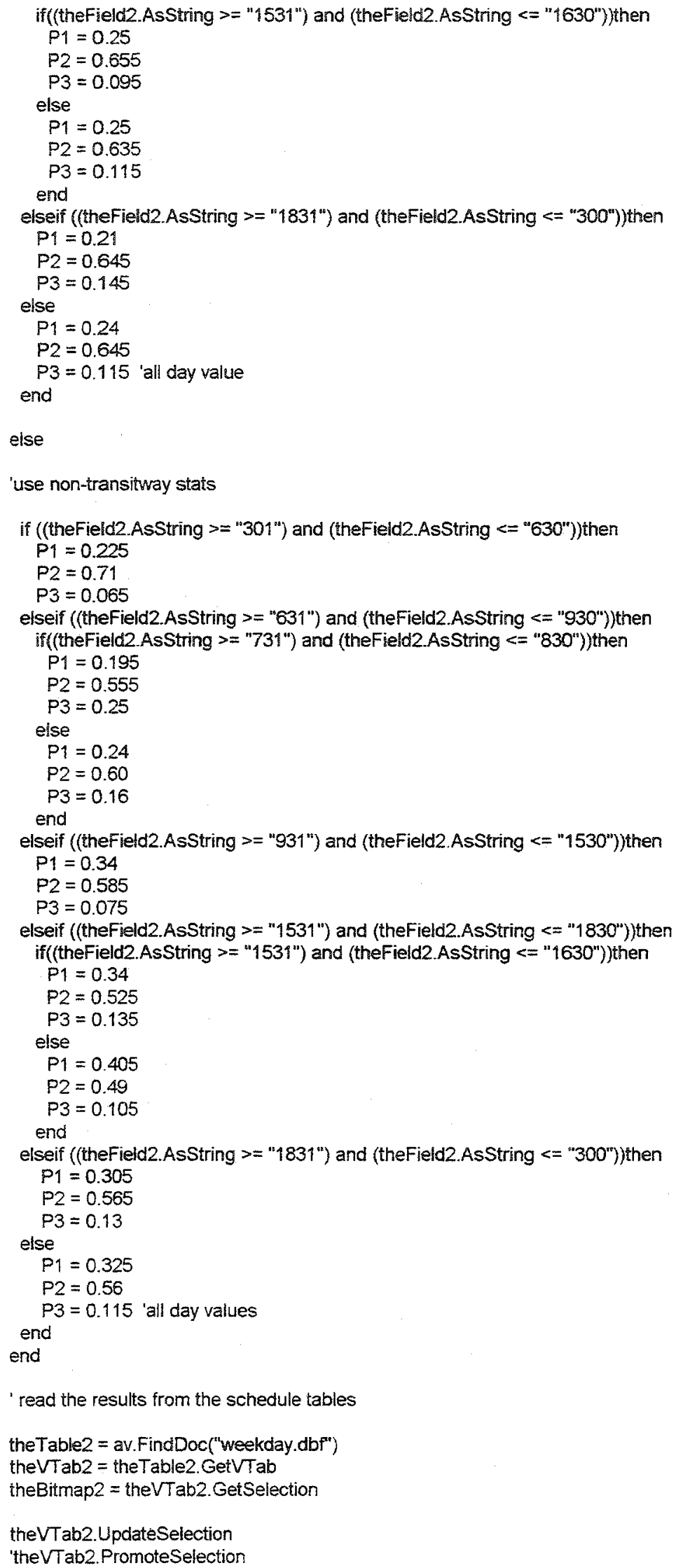




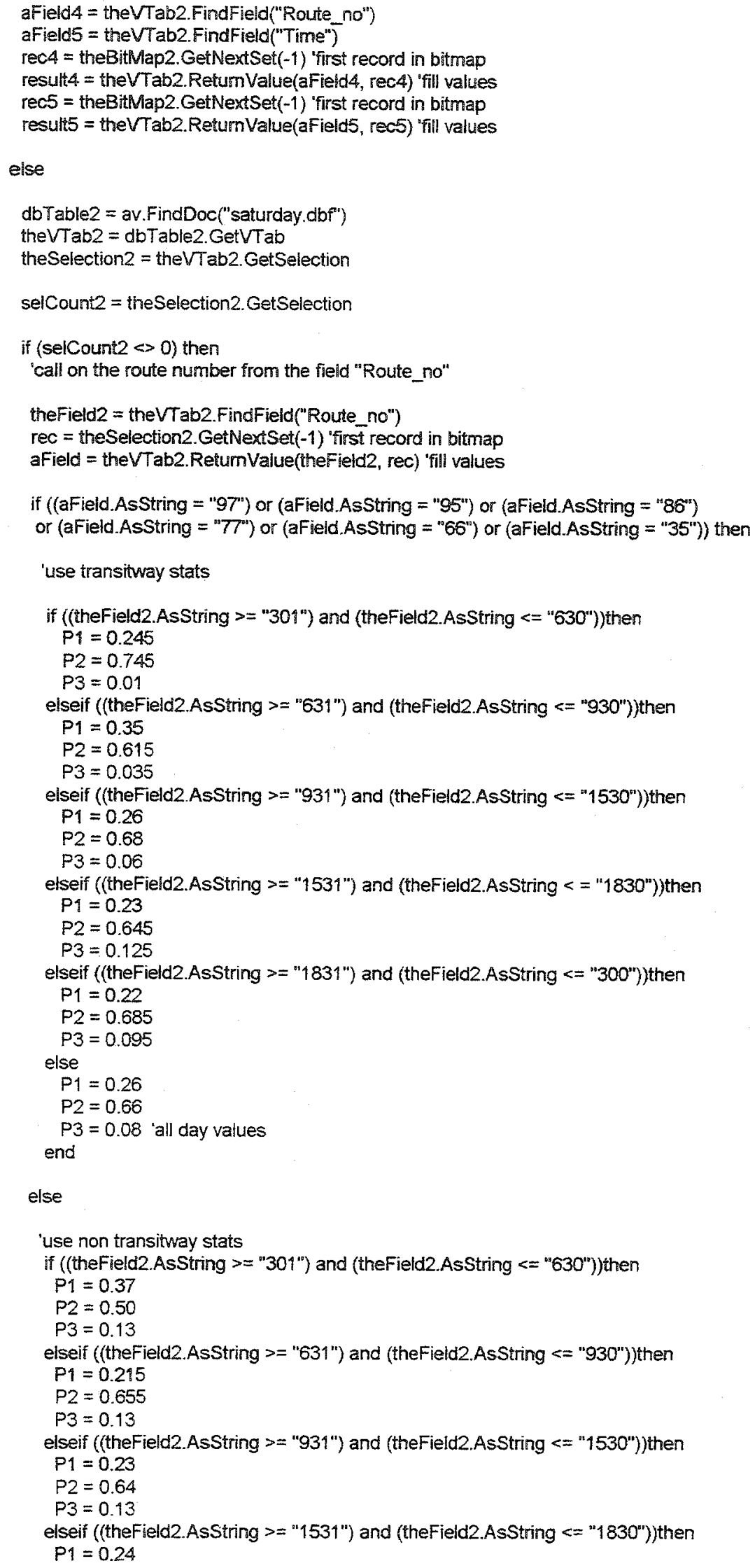




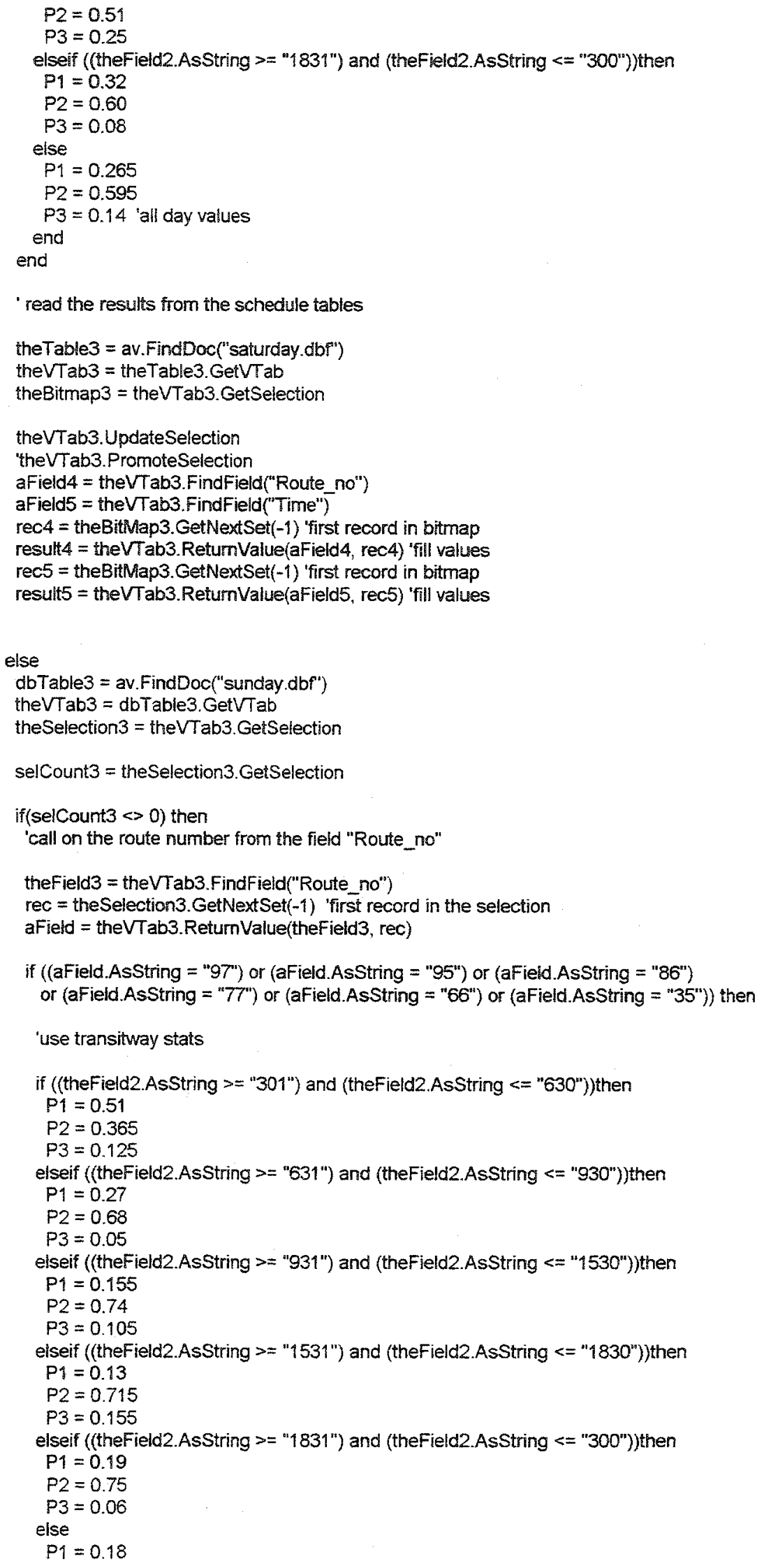




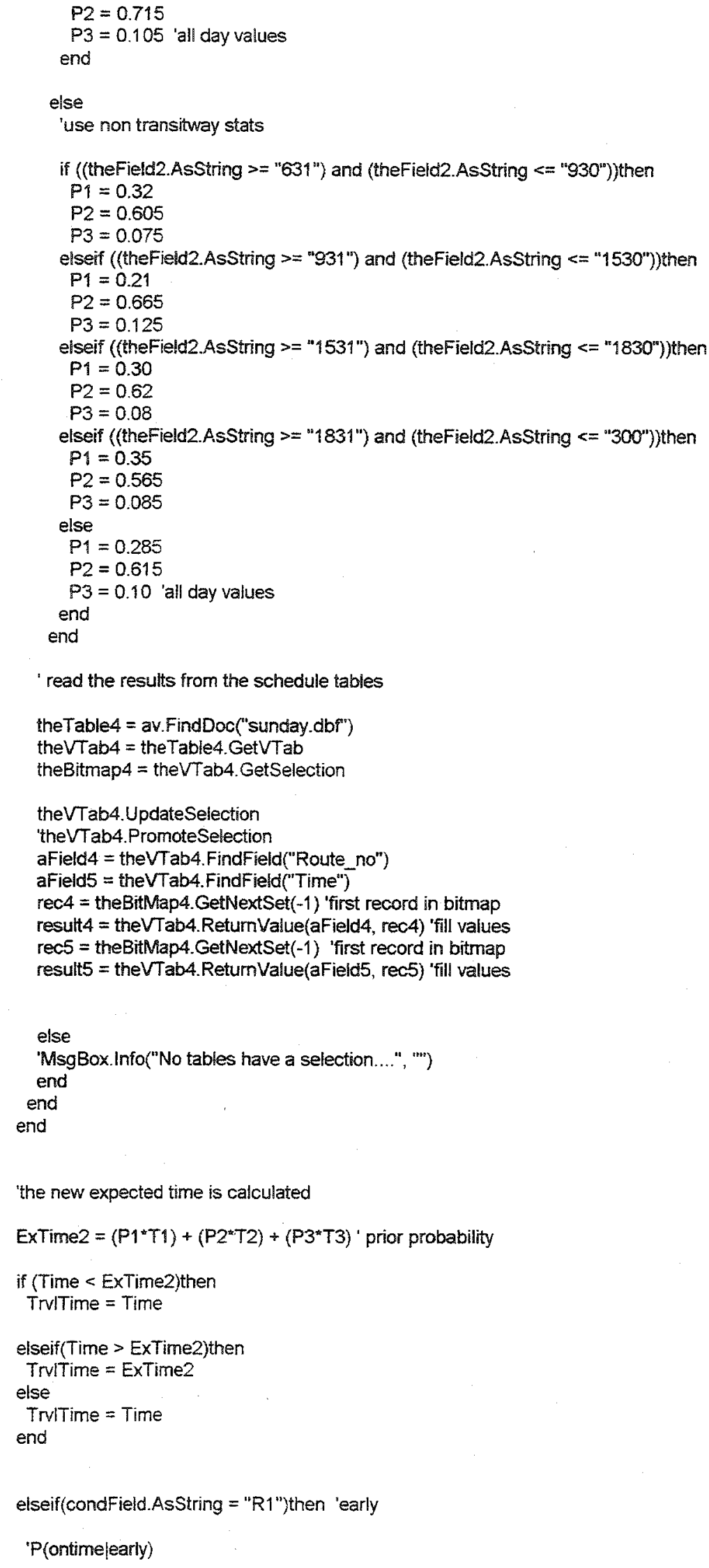

' read the results from the schedule tables

theTable4 $=$ av.FindDoc("sunday.dbf")

theVTab4 = theTable4.GetVTab

theBitmap4 $=$ theVTab4.GetSelection

theVTab4.UpdateSelection

'theVTab4.PromoteSelection

aField4 = theVTab4. FindField("Route no")

aField5 $=$ theVTab4.FindField("Time")

rec4 = theBitMap4.GetNextSet(-1) 'first record in bitmap

result4 $=$ theVTab4. RetumValue(aField4, rec4) 'fill values rec5 = theBitMap4.GetNextSet(-1) 'first record in bitmap result5 $=$ theVTab4.ReturnValue(aField5, rec5) 'fill values

\section{else}

'MsgBox.Info("No tables have a selection....", "."')

eno

end

end

'the new expected time is calculated

ExTime2 $=\left(P 1^{*} T 1\right)+\left(P 2^{*} T 2\right)+\left(P 3^{*} T 3\right) '$ prior probability

if (Time $<$ ExTime2)then

TrvlTime $=$ Time

elseif(Time > ExTime2)then

TrulTime $=$ ExTime2

else

TrviTime $=$ Time

end

elseif(condField.AsString = "R1")then 'early

'P(ontimelearly) 


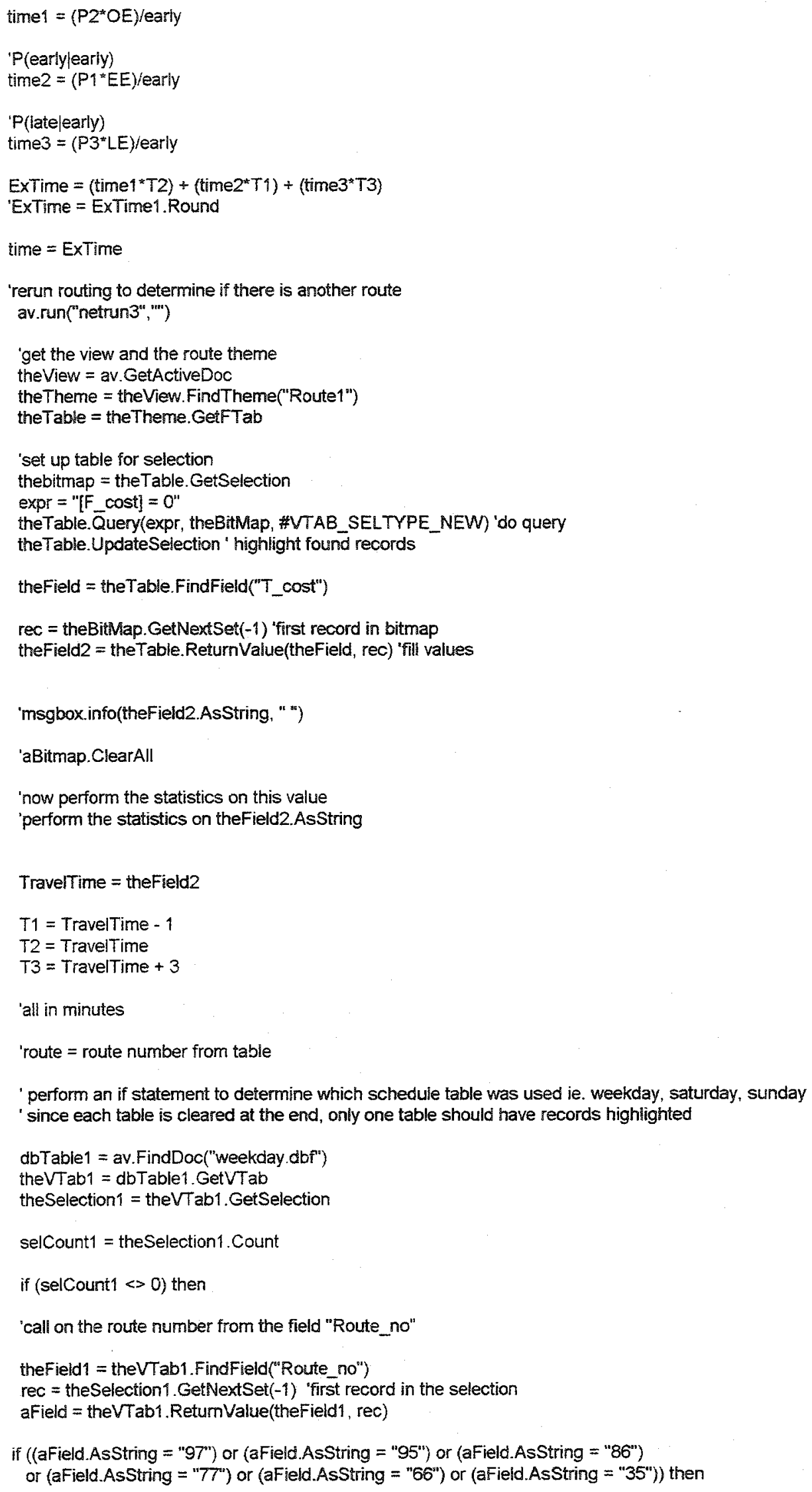




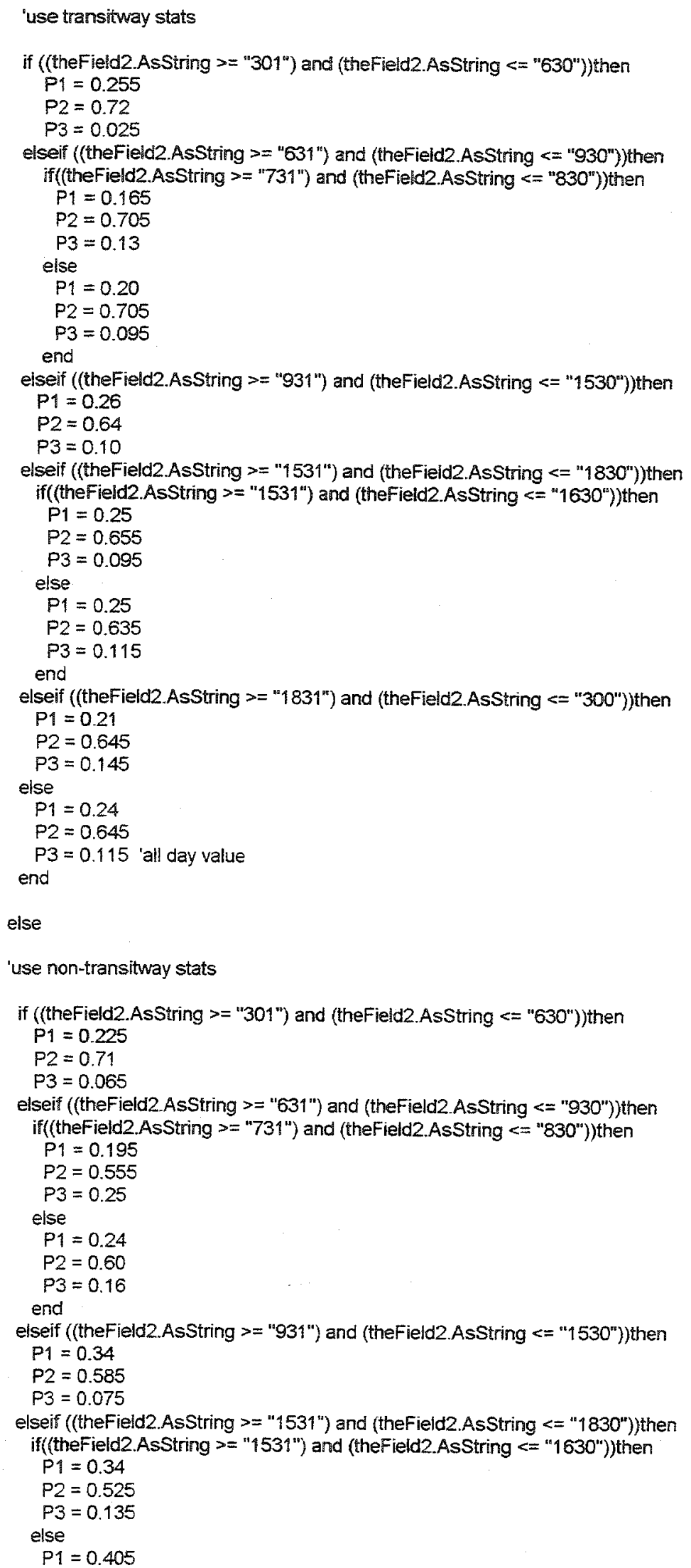

elseif ((theField2.AsString $>=" 1531 "$ ) and (theField2.AsString $<=" 1830 ")$ )then if(theField2.AsString $>=" 1531 "$ ) and (theField2.AsString $<=" 1630 ")$ )then

$\mathrm{P} 1=0.34$

$P 2=0.525$

$\mathrm{P3}=0.135$

else

$P 1=0.405$ 


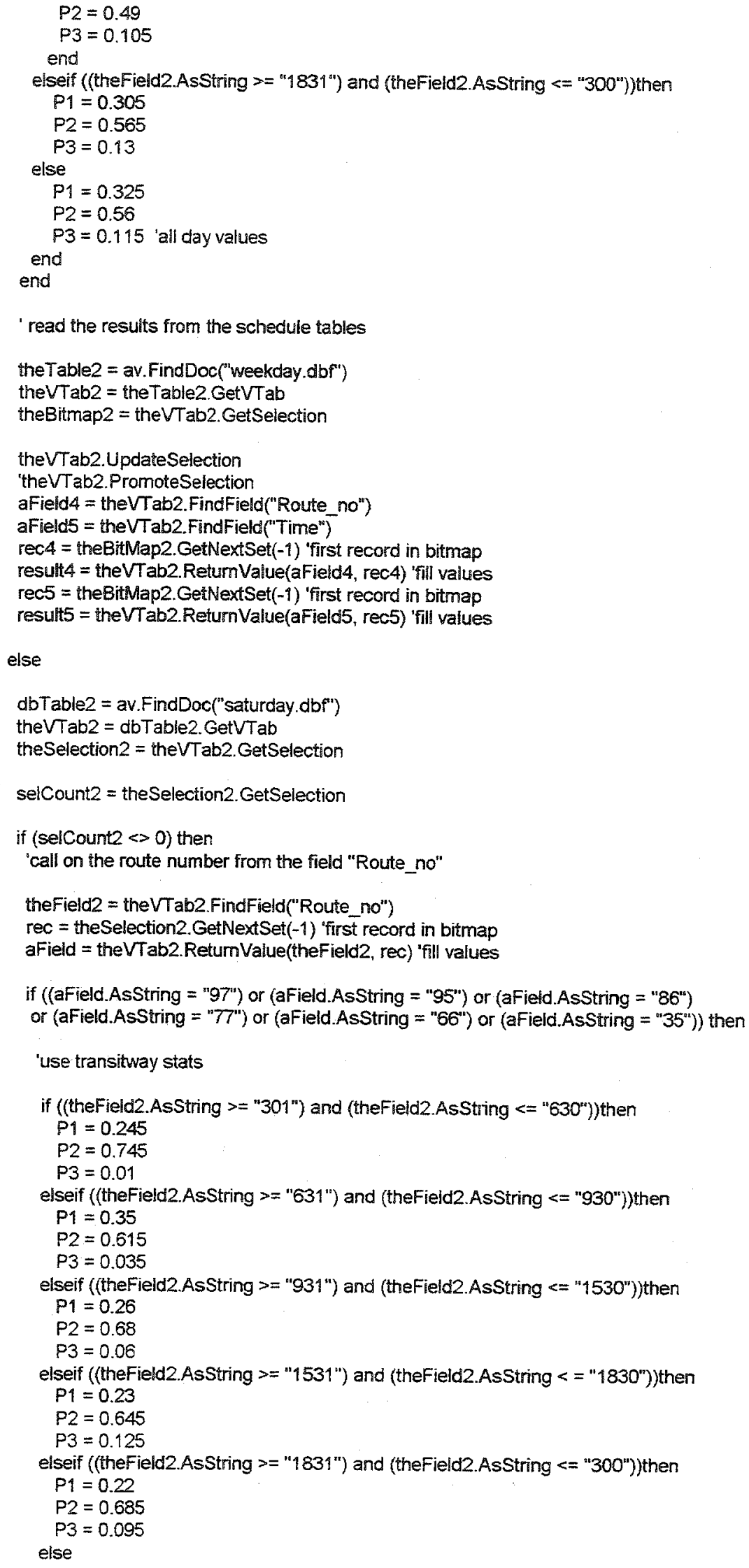




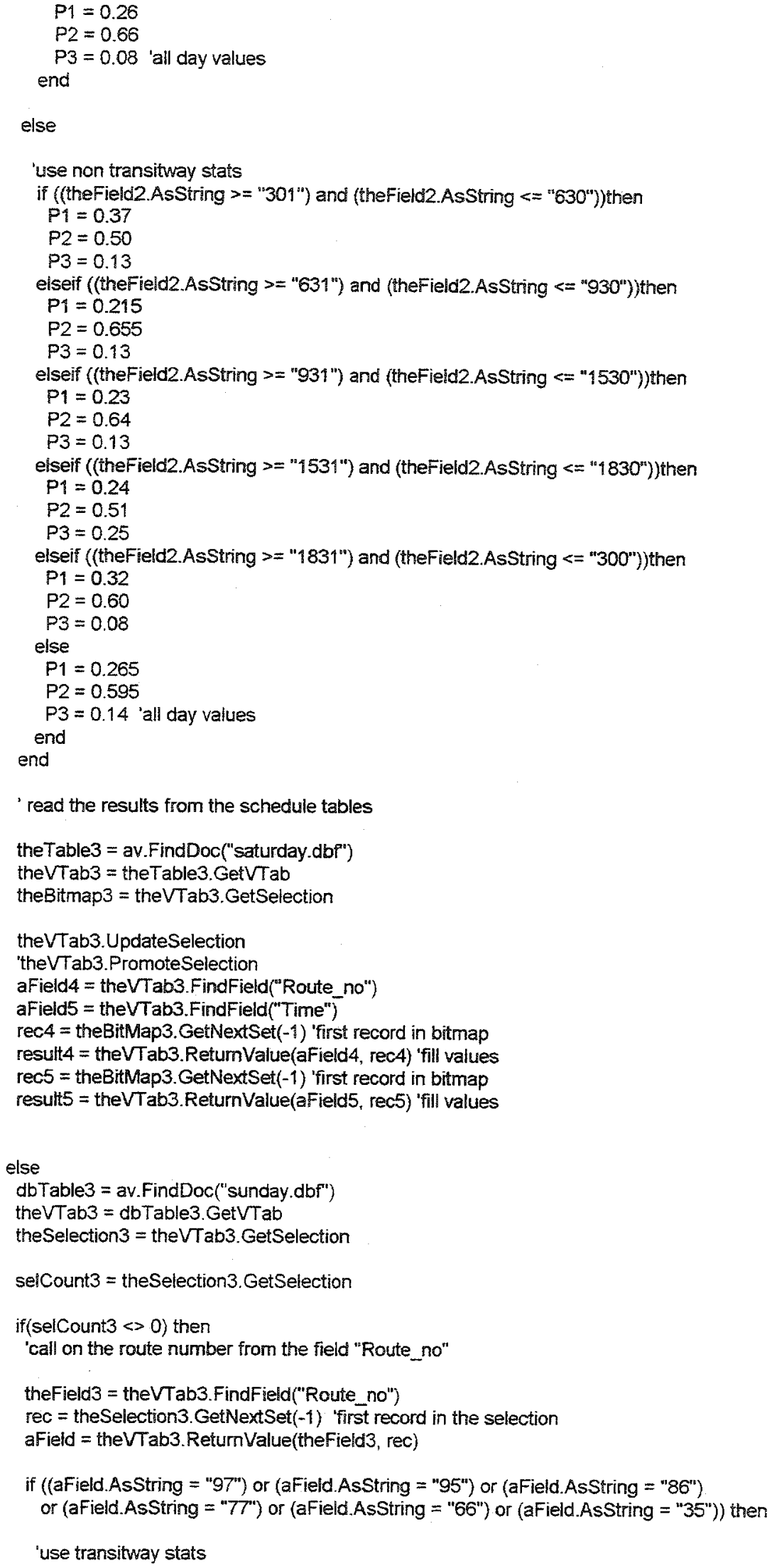




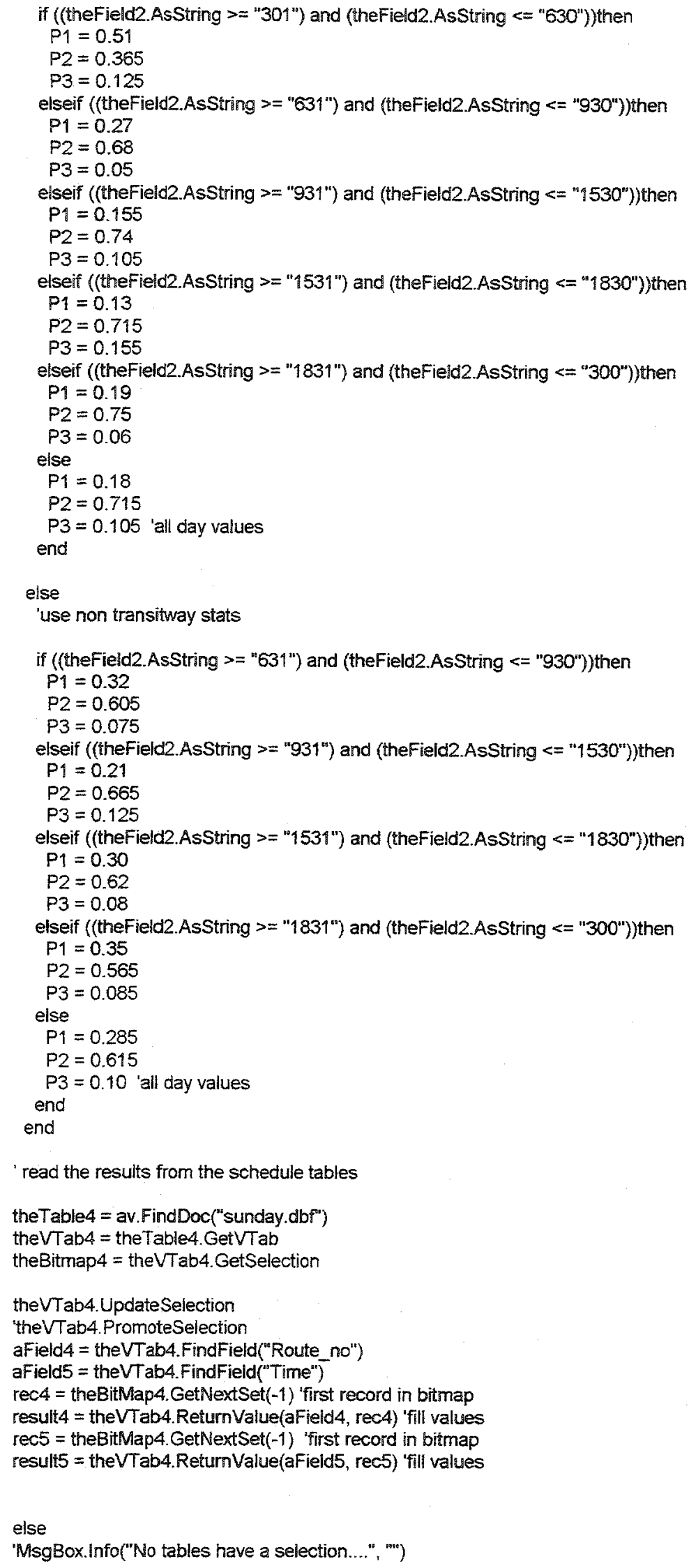




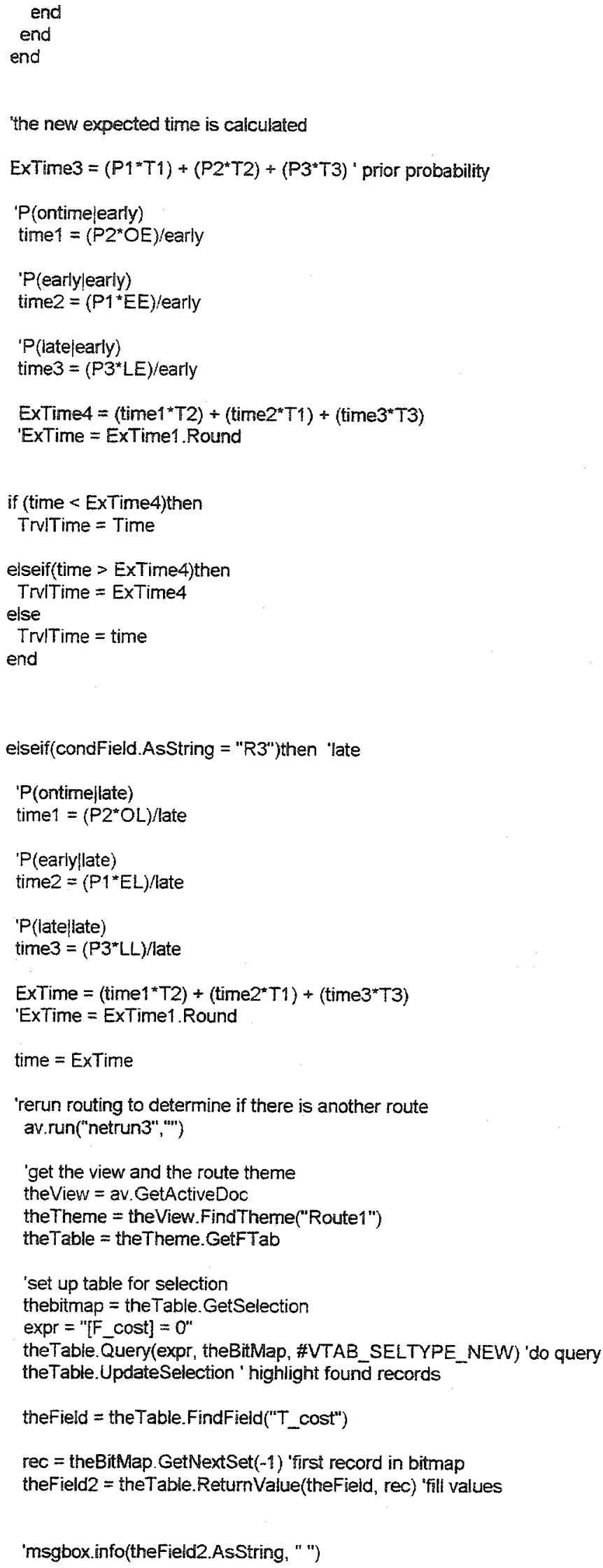




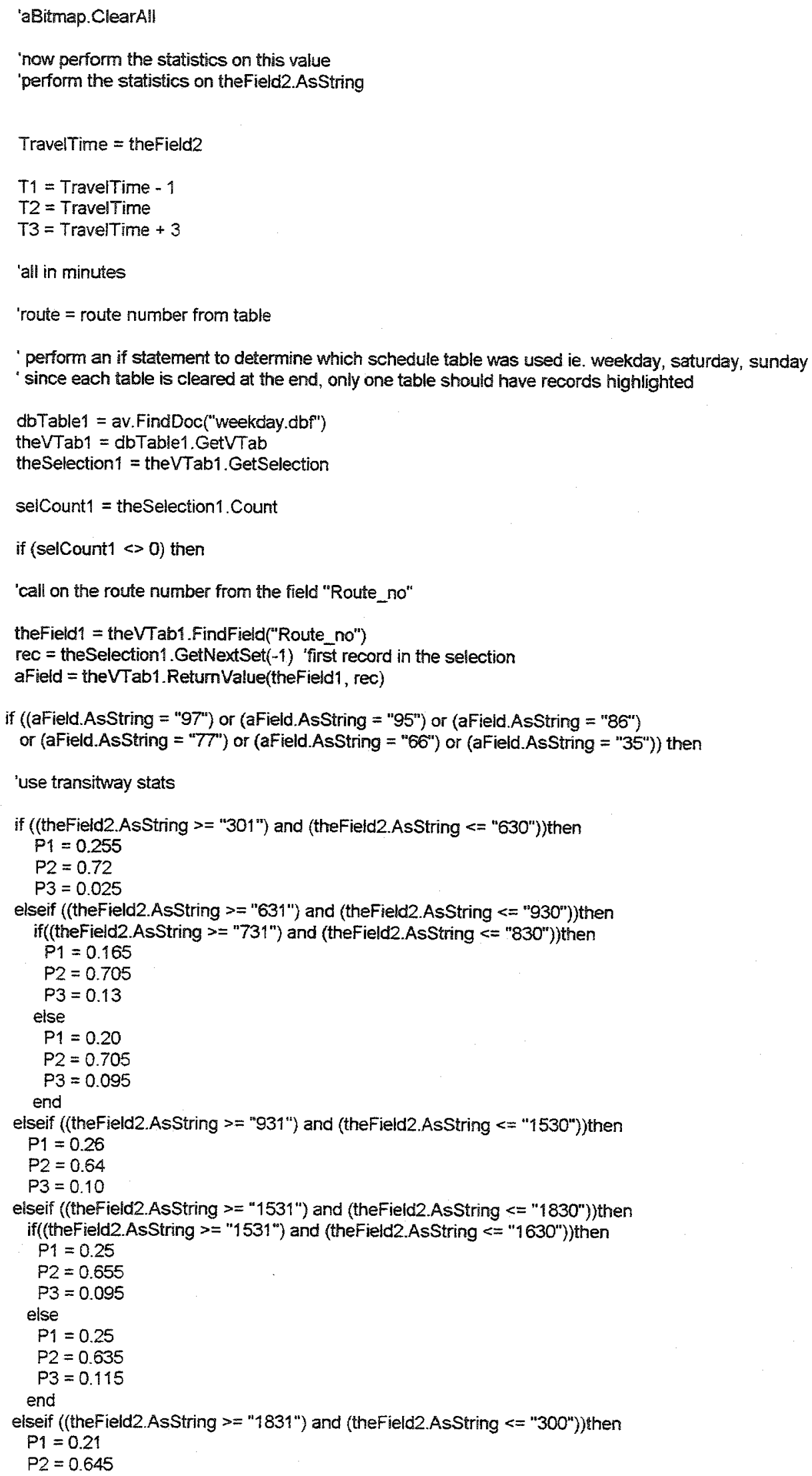




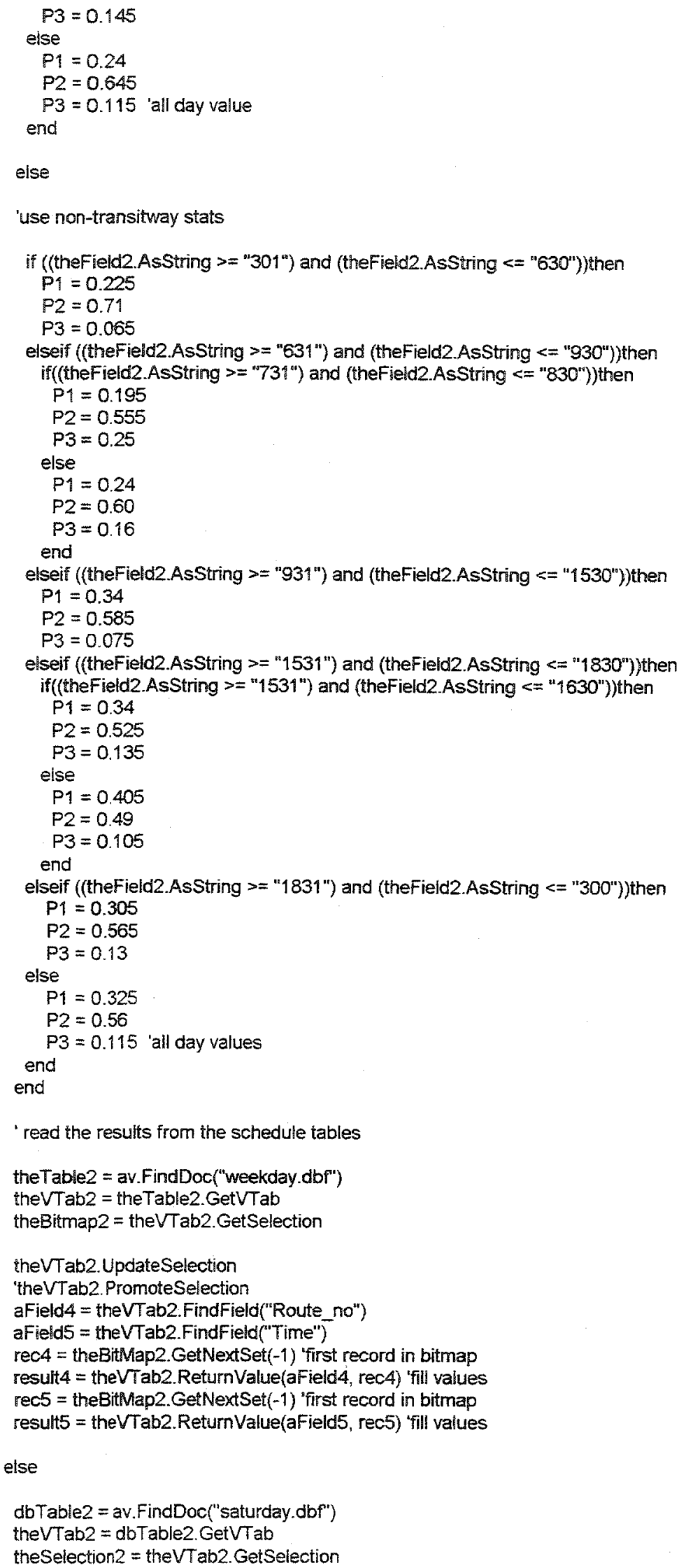




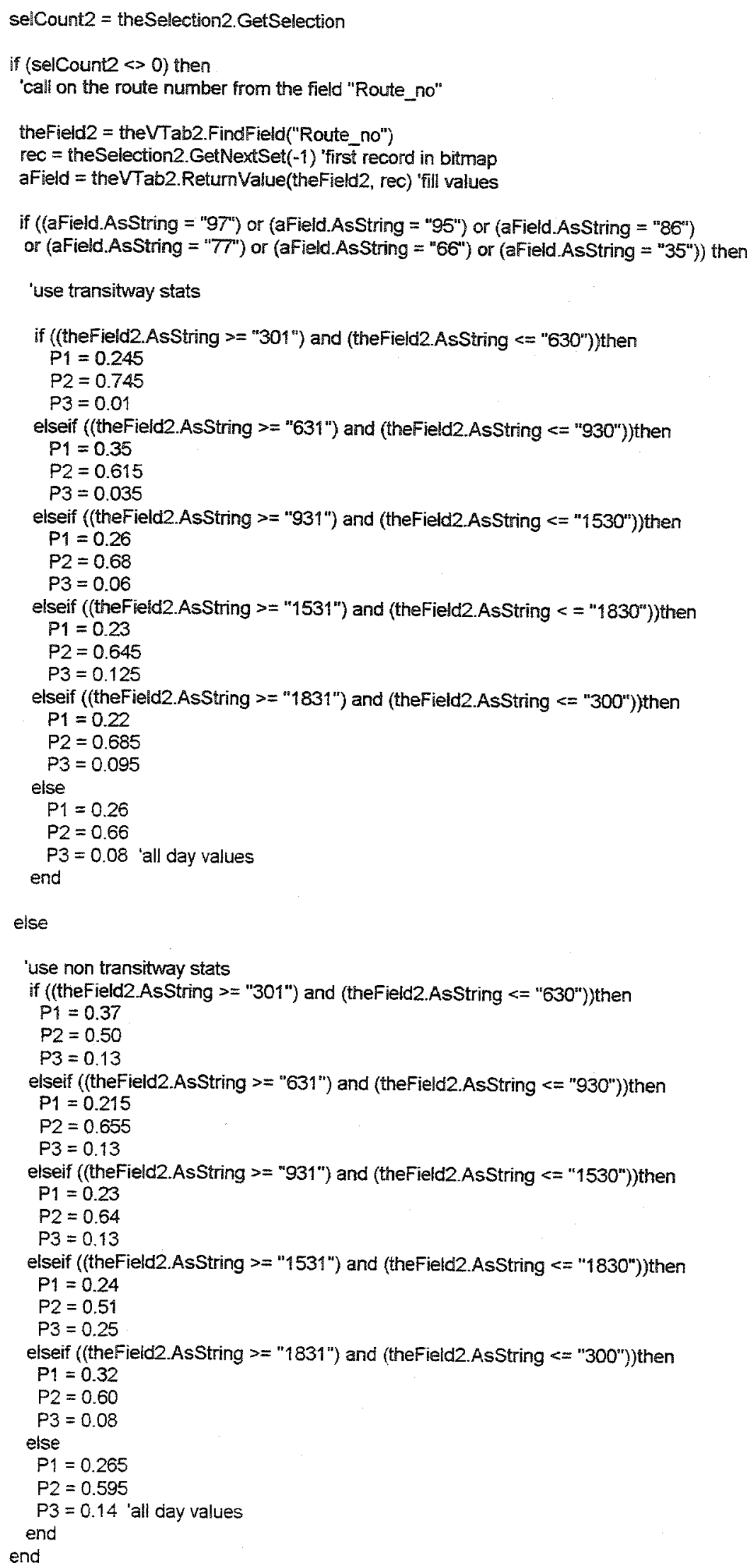


' read the results from the schedule tables

theTable $3=$ av.FindDoc("saturday.dbf")

theVTab3 = theTable3. GetVTab

theBitmap3 = theVTab3.GetSelection

theVTab3.UpdateSelection

'theVTab3.PromoteSelection

aField 4 = theVTab3.FindField("Route no")

aField5 = theVTab3.FindField("Time")

rec4 = theBitMap3.GetNextSet(-1) 'First record in bitmap

result4 = theVTab3.ReturnValue(aFieid4, rec4)' fill values

rec5 = theBitMap3. GetNextSet(-1) 'first record in bitmap

result5 $=$ theVTab3.ReturnValue(aField5, rec5) 'fill values

else

dbTable $=$ av. FindDoc("sunday.dbf")

theVTab3 = dbTable3. GetVTab

theSelection $3=$ theVTab3.GetSelection

selCount $3=$ theSelection 3. GetSelection

if(selCount $3<0$ ) then

'call on the route number from the field "Route no"

theField 3 = theVTab3.FindField ("Route no")

rec = theSelection3. GetNextSet(-1) 'first record in the selection

aField = theVTab3. ReturnValue(theField3, rec)

if ((aField.AsString = "97") or (aField.AsString = "95") or (aField.AsString = "86")

or (aField.AsString $=" 77 ")$ or (aField.AsString $=" 66 ")$ or (aField.AsString $=" 35 ")$ ) then

'use transitway stats

if ((theField2.AsString $>=" 301 ")$ and (theField2.AsString $<=$ "630"))then

$P I=0.51$

$P 2=0.365$

$P 3=0.125$

elseif ((theField2.AsString $>=$ "631") and (theField2.AsString $<=" 930 "$ ))then

$P 1=0.27$

$P 2=0.68$

$P 3=0.05$

elseif ((theField2.AsString $\left.>=" 931^{\prime \prime}\right)$ and (theFieid2.AsString $\left.<=" 1530 "\right)$ )then

$\mathrm{P} 1=0.155$

$P 2=0.74$

$\mathrm{P} 3=0.105$

elseif ((theField2.AsString $>=" 1531 ")$ and (theField2.AsString $\left.<=" 1830^{\prime \prime}\right)$ )then

$P 1=0.13$

$P 2=0.715$

$P 3=0.155$

elseif ((theField2.AsString $>=" 4831 "$ ) and (theField2.AsString $<=" 300 ")$ )then

$\mathrm{P} 1=0.19$

$P 2=0.75$

$P 3=0.06$

else

$\mathrm{P} 1=0.18$

$P 2=0.715$

$P 3=0.105$ 'all day values

end

else

'use non transitway stats

if ((thefield2.AsString >= "631") and (theField2.AsString $<=" 930 ")$ )then

$P 1=0.32$

$\mathrm{P} 2=0.605$

$\mathrm{P} 3=0.075$

elseif ((theField2.AsString $>=$ "931") and (theField2.AsString $\left.<=" 1530^{\prime \prime)}\right)$ then 


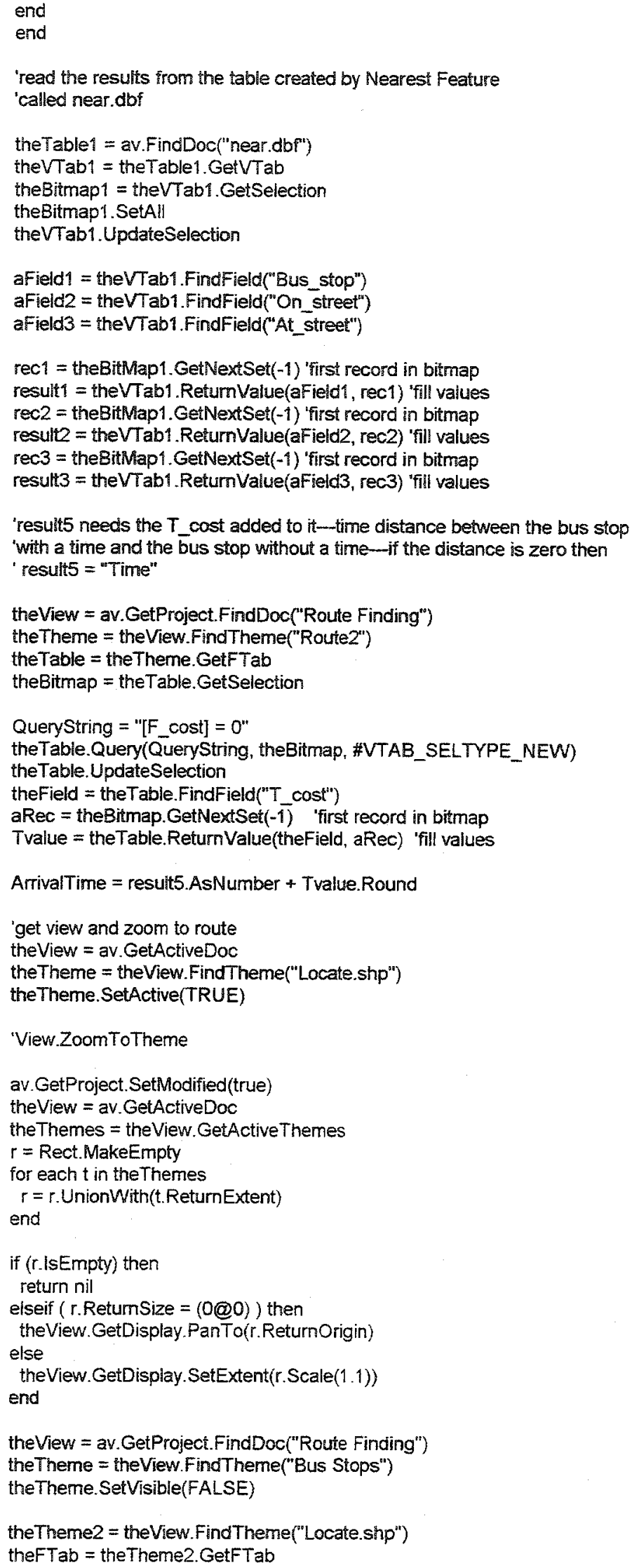




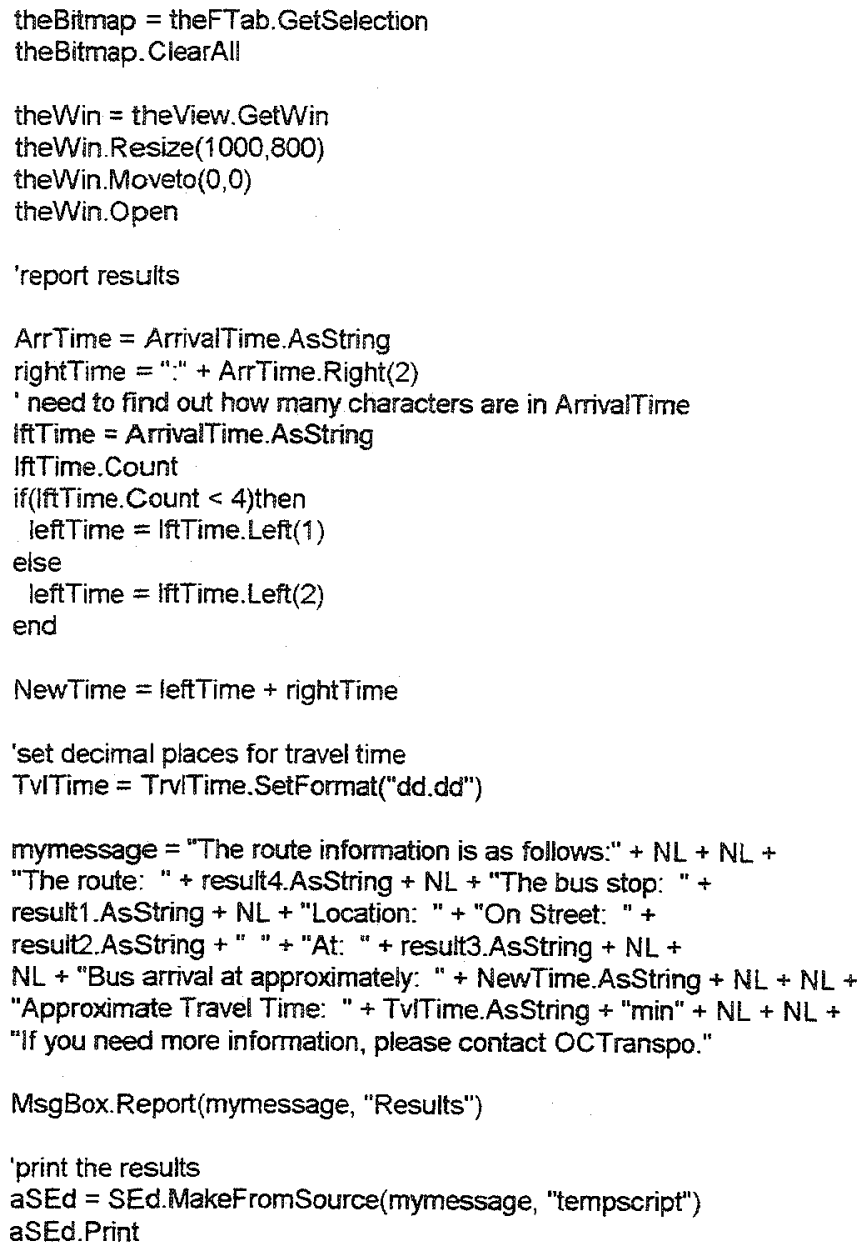




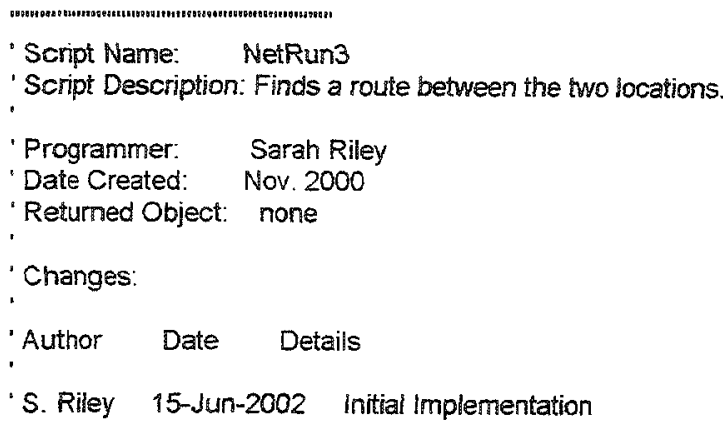

- Get a list of available costs for this line theme. Choose a cost from the list.

aNetCostFieidlist $=$ theNetDef.GetCostFields

aNetCostField = MsgBox.Choice(aNetCostFieldList, "Select Minutes", "Select Minutes")

"If a cost was chosen, set it.

if (aNetCostField <> ril) then the Network SetCostField(aNetCostField) end

"The above can be replaced by changing the hard code for Network Analyst wherein there will only be the chosen cost of MINUTES to select. there will only be

TravelDistance $=$ theNetwork. FindPath (thePointList, True, Faise) 'msgBox. Info( TravelDistance.AsString, "Total"-+reportUnitStr)

'theView.GetGraphics.add(GraphicShape.Make(theNetwork.ReturnPathShape))

aSymbol = Symbol.Make (\#SYMBOL_PEN)

aSymbol.SetSize(2.0)

aSymbol.SetColor(Color. GetMagente)

the $G L=$ theView. GetGraphics

theRoute=GraphicShape.Make(theNework.RetumPathShape)

theRoute.SetDisplay(av.GetActiveDoc.GetDisplay)

theroute.SetSymbol(aSymboi)

theGL.Add(the Route)

'theroute.GetSymbol.SetColor(color.GetMagenta).SetSymbol(aSymbol)

theRoute.Draw 
theroute.Invalidate

'set view size for public viewing

theView = av.getproject.finddoc("Route Finding")

theW in = the View. GetWin

"theW Win. Resize $(1025,600)$

'theWin.Moveto $(0,0)$

theWin.Open

- Write the route result FTab, this is necessary to get directons.

tmpFileName = FileName Make ("F:thesis data'Route.shp")

theNetwork. WritePath(tmpFileName)

resFTab = FTab.Make(SrcName.Make(tmpFileName.AsString))

'Show the directions then remove the temporary file

"the Directions $=$ "Your route directions are as follows" $+N L+N L+$ theNetwork.GetPathDirections(resFTab)

'msgBox.Repori(theDirections, "Route Information")

'add theme to view

newTheme $=$ FTheme.Make(resFTab)

theView.AddTheme(newTheme)

newTheme.SetName("Route1")

newTheme.SetVisible(True) 
"View. Hotlink

'Script Name: Theme.HotLink

' Script Description: Opens OC Transpo webpage linked to shapfile field "HotLink" of URL record

' Usage: As Tool under View Menu. Theme Properties linked to "HotLink" field of shapefile

'Author: Sarah Riley

Created: 15-Mar-2002

Changes:

Author Date Modifications

S. Riley 15-Mar-2002 Initial Implementation

' default project path should be "c:ischoof"

' default window header

Title = "HotLink to the Internet"

' Test Micro\$oft Operating system type, setup DLL's for appropriate system.

if (System. GetOSVariant $=$ \#SYSTEM OSVARIANT MSWNT) then dIIShell32 = DLL.Make("C: : winntlsystem32ishell32.dll".AsFileName) dilUser32 = DLL.Make("C: twinnt'system32luser32.dil".As FileName) elseif (System.GetOSVariant = \#SYSTEM_OSVARIANT_MSW95) then dllShell32 = DLL.Make("C:iwindowsisystemisheil32.dll".ÁsFileName) dllUser32 = DLL.Make("C: Iwindowstsystemiuser32.dI".AsFileName) else

MsgBox.Info("Windows is not being used.", Title)

exit

end

if $(($ dilShell32 $=$ nil $)$ or (dllUser32 $=$ nil $)$ ) then

MsgBox.Error("Can't find required DLL's. Check your setup.", Titte)

exit

end

' setup Win32API to talk with Avenue

dpGetActiveWindow = DLLProc.Make(dllUser32, "GetActiveWindow",\#DLLPROC_TYPE_INT32, \{0)

dpShellExecute $=$ DLLProc. Make

dIIShell32, "ShellExecuteA", \#DLLPROC_TYPE_INT32, \{

\#DLLPROC TYPE INT32

\#DLLPROC_TYPE_STR

\#DLLPROC TYPE STR

\#DLLPROC TYPE STR

\#DLLPROC TYPE STR

\#DLLPROC_TYPE_INT32

\}

' Get window handle of ArcView window

activeW in = DLL GetAWWindowHandle

' Get the URL off clicked area of theme

hotLink $=$ SELF

- Send info to default browser

hotLinkToBrowser = dpShellExecute.Call(\{ActiveWin, "Open", hotLink, Title, FileName.GetCWD.AsString, 1\})

if (hotLinkToBrowser $<=32$ ) then

MsgBox.Warning("Hotlink failed"', Title)

end 
'ESRI SCRIPT

'Get the project, get all themes and inactivate them except for ottawa streets

theProject $=$ av. GetProject

theView = the Project. FindDoc("Route Finding")

theThemeList=theView.getThemes

for each $i$ in (0.. (the ThemeList.count-1)

temp the ThemeList.get(i).setActive(FALSE)

end

theTheme=theView. Find Theme("Ottawa Street Network")

theTheme.setActive(TRUE)

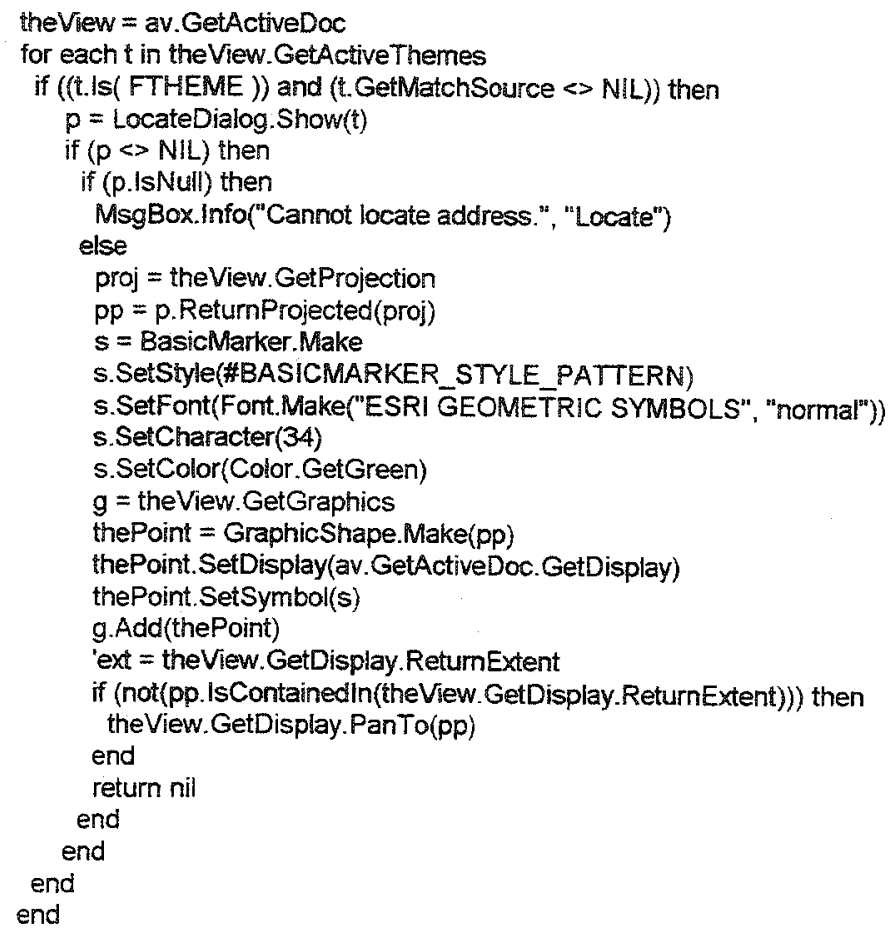




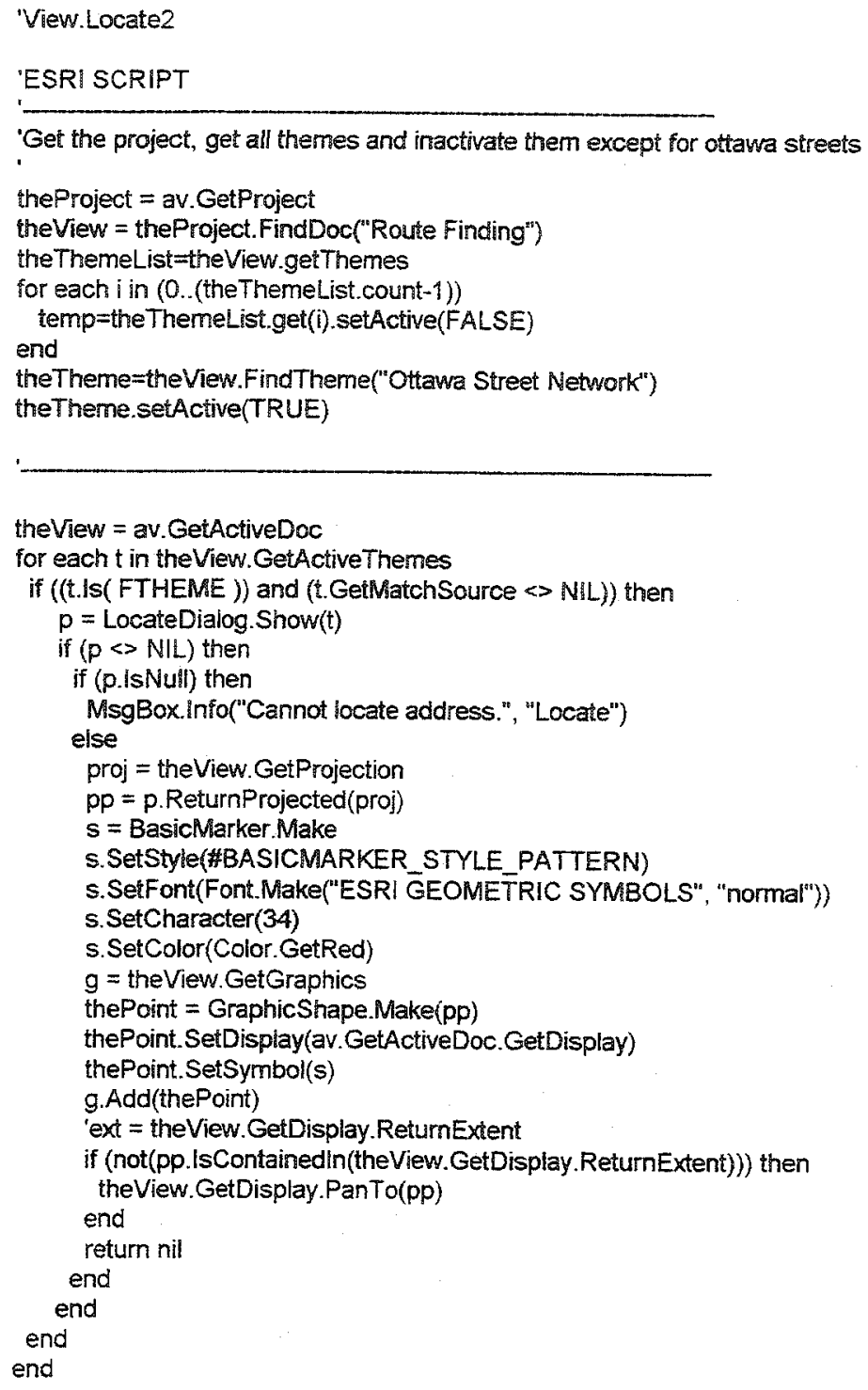




\section{Appendix D Attribute Tables}




\section{List of Tables}

Table D.1: Sample of Ottawa Street Network

Table D.2: Sample of Transit Routes

Table D.3: Sample of Bus Stops

Table D.4: Churches

Table D.5: Cinemas

Table D.6: Emergency

Table D.7: Grocery Stores

Table D.8: Hospitals

Table D.9: Hotels-Motels

Table D.10: Hotlink

Table D.11: Libraries

Table D.12: Location

Table D.13: Museums

Table D.14: Recreation Centers

Table D.15: Schools

Table D.16: Shopping Centers

Table D.17: Tourist Attractions 


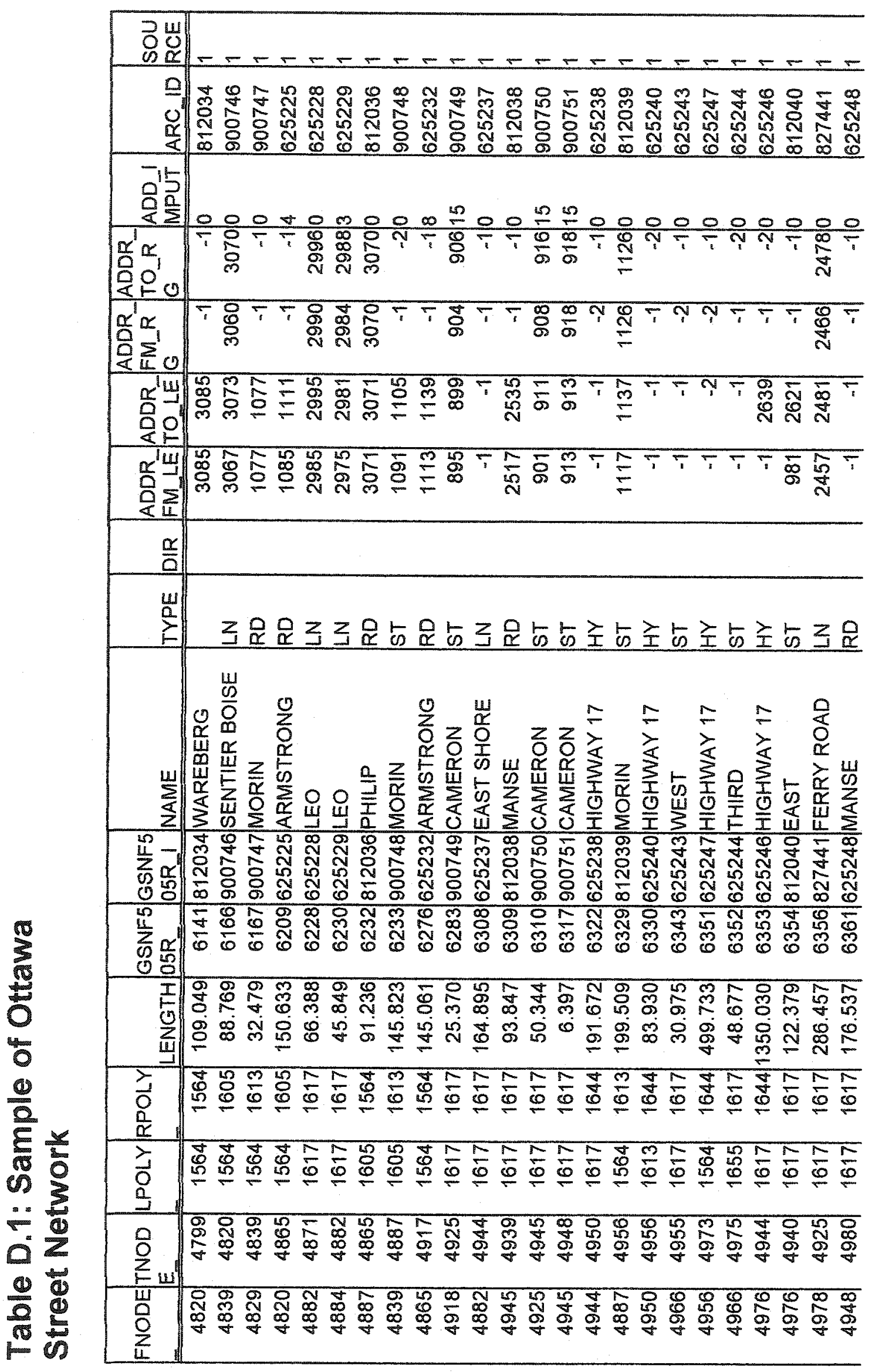




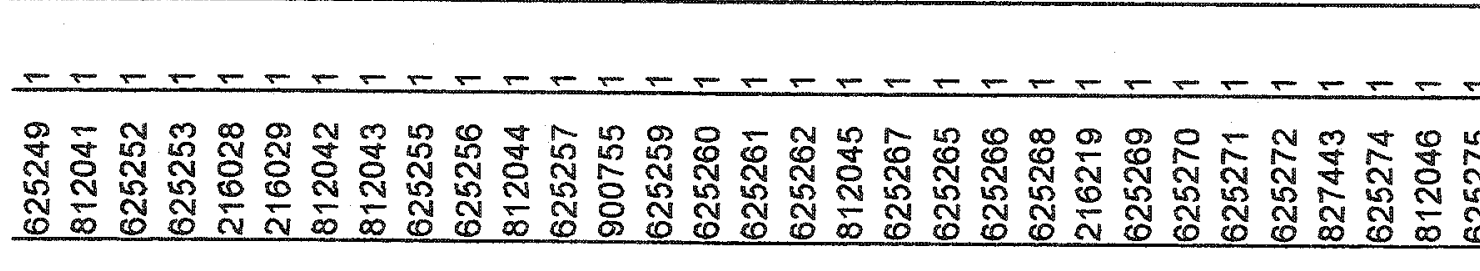

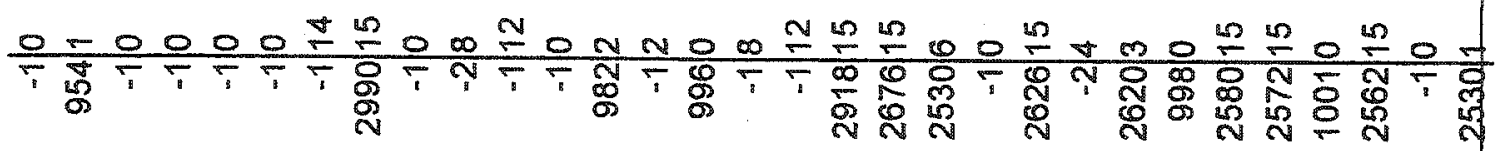

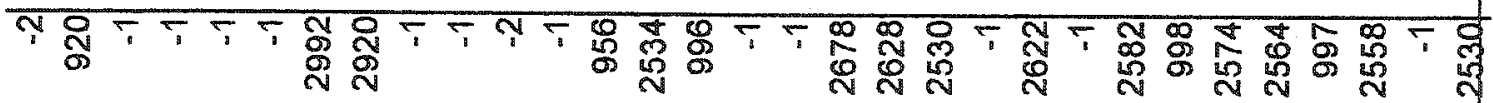

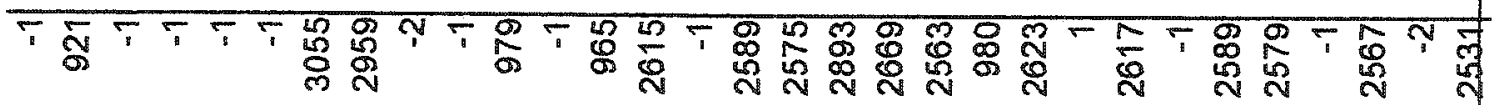

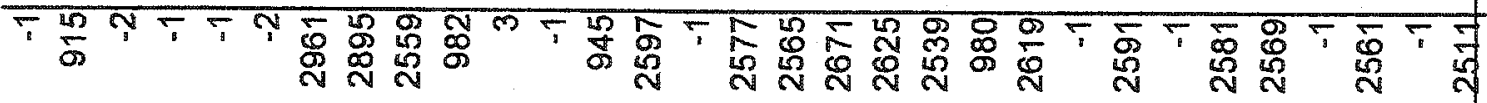

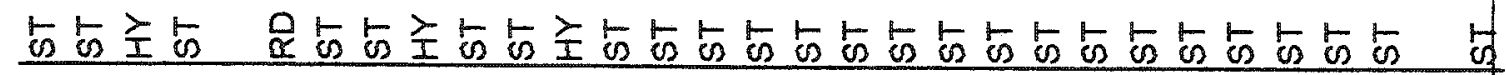

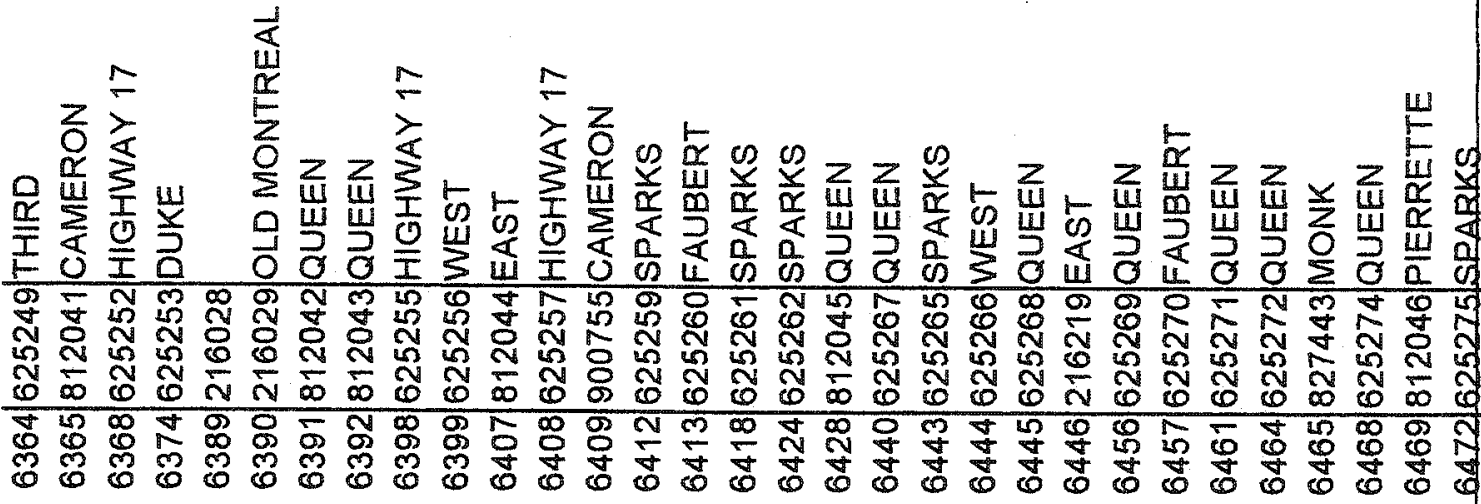

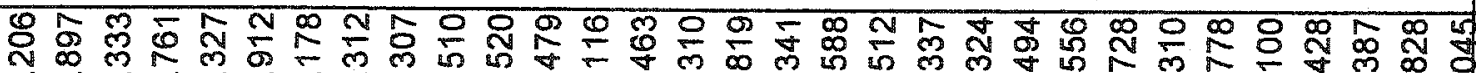
以

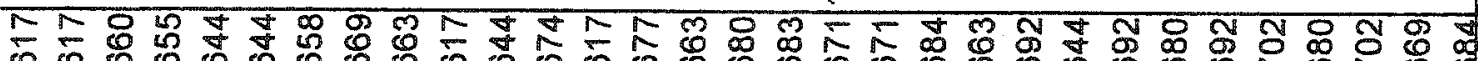

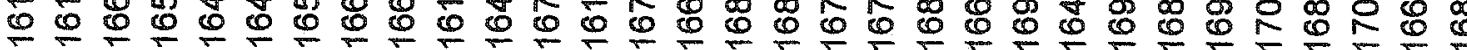
以下

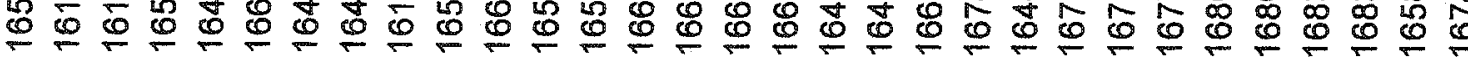

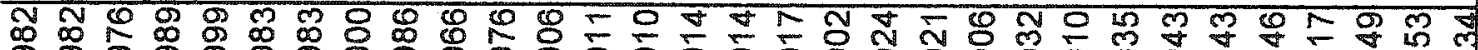

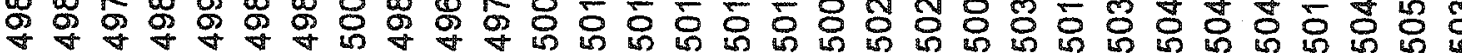

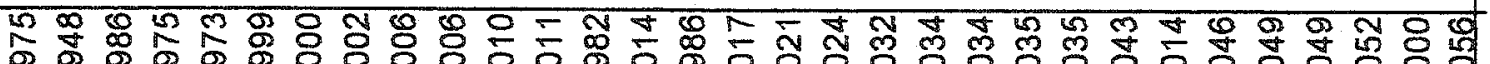

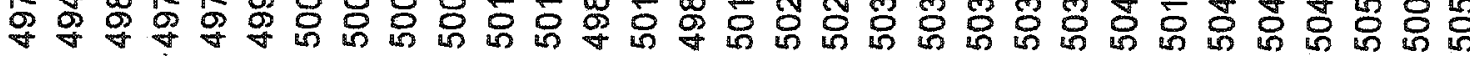




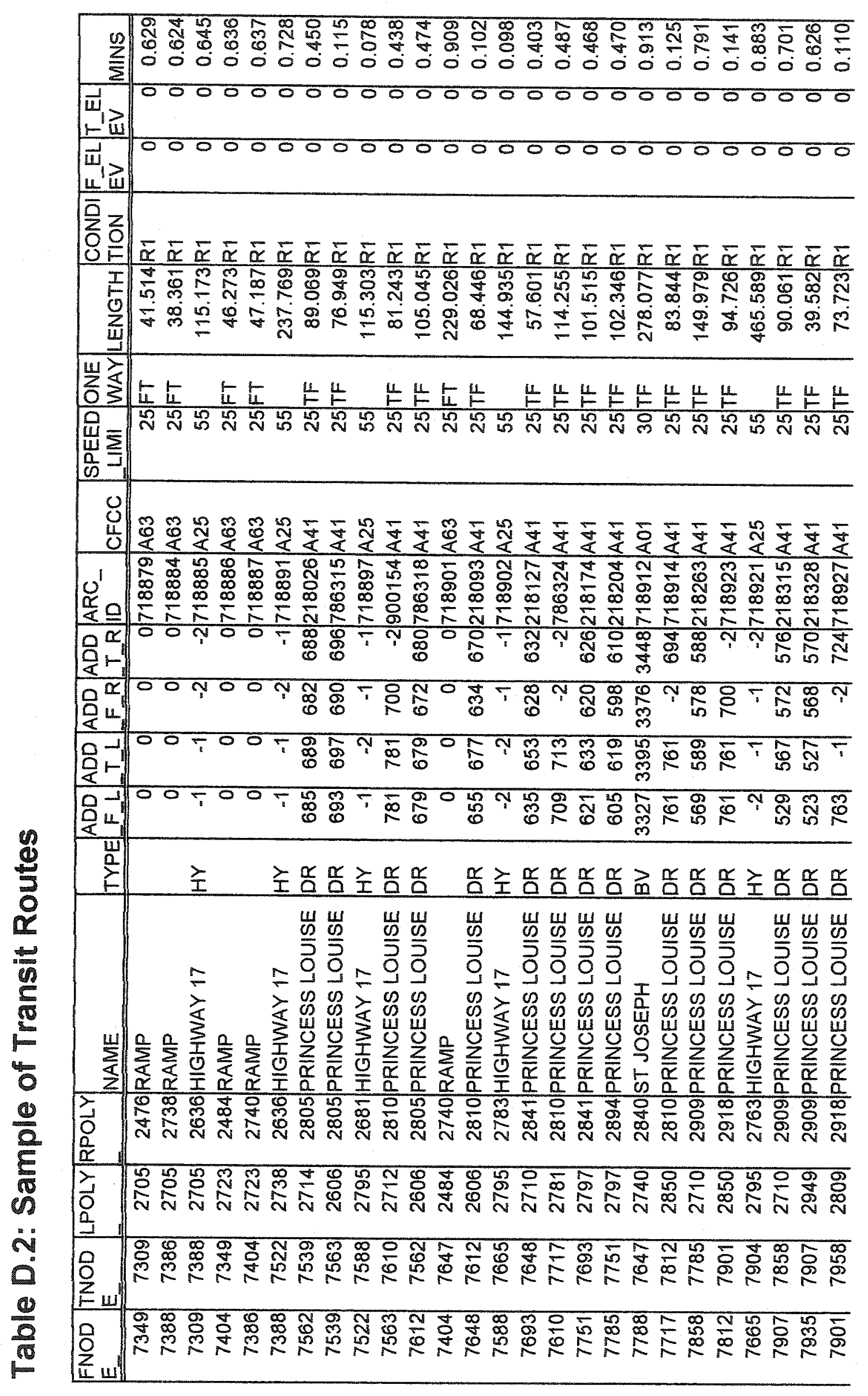


:

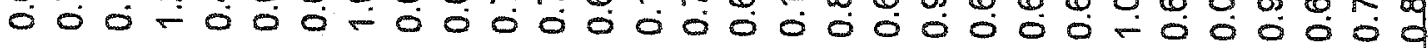

000000000000000000000000000000

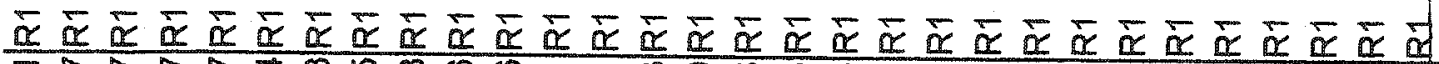

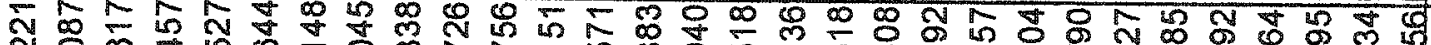

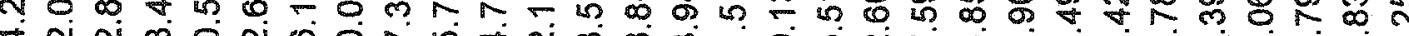

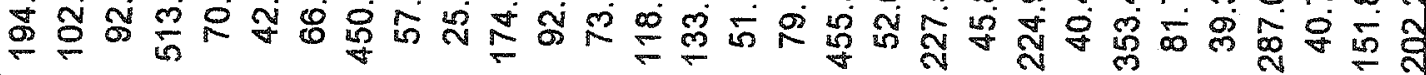

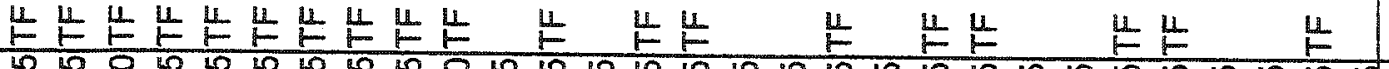

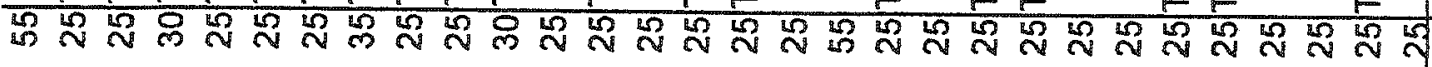

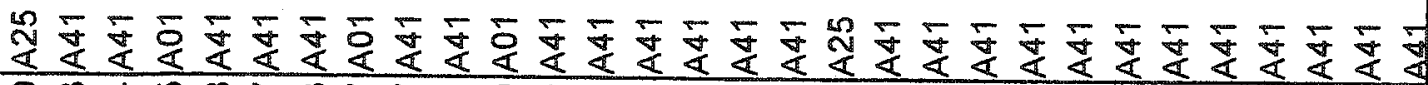

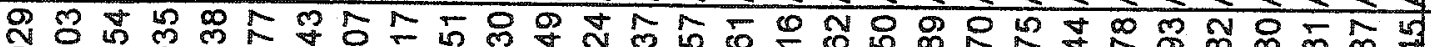

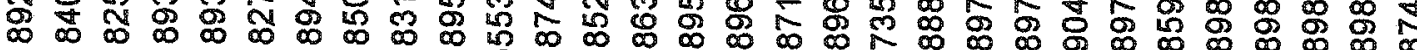

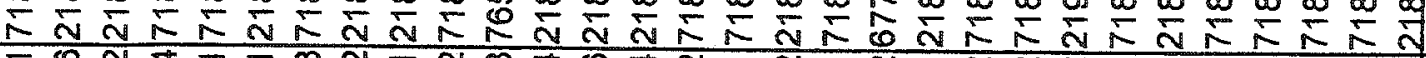

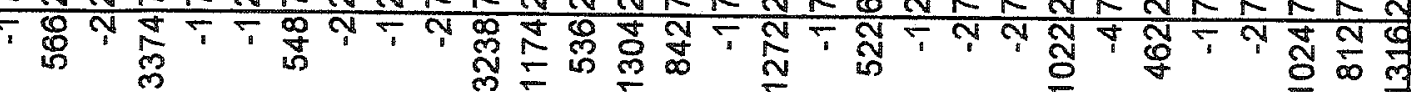

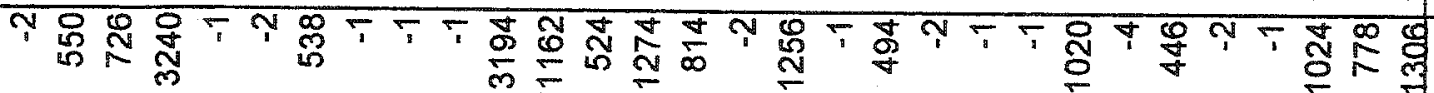

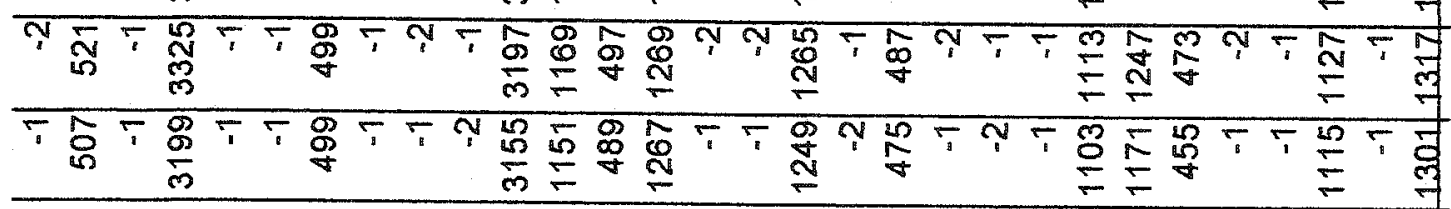

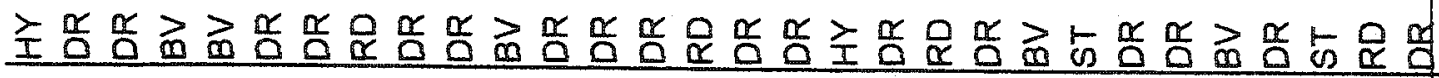

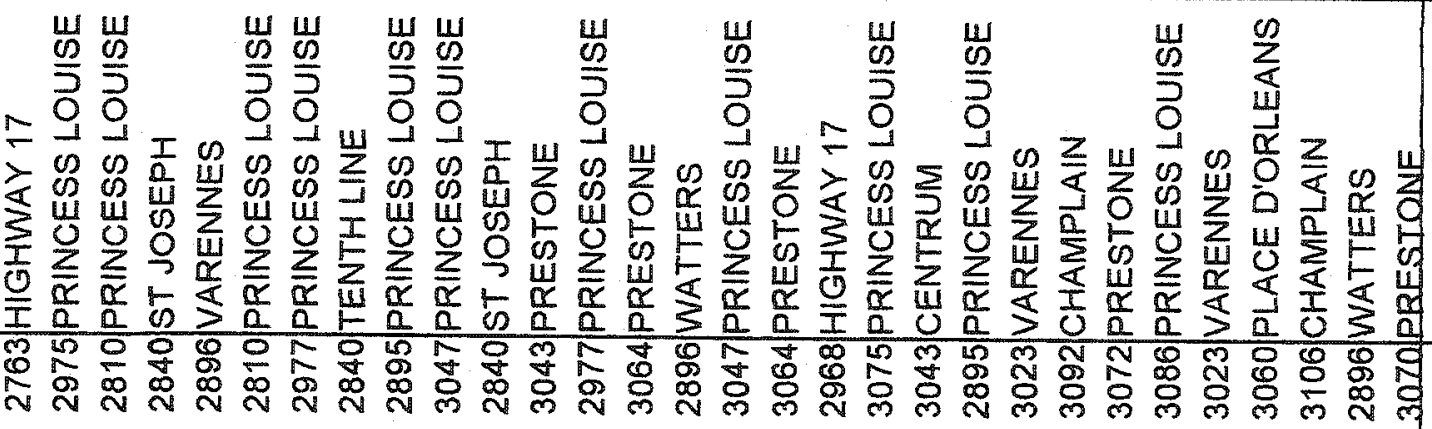

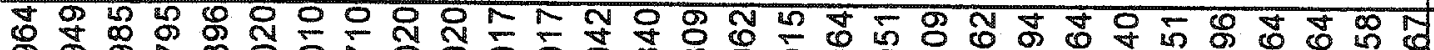
总 胥

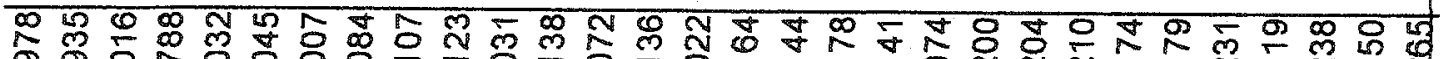

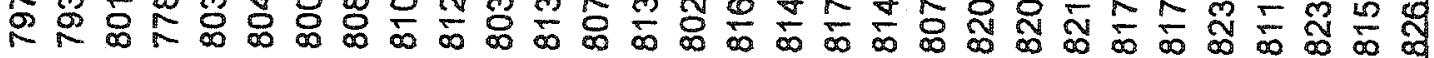

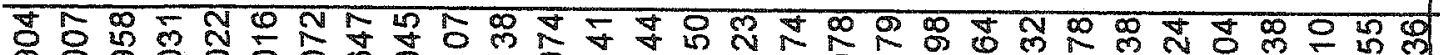

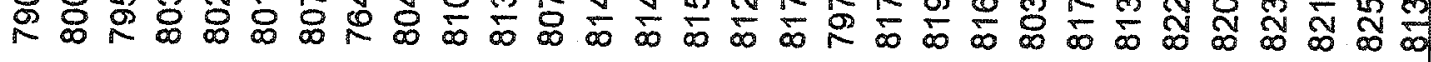


Table D.3: Sample of Bus Stops

\begin{tabular}{|c|c|c|c|c|c|c|}
\hline BUS STOF & JON_STREET & AT STREET & LATITUDE & LONGITUDE & OCO- & $\begin{array}{l}\text { DWELL } \\
\text { TIME }\end{array}$ \\
\hline WD564 & 417 RAMP & ACRES & 45.3448 & -75.8163 & 4 & 30 \\
\hline AF965 & ABBEY & $1 \mathrm{~A}$ & 45.4068 & -75.6648 & 4 & 60 \\
\hline AF970 & ABBEY & $2 A$ & 45.4068 & -75.6645 & 4 & 60 \\
\hline WJ620 & ABBEYHILL & CASTLEFRANK FS & 45.2944 & -75.8818 & 4 & 15 \\
\hline WJ650 & ABBEYHILL & BANNING NS & 45.2975 & -75.8794 & 4 & 15 \\
\hline WJ660 & ABBEYHILL & CHURCHM & 45.2969 & -75.8796 & 4 & 15 \\
\hline WJ670 & ABBEYHILL & HAZEL MALL ENT & 45.3000 & -75.8766 & 4 & 15 \\
\hline WD558 & ACRES & RICHMOND & 45.3429 & -75.8093 & 4 & 15 \\
\hline WD562 & ACRES & QUEENSWAY & 45.3444 & -75.8139 & 4 & 15 \\
\hline RE900 & AIRPORT & $1 \mathrm{~A}$ & 45.3201 & -75.6645 & 4 & 60 \\
\hline RE410 & AIRPORT PARKWAY & BRIDGE NS & 45.3287 & -75.6541 & 4 & 15 \\
\hline RE420 & AIRPORT PARKWAY & JOVERPASS FS & 45.3247 & -75.6602 & 4 & 15 \\
\hline RE430 & AIRPORT PARKWAY & DDEPARTURE & 45.3204 & -75.6654 & 4 & 15 \\
\hline CA920 & ALBERT & KENT & 45.4187 & -75.7034 & 4 & 60 \\
\hline CA930 & ALBERT & BAY & 45.4169 & -75.7076 & 4 & 60 \\
\hline CB900 & ALBERT & METCALFE & 45.4217 & -75.6961 & 4 & 60 \\
\hline CB910 & ALBERT & BANK & 45.4201 & -75.7001 & 4 & 60 \\
\hline C.J075 & ALBERT & TRANSITWAY FS & 45.4136 & -75.7117 & 4 & 60 \\
\hline CJU080 & ALBERT & BOOTHFS & 45.4125 & -75.7142 & 4 & 60 \\
\hline C.J085 & ALBERT & BOOTH NS & 45.4127 & -75.7135 & 4 & 60 \\
\hline CJ087 & ALBERT & PERKINS & 45.4133 & -75.7121 & 4 & 60 \\
\hline CJ090 & ALBERT & ROCHESTER FS & 45.4124 & -75.7141 & 4 & 60 \\
\hline AA035 & ALEXANDER & HOMAS & 45.4432 & -75.6930 & 4 & 15 \\
\hline AA050 & ALEXANDER & CHARLES NS & 45.4428 & -75.6921 & 4 & 15 \\
\hline$A B 300$ & ALFRED & MONTREAL FS & 45.4391 & -75.6549 & 4) & 15 \\
\hline AF090 & ALTA VISTA & POST OFFICE M & 45.4128 & -75.6562 & 4 & 30 \\
\hline AF 105 & ALTA VISTA & ROLLAND FS & 45.4116 & -75.6560 & 4 & 15 \\
\hline AF110 & ALTA VISTA & CALEDON FS & 45.4102 & -75.6573 & 4 & 15 \\
\hline AF120 & ALTA VISTA & CALEDON NS & 45.4101 & -75.6571 & 4 & 15 \\
\hline SD250 & AMBERWOOD & $\# 82$ FS & 45.3206 & -75.7073 & 4 & 15 \\
\hline$S D 260$ & AMBERWOOD & 86 NS & 45.3204 & -75.7073 & 4 & 15 \\
\hline SD270 & AMBERWOOD & LACEWOOD NS & 45.3205 & -75.7104 & 4 & 15 \\
\hline SD280 & AMBERWOOD & CALAIS NS & 45.3210 & -75.7106 & 4 & 15 \\
\hline SD290 & AMBERWOOD & \#43 NS & 45.3214 & -75.7132 & 4 & 15 \\
\hline SD300 & AMBERWOOD & $40 \mathrm{FS}$ & 45.3211 & -75.7130 & 4 & 15 \\
\hline SD310 & AMBERWOOD & WEATHERWD $1 \mathrm{~F}$ & 45.3203 & -75.7143 & 4 & 15 \\
\hline SD320 & AMBERWOOD & ERINLEA FS & 45.3194 & -75.7136 & 4 & 15 \\
\hline SD330 & AMBERWOOD & WEATHERWD \#2 F & 45.3187 & -75.7134 & 4 & 15 \\
\hline SD340 & AMBERWOOD & MERIVALE FS E & 45.3173 & -75.7148 & 4 & 15 \\
\hline EM580 & AMIENS & PRESTWICKNS & 45.4739 & -75.4990 & 4 & 15 \\
\hline SD640 & ANTARES & AURIGA NS & 45.3298 & -75.7083 & 4 & 15 \\
\hline SD660 & ANTARES & $56 \mathrm{M}$ & 45.3314 & -75.7096 & 4) & 15 \\
\hline
\end{tabular}




\begin{tabular}{|c|c|c|c|c|c|}
\hline SD670 & |ANTARES & 39 NS & 45.3315 & -75.7094 & 4 \\
\hline SD680 & ANTARES & CAPELLANS & 45.3340 & -75.7081 & 4 \\
\hline SD690 & ANTARES & CAPELLAFS & 45.3339 & -75.7078 & 4 \\
\hline SD700 & ANTARES & HUNT CLUB FS & 45.3350 & -75.7057 & 4 \\
\hline SD710 & ANTARES & HUNT CLUB NS & 45.3351 & -75.7048 & 4 \\
\hline SD620 & AURIGA & EPSILON NS & 45.3310 & -75.7035 & 4 \\
\hline SD635 & AURIGA & $46 \mathrm{MID}$ & 45.3304 & -75.7052 & 4 \\
\hline SD645 & AURIGA & $43 \mathrm{MID}$ & 45.3301 & -75.7058 & 4 \\
\hline SD650 & AURIGA & ANTARES NS & 45.3299 & -75.7075 & 4 \\
\hline EA700 & AVIATION MUSEUM & PLANTERS FS & 45.4584 & -75.6463 & 4 \\
\hline CAOBO & BANK & QUEEN NS & 45.4207 & -75.7011 & 4 \\
\hline CA090 & BANK & SLATER NS & 45.4193 & -75.6999 & 4 \\
\hline CA100 & BANK & SLATER NS & 45.4191 & -75.6997 & 4 \\
\hline CA110 & BANK & SPARKS FS & 45.4211 & -75.7013 & 4 \\
\hline CF 490 & BANK & RIVERDALE FS & 45.3914 & -75.6802 & 4 \\
\hline CF500 & BANK & CHESLEY FS & 45.3912 & -75.6802 & 4 \\
\hline CF510 & BANK & OSSINGTON M & 45.3923 & -75.6813 & 4 \\
\hline CF520 & BANK & GROVE FS & 45.3925 & -75.6819 & 4 \\
\hline CF530 & BANK & BELMONT NS & 45.3929 & -75.6821 & 4 \\
\hline CF540 & BANK & HOPEWELL NS & 45.3941 & -75.6836 & 4 \\
\hline CF550 & BANK & SUNNYSIDE FS & 45.3948 & -75.6838 & 4 \\
\hline CF560 & BANK & EUCLID NS & 45.3953 & -75.6844 & 4 \\
\hline CF570 & BANK & AYLMER OPP & 45.3957 & -75.6844 & 4 \\
\hline CF580 & BANK & WILTON NS & 45.3979 & -75.6858 & 4 \\
\hline CF590 & BANK & WILTON FS & 45.3981 & -75.6856 & 4 \\
\hline CF600 & BANK & COLLISEUM M OP & 45.3986 & -75.6862 & 4 \\
\hline CF610 & BANK & COLLISEUM M & 45.3990 & -75.6862 & 4 \\
\hline CF620 & BANK & HOLMWOOD FS & 45.3991 & -75.6865 & 4 \\
\hline CF630 & BANK & HOLMWOOD FS & 45.3998 & -75.6866 & 4 \\
\hline CF 640 & BANK & FIFTH NS & 45.4013 & -75.6877 & 4 \\
\hline CF650 & BANK & FIFTHNS & 45.4008 & -75.6872 & 4 \\
\hline CF660 & BANK & THIRD NS & 45.4026 & -75.6885 & 4 \\
\hline CF670 & BANK & THIRD FS & 45.4027 & -75.6883 & 4 \\
\hline CF680 & BANK & FIRST NS & 45.4039 & -75.6892 & 4 \\
\hline CF690 & BANK & FIRST NS & 45.4035 & -75.6887 & 4 \\
\hline CF700 & BANK & GLEBE NS & 45.4047 & -75.6896 & 4 \\
\hline CF710 & BANK & GLEBE NS & 45.4041 & -75.6891 & 4 \\
\hline CF 720 & BANK & STRATHCONA OPP & 45.4071 & -75.6911 & 4 \\
\hline CF730 & BANK & PATTERSON FS & 45.4062 & -75.6902 & 4 \\
\hline CF740 & BANK & CHAMBERLAIN FS & 45.4078 & -75.6914 & 4 \\
\hline CF750 & BANK & ISABELLA NS & 45.4084 & -75.6915 & 4 \\
\hline $\mathrm{CH} 010$ & BANK & LAURIER FS & 45.4183 & -75.6991 & 4 \\
\hline $\mathrm{CHO} 20$ & BANK & NEPEAN FS & 45.4176 & -75.6984 & 4 \\
\hline $\mathrm{CHO} 30$ & BANK & LISGAR NS & 45.4169 & -75.6978 & 4 \\
\hline $\mathrm{CHO} 40$ & BANK & SOMERSET FS & 45.4157 & -75.6967 & 4 \\
\hline $\mathrm{CH} 060$ & BANK & LEWIS NS & 45.4135 & -75.6947 & 4 \\
\hline
\end{tabular}




\section{Table D.4: Churches}

\begin{tabular}{|c|c|}
\hline NAME & ADDRESS \\
\hline All Saints (Sandy HiI) & 317 Chapel \\
\hline All Saints (Westboro) & 347 Richmond Rd \\
\hline Cathedral of the Annunciation & 289 Spencer \\
\hline Anglican Catholic Church of Canada & 190 Colonnade \\
\hline Epiphany & 1290 Ogilvie Rd \\
\hline St. Richard's & 8 Withrow \\
\hline St. John the Evangelist & 154 Somerset \\
\hline Christ Church Anglican Bells Corner & 3861 Richmond Rd \\
\hline St. George's & 152 Metcalfe \\
\hline St. Mark's & 1606 Fisher Ave \\
\hline Hawthome United & 2244 Russell Rd \\
\hline Barrhaven United & 3013 Jockvale \\
\hline Bell Street United & 384 Arlington \\
\hline Bell's Corners United & 3955 Richmond Rd \\
\hline Britannia United & 985 Pinecrest \\
\hline Calvary United & 1681 Cyrville Rd \\
\hline Carleton Memorial United & 740 Melia $\mathrm{Cr}$ \\
\hline City View United & 6 Epworth Ave \\
\hline Dominion Chalners United & 355 Cooper St \\
\hline Emmanuel United & 691 Smyth \\
\hline Fellowship United & 119 SteepleHill \\
\hline Glen Caim United & 140 Abbeyhill \\
\hline Woodroffe United & 207 Woodroffe \\
\hline Bilberry Creek & 480 Charlemagne $\mathrm{BI}$ \\
\hline Arabic Evangelical Baptist Church 0 & 887 Canterbury \\
\hline Bethany Baptist & 380 Centrepoint Dr \\
\hline Bromley Road Baptist & 1900 Bromley Rd \\
\hline Calvary Baptist & 107 Main St \\
\hline Eglise Baptiste Evangelique D'Ottaw & 284 King Edward \\
\hline Fellowship Baptist & 1078 Klondike \\
\hline Haven Baptist & 4000 Strandherd \\
\hline Nepean Baptist & 135 Woodfield \\
\hline Our Lady of Fatima & 153 Woodroffe Ave \\
\hline Annunciation - St. Nicholas Orthodo & 55 Clarey Ave \\
\hline Divine Infant Roman Catholic & 6658 Bilberry Dr \\
\hline Holy Redeemer & 44 Rothsay \\
\hline Our Lady of Perpetual Help & 15 Lebreton \\
\hline St. Patrick's Basillica & 281 Nepean St \\
\hline St. Anthony's & 427 Booth St \\
\hline St. Augustine's & 1060 Baseline Rd \\
\hline St. Barnabas & 70 James \\
\hline St. John the Apostle & 2340 Baseline Rd \\
\hline
\end{tabular}


St. John the Divine

105 Slack Rd

St. Joseph

St. Maurice

St. Patrick's - Fallowfield Barrhav

St. Thomas the Apostle

Abiding Word Evangelical Lutheran

All Saints

Christ Risen Lutheran

Divine Word Evangelical Lutheran

Faith Evangelical Lutheran

Trinity Presbyterian

Erskine Presbyterian

151 Laurier

4 Petry St

41 Weybridge

2345 Alta Vista Dr

1675 Belcourt Blvo

1061 Pinecrest

85 Leacock Dr

1081 Greenbank Rd

43 Meadowlands

110 McCurdy

343 Bronson

Grace Presbyterian

1220 Tenth Line Rd

Knox Presbyterian

120 Lisgar

St. Andrew's

82 Kent St 


\section{Table D.5: Cinemas}

\begin{tabular}{|l|l|}
\hline NAME & ADDRESS \\
\hline ByTowne Cinema & 325 Rideau St \\
Coliseum & 3090 Carling Ave \\
Famous Players & 50 Rideau St \\
IMAXIOMNIMAX & 100 Laurier \\
Mayfair Theatre & 1074 Bank St \\
World Exchange & 111 Albert St \\
Orlean Town Centre & 250 Centrum Blvd \\
South Keys & 2240 Bank St \\
Silvercity & 2385 City Park Dr \\
\hline
\end{tabular}




\section{Table D.6: Emergency}

\begin{tabular}{|c|c|c|}
\hline 10 & NAME & ADDRESS \\
\hline 36 & Barrhaven Base & 1073 Greenbank Road \\
\hline 38 & 3 lndustrial Base & 911 industrial Avenue \\
\hline 40 & oland Ambulance Services - Leased & 530 Tremblay Road \\
\hline 41 & Manotick Base & 5669 Main Street \\
\hline 42 & March Base & 1040 Riddell Drive \\
\hline 43 & 3) Metcalfe Base & 8011 Victoria Street \\
\hline 45 & Forleans Base & 1439 Youville Drive \\
\hline 47 & 7. Ottawa 01 Base & 738 Gladstone Avenue \\
\hline 48 & Ottawa 02 Base & 360 Hunt Club Road \\
\hline 49 & Ottawa Ambulance (CACC) & 2380 St Laurent Boulevard \\
\hline 50 & Queensway Base & 3045 Baseline Road \\
\hline 51 & Richmond Base & 6179 Perth Street \\
\hline 52 & Stittsville Base & 1631 Main Street \\
\hline 54 & Cumberland Fire Station 1 & 500 Charlemagne Boulevard \\
\hline 58 & Elections/Beaver Boxing & 1447 Carling Avenue \\
\hline 50 & Gloucester Fire Station 1 & 1700 Biair Road \\
\hline 60 & Gloucester Fire Station 2 & 6213 Jeanne D'Arc Boulevard \\
\hline 61 & Gloucester Fire Station 3 & 3202 Leitrim Road \\
\hline 62 & Gloucester Fire Station 4 & 3080 Old Innes Road \\
\hline & Goulbourn Fire Station 1 and Ambulance Base & \\
\hline 63 & Stittsville & 1631 Main Street \\
\hline 64 & Goulbourn Fire Station 2 - Richmond & 6280 Perth Street \\
\hline 65 & Kanata Fire Station 1 & 1021 Teron Road \\
\hline 66 & Kanata Fire Station 2 & 1040 Riddell Drive \\
\hline 67 & Kanata Fire Station 3 & 380 Eagleson Road \\
\hline 68 & Nepean Fire Station 1 - Bells Corners & 3845 Richmond Road \\
\hline 69 & Nepean Fire Station 2 - Viewmount & 230 Viewmount Drive \\
\hline 70 & Nepean Fire Station 3 - Barhaven & 1075 Greenbank Road \\
\hline 71 & Nepean Fire Station 4 - Knoxdale & 60 Knoxdale Road \\
\hline 72 & Osgoode Fire Station 1 - Metcalfe & 8011 Victoria Road \\
\hline 74 & Osgoode Fire Station 3 - Greely & 6891 Parkway \\
\hline 76 & Ottawa Fire Station 1 & 1443 Carling Avenue \\
\hline 77 & Ottawa Fire Station 2 & 135 Preston Street \\
\hline 78 & Ottawa Fire Station 3 & 530 King Edward Avenue \\
\hline 79 & Ottawa Fire Station 4 & 900 Industrial Avenue \\
\hline 80 & Ottawa Fire Station 5 & 900 Montreal Road \\
\hline 81 & Ottawa Fire Station 6 & 220 Beechwood Avenue \\
\hline 82 & Ottawa Fire Station 7 & 700 Brookfield Road \\
\hline 83 & Ottawa Fire Station 8 & 2355 Altavista Drive \\
\hline & Ottawa Fire Station 9 & 3336 McCarthy Road \\
\hline 85 & Ottawa Fire Station 10 & $6350^{\prime}$ Connor Street \\
\hline
\end{tabular}


Carlington Community Police Centre/ Carlington 102 poste de police communautaire

Central Police Headquarters / Poste de police 103 centrale

Cumberland Community Police Centre /

2E Lovell Lane

1309 Carling Ave

474 Elgin Street

105 Cumberland poste de police communautaire Cyrville Community Police Centre / Cyrville

106 poste de police communautaire

East Division Headquarters / Div. est poste de 107 police

Kanata Community Police Centre / Kanata

109 poste de police communautaire

Lowertown Foot Patrol / Patrouille p@destre de 110 la Basse-Ville

Manotick Community Police Centre/ Manotick

111 poste de police communautaire

Metcalfe Community Police Centre/ Metcalfe

112 poste de police communautaire

Orleans Community Police Centre / Poste de

113 police communautaire d'Orieans

Parkwood Hills Community Police Centre /

115 Parkwood Hills poste de police communautaire

116 Police Station - Leased

117) Police Station - Leased

Professional Development Centre/ Centre de

118 perfectionnement professionnel

260 Tompkins Road

1980 Ogilvie Road

4561 Highway 31

95 Abbeyhill Drive

368 Dalhousie Street

5542 Ann Street

8253B Victoria Street

5929 Jeanne D'Arc Boulevard

1343 Meadowlands Drive 1329 Wellington Street

21 Concourse Gate

1385 Woodroffe Avenue

111 Lisgar Street

Richmond Community Police Centre/ Richmond

120 poste de police communautaire

Rideau Street Community Police Centre / rue

121 Rideau poste de police communautaire Rockcliffe Park Community Police Centre /

122 Rockcliffe Park poste de police communautaire Somerset Community Police Centre / Somerset

123 poste de police communautaire

South Ottawa Community Police Centre /

124 Ottawa-Sud posie de police communautaire

Stittsville Community Police Centre / Stittsville

125. poste de police communautaire

\author{
6179 Perth Street \\ 362 Rideau Street \\ 360 Springfield Road \\ 393 Somerset Street West \\ 2870 Cedarwood Drive \\ 1532 Main Street
}


Vanier Community Police Centre / Vanier poste 126. de police communautaire

252 McArthur Road West Division Headquarters and Community

Police Centre / Div. ouest et poste de police

128. communautaire

245 Greenbank Road

Westboro Community Police Centre / Westboro

129 poste de police communautaire

418A Richmond Road

130 Youth Gym / Gymnase des jeunes

1463 Prince of Wales Drive 


\section{Table D.7: Grocery Stores}

\begin{tabular}{|c|c|}
\hline NAME & ADORESS \\
\hline A \& P/Superfresh McCarth & 3310 McCarthy Rd \\
\hline A \& P/Superfresh Heron & 1670 Heron Rd \\
\hline Bulk Barn & 2210 Bank St \\
\hline Costco & 1900 Cyrville Rd \\
\hline Farm Boy Fresh Merivale & 1642 Merivale Rd \\
\hline Farm Boy Fresh Montreal & 585 Montreal Rd \\
\hline Farm Boy Fresh Hazeldean & 457 Hazeldean Rd \\
\hline Farm Boy Fresh Centrum & 240 Centrum Rd \\
\hline Food Basics Kirkwood & 667 Kirkwood Ave \\
\hline Food Basics Charlemagne & 470 Charlemagne \\
\hline Food Basics Katimavik & 150 Katimavik \\
\hline IGA Barthaven & 900 Greenbank Rd \\
\hline Loblaws Ogilvie & 1980 Ogilvie Rd \\
\hline Loblaws Orleans & 1226 Orleans Blvd \\
\hline Loblaws Eagleson & 700 Eagleson Rd \\
\hline Loblaws Merivale & 1460 Merivale Rd \\
\hline Loblaws Robertson & 59 Robertson Rd \\
\hline Loblaws Strandherd & 3777 Strandherd \\
\hline Loblaws Bank & 2210 Bank St \\
\hline Loblaws Carling & 2085 Carling Ave \\
\hline Loblaws Iris & 2685 Iris \\
\hline Loblaws Isabella & 64 Isabella \\
\hline Loblaws Rideau & 363 Rideau St \\
\hline Loblaws Riverside & 2241 Riverside Dr \\
\hline Loblaws St. Laurent & 1910 St Laurent BI \\
\hline Loblaws McArthur & 100 McArthur Ave \\
\hline Loeb Merivale & 1512 Merivale $\mathrm{Rd}$ \\
\hline Loeb Booth & 314 Booth St \\
\hline Loeb Bank & 754 Bank St \\
\hline Loeb Rideau & 245 Rideau St \\
\hline Loeb Walkey & 2261 Walkley Rd \\
\hline Loeb Carling & 4048 Carling Ave \\
\hline Loeb Richmond & 3655 Richmond Rd \\
\hline Loeb Carleton & 345 Carleton \\
\hline Loeb South Keys & 2515 Bank St \\
\hline Loeb Gladwin & 2212 Gladwin $\mathrm{Cr}$ \\
\hline Loeb Beechwood & 50 Beechwood Ave \\
\hline Loeb Montreal & 1930 Montreal Rd \\
\hline Loeb St.Laurent & 1021 St. Laurent \\
\hline Loeb McArthur & 320 McArthur Ave \\
\hline Loeb Innes & 4510 Innes \\
\hline
\end{tabular}




Loeb 1360 Richmond
Loeb Greenbank
Loeb Hazeldean
Loeb Jeanne D'Arc
Loeb Tenth Line
Market Fresh St. Laurent
Market Fresh Selkirk
Sobeys
YIG Carling
YIG Laurier
YIG Eagleson
YIG Bank
YIG Grant Carmen
YIG Orieans
YIG Alta Vista
YIG 2210 Bank

1360 Richmond

250 Greenbank Rd

420 Hazeldean

6509 Jeanne d'ArC

1675 Tenth Line

1200 St. Laurent B

29 Selkirk

700 Terry Fox Dr

2148 Carling Ave

2737 Laurier Ave

300 Eagleson Rd

296 Bank St

200 Grant Carmen

1619 Orleans Blvd

2681 Alta Vista Dr

2210 Bank St 


\section{Table D.8: Hospitals}

\begin{tabular}{|r|l|l|}
\hline ID & NAME & ADORESS \\
\hline \hline 1 & Royal Ottawa Hospital & 1145 Carling Avenue \\
2 & Ottawa Hospital - Civic Campus & 1053 Carling Avenue \\
3 & Ottawa Hospital - Riverside Campus & 1967 Riverside Drive \\
4 & Children's Hospital of Eastem Ontario & 401 Smyth Road \\
5 & Ottawa Hosptial - General Campus & 501 Smyth Road \\
6 & Montfort Hospital & 713 Montreal Road \\
8 & Queensway-Carleton Hospital & 3045 Baseline Road \\
9 & Rehabilitation Centre & 505 Smyth Road \\
10 & Saint Vincent Hospital & 60 Cambridge Street North \\
11 & Elizabeth Bruyere Hospital & 43 Bruyere Street \\
\hline
\end{tabular}




\section{Table D.9: Hotels - Motels}

\begin{tabular}{|l|l|}
\hline NAME & ADDRESS \\
\hline Chateau Laurier & 1 Rideau St. \\
Crowne Plaza & 101 Lyon St. \\
Delta Ottawa Hotels and Suites & 361 Queen St. \\
Quality Hotel & 290 Rideau St. \\
Travelodge & 402 Queen St. \\
Westin Ottawa & 11 Colonel By Drive \\
WelcomiNNS & 1220 Michael St. \\
Travelodge and Convention Cent & 1376 Carling Ave \\
McKellar Park Apt/Hotel & 1983 Carling Ave \\
Adam'S Airport inn & 2721 Highway 31 \\
Monterey Motor inn & 2259 Highway 16 \\
Southway Inn & 2431 Bank St \\
\hline
\end{tabular}




\section{Table D.10: Hotlink}

\begin{tabular}{|l|l|}
\hline ID & HOTLINK \\
\hline & olhttp://wmm.octranspo.com \\
\hline
\end{tabular}




\section{Table D.11: Libraries}

\begin{tabular}{|c|c|c|}
\hline $1 \mathrm{D}$ & NAME & ADDRESS \\
\hline & 1) Main Library & 120 Metcalfe St \\
\hline & 2 Alta Vista & 2516 Alta Vista Dr \\
\hline & 3 Carlingwood & 281 Woodroffe AV \\
\hline & 4 Elmvale Acres & 1910 St. Laurent BI \\
\hline & 5/Rideau & 377 Rideau St \\
\hline & 6 Sunnyside & 1049 Bank St \\
\hline & 7) St. Laurent & 515 Cote St \\
\hline & 8Rosemount & 18 Rosemount Av \\
\hline & 9 Beaverbrook & 2500 Campeau Drive \\
\hline & ofHazeldean & 50 Castlefrank Road \\
\hline & 1 Nepean Central & 101 Centrepointe Dr \\
\hline & 2 Centenial & 3870 Richmond Road \\
\hline & 3 Emerald Plaza & 1547 Merivale Road \\
\hline & 4/Ruth E. Dickinson & 101 Malvern Dr \\
\hline & 5. Cumberland & 1599 Tenth Line Road \\
\hline & 6lBlossom Park & 2950 Highway 31 \\
\hline & 7) North Gloucester & 2036 Ogilvie Road \\
\hline & 8 Orleans & 1705 Orleans $\mathrm{BI}$ \\
\hline & 9. Blackburn Hamlet & 199 Glen Park Dr \\
\hline & ofGreely & 7008 Parkway Rd. \\
\hline & $1 /$ Metcalfe & 2782 Albert St. \\
\hline & 4/Manotick & 5499 South River Dr. \\
\hline & 6/Rockcliffe Park & 380 Springfield Road \\
\hline & 8 Stittsville & 1637 Main Street \\
\hline & O) Richmond & 6240 Perth Street \\
\hline
\end{tabular}


Table D.12: Location

\begin{tabular}{|l|l|l|}
\hline$D$ ADDRESS & NAME \\
\hline 1 & & \\
\hline 2 & \\
\hline
\end{tabular}

431 


\section{Table D.13: Museums}

\begin{tabular}{|l|l|}
\hline NAME & ADDRESS \\
\hline Canada Aviation Museum & 11 Aviation \\
Science and Technolgy & 1867 St Laurent \\
Museum of Nature & 240 McLeod \\
Billings Estate & 2100 Cabot \\
Museum of Contemporary Photography & 1 Rideau \\
Canadian War Museum & 330 Sussex \\
Carleton University Art Gallery & 1125 Colonel By \\
Currency Museum of the Bank of Cana & 245 Sparks street \\
Royal Canadian Mint & 320 Sussex \\
National Gallery of Canada & 380 Sussex \\
\hline
\end{tabular}




\section{Table D.14: Recreation Centers}

\begin{tabular}{|l|l|}
\hline NAME & ADDRESS \\
\hline RA Centre & 2451 Riverside Dr \\
Blackstar Community \& Resource Cent & 1800 Bank St \\
Coliseum Sports \& Recreation Centre & 1015 Bank St \\
Dovercourt Recreation Centre & 411 Dovercourt \\
Hunt Club - Riverside Community Cen & 3320 Paul Anka Dr \\
Kanata Leisure Centre & 70 Aird PI \\
James Patrick Sports Centre & 350 Hunt Club Rd \\
Nepean Sportsplex & 1701 Woodroffe Ave \\
Ottawa Boys \& Girls Club & 1463 Prince of Wal \\
Overbrook Community Recreation Asso & 33 Quill \\
Walter Baker Sports Centre & 100 Malvern Dr \\
\hline
\end{tabular}




\section{Table D.15: Schools}

\begin{tabular}{|c|c|}
\hline NAME & STREET \\
\hline A. Y. Jackson Secondary & 150 Abbeyhill Dr \\
\hline Adult High School & 300 Rochester St \\
\hline Agincourt Public School & 1250 Agincourt $\mathrm{Rd}$ \\
\hline Albert Street Secondary Alternative & 440 Albert Si \\
\hline Alexander Montessori & 1002 Karen Way \\
\hline Alta Vista Public School & 1349 Randall Av \\
\hline Arc-en-ciel (école) & 1830 Portobello Bl \\
\hline Arch Street Public School & $2129 \operatorname{Arch} S t$ \\
\hline Ashbury College & 362 Mariposa Av \\
\hline Assumption Catholic E.S. & 330 Lajoie St \\
\hline Barrhaven Public School & 80 Larkin Dr \\
\hline Bayshore Catholic E.S. & 50 Bayshore Dr \\
\hline Bayshore Public School & 145 Woodridge $\mathrm{Cr}$ \\
\hline Bayview Public School & 3071 Riverside Dr \\
\hline Bell High School & 40 Cedarview Rd \\
\hline Bell's Comers Public School & 3770 Richmond Rd \\
\hline Bishop Hamilton Chris. Montess. & 2199 Regency $T r$ \\
\hline Blessed Kateri Tekakwitha Catholic E.S. & 6400 Beausejour Dr \\
\hline Blossom Park Public School & 3810 Sixth St \\
\hline Briargreen Public School & 19 Parkfield $\mathrm{Cr}$ \\
\hline Bridlewood Community E.S. & 63 Bluegrass Dr \\
\hline Broadview Public School & 590 Broadview Av \\
\hline Brookfield High School & 824 Brookfield Rd \\
\hline Cadieux (école) & 345 St Denis St \\
\hline Cairine Wilson Secondary School & 975 Orleans $\mathrm{Bl}$ \\
\hline Cambridge St. Comm. Public School & 250 Cambridge St, $N$ \\
\hline Canadian Montessori Academy & 2 Peter St \\
\hline Canterbury High School & 900 Canterbury Av \\
\hline Carleton Heights Public School & 1660 Prince Of Wales Dr \\
\hline Carson Grove Elementary School & 1401 Matheson Rd \\
\hline Castlefrank Elementary School & 55 McCurdy Dr \\
\hline Cedarview Middle School & 2760 Cedarview Rd \\
\hline Centennial Public School & 376 Gloucester St \\
\hline Centre De Jour & 140 Genest St \\
\hline Centre Jules Leger & 281 Lanark St \\
\hline Century Public School & 8 Redpine Dr \\
\hline Chapel Hill Catholic E.S. & 1534 Forest Valley $\mathrm{Dr}$ \\
\hline Charles H. Hulse Public School & 2605 Alta Vista Dr \\
\hline Christie Public School & 2625 Draper Av \\
\hline Churchill School & 345 Ravenhill Av \\
\hline Clifford Bowey Public School & 1300 Kitchener AV \\
\hline
\end{tabular}


Colonel By Secondary School

Connaught Public School

Convent Glen Catholic E.S.

Convent Glen Elementary School

Corpus Christi Catholic E.S.

Counterpoint Children's Academy

Crystal Bay Centre for Special Ed.

D. A. Moodie Intermediate School

D. Roy Kennedy Public School

Des Pins (école)

Des Pionniers (école)

Des Villageois (école)

Des Voyageurs (école)

Devonshire Comm. Public School

Divine Infant Catholic E.S.

Dr. F. J. McDonald Catholic E.S.

Dunlop Public School

Dunning-Foubert Elementary School

É.é.p. Charlotte-Lemieux

É.é.p. Francojeunesse

É.é.p. Franco-Nations

É.é.p. Gabrielle-Roy

É.é.p. Jeanne-Sauvé

É.é.p. Le Trillium

É.é.p. L'Odyssée

É.é.p. Marie-Curie

É.é.p. Séraphin-Marion

É.p. Le Transit

É.s.p. De La Salle

É.s.p. de l'Ouest d'Ottawa

É.s.p. Louis-Riel

Eart Of March Secondary School

Éducation permanente

Éducation sans Frontières

Elgin Street Public School

Élisabeth-Bruyère (école)

Elizabeth Park Public School

Elizabeth Wyn Wood Site

Eimdale Public School

Elmridge Catholic E.S.

Elmwood School

Emily Carr Middle School

Fallingbrook Community E.S.

Featherston Drive Public School

Fernhill School

Fielding Drive Public School
2381 Ogilvie Rd

1149 Gladstone Av

6212 Jeanne D'arc Bi

1708 Grey Nuns Dr

157 Fourth Av

1151 River Rd

31 Moodie Dr

595 Moodie Dr

919 Woodroffe Av

1487 Ridgebrook Dr

720 Merkley Dr

1550 Verchere $S t$

6030 voyageur $\mathrm{Dr}$

100 Breezehill Av

8100 Jeanne D'arc $B I$

2860 Ahearn Av

1310 Pebble Rd

1610 Prestwick Dr

2093 Bel Air Dr

119 Osgoode St

1085 Grenon Av

1000 Brookfield Dr

1917 Gardenway Dr

135 Alice St

1770 Grey Nuns Dr

860 Colson AV

22 Steel St

140 Genest St

501 St Patrick St

1303 Fellows Rd

1655 Bearbrook Rd

948 Teron Rd

623 Smyth Rd

181 Donald St

310 Elgin St

100 Stonehaven Dr

15 De Niverville $\mathrm{Dr}$

20 Rossland Av

49 lona St

1923. Elmridge Dr

261 Buena Vista Rd

2681 Innes Rd

679 Deancourt $\mathrm{Cr}$

1801 Featherston Dr

1050 Naughton St

777 Fielding Dr 
First Avenue Public School

Fisher Park / Summit Public

Forest Valley Elementary School

Franco-Cité (école secondaire cath.)

Franco-ouest (Collège cath.)

Frank Ryan Catholic I.S.

Frederick Banting Site

Gameau (école secondaire cath.)

General Vanier Public School

Georges Vanier Catholic E.S.

Georges-Étienne-Cartier (école)

Glashan Public School

Glebe Collegiate Institute

Glen Cairn Public School

Glen Ogilvie Public School

Gloucester High School

Good Shepherd Catholic E.S.

Grant School

Greenbank Middle School

Hawthorne Public School

Head Start Academy (1st fl)

Henry Larsen Elementary School

Henry Munro Middle School

Heritage Academy

Hillcrest High School

Hillel Academy

Hilson Avenue Public School

Holy Cross Catholic E.S.

Holy Family Catholic E.S.

Holy Redeemer Catholic E.S.

Holy Trinity Catholic H.S.

Hopewell Avenue Public School

Immaculata Catholic I.S. and H.S.

immaculate Heart of Mary Catholic E.S.

J. H. Putman Public School

J. S. Woodsworth Secondary School

Jean Vanier Catholic I.S.

Joan Of Arc Academy

Jockvale Elementary School

John McCrae Secondary School

John Young Elementary School

Kanata Academy

Katimavik Elementary School

Knoxdale Public School

La Vérendrye (école)

Lady Evelyn Public School
73 First Av

250 Holland AV

1570 Forest Valley $\mathrm{Dr}$

623 Smyth Rd

411 Seyton Dr

128 Chesterton Dr

115 Terrence Matthews $\mathrm{Cr}$

6588 Carriere St

1025 Harkness Av

40 Varley $\mathrm{Dr}$

880 Thorndale $\mathrm{Dr}$

28 Arlington Av

212 Glebe AV

182 Morrena Dr

46 Centre Park Dr

2060 Ogilvie Rd

101 Bearbrook Rd

2720 Richmond Rd

168 Greenbank Rd

2158 St Laurent Blvd

88 Main St

1750 Sunview Dr

2105 Kender Av

2360 Virginia St

1900 Dauphin Rd

881 Broadview Av

407 Hilson Av

2820 Springland Dr

245 Owl Dr

$75 \mathrm{Mccurdy} \mathrm{Dr}$

180 Katimavik Rd

17 Hopewell Av

140 Main St

445 Pleasant Park Rd

2051 Bel Air Dr

159 Chesterton Dr

320 Lajoie St

209 Bayswater Av

101 Malvern Dr

103 Malvern Dr

5 Morton Dr

209 Bearverbrook Rd

64 Chimo Dr

170 Greenbank Rd

614 Eastvale Dr

63 Evelyn Av 


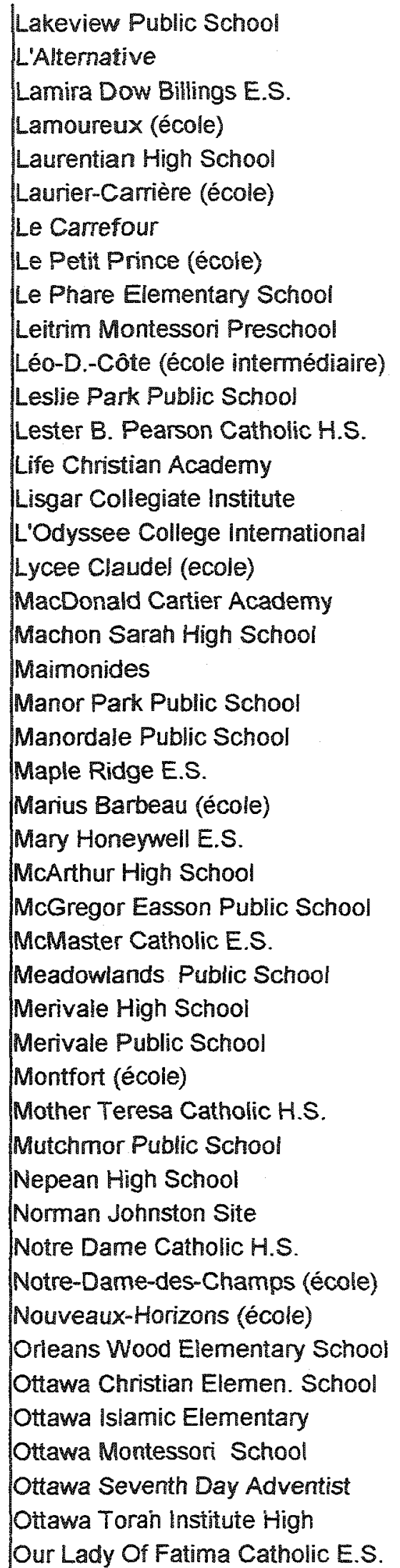

35 Corkstown Rd

307 Montgomery St

2147 Loyola Av

2540 Kaladar Ave

1357 Baseline Rd

14 Four Seasons Dr

$2315 \mathrm{St}$ Joseph Bl

349 OImstead St

1965 Naskapi Dr

4561 Highway 31

6664 Carriere St

20 Harrison St

2072 Jasmine $\mathrm{Cr}$

2214 Innes Rd

29 Lisgar St

839 Shefford St

1635 Riverside Dr

270 Crichton St

831 Broadview Av

25 Esquimault $A v$

100 Braemar St

16 Carola St

1000 Valin St

1345 Nottinghill Av

54 Kennevale Dr

485 Donald St

991 Dynes Rd

1760 Mcmaster Av

10 Fieldrow $S t$

1755 Merivale Rd

63 Slack Rd

641 Sladen Av

440 Longfields Dr

185 Fifth AV

574 Broadview Av

2401 Cleroux $\mathrm{Cr}$

710 Broadview Av

3349 Navan Rd

3681 Innes Rd

7859 Decarie Dr

2191 Benjamin Av

10 Coral St

335 Lindsay St

2200 Benjamin Av

151 Chapel St

2135 Knightsbridge Rd 
Jur Lady Of Mount Carmel Catholic E.S. 675 Gardenvale Rd Our Lady Of Peace Catholic E.S. Our Lady Of Victory Catholic E.S. Our Lady Of Wisdom Catholic E.S. Overbrook Public School Parkwood Hills Public School Parsifal (ecole)

Pauline-Vanier (école intermédiaire)

Pinecrest Public School

Pineview Catholic E.S.

Pleasant Park Public School

Pope John XXIII Catholic E.S. Préseault (école)

Prince Of Peace Catholic E.S. Queen Elizabeth Public School Queen Mary Street Public School Queen of the Angels Adult H.S. Queenswood Public School R. Byms Curry Public School Redeemer Christian High School Regina Public School Reine-des-Bois (école) Richmond Public School Rideau High School Ridgemont High School Riverview Public School (Ottawa) Robert Bateman Public School Robert E. Wilson Public School Robert Hopkins Elementary School Roberta Bondar Public School Rockcliffe Park Public School Roger-Saint-Denis (école) Roland Michener Public School Sainte-Anne (école) Sainte-Bernadette (école) Sainte-Geneviève (école) Sainte-Marie (école) Saint-François-d'Assise (école) Saint-Luc (école) Samuel-Genest (Collège cath.) Sawmill Creek Elem. School Severn Avenue Public School Sir Guy Carleton Secondary Schoo! Sir Robert Borden High School Sir Wilfrid Laurier Secondary School Sir Winston Churchill Public School

3877 Richmond Rd 1175 Soderlind St 1565 St George St 149 King George St 60 Tiverton Dr 630 island Park Dr 1837 Stonehenge $\mathrm{Cr}$ 1281 Pinecrest Rd 1500 Beaverpond Dr 564 Pleasant Park Rd 165 Knoxdale Rd 1080 St Pierre 1620 Heatherington $R d$ 689 St-Laurent BI 557 Queen Mary St 1461 Heron Rd 1445 Duford Dr 185 Owl Dr 82 Colonnade Rd 2599 Regina St 1450 Duford St 3499 Mcbean St 815 St-Laurent BI 2597 Alta Vista Dr $260 \mathrm{Knox} \mathrm{Cr}$ 1250 Blohm Dr 373 Mcarthur Av 2011 Glenfem Av 159 Lorry Greenberg Dr 350 Buena Vista Rd 186 Barrow $\mathrm{Cr}$ 100 Penfield Dr 340 York St 3781 Sixth Si 2198 Arch St 2599 Innes Rd 35 Melrose Av 2485 Dwight $\mathrm{Cr}$ 704 Carson Rd 3400 Daoust Av 2553 Severn Av 55 Centrepoint $\mathrm{Dr}$ 131 Greenbank Rd 1515 Tenth Line Rd 49 Mulvagh Av 
South Carleton High School

St. Andrew Adult H.S

St. Anne School

St. Anthony Catholic E.S.

St. Augustine Catholic E.S.

St. Bemard Catholic E.S.

St. Brigid Catholic E.S.

St. Clare Catholic E.S.

St. Daniel Catholic E.S.

St. Elizabeth Ann Seton Catholic E.S.

St. Elizabeth Catholic E.S.

St. Francis of Assisi Catholic E.S.

St. George Catholic E.S.

St. Gregory Catholic E.S.

St. Isidore Catholic E.S.

St. James Catholic E.S.

St. John the Apostle Catholic E.S.

St. Joseph Adult H.S.

St. Joseph Catholic I.S.

St. Jude's Academy (Home Study)

St. Luke Catholic E.S. (Nepean)

St. Luke Catholic E.S. (Ottawa)

St. Margaret Mary Catholic E.S.

St. Marguerite d'Youville Catholic ES.

St. Martin De Porres Catholic E.S.

St. Mary Catholic E.S. (Gloucester)

St. Mary Catholic E.S. (Ottawa)

St. Matthew Catholic H.S.

St. Michael Catholic E.S. (Ottawa)

St. Monica Catholic E.S.

St. Nicholas Adult H.S.

St. Patrick Adult H.S.

St. Patrick Catholic E.S. (Nepean)

St. Patrick Catholic H.S.

St. Patrick Catholic I.S.

St. Paul Catholic H.S.

St. Peter Catholic H.S.

St. Philip Catholic E.S.

St. Pius X Catholic H.S.

St. Rita Catholic E.S.

St. Thomas Catholic E.S.

St. Thomas More Catholic E.S.

Stephen Leacock Public School

3673 Mcbean St

1119 Lazard St

500 Stonehaven Dr

391 Booth St

1009 Amot Rd

1722 St Bemard St

200 Springfield Rd

2133 Gardenway Dr

1160 Maitland Av

41 Weybridge Dr

893 Admiral Av

795 Watters Rd

401 Picadilly Av

70 Fieldrow $S t$

1105March Rd

50 Stonehaven Dr

30 Costello Av

20 Graham St

130 Keyworth Av

112 Gardenview Private

60 Mountshannon Dr

2485 Dwight $\mathrm{Cr}$

88 Bellwood Av

89 Lorry Greenberg Dr

20 Mckitrick Dr

5536 Highway 31

175 Beech St

6550 Bilberry Dr

741 Bernard St

2000 Merivale Rd

130 Lotta Av

290 Nepean St

68 Larkin Dr

2525 Alta Vista Dr

1485 Heron Rd

2611 Draper Av

750 Charlemagne B!

79 Maitland AV

1481 Fisher Av

$\uparrow$ inverness Rd

9 Leeming Dr

1620 Blohm Dr

25 Leacock Dr

Terre-des-Jeunes (pav. Gaston-Vincent) 1313 Field St

Terre-des-Jeunes (pav. St-Bonaventure)

Terry Fox Elementary School

1366 Coldrey Av

6400 Jeanne D'arc BI 
Thomas D'Arcy McGee Catholic E.S.

Torah Academy of Ottawa

Trillium Elementary School

Tumbul Learning Center

Uplands Catholic E.S.

Vincent Massey Public School

Viscount Alexander Public School

Vision Jeunesse (école)

W. E. Gowling School

W. Erskine Johnston Public School

W. O. Mitchell Elementary School

Westboro Academy

Woodroffe Avenue Public School

Woodroffe High School

Yitzhak Rabin High School

York Street Public School
635 Laverendrye Dr

1231 Prince of Wales Dr

1515 Varennes $B$ l

1132 Fisher Av

17 De Niverville Dr

745 Smyth Rd

65 Mann Av

235 Mcarthur Ave

250 Anna Av

50 Varley $D r$

80 Steeple Chase Dr

130 Keyworth St

235 Woodroffe Av

2410 Georgina Dr

1755 Merivale Rd

310 York St 


\section{Table D.16: Shopping Centers}

\begin{tabular}{|l|l|}
\hline NAME & ADDRESS \\
\hline Rideau Centre & 73 Rideau St. \\
Bayshore Shopping Centre & 100 Bayshore Dr. \\
St. Laurent Shopping Centre & 1200 St. Laurent BV \\
Place d'Orleans & 110 Place d'Orleans \\
Merivale Mall & 1642 Merivale Rd. \\
Heron Gate Mall & 1670 Heron Rd. \\
Carlingwood Mall & 2121 Carling Ave \\
Westgate Shopping Centre & 1309 Carling Ave \\
\hline
\end{tabular}




\section{Table D.17: Tourist Attractions}

\begin{tabular}{|l|l|}
\hline SITE & ADDRESS \\
\hline Parliament Hill & Wellington St. \\
Supreme Court of Canada & Wellington St. \\
National Archives \& National Library of Canada & 395 Wellington S \\
Currency Museum & 245 Sparks St. \\
National Arts Centre & 53 Elgin St. \\
National War Memorial & Elgin St. \\
Museum of Contemporary Photography & 1 Rideau St. \\
Ottawa Congress Centre & 55 Colonel By Dr \\
Peacekeeping monument & 390 Sussex Dr. \\
National Gallery of Canada & 380 Sussex Dr. \\
Canadian War Museum & 330 Sussex Dr. \\
Royal Canadian Mint & 320 Sussex Dr. \\
Canadian Museum of Nature & 240 McLeod St. \\
Canadian Aggriculture Museum & 1 Experimental F \\
Prime Minister's House & 24 Sussex Dr. \\
Rideau Hall & 1 Sussex Dr. \\
Carleton University Art Gallery (St. Patrick Bld) & 1125 Colonel By \\
Billings Estate & 210 Cabot St. \\
Bytown Museum & 1 Rideau St. \\
Aviation Museum & 11 Aviation Park \\
Museum of Science and Technology & 1867 St. Laurent \\
Laurier House & 334 Laurier Ave \\
Vimy House & 221 Champagne Av \\
Civic Centre/Lansdowne Park & 1015 Bank St. \\
Corel Centre & 1000 Palladium D \\
St. Patrick's Basilica & 261 Nepean St. \\
Notre Dame Cathedral & 60 Guigues St. \\
\hline
\end{tabular}


Appendix E

Calalp.ave 
Name: View.CalculateFeatureGeometry

' Title: Calculates feature geometry values

' Topics: GeoData

'Description: Calculates area and perimeter for polygon themes and length

' for line themes. If the View has been projected the calculations are in

' projected meters. Otherwise the calculations are in 'native' map units.

'Modify the script to provide calculation in the current report units of

'the View. The script processes the list of active themes to calculate

' area and perimeter, or length, depending on the theme type.

'The script will add the fields: Area and Perimeter to polygon themes, Length

' to line themes if they do not exist. If the fields exist their values will

' be recalculated. Rerun the script if you change the projection of the view.

'Requires: A View with at least one active theme. You must have write access

' to the active theme(s).

' Self:

'

'Returns:

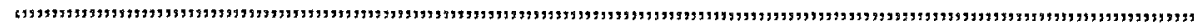

' Get the view and its projection if any.

theView $=$ av. GetActiveDoc

thePrj = theView.GetProjection

if (thePri.IsNull) then

hasPrj $=$ false

else

hasPrj $=$ true

end

' Get the list of active themes. if there aren't any, let the user know

' and exit.

theActivethemeList $=$ theView.GetActivethemes

if (theActivethemeList.Count $=0$ ) then

MsgBox.Error("No active themes.","')

Exit

end

'Loop through the list of active themes. if you can't edit the theme

' inform the user.

For Each thetheme in theActivethemeList

theFTab = thetheme. GetFTab

if (theFTab.CanEdit.Not) then

MsgBox.Info("Cannot edit table for theme:"++thetheme.AsString," ")

Continue

end

,

'Make the FTAB editable, and find out which type of feature it is. 


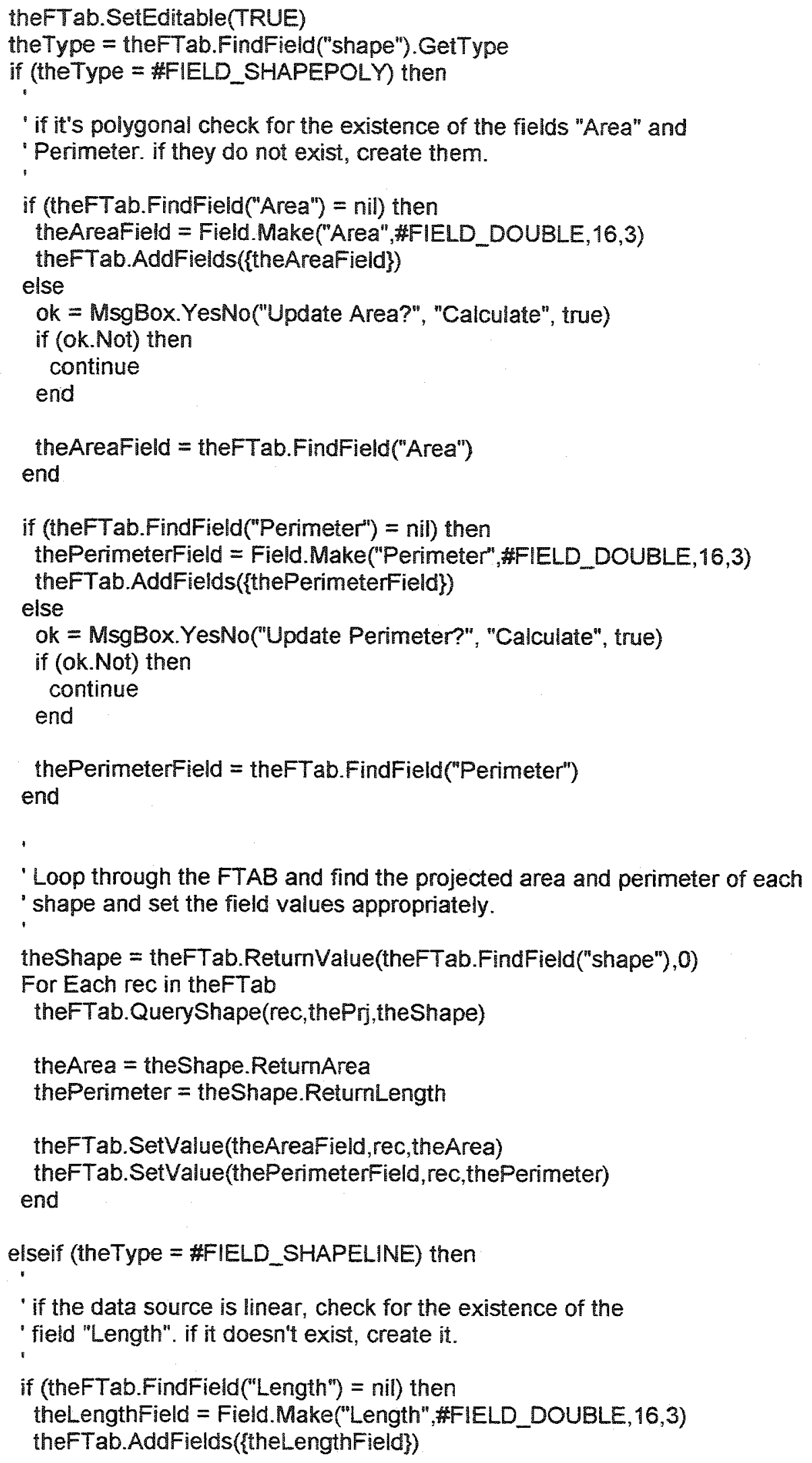




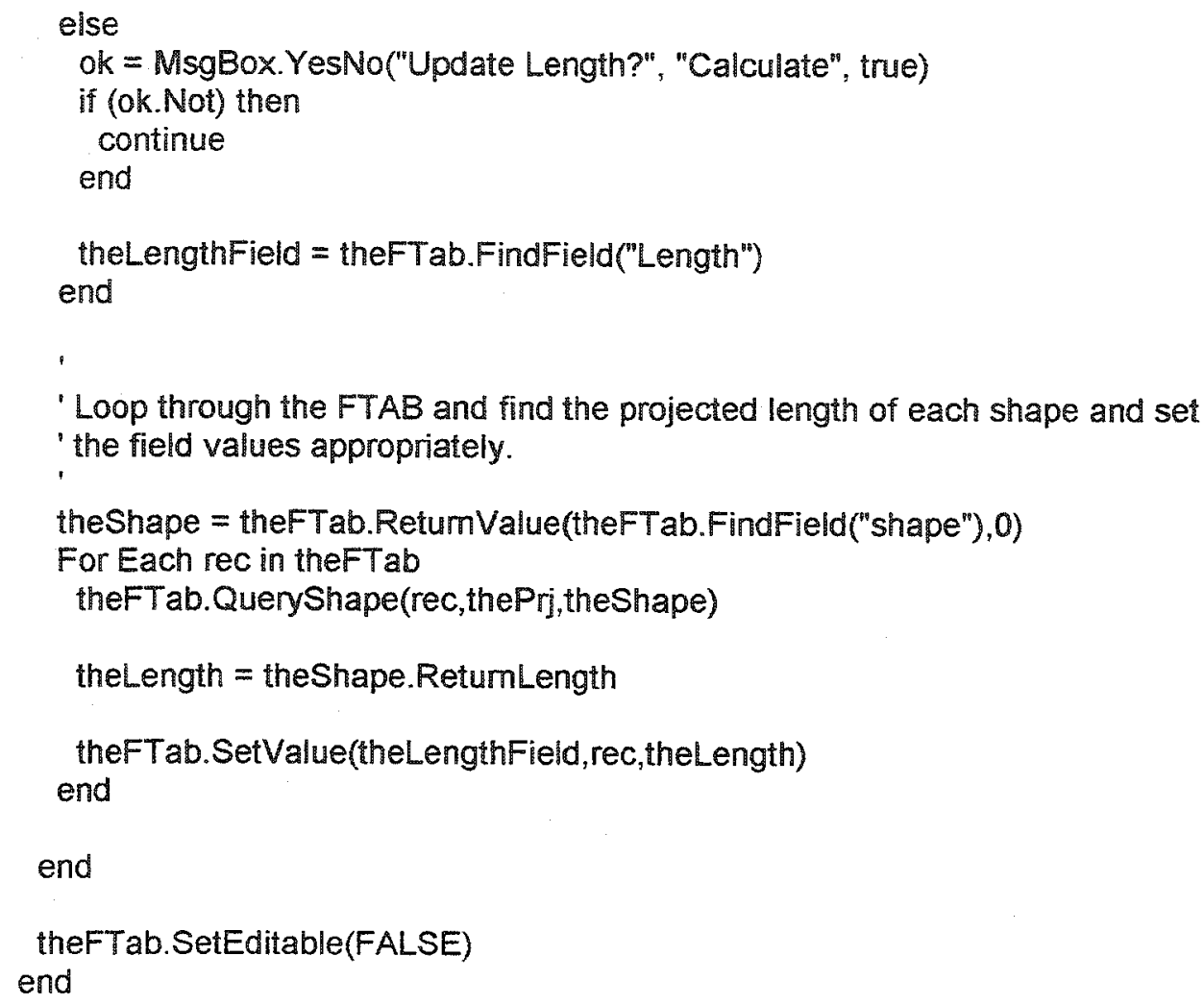


Appendix F Cfcc.dbf 
AOO Road, major and minor categories unknown

AO1 Road, unseparated

$40 \quad 4 \mathrm{AO1}$

AO2 Road, unseparated, in tunnel

$40 \quad 4 \mathrm{AO2}$

A03 Road, unseparated, underpassing

$40 \quad 4 \mathrm{AO3}$

A04 Road, unseparated, with rail line in center $\quad 40 \quad 4$ A04

A05 Road, separated

A06 Road, separated, in tunnel

$40 \quad 4 \mathrm{A05}$

A07 Road, separated, underpassing

$40 \quad 4$ A06

A08 Road, separated, with rail line in center

A10 Primary road with limited access or interstate highway

A11 Primary road with limited access or interstate highway, unseparated

A12 Primary road with limited access or interstate highway, unseparated, it

A13 Primary road with limited access or interstate highway, unseparated, L

A14 Primary road with limited access or interstate highway, unseparated, v

A15 Primary road with limited access or interstate highway, separated

A16 Primary road with limited access or interstate highway, separated, in t

A17 Primary road with limited access or interstate highway, separated, unc

A18 Primary road with limited access or interstate highway, separated, witt

A20 Primary road without limited access, U.S. and State highway

A21 Primary road without limited access, U.S. and State highways, unsepe

A22 Primary road without limited access, U.S. and State highways, unsepe

A23 Primary road without limited access, U.S. and State highways, unsepe

A24 Primary road without limited access, U.S. and State highways, unsepr

A25 Primary road without limited access, U.S. and State highways, separa

$40 \quad 4 \mathrm{AOT}$

$40 \quad 4 \mathrm{A08}$

$65 \quad 1$ A10

$65 \quad 1$ A11

$65 \quad 1 \mathrm{~A} 12$

$65 \quad 1 \mathrm{~A} 13$

$65 \quad 1 \mathrm{~A} 14$

$70 \quad 1 \mathrm{~A} 15$

$70 \quad 1 \mathrm{~A} 16$

$70 \quad 1 \mathrm{~A} 17$

$70 \quad 1 \mathrm{~A} 18$

$55 \quad 2 \mathrm{~A} 20$

$55 \quad 2 \mathrm{~A} 21$

$55 \quad 2$ A22

$55 \quad 2$ A23

$55 \quad 2$ A24

A26 Primary road without limited access, U.S. and State highways, separa

$60 \quad 2 \mathrm{~A} 25$

A27 Primary road without limited access, U.S. and State highways, separa

$60 \quad 2$ A26

$60 \quad 2$ A27

A28 Primary road without limited access, U.S. and State highways, separa

A30 Secondary and connecting road, State and county highways

A31 Secondary and connecting road, State and county highways, unsepar:

A32

A33

A34

A35

A36

A37

A38

Secondary and connecting road, State and county highways, unsepar:

Secondary and connecting road, State and county highways, unsepar:

Secondary and connecting road, State and county highways, unsepar:

Secondary and connecting road, State and county highways, separate

Secondary and connecting road, State and county highways, separate

Secondary and connecting road, State and county highways, separate

A40

Secondary and connecting road, State and county highway, separatec

A41

A42

A43 Local, neighborhood, and rural road, city street

Local, neighborhood, and rural road, city street, unseparated

Local, neighborhood, and rural road, city street, unseparated, in tunne

Local, neighborhood, and rural road, city street, unseparated, underpa

A44 Local, neighborhood, and rural road, city street, unseparated, with rail

A45 Local, neighborhood, and rural road, city street, separated

A46 Local, neighborhood, and rural road, city street, separated, in tunnel

A47 Local, neighborhood, and rural road, city street, separated, underpass

A48 Local, neighborhood, and rural road, city street, separated, with rail lin

A50 Vehicular trail, road passable only by four-wheel drive (4WD) vehicle

A51

Vehicular trail, road passable only by $4 \mathrm{WD}$ vehicle, unseparated

A52 Vehicular trail, road passable only by 4WD vehicle, unseparated, in tu

A53 Vehicular trail, road passable only by $4 \mathrm{WD}$ vehicle, unseparated, und 
A60 Road with characteristic unspecified

A61 Cul-de-sac, the closed end of a road that forms a loop or turn aro

A62 Traffic circle, the portion of a road or intersection of roads that fol

A63 Access ramp, the portion of a road that forms a cloverleaf or limit

A70 Other thoroughfare

A71 Walkway, nearly level road for pedestrians, usually unnamed

A72 Stainway, stepped road for pedestrians, usually unnamed

A73 Alley, road for service vehicles, usually unnamed, located at the !

B00 Railroad, major and minor categories unknown

B01 Railroad track, not in tunnel or underpassing

B02 Railroad track, in tunnel

B03 Railroad track, underpassing

B10 Railroad main track

B11 Railroad main track, not in tunnel or underpassing

B12 Railroad main track, in tunnel

B13 Railroad main track, underpassing

B20 Railroad spur track

B21 Railroad spur track, not in tunnel or underpassing

B22 Railroad spur track, in tunnel

B23 Railroad spur track, underpassing

B30 Railroad yard track

B31 Railroad yard track, not in tunnel or underpassing

B32 Railroad yard track, in tunnel

B33 Railroad yard track, underpassing

B40 Railroad ferry crossing

B50 Other rail line

B51 Carline, a track for street cars, trolleys, and other mass transit rai

B52 Cog railroad, incline railway, or logging tram

C0O Miscellaneous ground transportation, not road or railroad

C10 Pipeline, major category used alone

C20 Power transmission line, major category used alone

C30. Other ground transportation that is not a pipeline or a power trans

C31 Aerial tramway, monorail, or ski lift

D00 Landmark, major and minor categories unknown

D10 Military installation or reservation

D20 Multihousehold or transient quarters

D21 Apartment building or complex

D22 Rooming or boarding house

D23 Trailer court or mobile home park

D24 Marina

D25 Crew of vessel

D26 Housing facility for workers

D27 Hotel, motel, resort, spa, YMCA, or YWCA

D28 Campground

D29 Shelter or mission

D30 Custodial facility

D31 Hospital 
D32 Halfway house

D33 Nursing home, retirement home, or home for the aged

D34 County home or poor farm

D36 Jail or detention center

D37 Federal penitentiary, State prison, or prison farm

D40 Educational or religious institution

D41 Sorority or fratemity

D42 Convent or monastery

D43 Educational institution, including academy, school, college, and ur

D44 Religious institution, including church, synagogue, seminary, temp

D50 Transportation terminal

D51 Airport or airfield

D52 Train station

D53 Bus terminal

D54 Marine terminal

D55 Seaplane anchorage

D60 Employment center

D61 Shopping center or major retail center

D62 Industrial building or industrial park

D63 Office building or office park

D64 Amusement center

D65 Government center

D66 Other employment center

D70 Tower

D71 Lookout tower

D80 Open space

D81 Golf course

D82 Cemetery

D83 National park or forest

D84 Other Federal land

D85 State or local park or forest

D90 Special purpose landmark

D91 Post office box ZIP Code(R)

E00 Physical feature, tangible but not transportation or hydrographic.

E10 Fence line locating a visible and permanent fence between separe

E20 Topographic feature

E21 Ridge line, the line of highest elevation of a linear mountain

E22 Mountain peak, the point of highest elevation of a mountain

Foo Nonvisible boundary, major and minor categories unknown

F10 Nonvisible jurisdictional boundary of a legal or administrative entit

F11 Offiset boundary of a legal or administrative entity

F12 Corridor boundary of a legal or administrative entity

F13 Interpolated boundary of a legal or administrative entity used for $c$

F14 Superseded legal or administrative boundary

F15 Superseded legal or administrative boundary, corrected through $p$

F20 Nonvisible feature for data base topology

F22 Irregular feature extension determined manually to lengthen exis 
Closure extension to complete data base topological closure betwee

F24 Nonvisible separation line used with offset and corridor boundaries

F30 Point-to-point line, follows a line of sight and should not cross any vi

F40 Property line, nonvisible boundary of either public or private lands, $\varepsilon$

F50 ZIP Code(R)boundary, reserved for future use in delineating ZIP CO Map edge, now removed, used during data base creation

F70 Statistical boundary

F71 1980 statistical boundary

F72 1990 statistical boundary, used to hold collection and tabulation cen:

F73 1990 statistical boundary and extent of land use, it is not classifiable

F74 1990 statistical boundary, used to hold a tabulation census block boi

F80 Nonvisible other tabulation boundary, major category used when the

$0 \quad 0$ F74

F81

School district tabulation boundary

F82 Special census tabulation boundary

$0 \quad 0 F 80$

$0 \quad 0 F 81$

HOO

Water feature

$0 \quad 0$ F82

$0 \mathrm{OHOO}$

H01 Shoreline of perennial water feature

0 OHO1

$\mathrm{HO} 2$

Shoreline of intermittent water feature

$0 \quad 0 \mathrm{HO2}$

$\mathrm{H} 10$ Stream

H11 Perennial stream or river

H12 Intermittent stream, river, or wash

H13 Braided stream or river

H2O Canal, ditch, or aqueduct

H21 Perennial canal, ditch, or aqueduct

H22 Intermittent canal, ditch, or aqueduct

H3O Lake or pond

H31 Perennial lake or pond

H32 Intermittent lake or pond

$\mathrm{H} 40$ Reservoir

H41 Perennial reservoir

$\mathrm{H} 42$ Intermittent reservoir

H50 Bay, estuary, gulf, sound, sea, or ocean

$0 \mathrm{H} 10$

O $\mathrm{OH} 11$

0 OH12

$0 \quad 0 \mathrm{H} 13$

$0 \quad 0 \mathrm{H} 20$

$0 \quad 0 \mathrm{H} 21$

$0 \quad 0 H 22$

$0 \quad 0 \mathrm{H} 30$

$0 \quad 0 H 31$

$0 \quad 0 H 32$

$0 \quad 0 \mathrm{H} 40$

$0 \quad 0 \mathrm{H} 41$

$0 \mathrm{OH} 42$

$0 \quad 0 \mathrm{H} 50$

H51 Bay, estuary, gulf, or sound

H53 Sea or ocean

H60 Gravel pit or quarry filled with water

$0 \quad 0 \mathrm{H} 51$

$0 \quad \mathrm{H} H 53$

$\mathrm{H} 70$ Nonvisible water area definition boundary

H71 USGS closure line, used as maritime shoreline

$0 . \mathrm{H} 60$

O $\mathrm{OH}$ HO

H72 Census water center line, computed to use as median positional bou

$0 \quad 0 \mathrm{H} 71$

H73 Census water boundary, international in waterways or at 12-mile limi

$0 \quad 0 \mathrm{H} 72$

$0 \quad \mathrm{OH} 73$

H74 Census water boundary, separates inland from coastal or Great Lakł

O $0 \mathrm{H74}$

H75 Census water boundary, separates coastal from territorial at 3-mile I:

$0 \quad \mathrm{OH} 75$

H80 Special water feature, major category used when the minor category

H81 Glacier

X00 Feature not yet classified

$0 \quad 0 \mathrm{H} 80$

$0 \quad 0 \mathrm{H} 81$

$0 \quad 0 \times 00$ 


\section{Appendix G OC Transpo Operating Statistics}


As of December 31, 2000

Urban Transit Area

$368 \mathrm{sq} . \mathrm{km}$

Population served

695,000

Passengers

80.1 million

Average weekday ridership

325,000

\section{Service Facts}

Passenger km carried

689.5 million

Service $\mathrm{km}$ operated

50.2 million

(includes charters and extras, excludes special event)

Vehicle service hours

2.1 million

Average passenger trip length in $\mathrm{km}$

10

Total $\mathrm{km}$ of routes

5,335

Number of routes (includes school routes)

204

Employees

2,127

Bus Stops

5,500

Bus stops with map and schedule display boxes

2,970

Bus stops with shelters

1,064

Bus stops with benches

1,050

Telidon schedule display units

25

Telephone Centres calls (annual)

655,000 
"560" schedule information calls (annual)

$10,000,000$

Web site visits (annual)

$1,020,000$

\section{Transitway}

Weekday passenger volume

200,000

Peak hour one-way passenger volume

10,000

Buses per peak hour, through central area

190

Number of stations

34

Bike \& Ride locations

20 
Appendix H

Schedule Tables 


\section{List of Tables}

Table H.1: Weekday Schedule Sample

Table H.2: Saturday Schedule Sample

Table H.3: Sunday Schedule Sample 
Table H.1: Weekday Schedule Sample

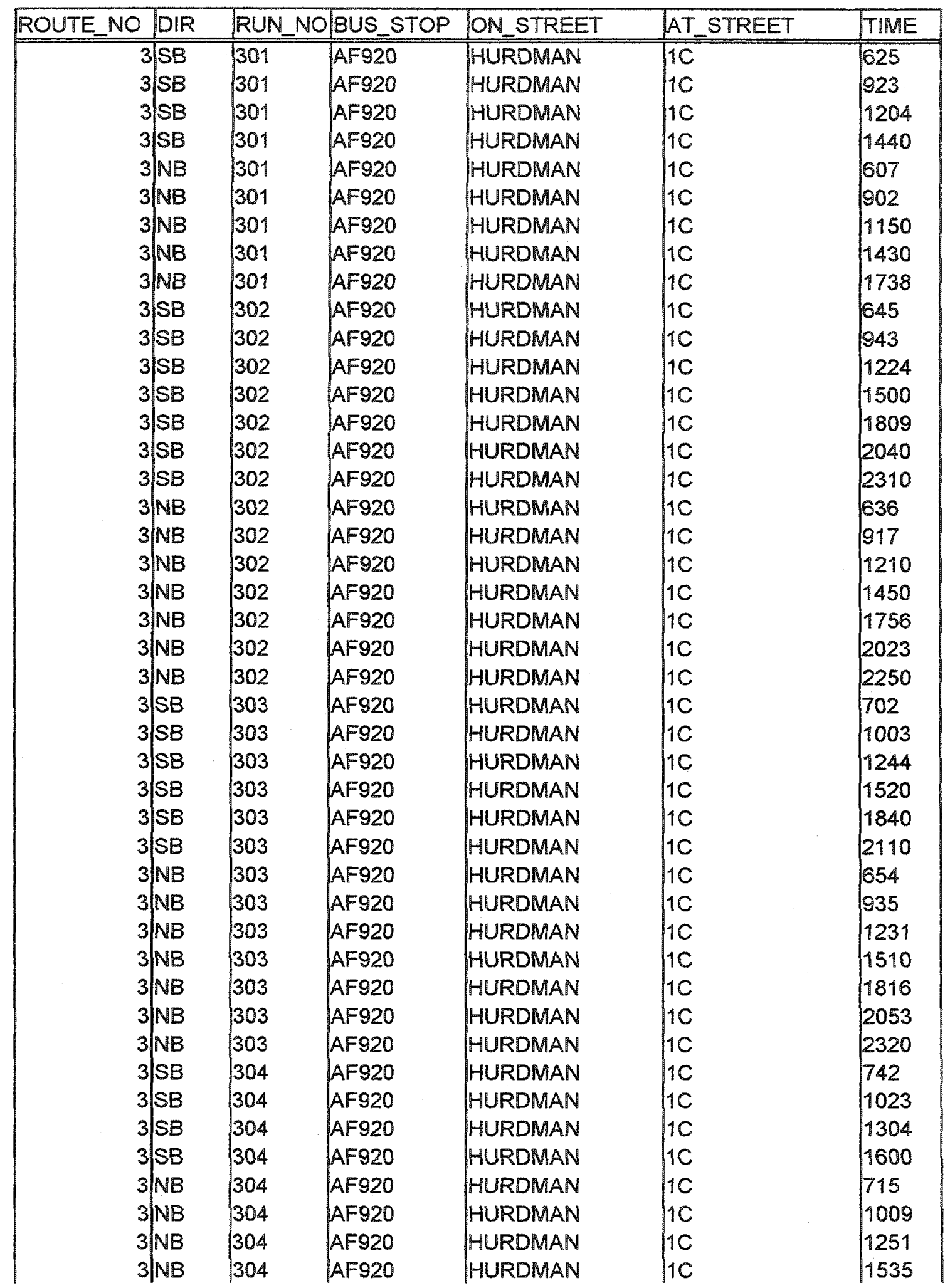




\begin{tabular}{|c|c|c|c|c|c|}
\hline $3 / N B$ & 304 & AF920 & |HURDMAN & 10 & 1834 \\
\hline $3 \mathrm{SB}$ & 305 & AF920 & HURDMAN & 10 & 802 \\
\hline $3 S B$ & 305 & $A F 920$ & HURDMAN & $1 \mathrm{c}$ & 1043 \\
\hline $3 \mathrm{SB}$ & 305 & AF920 & HURDMAN & 10 & 1324 \\
\hline $3 S B$ & 305 & AF920 & HURDMAN & 10 & 1620 \\
\hline $3 S B$ & 305 & AF920 & HURDMAN & $1 c$ & 1911 \\
\hline $3 \mathrm{SB}$ & 305 & AF 920 & HURDMAN & 10 & 2140 \\
\hline $3 / \mathrm{NB}$ & 305 & AF920 & HURDMAN & 10 & 737 \\
\hline 3 NB & 305 & AF 920 & HURDMAN & 10 & 1029 \\
\hline $3 / N B$ & 305 & AF920 & HURDMAN & 10 & 1311 \\
\hline $3 / N B$ & 305 & AF920 & HURDMAN & 10 & 1557 \\
\hline $3 / \mathrm{NB}$ & 305 & AF920 & HURDMAN & 10 & 1858 \\
\hline $3 / N B$ & 305 & AF920 & HURDMAN & 10 & 2122 \\
\hline $3 S B$ & 306 & AF920 & HURDMAN & 10 & 822 \\
\hline $3 / S B$ & 306 & AF920 & HURDMAN & 10 & 1103 \\
\hline $3 \mathrm{SB}$ & 306 & AF920 & HURDMAN & 10 & 1344 \\
\hline $3 S B$ & 306 & AF920 & HURDMAN & 10 & 1640 \\
\hline $3 S B$ & 306 & AF 920 & HURDMAN & $1 \mathrm{C}$ & 1940 \\
\hline $3 S B$ & 306 & AF920 & HURDMAN & 10 & 2210 \\
\hline 3 NB & 306 & AF920 & HURDMAN & 10 & 800 \\
\hline 3 NB & 306 & AF920 & HURDMAN & 10 & 1049 \\
\hline $3 / \mathrm{NB}$ & 306 & AF920 & HURDMAN & 10 & 1331 \\
\hline $3 / \mathrm{NB}$ & 306 & AF920 & HURDMAN & 10 & 1617 \\
\hline $3 / \mathrm{NB}$ & 306 & AF920 & HURDMAN & 10 & 1926 \\
\hline 3 NB & 306 & AF920 & HURDMAN & 10 & 2152 \\
\hline $3 \mathrm{SB}$ & 307 & AF920 & HUROMAN & 10 & 842 \\
\hline $3 S B$ & 307 & AF920 & HURDMAN & 10 & 1123 \\
\hline $3 S B$ & 307 & AF920 & HURDMAN & 10 & 1404 \\
\hline $3 / S B$ & 307 & AF920 & HURDMAN & 10 & 1720 \\
\hline $3 \mathrm{SB}$ & 307 & AF920 & HURDMAN & 10 & 2010 \\
\hline $3 /$ NB & 307 & AF920 & HURDMAN & 10 & 820 \\
\hline $3 \mathrm{NB}$ & 307 & AF920 & HURDMAN & 10 & 1109 \\
\hline $3 / \mathrm{NB}$ & 307 & AF920 & HURDMAN & 10 & 1350 \\
\hline 3 NB & 307 & AF920 & HURDMAN & $1 \mathrm{C}$ & 1658 \\
\hline $3 N B$ & 307 & AF920 & HURDMAN & 10 & 1953 \\
\hline 3 NB & 307 & AF 920 & HURDMAN & 10 & 2221 \\
\hline $3 S B$ & 308 & AF920 & HURDMAN & 10 & 603 \\
\hline $3 S B$ & 308 & AF920 & HURDMAN & 10 & 903 \\
\hline $3 S B$ & 308 & AF920 & HURDMAN & 10 & 1143 \\
\hline $3 \mathrm{SB}$ & 308 & AF920 & HURDMAN & 10 & 1421 \\
\hline $3 \mathrm{SB}$ & 308 & AF920 & HURDMAN & 10 & 1740 \\
\hline $3 / N B$ & 308 & AF920 & HURDMAN & 10 & 841 \\
\hline $3 / \mathrm{NB}$ & 308 & AF920 & HURDMAN & 10 & 1129 \\
\hline $3 / N B$ & 308 & AF920 & HURDMAN & 10 & 1410 \\
\hline $3 / N B$ & 308 & AF920 & HURDMAN & 10 & 1718 \\
\hline $3 \mathrm{SB}$ & 350 & AF920 & HURDMAN & 10 & 722 \\
\hline $3 / N B$ & 350 & AF920 & HURDMAN & 10 & 948 \\
\hline $3 / S B$ & $E-483$ & AF920 & HURDMAN & 10 & 1700 \\
\hline $3 / N B$ & E-491. & AF920 & HURDMAN & 10 & 1637 \\
\hline
\end{tabular}


Table H.2: Saturday Schedule Sample

\begin{tabular}{|c|c|c|c|c|c|}
\hline ROUTE_NODIR & RUN_NO & BUS_STOP & ON_STREET & AT_STREET & TIME \\
\hline $3 / \overline{S B}$ & & 1/AF920 & IHURDMAN & 10 & 640 \\
\hline $3 \mathrm{SB}$ & 30 & AF920 & HURDMAN & 10 & 740 \\
\hline $3 / S B$ & 30 & AF920 & HURDMAN & $1 \mathrm{C}$ & 810 \\
\hline $3 \mathrm{SB}$ & 30 & AF920 & HURDMAN & 10 & 840 \\
\hline $3 S B$ & 30 & AF920 & HURDMAN & 10 & 910 \\
\hline $3 \mathrm{SB}$ & 30 & AF920 & HURDMAN & 10 & 940 \\
\hline $3 \mathrm{SB}$ & 30 & AF920 & HURDMAN & 10 & 1010 \\
\hline $3 S B$ & 30 & AF920 & HURDMAN & 10 & 1040 \\
\hline $3 \mathrm{SB}$ & 30 & AF920 & HURDMAN & 10 & 1110 \\
\hline $3 S B$ & 30 & AF920 & HURDMAN & 10 & 1140 \\
\hline $3 \mid S B$ & 30 & AF920 & HURDMAN & 10 & 1210 \\
\hline $3 / S B$ & 30 & AF920 & HURDMAN & 10 & 1240 \\
\hline $3 \mathrm{SB}$ & 30 & AF920 & HURDMAN & 10 & 1310 \\
\hline $3 \mathrm{SB}$ & 30 & AF920 & HURDMAN & 10 & 1340 \\
\hline $3 \mathrm{SB}$ & 30 & AF920 & HURDMAN & 10 & 1410 \\
\hline $3 \mathrm{SB}$ & 30 & AF920 & HURDMAN & 10 & 1440 \\
\hline $3 \mathrm{SB}$ & 30 & AF920 & HURDMAN & 10 & 1510 \\
\hline $3 S B$ & 30 & AF920 & HURDMAN & 10 & 1540 \\
\hline $3 S B$ & 30 & AF920 & HURDMAN & 10 & 1610 \\
\hline $3 S B$ & 30 & AF920 & HURDMAN & 10 & 1640 \\
\hline $3 \mathrm{SB}$ & 30 & AF920 & HURDMAN & 10 & 1710 \\
\hline $3 \mid S B$ & 30 & AF920 & HURDMAN & 10 & 1740 \\
\hline $3 S B$ & 30 & AF920 & HURDMAN & 10 & 1810 \\
\hline $3 / \mathrm{SB}$ & 30 & AF920 & HURDMAN & 10 & 1840 \\
\hline $3 \mathrm{SB}$ & 30 & AF920 & HURDMAN & 10 & 1910 \\
\hline $3 S B$ & 30 & AF920 & HURDMAN & 10 & 1940 \\
\hline $3 \mathrm{SB}$ & 40 & AF920 & HURDMAN & 10 & 2010 \\
\hline $3 \mathrm{SB}$ & 40 & AF920 & HURDMAN & 10 & 2040 \\
\hline $3 \mathrm{SB}$ & 40 & AF920 & HURDMAN & 10 & 2110 \\
\hline $3 \mid \mathrm{SB}$ & 1750 & AF920 & HURDMAN & $1 \mathrm{C}$ & 2140 \\
\hline $3 \mathrm{SB}$ & 30 & AF920 & HURDMAN & 10 & 2210 \\
\hline 3 SB & 30 & AF920 & HURDMAN & 10 & 2240 \\
\hline $3 S B$ & 30 & AF920 & HURDMAN & 10 & 2310 \\
\hline $3 \mathrm{SB}$ & 30 & AF920 & HURDMAN & 10 & 2340 \\
\hline 3/NB & 30 & AF920 & HURDMAN & $1 \mathrm{C}$ & 714 \\
\hline 3) $\mathrm{NB}$ & 30 & AF920 & HURDMAN & 10 & 744 \\
\hline $3 / \mathrm{NB}$ & 30 & AF920 & HURDMAN & $1 \mathrm{C}$ & 814 \\
\hline $3 N B$ & 30 & AF920 & HURDMAN & 10 & 844 \\
\hline $3 \mathrm{NB}$ & 30 & AF920 & HURDMAN & 10 & 917 \\
\hline $3 / \mathrm{NB}$ & 30 & AF920 & HURDMAN & 10 & 947 \\
\hline $3 / N B$ & & AF920 & HURDMAN & 10 & 1018 \\
\hline $3 \mathrm{NB}$ & & AF920 & HURDMAN & $1 c$ & 1048 \\
\hline $3 / \mathrm{NB}$ & & AF920 & HURDMAN & 10 & 1123 \\
\hline $3 / \mathrm{NB}$ & 30 & AF920 & HURDMAN & 10 & 1153 \\
\hline
\end{tabular}




\begin{tabular}{|c|c|c|c|c|}
\hline 3|NB & $302 / A F 920$ & |HURDMAN & 10 & 1223 \\
\hline $3 / \mathrm{NB}$ & 303 AF920 & HURDMAN & $1 \mathrm{C}$ & 1253 \\
\hline $3 / \mathrm{NB}$ & 304 AF920 & HUROMAN & $1 c$ & 1323 \\
\hline $3 / N B$ & 301 AF920 & HURDMAN & $1 \mathrm{C}$ & 1353 \\
\hline $3 / \mathrm{NB}$ & 305 AF920 & HURDMAN & 10 & 1425 \\
\hline $3 N B$ & 302 AF920 & HURDMAN & 10 & 1455 \\
\hline 3 NB & 303 AF920 & HURDMAN & 10 & 1525 \\
\hline $3 / N B$ & 304 AF920 & HURDMAN & 10 & 1555 \\
\hline $3 / N B$ & 301 AF920 & HURDMAN & 10 & 1625 \\
\hline $3 \mathrm{NB}$ & 305 AF920 & HURDMAN & 10 & 1655 \\
\hline $3 / N B$ & 302 AF920 & HURDMAN & 10 & 1725 \\
\hline $3 \mathrm{NB}$ & 303 AF920 & HURDMAN & 10 & 1755 \\
\hline $3 / N B$ & 304 AF920 & HURDMAN & $1 \mathrm{C}$ & 1825 \\
\hline $3 \mathrm{NB}$ & 301 AF920 & HURDMAN & 10 & 1855 \\
\hline $3 / \mathrm{NB}$ & 305 AF920 & HURDMAN & 10 & 1919 \\
\hline $3 / \mathrm{NB}$ & 302 AF920 & HURDMAN & $1 \mathrm{C}$ & 1949 \\
\hline $3 / \mathrm{NB}$ & 303 AF920 & HURDMAN & 10 & 2009 \\
\hline $3 \widehat{N B}$ & 304 AF920 & HURDMAN & $1 \mathrm{C}$ & 2039 \\
\hline 3 NB & 301 AF920 & HURDMAN & 10 & 2109 \\
\hline $3 / N B$ & 305 AF920 & HURDMAN & 10 & 2139 \\
\hline 3/NB & 404/AF920 & HURDMAN & $1 \mathrm{C}$ & 2205 \\
\hline $3 / \mathrm{NB}$ & 403 AF920 & HURDMAN & 10 & 2235 \\
\hline $3 / N B$ & 401 AF920 & HURDMAN & 10 & 2305 \\
\hline $3 / N B$ & 303/AF920 & HURDMAN & $1 \mathrm{C}$ & 005 \\
\hline $3 \mathrm{SB}$ & $301 \mid$ AA170 & CRICHTON & BEECHWOOD NS & 650 \\
\hline $3 S B$ & 302/AA170 & CRICHTON & BEECHWOOD NS & 750 \\
\hline $3 / \mathrm{SB}$ & $303 / \mathrm{AA170}$ & CRICHTON & BEECHWOOD NS & 820 \\
\hline $3 \mathrm{SB}$ & $304 / A A 170$ & CRICHTON & BEECHWOOD NS & 850 \\
\hline $3 / \mathrm{SB}$ & 301 AA170 & CRICHTON & BEECHWOOD NS & 920 \\
\hline $3 \mathrm{SB}$ & 305 AA170 & CRICHTON & BEECHWOOD NS & 950 \\
\hline $3 / \mathrm{SB}$ & $302 / A A 170$ & CRICHTON & BEECHWOOD NS & 1020 \\
\hline $3 \mathrm{SB}$ & 303/AA170 & CRICHTON & BEECHWOOD NS & 1050 \\
\hline $3 / S B$ & 304/AA170 & CRICHTON & BEECHWOOD NS & 1122 \\
\hline $3 S B$ & 301 AA170 & CRICHTON & BEECHWOOD NS & 1152 \\
\hline $3 S B$ & 305 AA170 & CRICHTON & BEECHWOOD NS & 1222 \\
\hline $3 \mathrm{SB}$ & 302 AA 170 & CRICHTON & BEECHWOOD NS & 1252 \\
\hline $3 / S B$ & 303 AA170 & CRICHTON & BEECHWOOD NS & 1322 \\
\hline $3 \mathrm{SB}$ & 304 AA170 & CRICHTON & BEECHWOOD NS & 1352 \\
\hline $3 / S B$ & 301/AA170 & CRICHTON & BEECHWOOD NS & 1422 \\
\hline $3 / \mathrm{SB}$ & 305 AA170 & CRICHTON & BEECHWOOD NS & 1452 \\
\hline $3 / \mathrm{SB}$ & $302 / A A 170$ & CRICHTON & BEECHWOOD NS & 1522 \\
\hline $3 / 58$ & 303 AA170 & CRICHTON & BEECHWOOD NS & 1552 \\
\hline $3 \mathrm{SB}$ & 304 AA170 & CRICHTON & BEECHWOOD NS & 1622 \\
\hline $3 / S B$ & $301 / A A 170$ & CRICHTON & BEECHWOOD NS & 1652 \\
\hline $3 S B$ & 305 AA170 & CRICHTON & BEECHWOOD NS & 1722 \\
\hline $3 / \mathrm{SB}$ & 302 AA170 & CRICHTON & BEECHWOOD NS & 1752 \\
\hline $3 \mathrm{SB}$ & 303 AA170 & CRICHTON & BEECHWOOD NS & 1821 \\
\hline $3 S B$ & 304 AA170 & CRICHTON & BEECHWOOD NS & 1851 \\
\hline $3 \mid S B$ & 301 AA170 & CRICHTON & BEECHWOOD NS & 1919 \\
\hline
\end{tabular}


Table H.3: Sunday Schedule Sample

\begin{tabular}{|c|c|c|c|c|}
\hline ROUTE NOIDIR & RUN_NOBUS_STOP & ON_STREET & AT_STREET & TIME \\
\hline $3 \mid \mathrm{SB}$ & $308 /$ AF920 & HURDMAN & 10 & 823 \\
\hline $3 S B$ & 302 AF920 & HURDMAN & 10 & 853 \\
\hline $3 / S B$ & 307|AF920 & HURDMAN & 10 & 923 \\
\hline $3 S B$ & $304 \mid$ AF920 & HURDMAN & 10 & 953 \\
\hline $3 / \mathrm{SB}$ & 305 AF920 & HURDMAN & $1 \mathrm{C}$ & 1023 \\
\hline $3 \mathrm{SB}$ & 306 AF920 & HURDMAN & 10 & 1053 \\
\hline $3 \mid S B$ & 301 AF920 & HURDMAN & 10 & 1123 \\
\hline $3 S B$ & 303 AF920 & HURDMAN & 10 & 1153 \\
\hline $3 / S B$ & 308 AF920 & HURDMAN & 10 & 1223 \\
\hline $3 \mid S B$ & 302 AF920 & HURDMAN & 10 & 1253 \\
\hline 358 & 307 AF920 & HURDMAN & 10 & 1323 \\
\hline $3 / S B$ & 304|AF920 & HURDMAN & 10 & 1353 \\
\hline $3 \mathrm{SB}$ & 305|AF920 & HURDMAN & 10 & 1423 \\
\hline $3 \mathrm{SB}$ & 306/AF920 & HURDMAN & $1 \mathrm{C}$ & 1453 \\
\hline $3 \mathrm{SB}$ & 301/AF920 & HURDMAN & 10 & 1523 \\
\hline $3 / 58$ & 303/AF920 & HURDMAN & 10 & 1553 \\
\hline $3 / S B$ & 308 AF920 & HURDMAN & 10 & 1623 \\
\hline $3 / S B$ & 302 AF920 & HURDMAN & 10 & 1653 \\
\hline $3 \mathrm{SB}$ & 307 AF920 & HURDMAN & 10 & 1723 \\
\hline 3) $S B$ & 304 AF920 & HURDMAN & 10 & 1753 \\
\hline $3 \mathrm{SB}$ & 305 AF920 & HURDMAN & 10 & 1823 \\
\hline $3 S B$ & $306 \mid$ AF920 & HURDMAN & 10 & 1853 \\
\hline $3 S B$ & 301 AF920 & HURDMAN & 10 & 1923 \\
\hline 3 SB & 303 AF920 & HURDMAN & 10 & 1953 \\
\hline $3 \mathrm{SB}$ & 308 AF920 & HURDMAN & 10 & 2023 \\
\hline $3 S B$ & 307 AF920 & HURDMAN & 10 & 2123 \\
\hline $3 / S B$ & 305 AF920 & HURDMAN & 10 & 2223 \\
\hline $3 / N B$ & 302 AF920 & HURDMAN & 10 & 841 \\
\hline $3 / \mathrm{NB}$ & 303/AF920 & HURDMAN & 10 & 952 \\
\hline $3 / \mathrm{NB}$ & 308 AF920 & HURDMAN & $1 \mathrm{C}$ & 1022 \\
\hline $3 / \mathrm{NB}$ & 302 AF920 & HURDMAN & 10 & 1052 \\
\hline $3 / \mathrm{NB}$ & 307 AF920 & HURDMAN & 10 & 1122 \\
\hline $3 / \mathrm{NB}$ & 304 AF920 & HURDMAN & 10 & 1152 \\
\hline $3 / \mathrm{NB}$ & 305 AF920 & HURDMAN & 10 & 1222 \\
\hline $3 / \mathrm{NB}$ & 306 AF920 & HURDMAN & 10 & 1255 \\
\hline $3 / \mathrm{NB}$ & 301 AF920 & HURDMAN & 10 & 1325 \\
\hline $3 / \mathrm{NB}$ & 303/AF920 & HURDMAN & 10 & 1355 \\
\hline $3 / \mathrm{NB}$ & 308/AF920 & HURDMAN & 10 & 1425 \\
\hline $3 / \mathrm{NB}$ & 302 AF920 & HURDMAN & 16 & 1455 \\
\hline 3 NB & 307 AF920 & HURDMAN & 10 & 1525 \\
\hline $3 / \mathrm{NB}$ & 304.AF920 & HURDMAN & 10 & 1555 \\
\hline $3 / N B$ & 305 AF920 & HURDMAN & 10 & 1625 \\
\hline $3 / N B$ & $306 /$ AF920 & HURDMAN & 10 & 1655 \\
\hline $3 / N B$ & 301 AF920 & HURDMAN & 10 & 1725 \\
\hline
\end{tabular}




\begin{tabular}{|c|c|c|c|c|}
\hline $3 \mid \mathrm{NB}$ & $303 \mid A F 920$ & HURDMAN & 10 & 1755 \\
\hline 3) NB & 308 AF920 & HURDMAN & 10 & 1825 \\
\hline $3 / N B$ & 302 AF920 & HURDMAN & 10 & 1853 \\
\hline $3 / \mathrm{NB}$ & 307 AF920 & HURDMAN & 10 & 1923 \\
\hline $3 N B$ & 304 AF920 & HURDMAN & 10 & 1953 \\
\hline $3 / N B$ & 305 AF920 & HURDMAN & 10 & 2023 \\
\hline $3 / \mathrm{NB}$ & 306/AF920 & HURDMAN & 10 & 2053 \\
\hline $3 / N B$ & 301 AF920 & HURDMAN & 10 & 2123 \\
\hline $3 \mathrm{NB}$ & 303 AF920 & HURDMAN & 10 & 2153 \\
\hline $3 / \mathrm{NB}$ & 308 AF920 & HURDMAN & 10 & 2223 \\
\hline 3 NB & 307 AF920 & HURDMAN & 10 & 2323 \\
\hline $86 \mid E B$ & $8601 \mid A F 920$ & HURDMAN & 10 & 733 \\
\hline $86 \mid E B$ & 8603 AF920 & HURDMAN & 10 & 833 \\
\hline $86 E B$ & 8602 AF920 & HURDMAN & 10 & 903 \\
\hline $86 \mid \mathrm{EB}$ & 8605 AF920 & HURDMAN & 10 & 933 \\
\hline 86 EB & 8601 AF920 & HURDMAN & 10 & 1003 \\
\hline $86 \mid E B$ & 8604/AF920 & HURDMAN & $1 C$ & 1033 \\
\hline 86 $E B$ & 8603 AF920 & HURDMAN & $1 \mathrm{C}$ & 1105 \\
\hline 86 EB & 8602 AF920 & HURDMAN & 10 & 1135 \\
\hline $86 \mid E B$ & 8605 AF920 & HURDMAN & 10 & 1205 \\
\hline 86/EB & 8601 AF920 & HURDMAN & 10 & 1235 \\
\hline 86 EB & 8604 AF920 & HURDMAN & 10 & 1305 \\
\hline 86) EB & 8603 AF920 & HURDMAN & 10 & 1335 \\
\hline $86 \mid \mathrm{EB}$ & 8602 AF920 & HURDMAN & $1 \mathrm{C}$ & 1405 \\
\hline 86)EB & $8605 \mid A F 920$ & HURDMAN & 10 & 1435 \\
\hline 86)EB & 8601 AF920 & HURDMAN & 10 & 1505 \\
\hline 86 EB & 8604 AF920 & HURDMAN & 10 & 1535 \\
\hline 86/EB & 8603 AF920 & HURDMAN & 10 & 1605 \\
\hline $86 \mid E B$ & 8602/AF920 & HURDMAN & 10 & 1635 \\
\hline 86 EB & 8605 AF920 & HURDMAN & $1 \mathrm{C}$ & 1705 \\
\hline $86 \mid E B$ & 8601 AF920 & HURDMAN & 10 & 1735 \\
\hline 86 EB & 8604 AF920 & HURDMAN & 10 & 1805 \\
\hline 86|EB & 8603 AF920 & HURDMAN & 10 & 1835 \\
\hline 86 EB & 8602 AF920 & HURDMAN & $1 \mathrm{C}$ & 1905 \\
\hline 86|EB & 8605 AF920 & HURDMAN & $1 C$ & 1935 \\
\hline 86 EB & 8601 AF920 & HURDMAN & $1 \mathrm{C}$ & 2002 \\
\hline $86 \mid E B$ & $8604 \mid$ AF920 & HURDMAN & 10 & 2032 \\
\hline 86/EB & 8603 AF920 & HURDMAN & 10 & 2102 \\
\hline 86EB & 8602 AF920 & HURDMAN & 10 & 2132 \\
\hline 86 EB & 8605 AF920 & HURDMAN & 10 & 2202 \\
\hline 86 EB & 8601 AF920 & HURDMAN & 10 & 2232 \\
\hline 86EB & 1804 AF920 & HURDMAN & 10 & 2332 \\
\hline 86 WB & 8602 AF920 & HURDMAN & 10 & 712 \\
\hline 86/WB & 8601 AF920 & HURDMAN & 10 & 812 \\
\hline 86 WW & 8603/AF920 & HURDMAN & 10 & 912 \\
\hline $86 / W B$ & 8602 AF920 & HURDMAN & 10 & 942 \\
\hline 86/WB & 8605 AF920 & HURDMAN & 10 & 1012 \\
\hline
\end{tabular}

